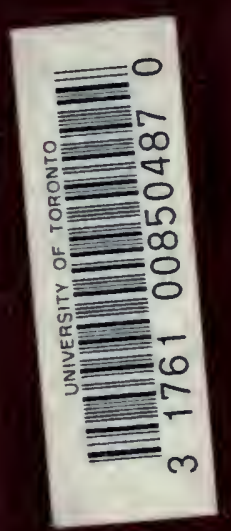



Digitized by the Internet Archive in 2008 with funding from Microsoft Corporation 
. 


THE "PRINCIPLES

OF

\section{B A C TE R I L L G Y:}

A PRACTICAL IIANUAL FOR STUDENTS

AND PHYSICIANS.

BY

A. C. 'АВBOT'T', M.D.,

PROFESSOR OF HYGIENE, AND DIRECTOR OF THE LABORATORY OF HYGIENE, UNIVERSITY OF PENSSYLVANIA.

FOURTH EDITION, ENLARGED AND THOROUGHLY REVISED.

With 106 Illustrations, of which 19 are colored.

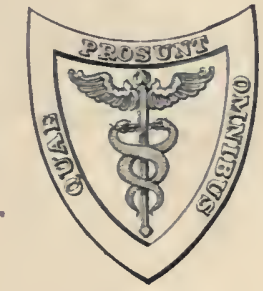

LEA BROTHERS \& CO., PHILADELPHIA AND NEW YORK. 1897 . MEDICAL LIBRARY NO, 33. 


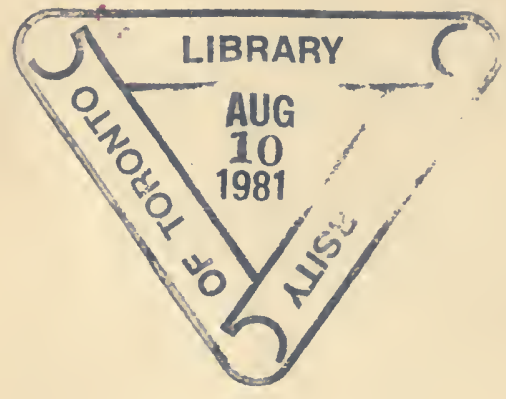

Entered according to the Act of Congress in the year 1897, by LEA BROTHERS \& CO,

In the Office of the Tibrarian of Congress. All rights reserved.

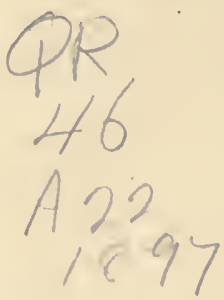

PHILADEI.PHIA:

1) O I NAN, I' II IT TI. 


\section{PREFACE TO THE FOUR'TH EDITION.}

IT becomes again the pleasant duty of the author to express his gratification at the farorable recognition that this book continues to receive, and to acknowledge his indebtedness to those of his readers who have kindly criticized its shortcomings and offered suggestions for its betterment.

In most cases such suggestions have been acted upon; in certain others they have been of such a mature that their adoption, while perhaps desirable, would have increased the size of the book too greatly for its purpose.

In this edition an effort has been made to include the more important of the newer ideas bearing directly upon the subjects under treatment, and, when deemed necessnry, opinions expressed in former editions have been made to conform to later views. In addition to the topics treated in the last edition there have been introduced illustrated descriptions of the bacillus of bubonic plague, of the bacillus of influenza, and of the micrococcus of gonorrhœa, as well as a number of new illustrations relating to descriptive passages in the text.

Philadelphia, May, 189i.

A. C. A. 



\section{PREFACE TO THE SECOND EDITION.}

THE cordial reception with which this book has met, and the demand for a second edition, afford the author no small degree of gratification. In revising The Principles of Bacteriology advantage has been taken of the valuable suggestions kindly offered by the reviewers of the first edition, for which the writer here acknowledges his indebtedness.

The section of the work devoted to deseriptive bacteriology has been somewhat extended, but no effort has been made to cover the entire field, only those species being introduced that are comparatively common or of importance in enabling the student to acquire a fundamental working knowledge capable of wider application. Wherever practicable, these descriptions liave been supplemented by illustrations, for the majority of which the author is responsible. The introduction of colored figures in the text is a new feature in this edition, and one which should increase its usefulness. A sketch of the evolution of our knowledge upon immunity and infection has been introduced, and an outline of apparatus necessary for a beginner's laboratory has bcen appended. 
vi PREFACE TO THE SECOND EDITION.

The original purpose of this book has been maintained, and it is hoped that the second edition, containing double the letterpress and treble the number of illustrations found in its predecessor, will in some corresponding measure improve upon the service which the work has apparently rendered to students and physicians.

A. C. A.

Philadelphia, July, 1894. 


\section{PREFACE TO THE FIRST EDITION.}

Is preparing this book the anthor has kept in mind the needs of the student and practitioner of medicine, for whom the importance of an acquaintance with practical bacteriology cannot be overestimated.

It is to advances made through bacteriological research that we are indebted for much of our knowledge of the conditions underlying infection, and for the elucidation of many hitherto obscure problems concerning the etiology, the modes of transmission, and the means of prevention of infections maladies.

Only within a comparatively short time have students and physicians been enabled to obtain the systematic instruction in this science that is of value in aiding them in their efforts to check disease. The rapid increase in the number who are availing themselves of these opportunities speaks directly for the practical value of the science.

As the majority of those undertaking the study of bacteriology do so with the view of utilizing it in medical practice, and as many of these can devote to it but a portion of their time, it is desirable that the subjectmatter be presented in as direct a manner as possible. 
Presuming the reader to be unfamiliar with the subject, the author has restricted himself to those fundamental features that are essential to its understanding. The object has been to present the important ideas and methods as concisely as is compatible with clearness, and at the same time to accentuate throughout the underlying prineiples which govern the work.

With the view of inducing independent thought on the part of the student, and of diminishing the frequency of that oft-heard query, "What shall I do next?" experiments have been suggested wherever it is possible. These have been arranged to illustrate the salient points of the work and to attract attention to the minute details, upon the observation of which so much in bacteriology depends.

A. C. A.

Philadelpuia, December, 1891. 


\section{CONTEN'TS.}

\section{I TRODUCTION.}

The overthrow of the doctrine of spontsneous generation- " Omne virum ex viro"-Earlier bacterinlogical studies-The birth of modern bacteriology

PAGE

\section{CHA P T E I.}

Definition of bacteria-Their place in nature-Difference between parasites and saprophytes-Nutrition of bacteria-Products of bacteria-Their relation to oxygen-Infuence of temperature upoil their growth .

CHA P T E R I I

Morphology of bacteria-Grouping-Mode of multiplicationSpore-formation-Motility .

\section{CHA PTER III.}

Principles of sterilization by heat-Methods employed-Discontinued sterilization-Sterilization under pressure-A pparatus employed-Chemical disinfection and sterilization

\section{C'H A P T E R I V.}

Principles involved in the methods of isolation of bacteria in pure culture by the plate method of Koch-Materials employed

CHA P T E R V.

Preparation of nutrient media-Bonillon, gelatin, agar-agar, potato, blood-serum, etc. . 


\section{CHAPTER VI.}

PAGE

Preparation of the tubes, flasks, etc., in which the media are to be preserved .

$109-112$

\section{CHA PTER VII.}

Technique of making plates-Esmareh tubes, Petri plates, etc. . 113-124

\section{CHAPTER VIII.}

The incubating-oven-Gas-pressure regulator-Thermo-regulatorSafety burner employed in heating the incubator.

\section{CHA P'TER IX.}

The study of colonies-Their naked-eye peculiarities and their appearance under different conditions-Differences in the structure of eolonies of different species of bacteria-Stab-cultures-Slantcultures .

\section{CH A PTER X.}

Methods of staining-Solutions employed-Preparation and staining of cover-slips-Preparation of tissues for section-cutling-Staining of tissues-Special staining-methods

\section{CHA PTER XI.}

Systemalic study of an organism-Polnts to be considered in identlfying an organism as a definite specles .

\section{CHAPपE R XII.}

Inoculation of animals-Subcutaneous Inoculation; intravenous injection-Inoculation into the great serous cavities, and into the an. terior chamber of the eye-Observation of animals aner inoculation

\section{CHA P'TER XIII.}

Post-mortem examination of animals-Bacteriological examinntion of the tissues-Disposal of tissues and disinfection of instruments aner the examluntion 


\section{APPLICATION OF THE METHODS OF BACTERIOLOGY. DESCRIPTIONS OF SOME OF THE MORE IM- PORTANT SPECIES.}

\section{II A P TER XIV.}

To obtain material with which to begin work.

\section{H A P T E X V.}

Various experiments in sterilization by steam and by hot air

\section{CHA P T E X XI.}

Suppuration-Staphylccoccus pyogenes aurcus-Staphylococcus pyogenes albus and citreus-Streplococcus pyogenes - Gonococcus-Bacillus pyocyaneus-Bacillus of Bubonic Plague.

$244-276$

\section{CH A P ER X VII.}

Sputum septicæmia-Septicæmia resulting from the presence of micrococcus tedragenus in the tissues.

\section{CH A P T R X V I I I.}

Tuberculosis-Microscopic appearance of miliars tubercles-Encapsulation of tuberculous foci-Diffuse caseation-Cavity-formation-Primary infection-Modes of infection-Location of the bacilli in the tissues-Staining-pecnliarities-Organisms with which bacillus luberculosis may be confounded-Points of differentiation-Bacillus of influenzs.

\section{CHA P T E R X X.}

Glanders-Characteristics of the disease-Histological structure of the glanders nodule-Susceptibility of different animals to glanders -The bacillus of glanders; its morphological and cultural peculiarities-Disgnosis of glanders

\section{CH A P T R X X.}

Bacillus diphtherix-Its isolation and cultivation-Morphological and cnltaral peculiarities-Pathogenic properties-Variations in virulence

\section{CHAPTER X XI.}

Typhoid fever-Stady of the organism concerned in its production-Bacterium coli commune-Its resemblance to the bscillus of typhoid ferer-Its morphological, cultural, and pathogenic properties-Its differentiation from bacillus typhi abdominalis . . . 342-36-4 


\section{CHAPTER XXII.}

The spirillum (comma bacillus) of Asiatic cholera-Its morphological and cultural peculiarities-Pathogenic properties-The bacteriological diagnosis of Asiatic cholera

\section{CHA PTER XXIII.}

Organisms of interest, historically and otherwise, that have been confounded with the spirillum of Asiatic cholera-Their peculiarities and differential features-Vibrio proleus, or bacllus of Finkler and Prior-Spirillum tyrogenum, or cheese spirillum of Deneke-The spirillum of Miller-Vibrio Meichnikovi

\section{CHA PTER X XIV.}

Study of bacillus anthracis, and the effects produced by its inoculation into animals-Peculiarities of the organlsm under varying conditions of surroundings

\section{CH A PTER X X V.}

The most important of the organisms found in the soil-The nitrifying bacteria-The bacillus of tetanus-The bacillus of malignant œdema-The bacillus of symptomatic anthrax . . . . .

\section{CHA P T E X X V L}

Infection and immunity-The types of infection; intimate naturc of infection-Septicæmia, toxæmia, variations in infectious processes-Immunity, natural and acquired-The hypotheses that have been advanced in explanation of Immunity-Conclusions . . .

\section{CHAPTER XXVII.}

Bacteriological study of water-Methods employed-Precautions to be observed-Apparatus used, and methods of using themMethods of investigating air and soil

\section{CHA PTER XXVIII.}

Methods of testing disinfectants and antiscptics-Experiments lllustrating the precautions to be taken-Experiments in skin-disinfection

\section{A P P N D I X.}

A plaratus necessary in a beginuer's bacteriological laboratory 527-532 


\title{
B ACTERIOLOGY.
}

\author{
INTRODUCTION.
}

"Omne vivun ex vivo"-The overthrow of the doctrine of spontaneous generation-Earlier bacteriological studies-The birth of modern bacterlology.

THE study of Bacteriology may be said to have had its beginning with the observations of Antony van Leeuwenhoek in the year 1675 . Though it is during the past decadc and a half that this line of research has received its greatest impulse, yet, by a review of the developmental stages through which it has passed in its life of more than two centuries, we see that it has a most interesting and instructive history. From the very outset its history is inseparably connected with that of medicine, and as it now stands its relations to hygiene and preventive medicine are of fundamental importance. It is, indeer, through a more intimate aequaintauce with the biological activities of the unicellular vegetable micro-organisms that modern hygiene has attained the prominence and importance now justly accorded to it. Through studies in the domain of bacteriology our knowledge of the causation, course, and prevention of infectious diseases is daily becoming more accurate, and it is needless to emphasize the relation of such knowledge to the manifold problems that present themselves to the student of preventive medicine. 
Though the contributions which have done most to place bacteriology on the footing of a science are those of rccent years, still, during the earlier stages of its development, many observations were made which formed the foundation-work for much that was to follow. Before regularly beginning our studies, therefore, it may be of advantage to acquaint ourselves with the more prominent of these investigations.

Antony van Leeuwenhoek, the first to describe the bodies now recognized as bacteria, was born at Delft, in Holland, in 1632. He was not considered a man of liberal education, having been during his early years an apprentice to a linendraper. During his apprenticeship he learned the art of lens-grinding, in which he became so proficient that he eventually perfected a simple lens by means of which he was enabled to see objects of much smaller dimensions than any hitherto seen with the best compound mieroscopes in existence at that date. At the time of his discoveries he was following the trade of linendraper in Amsterdam.

In 1675 he published the fact that he had succeded in perfecting a lens by means of which he could detect in a drop of rain-water living, motile " animalenles" of the most minute dimensions-smaller than anything that had hitherto been seen. Encouraged by this discovery, he continued to examine various substances for the presence of what he considered animal life in its most minute form. He found in sea-water, in wellwater, in the intestinal canal of frogs and birds, and in his own diarrhoeal evacuations, objects that differentiated themselves the one from the other, not only by their shape and size, but also by the peculiarity of movement which some of them were seen to possess. 
In the year 1683 he discovered in the tartar scraped from between the teeth a form of micro-organism upon which he laid special stress. This observation he embodied in the form of a contribution which was presented to the Royal Society of London on September 14, 1683. This paper is of particular importance, not only because of the careful, objective nature of the description given of the bodies seen by him, but also for the illustrations which accompany it. From a perusal of the text and an inspection of the plates there remains little room for doubt that Leeuwenhoek saw with his primitive lens the bodies now recognized as baeteria. ${ }^{1}$

Upon seeing these bodies he was apparently very much impressed, for he writes: "With the greatest astonishment I observed that everywhere throughout the material which I was examining were distributed animalcules of the most microseopic dimensions, which mored themselves about in a remarkably energetic way."

This discovery was shortly followed by others of an equally important nature. His field of observation appears to have increased rapidly, for after a time he speaks of bodies of much smaller dimensions than those at first described by him.

Throughout all of Leenwenhoek's work there is a conspicuous absence of the speculative. His contributions are remarkable for their purely objective nature.

After the presence of these organisms in water, in the mouth, and in the intestinal evacuations was made known to the world, it is hardly surprising that they were immediately seized upon as the explanation of the

1 See Arcana Naturæ detecta ab ANToNio VAN LEEUwExhorK; Delphis Batarorum, 1695. 
origin of many obscure diseases. So universal became the belief in a causal relation between these "animalcules" and disease that it amounted almost to a germmania. It became the fashion to suspect the presence of these organisms in all forms and kinds of disease, simply because they had been demonstrated in the mouth, intestinal evacuations, and water.

Though nothing of value at the time had boen done in the way of classification, and still less in separating and identifying the members of this large group, still, the foremost men of the day did not hesitate to ascribe to them not only the property of produeing pathological conditions, but some even went so far as to hold that variations in the appearance of symptoms of disease were the result of differences in the behavior of the organisms in the tissues.

Mareus Antonius Plenciz, a physieian of Viemua in 1762 , declared himself a firm believer in the work of Leenwenhoek, and based the doctrine which he taught upon the discoveries of the Dutch observer and upon observations of a confirmatory nature which he himself had made. The doctrine of Plenciz assumed a causal relation between the micro-organisms discovered and described by Leeuwenhoek and all infections discases. He claimed that the material of infection could be nothing else than a living substince, and endeavored on these grounds to explain the variations in the period of incubation of the different infectious discases. He likewise believed the living contagium to be capable of multiplication within the body, and spoke of the possibility of its transmission through the air. He claimed a special germ for each disease, holding that just as from a given cereal only one kind of grain ean grow, so by 
the special germ for each disease only that disease can be produced.

He found in all decomposing matters innumerable minute "aninalculæ," and was so firmly convinced of their etiological relation to the process that he formulated the law : that decomposition can only take place when the decomposable material becomes coated with a layer of the organisms, and can proceed only when they increase and multiply.

However convincing the arguments of Plenciz may appear, they seem to have been lost sight of in the course of subsequent events, and by a few were even regarded as the productions of an uubalanced mind. For example, as late as 1820 we find Ozanam expressing himself on the subject as follows: "Many authors have written concerning the animal nature of the contagion of infectious diseases; many have indeed assumed it to be developed from animal substances and that it is itself animal and possesses the property of life; I shall not waste time in efforts to refute these absurd hypotheses."

Similar expressions of opinion were heard from many other medical men of the time, all tending in the same direction, all doubting the possibility of these microscopic creatures belonging to the world of living things.

It was not until between the fourth and fifth decades of the present century that by the fortunate coincidence of a number of important discoveries the true relation of the lower organisms to infectious diseases was scientifically pointed out. With the investigations of Pasteur upon the cause of putrefaction in beer and the souring of wine; with the discovery by Pollender and Davaine of the presence of rod-shaped organisms in the blood of 
all animals dead of splenic fever, and with the progress of knowledge upon the parasitic nature of certain diseases of plants, the old question of "contagium animatum" again began to reccive attention. It was taken up by Henle, and it was he who first logically taught this doctrine of infection.

The main point, however, that had occupied the attention of seientific men from time to time for a period of about two hundred years subsequent to Leeuwenhoek's discoveries was the origin of these bodies. Do they generate spontaneously, or are they the descendants of pre-existing creatures of the same kind? was the allimportant question. Among the participants in this discussion were many of the most distinguished men of the day.

In 1749 Needham, who held firmly to the opinion that the bodies which were attracting such general attention developed spontaneously, as the result of vegetative changes in the substances in which they were found, attempted to demonstrate by experiment the grounds upon which he held this view. He maintained that the bacteria which were seen to appear around a grain of barley which was allowed to germinate in a watcherystal of water, which had been earefully covered, were the result of changes in the barley-grain itself incidental to its germination.

Spallanzani, in 1769, drew attention to the laxity of the methods employed by Needham, and demonstrated that if infusions of decomposable vegetable matter were placed in flasks, which were then hermetically sealed, and the flasks and their contents allowed to remain for a time in a vessel of boiling water, neither living organisms could be detected nor would decomposition 
appear in the infusions so treated. The objection raised by Treviranus, viz., that the high temperature to which the infusions had been subjected had so altered them and the air about them that the conditions farorable to spontaneous generation no longer existed, was met by Spallanzani by gently tapping one of the flasks, that had been boiled, against some hard object until a minute crack was produced; invariably organisms and decomposition appeared in the flask thus treated.

From the time of the experiments of Spallanzani until as late as 1836 but little advance was made in the elucidation of this obscure problem.

In 1836 Schulze attracted attention to the subject by the convineing nature of his investigations. He showed that if the air which gained access to boiled infusions was robbed of its living organisms by being caused to pass through strong acid or alkaline solutions no decomposition appeared, and living orgauisms could not be detected in the infusions. Following quickly upon this contribution came Schwann, in 1837, and somewhat later (1854) Schröder and Dusch, with similar results obtained by somewhat different means. Schwann deprived the air which passed to his infusions of its living particles by conducting it through highly heated tubes; whereas Schröder and Dusch, by means of cotton-wool interposed between the boiled infusion and the outside air, robbed the air passing to the infusions of its organisms by the sinple process of filtration. In 1860 Hoffmann and in 1861 Chevreul and Pasteur demonstrated that the preeautions taken by the preceding investigators for rendering the air which entered these flasks free from bacteria were not necessary; that all that was necessary to prevent the access of bacteria to the infu- 
sions in the flasks was to draw out the neck of the flask into a fine tube, bend it down along the side of the flask, and then bend it up again a few centimetres from its extremity, and leave the mouth open. The infusion was then to be boiled in the flask thus prepared and the mouth of the tube left open. The organisms which now fell into the open end of the tube were arrested by the drop of water of condensation which collected at its lowest angle, and none could enter the flask.

Though from our present-day standpoint the results of these investigations seem to be of a most convineing nature, yet there existed at the time many who required additional proof that "spontaneous generation" was not the explanation for the mysterious appearance of these minute living objects. The majority, if not all, of such doubts were subsequently dissipated through the wellknown investigations of Tyndall upon the floating matters of the air. In these studies he demonstrated by experiments that the presence of living organisms in decomposing fluids was always to be explained either by the pre-existence of similar living forms in the infusion or upon the walls of the vessel containing it, or by the infusion having been exposed to air which had not been deprived of its organisms.

'Throughout all the work bearing upon this subject, from the time of Spallanzani to that of 'Tyndall, eertain irregularities were constantly appearing. It was found that particular substances required to be heated for a much longer time than was necessary to render other substances free from living organisms, and even under the most careful precations decomposition would oceasionally appear.

In 1762 Bonnet, who was deeply interested in this 
subject, suggested, in reference to the results obtained by Needham, the possibility of the existence of "germs, or their eggs," which have the power to resist the tem. perature to which some of the infusions employed in Needham's experiments had been subjected.

More than a hundred years after Bonnet had made this purely speculative suggestion it became the happy privilege of Ferdinand Coln, of Breslau, to demonstrate its aecuracy.

Cohn repeated the foregoing experiments with like results. He concluded that the irregularities could only be due to either the existence of more resistant species of bacteria or to more resistant stages into which certain bacteria have the property of passing. After much work he demonstrated that certain of the rod-shaped organisms possess the power of passing into a resting or spore stage in the course of their life-cycle, and when in this stage they are much less susceptible to the deleterious action of high temperatures than when they are growing as normal vegetative forms. With the diseovery of these more resistant spores the doctrine of spontaneous generation received its death-blow. It was no longer difficult to explain the irregularities in the foregoing experiments, nor was it any longer to be doubted that putrefaction and fermentation were the result of bacterial life and not the cause of it, and that these bacteria were the offspring from pre-existing similar forms. In other words, the law of Harvey, Omne vivum ex ovo, or its modification, Omne vivum ex vivo, was shown to apply not only to the more highly organized members of the animal and vegetable kingdoms, but to the most microseopic, unicellular creatures as well.

The establishment of this point served as an impetus $2^{*}$ 
to further investigations, and as the all-important question was that concerning the relation of these microscopic organisms to disease, attention naturally turned into this channel of study. Even before the hypothesis of spontaneous generation had received its final refutation a number of observations of a most important nature had been made by investigators who had long since ceased to consider spontaneous generation as a tenable explanation of the origin of the microscopic living particles.

In the main, these studies had been eondueted npon wounds and the infections to which they are liable; in fact, the evolution of our knowledge of bacteriology to the point it now oceupies is so intimately associated with this particular line of investigation that a few historical facts in connection with it may not be without interest.

The observations of Rindfleiseh, in 1866, in which he deseribes the presence of small, pin-head points in the myocardium and general museulature of individuals that had died as a result of infeeted wounds, offer, probably, the first reliable contribution to this subject. He studied the tissue-changes round about these points up to the stage of miliary abseess formation. He refers to the organisms as "vibrios." Almost simultaneously Von Recklinghausen and Wakleyer described similar ehanges that they had observed in pyæmia and oecasionally sceondary to typhoid fever. Von Recklinghausen believed the grannles seen in the abscess-points to be mierococei and not tissue-detritus, and gave as the reason that they were regular in size and shape, and gave specifie reactions with partieular staining-fluids. Birch-Hirsehfeld was able to trace bacteria found in the blood and organs to the wound as the point of entrance, and believed both the local and constitutional 
condition to stand in direct ratio to the number of spherical bacteria present in the wound. He observed also that as the organisms increased in number they could often be found within the bodies of pus corpuscles. His studies of pyrmia led him to the important conelusion that in this condition micro-organisms were always present in the blood.

Of immense importance to the subject were the investigations of Klebs, made at the Military Hospital at Carlsruhe in 1870-71. He not only saw, as others before him had done, that bacteria were present in diseases following upon the infection of wounds, but described the manner in which the organisms had gained entrance from the point of injury to the internal organs and blood. His opinion was that the spherical and rodshaped bodies that he saw in the secretions of wounds were closely allied, and gave to them the designation "microsporon septicum." His opinion was that the organisms gained access to the tissues round about the point of injury both by the aid of the wandering leucocytes and by being forced through the connective-tissue lymph-spaces by the mechanical pressure of muscular contraction.

On erysipelatous inflammations secondary to injury important investigations were also being made, Wilde, Orth, Von Recklinghausen, Lukomsky, Billroth, Ehrlich, Fehleisen, and others agreeing that in these conditions micro-organisms could always be detected in the lymph-channels of the subeutaneous tissues; and through the work of Oertel, Nassiloff, Classen, Letzerich, Klebs, and Eberth the constant presence of bacteria in the diphtheritic deposits at times seen on open wounds was established. 
Simple and natural as all this may seem to us now, the stage to which the subject had developed when these observations were recorded did not admit of their meeting with unconditional acceptance. The only strong argument in favor of the etiological relation of the organisms that had been seen, to the diseases with which they were associated, was the constancy of this association. No efforts had been made to isolate them, and few or none to reproduce the pathological conditions by inoculation. Moreover, not a small number of investigators were skeptical as to the importance of these observations; many claimed that micro-organisms were normally present in the blood and tissues of the body, and some even believed that the organisms seen in the diseased eonditions were the result rather than the eause of the maladies. It is hardly necessary to do more than say that both of these views were purely speculative, and have never had a single reliable experimental argument in their favor. Billroth and Tiegel, who held to the former opinion, did endeavor to prove their position through experimental means; but the methods employed by them were of such an untrustworthy nature that the fallacy of deductions diawn from them was very quickly demonstrated by subsequent investigators. Their method for demonstrating the presence of microorganisms in normal tissues was to remove bits of organs from the healthy animal body with heated instruments and drop them into hot melted paraffin, holding that all living organisms on the surfice of the tissues would be destroyed by the high temperature, and that if decomposition should subsequently oceur it would prove that it was the result of the growth of bacteria in the depths of the tissue to which the heat had not penetrated. 
Decomposition did usually set in, and they accepted this as proof of the accuracy of their view. Attention was, however, shortly called to the fact that in cooling there was contraction of the paraffin, resulting usually in the production of small rents and cracks in which dust, and bacteria lodged upon it, could accumulate and finally gain access to the tissues, with the occurrence of decomposition as a consequence. Their results were thus explained after a manner analogous to that employed by Spallanzani, in 1769, in demonstrating to Treviranus the fallacy of the opinion held by him and the accuracy of his own views, viz., that it was always through the access of organisms from without that decomposition primarily originates. (See page 19.)

Under the most careful precautions, against which no objection could be raised, the experiments of Billroth and Tiegel were repeated by Pasteur, Burdon-Sanderson, and Klebs, but with failure in each and every instance to demonstrate the presence of bacteria in the healthy living tissues.

The fundamental researches of Koch (1881) upon pathogenic bacteria and their relation to the infectious diseases of animals differed from those of preceding investigators in many important respects. The scieutific methods of analysis with which each and every obscure problem was met as it arose served at once to distinguish the worker as a pioneer in this hitherto but partly cultivated domain. The outcome of these experiments was the establishment of a foundation upon which the bacteriology of the future was to rest. He, for the first time, demonstrated that distinet varieties of infection, as evidenced by anatomical changes, are due in many cases to the activities of specific micro-organisms, and 
that by proper methods it is possible to isolate these organisms in pure culture, to cultivate then indefinitely, to reproduce the eonditions by inoeulation of these pure cultures into susceptible animals, and, by continuous inoculation from an infected to a healthy animal, to continue the disease at will. By the methods that he employed he demonstrated a series of separate and distinct diseases that can be produced in mice and rabbits by the injection into their tissues of putrid substances. The discase known as septicremia of mice; also a disease eharacterized by progressive abscess-formation; and pyæmia and septicæmia of rabbits, are among the affections produced by him in this way. It was in the eourse of this work that the Abbe system of substage condensing apparatus was first used in bacteriology; that the aniline dyes suggested by Weigert were brought into general use; that the isolation and eultivation of bacteria in pure enlture on solid media were shown to be possible; and that animals were employed as a means of obtaining from mixtures pure enltures of pathogenic bacteria.

With the bounteous harvest of original and important suggestions that was reaped from Koch's classical series of investigations bacteriology reached an epoch in its development, and at this period modern bacteriology may justly be said to have had its birth.

Notr. - I have presented only the most prominent investigations that will serve to indicate the lines along which the subjeet has developed. For a more detailed aceount of the listorical development of the work the reader is referred to Lueffler's Vorlesungen über die geschichtliche Entwickelung der Lehre von den Bacterien, upon which I have drawn largely in preparing the foregoing sketeh. 


\section{CHAPTER I.}

Definition of bacteria-Their place in nature-Difference between parasites and saprophytes-Nutrition of bacteria-Products of bacteria-Their relation to oxygen-Influence of temperature upon their growth.

$\mathrm{Br}$ the term bacteria is understool that large group of minute regetable organisms the individual members of which multiply by a process of transverse division. They are spherical, oval, rod-like, and spiral in shape, and arc commonly devoid of chlorophyll. ${ }^{1}$ Owing to the absence of chlorophyll from their composition, the bacteria are forced to obtain their nutritive materials from organic malters as such, and lead, therefore, either a saprophytic ${ }^{2}$ or parasitic ${ }^{3}$ form of existence.

Their life-processes are so rapid, complex, and energetic that they result in the most profound alterations in the structure and composition of the materials in and upon which they are developing.

Decomposition, putrefaction, and fermentation result from the activities of the saprophytic bacteria, while the changes brought about in the tissues of their host by the

1 Chlorophrll is the green coloriug-matter possessed by the higher plants by means of which they are enabled in the presence of sunlight to decompose carbonic acid $\left(\mathrm{CO}_{2}\right)$ and ammonia $\left(\mathrm{NH}_{3}\right)$ into their elementary constituents.

2 A saprophyte is an organism that obtains its nutrition from dead organic matter.

${ }^{3}$ A parasite lives always at the expense of some other living, organic creature, known as its host, and in the strictest sense of the word cannot develop upon dead matter. There is, horever, a group of so-called "facultative" saprophytes and parasites which possess the power of accommodating themselves to existing surroundings-at one time leadiug a parasitic, at another time a saprophytic form of existence. 
pure parasitic forms find expression in disease-processes and not infrequently in complete death.

The rôle played in nature by the saprophytic bacteria is a very important one. Through their functional activities the highly complicated tissues of dead animals and vegetables are resolved into the simpler compounds, carbonic acid, water, and ammonia, in which form they may be taken up and appropriated as nutrition by the more highly organized members of the vegetable kingdom. It is through this ultimate production of carbonic acid, ammonia, and water by the bacteria, as end-products in the processes of decomposition and fermentation of the dead animal and vegetable tissues, that the demands of growing vegetation for these compounds are supplied.

The ellorophyll plants do not possess the power of obtaining their carbon and nitrogen from such highly organized and complicated substances as serve for the nutrition of bacteria, and as the production of these simpler compounds $\left(\mathrm{CO}_{2}, \mathrm{NH}_{3}, \mathrm{H}_{2} \mathrm{O}\right)$ by the animal world is not sufficient to meet the demands of the chlorophyll plants, the importance of the part played by bacteria in making up this deficit cannot be overestimated. Were it not for the activity of these mieroscopie living particles, all life upon the surface of the earth would cease. Deprive higher vegetation of the carbon and nitrogen supplied to it as a result of bacterial activity, and its development comes rapidly to an end; rob the animal kingdom of the food-stuffs supplied to it by the vegetable world, and life is no longer possible.

It is plain, therefore, that the saprophytes, which represent the large majority of all bacteria, must be looked upon by us in the light of benefactors, withont which existence would be impossible. 
With the parasites, on the other hand, the conditions are far from analogous. Through their activities there is constantly a loss, rather than a gain, to both the animal and vegetable kingdoms. Their host nust always be a living body in which exist conditions favorable to their development, and from which they appropriate substances that are necessary to the health and life of the organism to which they have found access; at the same time they eliminate substances as products of their nutrition that are directly poisonous to the tissues in which they are growing.

In their relations to humanity, the positions oceupied by the two biologically different groups, the saprophytes on the one hand and the parasites on the other, are diametrically opposite:-the saprophytic forms stand in the relation of benefactors, in resolving dead animal and vegetable bodies into their component parts, which serve as food for living vegetation, and, at the same time, they remove from the surface of the earth the remains of all dead organic substances; while the parasitic group exists only at the expense of the more highly organized members of both kingdoms. It is to the parasitic group that the pathogenic $c^{1}$ organisms belong.

In addition to the saprophytes that are concerned in the changes to which allusion has just been made, there exist other saprophytic forms whose life-processes result in specific changes of most interesting and important natures. Some of these are characterized by their property of producing pigments of different color; these are known as the chromogenic ${ }^{2}$ forms. Just what their

1 Pathogenic organisms are those which possess the property of producing disease.

2 Chromogenic :-possessing the property of generating color. 
exact rôle in nature is it is difficult to say; but it is probable that, in addition to their most conspicuous function of color-production, they are also in some way concerned in the omnipresent process of disintegration which is constantly going on in all dead organic substances.

Others, the so-called photogenic or phosphorescent baeteria, possess the property of producing light or of illuminating the medium on which they grow by a peculiar phosphorescence. These are found in sea-water and in decomposing phosphoreseent fish and meat.

Still others, the so-called zymogenic bacteria, are concerned in the various fermentations; while the putrefactive or saprogenic bacteria are those that produee the particular fermentation that we know as putrefaction. Another very important saprophytic group eomprises the so-called nitrifying and denitrifying bacteria, whose activities are concerned in specific forms of fermentation-the former oxidizing ammonia to nitrous and nitrie acids, the latter reducing nitric acid to nitrous acid and ammonia. It is through their association (symbiosis) with the nitrifying bacteria that certain plants, the leguminous, are enabled to make up their nitrogen deficit in part from the free nitrogen of the air. The discovery of this phenomenon gave to free atmospherie nitrogen a biological significance that had hitherto been denied it. The so-called thiogenic bacteria convert sulphuretted hydrogren into higher sulphur compounds.

We have said that through the agency of chlorophyll, in the presence of sunlight, the green plants are enabled to obtain the amount of nitrogen and carbon which is necessary to their growth from such simple bodies as carbon dioxide and ammonia, which they decompose into their elementary constituents. 'The baeteria, on 
the other hand, owing to the absence of chlorophyll from their tissue, do not possess this power. They must, therefore, have their carbon and nitrogeu presented as such, in the form of decomposable organic substances.

In general, the bacteria obtain their nitrogen most readily from soluble albumins, and, to a certain extent, but by no means so easily, from salts of ammonium. In some of Nïgeli's experiments it appeared probable that they could obtain the necessary amount of nitrogen from inorganic nitrates. At all events, he was able in certain cases to demonstrate a reduction of nitric to nitrous acid, and ultimately to ammonia. Nevertheless, in all of these experiments circumstances point to the probability that the nitrogen obtained by the bacteria for building up their tissues in the course of their development was derived from some source other than that of the nitric acid or the nitrates, and that the reduction of this acid was most probably a secondary phenomenon. It must be borne in mind, however, that there exists a specific group of bacteria, the nitrifying bacteria, that apparently increase and multiply without appropriating proteid nutrition. They are concerned in the particnlar form of fermentation that results in the oxidation of ammonia to nitrous and nitric acids, a process everywhere in progress in the superficial lavers of the soil.

For the supply of carbon many of the carbon compounds serve as sources upon which the bacteria can draw. The carbon deficit, for example, can be obtained from sugar and bodies of like composition; from glycerine and many of the fatty acids; and from the alkaline salts of tartaric, citric, malic, lactic, and acetic 
acids. In some instances carbon compounds which, when present in concentrated form, inhibit the growth of bacteria, may, when highly diluted, serve as nutrition for them. Salicylic acid and ethyl alcohol are of this class.

In addition to carbon and nitrogen, water is essential to the life and development of bacteria. Without it no development occurs, and in many cases drying the organisms results in their death. Certain forms, on the eontrary, though incapable of multiplying when in the dry stage, may be completely deprived of their water without causing them to lose the power of reproduction when favorable conditions reappear.

The closer study of the bacteria, and a more intimate acquaintanee with their nutritive changes, demonstrate an appreciable variability in the character of the substances best suited for the nutrition of different species, one requiring a tolerably concentrated form of nutrition, while another needs but a very limited amount of proteid substance for its development. Certain members bring about most profound alterations in the media in which they exist, while others produce but little apparent change. In one ease alterations in the reaction of the media will be conspicuous, while in another no such variation ean be detected. With the growth of some forms products resulting from speeific processes of fermentation appear. Other varieties produce poisons of remarkable degrees of toxicity, while the growth of others may be aceompanied by the bodies characteristic of putrefaction.

For the normal development of bacteria it is not only essential that the sourees from which they can obtain the necessary nutritive elements should exist, but ac- 
count must also be taken of the products of growth of the organisms in these substances. Nitrogen and carbon compounds in the proper form to be appropriated by bacteria may exist in sufficient quantities, and still their growth may be checked after a very short time by the accumulation of products of nutrition that are inhibitory to their further development. Most conspicuous are the changes that growing bacteria produce in the chemical reaction of the media. Since the majority of them grow best in media of a neutral or very slightly alkaline reaction, any excessive production of alkalinity or acidity, as a product of growth, arrests development, and no evidence of life or further multiplication can be detected until this deviation from the neutral reaction has been corrected.

Most favorable for the development of bacteria are neutral or very slightly alkaline solutions of proteid materials in one form or another.

Of considerable importance and interest in the study of the nutritive changes of bacteria is the difference in their relation to oxygen. With certain forms oxygen is essential to the proper performance of their functions, while with another group no evidence of life can be detected under the access of oxygen, and in a third group oxygen appears to play but an unimportant rôle, for development occurs as well with as without it. It was Pasteur who first demonstrated the existence of particular species of bacteria which not only grow and multiply and perform definite physiological functions without the aid of oxygen, but to the existence of which oxygen is positively harmful. To these he gave the name anaërobic bacteria, in contradistinction to the aërobic group, for the proper performance of whose 
functions oxygen is essential. In addition to these there is a third group, for the maintenance of whose existence the absence or presence of oxygen is apparently of no moment-development progresses as well with as without it; the members of this group comprise the class known as facultative in their relation to this gas. It is to this third group, the facultative, that the majority of bacteria belong. Though the multiplication of the facultative varieties is not interfered with by either the presence or absence of oxygen, yet experiments demonstrate that the produets of their growth are different under the varying conditions of absence or presence of this gas.

For example: in the ease of certain of the chromogenic forms the presenee or absence of oxygen has a very decided effect upon the production of the pigments by which they are characterized.

Nots.-Observe the difference between the intensity of color produced upon the surface of the medium and that along the track of the needle in stab-cultures of the bacillus prodigiosus and of the spirillum rubrum. With the former the red color is apparently a product dependent upon the presence of oxygen, while in the latter the greatest intensity of color oecurs at the point farthest removed from the action of oxygen.

Another factor which plays a highly important part in the biological functions of these organisms is the temperature under which they exist. The extremes of temperature between which the majority of bacteria are known to grow range from $5.5^{\circ}$ to $43^{\circ} \mathrm{C}$. At the former temperature development is hardly appreciable; it becomes more and more active until $38^{\circ} \mathrm{C}$. is reached, 
when it is at its optimum, and, as a rule, ceases with $43^{\circ} \mathrm{C}$.; though species exist that will multiply at as high a temperature as $70^{\circ} \mathrm{C}$. and others at as low as $0^{\circ} \mathrm{C}$. The studies of Globig, ${ }^{1}$ Miquel, ${ }^{2}$ and Macfadyen and Bloxall $^{3}$ have demonstrated that there exist in the soil, in water, in frees, in sewage, in dust, and, in fact, practically everywhere, bacteria that under artificial cultivation show no evidence of life at a temperature lower than $60^{\circ}$ to $65^{\circ} \mathrm{C}$., and would even grow at as high a temperature as $70^{\circ}$ to $75^{\circ} \mathrm{C}$., degrees of heat sufficient for the coagulation of albumin. Rabinowitsch ${ }^{4}$ has likewise described a number of species of these "thermophilic" bacteria, as they are called, but states that it was possible in her experiments to obtain evidence of their growth at a lower temperature $\left(34^{\circ}\right.$ to $44^{\circ} \mathrm{C}$.), as well as at the higher temperature mentioned ly preceding investigators. The most farorable temperature for the development of pathogenic bacteria is that of the human body, viz., $37.5^{\circ} \mathrm{C}$. There are a number of bacteria commonly present in water, the so-called normal water bacteria, that grow best at about $20^{\circ} \mathrm{C}$.

In general then, from what has been learned, it may be said that for the growth and development of bacteria organic matter of a neutral or slightly alkaline reaction, in the presence of moisture and at a suitable temperature, is necessary. From this can be formed some idea of the omuipresence in nature of these minute regetable forms. Everywhere that these conditions obtain bacteria can be found.

1 Globig: Zeitschrift für Hygiene, Bd. iii. S. 294.

2 Miquel : Annales de Micrographié, 15s8, pp. 4 to 10.

3 Macfadyen and Bloxall: Journal of Path. and Bact., vol. iii. Part $\mathrm{Y}$.

4abinowitsch : Zeitschrift für Hygiene n. Infectionskrankheiten, Bd. xx. Heft 1, S. 154 to 164. 


\section{CHAPTER II.}

Morphologyl of bacteria-Grouping-Mode of multiplication-Spore-formation-Motility.

Is structure the bacteria are unicellular; they are seen to occur as spherical, rod- or spiral-shaped bodies. They always develop from pre-existing cells of the same charaeter and never appear spontaneously.

The classifications of the older authors and of the botanists are usually upon purely morphological peculiarities, and, beeause of slight variations that are seen to occur in the size and shape of one and the same species, are more or less complieated. The present tendency is to simplify this morphological elassification, and to bring the bacteria into three great groups, with their subdivisions, the members of each group being determined by their individual outline, viz., that of a sphere, a rod, or a spiral.

To these three grand divisions are given the names cocci or micrococei, bacilli, and spirilla.

In the group micrococci belong all spherical forms, $i$. e., all those forms the isolated individual members of which are practically of the same diameter in all directions. (See Fig. 1, $a, b, c, d$.)

The bacilli comprise all oval or rod-formed bacteria. (See Fig. 2.)

To the spirilla belong all organisms that are curved

1 Morphology :-pertaining to shape, outline, structure. 
when seen in short segments, or when in longer threads are twisted in the form of a corkscrew. (See Fig. 3.)

Fio. 1.

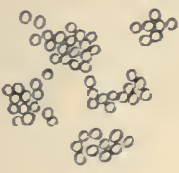

(a)

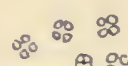

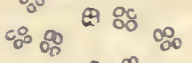
188 요 1000

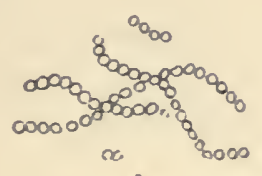

b

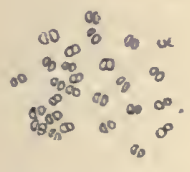

$c$ d

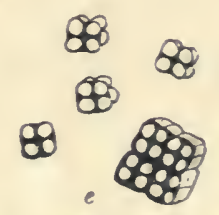

a. Staphylococcl. b. Streptococel. c. Diplococci. d. Tetrads.

FIG. 2.

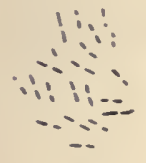

$a$

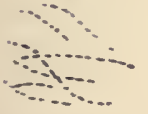

d

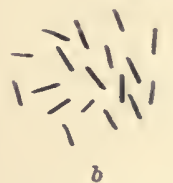

$-\left.\operatorname{li}_{-\infty}\right|^{--}$
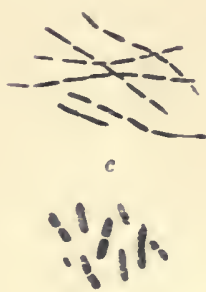

$f$

n. Bacilli in pairs, $b$. Single bacilli. $c$ and $d$. Bacilli in threads. $e$ and $f$. Bacilli of variable morphology.

FIG. 3.

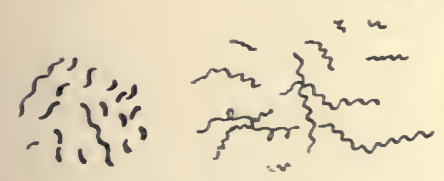

a

b.

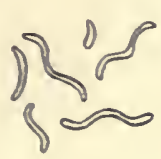

c

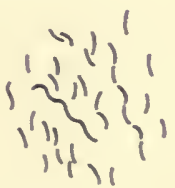

d

$a$ and $d$. Spirilla in short segments and longer threads-the so-called comma forms and spirals. $b$. The forms known as spirocbrta. $c$. The thick spirals sometimes known as vibrios. 
The micrococei are subdivided according to their grouping, as seen in growing cultures, into staphylococci -those growing in masses like clusters of grapes (see Fig. 1, a); streptococci-those growing in chains consisting of a number of individual cells strung together like beads upon a string (see Fig. 1, b); diplococci -those growing in pairs (Fig. 1, c); tetrads-those developing as fours (Fig. 1, d); and sarcina-those dividing into fours, eights, etc., as cubes-that is, in contradistinction to all other forms, the segmentation, which is rarely complete, takes place regularly in three directions of space, so that when growing the bundle of segmenting cells presents somewhat the appearance of a bale of cotton (Fig. 1,e).

To the bacilli belong all straight, rod-shaped bacteria -i.e., those in which one diameter is always greater than the other.

FIG. 4.

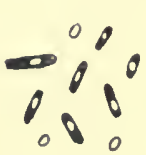

$\boldsymbol{a}$

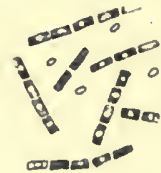

$b$

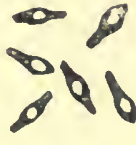

c

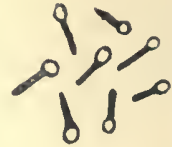

d

a. Baclllus subtlils with spores. b. Bacillus anthracis with spores. c. Clostridium form with spores. d. liacllius of tetanus with end spores.

In this group are found those organisms the life-cycle of many of which presents deviations from the simple rod shape. Many of them in the course of development increase in length into long threarls, along the course of which traces of segmentation may usually be found-the anthrax bacillus and bacillus subtilis are conspicuous examples of this. Again, under certain conditions, many of them possess the property of form- 
ing within the body of the rods oval, glistening spores (see Fig. 4), and, if the conditions are not altered, the rods may entirely disappear, and nothing be left in the culture but these oval spores. In some of them this phenomenon of spore-formation is accompanied by an enlargement or swelling of the bacillus at the point at which the spore is located (see Fig. 4, $c$ and $d$ ). Again, many of them, from unfavorable conditions of nutrition, aëration, or temperature, undergo pathological changes-that is, the individuals themselves experience degeneration of their protoplasm with coincident distortion of their outline; they are then usually referred to as "involution forms" (see Fig. 5, $a$ and b). In

Fig. 5.

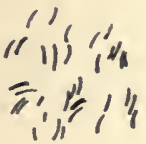

a

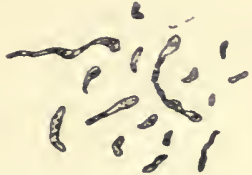

$b$

a. Spirillum of Asiatic cholera (comma bacillus). b. Involution forms of this organism as seen in old cultures.

all of these conditions, however, so long as death has not actually occurred, it is possible to cause these forms to revert to the rod-shaped ones from which they originated, by the renewal of the conditions favorable to their normal vegetation.

It must be borne in mind, though, that it is never possible by any means to bring about changes in these organisms that will result in the permanent conversion of the morphology of the members of one group into that of another-that is, one can never produce bacilli from micrococci, or vice versa, and any evidence which 
may be presented to the contrary is based upon untrustworthy methods of observation.

Not infrequently bacteria may be observed irregularly massed together as a pellicle. When in this condition they are held together by a gelatinous material, and are known as zoogloea of bacteria. (See Fig. 6.)

FIG. 6.

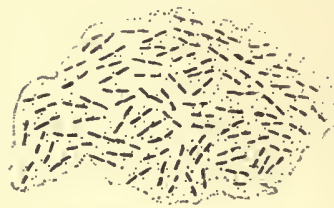

Zoogloea of bacilli.

Very short oval bacilli may sometimes be mistaken for micrococci, and at times micrococci in the stage of segmentation into diplococci may be mistaken for short bacilli; but by careful inspection it will always be possible to detect a continuous outline along the sides of the former, and a slight transverse indentation or partition-formation between the segments of the latter. The high index of refraction of spores, the property which gives to them their glistening appearance, will always serve to distinguish them from micrococci. This difference in refraction is especially noticeable if the illumination from the reflector of the microscope with which they are examined be reduced to the smallest possible bundle of light-rays. The spores, moreover, take up the staining reagents much less readily than do the inicrocoeci. The most reliable differential points, however, are: the infallible property possessed by the spores of developing into bacilli, and that of the spherical organism with which they may have been con- 
founded of always producing other micrococci of the same round form.

For convenience, a common classification of the bacilli is that based upon constant characteristics which are seen to appear in the course of their development under special conditions-certain of them possessing the power of forming spores, while from others this peculiarity is absent.

We have less knowledge of the life-history of the spiral forms. Efforts toward their cultivation under artificial conditions have thus far been successful in only a comparatively limited number of cases. Morphologically, they are thread- or rod-like bodies which are twisted into the form of spirals. In some of them the turns of the spiral are long, in others quite short. They are motile, and multiply apparently by the simple process of fission. ${ }^{1}$ In most respects, save form and the power of producing spores, they are analogons in their mode of growth to the bacilli.

The micrococei develop by simple fissiou. When development is in progress a single cell will be seen to elongate slightly in one of its diameters. Over the centre of the long axis thus formed will appear a slight indentation in the outer envelope of the cell; this indentation will increase in extent until there exist eventually two individuals which are distinctly spherical, as was the parent from which they sprang, or they will remain together for a time as diplococci; the surfaces now in juxtaposition are flattened against one another, and not infrequently a fine, pale dividing-line may be seen between the two cells. (See Fig. 1, $c$ and $d$.) A similar

1 Dividing into two transversely. 
division in the other direction will now result in the formation of a group of forms as tetrads.

In the formation of staphylococei sneh division oceurs irregularly in all directions, resulting in the production of the clusters in which these organisms are commonly seen. (See Fig. 1, a.) With the streptocoeci, however, the tendeney is for the segmentation to continue in one direction only, resulting in the production of long chains of 4,8 , and 12 individuals. (See Fig. 1, b.)

The sarcinæ divide more or less regularly in three directions of space; but instead of becoming separated the one from the other as single cells, the tendency is for the segmentation to be incomplete, the cells remaining together in masses. The indentations upon these masses or eubes, which indicate the point of incomplete fission, give to these bundles of cells the appearance eommonly aseribed to them-that of a bale of cotton or a packet of rags. (See Fig. 1, e.)

The multiplication of bacilli is in the main similar to that given for the micrococei. A dividing cell will elongate slightly in the direetion of its long axis; an indentation will appear about midway between its poles, and will become deeper and deeper until eventually two daughter cells will be formed. This process may occur in such a way that the two young bacilli will adhere together by their adjacent ends in much the same way that sausages are seen to be held together in strings (Fig. $2, f$ ), or the segmentation may take place more at right angles to the long axis, so that the proximal ends of the young cells are flattened, while the distal extremities may be rounded or slightly pointed (Fig. $2, e)$. The segmentation of the anthrax bacillus, with which we are to become acquainted later, results, when 
completed, in an indentation of the adjacent extremities of the young segments, so that by the aid of high magnifying powers these surfaces are seen to be actually concave. Bacilli never divide longitudinally.

With the spore-forming bacilli, under favorable conditions of nutrition and temperature, the same is seen to occur during vegetation; but as soon as these conditions become altered by the exhaustion of nutrition, the presence of detrimental substances, unfarorable temperatures, etc., there appears the stage in their lifecycle to which we have referred as "spore-formation." This is the process by which the organisms are enabled to euter a stage in which they resist deleterious influences to a much higher degree than is possible for them when in the growing or vegetative condition.

In the spore, resting, or permanent stage, as it is called, no evidence of life whatever is given by the spores, though as soon as the conditions which favor their germination have been renewed, these spores develop again iuto the same kind of cells as those from which they originated, and the appearances observed in the vegetative or growing stage of their history are repeated.

Multiplication of spores, as such, does not occur; they possess the power of developing into individual rods of the same nature as those from which they were formed, but not of giving rise to a direct reproduction of spores.

When the conditions which faror spore-formation present, the protoplasm of the regetative cells is seen to undergo a change. It loses its normal homogeneous appearance and becomes marked by granular, refractive points of irregular shape and size. These eventually coalesce, leaving the remainder of the cell clear and 
transparent. When this coalescence of highly refractive particles is complete the spore is perfected. In appearance the spore is oval or round, and very highly refractive-glistening. It is easily differentiated from the remainder of the cell, which now consists only of a cell-membrane and a transparent, clear fluid which surrounds the spore. Eventually both the cellmembrane and its fluid contents disappear, leaving the oval spore free; it then gives the impression of being surrounded by a dark, sharply defined border. It evinces no motion other than the mechanical tremor common to all insoluble microseopie particles suspended in fluids, and it remains quiescent until there appear conditions favorable to its subsequent development into a vegetative form similar to that from which it originated. Occasionally the membrane of the vegetative cell in which the spore is formed does not disappear from around it, and the spore may then be seen lying in a very delicate tubular envelope. Now and then, remnants of the envelope may be noticed adhering to a spore which has not yet beeome completely free.

In staining, the spore-containing cells do not take up the dyes in a bomogeneous way. By the ordinary methods the spores do not stain, so that they appear in the stained cells as pale, transparent, oval bodies, surrounded by the remainder of the eell, which has taken up the staining.

A single cell produces but one spore. This may be located either at an extremity or in the eentre of the eell. (Fig. 4.)

Occasionally spore-formation is accompanied by an enlargement of the cell at the point at which the proeess is in progress. As a result, the outline of the cell 
loses its regular rod shape and becomes that of a club, a drum-stick, or a lozenge, depending upon whether the location of the spore is to be at the pole or in the centre of the cell. (See Fig. 4, $e$ and $d$.)

In addition to the property of spore-formation there is another striking difference between the rod-shaped organisms, namely, the property of motility which many of them are seen to possess. This power of motion is due to the possession by the motile bacilli of very

Fra. 7.

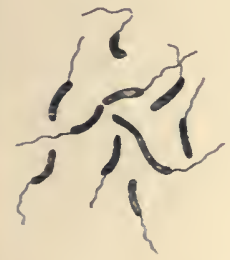

a

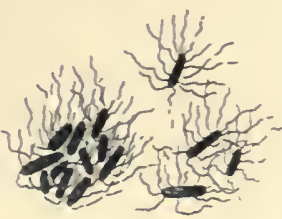

$b$

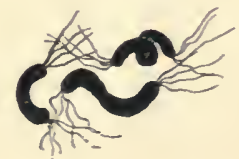

c

a. Spiral forms with a flagellnm at only one end. $b$. Bacillus of typhoid fever with flagella given off from all sides. c. Large spirals from stagnant water with wisps of flagella at their ends (spirillum undula).

delicate, hair-like appendages or flagella, by the lashing motions of which the rods possessing them are propelled through the fluid. In some cases the flagella are located at but one end of a bacillus, either singly or in a bunch; again, they may be seen at both poles, and in some cases, especially with the bacillus of typhoid fever, they are given off from the whole surface of the rod. (See Fig. 7.) In a few instances similar locomotive organs have been detected on spherical bacteria-i.e., motile micrococci have been observed.

For a long time this property of independent motion that is peculiar to certain species of bacteria was sup- 
posed to be due to the possession of some such form of locomotive apparatus, because similar appendages had been seen in some of the large, motile spirilla found in staguant water, and it was not until recently that the accuracy of this supposition was actually demonstrated. By a special method of staining Loffler has been able, in a number of cases, to render visible these hair-like appendages. His method consists in the employment of a mordant, by the aid of which the flagella are caused to retain the staining, and thus become visible. Lœefler's method of staining will be found in the chapter devoted to this part of the technique. 


\section{CHA PTER III.}

Principles of sterilization by heat-Methods employed-Discontinued sterilization-Sterilization under pressure-Apparatus employed-Chemical disinfection and sterilization.

Most important for the proper performance of bacteriological manipulations are acquaintance with the principles underlying the methods of sterilization and disinfection, and familiarity with the approved methods of applying these principles in practice.

In many laboratories it is eustomary to employ the term sterilization for the destruction of bacteria by heat, and the term disinfection for the accomplishment of the same end through the use of chemical agents. This distinction in the use of the terms is not strictly correct, as we shall endeavor to explain.

The laboratory application of the word sterilization for the destruction of bacteria by high temperatures probably arose from the circumstance that culture media, and certain other articles that it is desirable to render absolutely free from bacterial life, are not treated by chemical agents for this purpose, but are exposed to the influeuce of heat in various forms of apparatus known as sterilizers; and the process is, therefore, known as sterilization. On the other hand, cultures no longer useful, bits of infected tissue, aud apparatus generally, that it is desirable to render free from danger, are commonly subjected for a time to the action of chemical compounds possessing germicidal properties-i.e., 
to the action of disinfectants; and the process is, therefore, known as disinfection, though the same end can also be reached by the application of heat to these articles. Strictly speaking, sterilization implies the complete destruction of the vitality of all micro-organisms that may be present in or upon the substance to be - sterilized, and can be accomplished by the proper application of both thermal and chemical agents; while disinfection, though it may, need not of necessity, insure the destruction of all living forms that are present, but only of those possessing the power of infecting; it may or may not, therefore, be complete in the sense of sterilization. From this we see it is possible to accomplish both sterilization and disinfection as well by chemical as by thermal means.

In practice the employment of these means is governed by circumstances. In the laboratory it is essential that all culture media with which the work is to be conducted should be frec from living bacteria or their spores-they must be sterile; and it is equally important that their original chemical composition should remain unchanged. It is evident, therefore, that sterilization of these substances by means of chemicals is out of the question, for, while the media could be thus sterilized, it would be necessary, in order to accomplish this, to add to them substances capable not only of destroying all micro-organisms present, but whose presence wonld at the same time prevent the growtl of bacteria that are to be subsequently cultivated in these medial一that is to say, after performing their sterilizing or germicidal function the chemical disinfectants would, by their further presence, exhibit their antiseptic properties and thus render the material useless as a culture 
medium. Exceptions to this are seen, however, in the case of certain volatile substances possessing disinfectant powers-chloroform and ether, for instance; these bodies, after performing their germicidal activities, may be driven off by gentle heat, leaving the media quite suitable for purposes of cultivation. They are not, however, in general use in this capacity.

The circnmstances under which chemical sterilization or disinfection is practised in the laboratory are, ordinarily, either those in which it is desirable to render materials free from danger that are not affected by the chemical action of the agents used, such as glass apparatus, etc., or where destructive changes in the composition of the substances to be treated, as in the case of old eultures, infected tissues, etc., are a matter of no consequence. On the other hand, for the sterilization of all materials to be used as culture media heat only is employed. ${ }^{1}$

The two processes will be explained in this chapter, beginning with

\section{STERILIZATION BY HEAT.}

Sterilization by means of high temperature is accomplished in several ways, viz., by subjecting the articles to be treated to a high temperature in a properly constructed oven-this is known as dry sterilization; by subjecting them to the action of streaming or live steam at the temperature of $100^{\circ} \mathrm{C}$.; and by subjecting them to the action of steam under pressure, under which

1 An exception to this is the use of chloroform, a volatile disinfectant, that may easily be eliminated after haring exercised its germicidal properties. This is, however, not \& commonly employed method. 
circumstance the temperature to which they are exposed becomes more and more elevated as the pressure increases.

Experiments have taught us that the process of sterilization by dry heat is of limited application because of its many disadvantages. For successful sterilization by the method of dry heat not only is a relatively high temperature essential, but the substances under treatment must be exposed to this temperature for a comparatively long time. Its peuetration into materials which are to be sterilized is, moreover, much less thorough than that of steam. Many substances of vegetable and animal origin are rendered useless by subjection to the dry method of sterilization. For these reasons there are comparatively few materials that can be sterilized in this way without seriously impairing their further usefulness.

Successful sterilization by dry heat cannot usually be accomplished at a temperature lower than $150^{\circ} \mathrm{C}$., and to this degree of heat the objects should be subjected for not less than one hour. For the sterilization, therefore, of the organic materials of which the media employed in bacteriological work are composed, and of domestic articles, such as cotton, woollen, wooden, and leather articles, this method is wholly unsuitable. In bacteriological work its application is limited to the sterilization of glassware principally-such, for example, as flasks, plates, small dishes, test-tubes, pipettesand such metal instruments as are not seriously injured by the high temperature.

Sterilization by moist heat-steam-offers conditions much more favorable. The penetrating power of the steam is not only more complete, but the tempera- 
ture at which sterilization is ordinarily accomplished is, as a rule, not destructive to the objects under treatment. This is conspicuously seen in the work of the laboratory; the culture media, composed in the main of decomposable organic materials that would be rendered entirely worthless if exposed to the dry method of sterilization, sustain no injury whatever when intelligently subjected to an equally effective sterilization with steam. The same may be said of cotton and woollen fabrics, bedding, clothing, etc.

Aside from the relations of the two methods to the materials to be sterilized, their action toward the organisms to be destroyed is quite different. The penetratiug power of the steam renders it by far the more efficient agent of the two. The spores of several organisms which are killed by an exposure of but a few moments to the action of steam, resist the destructive action of dry heat at a higher temperature for a much greater length of time.

These differences will be strikingly brought out in the experimental work on this subject. For our purposes it is necessary to remember that the two methorls have the following applications :

The dry method, at a temperature of $150^{\circ}-180^{\circ} \mathrm{C}$. for one hour, is employed for the sterilization of glassware : flasks, test-tubes, culture-dishes, pipettes, plates, etc.

The sterilization by steam is practised with all culture media, whether fluid or solid. Bouillon, milk, gelatin, agar-agar, potato, etc., are under no circumstances to be subjected to dry heat.

The manner in which heat is employed in processes of sterilization varies with circumstances. When used 
in the dry form its applieation is always continuousi.e., the objects to be sterilized are simply exposed to the proper temperature for the length of time necessary to destroy all living organisms which may be upon them. With the use of steam, on the other hand, the articles to be sterilized are frequently of such a nature that a prolonged application of heat might materially injure them. For this and other reasons steam is usually applied intermittently and for short periods of time. The principles involved in this method of sterilization depend upon differences of resistance to heat which the organisms to be destroyed are known to possess at different stages of their development. During the lifeeycle of many of the bacilli there is a stage in which the resistance of the organism to the action of both chemical and thermal agents is much greater than at other stages of their development. This increased power of resistance appears when these organisms are in the spore or resting stage, to which reference has already been made. When in the vegetative or growing stage most bacteria are killed in a short time by a relatively low temperature, whereas, under eonditions which favor the production of spores, the spores are secn to be capable of resisting very mueh higher temperatures for an appreciably longer time; indeed, spores of eertain bacilli have been encountered that retain the power of germinating after an exposure of from five to six hours to the temperature of boiling water. Such power's of resistance have never been observed in the vegetative stage of development. These differences in resistance to heat which the spore-forming organisms possess at their different stages of development are taken advantage of in the process of sterilization by 
steam known as the fractional or intermittent method, and are the essential feature of the principles on which the method is based.

As the culture media to be sterilized are dependent for their usefulness upon the presence of more or less unstable organic compounds, the object aimed at in this method is to destroy the organisms in the shortest time and with the least amount of heat. It is accomplished by subjecting them to the elevated temperature at a time when the bacteria are in the vegetating or growing stage -i.e., the stage at which they are most susceptible to detrimental influences. In order to accomplish this it is necessary that there should exist conditions of temperature, nutrition, and moisture which favor the vegetation of the bacilli and the germination of any spores that may be present. When, as in freshly prepared nutrient media, these surroundings are found, the spore-forming organisms are not only less likely to enter the sporestage than when their environments are less favorable to their vegetation, but spores which may already exist develop very quickly into mature cells.

It is plain, then, that with the first application of steam to the substance to be sterilized the mature vegetative forms are destroyed, while certain spores that may be present resist this treatment, providing the sterilization is not continued for too long a time. If now the sterilization is discontinued, and the material which presents conditions favorable to the germination of the spores is allowed to stand for a time, usually for about twenty-four hours, at a temperature of from $20^{\circ}-30^{\circ}$ C., those spores which resisted the action of the steam will, in the course of this interval, germinate into the less resistant vegetative cells. A second short 
exposure to the steam kills these forms in turn, and by a repetition of this process all bacteria that were present may be destroyed withont the application of the steam having been of long duration at any time. It should be remembered that while spores which may be present are not directly killed by the exposure to heat that they experience in the intermittent method of sterilization, still their power of germination is somewhat inhibited by this treatment. In this method, therefore, if the temperature of $100^{\circ} \mathrm{C}$. be employed for too long a time, it is possible so to retard the germination of the spores as to render it impossible for them to develop into the vegetating stage during the interval between the heatings. By cxcessively long exposures to high temperature, but not long enough to destroy the spores directly, the object aimed at in the method may be defeated, and in the end the substance undergoing sterilization be found still to contain living bacteria. In this process the plan that has given most satisfactory results is to subject the materials to be sterilized to the action of steam, under the ordinary conditions of atmospheric pressure, for fifteen minutes on each of three successive days, and during the intervals to maintain them at a temperature of about $25^{\circ}-30^{\circ} \mathrm{C}$. At the end of this time all living organisms which were present will have been destroyed, and, unless opportunity is given for the acess of new organisms from without, the substances thus treated remain sterile.

As an exception to this, one occasionally cncounters certain species of spore-forming bacteria that are not readily destroyed by this mode of treatment. 'They are, presumably, of the group of so-called "soil organ- 
isms," and represent the forms most resistant to the influence of heat. We are not as yet sufficiently familiar with all their peculiarities to warrant our speaking with certainty as to a means of sterilizing media in which they are present. It does not seem unlikely that they are of the thermophilic (possibly facultative thermophilic) variety, and they show little tendency to develop into the vegetative stage between the heatings, germinating perhaps so slowly at the temperature under which they find themselves as not to leave completely the spore stage before another exposure to the steam, but manifesting after a time properties of life in the media that are thought to be sterile and which have been placed aside for subsequent use. This is a mere hypothesis, however, and is as yet entirely wanting in experimental proof.

Fortunately, these undesirable experiences are rare; but that they do occur, and result in no small degree. of annoyance, is an experience that has probably been had by most bacteriologists.

It must be borne in mind that this method of sterilization is only applicable in those eases which present conditions favorable to the germination of the spores into mature regetative cells. Dry substances, such as instruments, bandages, apparatus, etc., or organic materials in which decomposition is far advanced, where conditions of nutrition favorable to the germination of spores are not present, do not offer the conditions requisite for the suceessful operation of the principles underlying the intermittent method of sterilization.

The process of fractional sterilization at low temperatures is based upon exactly the same principle, but differs from the foregoing in the method by which it is 
practised in two respects, viz., it requires a greater number of exposures for its accomplishment, and the temperature at which it is conducted is not raised above $68^{\circ}-70^{\circ} \mathrm{C}$. It is employed for the sterilization of easily decomposable materials, which would be rendered useless by steam, but which remain intact at the temperature employed, and for certain albuminous eulture media that it is desirable to retain in a fluid condition during sterilization, but which would be coagulated if exposed to high temperatures. This process requires that the material to be sterilized should be subjected to a temperature of $68^{\circ}-70^{\circ} \mathrm{C}$. for one hour on each of six successive days, the interval of twenty-four hours between the exposures admitting of the germination of spores into mature cells. During this interval the substances under treatment are kept at about $25^{\circ}-30^{\circ}$ C. The temperature employed in this process suffices to destroy the vitality of almost all organisms in the vegetative stage in about one hour. Until recently blood-serum was always sterilized by the intermittent method at low temperature.

Sterilization by steam is also practised by what may be called the direct method. That is to say, both the mature organisms and the spores which may be present in the material to be sterilized are destroyed by a single exposure to the steam. In this method steam at its ordinary temperature and pressure-live steam or streaming steam, as it is called-is employed just as in the first methol described, but it is allowed to act for a much longer time, usually not less than an hour; or steam under pressure, and consequently of a higher temperature, is now frequently employed. By the latter procedure a single exposure of fifteen minutes is sufficient 
for the destruction of practically all bacilli and their spores, providing the pressure of the steam is not less than one atmosphere over and above that of normal; this is approximately equivalent to a temperature of $122^{\circ} \mathrm{C}$. to which the organisms are exposed.

The objection that has been urged to both of these methods, particularly that in which steam under pressure is employed, is that the properties of the media are altered. Gelatin is said to become cloudy and lose the property of solidifying; in bouillon and agar-agar fine precipitates are thought to occur, and some think the reaction undergoes a change. In the experience of those who have used steam under pressure, not exceeding one or one and one-half atmospheres for ten to fifteen minutes, these obstacles have rarely been encountered. There is one point to be borne in mind, however, in using steam under pressure, viz., it is not possible to regulate the time of exposure to the same degree of nicety as where ordinary live steam is used. The reason for this is that if the apparatus be opened to remove the objects being sterilized while the steam within it is under pressure, the escape of steam will be so rapid that all fluids within the chamber, thus suddenly relieved of pressure, will begin to boil violently, and, as a rule, will boil quite out of the tubes, flasks, etc., containing them. For this reason the apparatus must be kept closed until cool, or until the gauge indicates that pressure no longer exists within the chamber, and even then it should be opened very cautiously. It is patent from this that the temperature and time of exposure of articles sterilized by this process cannot usually be controlled with accuracy. It requires some time to reach a given pressure after 
the apparatus is closed, and it also requires time for cooling after the desired exposure to such pressure before the apparatus can be opened.

It is manifest that during these three periods, viz., (a) reaching the pressure desired, (b) time during which the pressure is maintained, and $(c)$ time for fall of pressure before the chamber can be opened, it is difficult to say certainly to what temperature and pressure the articles in the apparatus have, on the whole, been subjected.

\section{Fig. 8.}
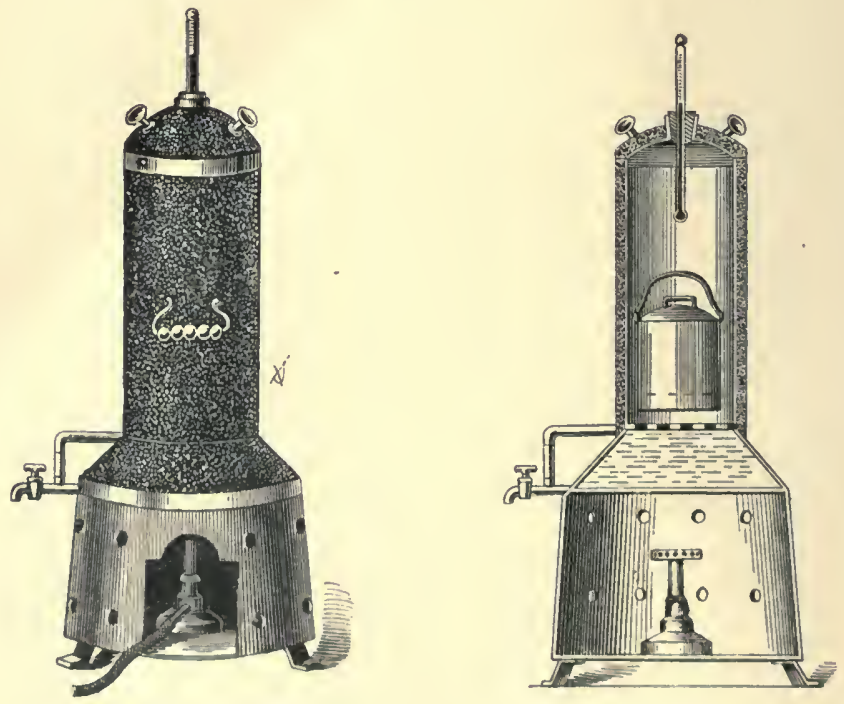

Steam sterilizer, pattern of Koch.

Clearly, if the desired pressure and temperature have been maintained for ten minutes, one cannot say that this is all the heat to which the articles have been subjected during their stay in the chamber. In this light, 
while steam under pressure may auswer very well for routine sterilization, still it presents insurmountable obstacles to its use in more delicate experiments where time-exposure to definite temperature is of importance.

For sterilization by live steam the apparatus commonly employed has, until recently, been the cylindrical boiler recommended by Koch. (See Fig. 8.)

Its construction is very simple, essentially that of the ordinary potato-steamer used in the kitcheu. It consists of a copper cylinder, the lower fifth of which is somewhat larger in circumference than. the remaining four-fifths, and acts as a reservoir for the water from which the steam is to be generated. Covering this section of the cylinder is a wire rack or grating through which the steam passes, and which serves to support the articles to be sterilized. Above this, comprising the remaining four-fifths of the cylinder, is the chamber for the reception of the materials over and through which the steam is to pass. The cylinder is closed by a snugly fitting cover throngh which are usually two perforations into which a thermometer and a manometer may be inserted. The whole of the outer surface of the apparatus is encased in a non-conducting mantle of asbestos or felt.

The water is heated by a gas-flame placed in an enclosed chamber, upon which the apparatus rests, which serves to diminish the loss of heat and deflection of the flame through the action of draughts. The apparatus is simple in construction, and the only point which is to be observed while using it is the level of the water in the reservoir. On the reservoir is a water-gange which indicates at all times the amount of water in the apparatus. The amount of water should never be too 
small to be indicated by the gauge; otherwise there is danger of the reservoir becoming dry and the bottom of the apparatus being destroyed by the direct action of the flame.

A sterilizer that has come into very general use in bacteriological laboratories is one originally intended for use in the kitchen. It is the so-called "Arnold Steam Sterilizer." It is very ingenious in its construction as well as economical in its employment.

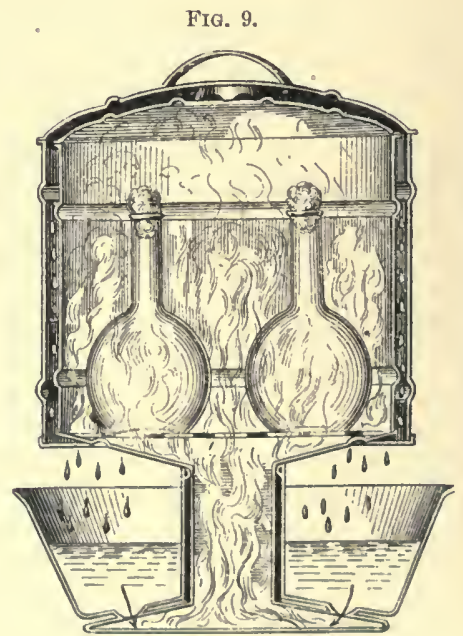

Arnold steam sterilizer.

The difference between this apparatus and that just deseribed is that it provides for the condensation of the steam after its escape from the sterilizing chamber, and returns the water of condensation automatically to the reservoir, so that in practice the apparatus requires but little attention, as with ordinary eare there is no fear of 
the water in the reservoir becoming exhausted and the consequent destruction of the sterilizer.

Fig. 9 shows a section through this apparatus.

STERILIZATION UNDER PRESSURE.

For sterilization by steam under pressure several special forms of apparatus exist. The principles involved in them all are, however, the same. They provide for

Fig. 10 .

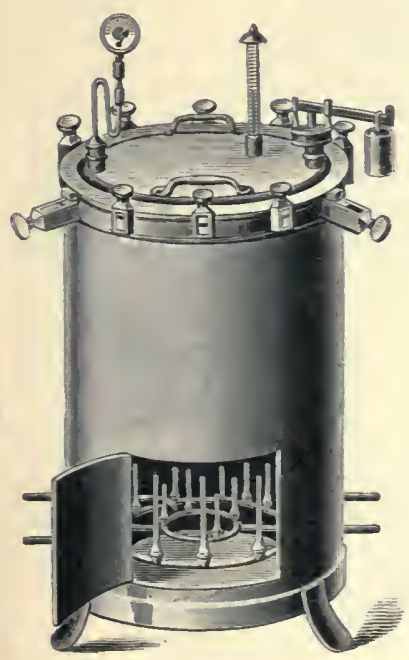

$A$

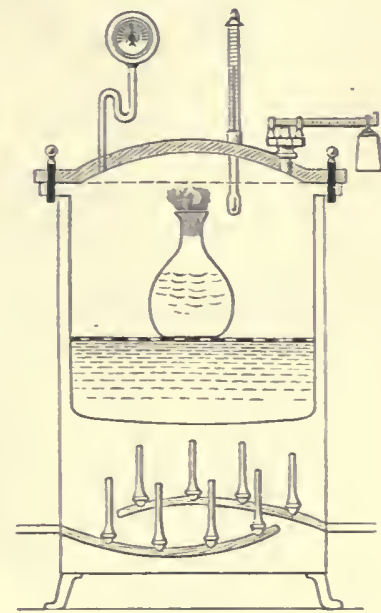

$B$

Autoclave, pattern of Wiesnegg. A. External appearance. B. Section.

the generation of steam in a chamber from which it cannot escape when the apparatus is closed. Upon the cover of this chamber is a safety-valve, which can be 
regulated so that any degree of pressure (and coincidently of temperature) that is desirable can be maintained within the sterilizing chamber. These sterilizers

Fig. 11.

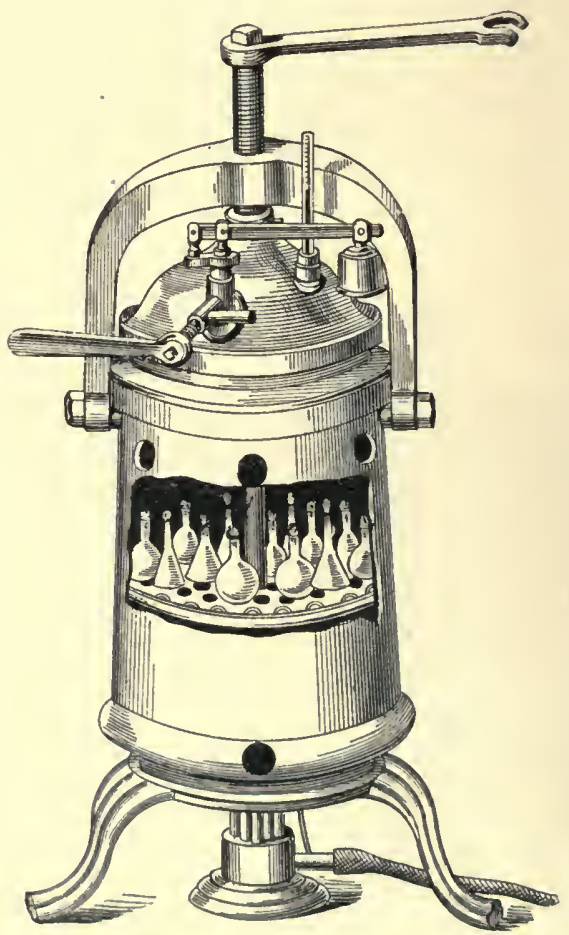

Autoclave or digester for sterllizing by steam under pressure.

are known as "digesters" and as "autoclaves." Their eonstruction ean best be understood by referenee to Figs. 10 and 11. 


\section{STERILIZATIOY BY HOT AIR.}

The hot-air sterilizers used in laboratories are simply double-walled boxes of Russian or Swedish iron (Fig. 12), having a double-walled door, which closes tightitly, and a heavy copper bottom. They are arranged with ventilating openings for the escape of the contained air and the entrance of the heated air. The flame, usually from a rose burner (Fig. 13), is applied directly to the bottom. The heat circulates from the lower surface around about the apparatus through the space between its walls.

\section{FIG. 12.}

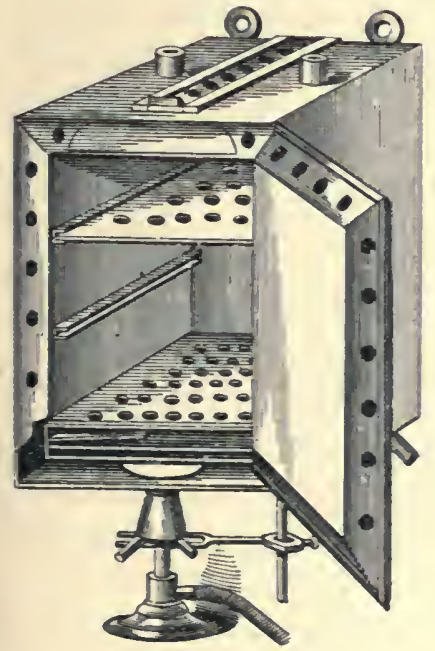

Frg. 13.

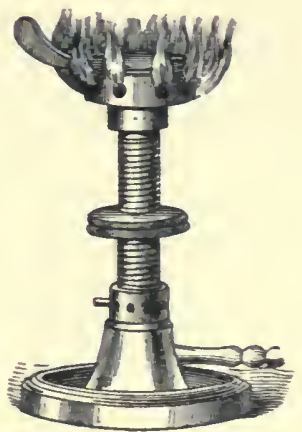

The construction of the copper bottom of the apparatus upon which the flame impinges is designed to prevent the direct action of the flame upon the sheet-iron bottom of the chamber. It consists of several copper 
plates placed one above the other, but with a space of about 4 to $5 \mathrm{~mm}$. between the plates. These copper bottoms after a time become burned out, and unless they are replaced the apparatus is useless. The older forms of hot-air sterilizers are so constructed that their repair is a matter involving some time and expense. To meet this objection I have had constructed a sterilizer in all respects similar to the old form except in the arrangement of this copper bottom. This is made in such a way that it can be easily removed, so that by keeping several sets of copper plates on hand a now one can readily be inserted when the old one is burned out.

In the employment of the hot-air sterilizer care should always be given to the condition of the copper bottom; for the direct application of the heat to the sheet-iron plate upon which the substances to be sterilized stand results not only in destruction of the apparatus, but frequently in destruction of the substances undergoing sterilization.

Since the temperature at which this form of sterilization is usually accomplished is high, from $150^{\circ}$ to $180^{\circ} \mathrm{C}$., it is well to have the apparatus encased in asbestos boards, to diminish the radiation of heat from its surfaces. This not only confines the heat to the apparatus, but guards against the destructive action of the radiated heat on woodwork, furniture, etc., that may be in the neighborhood.

\section{CHEMICAL STERILIZATION AND DISINFECTION.}

As has already been stated, it is possible by means of certain chemical substances to destroy all bacteria and their spores that may be within or upon various 
materials and objects-i.e., to sterilize them; and it is also possible by the same means to rob infected objects of their dangerous infective properties without at the same time sterilizing them-i.e., to disinfect them. This latter process depends upon the fact that the vitality of many of the less resistant pathogenic organisms is easily destroyed by an exposure to particular chemical substances that may be without effect upon the more resistant saprophytes and their spores that are present.

In general, the use of chemicals for sterilization is not to be considered in connection with substances that are to be employed as culture media, and their employment is restricted in the laboratory to materials that are of no further value, and to infected articles that are not injured by the action of the agents used, though for particular purposes such volatile germicides as chloroform and ether may serve as exceptions to this. (See Preservation of Blood-serum with Chloroform.) In short, they are mainly of value in rendering infected waste materials free from danger. For the successful performance of this form of disinfection there is one fundamental rule always to be borne in mind, viz., it is absolutely essential to success that the disinfectant used should come in direct contact with the bacteria to be destroyed, otherwise there is no disinfection.

For this reason, one should always remember, in selecting the disinfecting agent, the nature of the materials containing the bacteria upon which it is to act, for the majority of disinfectants, and particularly those of an inorganic nature, vary in the degree of their potency with the chemical nature of the mass to which they are applied. Often the materials containing the bacteria 
to be destroyed are of such a character that they combine with the disinfecting agent to form insoluble precipitates; these so interfere with the penetration of the disiufectant that many bacteria may escape its destructive action entirely and no disinfection be accomplished, though an agent might have been employed that would, under other circumstances, have given entirely satisfactory results.

In the destruction of bacteria by means of chemical substances there occurs, most probably, a definite chemical reaction-that is to say, the characteristies of both the bacteria and the agent employed in their destruction are lost in the production of an inert third body, the result of their combination. It is impossible to say with absolute certainty, as yet, that this is the case; but the evidence that is rapidly accruing from the more recent studies upon disinfectants and their mode of action points strongly to the accuracy of this belief. This reaction, in which the typical structures of both bodies concerned are lost, takes place between the agent employed for disinfection and the protoplasm of the bacteria. For example, in the reaction that is seen to take place between the salts of mercury and albuminous bodies there results a third compound, which has the characteristics neither of mercury nor of albumin, but partakes of the peculiarities of both; it is a combination of albumin and mercury known by the indefinite term "albuminate of mercury." Some such reaction as this oceurs when the soluble salts of mercury are brought in contact with bacteria. This view has recently been strengthened by the experiments of Geppert, in which the reaction was cansed to take place between the spores of the anthrax bacillus and a solu- 
tion of mercuric chloride, the result being the apparent destruction of the vitality of the spores by the formation of this third compound. In these experiments it was shown that though this combination had taken place, still it did not of necessity imply the complete death of the spores, for if by proper means the combination of mercury with their protoplasm was broken up, many of the spores returned from their condition of apparent death to that of life, with all their previous disease-producing and cultural peculiarities. Geppert employed a solution of ammonium sulphide for the purpose of destroying the combination of sporeprotoplasm and mercury; the mercury was precipitated from the protoplasm as an insoluble sulphide, and the protoplasm of the spores returned to its original condition. These and other somewhat similar experiments have given an entirely new impulse to the study of disinfectants, and in the light shed by them many of our previously formed ideas concerning the action of disinfecting agents must be modified. The process is not a catalytic one-i.e., occurring simply as a result of the presence of the disinfecting body, which is not itself decomposed during its process of destruction -but is, as said, a definite chemical reaction occurring within more or less fixed limits-that is to say, with a given amount of the disinfectant employed just so much work, expressed in terms of disinfection-destruction of bacteria-can be accomplished.

Another point in favor of this view is the increased energy of the reaction with elevation of temperature. Just as in many other chemical phenomena the intensity of the reaction becomes greater under the influence of heat, so in the process of disinfection the combination 
between the disinfectant and the organisms to be destroyed is much more energetic at a temperature of $37^{\circ}$ to $39^{\circ} \mathrm{C}$. than it is at $12^{\circ}$ to $15^{\circ} \mathrm{C}$.

What has been said refers more particularly to the inorganic salts which are employed for this purpose. It is probable that the organic bodies possessing disinfectant properties owe this power to some such similar reaction, though, as yet, these substances have not been so thoroughly studied in this relation.

The reaction between the inorganic salts and albuminous bodies is not selective; they combine in most instances with any or all protoplasmic bodies present. For this reason the employment of many of the commoner disinfectants in general practice is a matter of doubtful advantage. For example, the disinfection of excreta, sputum, or blood, containing pathogenic organisms, by means of corrosive sublimate, is a procedure of questionable success. The amount of sublimate employed may be entirely used up and rendered inactive as a disinfectant by the ordinary protoplasmic substances present, without having any appreciable effeet upon the bacteria which may be in the mass.

These remarks are introduced in order to guard against the implicit confidence so often placed in the disinfecting value of corrosive sublimate. In many bacteriological laboratories, where there is constantly more or less of infectious material, it is the custom, with few exeeptions, to keep vessels containing solutions of corrosive sublimate at haud, into which infectious materials may be placed. The value of this procedure, as we have just learned, may be more or less questionable, especially in those cases in which the substance to be disinfected is of a proteid nature and 
where the solution used is not freshly prepared. Witl the introduction of such substances into the sublimate solution the mercury is quickly precipitated by the albumin, and its disinfecting properties may be entirely destroyed; we may in a very short time have little else than water containing a precipitate of albumin and mercury, in so far as its value as a disinfectant is concerned.

Though the other inorganic salts have not been so thoroughly studied in this connection, it is nevertheless probable that the same precautions should be taken in their employment as we now know to be necessary in the use of the salts of mercury.

Where it is desirable to use chemical disinfectants in the laboratory much more satisfactory results can usually be obtained through the employment of carbolic acid in solution. A three or four per cent. solution of commercial carbolic acid in water requires a somewhat longer time for disinfection; but it is, at the same time, open to fewer objections than are solutions of the inorganic salts, though here, too, we find a somewhat analogous reaction between the carbolic acid and proteid matters. Under ordinary circumstances its action is complete in from twenty minutes to one-half hour. It is not reliable for the disinfection of resistant spores, such, for instance, as those of bacillus anthracis.

In the laboratory heat is the surest agent to employ. All tissues containing infectious organisms should be burned, and all cloths, test-tubes, flasks, and dishes should be boiled in 2 per cent. soda (ordinary washingsoda) solution for fifteen to twenty minutes, or placed in the steam sterilizer for half an hour.

Intestinal evacuations may best be disinfected with 
boiling water or with milk of lime, a mixture eomposed of lime in solution and in suspension-ordinary fluid "white-wash." This should be thoroughly mixed with the evacuations until the mass reacts distinetly alkaline, and should remain in eontact with the infective substance for one or two hours. If boiling water be used, the amount should be about double the volume of the mass to be disinfected. They should be thoroughly mixed and allowed to stand, covered, until cold.

Sputum in which tubercle bacilli are present, as well as the vessel containing it, must be boiled in 2 per cent. soda solution for fifteen minutes, or steamed in the sterilizer for at least half an hour.

On the whole, in the laboratory we should as yet rely more upon the destructive properties of heat than upon those of chemical agents.

From what has been said, the absurdity of sprinkling about, here and there, a little carbolic acid or in placing about apartments in which infections diseases are in progress little vessels of earbolic acid, must be plain. The disinfection of water-closets and cesspools by allowing now and then a few eubic centimetres of some so-called disinfectant to trickle through the pipes is ridiculons. A disinfectant must be applied to the bacteria, and must be in contact with them for a long enough time to insure the destruction of their life.

In the light of the latest experiments upon disinfectants, the place formerly oceupied by many agents in the list of substanees employed for the purpose will most likely be changed as they are studicd more closely. The agents, then, which will prove of most value in the laboratory for the purpose of rendering infectious materials harmless are: heat, either by burning, by 
steaming for from half an hour to an hour, or by boiling in a 2 per cent. sodium carbonate solution for fifteen minutes; 3 to 4 per cent. solution of commercial carbolic acid; milk of lime, and a solution of chlorinated lime ("chloride of lime") containing not less than 0.25 per cent. of free chlorine. The chloride of lime from which such a solution is to be made should be fresh and of good quality. Good chlorinated lime, as purchased in the shops, should contain not less than 25 to 30 per cent. of available chlorine. The materials to be disinfected in either of the lime solutions should remain in them for about two hours. The solutions should be freshly prepared when needed, as they rapidly decompose upon standing.

Antiseptic. An antiseptic is a body which, by its presence, prevents the growth of bacteria without of necessity killing them. A body may be an antiseptic without possessing disinfecting properties to any very high degree, but a disinfectant is always an antiseptic as well. A germicide is a body possessing the property of killing bacteria. 


\section{CHAPTER IV.}

Principles involved in the methods of isolation of bacteria in pure culture by the plate method of Koch-Materials employed.

As was stated in the introductory chapter, the isolation in pure cultures of the different species that may be present in mixtures of bacteria was rendered possible only through the methods suggested by Koch. Since the adoption of these methods they have undergone many modifications, but the prineiple originally involved has remained unaltered. The observation which led to their development was a very simple one, and one that is eommonly before us. Koch noticed that on solid substances, such, for example, as a slice of potato or of bread, which had been exposed for a time to the air and which afforded proper nourishment for the lower organisms, there developed after a short time small patches of material which proved to be colonies of bacteria. Each of these colonies on eloser examination showed itself to be, as a rule, composed of but a single species. There was little tendeney on the part of these colonies to become confluent, and from the differences in their naked-eye appearances it was easy to see that they were mostly the ontgrowth of different species of bacteria.

The question that then presented itself was: If from a mixture of organisms floating in the air it is possible in this way to obtain in pure eultures the component individuals, what means ean be employed to obtain the same results at will from mixtures of different species of 
bacteria when found together under other conditions? It was plain that the organisms were to be distingnished primarily, the one from the other, only by the structure and general appearance of the colonies growing from them, for by their morphology alone this is impossible. What means could be devised, then, for separating the individual members of a mixture in such a way that they would remain in a fixed position, and be so widely separated, the one from the other, as not to interfere with the production of colonies of characteristic appearance, which would, under the proper conditions, develop from each individual cell ?

If one take in the hand a mixture of barley, rye, corn, oats, etc., and attempt to separate the mass into its constituents by picking out the different grains, much difficulty is experienced; but if the handful of grain be thrown upon a large flat surface, as upon a table, the grains become more widely separated and the task is considerably simplified; or, if sown upon proper soil, the various grains will develop into growths of entirely different external appearance by which they can readily be recognized as unlike in nature. Similarly, if a testtube of decomposed bouillon be poured out upon a large flat surface, the individual bacteria in the mass are very much more widely separated the one from the other than they were when the bouillon was in the tube; but they are in a fluid medium, and there is no possibility of their either remaining separated or of their forming colonies under these conditions, so that it is impossible by this means to pick out the individuals from the mixture.

If, however, it is possible to discover some substance which possesses the property of being at one time fluid 
and at another time solid, and which can be added to this bouillon without in any way interfering with the life-functions of the bacteria, then, as solidification sets in, the organisms will be fixed in their positions and the conditions will be analogous to those seen on the bit of potato.

FIg. 14.

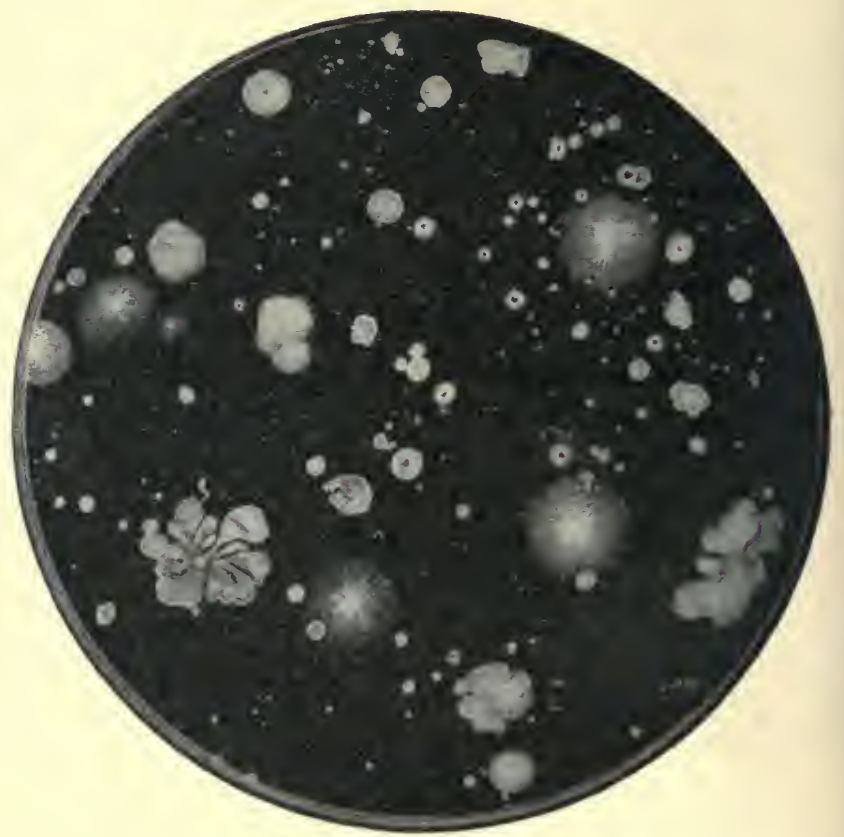

Plate showing certain macroscopic characteristics of colonies. Natural size.

Gelatin possesses this property. At a temperature which does not interfere with the life of the organisms it is quite fluid, whereas when subjected to a lower temperature it solidifies. When onee solid it may be kept 
at a temperature favorable to the growth of the bacteria and will remain in its solid condition.

Gelatin was added to the fluids containing mixtures of bacteria, and the whole was then poured upon a large flat surface, allowed to solidify, and the results noted. It was found that the conditions seen on the slice of potato could be reproduced; that the individuals in the mixture of bacteria grew well in the gelatin, and, as on the potato, grew in colonies of typical macroscopic structure, so that they could easily be distinguished the one from the other by their naked-eye appearances. (See Fig. 14.) It was necessary, however, to use a more dilute mixture of bacteria than that seen in the original decomposed bouillon. The number of individuals in the tube was so enormous that on the gelatin plate they were so closely packed together that it was not only impossible to pick them out because of their proximity the one to the other, but also because this packing together materially interfered with the production of those characters by means of which differences can be seen with the naked eye. The numbers of organisms were then diminished by a process of dilution, consisting of transferring a small portion of the original mixture into a second tube of sterilized bouillon to which gelatin had been added and liquefied; from this a similar portion was added to a third gelatin-bouillon tube, and so on. These were then poured upon large surfaces and allowed to solidify. 'The results were entirely satisfactory. On the gelatin plates from the original tube, as was expected, the colonies were too numerous to be of any use; on the plates made from the first dilution they were much fewer in number, but still they were usually too numerous and too closely packed to permit of charac- 
teristic growth; but on the second dilution they were, as a rule, fewer in number and widely separated, so that the individuals of each species were in no way prevented by the proximity of their neighbors from growing each in its own typical way. (Fig. 15.) There was then no difficulty in picking out the colonies resulting from the growth of the different individual bacteria.

FIG. 15.

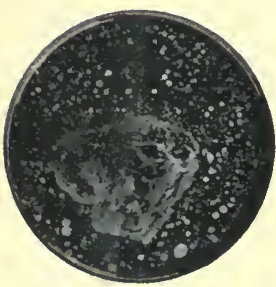

$A$

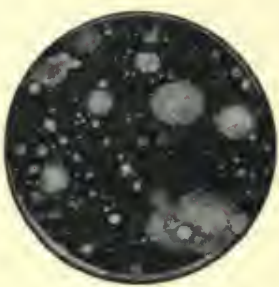

$B$

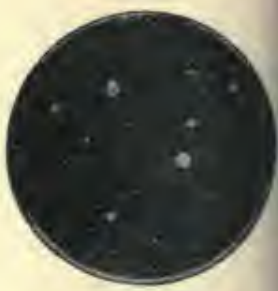

0

Series of plates showing the results of dilution upon the number of colonies : $A$. Plate No. 1, or "original." $B$. First dilution, or Plate No. 2. C. Second dilution, or Plate No. 3. About one-fourth natural size.

This, then, is the principle underlying Koch's method for the isolation of bacteria by means of solid media.

The fundamental part of the media employed is the bouillon, which contains all the elements necessary for the nutrition of most bacteria, the gelatin being employed simply for the purpose of rendering the bouillon solid. The medium on which the organisms are growing is, therefore, simply solidified bouillon, or beef tea.

In practice, two forms of grelatin are employed-the one an animal or bone grelatin, the ordinary table gelatin of good quality; and the other a vegetable gelatiu, known as agar-agar, or Japanese gelatin, which is obtained from a group of algæ growing in the sea along 
the coast of Japan, where it is employed as an article of diet by the natives.

Aside from these differences in origin of the two forms of gelatin employed, their behavior under the influence of heat and of bacterial growth renders them of different application in bacteriological work. The animal gelatin liquefies at a much lower temperature, and also requires a lower temperature for its solidification, than does the agar-agar. Ordinary gelatin, in the proportion commonly used in this work, liquefies at about $24^{\circ}-26^{\circ} \mathrm{C}$, and becomes solid at from $8^{\circ}-10^{\circ} \mathrm{C}$. It may be employed for those organisms which do not require a higher temperature for their development than $22^{\circ}-24^{\circ} \mathrm{C}$. Agar-agar, on the other hand, does not liquefy until the temperature has reached about $98^{\circ}-99^{\circ}$ C. It remains fluid ordinarily until the temperature has fallen to $38^{\circ}-39^{\circ} \mathrm{C}$., when it rapidly solidifies. For our purposes, only that form of agar-agar can be used which remains fluid at from $38^{\circ}-40^{\circ} \mathrm{C}$. Agar-agar which remains fluid only at a temperature above this point would be too hot, when in a fluid state, for use; many of the organisms introduced into it would either be destroyed or checked in their development by so high a temperature. Agar-agar is for use in those cases in which the cultivation must be conducted at a temperature above the melting-point of gelatin.

In addition to their differences when under the influence of various temperatures, the relations of these two gelatins to bacteria are quite distinct. Many bacteria bring about alterations in gelatin which cause it to become liquid (a process analogons to peptonization), in which state it remaius. There are no known organisms that bring about such a change in agar-agar. 
As a rule, the colony-formations seen upon gelatin are much more characteristic than those which develop on agar-agar, and for this reason gelatin is to be preferred when circumstances will permit. Both gelatin and agar-agar may be used in the preparation of plates and Esmarch tubes, subsequently to be described. 


\section{CHAPTER V.}

Preparation of media-Bouillon, gelatin, agar-agar, potato, blood-serum, etc.

As has been stated, the fundamental constituent of our culture media is beef tea, or bouillon.

BourLlox.-The directions of Koch for the preparation of this medium have undergone many modifications to meet special cases, but for general use his original formula is still retained. It is as follows : five hundred grammes of finely chopped lean beef, free from fat and tendons, are to be soaked in one litre of water for twenty-four hours. During this time the mixture is to remain in the ice-chest or to be otherwise kept at a low temperature. It is then to be strained through a coarse towel and pressed until a litre of fluid is obtained. To this are to be added ten grammes (1.0 per cent.) of dried peptone and five grammes ( 0.5 per cent.) of common salt $(\mathrm{NaCl})$. It is then to be rendered exactly neutral or very slightly alkaline with a few drops of saturated sodium carbonate solution. The flask containing the mixture is then to be placed either in the steam sterilizer or in a water-bath, or over a free flame, and kept at the boiling-point until all the albumin is coagulated and the fluid portion is of a clear, pale straw color. It is then filtered through a folded paper filter, and sterilized in the steam sterilizer by the fractional method. Certain of the modifications of this method are of sufficient value to justify mention. Most 
important is the neutralization. Ordinarily, this is accomplished with the saturated sodium carbonate solution, and the reaction is determined with red and blue litmus papers, and for the beginner this method serves most purposes.

The sodium carbonate solution is not so good, however, as a strong solution of caustic soda or potash, because the carbonic acid liberated from the sodium carbonate is frequently seen to give rise to confusing, temporary acid reaction which disappears on heating, nor is litmus the most reliable indicator to employ. To obviate this, Schultz (Centralb.f. Bakt. u. Parasitenkunde, 1891, Bd. x., Nos. 2 and 3) recommends exact titration with a solution of caustic soda. For this purpose a 4 per cent. solution of caustic soda is prepared. From this a 0.4 per cent. solution is made, and with it the titration is practised. After the bouillon has been deprived of all coagulable albumin and blood-coloringmatter by boiling and filtration, and has cooled down to the temperature of the air, its whole volume is exactly measured.

From it a sample of exactly 5 or 10 c.c. is then taken, and to this a few drops of one of the indicators commonly employed in analytical work are added. Sehultz reeommends 1 drop of phenolphtalein solution (1 gramme phenolphtalein in 300 c.c. of alcohol) to 1 e.c. of bouillon. The beaker containing the sample is placed upon white paper, and the dilute caustic soda solution is then allowed to drop into it, very slowly, from a burette, until there appears a very delicate rose color, which indicates the begimning of alkaline reaction. A second sample of the bouillon is treated in the same way. If the amounts of caustic soda solution required 
for each sample deviate but very slightly or not at all the one from the other, the mean of these amounts is taken as the amount of alkali necessary to neutralize the quantity of bouillon employed. If 10 c.c. of bouillon were employed, then, for the whole amount of 1 litre, just 100 times as much, minus that for the two samples used in titration, will be needed. For example: to neutralize 10 c.c. of bouillon 2 c.c. of the diluted (0.4 per cent.) caustic soda solution were employed. For the remaining 980 c.c. of the litre of bouillon, then, 196 c.c. (200 c.c. -4 c.c., the amount employed for the two samples of 10 c.c. each of bouillon) are needed of the 0.4 per cent. solution, or one-tenth of this amount of the 4 per cent. caustic soda solution.

For the neutralization of the whole bulk of the bouillon it is better to employ the stronger alkaline solution, as by its use the volume is not increased to so great an extent as when the dilute solution is nsed.

It is evident that this method is much more exact than that ordinarily employed, but at the same time it must be remembered that for its success exactness in the measurement of the volumes and in the preparation of the dilutions is required. To obviate error, it is better to employ this method when the solutions are all cool and of nearly the same temperature, so that rapid fluctuations in temperature, and consequent alterations in volume, will not materially interfere with the accuracy of the results.

This method of neutralization, as suggested by Schultz, is to be adopted for those experiments in which it is desirable to have the reaction of the medium accurate and constantly of the same degree.

For the ordinary purposes of the beginner, however, 
results quite satisfactory in their nature may be obtained by the employment of the saturated sodium carbonate solution for nentralization and litmus paper as the indicator. For some time, however, it has been our practice to employ the yellow curcuma paper for the detection of alkalinity, rather than the red litmus paper.

In the exhanstive paper of Fuller on this point it was shown that the results obtained by titrating the same culture medium with the same alkali solution differed very markedly with the indicator employed. For instance, a litre of ordinary meat-infusion nutrient agar-agar required 47 c.c. of a normal caustic alkali solution to neutralize it when phenolphtalein was the indicator used, 28 c.c. when blue litmus was employed, and 5 c.c. when rosolic acid was substituted. It is manifest from this that the actual reactions of media, in the neutralization of which different indicators have been used, may differ very widely from one another, and that the results of cultivation on a medium neutralized by one method are not fairly comparable with those obtained when another indicator has been used. For the sake of uniformity Fuller suggests that bacteriologists should agree upon some one trustworthy method of neutralization and employ it to the exclusion of other methods. He recommends, as the procedure that has given the most satisfactory results in his hands, a modification of Schultz's method, viz., 5 c.c. of the culture medium are to be mixed with 45 c.c. of distilled water in a porcelain evaporating dish and boiled for three minutes, after which 1 c.c. of phenolphtalein

1 Fuller: On the Proper Reaction of Nutrient Media for Bacterial Cultjration. "Public Health" (Journal of the American Public Health Association), Quarterly Series, 1895, vol. i. p. 381. 
solution $^{1}$ is added and the titration with one-twentieth normal canstic alkali solution is quickly made. The neutral point (slightly on the side of alkalinity) is indicated by the appearance of a pink color, the effect of the alkali on the phenolphtalein. From the amount of one-twentieth normal alkali solution needed for 5 c.c. of the medium it is casy to calculate the number of cubic centimetres of the normal solntiou that will be required to neutralize the entire mass.

The phenolphtalein neutral point lies so high, averaging 47 c.c. of normal canstic alkali solution per litre for nutrient meat-infusion agar-agar, and 56 c.e. per litre for nutrient gelatin, that it is improbable from experience gained by the older methods that the conditions offered by media neutral to this indicator are suitable for the growth of all bacteria, so that with particular species it may be necessary to determine by experiment the degree of deviation from the neutral poiut that is best suited for development. In Fuller's experience the degree of deviation from the phenolphtalein neutral point that gives in general the best results is represented by from 15 to 20 of his scale-i.e., there should remain enough uncombined acid in a litre of the finished medium to require the further addition of caustic alkali to the extent of from 15 to 20 c.c. of a normal solution to bring the reaction of the mass up to the phenolphtalein neutral point. Thus, for example, if upon titration it should be found that to neutralize a litre of nutrient meat-infusion gelatin by the phenolphtalein process 5 j c.c. of normal caustic alkali solution would be needed, the amount actually added would be from 35 to 40 c.c.-

1 A 0.5 per cent. solution of the powder in 50 per cent. alcohol. 
i. e., from 15 to 20 c.c. less than the amount needed to bring the reaction up to the neutral point.

Not infrequently the filtered bouillon, neutralized and sterilized, will be seen to contain a fine, flocculent precipitate. This may be due either to excess of alkalinity or to incomplete precipitation of the albumin. The former may be correeted with dilute acetic or hydrochloric acid, and the bonillon again boiled, filtered, and sterilized; or, if due to the latter cause, subsequent boiling and filtration usually result in ridding the bouillon of the precipitate.

Another modification now generally employed is the use of meat-extracts instead of the infusion of meat. Almost any of the meat-extracts of commerce answer the purpose, though we usually employ Liebig's. It is used in the strength of from two to four grammes to the litre of water. Peptone and sodium chloride are added as in the bouillon made from the meat-infusion. The advantages of meat-extract are: it takes less time; affords a solution of more uniform composition if used in fixed proportions, and in general use gives results that are equally as satisfactory as those obtained from the employment of infusion of meat.

Nutrient Gidatis.-For the preparation of gelatin the bonillon is first prepared in exactly the same way as has just been described, except that the neutralization takes place after the gelatin has been completely dissolved, which oceurs very rapidly in hot bonillon. The reaction of the gelatin as it comes from the manufactories is frequently quite acid, so that a much larger amount of alkali is need for its neutralization than for other media. It is possible, however, to obtain from the makers an excellent grade of gelatin from 
which all acid has been carefully washed.' The gelatin is added in the proportion of 10 to 12 per cent. Its complete solution may be accomplished either over the water-bath, in the steam sterilizer, or orer a free flame. If the latter method be practised, care must be taken that the mixture is constantly stirred to prevent burning at the bottom and consequent breaking of the flask, if a flask is employed.

For some time it has been our practice to use, for the purpose of making both gelatin and agar-agar, enamelled iron saucepans instead of glass flasks; by this means the free flame may be employed without danger of breaking the ressel, and, with a little care, without fear of burving the media. Under any conditions it is better to protect the bottom of the vessel from the direct action of the flame by the interposition of sereral layers of wire gauze, a thin sheet of asbestos-board, or an ordinary cast-iron stove-plate.

When the gelatin is completely melted it may be filtered through a folded paper filter supported on an ordinary funnel; if the solution is perfect, this should be very quickly accomplished.

For the filtration of such substances as gelatin and agar-agar it is of much importance to have a properly folded filter. To fold a filter correctly, proceed as follows: a circular piece of filter paper is folded exactly through its centre, forming the fold $1,1^{\prime}$ (Fig. 16); the end 1 is then folded over to $1^{\prime}$, forming the fold 5 ; 1 and $1^{\prime}$ are each then brought to 5 , thus forming the folds 3 and $7 ; 1$ is then carried to the point $\overline{7}$, and the fold 4 is formed, and by carrying $1^{\prime}$ to 3 the fold 6 is

1 Hesteberg's acid-free, gold label gelatin has given us entire satisfaction in this respect. 
produced; and by bringing 1 to 3 and $1^{\prime}$ to 7 the folds 2 and 8 result.

Fig. 16.

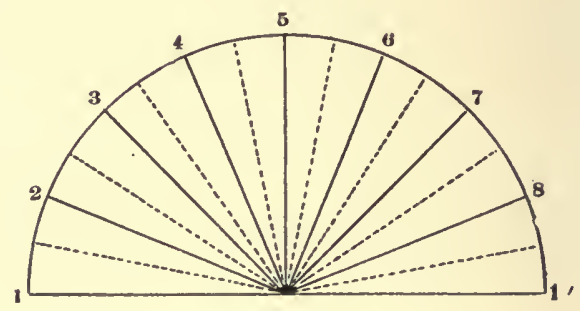

Thus far the ridges of all folds are on the side of the paper next to the table on which we are folding. The paper is now taken up, and each space between the seams just produced is to be subdivided by a seam or fold through its centre, as indicated by the dotted lines in Fig. 16, but with the creases on the side opposite to that

FIG. 17.
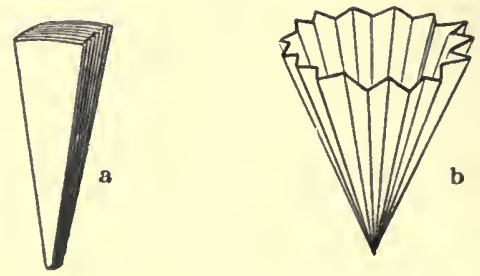

- occupied by the creases $1,2,3,4$, etc., first made. As each of these folds is made the paper is gradually folded into a wedge-shaped bundle (Fig 17,a), which when opened assumes the form of a properly folded filter (seen in $b$, Fig. 17). Before placing it upon the funnel it is well to go over each crease again and see that it is 
as tightly folded as possible, without tearing it. The advantage of the folded filter is that by its use a much greater filtering surface is obtained, as it is in contact with the funnel only at the points formed by the ridges, leaving the majority of the flat surface free for filtration.

The employment of the hot-water funnel, so often recommended, has been dispensed with in this work to a very large extent, as we know that, if the solution of the gelatin is complete, filtration is so rapid as not to necessitate the use of an apparatus for maintaining the high temperature. The temperature at which the hotwater funnel retains the gelatin is so high that evaporation and concentration rapilly occur, and in consequence the filtration is, as a rule, retarded. The filtration is frequently done in the steam sterilizer, but this too is unnecessary if the gelatin is quite dissolved. At the ordinary temperature of the room, and by the means commonly employed for the filtration of other substances, both gelatin and agar-agar may be rapidly filtered if they are completely dissolved.

It not infrequently occurs that, even under the most careful treatment, the filtered gelatin is not perfectly transparent (the condition in which it must exist, otherwise it is useless), and clarification becomes necessary. For this purpose the mass must be redissolved, and when at a temperature between $60^{\circ}$ and $70^{\circ} \mathrm{C}$. an egg, which has been beaten up with about 50 c.c. of water, is added. The whole is then thoroughly mixed together and agaiu brought to the boiling-point, and kept there until coagulation of the albumin occurs. It is better not to break up the large masses of coagulated albumin if it can be avoided, as when broken up into 
fine flakes they clog the filter and materially retard filtration.

The practice sometimes recommended of removing these albuminous masses by first filtering the gelatin through a cloth, and then finally through paper, is not only superfluous, but in most instances renders the process of filtration much more difficult, because of the disintegration of these masses into finer particles, which have the effect just mentioned, viz., of clogging the filter.

Under no circumstances is a filter to be used without first having been moistened with water. If this is not done, the pores of the paper, which are relatively large when in a dry state, when moistened by the gelatin not only diminish in size, but in contracting are often entirely oceluded by the finer albuminous flakes which become fixed within them, and filtration practically ceases. The preliminary moistening with water causes diminution of the size of the pores to such an extent that the finer particles of the precipitate rest on the surface of the paper, instead of becoming fixed in its meshes.

During boiling it is well to filter, from time to time, a few cubic centimetres of the gelatin into a test-tube and boil it over a free flame for a minute or so; in this way one can detect if all the albumin has been coagulated-i.e., if the solution is ready for filtration.

Gelatin should not, as a rule, be boiled over ten or fifteen minutes at one time, or left in the steam sterilizer for more than thirty to forty-five minutes, otherwise its property of solidifying may be impaired.

As soon as the gelatin is complete, whether it is retained in the flask into which it has been filtered or decanted off into sterilized test-tubes, it should be sterilized in the steam sterilizer on three successive days, 
for fifteen minutes each day-the mouth of the flask or the test-tubes containing it having been previously closed with cotton plugs.

Nutriest Ag.AR-AGaR.-The preparation of nutrient agar-agar by the beginner is far too frequently a tedious and time-taking experience. This is due mainly to lack of patience and to deviation from the rules laid down for the preparation of this medium. If the directions given below for the preparation of nutrient agar-agar be strictly observed, no difficulty whatever should be encountered. Many methods are recommended for its preparation; almost every worker has some slight modification of his own.

The methods that have given us the best results, and from which we have no good grounds for departing, are as follows:

Prepare the bouillon in the usual way. Agar-agar reacts neutral or very slightly alkaline, so that the bouillon may be neutralized before the agar-agar is added. Then add finely chopped or powdered agaragar in the proportion of 1 to 1.5 per cent. Place the mixture in a porcelain-lined iron vessel and make a mark on the side of the ressel at which the level of the fluid stands; if a litre of medium is being made, add about 250 c.c. to 300 c.c. more of water and allow the mass to boil slowly, occasionally stirring, over a free flame, for from one aud one-half to two hours; or, in other words, until the excess of water $-i$.e., the 250 or 300 c.c. that were added - has evaporated. Care must be taken that it does not boil over the sides of the vessel. From time to time observe if the fluid has fallen below the mark of its original level; if it has, add water until its volume of 1 litre is 
restored. At the end of the time given remove the flame and place the vessel containing the mixture in a large dish of cold water; stir the agar-agar continuously until it has cooled down to about $68^{\circ}-70^{\circ} \mathrm{C}$., and then add the white of one egg which has been beaten up in about 50 e.c. of water; or the ordinary dried albumin of commerce may be dissolved in cold water in the proportion of about 10 per cent., and used; the results are equally as good as when eggs are employed. Mix this carefully throughout the agar-agar, and allow the mass to boil slowly for about another half-hour, observing all the while the level of the fluid, which should not fall below the litre mark. It is necessary to reduce the temperature of the mass to the point given, $68^{\circ}-70^{\circ} \mathrm{C}$; otherwise the coagulation of the albumin will occur suddenly in lumps and masses as soon as it is added, and its clearing action will not be homogeneous. The process of clarification with the egg is purely mechanieal - the fincr particles, which would otherwise pass though the pores of the filter, being taken up by the albumin as it coagulates and retained in the coagula.

At the end of one-half hour the boiling mass may be easily and quickly filtered through a heavy, folded paper filter at the room temperature, and, as a rule, the filtrate is as clear and transparent as agar-agar usually appears.

It might be well to emphasize the fact that for the filtration of agar-agar a hot-water funnel, or any other special device for maintaining the temperature of the mass, is totally unnecessary. Agar-agar prepared after the methods just given should filter through a properly folded paper filter at the rate of a litre in from twelve to fifteen minutes.

Another plan that insures complete solution of the 
agar-agar without causing the precipitates that are commonly seen when all the ingredients are added at first and boiled for a long time, is to weigh out the necessary amount of agar-agar, 10 or 15 grammes, and place this in 1300 or 1400 c.c. of water and boil down over a free flame to 1000 c.c. The peptone, salt, and beef-extract are then added and the boiling again continued until they are dissolved. The clarification with egg-albumin may then be done, and usually the mass filters quite clear and does not show the presence of precipitates upon cooling. If the mixture is positively alkaline, it is not only cloudy, but it filters with difficulty; if it is acid, it is usually quite clear, filters more quickly, but, as Schulze has pointed out, loses at the same time some of its gelatinizing properties. The bouillon should always be neutralized before the agar-agar is added to it, for if the bouillon be acid, from the acid of the meat, it robs the agar-agar, under the influence of heat, of some of its gelatinizing powers, which canuot be regained by subsequent neutralization.

Another method by which the agar-agar can easily and quickly be melted is by steam under pressure. If the flask containing the mixture of bouillon and agaragar be kept in the digester or antoclave, with the steam under a pressure of about one atmosphere, as shown by the gauge, for ten minutes, the agar-agar will be found at the end of this time completely melted, and filtration may then be accomplished with but little difficulty.

If glycerin is to be added to the agar-agar, it is done after filtration and before sterilization. The nutritive properties of the media for certain organisms, particularly the tubercle bacillus, are improved by the addition of glycerin in the proportion of 5 to 7 per cent. 
If after filtration a fine floceulent precipitate is seen, look to the reaction of the medium. If it is quite alkaline, neutralize, boil, and filter again. If the reaction is neutral or only very slightly acid, dissolve and clarify again with egg-albumin by the method given.

The most important point in all the media, aside from the correct proportion of the ingredients, is their reaction. They must be neutral or very slightly alkaline to litmus. (See remarks on Nentralization of Media.) Only a few organisms develop well on media of an acid reaction. In all of the above media the meat-extracts now on the market may usually be substituted for the meat itself in preparing the bouillon. They may be employed in the proportion of from two to four grammes to the litre of water.

Preparation of Potatoes. - Potatoes are prepared for use in two ways:

1. They are taken as they come to the market—old potatoes being usually recommended, and carefully scrubbed under the water-tap with a stiff brush until all adherent dirt has been removed; "the eyes" and all discolored or decayed parts are carefully removed with a pointed knife. They are then to be placed in a solution of corrosive sublimate of the strength of $1: 1000$ and allowed to remain there for twenty minutes; at the end of this time, without rinsing off the sublimate, they are placed in a covered tin bucket with a perforated bottom and sterilized in the steam sterilizer for fortyfive minutes. On the second and third days the sterilization is repeated for fifteen to twenty minutes each day. They must not be removed from the sterilizing bucket until sterilization is complete. At the end of this time they are ready for use. When prepared in this way 
they are usually intended to be cut in half, and the cultivation of the organisms is to be conducted upon the flat surfaces of the sections. (Koch's original method.)

This method requires some care to prevent contamination during manipulation. The hand which is to take up the potato from the bucket, which until now has remained covered, is first disinfected in the sublimate solution for ten minutes, the potato is then taken up between the thumb and index finger and severed into two by a knife which has just been sterilized in the free flame until it is quite hot. The blade of the knife is passed not quite through the potato, but nearly so. A large glass culture-dish for the reception of the two halves of the potato, having been disinfected for twenty minutes with 1:1000 sublimate solution and then drained of all the adherent solution, is at hand ready for the bits of potato; the cover is removed, and by twisting the knife gently the two halves of the potato may be cansed to fall apart in the dish and usually to fall upon their convex surfaces, leaving the flat sections uppermost. The cover is placed upon the dish and the potatoes are ready for inoculation.

2. Preparation of potatoes for test-tube cultures. Method of Bolton. If the potatoes are to be employed for testtube cultures, one simply scrubs off the coarser particles of dirt with water and a brush, and with a cork-borer punches out eylindrical bits of potato which will fit loosely into the test-tubes to be used. On each bit of potato is then to be cut a slanting surface running from about the junction of the first and second thirds of the cylinder to the diagonally opposite end. These cylin-

1 Medical News, 1857, vol. i. p. 133. 
ders of potato are now to be left in running water over night, otherwise they will be very much discolored by the sterilization to which they are to be subjected. At the end of this time they are placed in previously prepared test-tubes, one piece in each tube, with the slanting surface up, the cotton plugs of the tubes replaced, and they are then to be sterilized in the steam for fifteen to twenty minutes on each of three successive days.

Or the entire sterilization may be accomplished in the autoclave, with the steam under a pressure of one atmosphere, by a single exposure of twenty Frg. 18. to twenty-five miuutes. When finished

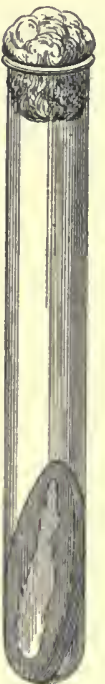

Potato in testtube. they have the appearance seen in Fig. 18, except that there is no growth upon the surface as is shown in the ent.

For some purposes potatoes may be advantageously peeled, sliced into disks of about $1 \mathrm{~cm}$. in thickness, and placed in small glass dishes provided with covers, similar to the ordinary Petri dishes. The dish and its contents are then sterilized by steam in the usual way (method suggested by von Esmarch). By this plan a relatively large area for cultivation is obtained.

Potatoes may also be boiled, or steaned, and mashed, and the mass placed in covered dishes, test-tubes, or flasks, and sterilized. By this method one obtains in the mass a mean of the composition of the several potatoes, or bits of potatoes, used in making it, an advantage where uniformity is desired.

Care must be given to the sterilization of potatoes, because they always have adhering to them the organ- 
isms commonly found in the ground, the spores of which are among the most resistant known. The so-called "potato bacillus" is one of this group; it is an organism which is not infrequently more or less of an obstacle to the work of the beginner.

BLOOD-SERUM. - Originally blood-serum required special care in its preparation; it was always necessary to reduce the unavoidable contamination, which to a certain extent occurs when the blood is obtained, to the minimum degree.

It is possible to collect serum from small animals and in small quantities under such precautions that it is perhaps not contaminated; but, ordinarily, for laboratory purposes a larger quantity is needed, so that the slaughter-houses form the souree from which it is usually obtained, and here a certain amount of contamination is unavoidable, though its degree may be limited by proper precaution.

The steps that were formerly thought to be essential to the successful collection of blood and the preparation of serum for culture purposes were about as follows :

The animal from which the blood is to be collected should be drawn up to the ceiling by the hind legs, the head should be held well back, and with one pass of a very sharp knife the throat should be completely cut through. The blood which spurts from the severed vessels should be collected in large glass jars which have been previously cleaned, disinfected, and all traces of the disinfectant removed with alcohol and, finally, ether. The latter evaporates very quickly and leaves the jar quite dry. The jars should be provided with covers which close hermetically-these, too, should be carefully disinfected. The best form of glass vessels 
for the purpose is the large glass museum jar of about one gallon capacity, which closes by a cover that can be tightly serewed down upon a rubber joint. From two such jarfuls of blood one can recover quite a large quantity of clear serum, ordinarily from 500-700 c.c. The jars having been filled with blood, their covers are placed loosely upon them and they are allowed to stand for about fifteen minutes until elotting has begun. At the end of this time a clean glass rod is passed around the edges of the surface of the clot to break up any adhesions to the side of the jar that might have formed, and which would prevent the sinking of the elot to the bottom. The covers are then replaced and tightly clamped in position, and with as little agitation as possible the jars are placed in an ice-chest, where they remain for twenty-four to forty-eight hours. The temperature should, however, not be low enough to prevent coagulation, but should be sufficiently low to interfere with the development of any living organisms that may be present. The temperature of the ordinary domestic refrigerator is sufficient for the purpose. After twenty-four to forty-eight hours the clot will have become firm, and will be seen at the bottom of the jar. Above it is a quantity of dark straw-colored serum. The serum may then be drawn off with a sterilized pipette and placed in tall cylinders that have previonsly been plugged with eotton wadding and sterilized. After treating all the serum in this way, care having been taken to get as little of the coloring matter of the blood as possible, it may be placed again in the ice-chest for twenty-four hours, during which time the eorpuscular elements will sink to the bottom, leaving the supernatant fluid quite clear. This may 
then be pipetted off, either into sterilized test-tubes, about 8 c.c. to each tube, or into small sterilized flasks of about 100 c.c. capacity. It is then to be sterilized by the intermittent method at low temperatures, viz., for one hour on each of five consecutive days at a temperature of $68^{\circ}-70^{\circ} \mathrm{C}$. During the intervening days it is to be kept at the room temperature to permit of the development of any spores that may be present into their vegetative forms, in which condition they are killed by an hour's exposure to the temperature of $70^{\circ} \mathrm{C}$.

Fig. 19.

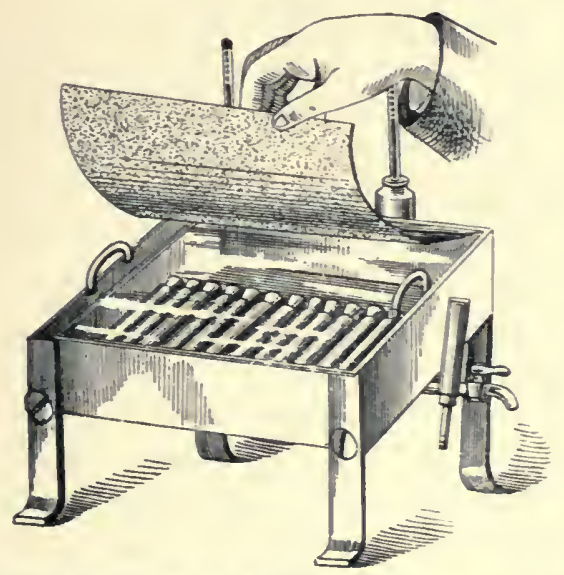

Chamber for sterilizing and solidifying blood-serum. (Косн.)

At the end of this time the serum in the tubes may either be retained as fluid serum or solidified at between $76^{\circ}-80^{\circ} \mathrm{C}$. In solidifying the serum the tubes should be placed in an inclined position so that as great a surface as possible may be given to the serum. The process of solidification requires constant attention if good 
results are to be obtained-i.e., if a translucent, solid medium is to result. If the old, small form of apparatus be employed (Fig. 19), then the solidification can be accomplished in a shorter time than if the larger forms commonly employed are used. No definite rule for the time that will be required ean be laid down, for this is not constant. If the small solidifying apparatus be used, very good results may be obtained in about two hours. at $78^{\circ} \mathrm{C}$. It frequently requires a longer time at a higher temperature than has been mentioned. This is espeeially the ease with Loeffler's serum mixture.

The best results are obtained when a low temperature is employed for a long time. Under any circumstances the tubes must be observed from time to time through the glass door or cover with which the solidifying oven is provided, and each time the oven should be slightly jarred with the hand to see if solidification, as indieated by the disappearance of tremors from the serum, is beginning. If the temperature gets too high, or the exposure is too long, an opaque medium results. The temperature to be observed is that of the air inside the chamber, and also that of the water surrounding it. The latter is usually a degree or two higher than the former. The tubes should not rest directly upon the lieated bottom or against the heated sides of the ehamber, but should lie upon racks of wood or wire, and be protected from the sides by a wire sereen of gauze: in this way the tubes are all exposed to about the same temperature. The thermometer which indicates the temperature inside the chamber should not toueh the surfaces, but should either be suspended free from above through a cork in the top of the apparatus, if 
the large form of apparatus be used, or should lie upon a rack of cork or wood, its bulb being free and a little lower than the other extremity, if the small, old-fashioned apparatus of Koch be employed. The latter form is preferable, as it is more easily managed.

When solidification is complete the tubes are to be retained in the erect position, and, unless they are intended for immediate use, must be prevented from drying. The superfluous ends of the cotton plugs should be burned off, and the mouths of the tubes may then be covered by sterilized rubber caps, or, as Ghriskey suggests, they may be closed with sterilized corks pushed in on top of the cotton plugs. Even with the greatest care it not uncommonly happens that one or two of the lot of tubes thus prepared and protected will become contaminated. This is usually due to spores of moulds that have fallen into the rubber caps or on the cotton plugs during manipulation, and, finding no means of outward growth, lave thrown their hyphæ downward through the cotton into the tube, and their spores have fallen on the surface of the serum and developed there.

The foregoing is, in the main, the plan originally recommended by Koch for the preparation of this medium. In recent times, however, particularly since the study of diphtheria by the method of Lœffler has become so general, and large quantities of serum tubes were found to be necessary, a modification has been suggested that has, in this country at least, almost entirely supplanted the method by Koch. The popularity of the Councilman-Mallory method is due to the following facts: by it the serum is more quickly and easily prepared; rigid precautions against contamination 
during collection of the serum are not so necessary, and the resulting medium, while not transparent or even translucent (points aimed at in the original method), fully meets all the requirements.

The special points in the method are: the serum is decanted into test-tubes as soon as obtained; it is then firmly coagulated in a slanting position in the dry-air sterilizer at from $80^{\circ}$ to $90^{\circ} \mathrm{C}$; ; it is then sterilized in the steam sterilizer at $100^{\circ} \mathrm{C}$. on three suceessive days, as in the case of other culture media. It may then be protected against evaporation by sterilized rubber caps or sterilized corks in the way already described, and set aside until needed.

Unless the coagulation in the dry sterilizer be complete, the surface of the serum will be found to be blistered and pitted by bubbles and cavities after it has been subjected to the steam sterilization. A similar formation of cavities over the surface of the serum will occur if the temperature of the hot-air sterilizer, in which it is solidified, is allowed to get above $90^{\circ} \mathrm{C}$., or if it be elevated to this point too quickly.

It is of no special advantage to have the serum clear, as the admixture of blood-coloring-matter does not affect its nutritive properties.

It is often desirable to obtain blood-serum in small quantities, either for culture purposes or for the study of the serum of different animals in its relation to bacteria, and for this purpose Nuttall (Centralb. für Balt. u. Parasitenkunde, 1892, Bd. xi. p. 539) suggests a very convenient method. By the use of a sterilized vessel, of the shape given in Fig. 20, from ten to one hundred enbic centimetres of blood can be collected, and if proper precautions are observed no contamination by bacteria 
need occur. The collecting bulb is used in the following way: an artery, either femoral or carotid, is exposed, and around it two ligatures are placed; that distant from the heart is tightened, while the one nearest the heart is left loose; between the latter and the heart the artery is clamped. A small slit is then made in its wall, into which the point $a$ of the bulb is introduced and the artery bound tightly around it with the

\section{FIG. 20.}

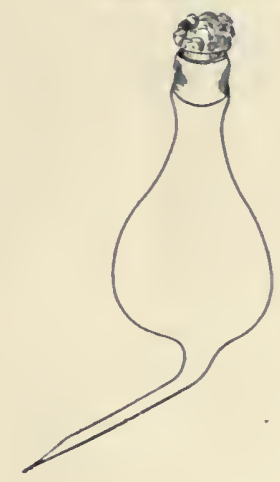

a

Nuttall's bulb for collecting blood-serum under antiseptic precautions.

hitherto loose ligature; the clamp is removed and the bulb quickly fills with blood. The clamp is now again put in position, the point of the bulb removed and sealed in the gas-flame, the loose ligature tightened, the wound closed, and the bulb containing the blood is set aside in a cool place until coagulation has occurred. The serum is most easily withdrawn from the bulb by means of a pipette, closed above with a cotton-plug, and supplied with a bit of rubber tubing, about one-half metre long, with glass mouth-piece. By holding the 
pipette in the hand and sucking upon the rubber tube one can more easily direct the point of the pipette than if it is used in the ordinary way.

The bulbs are easily blown, and after having been sealed at the point and plugged with cotton can be kept on hand just as are sterilized test-tubes.

It is sometimes desirable to preserve blood-serum in a fluid state. This can be done by the fractional method of sterilization at low temperatures, already described, or with much less effort, and without the use of leat, by a method that we have found to be very satisfactory. In the studies of Kirchner chloroform was shown to possess decided disinfectant properties; as it is quite volatile, it is easily removed when its disinfectant or antiseptic functions are no longer required. If, therefore, the serum to be preserved be placed in a closely stoppered flask and enough chloroform added to form a thin layer, about $2 \mathrm{~mm}$., on the bottom, the serum may be kept indefinitely without contamination, so long as the chloroform is not permitted to evaporate. When required for use the serum is decanted into test-tubes, which are then placed in a water-bath at about $50^{\circ} \mathrm{C}$. until all the chloroform has been driven off; this can be determined by the disappearance of its characteristic odor. The serum may then be solidified, sterilized by heat, and employed for culture purposes. We have found serum so preserved to answer all requirements as a culture medinm.

Special Media.-The media just described-bouillon, nutrient gelatin, nutrient agar-agar, potato, and blood-serum-are those in general use in the laboratory for purposes of isolation and study of the ordinary forms of bacteria. For the finer points of differentia- 
tion special media have been suggested; a few of them will be mentioned.

Milk. Fresh milk should be allowed to stand over night in the ice-chest, the cream theu removed, and the remainder of the milk pipetted into test-tubes, about 8 c.c. to each tube, and sterilized by the intermittent process, at the temperature of steam, for three successive days.

The separation of the cream may be accelerated and rendered more complete by one sterilization of the milk in the crlinder before it is placed in the ice-chest.

The cream is best separated from the milk by the use of a cylindrical vessel with stopcock at the bottom, by means of which the milk, devoid of cream, may be drawn off. A Chevalier creamometer with stopcock at the bottom serves the purpose very well. It should be covered while standing. ${ }^{1}$

Milk may be used as a culture medium without any addition to it, or, before sterilizing, a few drops of litmus tincture may be added, just enough to give it a pale blue color. By this means it will be seen that different organisms bring about different reactions in the medium; some producing alkalies which canse the blue color to be intensified, others producing acids which change it to red, while others bring about neither of these changes. Similarly litnus solution is often added to gelatin and agar-agar for the same purpose.

Milk may also be employed as a solid culture medium by the addition to it of gelatin or agar-agar in the proportions given for the preparation of the ordinary nutri-

${ }^{1}$ For some time past vye have been using what is technically known as "separator milk"-i. c., the fluid left after milk has been deprived of its fat (cream) by centrifugal force. 
ent gelatin or agar-agar. It has, however, in this form the disadvantage of not being transparent, and can therefore best be used for the study of those organisms which grow upon the surface of the medium without causing liquefaction.

Nutrient gelatin and agar-agar can also be prepared from neutral milk whey, obtained from milk after precipitation of the casein.

Dunham's peptone solution. The medium usually known as Dunham's solution is prepared according to the following formula:

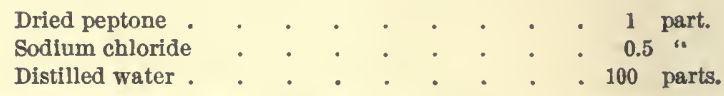

It is usually of a neutral or slightly alkaline reaction, and neutralization is not, therefore, necessary. It is filtered, decanted into tubes or flasks, and sterilized in the steam sterilizer in the ordinary way. The most common use to which this solution is put is in determining if the organism under consideration possesses the property of producing indol as one of its products of nutrition. It is essential for accuracy that the preparation of dried peptone employed should be of as nearly chemical purity as is possible, and indeed the other ingredients should be correspondingly free from impurities. Gorini (Centralblatt für Bakteriologie und Parasitenkunde, 1893, Bd. xiii. p. 790) calls attention to the fact that impuritics in the peptone, particularly the presence of carbohydrates, so interfere with the production of indol by certain bacteria that otherwise produce it, that it is ofttimes impossible, when such preparations have been employed, to obtain the characteristic color-reaction of 
this body, and where it is obtained it is always after a much longer time than is the case where peptone free from these substances has been used. He suggests the advisability of testing the purity of all peptone preparations before using them, by means of the reaction that they exbibit when acted upon by Fehling's alkaline copper solution. Under the influence of this agent pure peptone in solution gives a violet color (the biuret reaction), which remains permanent even after boiling for five minutes. If, instead of a violet color, there appears a red or reddish-yellow precipitate, the peptone should be discarded, as in lis experience no indol is produced from peptone giving this reaction. Both the peptone solution and that of the copper (particularly the latter) should be relatively dilute in order for the reaction to be successful.

Peptone rosolic acid solution. Peptone solution containing rosolic acid serves well for the detection of alterations in reaction. It consists of the peptone solution of Dunbam, to each 100 c.c. of which 2 c.c. of the following solution are added:

Rosolic acid (coralline) . . . . . 0.5 gramme. Alcohol (80 per cent.) • . . . . 100 c.c.

This is to be boiled, filtered, and decanted into clean, sterilized test-tubes, about 8 to 10 c.c. to each tube. The tubes are then to be sterilized in the usual way by steam. When sterilization is completed and the tubes cooled the solution will be of a very pale rose color, which disappears entirely under the action of acids, and becomes much more intense when alkalies are produced. We have used this solution for some time for the study of the reactions produced by different organisms, and 
find it a valuable addition to our means of differentiating bacteria.

Rosolic acid cannot be used with safety in solutions containing glucose, as the reducing action of the latter deprives it of its color.

Lactose-litmus-agar, or gelatin of Wurtz. A medium of much use in the differentiation of bacteria is that recommended by Wurtz, consisting of ordinary nutrient, slightly alkaline agar-agar, to which from 2 to 3 per cent. of lactose and sufficient litmus tincture to give it a pale blue color have been added. Bacteria capable of causing fermentation of lactose when grown on this medium develop into colonies of a pale pink color and cause, likewise, a reddening of the surrounding medium, owing to the production of acid as a result of their action upon the lactose; while other bacteria, incapable of such fermentative activities, grow as pale blue colonies and cause no reddening of the surrounding medium. It is an especially uscful aid in the differentiation of the bacillus of typhoid fever, which does not possess the property of bringing about fermentation of lactose, from other organisms that simulate it in many other respects, but which do possess this property.

Its preparation is as follows: to nutrient agar-agar or gelatin, the alkalinity of which is such that one cubic centimetre will require 0.1 c.c. of a $1: 20$ normal sulphuric acid solution to neutralize it, lactose is added in the proportion of 2 or 3 per cent; ; it is then decanted into test-tubes and sterilized in the usual way. When sterilization is complete there is to be added to each tube enough sterilized litmus tincture to give a decided though not very intense blue color. This must be done carefully, to avoid contamination of the tubes during ma- 
nipulation. It is better not to add the litmus tincture before sterilizing the tulbes, as its color-characteristics are in some way altered by its contact with organic matters under the influence of heat.

When ready it may be used as ordinary agar-agar or gelatin, either for plates or slant-cultures.

Loeffler's blood-serum mixture. Lœffler's blood-serum mixture consists of one part of neutral meat-infusion bouillon, containing 1 per cent. of grape-sugar, and three parts of blood-serum. This mixture is placed in test-tubes, sterilized, and solidified in exactly the way given for blood-serum. It requires for its solidification a somewhat higher temperature and a longer exposure to this temperature than does blood-serum to which no bonillon has been added. (See also the CouncilmanMallory method.)

Guarniari's agar-gelatin:

Meat-infusion

Sodium chloride .

Peptone.

Gelatin .

Agar-agar

Water.
950 c.c.

5 grammes.

$25-30$ "

$40-60$ "a

3-4 "

50 c.c.

The point in the preparation of this medium is its reaction, which should be exactly neutral.

The full list of special media is too great to be given in a work of this size. For their description the reader is referred to the eurrent literature on the subject. Those that have been given above will suffice for obtaining a clear understanding of the principles of the work.

NoтE. - The term "meat-infusion" always implies a watery extract of meat made by mixing 500 grammes 
of finely chopped lean meat and 1 litre of water together, and allowing them to stand in a cool place for twenty-four hours. At the end of this time the fluid portion is strained off through a coarse towel. This represents the infusion. 


\section{CHAPTER VI.}

Preparation of the tubes, flasks, etc., in which the media sre to be preserved.

WHILE the media are in course of preparation it is well to get the test-tubes and flasks ready for their reception, and it is essential that they should be as clean as it is possible to make them. For this purpose it is advisable that both new tubes and those which have previously been used should be boiled for some time, about thirty to forty-five minutes, in a 2 to 3 per cent. solution of common soda; it is not necessary to be exact as to strength, but it should not be weaker than this. At the end of this time they are to be carefully swabbed out with a cylindrical bristle. brush, preferably one having a reed handle (Fig. 21), as those with wire

FIG. 21.

Brush for cleaning test-tubes.

handles are apt to break through the bottoms of the tubes. All traces of adherent material should be carefully removed. When the tubes are quite clean they may be rinsed in a warm solution of commercial hydrochloric acid of the strength of about 1 per cent. This is to remove the alkali. They are then to be thoroughly rinsed in clear, running water, and stood top down until the water has drained from them. When 
dry they are to be plugged with raw cotton. The plugging with the cotton requires a little practice before it can be properly done. The cotton should be introduced into the mouths of the tubes in such a way that no cracks or creases exist, but should fill them quite regularly all around. The plug should fit neither too tightly nor too loosely, but should be just firmly enough in position to sustain the weight of the tube into which it is placed when held up by the portion which projects from and overhangs the mouth of the tube. The tubes thus plugged with cotton are now to be placed upright in a wire basket and heated for one hour in the hot-air sterilizer at a temperature of about $150^{\circ} \mathrm{C}$. A very good rule for this process of sterilization is to observe the tubes from time to time, and as soon as the cotton has become a very light brown color, not deeper than a dark-cream tint, to consider sterilization complete. The tubes are then removed and allowed to cool.

The cotton used for this purpose should be the ordinary cotton batting of the shops, and not absorbent cotton; the latter becomes too tightly. packed, and is, moreover, much too expensive for this purpose.

Care should be taken not to burn the cotton, otherwise the tubes will become coated with a dark-colored, empyrenmatic, oily deposit, which renders them unfit for use until they have been cleaned again.

Filing the Tubes.-When the tubes are cold they may be filled. This is best accomplished by the use of a spherical form of funnel, such as is shown in Fig. 22. The liquefied medium is poured into this funnel, which has been carefully washed, and by pressing the pincheock with which the funnel is provided the desired amount of material (5-10 c.e.) 
may be allowed to flow into the tubes held under its opening.

It is not necessary to sterilize the funnel, for the medium is to be subjected to this process as soon as it is in the test-tubes.

Fig. 22.

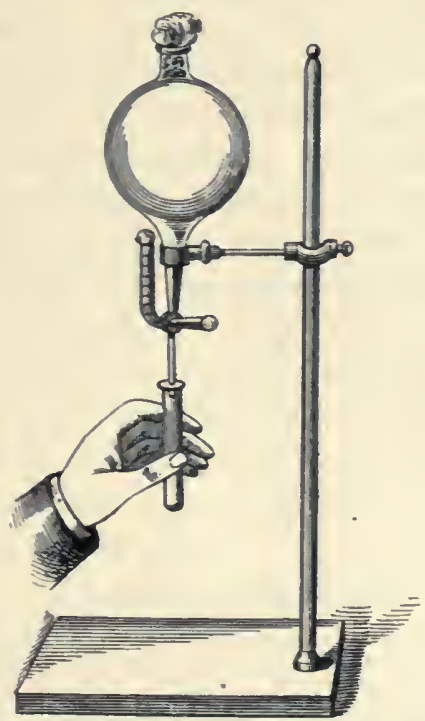

Funnel for flling tubes with culture medis.

Care should be taken that none of the medium is dropped upon the mouth of the test-tube, otherwise the cotton plug becomes adherent to it, and is not only difficult to remove, but presents a very untidy appearance, and interferes, indeed, with the proper manipulations.

As soon as the tubes have been filled they are to be 
sterilized in the steam sterilizer for fifteen minutes on each of three successive days. During the intervening days they may be kept at the ordinary room temperature.

When sterilization is complete, and the medium in the tubes is still liquid, some of them may be placed in a slanting position, at an angle of about ten degrees with the surface on which they rest, and the medium allowed to solidify in this position. These are for the so-called slant-cultures. The remainder may solidify in the erect position; these serve for making plates.

For Esmarch tubes not more than 5 c.c. of material should be placed in each tube, as more than this renders it difficult to distribute the gelatin evenly over the inner surface of the tubes when they are rolled. 


\section{CHA PTER VII.}

Technique of making plates-Esmarch tubes, Petri plates, etc.

Plates. - The plate method can be practised with both agar-agar and gelatin. It cannot be practised with blood-serum, because the serum, when once solidified, cannot be again liquefied.

Plates are usually referred to as "a set." This term implies three individual plates, each representing the mixture of organisms in a higher state of dilution. The first plate is known usually as "the original," or "plate 1," the first dilution from this as "plate 2," and the second as "plate 3."

In the preparation of a set of plates the following are the steps to be observed:

Three tubes, each containing from 7 to 9 c.c. of gelatin or agar-agar, are placed in the warm water-bath until the medium has become liquid. If agar-agar is employed, this is accomplished at the boiling-point of water; if gelatin is used, a much lower temperature suffices $\left(35^{\circ}-40^{\circ} \mathrm{C}\right.$.). When liquefaction is complete the temperature of the water, in the case of agar-agar, must be reduced to $41^{\circ}-42^{\circ} \mathrm{C}$., at which temperature the agar-agar remains liquid, and the organisms may be introduced into it without fear of destroying their vitality. The medium being now liquid and of the proper temperature, a very small portion of the mixture of organisms to be studied is takeu up with a sterilized, 
looped platinum wire (Fig. 23, a). This is nothing more than a piece of platinum wire about $5 \mathrm{~cm}$. long, twisted into a small loop at one end and fused into a bit of glass rod, which acts as a handle, at the other extremity. This loop is one of the most useful of bacteriological instruments, as there is hardly a manipulation in the work into which it does not enter. Under no conditions is it to be employed without having been passed through the gas-flame until quite hot; this is for the purpose of sterilization. One should form a habit

FIG. 23.

Looped and straight platinum wires in glass handles.

of never taking up one of these platinum-wire needles, as they are called, for they are curved and straight as well as looped (Fig. 23, b), without passing it through the flame, and the sooner the beginner learns to do this as a matter of reflex, the sooner does he rid himself of one of the possible sources of error in his work. It must be remembered, though, that it should not be used when hot, otherwise the organisms taken upon it are killed by the high temperature; after sterilization in the flame one waits for a few seconds until it is cool before using.

The bit of material under consideration is transferred with the sterilized loop into tube No. 1, "the original," where it is carefully disintegrated by gently rubbing it against the sides of the tube. The more carefully this is done the more homogeneous will be the distribution 
of the organisms and the better the results. The loop is then again sterilized, and three of its loopfuls are passed, without touching the sides of the tube, from "the original" into tube No. 2, where they are carefully mixed. Again the loop is sterilized, and again three dips are made from tube 2 into tube 3 . This completes the dilution. The loop is now sterilized before laying it aside.

FIG. 24.

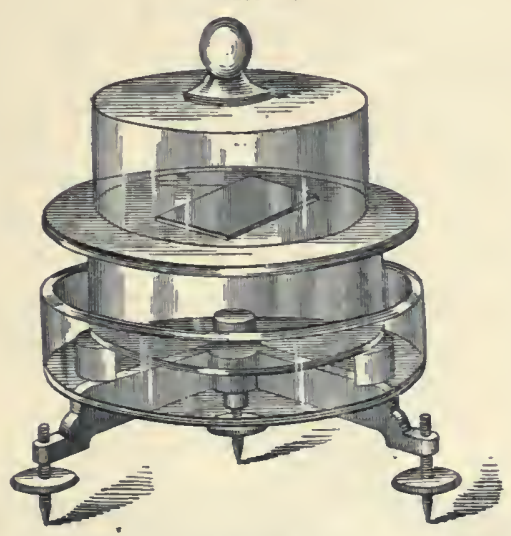

Levelling-tripod with glass chamber for plates.

During this manipulation, which must be done quickly if agar-agar be employed, the temperature of the water in the bath in which the tubes stand should never get lower than $39^{\circ} \mathrm{C}$, and never higher than $43^{\circ} \mathrm{C}$. If it falls too low, below $38^{\circ} \mathrm{C}$, , the agar-agar gelatinizes, and can only be redissolved by a temperature that would be destructive to the organisms which may have been introduced into the tubes. This is not of so much moment with gelatin, as it may readily be redissolved at a temperature not detrimental to the 
organisms with which the tubes may have been inoculated.

The Cooling-STAge and Leveluing-Tripod.While the medium of which the plates are to be made is melting, it is well to arrange the cooling-stage (Fig. 24) upon which the gelatin or agar-agar is to be subsequently solidified.

This stage consists of a glass dish filled with icewater and covered with a ground-glass plate, which in turn has a dome-shaped cover. The dish rests upon a tripod which can be brought to an exact level, as indicated by the spirit-level, by raising or lowering its legs by means of thumb-screws, with which they are provided. Three stages are usually employed. When ready for use they should be exactly level.

The Glass Plates.-On each of the stages is to be placed a glass plate upon which the liquefied gelatin or

FIG. 25.

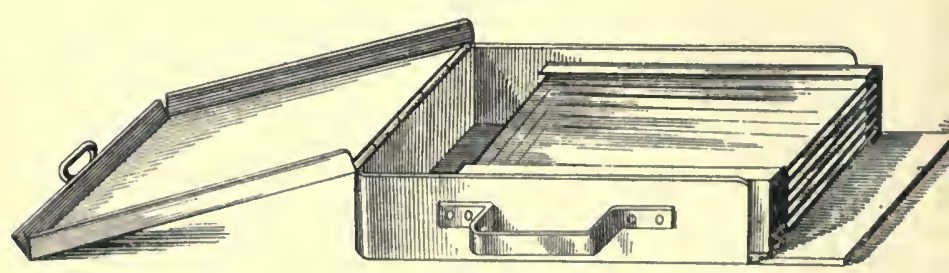

Russia irou box for holding plates, ete., during sterilization in dry heat.

agarr-agar is to be poured and allowed to solidify. It is, therefore, necessary that the plates should not only be sterile when placed upon the stages, but they should be earefully protected by a cover against dust and bacteria from outside somrees during manipulation.

$\Lambda$ number of plates at a time are usnally sterilized in 
the dry sterilizer at a temperature of $150^{\circ}$ to $180^{\circ} \mathrm{C}$. for one hour. During sterilization and until used they are retained in an iron box (Fig. 25), which is especially designed for the purpose.

They should never be placed upon the stage until cold; otherwise they crack.

When the plates which have been placed upon the stages are quite cold the melted gelatin or agar-agar in the tubes which represent the three dilutions should be poured upon them, each tube being emptied upon a separate plate. If the medium is quite fluid, it spreads over the surface of the plates in a thin, even layer. Sometimes it may be more evenly spread as it flows from the tube by the aid of a sterilized glass rod.

FIG. 26.

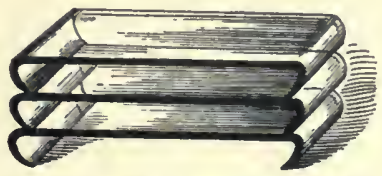

Glass benches for supporting plates.

As the contents of each tube are emptied upon a plate the cover of the cooling-stage is quickly replaced and the plate allowed to stand until the gelatin or agar-agar is quite solid. This takes longer with gelatin than with agar. When quite solid they are placed upon little glass benches (Fig. 26), and each bench is labelled with the number of the plate in the series of dilutions. The benches, with the plates upon them, are then piled one above the other in a glass dish, the so-called "culturedish," in which the plates are to be kept during the growth of the bacteria. The benches are sterilized before using, in the way given for the plates. 
Culiture-Dish.-This dish, which is about $22 \mathrm{~cm}$. in diameter and has vertical sides of about $6 \mathrm{~cm}$. in height, is provided with a cover of exactly the same design, but of a little larger diameter. This cover, when placed upon the dish containing the plates, fits over it and prevents the access of dust. Prior to using, the dish and eover should have been disinfected for onehalf an hour with $1: 1000$ sublimate, and then all the sublimate solution allowed to drain from it.

In the bottom of this dish is sometimes placed a disk of sterilized filter-paper moistened with sterilized water, which serves to prevent the drying of the medium. This, however, is not necessary.

If agar-agar be employed, the dish and its eontents may be kept at a temperature of $37^{\circ}-38^{\circ} \mathrm{C}$.; if gelatin, the temperature at which the plates are to be maintained should not be over $22^{\circ} \mathrm{C}$, , otherwise the gelatin becomes liquefied and the plates are rendered useless.

When development has oecurred the object of the dilution will be easily seen, and the different species of bacteria in the mixture will be recognized by differences in the charaeter of the eolonies growing from them.

This, in short, is the plate method of Koch for the separation of the individual species contained in a mixture of bacteria. Many modifications of this method exist; all, however, are based upon the same principles. The modifications have for their object the aecomplishment of the same end, but with a smaller armamentarium of apparatus, and in general the one or the other of these modifications has entirely supplanted the original plate method as practised and reeommended by Kkoch. 
Petri's Modification of the Plate Method.The modification which approaches nearest to the original method, and at the same time lessens very materially the number of steps in the process, is that suggested by Petri. It consists in substituting for the plates small, round, double glass dishes, which have about the same surface-area as the plates. The liquid medium may be poured directly into these little dishes without their being exactly level. Each dish acts as a plate. Their covers are then to be replaced, and they are set aside for observation. In all other respects the steps are the same as those given for Koch's original method. Petri's dishes are flat, double dishes of glass

Fig. 27 .

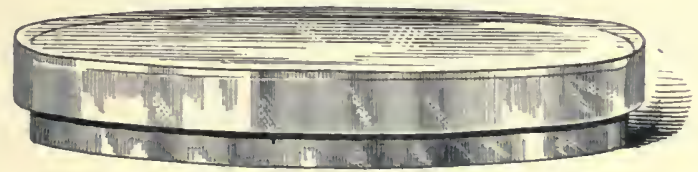

Petri double dish, now generally used instead of plates.

(Fig. 27). They are about $8 \mathrm{~cm}$. in diameter and about 1.5 to $2 \mathrm{~cm}$. in height, the walls being vertical. They may readily be sterilized either by the hot-air or steam methods of sterilization. They are very useful for this work, as they do away with the necessity for the cooling-stage and levelling-tripod, though in warm weather the cooling-stage may be used to hasten the solidification of gelatin. A cooling-stage of very convenient design for use with these dishes consists of a closed, flat metal box, either of copper or block tin, and either round or square in shape, so arranged that it can be 
filled with cold water, or that eold water can constantly be passed throngh it by means of a rubber connection with a spigot. The inlet for the water should be just above the bottom of the box, and the outlet just beneath the top and slightly turned upward and then downward, so as to insure the complete filling of the space with water. The box should be sufficiently strong to resist the pressure of the water. A convenient size is from 20 to 25 $\mathrm{cm}$. in diameter, and of about 1.5 to $2 \mathrm{~cm}$. high. It is simple in eonstruetion, and can be made by any copper spinner. An idea of its construetion is given in Fig. 28.

FIG. 28.

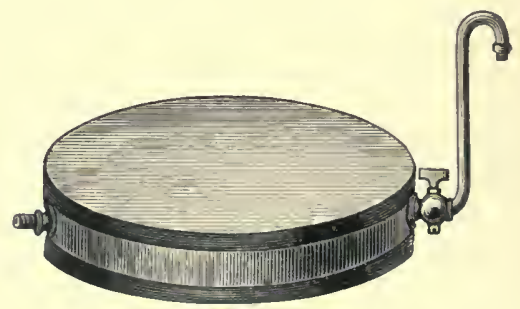

Metal cooling-stage.

When gelatin or agar-agar is to be cooled it is only necessary to place the dishes containing it on top of this box and start cold water cireulating through it.

Esmarch Tubes. - The modifieation of Koch's method which insures the greatest secnrity from contamination by outside organisms and requires the smallest supply of apparatus is that suggested by v. Esmarch. It differs from the other methods thus: the dilutions having been prepared in tubes containing a smaller amount of medium than usual-as a rule, not more than 5 to 6 c.c. - are, instead of being poured out upon 
plates or into dishes, spread over the immer surface of the tube containing them, and without removing the cotton plugs are caused to solidify in this position. The tubes then present a thin cylindrical lining of gelatin or agar-agar, upon which the colonies develop. In all other respects the conditions for the growth of the organisms are the same as in flat plates.

Esmarch directs that after completion of the dilutions the tops of the cotton plugs in the test-tubes should be cut off flush with the mouths of the tubes and sterilized rubber caps be placed over them. They are then to be held in the horizontal position and twisted between the fingers upon their long axis under icewater. The gelatin becomes solidified thereby and adheres to the sides of the tube. When the gelatin is quite hard the tubes are removed from the water, wiped dry, the rubber caps removed, and the tubes set aside for observation.

For some time past we have deviated from the direction given by v. Esmarch for this part of his method, and instead of rolling the tubes under ice-water, we roll them upon a block of ice (Fig. 29), after the method devised by Booker in the Pathological Laboratory of the Johns Hopkins University in 1887. In this method a small block of ice only is needed. It is arranged nearly level, and is held in position by being placed in a dish upon a towel. A horizontal groove is melted in the surface of the ice with a test-tube full of hot water. The tubes to be rolled are then held in an almost, not quile, horizontal position and twisted between the fingers until the sides are moistened by the contents to within about $1 \mathrm{~cm}$. of the cotton plug, care being taken that the gelatin does nol touch the cotton; otherwise the latter 
becomes adherent to the sides of the tube and is difficult to remove. The tube is then placed in the groove in the ice and rolled, neither rubber cap nor cutting off of the cotton plug being necessary.

FTG. 29.

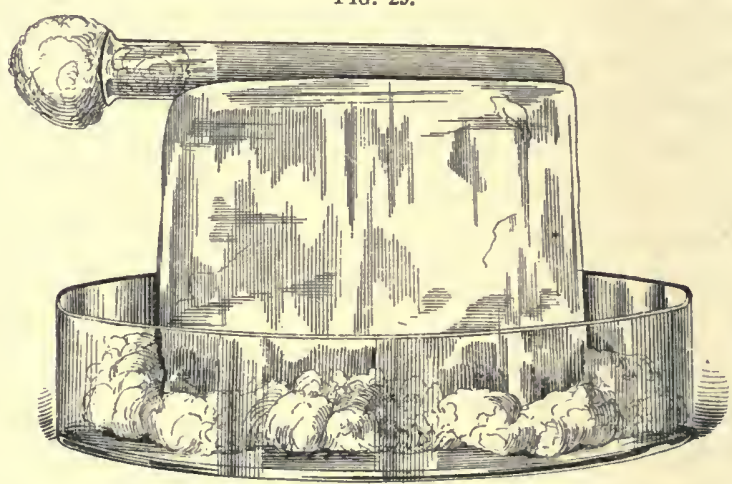

- Demonstrating Booker's method of rolling Esmarch tubes on a block of ice.

The advantages of this process over that followed by v. Esmarch are that it requires less time, is clcaner, no rubber caps are needed, the rolled tubes are more regular, and the gelatin does not touch the cotton plug, as is always the case in the tubes rolled under water, because of the impossibility of holding them steady at one level.

There is an impression that Esmarel tubes are not a suecess when made from ordinary mutrient agar-agar because of the tendency of this medium to collapse and fall to the bottom of the tube. This slipping down of the agar-agar is due to the water that is squeezed from it during solidification getting between the medium and the walls of the tube. This can easily be overcome by allowing the rolled tubes to remain in nearly 
a horizontal position, the cotton end of the tube about $1 \mathrm{~cm}$. higher than the bottom of the tube, for twentyfour hours after rolling them. During this time the edge of the agar-agar nearest the cotton plug becomes dried and adherent to the walls of the tube, while the water collects at the most dependent point-i.e., the bottom of the tube. After this they may be retained in the upright position without fear of the agar-agar slipping down. We have followed this process for several years with entire satisfaction. ${ }^{1}$ In all these processes, if the dilutions of the number of organisms have been properly conducted, the results will be the same. The original plate or tube, as a rule, will be of no use because of the great number of colonies in it. Plate or tube No.' 2 may be of service, but plate or tube 3 will nsually contain the organisms in such small numbers that the colonies originating from them will have nothing to prevent their characteristic development.

For reasons of economy the "original," tube 1, is sometimes substituted by a tube containing normal saltsolution ( 0.6 to 0.7 per cent. of sodium chloride in water), which is thrown aside as soon as the dilutions are completed, and only plates or tubes 2 and 3 are made.

Another method for the separation of bacteria and their isolation as single colonies consists in the making of dilutions upon the surface of solid media, such as potato, coagulated blood-serum, agar-agar, and gelatin. For the performance of this method one selects a number of tubes containing the medium to be employed in

1 The impression that agar-agar is not suitable for rolled tubes was shown to be erroneous, and the abore method was developed in the Pathological Laboratory of the Johns Bopkins University. 
a slanting position. With a platinum needle a bit of the substance to be studied is smeared upon tube No. 1 ; without sterilizing the needle it is passed thoroughly over the surface of the medium in tubes 2,3 , 4, etc., etc., in succession. When development has occurred essentially the same conditions as regards separation of the colonies will be found as is the case when plates are poured. If a slanted medium be employed, about the most dependent angle of which water of condensation has accumulated, as blood-serum, agar-agar, and potato, the dilutions may be made in this fluid, and this is then to be earefully smeared over the solid surface of the medium. The tubes thus treated should be kept in an upright position to prevent the fluid from flowing over the surface. Wheu sufficiently developed, single colonies may be isolated from tubes prepared in this manner with comparative ease. (See also method for the isolation of bacillus diphtherice on blood-serum.) 


\section{CHAPTER VIII.}

The incubating oven - Gas-pressure regulator - Thermo-regulator - The safety burner employed in heating the incubator.

The Incubator. - When the plates have been made it must be borne in mind that for the development of certain forms of bacteria a higher temperature is necessary than for the growth of others. The pathogenic or disease-producing organisms all grow more luxuriantly at the temperature of the human body $\left(37.5^{\circ} \mathrm{C}\right.$. $)$ than at lower temperatures; whereas, with the ordinary saprophytic forms almost any temperature between $18^{\circ} \mathrm{C}$. and that of the body is suitable. It therefore becomes necessary to provide some place in which a constant temperature favorable to the growth of the pathogenic organisms can be maintained. For this purpose there have been devised a number of different forms of apparatus. Fundamentally they are all based upon the same principles, however, and a general description of the essential points involved in their construction will be all that is needed here.

This apparatus has the names thermostat, incubator, and brooding-oven. It is a copper chamber (Fig. 30) with double walls, the space between which is filled with water. The incubating chamber may be opened or closed by a closely fitting donble door,inside of which is usually a false door of glass through which the contents of the chamber may be inspected without actually opening it. The whole apparatus is encased in either 
asbestos boards or thick felt, to prevent radiation of heat and consequent fluctuations in temperature. In the top of the chamber is a small opening through which a thermometer projects into its interior. At either corner, leading into the space containing the water, are

FIG. 30.
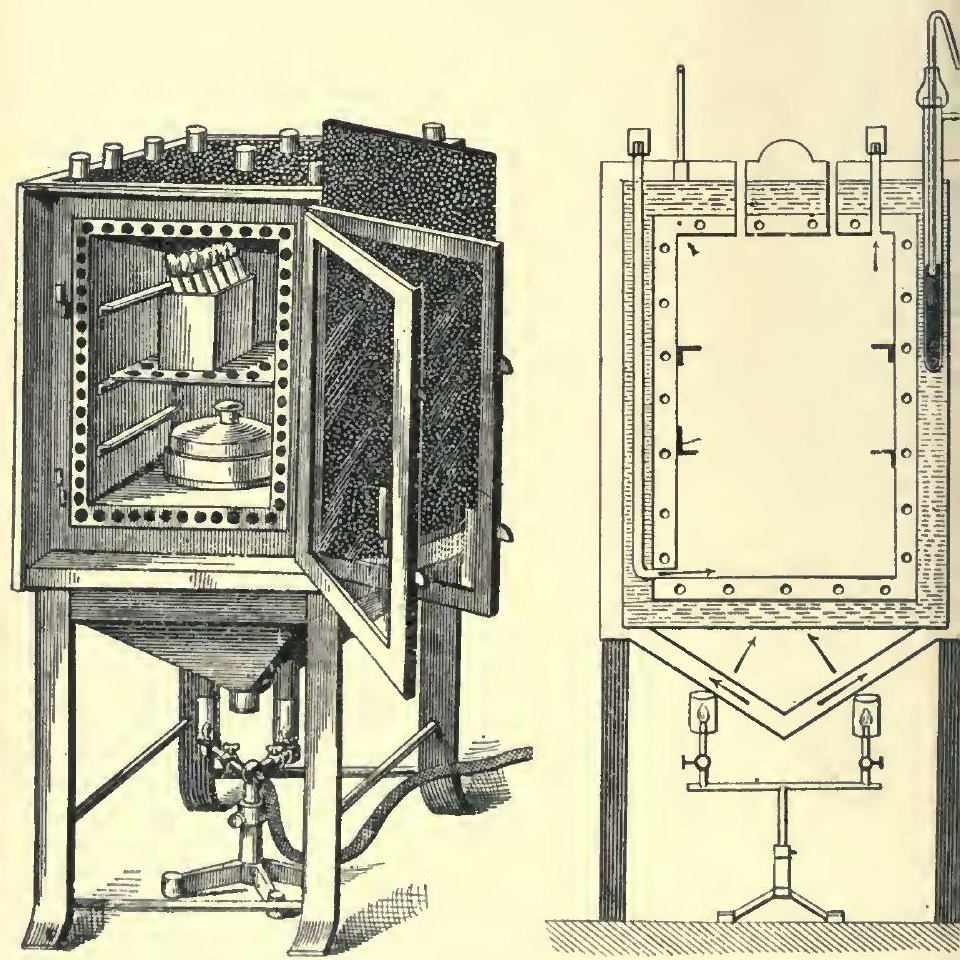

lncubator used in bacteriological work.

other openings for the reception of another thermometer and a thermo-regulator, and for refilling the apparatus as the water evaporates. On the side is a water-gange 
for showing the level of the water between the walls. The object of the water chamber, which is formed by the double wall arrangement, is to insure, by means of the warmed water, an equable temperature at all parts of the apparatus-at the top as well as at the sides, back, and bottom-and the apparatus should be kept filled with water, otherwise the purpose for which it is constructed will not be accomplished. When the chamber between the walls is filled with water heat is supplied from a gas-flame placed beneath it.

FIG. 31.

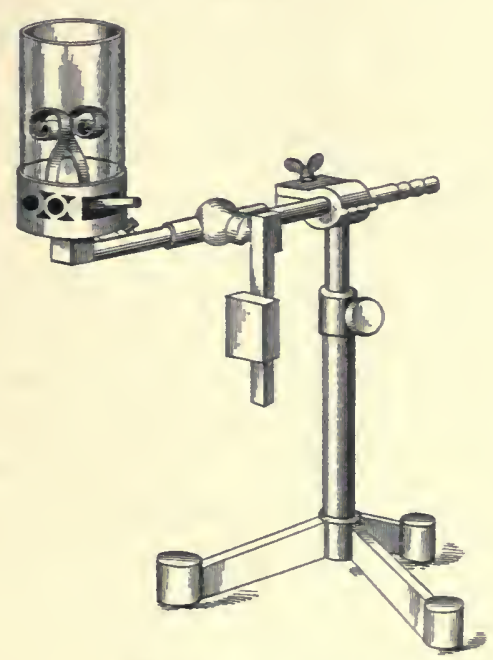

Koch's safety burner.

The burner employed in heating the incubator was originally devised by Koch, and is known as "Koch's safety burner"' (Fig. 31). . It is a Bunsen burner provided with an arrangement for automatically turning 
off the gas-supply and thus preventing accidents should the flame become extinguished at a time when no one is near. The gas-cock by which the gas is turned on and off is provided with a long arm which is weighted, and which, when the gas is turned on and burning, rests upon an arm attached to the side of a revolving, horizontal disk that is connected with the free ends of two metal spirals which are fixed by their other ends in opposite directions on either side of the flame and heated by it. If by draughts or any other accident the flame becomes extinguished, the metal spirals cool, and in cooling contract, twist the horizontal disk in the opposite direction, and allow the weighted arm of the gas-cock to fall. By its falling the gas-supply is turned off.

Thermo-regulators. - The regulation and maintenance of the proper temperature within the incubator are accomplished by the employment of an antomatic thermo-regulator.

The common form of thermo-regulator used for this purpose is constructed upon principles involving the expansion and contraction of fluid substances under the influence of heat and cold. By means of this expansion and contraction the amount of gas passing from the source of supply to the burner may be either diminished or increased as the temperature of the substance in which the regulator is placed either rises or falls.

The simplest form of thermo-regulator which serves to illustrate the principles involved is seen in Fig. 32.

It consists of a glass cylinder $e$, having a communicating branch tube $b$, and rubber stopper $f$, through which projects the bent tube $a$. The tube $a$ is ground to a slanting point at the extremity which projects into 
the tube $e$, and is provided a short distance above this point with a capillary opening, $g$, in one of its sides.

When ready for use the cylinder $e$ is filled with mercury up to about the level shown in the figure. It is

Fra. 32.

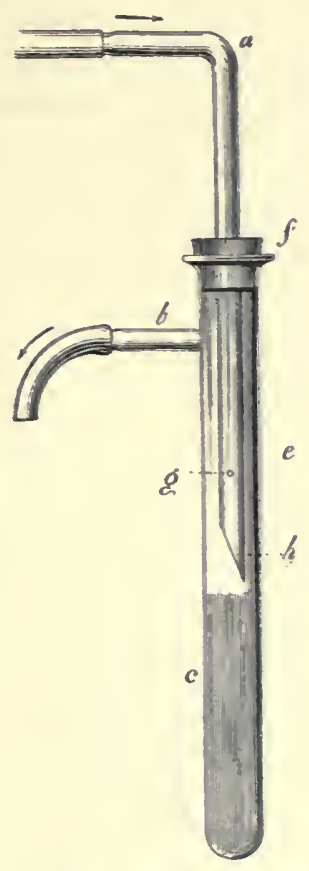

Mercurial thermo-regulator.

then allowed to stand, or is suspended, in the bath the temperature of which it is to regulate. The rubber tubing coming from the gas-supply is attached to the outer end of the glass tube $a$, and the tube going to the burner is slipped over the branch tube $b$. The gas is 
turned on and the burner lighted and placed under the bath. The gas now streams through the tube $a$ into the cylinder $e$ and out at $b$ to the burner, but as the temperature of the bath rises the mercury contained in the cylinder $e$, under the influence of the elevation of temperature, begins to expand, and, as a continuous rise in temperature proceeds, the expansion of the fluid accompanies it and gradually closes the slanting opening $h$ of tube $a$. In this way the supply of gas becomes diminished and the rise in temperature of the bath will be less rapid, until finally the opening at $h$ will be closed entirely, when the supply of gas to the burner will now be limited to that passing through the capillary opening $g$. This is not sufficient to maintain the highest temperature reached, and as cooling begins a gradual coutraction of the mercury occurs until there is again an outflow of gas from the opening $h$, when again the temperature rises. This contraction and expansion of the mercury in the regulator continues until eventually a point is reached at which its position in the cylinder $e$ allows of the passage of just enough gas from the opening $h$ to maintain a constant temperature; and, therefore, a constant degree of expansion of the mercury in the tube $e$. This, in short, is the principle on which thermo-regulators are constructed; but it must be borne in mind that a great deal of detail exists in the construction of an accurate instrument. The number of different forms of this apparatus is comparatively large, and each form has its special merits.

The value-that, is, the delicacy - of the thermo-regulator depends upon a number of factors, all of which it would be useless to introduce into a book of this kind; but in general it may be said that the essential points to 
be observed in selecting a thermo-regulator depend in the main upon the temperatures to which it is to be applied. For low temperatures, regulators containing such fluids as ether, alcohol, and calcium chloride solution, which expand and contract rapidly and regularly under slight variations in temperature, are commonly employed; whereas for temperatures approaching the boiling-point of water mercury is most frequently used.

The temperature of the incubator is to be regulated, then, by the use of some such form of apparatus as that just described. It should be of sufficient delicacy to prevent a fluctuation of more than $0.2^{\circ} \mathrm{C}$. in the temperature of the air within the chamber of the apparatus.

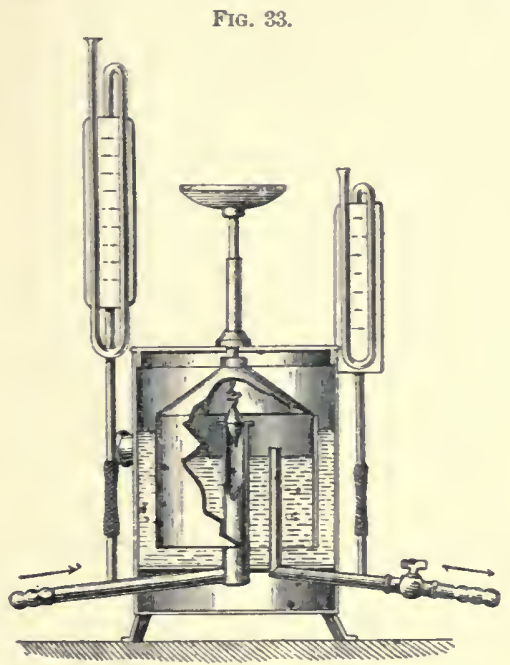

Moitessier's gas-pressure regulator.

Gas-Pressure Regulators.-A gas-pressure regulator is not rarely intervened between the gas-supply 
and the thermo-regulator. This apparatus has for its object the maintenance of a constant pressure of the gas going to the thermo-regulator. There are several instruments of this form in use, but they do not accomplish the object for which they are designed.

The instrument most commonly employed, the apparatus of Moitessier (Fig. 33), is based on somewhat the same principles as the large regulators seen at the manufactories of illuminating gas. Such apparatus act very well when employed on the large scale, as one sees them at the gas-works; but when applied to the limited and sndden fluctuations seen in the gas coming from an ordinary gas-cock are practically useless. They are too gross in their construction, and act only under comparatively great and gradual fluctuations in pressure. If a good form of thermo-regulator be employed, there is no necessity for the use of any of the pressure-legulators thus far introduced. 


\section{CHAPTER IX.}

The study of colonies-Their naked-eye peculiarities and their appearance under different conditions-Differences in the structure of colonies from different species of bacteria-Stab-cultures-Slant-cultures.

THE plates of agar-agar which have been prepared from a mixture of organisms and have been placed in the incubator, and those of gelatin which have been maintained at the ordinary temperature of the room, are usually ready for examination after twenty-four to forty-eight hours. They will be found marked here and there by small points or little islands of more or less opaque appearance. In some instances these will be so trausparent that it is with difficulty one can see them with the naked eye. Again, they may be of a dense, opaque appearance, at one time sharply circumscribed and round, again irregular in their ontline; here a point will present one color, there perhaps another. On gelatin some of the points will be seen to be lying on the surface of the medium, others will have sunk into little depressions, while at still other points the clear gelatin will be marked by more or less saucershaped pits containing opaque fluid.

Place the plate containing these points upon the stage of the microscope and examine them with a lowpower objective, and again differences will be observed. Some of these minute points will be finely granular, others coarsely so; some will present a radiated appearance, while a neighbor may be concentrically arranged; 
here nothing particularly characteristic will present, there the point may resolve itself into a little mass having somewhat the appearance of a very small pellicle of raw cotton. All these differences, and many more, aid us in saying that these little points must be different in their nature. With a pointed platinum needle take up a bit of one of these little islands, prepare it for microscopic examination (see chapter on stained cover-slip preparations), and examine it under the high-power oilimmersion objective, with access of the greatest amount of light afforded by the illuminator of the microscope. The preparation will be seen to be made up entirely of bodies of the same shape; they will all be spheres, or ovals, or rods, but not a mixture of these forms, if proper eare in the manipulation has been taken. Examine in the same way a neighboring spot which possesses different naked-eye appearances, and it will often be found to consist of bodies of an entirely different appearance from those seen in the first preparation.

These spots or islands on the surface of the plates are colonies of bacteria, differing severally, not only in ontward appearances, the one from the other, but, as our cover-slip preparations show, in the morphological characteristics of the individual organisms composing them.

If from one of these colonies a second set of plates be prepared, the peeuliarities which were first observed in this colony will be reproduced in all of the new set of colonies which develop; each will be found to consist of the same organisms as the colony from which the plates were made. In other words, these peculiarities are constant under like conditions.

With all organisms differences in the appearance of the colonies dependent upon their location in the me- 
dium ean usually be detected. When deep down in the medium, owing to surrounding pressure, they are quite round, oval, or lozenge-shaped; whereas when they are on the surface of the gelatin or agar they may take quite a different form. This is purely a mechanical effect due to the pressure of, or resistance offered by, the medium surrounding them, and is always to be borne in mind, otherwise errors are apt to arise.

Pure Cultures. - If from one of these small colonies a bit be takeu upon the point of a sterilized platinum needle and introduced into the tube of sterilized gelatin or agar-agar, the growth that results will be what is known as a "pure culture," the condition to which all organisms must be brought before a systematic study of their many peculiarities is begun. Sometimes several series of plates are necessary before the organism can be obtained pure, but by patiently following this plan the results will ultimately be satisfactory.

Test-tube Culatures; Stab-cultures; SimearCULTURES.-After separating the organisms the one from the other by the plate method just described, they must be isolated from the plates as pure stab- or smearcultures.

This is done in the following way: decide upon the colony from which the pure culture is to be made. Select preferably a small colony and one as widely separated from other colonies as possible. Sterilize in the gas-flame a straight platinum-wire needle. The glass handie of the needle should be drawn through the flame as well as the needle itself, otherwise contamination from this source may occur. When it is cool, which is in five or ten seconds, take up carefully a portion of the colony. Guard against touching anything but the colony. If 
during manipulation the needle touches anything else whatever than the colony from which the culture is to be made, it must be sterilized again. This holds not only for the time before touching the colony, but also during its passage into the test-tube from the colony, otherwise there is no guarantee that the growth resulting from the inoculation of this bit of colony into a fresh sterile medium will be pure.

In the meantime have in the other hand a test-tube of sterile medium: gelatin, agar-agar, or potato. This tube is held across the palm of the hand in an almost horizontal position with its mouth pointing out between the thumb and index finger and its contents toward the body of the worker. With the disengaged fingers of the other hand holding the needle the cotton plug is removed from the tube by a twisting motion and placed between the index and second fingers of the hand holding the tube, in such a way that the portion of the plug which fits into the mouth of the test-tube looks toward the dorsal surface of the hand and does not touch any portion of the hand; this is accomplished by placing only the overhanging portion of the plug between the fingers. The needle containing the bit of colony is now to be thrust into the medium in the tube if a stab-culture is desired, or rubbed gently over its surface if a smearculture is to be made. The needle is then withdrawn, the cotton plug replaced, and the needle sterilized before it is laid down. Neither the needle nor its handle should touch the imner sides of the test-tube if it can be avoided.

The tube is then labelled and set aside for observation. The growth which appears in the tube after twenty-four to thirty-six hours will be a pure culture of the organisms of which the colony was composed. 
TEST-TUBE, STAB-AND SMEAR-CULTURES. 137

Cultures of this form are not only useful as a means of preserving the different organisms with which we may be working, but serve also to bring out certafn

Fig. 34.

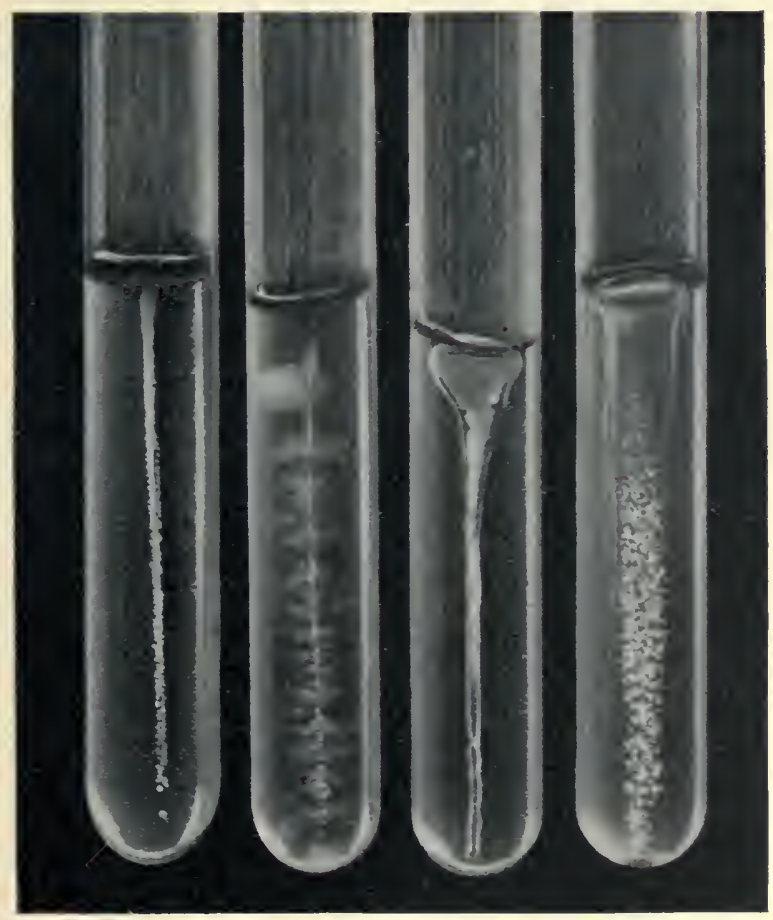

Series of stab-cultures in gelatin, showing modes of growth of different species of bacteria.

characteristics of different organisms when grown in this way.

If gelatin be employed and the organism which has been introduced into it possesses the power of bringing 
about liquefaction, it will soon be diseovered that this result is by no means of the same appearance for all organisms. Some organisms cause a liquefaetion which spreads across the whole upper surface of the gelatin and continues gradually downward; again, it oecurs in a funnel-shape, the broad end of the funnel being uppermost and the point downward, corresponding to the track of the needle. At times a stocking- or sac-like liquefaetion may be noticed. (See Fig. 34.)

Note.-Obtain a number of organisms from different sources in pure eultures by the method given. Plant them as pure cultures, all at the same time, in gelatinpreferably gelatin of the same making-retain them under the same conditions of temperature, and sketch the finer differenees in the way in which liquefaetion oceurs. 


\section{CHA PTER X.}

Methods of staining - Solntions employed - Preparation and staining of cover-slips-Preparation of tissues for section-cntting-Staining of tissuesSpecial staining-methods.

THE entire list of solutions and methods that are recommended for the staining of bacteria is not essential to the work of the beginner, so that only those which are of most common application will be given in this book. In general, it suffices to say that bacteria stain best with watery solutions of the basic aniline dyes; and of these, fuchsin, gentian-violet, and methylene-blue are those most frequently employed.

In practical work bacteria require to be stained in two conditions: either dried upon cover-slips and then stained, or stained in sections of tissues in which they have been deposited during the course of disease. In both processes the essential point to be borne in mind is that the bacteria, because of their microscopic dimensions, require to be more conspicuously stained than the surrounding materials upon the cover-slips or in the sections, otherwise their differentiation is a matter of the greatest difficulty, if not of impossibility. For this reason, especially in the case of section staining, it frequently becomes necessary to decolorize the tissues after removing them from the staining-solutions, in order to render the bacteria more prominent, and for this purpose special methods, which provide for decolorization of the tissues without robbing the bacteria of their color, are 
employed. The ordinary method of cover-slip examination of bacteria, constantly in use in these studies, is performed in the following way:

Cover-slip Preparations.-In order that the distribution of the organisms upon the cover-slips may be uniform and in as thin a layer as possible it is essential that the slips should be elean and free from grease. For cleansing the slips several methods may be employed.

'The simplest plan with new cover-slips is to immerse them for a few hours in strong nitric acid, after which they are rinsed in water, then in alcohol, then ether, and, finally, they may be kept in alcohol to which a little ammonia has been added. When about to be used they should be wiped dry with a clean cotton or silk handkerchief.

If the slips have been previously used, boiling in strong soap solution, followed by rinsing in clean warm. water, then treated as above, renders them clean enough for ordinary purposes.

A method commonly employed is to remove all coarse adherent matter from slips and slides by allowing them to remain for a time in strong nitric acid or sulphuric aeid. They are removed from the acid after several days, rinsed off in water, and treated as above. Knauer has recently suggested the boiling of soiled cover-slips and slides for from twenty to thirty minutes in a 10 per cent. watery solution of lysol, after which they are to be earefully rinsed in water until all trace of the lysol has disappeared. They are then to be wiped dry with a clean handkerehief.

Loffler's method, which provides for the complete removal of all grease, is to warm the cover-slips in concentrated sulphuric acid for a time, then rinse them in 
water, after which they are kept in a mixture of equal parts of alcohol and ammonia. They are to be dried on a cloth from which all fat has been extracted.

Steps in making the preparations. Place upon the centre of one of the clean, dry cover-slips a very small drop of distilled water or physiological salt-solution. With a platinum needle, which has been sterilized in the gas-flame just before using and allowed to cool, take up a very small portion of the colony to be examined and mix it carefully with the drop on the slip until there exists a very thin homogeneous film over the larger part of the surface. This is to be dried upon the slip by either allowing it to remain upon the table in the horizontal position under a cover, to protect it from dust, or by holding it between the fingers (not with the forceps), at some distance above the gas-flame, until it is quite dry. If held with the forceps over the flame at this stage, too much heat may be unconsciously applied, and the morphology of the organisms in the preparation distorted. When held between the fingers with the thin layer of bacteria away from the flame no such accident is likely to occur. When the whole pellicle is completely dried the slip is to be taken up with the forceps, and, holding the side upon which the bacteria are deposited away from the direct action of the flame, is to be passed through the flame three times, a little more than one second being allowed for each transit. Unless the preliminary drying at the low temperature has been complete, the preparation will be rendered worthless by the subsequent "fixing" at the higher temperature, for the reason that the protoplasm of bacteria when moist coagulates at these temperatures, and in doing so the normal outline of the cells is 
altered. If carefully dried before fixing, this does not oceur and the morphology of the organism remains unchanged. A better plan for the process of fixing is to employ a copper plate about $35 \mathrm{~cm}$. long by $10 \mathrm{~cm}$. wide by $0.3 \mathrm{~cm}$. thick. This plate is laid upon an iron tripod and a small gas-flame is placed beneath one of its extremities. By this arrangement one can get a graduated temperature, beginning at the point of the plate above the gas-flame where it is hottest, and becoming gradually cooler toward the other end of the plate, which may be of a very low temperature. By dropping water upon the plate, beginning at the hottest point and proceeding toward the cooler end, it is easy to determine the point at which the water just boils; it is at a little below this point that the eover-slips are to be placed, bacteria side up, and allowed to remain about ten minutes, when the fixing will be eomplete. The same may be accomplished in a small eopper drying-oven, which is regulated to remain at the temperature of $95^{\circ}$ to $98^{\circ} \mathrm{C}$. In very particular work this plan is to be preferred to the process of passing the eover-slips through the flame, as the organisms are always subjected to the same degree of heat, and the distortions which sometimes oceur from the too great and irregular application of high temperatures may in part be eliminated, or, if not, will be more nearly constant. The fixing consists in drying or coagulating the gelatinous envelope surrounding the organisms, by which means they are eaused to adhere to the surface of the cover-slip. When fixed, the staining is usually a simple matter. The majority of baeteria with whieh the beginner will have to deal stain readily with solutions of any of the basic aniline dyes. 
To stain the fixed cover-slip preparation it is taken by one of its edges between the forceps, and a few drops of a watery solution of fuchsin, gentian-violet, or methylene-blue are placed upon the film and allowed to remain there twenty to thirty seconds. The slip is then carefully rinsed in water, and without drying is placed bacteria down upon a slide; the excess of water is taken up by covering it with blotting-paper and gently pressing upon it, and the preparation is ready for examination.

Another plan that is sometimes used is to bring the slip upon the slide, bacteria down, without rinsing off the staining-fluid; the excess of fluid is removed with blotting-paper and the preparation is ready for examination with the microscope. This method is satisfactory and time-saving, but must always be practised with care. The staining-fluid should always be carefully filtered before using, to rid it of insoluble particles which might be taken for bacteria. If upon examination the preparation proves to be of particular interest, so that it is desirable to preserve it, then it is to be mounted permanently. The drop of immersion oil is to be removed from the surface of the slip with blotting-paper, and the slip loosened, or rather floated, from the slide by allowing water to flow around its edges. It is then taken up with the forceps, carefully deprived of the water adhering to it by means of blotting-paper and then allowed to dry. When dry it is mounted in xylol-Canada-balsam by placing a small drop of the balsam upon the surface of the film, and then inverting the slip upon a clean glass slide. It is sometimes desirable to have the balsam harden quickly, and a method that is commonly employed to induce this is as follows: 
the slide, held by one of its ends between the fingers, is warmed over the gas-flame until quite hot; a drop of balsam is then placed on the centre of it, and it is again warmed; the cover-slip is then placed in position, and when the balsam is evenly distributed the temperature is rapidly reduced by rubbing the bottom of the slide with a towel soaked in cold water. Usually the preparation is firmly fixed after this treatment; a little practice is necessary, however, in order not to overheat and not to crack the slide. The method is applicable only to cover-slip preparations, and cannot be safely used with tissues.

Impression Cover-slip Preparations. - The impression preparations differ in value from the ordinary cover-slip preparations only in one respect: they present an impression of the organisms as they were arranged in the colony from which the preparation is made. They are made by gently eovering the colony with a thin, clean cover-slip, lightly pressing upon it, and, without moving the slip laterally, lifting it up by one of its edges. The organisms adhere to the slip in the same relation to one another that they had in the colony. The subsequent steps of drying, fixing, staining, and mounting are the same as those just given for the ordinary cover-slip preparations.

By this method constancies in the arrangement and grouping of the individuals in a colony ean often be made out. Some will always appear irregularly massed together, others will grow in parallel bundles, while others, again, will be seen as long, twisted threads.

Note.-From a colony of bacillus subtilis make a cover-slip preparation in the ordinary way; now make 
an impression cover-slip preparation of another colony of the same organism. Compare the results.

The Ordinary Staining-Solutions. - The solutions commonly employed in staining cover-slip preparations are, as has been stated, watery solutions of the basic aniline dyes-fuchsin, gentian-violet, and methylene-blue. These solutions may be prepared either by directly dissolving the dyes in substance in water until the proper degree of concentration has been reached, or by preparing them from concentrated watery or alcoholic solutions of the dyes which may be kept on hand as stock. The latter method is that commonly practised.

The solutions of the colors which are in constant use in staining are prepared as follows:

Prepare as stock, saturated alcoholic or watery solutions of fuchsin, gentian-violet, and methylene-blue. These solutions are best prepared by pouring into clean bottles enough of the dyes in substance to fill them to about one-fourth of their capacity. Each bottle should then be filled with alcohol or with water, tightly corked, well shaken, and allowed to stand for twenty-four hours. If at the end of this time all the staining-material has been dissolved, more should be added, the bottle being again shaken and allowed to stand for another twentyfour hours; this must be repeated until a permanent sediment of undissolved coloring-matter is seen upon the bottom of the bottle. The bottles are then to be labelled saturated alcoholic or watery solution of fuchsin, gentian-violet, or methylene-blue, as the case may be. The alcoholic solutions are not directly employed for staining-purposes. 
The solntions with which the staining is accomplished are made from these stock solutions in the following way:

An ordinary test-tube of about $13 \mathrm{~mm}$. diameter is three-fourths filled with distilled water and the concentrated alcoholic or watery solution of the dye is then added, little by little, until one can just see through the solution. It is then ready for use. Care must be taken that the color does not become too dense. The best results are obtained when it is just transparent as viewed through a layer of about 12 to $14 \mathrm{~mm}$. thick.

These represent the staining-solutions in everyday use. They are kept in bottles supplied with stoppers and pipettes (Fig. 35), and when used are dropped upon

FIG. 35.

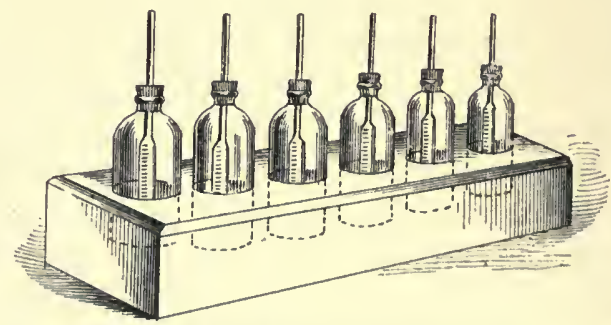

Rack of bottles for staining-solutious.

the preparation to be stained. After remaining upon the preparation for from twenty to thirty seconds they are washed off in water, and the preparation can then be examined.

For certain baeteria which stain only imperfectly with these simple solutions it is necessary to employ some agent that will increase the penetrating action of the dyes. Experience has taught us that this can be 
accomplished by the addition to the solutions of small quantities of alkaline substances, or by dissolving the staining-materials in strong watery solutions of either aniline oil or carbolic acid, instead of simple water-in other words, by employing special solvents and mordants with the stains.

Of the solutions thus prepared which may always be employed upon bacteria that show a tendency to stain imperfectly, there are three in common use-Loffler's alkaline methylene-blue solution; the Koch-Ehrlich aniline-water solution of either fuchsin, gentian-violet, or methylene-blue; and Ziehl's solution of fuchsin in carbolic acid. These solutions are as follows:

Loefler's alkaline methylene-blue solution:

Concentrated alcoholic solution of methylene-blue - 30 c.c.

Caustic potash in $1: 10,000$ solution . . . . . 100 c.c.

Koch-Ehrlich aniline-water solution. To about 100 c.c. of distilled water aniline oil is added, drop by drop, and the solution thoroughly shaken after each addition, until it is of an opaque appearance. It is then filtered through moistened filter-paper until the filtrate is perfectly clear. To 100 c.c. of the clear filtrate add 10 c.c. of absolute alcohol and 11 c.c. of the concentrated alcoholic solution of either fuchsin, methylene-blue, or gentian-violet, preferably fuchsin or gentian-violet.

Ziehl's carbol-fuchsin solution:

Distilled water

Carbolic acid (crystalline) .

Alcohol .

Fuchsin in substance.
100 c.c.

5 grammes.

10 c.c.

1 gramme.

Or it may be prepared by adding to a 5 per cent. watery solution of carbolic acid the saturated alcoholic solution of fuchsin until a metallic lustre appears on the surface of the fluid. 
The Koch-Ehrlich solution decomposes after having been made for a time, so that it is better to prepare it fresh in small quantities when needed than to employ old solutions. Solutions older than fourteen days should not be used.

The three solutions just given may be used for coverglass preparations in the ordinary way.

In some manipulations it becomes necessary to stain the bacteria very intensely, so that they may retain their color when exposed to the action of decolorizing agents. These methods are usually employed when it is desirable to deprive surrounding objects or tissues of their color, in order that the stained bacteria may stand out in greater contrast. It is in these cases that the staining-solution with which the bacteria are being treated is to be warmed, and in some cases boiled, so as to further increase its penetrating action. When so treated, certain of the bacteria will retain their color, even when exposed to very strong decolorizers. The tubercle bacillus is distinguished from the great majority of other bacteria by the tenacity with which it retains its color when treated in this way. It is an organism that is difficult to stain, but when once stained is equally difficult to rob of its color.

Method of Staining the Tumercle Baciluus. -Select from the sputum of a tuberculous subject one of the small, white, cheesy masses which it is seen to contain. Spread this upon a eover-slip and dry and fix it in the usual way. The slip is now to be taken by its edge with the forceps and the film covered with a few drops of either the solution of Koch-Ehrlich or that of Ziehl. It is then held over the gas-flame, at first some distance away, gradually being brought nearer, 
until the fluid begins to boil. After it has bubbled up once or twice it is removed from the flame, the excess of stain washed away in a stream of water, then immersed in a 30 per cent. solution of nitric acid in water, and allowed to remain there until all the color has disappeared. In some cases this takes longer than in others. One can always determine if decolorization is complete by washing off the acid in a stream of water. If the preparation is still quite colored, it shonld be again immersed in the acid; if of only a very faint color, it may be dipped in alcohol, again washed off in water, and may now be stained with some contrast-color. If, for example, the tubercle bacilli have been stained with fuchsin, methylene-blue forms a good contrast-stain. In making the contrast-stain the steps in the process are exactly those followed in the ordinary staining of cover-slip preparations in general: the slip containing the stained tubercle bacilli is rinsed off carefully in water, and a few drops of the methylene-blue solution are placed upon it and allowed to remain for thirty or forty seconds, when it is again rinsed in water and examined microscopically. For the purpose of observing the difference between the behavior of the tubercle bacilli and the other organisms present in the preparation toward this method of staining, it is well to examine the preparation microscopically before the contraststain is made, then remove it, give it the contrast-color, and examine it again. It will be seen that before the contrast-color has been given to the preparation the tubercle bacilli will be the only stained objects to be made out, and the preparation will appear devoid of other organisms; but upon examining it after it has received the contrast-color a great many other organisms 
will now appear; these will take on the second color employed, while the tubercle bacilli will retain their original color. Before decolorization all organisms in the preparation were of the same color, but during the application of the decolorizing solution all except the tubercle bacilli gave up their color. This characteristic, together with reactions to be described, as said, serves to differentiate the tuberele bacillus from other organisms with which it might be confounded. A number of different methods have been suggested for the staining of tubercle bacilli, but the original method as enployed by Koch is so satisfactory in its results that it is not advisable to substitute others for it. The above differs from the original Koch-Ehrlich method for the staining of tubercle bacilli in sputum only in the oceasional employment of Ziehl's carbol-fuchsin solution and in the method of heating the preparation with the staining-fluid upon it.

As Nuttall has pointed out, however, the strong aeid decolorizer used in this method can, with advantage, be replaced by much more dilute solutions, as a certain number of the bacilli are entirely decolorized by the too energetic action of the strong acids. He recommends the following method of decolorization: after staining the slip or section in the usual way, pass it through three alcohols; it is then to be washed out in a solution composed of

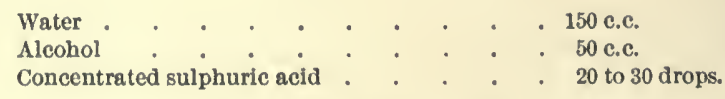

From this it is removed to water and carefully rinsed. The remaining steps in the process are the same as those given in the other methods. 
Gabbetr's Method for the staining of tubercle bacilli recommends itself because of its simplicity and the rapidity with which it can be performed. By many it is considered the best method for routine employment. It consists in staining the cover-slips, prepared in the manner given, for from two to five minutes in a cold carbol-fuchsin solution, after which they are subjected to the action of Gabbett's methylene-blue sulphuric acid solution. This latter consists of

Sulphuric acid, strength 25 per cent. - . 100 c.c.

Methylene-blue, in substance . . . . 1 to 2 grammes.

They are then rinsed off in water and are ready for examination. The tubercle bacilli will be stained red by the fuchsin, while all other bacteria, cell nuclei, etc., will be tinted blue.

Gray's Method.-Another differential method of staining which is very commonly employed is that known as Gram's method. In this method the objects to be stained are treated with an aniline-water solution of gentian-violet made after the formula of KochEhrlich. After remaining in this for twenty to thirty minutes they are immersed in an iodine solution composed of

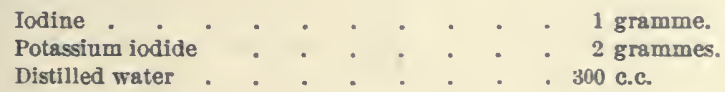

In this they remain for about five minutes; they are then transferred to alcohol and thoroughly rinsed. If they are still of a violet color, they are again treated with the iodine solution, followed by alcohol, and this is continued until no trace of violet color is visible to the naked eye. "They may then be examined, or a contrastcolor of carmine or Bismarck-brown may be given them. 
This method is particularly useful in demonstrating the capsule which is seen to surround some bacteria, particularly the micrococeus lanceolatus of pneumonia.

Glacial Acetic Acid Method.-Another method which may be employed for demonstrating the presence of the capsule surrounding certain organisms is to prepare the cover-slips in the ordinary way, then cover the layer of bacteria upon them with glacial acetic acid, which is instantly poured off (not washed off in water), and the aniline-water gentian-violet solution dropped upon them; this is allowed to remain three or four minutes, is poured off, and a few drops more are added, and lastly the slip is washed off in a solution of sodium chloride. Usually this is of the strength of the ordinary physiological salt-solution, viz., 0.6 to 0.7 per cent., but at times the strength must be greater, sometimes increased to from 1.5 to 2 per cent. of salt. The reason for this is that if the slips be washed in water, or in salt-solution that is too weak, the mucin eapsule that has been coagulated by the acetic acid is redissolved and rendered invisible. This does not occur when the salt-solution is of the proper strength-a point that can be determined only after a few trials with solutions of different strengths. (Welch.) A very clear, sharply cut picture usually follows this method of procedure.

Staining of Spores. - We have learned that one of the points by which spores may be recognized is their refusal to take up staining-substances when applied in the ordinary way. They may, however, be stained by special methods; of these, one that has given very satisfactory results in our hands is as follows: the coverslip is to be prepared from the material containing the spores in the ordinary way, dried, and fixed. It is then 
to be held by its edge with the forceps, and its surface covered with Lœffler's alkaline methylene-blue solution. It is then held over the Bunsen flame until the fluid boils; it is then removed, and after a few seconds is heated again. This is continued for about one minute, after which it is washed off in water and dipped five or six times in alcohol containing about 0.2 to 0.3 per cent. of hydrochloric acid. This is rinsed off in water and the preparation is now stained for from eight to ten seconds in aniline-water fuchsin solution (KochEhrlich solution), and finally again washed in water. By this method the spores are of a blue color and the body of the cell red.

By another process the cover-slip is floated, bacteria down, upon the surface of freshly prepared KochEhrlich solution of fuchsin contained in a watch-crystal. This is then held by its edge with the forceps about $2 \mathrm{~cm}$. above a very small flame of a Bunsen burner, care being taken that the flame touches only the centre of the bottom of the crystal. After a few seconds the erystal is elevated gradually until it is about 6 to $8 \mathrm{~cm}$. above the flame, then it is slowly moved down to the flame again, and this up-and-down movement is continued until the staining-fluid begins to boil. As soon as a few bubbles have been given off it is held aside for a minute or two, when the process of heating is repeated. When the boiling begins the crystal is held aside again for a minute or two. The crystal is heated in this way for about five or six consecutive times. When the fluid has stood for about five minutes after the last boiling the preparation is transferred, without washing in water, into a second wateh-crystal containing the following decolorizing solution: 
In this solution it is placed, bacteria up, and the vessel is tilted from side to side for about one minute. It is then removed, washed in water, and stained with the methylene-blue solution. The spores will be stained red and the body of the cells will be blue.

Moeller's Method for Staining Sporfs.-A method that has recently been published by Moeller is designed to favor the penetration of the coloringmaterial through the spore membrane by macerating the spores in a solution of chromic acid before staining them. It is as follows:

The cover-slips are prepared in the usual way, or the fixing may be accomplished with absolute alcohol instead of high temperatures. The preparation is then leeld for two minutes in chloroform, then washed off in water, then placed for from one-half to two minutes in a 5 per cent. solution of chromic acid; again washed off in water, and now stained in carbol-fuchsin. In the process of staining, the slip is taken by the corner with the forceps, and carbol-fuchsin is dropped upon the side containing the spores. It is then held over the flame until it boils, and then held some distance above the flame for one minute. The staining-fluid is then poured off and the preparation is completely decolorized in 5 per cent. sulphuric acid, again washed off in water, and finally stained for thirty seconds in the watery methylene-blue solution. The spores will be red, the body of the cells blue.

In this method the object of the preliminary exposure to chloroform is to dissolve away any erystals of lecithin, cholesterin, or fat that may be in the pre- 
paration, and which when stained might give rise to confusion.

It must be remembered that there are conspicuous differences in the behavior of spores of different bacteria to staining-methods. Some stain readily by either of the methods especially devised for this purpose, while others can hardly be stained at all, or only with the greatest difficulty, by any of the known processes.

Leffieris Method for Staining Flagella. For the demonstration of the locomotive apparatus possessed by motile bacteria we are indebted to Lœffler. By a special method of staining, in which the use of mordants played the essential part, he has shown that these organisms possess very delicate, hair-like appendages, by the lashing movements of which they propel themselves through the fluid in which they are located. The method as given by Loeffler is as follows:

It is essential that the bacteria be evenly and not too numerously distributed upon the cover-slip. The slips must therefore be carefully cleansed. (See Loeffler's method of cleaning cover-slips.) Five or six of the carefully cleansed cover-slips are to be placed in a line on the table, aud on the centre of each slip a very small drop of tap-water is placed. From the culture to be examined a ninute portion is transferred to the first slip and carefully mixed with the drop of water; from this mixture a small portion is transferred to the second, and from the second to the third slip, and so on-in this way insuring a dilution of the number of organisms present in the preparation.

These slips are then dried and fixed in the ordinary way. They are next to be warmed in the following solution: 
Tannic acid solution in water (20 acid, 80 water $)$. . 10 c.c.

Cold saturated solution of ferro-sulphate . . . . 5 c.c.

Saturated watery or alcoholic solution of fuchsin . . 1 c.c.

This solution represents the mordant. A few drops of it are to be placed upon the film of bacteria on the cover-slip, which is then to be held over the flame until the solution begins to steam. It should not be boiled. After steaming, the mordant is washed off in water and finally in alcohol. The bacteria are then to be stained in a saturated aniline-water-fuchsin solution.

When treated in this way different bacteria behave differently: the flagella of some stain readily in the above solutions; others require the addition of an alkali in varying quantities; while others stain best after the addition of acids. To meet these conditions an exact 1 per cent. solution of caustic soda in water must be prepared, and also a solution of sulphuric acid in water of snch strength that one cubic centimetre will be exactly neutralized by one cubic centimetre of the alkaline solution.

For different bacteria which have been studied by this method the one or the other of these solutions is to be added to the mordant in the following proportions.

Of the acid solution:

For the bacllus of Asiatic cholera . . . . $\frac{1}{2}$ to 1 drop. For the spirillum rubrum . . . . . . 9 drops.

Of the alkaline solution:

For the bacillus of typhoid fever . . . . 1 c.c.

For the baclllus subtilis . . . . . 28 to $30 \mathrm{drops}$

For the baclllus of mallgnant oedema . . . 36 to 37

For other organisms one must determine whether the results are better after the addition of acid or alkali, and how much of either is required. In general, it may 
be said that bacteria which produce acids in the media in which they are growing require the addition of alkalies to the mordant, while those that produce alkalies require acids to be added. By following Lœefler's directions the delicate, hair-like flagella on motile organisms may be rendered plainly visible.

There are several points and slight modifications in connection with this method that require to be emphasized in order to insure success: the culture to be employed should be young, not over 18-20 hours old. It should have developed for this time on fresh agar-agar at $37^{\circ}$ to $38^{\circ} \mathrm{C}$.; the mordant should not be perfectly fresh, as the best results are obtained from the use of old solutions that have stood exposed to the air, and that have been filtered just before using; when placed on the cover-slip and held over the flame never heat the mordant to the boiling-point; indeed, the best resulls are obtained when the preparation is held high above the flame and removed from it at the first evidence of vaporization, or, better still, a little before this point is reached. We have derived no advantage from the addition of acids or alkalies to the mordant, as recommended by I Leffler; but obtain, with a fair degree of regularity, satisfactory results through the use of the nentral mordant alone. ${ }^{1}$

Buxge's Method.-A useful modification of Loeffler's method is that recommended by Bunge: prepare a saturated solution of tannin, and a solution of liquor ferri sesquichlor. of the strength of 1:20 of distilled water. To 3 parts of the tannin solution add 1 part of the dilute iron solution. To 10 c.c. of such a mixture

1 I am indebted to Dr. James Homer Wright, Thomas Scott Fellow in $\mathbf{H y}$ giene, 1892-'93, Unirersity of Pennsylvania, for some of the suggestions in connection with the modification of this method. 
add 1 c.c. of concentrated watery solution of fuchsin. This mordant is not to be used fresh, but only after standing exposed to the air for several days (better for several weeks). After preparing the cover-slip with all precautions necessary to cleanliness the filtered mordant is allowed to act cold for about five minutes, after which it is slightly warmed; the slip is then washed off in water, dried, and faintly stained with carbol-fuchsin. No addition of acid or alkali to the mordant is necessary.

The Method of Van Ermengem. - Another method of demonstrating the presence of flagella is that suggested by Van Ermengem. It is somewhat more complicated than either of the preceding methods. The steps in the process are as follows:

In the centre of a perfectly cleaned cover-slip place a drop of a very dilute suspension, in physiological saltsolution, of a 10-to 18-hour old agar-agar culture of the organism to be studied. The suspension of the organisms in the salt-solution should be very dilute in order to favor the isolation of single cells on the slip and also to obviate the occurrence of excessive precipitation. The slips are then to be dried in the air and in the gas-flame in the usual manner.

The mordant used consists of:

Osmic acid (2 per cent. solution) . . . . . 1 part.

Tanuin (10-25 per cent, solution) . . . . . 2 parts.

To this 4 or 5 drops of glacial acetic acid may be added, but experience has shown this to be hardly necessary.

1'lace a drop or two of this mordant on the cover-slip to be stained, and allow it to act for one-half hour at room temperature, or for five minutes at $50^{\circ}$ to $60^{\circ} \mathrm{C}$. 
Wash carefully in water and alcohol, and then immerse for a few seconds in the " sensitizing bath," viz., a $0.25-0.5$ per cent. solution of silver nitrate. Without washing, bring the slip into a watch-crystalful of the "reducing and reinforcing bath," viz.:

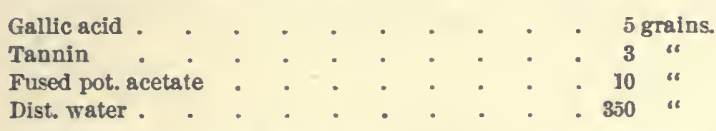

After a few seconds pass the slip back into a watchcrystal containing the dilute silver bath (0.25-0.5 per cent. solution of silver nitrate in water) and keep it in constant motion until the solution begins to take on a brown or blackish color. Wash in water thoroughly; dry with blotting-paper, and mount in balsam.

\section{STAINING IN GENERAL.}

The physics of staining and decolorization is hardly a subject to be discussed at length in a book of this character; but, as Kühne has pointed out, it may be said that solutions which favor the production of diffusion eurrents facilitate intensity of staining, and by a similar process increase the energy of decolorizing agents. For example, tissues which are transferred from water into watery solntions of the coloring matters are less intensely stained and more easily decolorized than when transferred from alcohol into watery staining-fluids; for the same reason tissues stained in watery solutions of the dyes do not become decolorized so readily when placed in water as when placed in alcohol.

The diffusion of staining-solutions into the protoplasm of dried bacteria, as found upon cover-slip preparations, 
is much greater and more rapid than when the same bacteria are located in the interstices of tissues. These differences are not in the bacteria themselves, but in the obstruction to diffusion offered by the tissues in which they are located.

The result of absence of diffusion may easily be illustrated. Prepare a cover-slip preparation, dry it carefully, fix it, and, without allowing water to get on it from any source, attempt to stain it with a solution of the dyes in absolute alcohol, washing it out subsequently with absolute alcohol; the result is negative. The absolute alcohol does not possess the property of diffusing into the dried tissues, and hence, as has been stated before, alcoholic solutions of the staining-dyes should not be employed. The staining-dyes should always be watery. ${ }^{1}$

Decolorizing-Solutions.-As regards the employment of decolorizing-agents, it must always be borne in mind that objects which are easily stained are also easily decolorized, and those that can be caused to take up the staining-material only with difficulty are also very difficult to rob of their color. The most common decolorizer in use is probably alcohol-not absolute alcohol, but alcohol containing more or less of water. Water alone has this property, but in a much lower degree than dilute alcohol. On the other hand, a much more energetic decolorization than that possessed by either alone can be obtained by alternate exposures to alcohol and

1 In the beginning of thls ehapter it was stated that the saturated aleoholic solutions of the dyes do not serve as stains for bacteria. It must be remembered that this holds only when absolute aleohol and perfeetly dry coloring matters have been used. If but a small proportion of water is present, the bacterla may be stalned with these solutions, though the results are, as a rule, unsatisfaetory. 
water. More energetic in their decolorizing action than either water or alcohol are solutions of the acids. They appear, particularly when they are alcoholic solutions, to diffuse rapidly into tissues and bacteria and very quickly extract the staining-materials which have been deposited there. For this reason these solutions should be employed with much care.

Very dilute acetic acid robs tissues and bacteria of their stain with remarkable activity; still more energetic are solutions of the mineral acids, and particularly, as has-been said, when this action is accompanied by the decolorizing-properties of alcohol.

The acid solutions that are commonly employed are:

Acetic acid in from 0.1 per cent. to 5 per cent. watery solution.

Nitric acid in from 20 per cent. to 30 per cent. watery solution.

Hydrochloric acid in 3 per cent. solution in alcohol.

STAINING OF BACTERIA IN TISSUES.

In staining tissues for the purpose of demonstrating the bacteria which they may contain a number of points must be borne in mind: the conditions which favor the diffusion of the staining-fluids into the bacteria are now not so farorable to rapid staining as they were when the bacteria alone were present upon cover-slips; the staining of tissues, therefore, requires a longer exposure to the dyes than does that of cover-slips. In tissues, too, there are other substances beside the bacteria which become stained, and these, unless robbed in whole or in part of their color, may so mask the stained bacteria as to render them difficult, if not impossible, of detection. 
Tissues must, therefore, always be subjected to some degree of decolorization, and this must be accomplished without depriving the bacteria of their color.

The details of the method of decolorization will be deseribed in the section on the technique of staining.

Another point to be remembered in staining tissues is that they cannot be heated and retain their structure in the same way that one heats cover-slips. The best results are not obtained in efforts to hasten the staining by subjection to high temperatures, but rather by longer exposures to lower temperatures.

Hardening the 'Tissues. - The bits of tissue-not greater than one cubic centimetre-are to be placed, as fresh as possible, in absolute alcohol. The bit of tissue should rest upon a pad of cotton or filter-paper in the bottle containing the alcohol, in order that it may be elevated and surrounded by the part of the alcohol which is specifically the lightest, and consequently contains least water. The alcohol abstracts water from the tissue, and, as the dehydration proceeds, the tissue becomes accordingly more and more dense. When of about the consistency of fresh solid rubber, or preferably not quite so dense, it is ready to eut. A small portion, about half a cubic centimetre, should be cemented to a bit of cork with ordinary mucilage, and allowed to remain in the open air for a minute or two for the mucilage to harden. Alcohol should be dropped upon it occasionally to prevent drying of the tissue. When the mucilage is hard the cork with the piece of tissue upon it may be left in alcohol over night, and on the following day the sections may be eut.

SECrION-CUTTING.- This is accomplished by the use of an instrument known as a microtome. In Fig. 36 
is seen the form now commonly employed. It is known by the name of the maker, as Schanze's microtome. It is an apparatus provided with a clamp for holding the cork upon which the tissue is cemented, and also a sliding clamp which carries a knife. The tissue is clamped horizontally, and the knife is caused to slide across its upper surface, also in a horizontal plane. Beneath the clamp for holding the tissue is a milled disk,

FIf. 36.

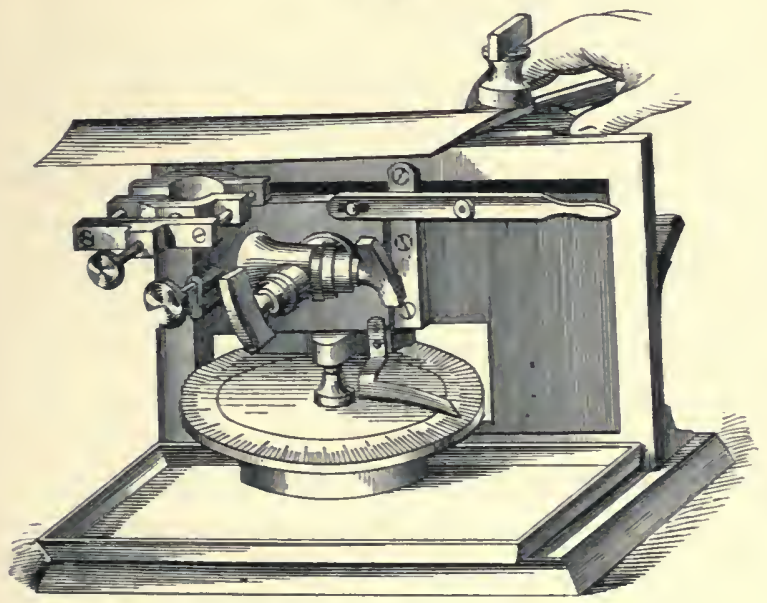

Schanze's microtome.

by means of which a screw is caused to revolve, and in revolving raises or lowers the clamp holding the tissue, so that the tissue may be brought closer to or farther from the plane in which the knife slides. By this arrangement sections of any desired thickness can be cut by turning the milled disk with the one hand and causing the knife to traverse the tissue with the other.

The tissue and the knife-blade shonld be kept wet 
with alcohol, so that the sections may float upon the blade of the knife, from which they can be easily removed, without tearing, with a curved needle or a eamel-hair pencil. As the sections are cut they are placed in a dish containing alcohol.

There are some tissues which, by reason of their histological structure, do not become sufficiently dense when exposed to alcohol to permit of their being cut in the above way. It becomes necessary to render them more solid by filling their interstices with some substance that neither interferes with their structure, nor prevents their being cut into sections. They nust be " imbedded," as this process is called.

Imbedding in celloidin. Most convenient for this purpose is celloidin, a body somewhat similar to collodion, soluble in a mixture of equal parts of alcohol and ether, as well as in absolute alcohol.

After hardening in alcohol the tissue to be imbedded is placed in a mixture of equal parts of absolute alcohol and ether and left there for twenty-four hours. It is then transferred to celloidin. Two solutions of celloidin are to be employed, the one a thin solution in a mixture of equal parts of absolute alcohol and ether, the other a thick solution in the same solvent. Into the thin solution, which should be of about the consistence of very thin syrup, the tissue is placed from the absolute alcohol and ether, and allowed to remain there for twenty-four hours. It is then placed in a thick solution for about a day. From this it may be removed and placed inmediately upon a bit of cork or a block of wood. The adherent celloidin will act as a cement, and as it hardens rapidly the tissue is soon fast to the cork. It is then left in 60 per cent. aleohol for twenty-four hours to 
complete the solidification of the celloidin, after which sections may be cut in the way just described for tissues not so treated.

Imbedding in paraffin. After bits of the tissue not larger than a cubic centimetre have been hardened in the usual way they are placed in fresh absolute alcohol for twenty-four hours to complete the process. From this they are transferred to pure turpentine, and kept in a warm oven at a temperature not exceeding $35^{\circ}$ to $38^{\circ} \mathrm{C}$. Here they remain for a time sufficient for them to become thoroughly saturated with the turpentine, as is recognized by the transparent appearance that they assume. From this they are placed in paraffin that is melted at $53^{\circ} \mathrm{C}$., and allowed to remain in this for three or four hours. They are then transferred to a small paper or metal mould, or a pill-box, and melted paraffin is poured over them. When the paraffin has become solid the mould or pill-box is removed from around it, the excess of paraffin removed from about the imbedded tissue, and the latter is ready for cutting.

When the sections are cut they are freed from paraffin by exposing them to turpentine; the latter is removed by washing in alcohol and the sections can now be stained in the ordinary way. In cutting sections from tissues that have been imbedded in paraffin the long axis of the knife should be at nearly right angles to the direction in which the kuife travels. For bacteriological purposes the method of imbedding in paraffin does not, as a rule, give such good results as when the celloidin method is employed. In this work, therefore, the latter is usually preferred.

Staining of the Sections. - The sections when cut may be stained in a variety of wass. The ordinary 
watery solutions of the three common basic aniline dyes -fuchsin, gentian-violet, and methylene-blue-or, what is better, the alkaline methylene-blue solution of Lœefler may be employed for general use.

Some of the acid aniline dyes, as well as some of the vegetable coloring matters, are essentially nuclear stains, and are not applicable to the staining of bacteria.

Into a watch-glass containing either of the stainingsolutions mentioned the sections are to be placed after having been in water for about one minute. They remain in the staining-solutions for from five to eight minutes. They are then removed, rinsed in water, and partly decolorized in 0.1 per cent. solution of acetic acid for only a few seconds; again washed out in water, then in absolute alcohol for a few seconds, and from this again into absolute alcohol for the same time, and finally into cedar oil or xylol. Here they remain for from one-half to three-fourths of a minute. They are now to be carefully spread out upon a spatula, which is held in the fluid under them, and, without draining off the fluid, are transferred to a clean glass slide. This must be done carefully to avoid tearing. The easiest way to do this is to hold the spatula on which the section floats in one hand, with its point just touching the surface of the glass slide, and then with a needle pull the seetion gently off upon the slide. The fluid comes with it, and the floating seetion may be easily spread out into a flat surface. The excess of fluid is taken up with blotting-paper, after which a drop of xylol-balsam is placed upon the centre of the section, and is then covered with a thin, clean cover-slip. It is now ready for examination.

Each step in the above process has its definite object. 
The sections are placed in water before staining in order that the diffusion of the staining-solution into the tissues may be diminished; otherwise our efforts at rendering the bacteria more conspicuous by decolorizing the tissues in which they are located would rob the bacteria of their color as well.

The acetic acid and also the alcohol are decolorizers, and are directed toward the excess of stain in the tissues, though they have also some decolorizing action upon the bacteria. The cedar oil and xylol are bodies which mix on the one hand with alcohol, and on the other with balsam. They are known as "clearing fluids," and not only serve to differentiate the component parts of the tissue, but fill up the gap that would otherwise be left in the process, for a section cannot be mounted in balsam directly from alcohol; the two bodies do not mix perfectly.

A number of clearing agents are in general use; in fact, almost all the essential oils come under this head. There is one-oil of cloves-which is very commonly used in histological work; but it must not be employed in tissues containing bacteria. It not only extracts too much color from the bacteria, but causes them to fade after the sections have been mounted for a time.

When the section thus stained and mounted is examined microscopically it may be found that the tissues still possess so much color that the bacteria are not visible, in which case they have not been decolorized sufficiently; or, on the other hand, both bacteria and tissues may have parted with their stains-then decolorization has been carried too far. In either case the fault must be remedied in the manipulation of the next section to be mounted. 
In short, the steps in the process of staining sections in general are these:

a. From alcohol into distilled water for one minute.

$b$. Into the staining-fluid for from five to eight minutes.

c. Into water for from three to five minutes.

d. Into 0.1 per cent. acetic acid for about one-half minute.

$e$. Into absolute alcohol for a few seconds.

$f$. Into absolute alcohol again for a few seconds.

g. Xylol for about one-half minute.

$h$. Removal with spatula or section-lifter to slide.

$i$. Removal of excess of xylol.

$j$. Mounting in xylol-balsam.

The section must be lifted from one vessel to the other by means of either a curved needle or a glass rod drawn out to a fine end and bent in the form of a curved needle.

By the above process of staining, which can be practised as a routine method for most bacteria in tissues, the nuclei of the tissue cells, as well as the bacteria, will be more or less deeply stained.

Special Methods of Staining Bacteria in Tissues. - For purposes of contrast-stains it sometimes becomes necessary to decolorize completely, or nearly completely, the tissues and leave the bacteria unaltered in color. For this purpose special methods depending on the staining-peculiarities of the bacteria under consideration have been devised.

Gram's method with tissues. One of the most commonly employed differential stains is that of Gram. In general, it is practised in the way given for its employment on cover-slip preparations, with some slight modifications. 
In this method the sections are to be placed from water into a solution of aniline-water gentian-violet, as prepared by the Koch-Ehrlich formula, but which has been diluted with about one-third its volume of water. In this the sections remain for about ten minutes, preferably in a warm place, at a temperature of about $40^{\circ} \mathrm{C}$. They should never, under any conditions, be boiled.

From this they are washed alternately in the iodine solution and alcohol, occasionally renewing the stained with clean alcohol, until all color has been extracterl from them. They are then brought for one minute into a dilute watery solution of eosin or safranin, or Bismarck-brown, again washed out for a few seconds in alcohol, and finally for one-fourth minute in absolute alcohol. From this they are transferred to xylol for a half-minute. The remaining steps in the process are the same as those given in the general method. In some cases better results are obtained by reversing the steps in the process and staining the bacteria last, for then the frequent decolorizing action of the alcohol on the bacteria is diminished; thus, place the sections from alcohol into eosin, safranin, or Bismarck-brown for a few minutes, then wash out in 50 per cent. alcohol, then for from three to five minutes in the dilute aniline-water gentian-violet solution, then into the iodine bath, after three minutes wash out in alcohol, and, finally, for onefourth minute in absolute alcohol, and then into the xylol, from which they may be mounted. Some of the organisms which may be stained by this method are micrococcus tetragenus, $b$. diphtherice, $b$. anthracis, and staph. pyogenes aureus. It cannot be successfully employed with the bacillus of typhoid fever. 
Staining with dahlia and decolorizing with sodium carbonate solution. Another method that is not very commonly employed, though the results obtained by its use are in many cases very satisfactory, is to stain the tissues in a strong watery solution of dahlia (about onefourth saturated) for from ten to fifteen minutes; from this they are transferred into a 2 per cent. solution of sodium or potassium carbonate, and from this into alcohol, altemating from the one to the other until the section is almost colorless. From the alcohol they are rinsed out in water and then put into a dilute watery solution of either eosin, Bismarck-brown, or safranin for one minute, then washed out in alcohol, finally in absolute alcohol, and then in xylol, from which they may be mounted in the manner given.

Especially brilliant results are obtained when tissues containing anthrax bacilli are stained by this process; the bacilli will be of a deep blue color, while the surrounding tissues will be of the color used as contrast.

Kühne's carbolic methylene-blue method. Stain the sections in the following solution for from one-half to one hour:

Methylene-blue, in substance . . . . . 1.5 grammes.

Absolute alcohol . . . . . . . 10 c.c.

Rub up thoroughly in a mortar, and when the blue is completely dissolved add gradually 100 c.c. of a 5 per cent. solution of carbolic acid. (The solution decomposes after a short time; it should be made fresh when necded.) From this the sections are washed out in water, then in 1.5 to 2 per cent. hydrochloric acid in water, from this they are transferred to a solution of lithium carbonate of the strength of six to eight drops of a concentrated watery solution of the salt to ten drops 
of water, and from this they are again thoroughly washed in water, then in absolute alcohol containing enough methylene-blue in substance to give it a tolerably dense color, then for a few minutes in aniline oil to which a little methylene-blue in substance has been added, then completely rinse out in pure aniline oil; from this they are passed into thymol or oil of turpentine for two miuutes, and then into xylol, from which they are mounted in xylol-balsam. The advantages of this method are that it is generally applicable, and by its use the bacteria are not robbed of their color, whereas the tissues are sufficiently decolorized to render the bacteria visible and admit of the use of contraststains.

Weigert's modification of Gram's method for sections. Stain the sections in the Koch-Ehrlich aniline-water gentian-violet solution for five or six minutes; wash out in water or physiological salt-solution ( 0.6 to 0.7 per cent. solution of sodium chloride in distilled water); transfer them with the section-lifter to the slide; take up the excess of fluid by gently pressing upon the flat section with blotting-paper; treat the section with the iodine solution used by Gram; take up the excess of the solution with blotting-paper; cover the section with aniline oil-this not only differentiates the component parts of the section, but dehydrates as well; wash out the aniline oil with xylol, and mount in the usual way in xylol-balsam. Or, decolorization with iodine may be omitted, and the sections, after staining in the aniline-water gentian-violet for five or six minutes-or longer, if necessary - are transferred to the slide without being washed in water or salt-solution (or, if so, only very slightly and rapidly), dried as completely as possi- 
ble with filter-paper, and then decolorized with a mixture of aniline oil (one part) and xylol (two parts). This is the delicate part of the process, and can be watched under the low power of the microscope. When decolorization is sufficient (repeated applications of the aniline oil and xylol mixture are generally necessary) pure xylol replaces the mixture, and the specimen is finally mounted in xylol-balsam. Unless all the aniline oil is replaced by the xylol the specimen will not keep well. In this process the aniline oil is really the decolorizer, and has the valuable property of absorbing a certain amount of water, so that dehydration with alcohol is avoided. This method, while it stains certain bacteria in tissues very satisfactorily, is nevertheless designed especially for the staining of fibrin. Fibrin and hyaline material will be stained deep blue, bacteria a dark violet.

Staining of Tubercle Bacilli in 'Tissues.-As for the staining of cover-slips, only those methods most commonly employed will be given.

The method of Ehrlich. Stain the sections in anilinewater fuchsin or gentian-violet for twenty-four hours; decolorize in 20 per cent. nitric acid for a few seconds only - the color need not be entirely extracted; then into 70 per cent. alcohol until no more color can be extracted by the alcohol; stain as contrast-color in dilute watery methylene-blue, malachite-green, or Bismarck-brown solution; wash out in 90 per cent. alcohol, then in absolute alcohol for a few seconds; clear up in xylol and mount in xylol-balsam.

Method of Ziehl-Neelsen. Stain the sections in warmed carbol-fuchsin solution for one hour; temperature to be about $45^{\circ}$ to $50^{\circ} \mathrm{C}$. Decolorize for a few seconds in 5 
per cent. sulphuric acid, then in 70 per cent. alcolol, and from this on as by the Ehrlich method.

Dry method. For tuberele bacilli, as for many other organisms in tissues, the following method may be employed if only the presence of organisms is to be detected and the histological condition of the tissues is a matter of no consequence: bring the sections from water upon a slide or cover-slip, dry, fix, and stain by the methods for cover-slip preparations.

Gray's method. The method employed by Gray at the Army Medical Museum, Washington, D. C., a description of which is given by Borden, is as follows: the tissue to be stained should be hardened, preferably in alcohol, in pieces not exceeding 1.5 by $1.5 \mathrm{by} 1 \mathrm{~cm}$. in size, though tissues hardened by any other of the regular methods can be stained. Alcohol is to be preferred, however, as after its use the bacilli stain more quickly and brilliantly than when one of the other hardening fluids-Müller's, for instance-is employed.

After the tissue has been hardened it is imbedded in paraffin, and cut in the usual manner. The sections are then cemented to the slides with a filtered $\frac{1}{2}$ per cent. solution of gold-label gelatin, to which is added chloral hydrate in the proportion of 1 per cent., as a preservative. Several drops of this are placed on each slide, a section laid on top, and the slides placed in a warming-oven kept at a temperature slightly below the melting-point of the paraffin. In about five minutes all wrinkles will have been taken out of the sections, which will lie perfectly flat and smooth on the surface of the gelatin solution. The slides are then removed from the oven and the surplus fluid poured from them, thus bringing the sections in contact with their surface, after which they 
are set aside in a place protected from dust, to remain until the sections are firmly cemented to them by the drying of the gelatin solution. The drying may be lastened by keeping the slides in an oven below the melting-point of the paraffin, but it is best to set the slides aside until the next day, when the sections will be found to be perfectly cemented to them. The paraffin is then removed from the sections by turpentine, the turpentine by absolute alcohol, the absolute alcohol by 50 per cent. alcohol, and this by water, after which the slides are placed in a 5 per cent. aqueous solution of potassium bichromate for five minutes. This renders the gelatin insoluble, and prevents the sections from leaving the slides during their necessarily more or less prolonged immersion in the fuchsin stain. The potassium bichromate is washed out with water, and the slides are then placed in a fuchsin stain, which is prepared as follows:

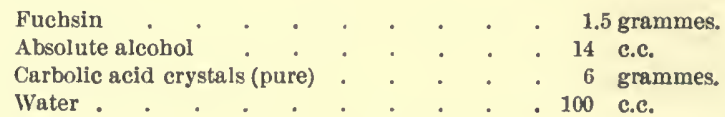

Dissolve the fuchsin in the alcohol and the earbolic acid in the water. Mix the two solutions and let stand for twelve hours, with occasional shaking or stirring; then filter.

The length of time that the slide remains in this solution varies with circumstances. The tuberele bacilli stain very quickly; in tissues properly hardened in alcohol five minutes are generally sufficient to stain them deeply.

Prolonged immersion in the fuchsin does no harm and insures certainty of results. After a section las 
been in the stain a sufficient length of time it, with the slide to which it is cemented, is washed in water until the surplus stain is removed; it is then subjected to the action of a combined decolorizer and contrast-stain made as follows:

Methrl-blue

Absolute alcohol

Sulphuric acid .

Water (distilled)

2.25 grammes.
30 c.c.
12 ..
100 s.

Dissolve the methyl-blue in the alcohol, add the acid to the water, mix the two solutions, and let stand, with occasional shaking, for twelve hours; then filter.

This solution is allowed to act upou the tissue for a few seconds, and as soon as the blue color predominates over the red, as seen by transmitted light, the section is immediately washed in water. Generally the red color reappears, and the section must be again subjected to the action of the blue solution and again washed in water. This must be repeated until the blue almost, if not quite completely and permanently, replaces the red stain. This is the most important part of the process, and entirely satisfactory results are only obtained after some practice. The tendency is usually not to replace sufficiently the fuchsin with the methyl-blue, and in consequence the red color of the bacilli is masked by the red of the surrounding tissues. Unless all acid is thoroughly removed by the fiual washing in water. the stain is not permanent. The section is then completely dehydrated with absolute alcohol, after taking up the excess of water on the slide with blotting-paper. The alcohol is followed by turpentine, and the process is completed by mounting in xylol-balsam.

In case it is desired to stain sections cut by the freez- 
ing method, they are placed upon a slide on which a few drops of the grelatin fixative have been placed, and after about five minutes, during which the fixative will have penetrated the section, the surplus is poured from beneath the section. The slides are then set aside for the gelatin to harden by drying, and after drying they are placed in bichromate fluid to render the gelatin insoluble. They are then manipulated in exactly the same manner as the sections cut by the paraffin method. This method gives equally as good results with tissues containing the lepra bacillus as with those containing tubercle bacilli. 


\section{CHAPTER XI.}

Systematic study of an organism-Points to be considered in identifying an organism as a definite species.

AFTER isolating an organism by the plate method considerable work is necessary in order to establish its identity as a definite species.

It must possess certain morphological and cultural peculiarities, which must be constant under constant conditions.

Its form at different stages must always be the same. Its ability or inability to produce spores must not vary under proper conditions. Its growth upon the different media under constant conditions of temperature and reaction must always present the same ontward appearances. The changes brought about by it in the reaction of the media in which it is growing must follow a fixed rule. Its power to produce liquefaction of the gelatin, or to grow upon it without bringing about this change, must always be the same. Its motility or non-motility, and, if motile, the approximate number and position of its organs of locomotion, must be determined. Its production of certain chemical products must be detected by chemical analysis. Its behavior toward oxygeni.e., Does it require this gas for its growth? Is this gas an indifferent factor? or, By its presence are the lifeprocesses of the organism checked ?-must be decided. Its behavior under varying conditions of temperature and under the influence of different chemical bodies, as 
well as its growth in media of different reactions, is to be studied. The property of producing fermentation with the liberation of gases, and the character and quantitative relations of these gases, must be ascertained; if it produces pigment, what are the conditions favorable and unfavorable to this function; and, lastly, we must consider its behavior when introduced into the bodies of animals used for experimental work-i.e., Is it a disease-producing organism, or does it belong to the group of innocent saprophytes?

We have learned the methods of obtaining colonies, and have acquainted ourselves with some of the peculiarities by which they are distinguished from one another. The next important steps are to determine the morphology of the individuals composing these colonies, as well as their relation to each other in the colony. These points are decided by microscopic examination of bits of the colony which are transferred to thin glass cover-slips, upon which they are dried, stained, and monnted. Cover-slips for this purpose are prepared in two ways: either by taking up a bit of the colony on a platinum needle, smearing it upon a cover-slip, staining it, and examining it-by which only the morphology of the individual bacteria can be made out-or by the method of "impression cover-slip preparations," by which not only the morphology, but also the relation of the organisms to one another in the colony can be determined. The details of these methods will be found in the chapter on the method of staining.

MICROSCOPIC FXAMINATION OF PREPARATIONS.

The Different Parts of the Microscope.Before deseribing the process of examining prepara- 
tions microscopically, a few definitions of the terms used in referring to the microscope may not be out of

FIG. 37.

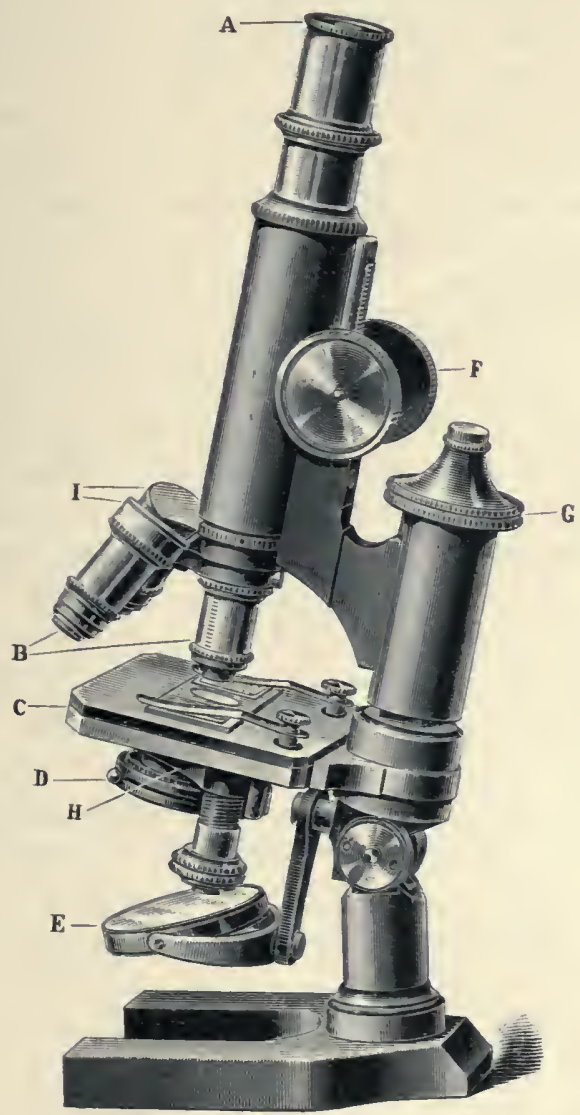

place. (The different parts of the microscope referred to below are indicated by letters in Fig. 37.) 
The ocular or eye-piece $(\mathrm{A})$ is the lens at which the eye is placed in looking through the instrument. It serves to magnify the image projected through the objective.

The objective (B) is the lens which is at the distal end of the barrel of the instrument, and which serves to magnify the object to be examined.

The stage (c) is the shelf or platform of the mieroscope on which the object to be examined rests.

The diaphragms are the perforated stops that fit in the centre of the stage. They vary in size, so that different amounts of light may be admitted to the object by using diaphragms with larger or smaller openings.

The "Iris" diaphragm (D) opens and eloses like the iris of the eye. It is so arranged that its opening for admission of light can be increased or diminished by moving a small lever in one or another direction.

The reflector $(\mathrm{E})$ is the mirror placed beneath the stage, which serves to direet the light to the object to be examined.

The coarse adjustment $(\mathrm{F})$ is the rack-and-pinion arrangement by which the barrel of the microscope can be quickly raised or lowered.

The fine adjustment $(\mathrm{G})$ serves to raise and lower the barrel of the instrument very slowly and gradually.

For the microseopic study of baeteria it is essential that the microseope be provided with an oil-immersion system and a sub-stage condensing apparatus.

The oil-immersion or homogeneous system consists of an objective so eonstrueted that it can only be used when the transparent media through which the light passes in entering it are all of the same index of refraction-i.e., are homogeneous. 'This is accomplished by interposing between the face of the lens and the cover-slip covering 
the object to be examined a body which refracts the light in the same way as do the glass slide, the coverslip, and the glass of which the objective is made. For this purpose a drop of oil of the same index of refraction as the glass is placed upon the face of the lens, and the examinations are made through this oil. There is thus no loss of light from deflection, as is the case in the dry system.

The sub-stage condensing apparatus (H) is a system of lenses situated beneath the central opening of the stage. They serve to condense the light passing from the reflector to the object in such a way that it is focussed upon the object, thus furnishing the greatest amount of illumination. Between the condenser and reflector is placed the "Iris" diaphragm, the aperture of which ean be regulated, as circumstances require, to permit of either a very small or very large amount of light passing to the object.

The nose-piece (I) consists of a collar, or group of collars joined together (two or more), that is attached to the distal end of the tnbe of the microscope. It enables one to attach several objectives to the instrument in such a way that by simply rotating the nose-piece the various lenses of different power may be conveniently used in succession.

Microscopic Examination of Cover-slips.-The stained cover-slip is to be examined with the oil-immersion objective, and with the diaphragm of the sub-stage condensing apparatus open to its full extent. The object gained by allowing the light to enter in such a large volume is that the contrast produced by the colored bacteria in the brightly illuminated field is much more conspicuous than when a smaller amount of light is thrown upon 
them. This is true not only for stained bacteria on cover-slips, but likewise for their differentiation from surrounding objects when they are located in tissues. With unstained bacteria and tissues, on the contrary, the structure can best be made out by redneing the bundle of light-rays to the smallest amount compatible with distinct vision, and in this way favoring, not color-contrast, but contrasts which appear as lights and shadows, due to the differences in permeability to light of the various parts of the material under examination.

Steps in Examining Stained Preparations with the Orl-Immersion-Sr'stem.-Place upon the centre of the cover-slip which covers the preparation a small drop of immersion oil. Place the slide upon the centre of the stage of the microscope. With the coarse adjustment lower the oil-immersion objective until it just touches the drop of oil. Open the illuminating apparatus to its full extent. Then, with the eye to the ocular and the hand on the fine adjustment, turn the adjusting-serew toward the right until the field becomes somewhat colored in appearance. When this is seen proceed more slowly in the same direction, and, after one or two turns, the object will be in focus. Do not remove the eye from the instrument until this has been accomplished.

Then, with one hand upon the fine adjustment and the thrmb and index finger of the other hand holding the slide lightly by its end, the slide may be moved about under the objective. At the same time the screw of the fine adjustment must be turned back and forth so that the different planes of the preparation may be brought into foens one after the other. In this way the whole section or prejaration may be inspected. When 
the examination is finished raise the objective from the preparation by turning the screw of the coarse adjustment toward you. Remove the preparation from the stage, and, with a fine silk cloth or handkerchief, wipe very gently and carefully the oil from the face of the lens. The lens is then unserewed from the microscope and placed in the case intended for its reception.

During work, of course, the lens need not be cleaned and put away after each examination; but when the work for the day is over an immersion lens must always be protected in this way. Under no circumstances should it be allowed to remain in the immersion oil or exposed to dust for any length of time.

Examination of Uxstained Preparations."Hanging drops." It frequently becomes necessary to examine bacteria in the unstained condition. The circumstances calling for this arise whilc studying the multiplication of cells, the germination of spores, the formation of spores, and the absence. or presence of motility.

In this method the organisms to be studied are suspended in a drop of physiological salt-solution or bouillon in the centre of a cover-slip. This is then placed, drop down, upon a slide in the centre of which a hollow or depression is ground (Fig. 38). The slip is held in

FIG. 38.

Longitudinal section of hollow-ground glass slide for observing bacteris in banging drops.

position by a thin layer of vaselin, which may be painted around the margins of the depression. This 
not only prevents the slip from moving from its position during examination, but also prevents drying by evaporation if the preparation is to be observed for any length of time. This is known as the "hanging-drop" method of examination or cultivation. It is indispensable for the purposes mentioned, and at the same time requires considerable care in its manipulation. 'The fluid is so transparent that the cover-slip is often broken by the objective being brought down upon the preparation before one is aware that the focal distance has been reached. This may be avoided by grasping the slide with the left hand and moving it back and forth under the objective as it is brought down toward the object. As soon as the least pressure is felt upon the slide the objective must be raised, otherwise the cover-slip will be broken and the lens may be rendered worthless.

A safer plan is to bring the edge of the drop into the centre of the field with one of the ligher power dry lenses. When this is accomplished substitute the immersion for the dry system, and the edge of the drop should now be somewhere near the centre of the field.

In examining baeteria by this method there is a possibility of error that must be guarded against. All microscopic insoluble particles in suspension in fluids possess a peculiar tremor or vibratory motion, the socalled "Brownian motion." 'This is very apt to give the impression that the organisms under examination are motile, when in truth they are not so, their movement in the fluid being only this moleenlar tremor.

The difference between the motion of bodies undergoing this molecular tremor and that possessed by certain living bacteria is that the former particles never move from their place in the field, while the living 
bacteria alter their position in relation to the surrounding organisms, and may dart from one position in the field to another. With some cases the true movement of bacteria is very slow and undulating, while in others it is rapid and darting. The molecular tremor may be seen with non-motile and with dead organisms.

Note.-Prepare three hanging-drop preparationsone from a drop of dilute India-ink, a second from a culture of micrococci, and a third from a culture of the bacillus of typhoid fever. In what way do they differ?

Study of Spore-formatiox.-The hanging-drop method just mentioned is not only employed for detecting the motility of an organism, but also for the study of its spore-forming properties.

Since with aërobic organisms spore-formation occurs, as a rule, only in the presence of oxygen, and is induced more by limitation of the nutrition of the organisms than by any other factor, it is essential that these two points should be borne in mind in preparing the drop cultures in which the process is to be studied. For this reason the drop of bouillon should be small and the air-chamber relatively large.

The cover-slip and hollow-ground slide should be carefully sterilized, and with a sterilized platinum loop a very small drop of bouillon is placed in the centre of the cover-slip. The slip is then inverted over the hollow depression in the sterilized object-glass and sealed with vaselin. The most convenient method of performing this last step in the process is to paint a ring of vaselin around the edges of the hollow in the slide, and then, without taking the cover-slip up from 
the table upon which it rests, invert the hollow over the drop and press it gently down upon the cover-slip. The vaselin causes the slip to adhere to the slide, so that it can be easily taken up. The drop now hangs in the centre of the small air-tight ehamber which exists between the depression in the slide and the cover-slip. (See Fig. 38.)

A very thin drop of sterilized agar-agar may be substituted for the bouillon. It serves to retain the organisms in a fixed position, and the process may be more easily followed.

As soon as finished the preparation is to be examined microscopically and the condition of the organisms noted. It is then to be retained in a warm chamber especially devised for the purpose, and kept under continuous observation. The form of ehamber best adapted for the purpose is one which envelops the whole microscope. It is provided with a window through which the light enters, and an arrangement for moving the slide about from the outside. The formation of spores requires a much longer time than the germination of spores into baeilli, but with patienee both processes may be satisfactorily observed.

It will be noticed that the deseription of this proeess is very mueh like that which immediately precedes, but differs from it in one respect, viz., that in this manipulation we are not making a preparation which is simply to be examined and then thrown aside, but it is an actual pure culture, and must be kept as sueh, otherwise the observation will be worthless. For this reason the greatest eare must be observed in the sterilization of all objects employed. Studies upon spore-formation by this method frequently continue over hours, and some- 
times days, and coutaniuation must, therefore, be carefully guarded against. The study should be begun with the vegetative form of the organisms; the hanging-drop preparation shonld, for this reason, always be made from a perfectly fresh culture of the organism under consideration before time has elapsed for spores to form.

The simple detection of the presence or absence of spore-formation can in many cases be made by other methods. For example, many species of bacteria which possess this property form spores most readily upon media from which it is somewhat difficult for them to obtain the necessary nutrition; potatoes and agar-agar that have become a little dry offer very favorable conditions, because of the limited area from which the growing bacteria can draw their nutritive supplies and because of the free access which they have to oxygen; for, their growth being on the surface, they are surrounded by this gas unless means are taken to prevent it. By the hanging-drop method, however, more than this simple property may be determined. It is possible not only to detect the stages and steps in the formation of endogenous spores, but when the spores are completely formed by transferring them to a fresh bouillondrop or drop of agar-agar, preserved in the same way, their germination into mature rods may be seen. The word rods is used because as yet we have no evidence that endogenous spore-formation occurs in any of the other morphological groups of bacteria.

Study of Gelatix Culdures. - As has been previously stated, the behavior of bacteria toward gelatin differs-some of them producing apparently no alteration in the medium, while the growth of others is accompanied by an enzymotic action that results in 
liquefaction of the gelatin at and around the place at which the colonies are growing. In some instances this liquefaction spreads laterally and downward, causing a saucer-shaped cxcavation, while in others the colony sinks directly down into the gelatin and may be seen lying at the bottom of a fumnel-sbaped depression. These differences are constantly employed as one of the means of differentiating otherwise closely allied members of the same family of bacteria. (See Fig. 34.) Studies upon the spirillum of Asiatic cholera and a number of closely allied species, for example, reveal a decided difference in the form of liquefaction produced by these different organisms. The slightest detail in this respect must be noted, and its frequency or eonstancy under different eonditions determined.

Cultures on Potato.-A very important feature in the study of an organism is its growth on sterilized potato. Many organisms present appearances under this method of cultivation which alone can almost be eonsidered characteristic. In some eases coarsely lobulated, elevated, dry or moist patehes of development occur after a few hours; again, the growth may be finely granular and but slightly elevated above the surface of the potato; at one time it will be dry and dull in appearance, again it may be moist and glistening. Sometimes there is a production of bubbles, owing to fermentation brouglit about by the growth of the organisms.

A most striking form of development on potato is that possessed by the bacillus of typhoid fever and the bacillus of diphtheria. After the inoculation of a potato with either of these organisms there is usually no nakedcye evidence of a growth in either instance, thongh microseopic examination of scrapings from the surface 
of the potato reveals an active multiplication of the organisms which had been planted there. The potato is one of the most important differential media which we possess for this work.

REACTIONS PRODUCED BY BACTERIA DURING THFir GrowTH.-The reactions produced in the media by different species of bacteria in the course of their growth are very valuable as means of differentiation.

In some cases these changes are so marked that they are readily detected by the coarser reagents; again, they are so slight as to require the employment of the most delicate indicators. They are sometimes seen to produce at one period of their growth an alkaline, at another period an acid reaction. This is seen in the cultures of the bacillus diphtherice of Loffler.

These differences are best seen after the addition to the media in which the organisms are to grow of some of the chemical substances which do not interfere with the development of the organisms, but, which under one reaction are of one color, and with an alteration of the reaction become a different color, the change being indicated by the play of colors. Such substances as litmus in the form of the so-called " litmus tincture," and coralline (rosolic acid) in alcoholic solution, are commonly employed for this purpose. They may be added to the media in the proportions given in the chapter on media, and the alterations in their colors studied with different bacteria. Milk and litmus tincture or peptone solution to which rosolic acid has been added are very favorable media for this experiment.

In milk coagula will now and then appear as a result of the influence of acids, produced from milk sugar by bacterial action, upon the casein of the milk, while 
again acids may be produced and yet no coagulation be noticed.

Aniline Dyes for Differential Diagnosis.The addition to solid media of some of the aniline dyes, fuchsin, methylene-blue, methylene-green, and several others, as well as combinations of these dyes, has been recommended as a means of differentiation of bacteria. The differences that are said to be produced consist of alterations in the color of the media due to oxidizing or reducing properties of the growing bacteria. As yet but little has come from this method of work. It cannot at present be recommended as a reliable means of diagnosis.

Behavior toward Staining-Reagents. - The behavior of certain bacteria toward the different dyes and their reactions under special methods of aftertreatment serve as aids to their diagnosis. With very few exceptions bacteria stain readily with the common aniline dyes, but they differ materially in the tenacity with which they retain these colors under the subsequent treatment with decolorizing-agents.

The tubercle bacillus and the bacillus of leprosy, for example, are difficult to stain, but when once stained retain their color under the action of such energetic decolorizing-agents as alcohol, nitric acid, oxalic acid, etc.

Certain other organisms when stained with a solution of gentian-violet in aniline-water retain their color when treated with such decolorizing-bodies as iodine solution and aleohol (Gram's method), while again others are completely decolorized by this method.

Many of them can only be treated with water, or but for a few seconds with alcohol, without losing their color. 
It is essential that these peculiarities should be carefully noted in studying an organism.

Fermentatios. - The production of gas as an indication of fermentation is an accompaniment of the growth of some bacteria. This is best studied in media to which 1 to 2 per cent. of grape sugar (glucose) has been added.

In this experiment the test-tube should be filled to about one-half its volume with agar-agar. The medium is then liquefied, and when reduced to the proper temperature a small quantity of a pure culture of the organism under consideration should be carefully distributed throngh it. The tube is then placed in ice-water and rapidly solidified in the vertical position. When solid it is placed in the incubator. After twenty-four to thirty-six hours, if the organism possesses the property of causing fermentation of glucose, the medium will be dotted everywhere with very small cavities containing the gas that has resulted.

This property of fermentation with production of gas is of such importance as a differential means that latterly considerable attention has been given to it, and those who have been most intimately concerned in the 'development of our knowledge on the subject do not consider it enough to say that the growth of an organism " is accompanied by the production of gas-bubbles," but that under given conditions we should determine not only the amount of gas or gases produced by the organism under consideration, but also their nature and quality. For this purpose $\mathrm{Smith}^{1}$ recommends the

1 An excellent and exhaustive contribution to this subject has been made by Theobald Smith in "The Wilder Quarter-Century Book," Ithaca, X. Y., 1893. 
employment of the fermentation-tube. It is a tube bent at an acute angle, closed at one end and enlarged with a bulb at the other. At the bend the tube is constricted. To it a glass foot is attached so that the tube may stand upright. (See Fig. 39.) To fill the tube the fluid (it is only used with fluid media)

Fig. 39.

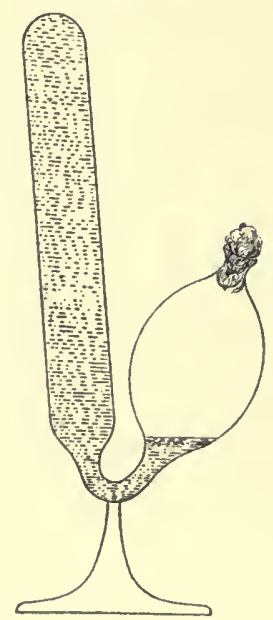

F'ermentation-tube.

is poured into the bulb until this is about half full. The tube is then tilted until the closed arm is nearly horizontal, so that the air may flow out into the bulb and the fluid flow into the elosed arm to take its place. When this has been completely filled enough fluid should be added to cover the lowest expanding portion of the bulb, and the opening of the bulb plugged with cotton. The. tubes thus filled are then to be sterilized. During sterilization they are to be maintained 
in the upright position. Under the influence of heat the tension of water-vapor in the closed arm forces most of the fluid into the bulb. As the tube cools the fluid returns to its place in the closed arm and fills it again, with the exception of a small space at the top, which is occupied by the air originally dissolved in the liquid and which has been driven ont by the heat. The airbubble should be tilted out after each sterilization, and finally, after the third exposure to steam, this arm of the tube will be free from air.

The medium employed is bouillon containing some fermentable carbohydrate, as glucose, lactose, or saceharose. After inoculation the flasks are placed in the incubator and the amount of gas that collects in the closed arm is, from day to day, noted.

From studies that have been made this gas is found to consist usually of about one part by volume of carbonic acid and two parts by volume of an explosive gas consisting largely of hydrogen. For determining the nature and quantitative relations of these gases Smith ${ }^{1}$ recommends the following procedure: "The bulb is completely filled with a 2 per cent. solution of sodium hydroxide $(\mathrm{NaOH})$ and closed tightly with the thumb. The fluid is shaken thoroughly with the gas and allowed to flow back and forth from bulb to closed branch and the reverse several times to insure intimate contact of the $\mathrm{CO}_{2}$ with the alkali. Lastly, before removing the thumb all the gas is allowed to collect in the closed branch, so that none may escape when the thumb is removed. If $\mathrm{CO}_{2}$ be present, a partial vacuum in the closed branch causes the fluid to rise suddenly when the thumb is re- 
moved. After allowing the layer of foam to subside somewhat the space occupied by gas is again measured, and the difference between this amount and that measured before shaking with the sodium hydroxide solution gives the proportion of $\mathrm{CO}_{2}$ absorbed. The explosive character of the residue is determined as follows: the cotton plug is replaced and the gas from the closed branch is allowed to flow into the bulb and mix with the air there present. The plug is then removed and a lighted match inserted into the mouth of the bulb. The intensity of the explosion varies with the amount of air present in the bulb."

Cultivation without Oxygen,-As we have already learned, there is a group of organisms to which the name "anaërobie organisms" has been given, which are characterized by their inability to grow in the presence of oxygen. For the cultivation of the members of this group a number of devices are employed for the exclusion of oxygen from the cultures.

Koch's method. Koch covered the surface of a gelatin plate, which had been previonsly inoculated, with a thin shect of sterilized isinglass. The organisms which grew beneath it were supposed to grow without oxygen.

Hesse's method. Hesse poured sterilized oil upon the surface of a culture made by stabbing into a tube of gelatin. The growth that occurred along the track of the needle was supposed to be anaërobic in nature.

Methods of Liborius. Liborius has suggested two useful methods for this purpose. The one is to fill a test-tube about three-quarters full of gelatin or agaragar, which, after having been sterilized, is to be kept in a vessel of boiling water for teu minutes to expel all 
air from it. It is then rapidly cooled in ice-water, and when between $30^{\circ}$ and $40^{\circ} \mathrm{C}$, still fluid, is to be inoculated and very rapidly solidified. It is then sealed up in the flame. Anaërobic bacteria develop only in the lower layers of the medium. His other method is that in which he employs a special tube, known as " the Liborius tube." Its construction is shown in Fig. 40.

Fig. 40.

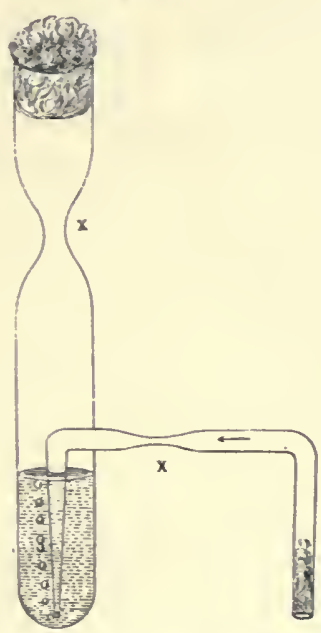

Liborius tube for anaërobic cultures.

Through the side tube hydrogen is passed until all air is expelled; the contracted parts, both of the neck of the tube and the side arm, are then sealed in the flame. ${ }^{1}$ This tube can be used for either solid or liquid

1 As the tubes come from the maker the contracted parts marked $\mathrm{x}$ in the cut are usually so thick as to render the sealing in the flame during the passage of hydrogen somewhat troublesome; it is better to draw them out in the flame quite thin before passing the hydrogen into the tube This makes the final sealing a matter of no difficulty. 
media, but, owing to its usual small capacity, gives better results with fluid media. (For precautions in using hydrogen see note to Fränkel's method, page 198.)

Method of Buchner. The plan suggested by Buchner of allowing the cultures to develop in an atmosphere robbed of its oxygen by pyrogallic acid gives very good results. In this method the culture, which is either a slant- or stab-culture in a test-tube, is placed-tube, cotton plug, and all-into a larger tube in the bottom of which have been deposited 1 gramme of pyrogallic acid and 10 c.c. of $\frac{1}{T_{0}}$ normal $^{1}$ caustic potash solution. The larger tube is then tightly plugged with a rubber stopper. The oxygen is quickly absorbed by the pyrogallic acid, and the organisms develop in the remaining constituents of the atmosphere, viz., nitrogen, a small amount of $\mathrm{CO}_{2}$, and a trace of ammonia.

Method of C. Fränkel. Carl Fränkel suggests the following as a modification of or substitute for the tubes of Liborius: the tube is first inoculated as if it were to be poured as a plate or rolled as an ordinary Esmarch tube. The cotton plug is then replaced by a rubber stopper, through which pass two glass tubes. These

1 A normal solution is one that contalns in a litre as many grammes of the dissolved substance as are indicated by its molecular equivalent. The equivaleut is that amount of a chemical compound which possesses the same ehemical value as does one atom of hydrogen. For example: one molecule of hydrochlorle acid $(\mathrm{HCl})$ has a molecular weight and also an equivalent weight of $36.5 ;$ a molecule of this aeld has the same chemical value as one atom of hydrogen. Its normal solution is therefore 36.5 grammes to the litre. On the other hand, sulphurie acid $\left(\mathrm{H}_{2} \mathrm{SO}_{4}\right)$ contains in each moleeule two replaceable hydrogen atoms; its normal solution is not, therefore, 80 grammes (its moleeular weight) to the litre, but that amount whlch would be equivalent ehemleally to one hydrogen atom, viz., 40 grammes (one-half its molecular welght) to the litre. A normal solution of caustic potash contains as many grammes to the litre as the number of its molecular weight -56.1 grammes to the litre of water. 
must all have been sterilized in the steam sterilizer hefore using. On the onter side of the stopper these two tubes are bent at right angles to the long axis of the test-tube into which they are to be placed, and both are slightly drawn out in the gas-flame. Both of these

Fia. 41.

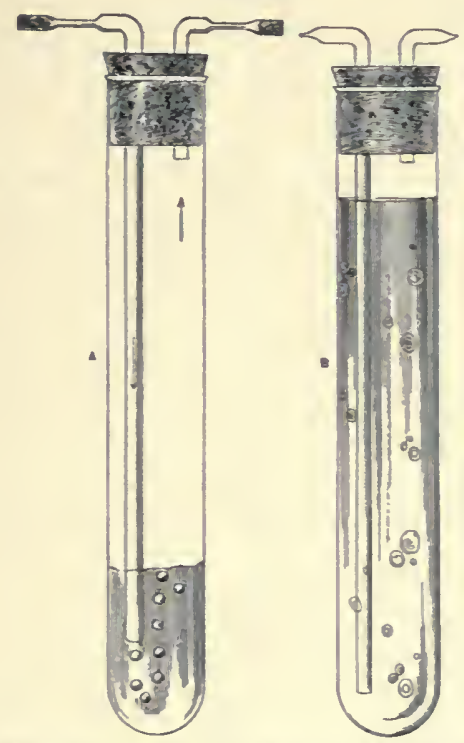

Fränkel's method for the cultivation of ansërobic bacteria.

tubes must be provided, before sterilization, with a plug of cotton; this is to prevent the access of foreign organisms to the medium during manipulations. At the inner side of the rubber stopper-that is, the end which is to be inserted into the test-tube-the glass tubes are of different lengths: one reaches to within $0.5 \mathrm{~cm}$. of the bottom of the test-tube, the other is cut off flush with the under surface of the stopper. The 
onter end of the longer glass tube is then connected with a hydrogen generator and hydrogen is allowed to bubble throngh the gelatin (Fig. 41, A) in the tube until all eontained air has been expelled and its place taken by the hydrogen. ${ }^{1}$ When the hydrogen has been bubbling through the gelatin for about five minutes (at least) one can be reasonably sure that all oxygen has been expelled. The drawn-out portions of the tubes can then be sealed in the gas-flame without fear of an explosion. The protruding end of the rubber stopper is then painted around with melted paraffin and the tube rolled in the way given for ordinary Esmareh tubes. A tube thus prepared and containing growing colonies is shown in Fig. 41, B.

The development that now occurs is in an atmosphere of hydrogen, all oxygen having been expelled. During the operation the tube containing the liquefied gelatin should be kept in a water-bath at a temperature sufficiently high to prevent its solidifying, and at the

1 Before beginning the experiment it is always wise to test the bydrogen$i . e$. , to see that it is free from oxygen and there is no danger of an explosion, for unless this be done the entire apparatus may be blown to pieces and a serfous accident occur. The agents used should be pure zinc, and pure sulphuric acid of about 25 to 30 per cent. strength. With the primary evolution of the gas the outlet of the generator should be closed and kept elosed until the gas reservoir is quite filled with hydrogen. The outlet should then be opened and the entire volume of gas allowed to escape, care being taken that no flame is in the neighborhood. This shon!d be repeated again, after which a sample of the bydrogen generated should be collected in an inverted test-tube in the ordinary way for collecting gases over water, viz., by filling a test-tube with water, closing Its inouth with the thumb, inverting it, and placing its mouth under water, when, after removing the thumb, the water will be kept in it by atmospheric pressure. The bydrogen which is flowing from the open generator may be conducted to the test-tube by a bit of rubber tubing. When the watcr has been replaced try the gas by holding a flame near the open mouth of the test-tube. If no explosion occurs, the hydrogen is safe to use. Shonld there be an explosion the gencration of bydrogen must be continued in the apparatus uutil it simply burns with a colorless flame when tested in a test-tube. 
same time not high enough to kill the organisms with which it has been inoculated.

One of the obstacles to the successful performance of this method is the bubbling of the gelatin, the foam from which will often fill the exit tube and sometimes be forced from it. This may be obviated by reversing the order of proceeding, viz.: roll the Esmarch tube in the ordinary way with the organisms to be studied, using a relatively small amount of gelatiu, so as to have as thin a layer as possible when it is rolled. Theu replace the cotton plug with the sterilized rubber stopper carrying the glass tubes through which the hydrogen is to be passed, and allow the hydrogen to flow through just as in the method first given. The gas now passes over the gelatin instead of through it, and consequently no bubbling results. In all other respects the procedure is the same as that given by Frankel.

Method of Kitasato and Weil. For faroring the anaërobic conditions Kitasato and Weil have suggested the addition to the culture media of some strong reducing agent. They recommend formic acid in 0.3 to 0.5 per cent.; glucose in 1.5 to 2 per cent.; or blue litmus tincture in 5 per cent. by volume. This is, of course, in addition to an atmosphere from which all oxygen has been expelled.

Esmarch's method. Esmarch's plan is to prepare in the usual way a roll tube of the organisms; subject it to a low temperature, and while quite cold fill it with liquefied gelatin, which is caused to solidify rapidly. In this method the colonies develop along the sides of the tubes, and can more easily be studied than where they are mixed through the gelatin, as in the method of Liborius. 
By some workers the oxygen is removed from the culture medium by the use of the air-pump.

Many other methods exist for this special purpose, but for the beginner those given will suffice.

From what has been said it may be inferred that the cultivation of anaërobic bacteria is a simple matter and attended with but little difficulty. Such an inference will, however, be quickly dispelled when the beginner attempts this part of his work for the first time, and particularly when his efforts are directed toward the separation of these forms from other organisms with which they are associated. The presence of sporeforming, facultative anaërobes in mixed cultures is always to be suspected, and it is this gronp that renders the task so diffienlt. At best the work requires undivided attention and no small degree of skill in bacteriological technique.

Indol Production. - The production of products other than those that give rise to alterations in the reaction of the media, and whose presence may be detected by chemical reactions, is now a recognized step in the identification of different species of bacteria. Among these chemical products there is one that is produced by a number of organisms, and whose presence may easily be detected by its characteristic behavior when treated with certain substances. I refer to the body nitroso-indol, the reactions of which were described by Beyer in 1869, and the presence of which as a product of the growth of certain bacteria has since furnished a topic for considerable discussion.

Indol, the name by which this body is now generally known, when acted upon by reducing agents, is seen to become of a more or less conspicuous rose color. This 
body was recognized as one of the products of growth of the spirillum of Asiatic cholera first by Poel, and a short time subsequently by Bujwid and by Dunham, and for a time was thought to be peculiarly eharacteristic of the growth of this organism. It has since been found that there are many other bacteria which also possess the property of producing indol in the course of their development.

The method employed for its detection is as follows: cultivate the organism for twenty-four to forty-eight hours at a temperature of $37^{\circ} \mathrm{C}$., in the simple peptone solution known as "Dunham's solution" (see formula for this medium). This solution is preferred because its pale color does not mask the rose color of the reaction when the amount of indol present is very small.

Four tubes should always be inoculated and kept under exactly the same conditions for the same length of time.

At the end of twenty-four or forty-eight hours the test may be made. Proceed as follows: to a tube containing 7 c.c. of the peptone solution, but which has not been inoculated, add 10 drops of concentrated sulphuric acid. To another similar tube add 1 c.c. of a 0.01 per cent. solution of sodium nitrite, and afterward 10 drops of concentrated sulphuric acid. Observe the tubes for five to ten minutes. No alteration in their color appears, or at least there will be no production of a rose color. They contain no indol.

Treat in the same way, with the acid alone, two of the tubes which have been inoculated. If no rose color appears after five or ten minutes, add 1 c.c. of the sodium nitrite solution. If now no rose color is pro- 
duced, the indol reaction may be considered as negative. No indol is present.

If indol is present, and the rose color appears after the addition of the acid alone, it is plain that not only indol has been formed, but likewise a reducing body. This is found, by proper means, to be salts of nitrous acid. The sulphuric acid liberates this acid from its salts and permits of its reducing action being brought into play.

If the rose color appears only after the addition of both the acid and the nitrite solution, then indol has been formed during the growtl of the organisms, but no nitrites.

Control the results obtained by trating the two remaining cultures in the same way.

The test is sometimes made by allowing concentrated acid to flow down the sides and collect at the bottom of the tube; the reaction is then seen as a rose-colored zone overlying the line of contact of the acid and culture medium. This method is open to the objection that, if indol is present in only a very limited amount, the rose color produced by it is apt to be masked by a brown color that results from the charring action of the concentrated acid on the other organic matters in the culture medium, so that its presence may in this way escape detection. In view of this, Petri recommends the use of dilute sulphuric aeid. He states that when indol is present the characteristic rose color appears : little more slowly with the dilute acid, but is more permancnt, and there is never any danger of its presence being masked by the occurrence of other color reactions.

Test for Nitrites. For this purpose Lunkewiez has recently recommended the employment of Ilosvay's 
morlification of the method of Griess. As reagents the following solutions are employed:
a. Naphthylamine
Dist. water
0.1 gramme.
20.0 c.c.
Acetic acid ( 25 per cent. sol.)
150.0 c.c.
b. Sulfanilic acid
Acetic acid ( 25 per cent. sol.)
0.5 gramme.
150.0 c c.

In preparing solution $a$ the naphthylamine is dissolved in 20 c.e. of boiling water, filtered, allowed to cool, and mixed with the dilute acetic acid.

Solutions $a$ and $b$ are then mixed. The resulting mixture should be colorless. It is best to prepare it fresh as it is needed, though if kept in a closely stoppered flask it retains it virtues for some time.

When added to cultures containing nitrites, in the proportion of one volume to five volumes of the culture, a deep red color appears in a few seconds. If the nitrites are not present, no color reaction occurs. In making the test on cultures always control the results by tests on the same medium not inoculated, as some of the ingredients of which the medium is composed may eontain nitrites. Lunkewicz recommends the use of Merck's peptone for this test, elaiming that nitrites are always to be found in Witte's peptones.

POINTS TO BE OBSERVED IN DESCRIBLNG AN ORGANISM.

The following is an outline of points to be considered in describing a new organism or in identifying an organism with one already described:

1. Its source-as air, water, or soil. If found in the animal body, is it normally present or only in pathologieal conditions?

2. Its form, size, mode of development, occurrenee of 
involution-forms or other variations in morphology. Grouping, as in pairs, chains, elumps, zoogloea; presence of capsule; development and germination of spores; arrangement of flagella.

3. Staining-peculiarities-especially its reactions with Gram's (or Weigert's fibrin) stain, and peculiar or irregular modes of staining.

4. Motility - to be determined on very fresh cultures and on cultures in different media.

5. Its relation to oxygen-is it aërobic, anaërobic, or facultative? Does it develop in other gases, as earbonic aeid, hydrogen, etc. ?

6. Both the macroscopic and microscopic appearance of its colonies on nutrient gelatin and on nutrient agaragar.

7. The appearance of its growth in stab-and slantcultures on gelatin, agar-agar, blood-serum, and on potato.

8. The character of its growth in fluid media, as in bouillon, milk, litmus milk, rosolic-acid-peptone solution, and in bouillon containing glucose.

9. Does it grow best in acid, alkaline, or neutral media?

10. Is the normal reaction of the medium altered by its growth? Is its growth accompanied by the production of indol; is the indol associated with the coincident production of nitrites?

11. Is its growth accompanied by the production of gas, as evideneed by the appearance of gas-bubbles in the media-both in media containing fermentable sugars and those from which these bodies are absent? When cultivated in sugar-bouillon in the fermentation-tube, what production of gas is evolved under known condi- 
tions? How much of this gas is carbonic acid and how much is explosive?

12. At what temperature does it thrive best, and the lowest and highest temperatures at which it will develop? What is its thermal death-point, both by steam and dry-air methods of determining this point?

13. What is its behavior when exposed to chemical disinfectants and antiseptics? Does it withstand drying and other injurious influences, both in the vegetative and spore stages? The germicidal value of the blood-serum of different animals may also be tried upon it.

14. Its pathogenic powers-modes of inoculation by which these are demonstrated; quantity of material used in inoculation; duration of the disease and its symptoms; lesions produced, and distribution of the bacteria in the inoculated animal; which animals are susceptible and which immune, and the character of its pathogenic activities? Variations in virulence, and the probable cause to which they are due. Can they be produced artificially and at will?

15. The detection of specific, toxic, and immunizing products of growth.

16. Its behavior when exposed to the influence of blood-serum of animals immunized from it; also its behavior when mixed with serum from an animal in the height of infection by it. Are the relations between the organism and the serum constant and specific? 


\section{CHAPTER XII.}

Inoculation of animals-Subcutaneous inoculation; intravenous injection -Inoculation into the great serous cavities, and Into the anterior chamber of the eye-Observation of animals after inoculation.

AFTER subjecting an organism to the methods of study that we have thus far reviewed there remains to be tested its action upon animals-i.e., to determine if it possesses the property of producing disease or not, and, if so, what are the pathological results of its growth in the tissues of these animals, and in what way must it gain entrance to the tissues in order to produce these results? The mode of deciding these points is by inoculation, which is practised in different ways according to circumstances. Most commonly a bit of the culture to be tested is simply introduced beneath the skin of the animal, but in other eases it may be necessary to introduce it directly into the vaseular or lymphatic circulation or into one or the other of the great serous eavities; or, for still other purposes of observation, into the anterior chamber of the eye, upon the iris.

Subcutanfous Iroculation of Animats.-The animals usually employed in the laboratory for purposes of inoeulation are white mice, gray house-miee, guineapigs, rabbits, and pigeons.

For simple subeutaneous inoeulation the steps in the process are practically the same in all cases. The hair or feathers are to be carefully removed. If the skin is very dirty, it may be scrubbed with soap and water. 
Sterilization of the skin is impossible, so that it need not be attempted. If the inoculation is to be by means of a hypodermic syringe, then a fold of the skin may be lifted up and the needle inserted in the way common to this procedure. If a solid culture is to be inoculated, a fold of the skin may be taken up with the forceps and a pocket cut into it with scissors which have previously been sterilized. This pocket must be ent large enough to admit the end of the needle without its touching the sides of the opening as it is inserted. Beneath the skin will be found the superficial and deep ronnective-tissue fasciæ. These must be taken up with sterilized forceps, and with sterilized scissors incised in a way corresponding to the opening in the skin. The pocket is then to be held open with the forceps and the substance to be inserted is introduced as far back under the skin and fasciæ as possible, care being taken not to touch the edges of the wound if it can be avoided. The wound may then be simply pulled together and allowed to remain. No stitching or efforts at closing it are necessary, though a drop of collodion over the point of operation may serve to lessen contamination.

During manipulation the animal must be held still. For this purpose special forms of holders have been devised, but, if an assistant is to be obtained for the operation, the simple subcutaneous inoculation may be made without the aid of a mechanical holder.

It is at times, however, more convenient to dispense with the presence of an assistant, and several forms of apparatus have been devised for holding guinea-pigs, rats, rabbits, etc. For small animals, such as mice and rats, the holder suggested by Kitasato is very useful. It is simply a metal plate attached to a stand by a 
clamped ball-and-socket joint, so that it can be fixed in any position. It is provided with a spring-clip at one

\section{FIG. 42.}

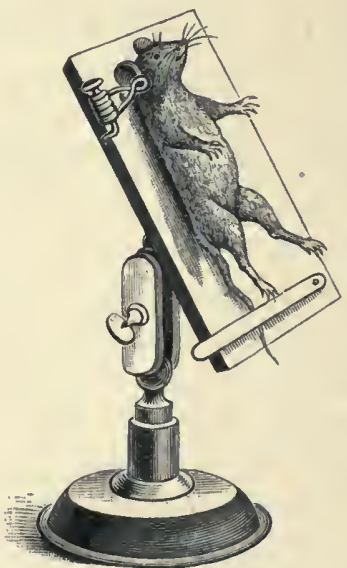

Kitasato's mouse-holder.

FiG. 43.

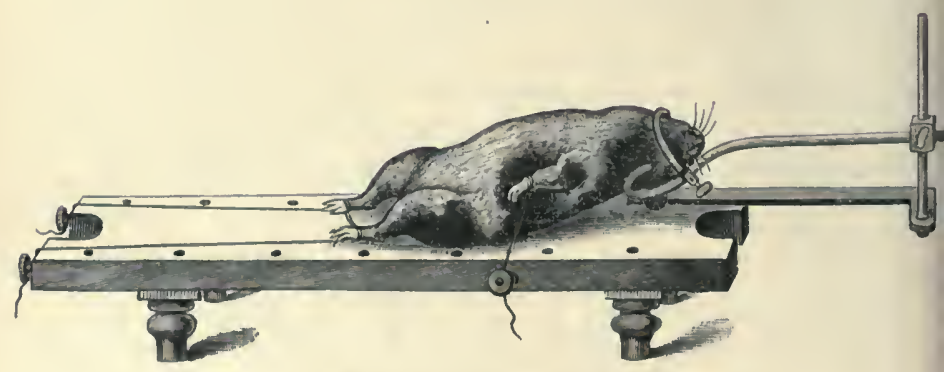

IIolder for larger animals.

end that holds the animal by the skin of the neck, and at the other end with another elamp that holds the tail of the animal. 'This holder is shown in Fig. 42. 
For larger animals the form of holder shown in Fig. 43 is commonly used.

A very simple and useful holder for guinea-pigs consists of a metal cylinder of about $5 \mathrm{~cm}$. in diameter

Frg. 44.

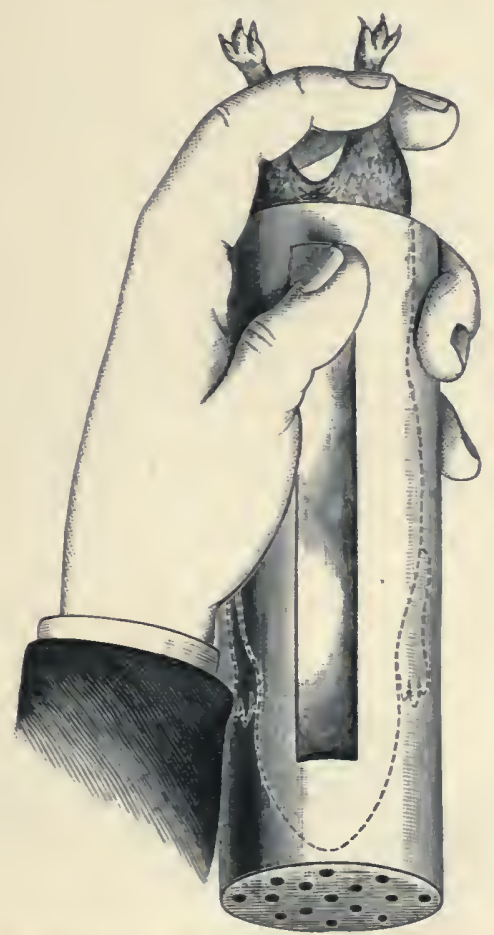

The Voges-Rabinowitsch holder for guinea-pigs.

and about $13 \mathrm{~cm}$. long; closed at one end by a perforated cap of either tin or wire netting. Along the side of this box is a longitudinal slit of $12 \mathrm{~mm}$. wide that runs for $9.5 \mathrm{~cm}$. from within $0.5 \mathrm{~mm}$. of the open extremity of the cylinder. 
The animal is placed in such a cylinder with its head toward the perforated bottom. It is then easily possible to make subcutaneous inoculation by taking up a bit of skin through the slit in the side of the box, or to make intraperitoneal injection by drawing the posterior extremities slightly from the box and holding them steady between the index and second finger, as seen in Fig. 44. It is also very convenient for use when the rectal temperature of these small animals is to be taken. The manipulations can easily be made without the aid of an assistant. Its construction is best seen in Fig. $44{ }^{1}$

For ordinary subcutaneous inoculations at the root of the tail in mice a simple piece of apparatus consists of a bit of board about $7 \times 10 \mathrm{~cm}$. and $2 \mathrm{~cm}$. thick, upon which is tacked a hollow, tapering roll of wire gauze, a truncated cone, about $6 \mathrm{~cm}$. long and about $1.5 \mathrm{~cm}$. in diameter at one end and $2 \mathrm{~cm}$. at its other

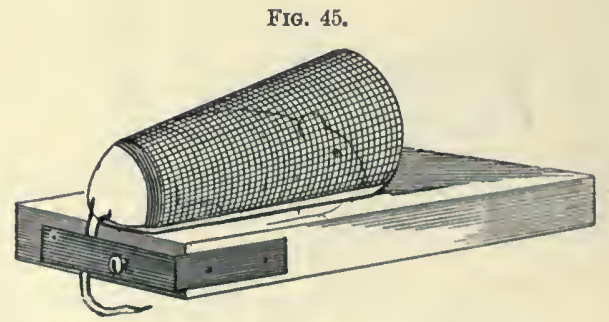

Mouse-holder, with mouse in proper position.

end. This is tacked upon the board in such a position that its long axis rums in the long axis of the board, being equidistant from its two sides. Its small end is placed

1 Centralblatt für Bakteriologie und Parasitenkunde, Bd. 18, 1895, p. 530. 
at the edge of the board. The mouse is taken up by the tail by means of a pair of tongs and allowed to crawl into the smaller end of this wire cone. When so far in that only the root of the tail projects the animal is then fixed in this position by a clamp and thumb-screw, with which the apparatus (Fig. 45) is provided. The animal usually remains perfectly quiet and may be handled without difficulty.

The hair from over the root of the tail is to be carefully cut away with the scissors, and a pocket cut through the skin at this point. The inoculation is then made into the loose tissue under the skin over this part of the back in the way that has just been described. It is always best to insert the needle some distance along the spinal column, and thus deposit the material as far from the surface-wound as possible.

As the subcutaneous operation is very simple and takes only a few moments, guinea-pigs, rabbits, and pigeons may be held by an assistant. The front legs in the one hand and the hind legs in the other, with the animal stretched upon its back on a table, is the usual position for the operation when practised upon guineapigs and rabbits. The point at which the inoculations are commonly made is in the abdominal wall either to the right or left of the median line and about $3 \mathrm{~cm}$. distant. When pigeons are used they are held with the legs, tail, and ends of the wings in the one hand, and the head and anterior portion of the body in the other, leaving the area occupied by the pectoral muscles, over which the inoculation is to be made, free for manipnlation. The hair over the point selected for the inoculation should be closely eut with the scissors in the case of guinea-pigs and rabbits, and from a small 
area the feathers should be plucked in the case of the pigeon.

Injection rnto the Circulation. - It is not infrequently desirable to inject the material under consideration directly into the circulation of an animal. If a rabbit is to be employed for the purpose, the operation is usually done upon one of the veins in the ear.

To those who have had no practice in this procedure it offers a great many difficulties; but if the directions which will be given are strictly observed, the greatest of these obstacles to the successful performance of the operation may be overcome.

When viewing the circulation in the ear of the rabbit by transmitted light three conspicuous branches of the main vessel (vena auricularis posterior) will be seen. One runs about centrally in the long axis of the ear, one runs along its anterior margin, and one along its posterior margin. The central branch (ramus anterior of the vena auricularis posterior) is the largest and most conspicuous vessel of the ear, and is, therefore, selected by the inexperienced as the branch into which it would appear easiest to insert a hypodermic needle. This, however, is fallacious. This vessel lies very loosely imbedded in connective tissue, and, in efforts to introduce a needle into it, rolls about to such an extent that only after a great deal of difficulty does the experiment suceed. On the other hand, the posterior branch (ramus lateralis posterior of the vena auricularis posterior) is a very fine, delicate vessel which runs aloug the posterior margin of the ear, and which is so firmly fixed in the dense tissues which surround it that it is prevented from rolling about under the point of the needle. The further away from the mouth of the vessel -that is, the 
nearer we approach its capillary extremity-the more favorable become the conditions for the success of the operation.

Select, then, the very delicate vessel lying quite close to the posterior margin of the ear, and make the injection as near to the apex of the ear as possible. From time to time the ressels of the ear will be found to contain so little blood that they are hardly distinguishable, making it very difficult to introduce the needle. This is sometimes overcome by pressure at the root of the ear, causing, thereby, stasis of the blood and distention of the vessels. A very satisfactory method of causing the veins to become more prominent is to lightly press or gently prick with the point of a needle the skin over the ressel to be used. In a few seconds, as a result of this irritation, the vessel will have become dilated, distended with blood, readily distinguished from the surrounding tissues, and may then be easily punctured by the needle of the syringe. The injection is always to be made from the dorsal surface of the ear.

Of no less importance than the selection of the proper vessel is the shape of the point of the needle employed.

The hypodermic needles as they come from the makers are not suited at all for this operation, because of the way in which their points are ground. If one examine carefully the point of a new hypodermic needle, it will be seen that the long point, instead of presenting a flat, slanting surface when viewed from the side, has a more or less curved surface. Now, in efforts to introducc such a needle into a vessel of very small calibre, it is commonly seen that the extreme point of the needle, instead of remaining in the vessel, as it would do were it straight, very commonly projects into 
the opposite wall, and as the needle is inserted further and further into the tissues it is usually pushed through the vessels into the loose tissues beyond, and the material to be injected is deposited into these tissues, instead of into the circulation. If, on the contrary, the slanting point of the needle be ground down until its surface is perfectly flat when viewed from the side, and no more curvature exists, then when once inserted into a vessel it usually remains there, and there is no tendency to penetrate through the opposite wall. We never use a new hypodermic needle until its point is carefully ground down to a perfectly flat, slanting surface and no more curvature exists.

These differences may perhaps come out clearer if represented diagrammatically.

FIG. 46.

a

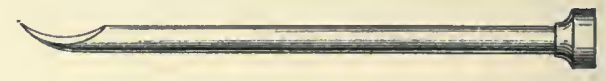

$b$

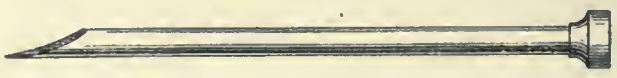

Hypodermic needles magnified, a. Improper point. b. Proper shape of point.

In Fig. $46, a$, the needle has the point usually seen when new.

In Fig. 46, $b$, the point has been ground down to the shape best suited for this operation.

The needles need not be returned to the maker. One can grind them to the shape desired in a few minutes upon an oilstone.

The size of the needle is that commonly employed for subcutaneons injections. 
When the operation is to be performed an assistant holds the animal gently but firmly in the cronching position upon a table. If the animal does not remain quiet, it is best to wrap it in a towel, so that nothing but its head protrudes; though in most cases we have not found this necessary, and particularly if the animal has not been excited prior to the beginning of the operation.

The animal should be placed so that the ear upon which the operation is to be performed comes between the operator and the source of light. This renders visible by transmitted light not only the coarser vessels of the ear, but also their finer branches. The point at which the injection is to be made is to be shaved clean of hair, by means of a razor and soap.

The filled hypodermic syringe is taken in one hand and with the other hand the ear is held firmly. The point of the needle is then inserted through the skin and into the finest part of the ramus posterior, the part nearest the apex of the ear, where the course of the vessel is nearly straight. When the point of the needle is in this vessel it gives to the hand a sensation quite different from that felt when it is in the midst of connective tissue. As soon as one thinks the point of the needle is in the vessel a drop or two of the fluid may be injected from the syringe, and, if his suspicions are correct, the circulation in the small ramifications and their anastomoses will quickly alter in appearance. Instead of their containing blood, the colorless fluid which is being injected will now be seen to circulate. This must be carefully observed, for sometimes when the needle-point is not actually in the vessel, but is in the lympli-spaces surrounding it, an appearance somewhat similar is to be seen. It may always be differen- 
tiated, however, by continuing the injection, when the circulation of clear fluid through the vessels will not only fail to take the place of the circulating blood, but there will at the same time appear a localized swelling under the skin about the point of the needle. The needle must then be withdrawn and inserted into the vessel at a point a little nearer to its proximal end.

Care must be taken that no air is injected.

The hypodermic syringe and needle must, previous to operation, have been carefully sterilized in the steam sterilizer or in boiling water. The animal must be kept under close observation for about an hour after injection.

The operation is one that cannot be learned from verbal description. It can only be successfully performed after actual practice.

If the precautions which have been mentioned are observed, but little difficulty in performing the operation will be experienced.

Its greater convenience and simplicity as compared with other methods for the introduction of substances into the circulation commend it as an operation with which to make one's self familiar. The animals sustain practically no wound, they experience no pain-at least they give no evidence of pain-and 110 anesthetic is required.

The form of syringe best suited for this operation is of the ordinary design, but one that permits of thorough sterilization by steam. It should be made of glass and metal, with packings that may be sterilized by steam without injury. The syringes commonly employed are those shown in Fig. 47-A, Koch's; $B$, Strohschein's; $C$, Overlack's. 
For operations requiring exact dosage experience has led me to prefer a syringe after the pattern of $C$, in Fig. 47-i.e., of the form commonly used by physicians. The reason for this is as follows: in making hypodermic injections or injections into the circulation there is a certain amount of resistance to the passage of fluid from the needle. If one overcomes this resistance

F10. 47.
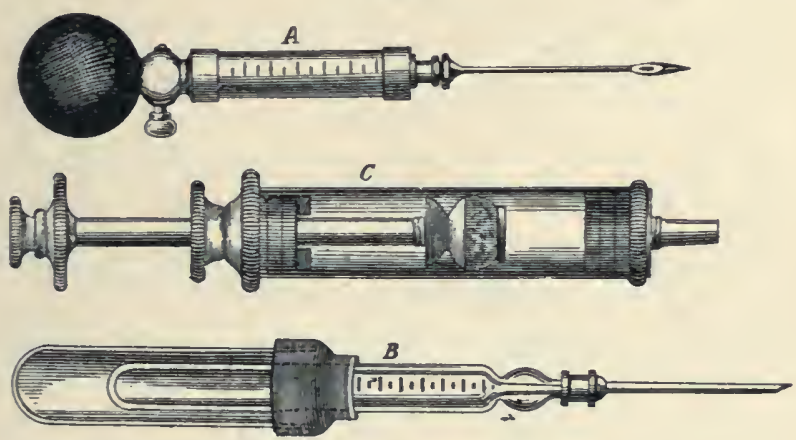

Forms of hypodermic syringe.

A. Koch's syringe. B. Syringe of Strohschein. C. Overlack's form.

by means of a cushion of compressed air, as is the case in syringes $A$ and $B$ of $\mathrm{Fig} .47$, the sudden expansion of the air in the body of the syringe when resistance is overcome frequently causes a larger amount of fluid to be ejected from the needle than is desired. No such accident is likely to occur when the fluid is forced from the barrel of the syringe by the head of a closefitting piston, with no air intervening between the fluid and the head of the piston. With such an instrument, properly manipulated, the dose can always be controlled with aceuracy. 
Inoculation into the Lymphatic Circulation. - Fluid cultures or suspensions of bacteria may be injected into the lymphatics by way of the testicles. The operation is a simple one. One simply plunges the point of the hypodermic needle directly into the substance of the testicle and then injects the amount desired.

Injections made in this manner are sometimes followed by interesting pathological lesions of the lymphatic apparatus of the abdomen.

\section{INOCULATION INTO THE GREAT SEROUS CAVITIES.}

Inoculation into the peritoneum presents no difficulties if fluids are to be introduced. In this case one makes, with a pair of sterilized scissors, a small nick through the skin down to the underlying fasciæ, and, taking up a fold of the abdominal wall between the fingers, plunges the hypodermic needle through the opening just made directly into the peritoneal cavity... There is no fear of penetrating the intestines or other internal viscera if the puncture be made along the median line at about midway between the end of the sternum and the symphysis pubis. Though this may scem a rude method, it is, nevertheless, the rarest of accidents to find that the intestines have been penetrated. The object of the primary incision is to lessen the chances of contaminating the inoculation by bacteria located in the skin, some of which would adhere to the needle if it were plunged directly through the skin, and might complicate the results.

If solid substanecs, bits of tissue, etc., are to be introduced into the peritoneum, it becomes necessary to conduct the operation upon the lines of a laparotomy. 
The hair should be shaved from a small area over the median line, after which the skin is to be thoroughly washed. A short longitudinal incision (about $2 \mathrm{~cm}$. long) is then to be made in the median line through the skin, and down to the fasciæ. Two subcutaneous sutures, as employed by Halsted, are then to be introduced transversely to the line of incision at about $1 \mathrm{~cm}$. apart, and their ends left loose. This particular sort of suture does not pass through the skin, but, instead, the needle is introduced into the subcutaneous tissues along

Fig. 48.

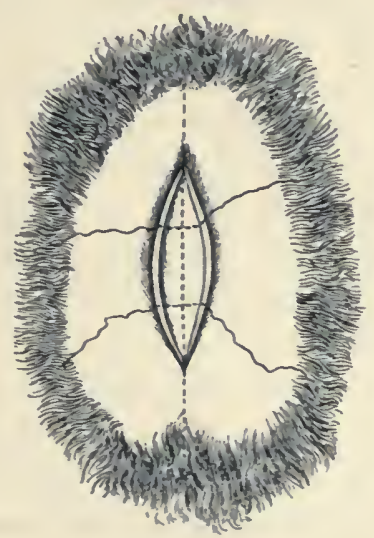

the edge of the incision. In this case they are to pass into the abdominal cavity and out again, entering at one side of the line of incision and leaving at the other, as indicated by the solid and dotted lines in Fig. 48 . (This figure indicates the primary opening through the skin. By the longitudinal dotted line is seen the opening to be made into the abdomen; by the transverse dotted lines, with their loose ends, the sutures as placed 
in position before the abdomen is opened; it will be seen that these sutures in all cases pass through the subcutaneous tissues only and do not penetrate the skin proper.)

The opening through the remaining layers may now be completed; the bit of tissue deposited in the peritoneal cavity, under precautions that will exclude all else; the edges of the wound drawn evenly and gently together by tying the sutures, and the lines of incision dressed with collodion. It should be needless to say that this operation must be conducted under the strictest precautions, to avoid complications. All instruments, sutures, ligatures, ctc., must be carefully sterilized either in the steam sterilizer for twenty minutes, or by boiling in 2 per cent. sodium carbonate solution for ten minutes; the hands of the operator, though they should not touch the wound, should be carefully cleansed, and the material to be introduced into the abdomen should be handled with only sterilized instruments.

Inoculation into the pleural eavity is much less frequently called for-in fact, it is not a routine method employed in this work. It is not easy to enter the pleural cavity with a hypodermic needle without injuring the lung, and it is rare that conditions call for the introduction of solid particles in this locality.

Inoculation into the anterior chamber of the eye is performed by making a puncture through the cornea just in front of its junction with the sclerotic, the knife being passed into the anterior chamber in a plane parallel to the plane of the iris. By the aid of a fine pair of forceps the bit of tissue is passed through the opening thus made and is deposited upon the iris, where it is allowed to remain, and where its pathogenic properties upon the iris can be conveniently studied. It is a mode of inoc- 
ulation of very limited application, and is therefore but rarely practised. It was employed in the classical experiments of Cohnheim in demonstrating the infectious nature of tuberculous tissues, tuberculosis of the iris being the constant result of the introduction of tuberculous tissue into the anterior chamber of the eye of rabbits.

OBSERVATION OF ANIMALS AFTER INOCULATION.

After either of these methods of inoculation, particularly when unknown species of bacteria are being tested, the animal is to be kept under constant observation and all that is unusual in its conduct noted-as, for iustance, elevation of temperature; loss of weight ; peculiar position in its cage; loss of appetite; roughening of the hair ; excessive secretions, either from the air-passages, conjunctiva, or kidneys; looseness of or hemorrhage from the bowels; tumefaction or reaction at site of inoculation, etc. If death ensue in from two to four days, it may reasonably be expected that at autopsy evidence of either acute septic or toxic processes will be found. It sometimes occurs, however, that inoculation results in the production of chronic conditions, and the animal must be kept under observation often for weeks. In these cases it is important to note the progress of the chauges by their effect upon the physical conditions of the animal, viz., upon the nutritive processes as evidenced by fluctuation in weight, and upon the body temperature. For this purpose the animal is to be weighed daily, always at about the same hour and always about midway between the hours of feeding; at the same time its temperature as indicated by a ther- 
mometer placed in the rectum is to be recorded. ${ }^{1}$ By the comparison of these daily observations with one

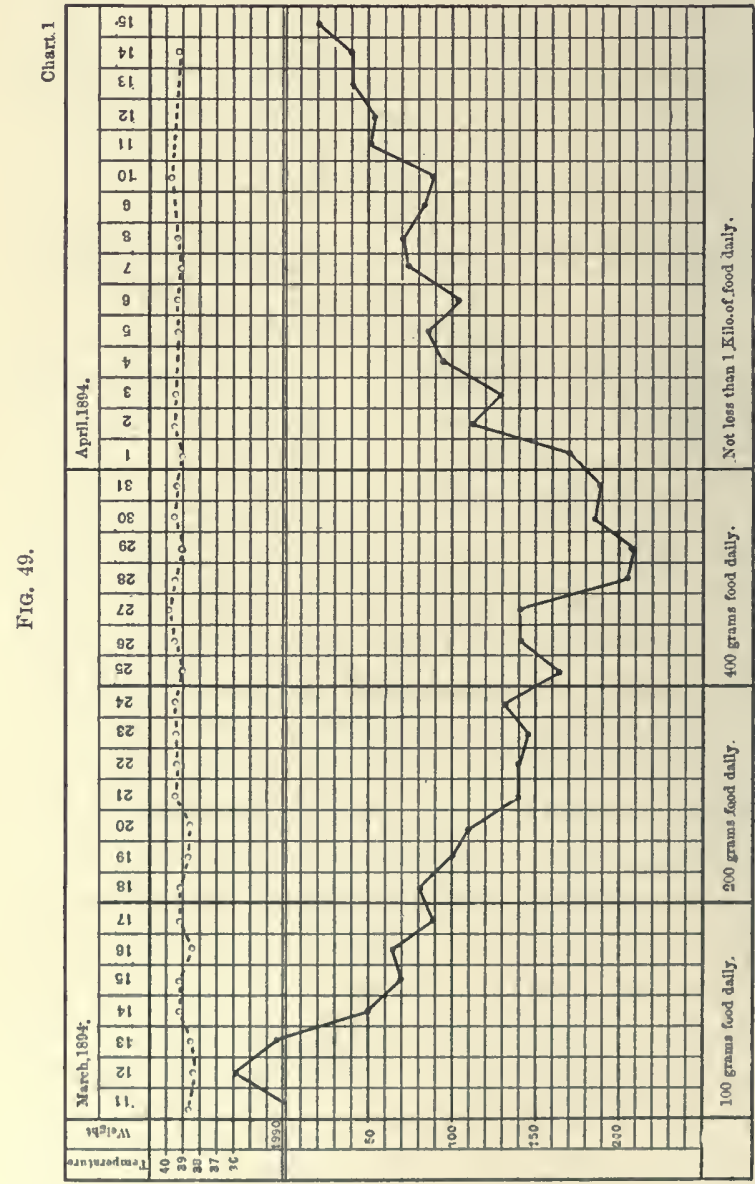

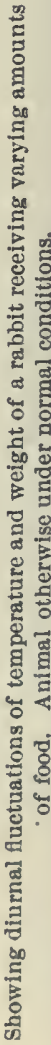

I The thermometer must be inserted into the reetum beyond the grasp of the sphineter, otherwise pressure upon its bulb by contraction of this musele may force up the mercurial column to a point higher than that resulting from the actual body temperature. 
another, one is aided in observing the course the infection is taking.

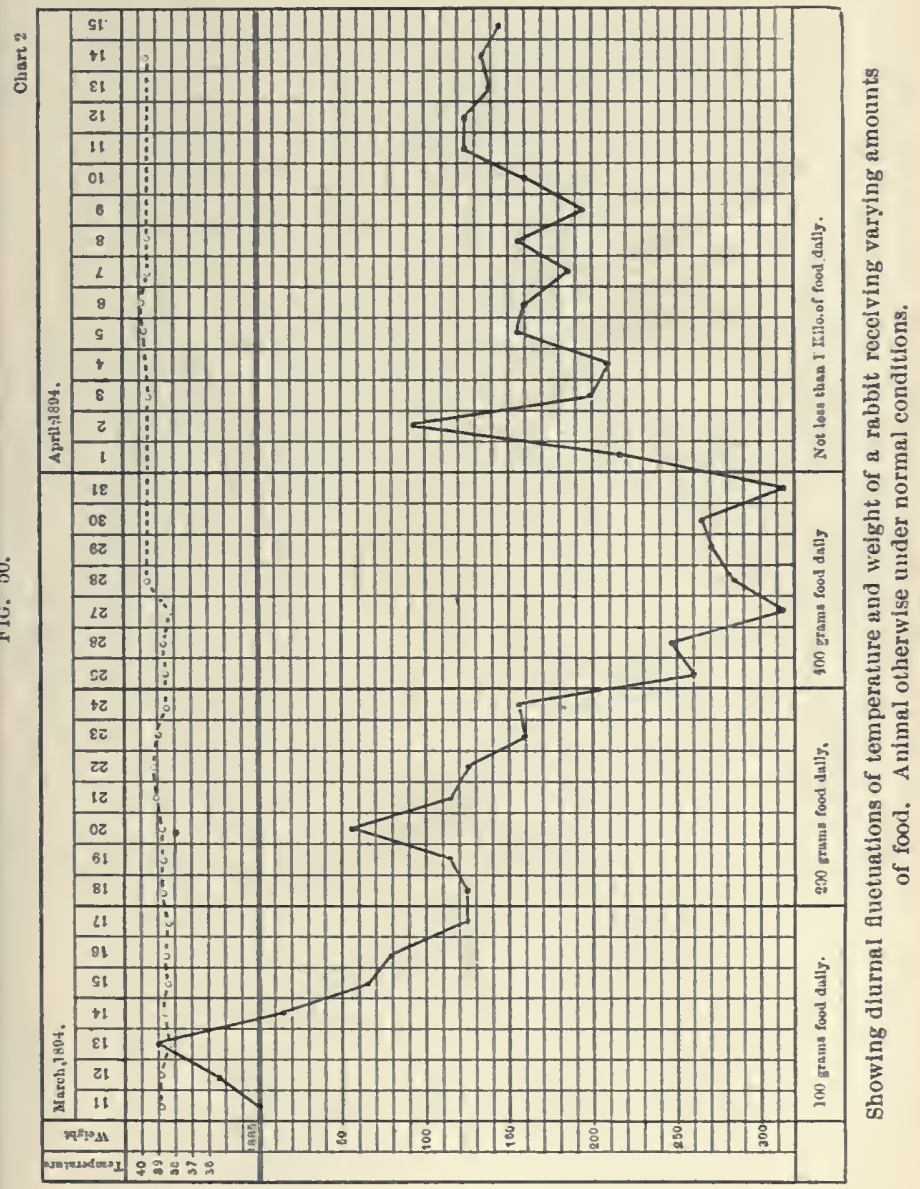

Too much stress must not, however, be laid upon moderate and sudden daily fluctuations in either temperature or weight, as it is a common observation that 
presumably normal animals when confined in cages and fed regularly often present very striking temporary

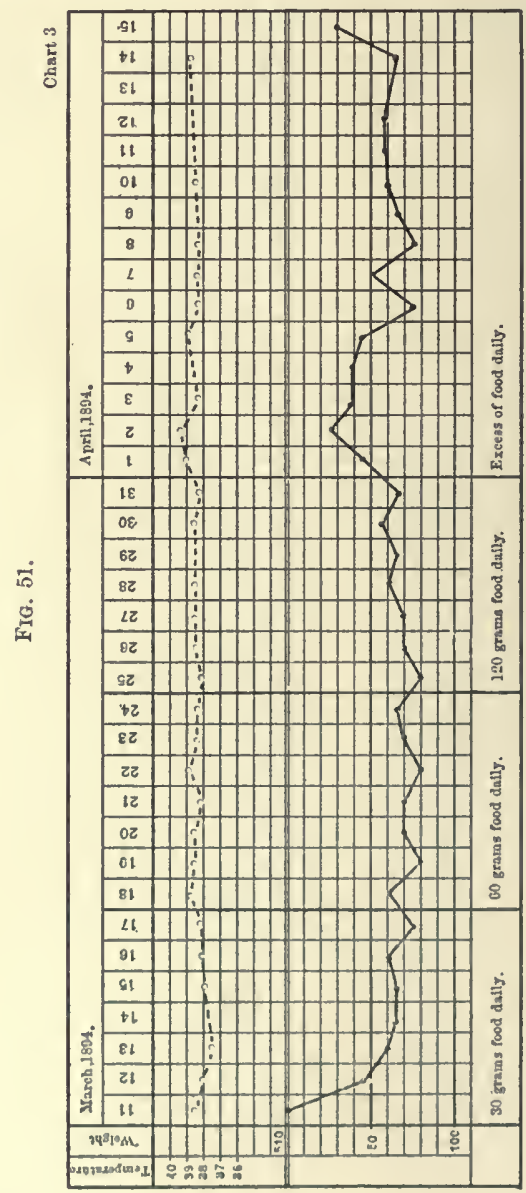

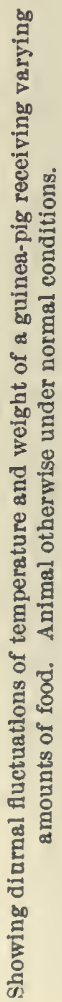

gains and losses in weight, often amounting to 50 or 100 grammes in twenty-four hours, even in animals 
whose total weight may not exceed 500 or 600 grammes; similarly they are seen to experience-unexplainable rises

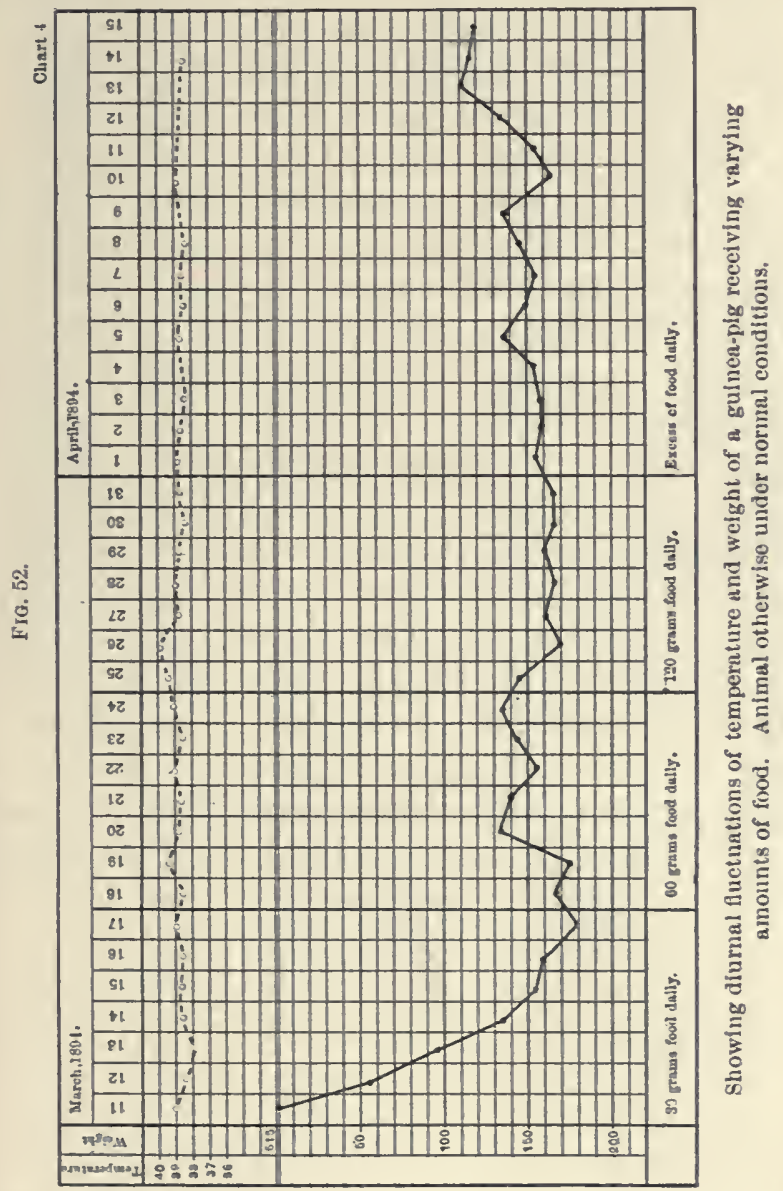

and falls of temperature, often as much as a degree from one day to another. Such fluctuations have apparently 
no bearing upon the general condition of the animal, but are probably due to transient causes, such as overfeeding or scarcity of food, improper feeding, lack of exercise, excitement, fright, etc.

The accompanying charts (Figs. 49, 50, 51, 52) will serve to illustrate some of these points. The animals, two rabbits and two guinea-pigs, were taken at random from among the stock animals and placed each in a clean cage, the kind used for animals under experiment, and kept under as good general conditions as possible. For the first week the rabbits received each 100 grammes of green food (cabbage and turnips) daily, and the guinea-pigs 30 grammes each of the same food. During the second week this daily amount of food was doubled; during the third week it was quadrupled; and for the fourth and fifth weeks they each received an excess of food daily, consisting of green vegetables and grain (oats and corn). By reference to the charts sudden diurnal fluctuations in weight will be observed that do not correspond in all instances with scarcity or sufficiency of food. With the rabbits there is a gradual loss of weight with the smaller amounts of food, which losses are not totally recovered as the food is increased. With the guinea-pigs there is likewise at first a loss, but after a short time the weight remains tolerably constant, and is not so conspicuously affeeted by the increase in food as one might expect. From the recorded temperatures one sees the peculiar fluctuations mentioned. To just what they are due it is impossible to say. It is manifest that the normal temperature of these animals, if we cun speak of a normal temperature for animals presenting such fluctuations, is abont a degree or more, Centigrade, higher than that of human beings. The animals 
from which these charts were made were not inoculated, nor were they subjected to any operative procedures whatever, the only deviations from normal conditions being the variations in the daily amount of food given.

In certain instances, however, there will be noticed a constant tendency to diminution in weight, notwithstanding the daily fluctuations, and after a time a condition of extreme enraciation may be reached, the animal often being reduced to from 50 to 60 per cent. of its original weight. In other cases, after inoculations to which the animal is not susceptible, rabbits in particular, if properly fed, will frequently gain steadily in weight. The condition of progressive emaciation just mentioued is conspicuously seen after intravenous inoculation of rabbits with cultures of the bacillus typhi abdominalis and of the bacterium coli commune referred to in the chapter on the latter organism, and if looked for will doubtless be seen to follow inoculation with other organisms capable of producing chronic forms of infection, but which are frequently considered nonpathogenic because of their inability to induce acute conditions. Not infrequently in chrouic infections there may be hardly any marked and constant temperature variations until just before death, when there will sometimes be a rise and at other times a fall of temperature. In the majority of cases, however, one must be very cautious as to the amount of stress laid upon changes in weight and temperature, for unless they are progressive or continuous in one or another direction they may have little or no significance in indicating the existence or absence of disease. 


\section{CHA PTER XIII.}

Post-mortem examination of animals-Bacteriological examination of the tissues-Disposal of tissues and disinfection of instruments after the examination.

During the bacteriological examination of the tissues of dead animals certain rigid precautions must be observed in order to avoid error.

The antopsy should be made as soon as possible after death. If delay cannot be avoided, the animal should be kept on ice until the examination can be made, otherwise decomposition sets in, and the saprophytic bacteria now present may interfere with the accuracy of results. When the autopsy is to be made the animal is first inspected externally, and all visible lesions noted. It is then to be fixed upon its back upon a board with nails or tacks. The four legs and the end of the nose, through which the tacks are driven, are to be moderately extended. Plates are now to be made from the site of inoculation, if this is subcutaneous. The surfaces of the thorax and abdomen are then to be moistened to prevent the fine hairs, dust, ete., from floating about in the air and interfering with the work. An incision is then made through the skin from the chin to the symphysis pubis. This is only a skin incision, and does not reach deeper than the muscles. It is best done by first making a small incision with a scalpel, just large enough to permit of the introduetion of one blade of a blunt-pointed seissors. It is 
then completed with the scissors. The whole of the skin is now to be carefully dissected away, not only from the abdomen and thorax, but from the axillary, inguinal, and cervical regions, and the fore and hind legs as well. The skin is then pinned back to the board so as to keep it as far from the abdomen and thorax as possible, for it is from the skin that the chances of contamination are greatest.

It now becomes necessary to proceed very carefully. All incisions from this time on are to be made only through surfaces that have been sterilized. The sterilization is best accomplished by the use of a broad-bladed common table knife that has been heated in the gasflame. The blade, made quite hot, is to be held upon the region of the linea alba until the skin at that region begins to burn; it is then held transverse to this line over about the centre of the abdomen, thus making two sterilized tracks through which the abdomen may be opened by a crucial incision. The sterilization thus accomplished is, of course, directed only against organisms that may have fallen upon the surface from without, and it therefore need not extend deep down through the tissues.

In the same way two burned lines may be made from either extremity of the transverse line up to the top of the thorax.

With a hot scissors the central longitudinal incision extending from the point of the sternum to the genitalia, is to be made without touching the internal viscera. The abdominal wall must therefore be held up during the operation with sterilized forceps or hook.

The cross incision is made in the same way. When this is completed an incision throngh the ribs with a 
pair of heavy, sterilized scissors is made along the scorched tracks on either side of the thorax.

After this the whole anterior wall of the thorax may easily be lifted up, and by severing the connections with the diaphragm it may be completely removed.

When this is done and the abdominal flaps laid back, the coutents of both cavities are to be inspected and their condition noted without disturbing them.

After this the first steps to be taken are to prepare plates or Esmarch tubes from the blood, liver, spleen, kidneys, and any exudates that may exist.

This is best done as follows:

Heat a scalpel quite hot and apply it to a small surface of the organ from which the cultures are to be made. Hold it upon the organ until the surface directly beneath it is visibly scorched. Then remove it, heat it again, and while quite hot insert its point through the capsule of the organ. Into the opening thus made insert a sterilized platinum-wire loop, made of wire a little heavier than that commonly employed. Project this deeply into the tissues of the organ; by twisting it about enough material from the centre of the organ ean be obtained for making the cultures.

As the resistance offered by the tissues is sometimes too great to permit of a puncture with the ordinary wire loop, Nuttall (Centralblatt für Bakteriologie und Parasitenkunde, 1892, Bd. xi. p. 538) has devised for the purpose a platinum-wire spear which passesses considerable advantage over the loop. It is of the form seen in Fig. 53. It is easily made by beating a piece of heavy platinum wire into a spear-head at one end, and perforating this with a small drill, as seen in the ent. It is attached by the other end to either a metal 
or glass handle, preferably the former. It can readily be thrust into the densest of the soft tissues, and by twisting it about after its introduction particles of the tissue sufficient for examination are withdrawn in the eye of the spear-head.

FiG. 53.

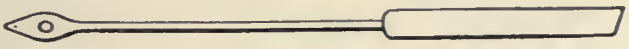

Nuttall's platinum spear for use at autopsies.

The cultures from the blood are usually made from one of the cavities of the heart, which is always entered through a surface which has been burned in the way given.

In addition to cultures, cover-slips from the site of inoculation, from each organ, and from any exudates that may exist, must be made. These, however, are prepared after the materials for the cultures have been obtained.

They need not be examined immediately, but may be placed aside, under cover, on bits of paper upon which the name of the organ from which they were prepared is written.

When the autopsy is complete and the gross appearances have been carefully noted, small portions of each organ are to be preserved in 95 per cent. alcohol for subsequent examination. Throughout the entire autopsy it must be borne in mind that all cultures, cover-slips, and tissues must be carefully labelled, not only with the name of the organ from which they originate, but with the date, designation of the animal, etc., so that an account of their condition 
after closer study may be subsequently inserted in the protocol.

The cover-slips are now to be stained, mounted, and examined microscopically, and the results carefully noted.

The same may be said for the subsequent study of the cultures and the hardened tissues which are to bc stained and subjected to microscopic examination. The results of microscopic study of the cover-slip preparations and of those obtained by cultures should in most cases correspond, though it not rarely occurs that bacteria are present in such small numbers in the tissues that their presence may be overlooked microscopically, and still they may appear in the cultures.

If the autopsy has been performed in the proper way, under the precautions given, and sufficiently soon after death, the results of the bacteriological examination should be either negative or the organisms which appear should be in pure cultures.

This is particularly the case with cultures made from the internal viscera.

Both the cover-slips and cultures made from the point of inoculation are apt to contain a variety of organisms.

If the organism obtained in pure culture from the intermal viscera, or those predominating at the point of inoculation of the animal, have caused its death, then subsequent inoculation of pure cultures of this organism into the tissues of a second animal should produce similar results.

When the antopsy is quite finished the remainder of the animal should be burned; all instruments subjected to either sterilization by steam or boiling for fifteen minutes in a 1 to 2 per cent. soda solution, and the board 
upon which the animal was tacked, as well as the tacks, towels, dishes, and all other implements used at the autopsy, are to be sterilized by steam. All cultures, coverslips, and, indeed, all articles likely to have infectious material upon them, must be thoroughly sterilized as soon as they are of no further service. 



\section{APPLICATION OF THE METHODS OF BACTERIOLOGY.}

\section{CHAPTER XIV.}

To obtain material with which to begin work.

Expose to the air of an inhabited room a slice of freshly steamed potato or a bit of slightly moistened bread upon a plate for about one hour. Then cover it with an ordinary water-glass and place it in a warm spot (temperature not to exceed that of the human body $-37.5^{\circ} \mathrm{C}$.), and allow it to remain unmolested. At the end of twenty-four to thirty-six hours there will be seen upon the cut surface of the bread or potato small, round, oval, or irregularly round patches which present various appearances.

These differences in macroscopic appearance are due, in some cases, to the presence or absence of color; in others to a higher or lower degree of moisture; in some instances a patch will be glistening and smooth, while its neighbor may be dull and rough or wrinkled; here will appear an island regularly round in outline, and there an area covered by an irregular ragged deposit. All of these gross appearances are of value in aiding us to distinguish between these colonies-for colonies they are-and under the same conditions the organisms composing each of them will always produce 
growth of exactly the same appearance. It was just such an experiment as this, accidentally performed, that suggested to Koch a means of separating and isolating from mixtures of bacteria the component individuals in pure cultures, and it was from this observation that the methods of cultivation on solid media were evolved.

If, without molesting our experiment, we continue the observation from day to day, we shall notice changes in the colonies due to the growth and multiplication of the individuals, composing them. In some cases the colonies will always retain their sharply cut, round, or oval outline, and will increase but little in size beyond that reached after forty-eight to seventy-two hours, whereas others will spread rapidly, and will very quickly overrun the surface upon which they are growing, and, indeed, grow over the smaller, less rapidly developing colonies. In a number of instances, if the observation be continued long enough, many of these rapidly growing colonies will, after a time, lose their lustrous and smooth or regular surface and will show, at first here and there, elevations which will continue to appear until the whole surface takes on a wrinkled appearance. Again, bubbles may be seen seattered through the colonies. These are due to the escape of gas resulting from fermentation which the organisms bring about in the medium upon which they are growing. Sometimes peculiar odors resulting from the same cause will be noticed.

Note carefully all these changes and appearances, as they must be employed subsequently in identifying the individual organisms from which each colony on the medium has developed.

If now we examine these points upon our bread or 
potato with a hand-lens of low magnifying power, we will be enabled to detect differences not noticeable to the naked eye. In some cases we shall still see nothing more than a smooth non-characteristic surface; while in others minute, sometimes regularly arranged, corrugations may be observed. In one colony they may appear as tolerably regular radii, radiating from a central spot; and again they may appear as concentric rings; and if by the methods which have been described we obtain from these colonies their individual components in pure culture, we shall see that this characteristic arrangement in folds, radii, or concentric rings, or the production of color, is under normal conditions constant.

So much for the simplest naked-eye experiment that can be made in bacteriology, and which serves to furnish the beginner with material upon which to begin his studies. It is not necessary at this time for him to burden his mind with names for these organisms; it is sufficient for him to recognize that they are mostly of different species and that they possess characteristics which will enable him to differentiate the one from the other.

In order now for him to proceed it is necessary that he should have familiarized himself with the methods by which his media are prepared aud the means employed in sterilizing them and retaining them sterilei.e., of preventing the access of foreign germs from without-otherwise his efforts to obtain and retain his organisms as pure cultures will be in vain.

Exposure and Contact.-Make a number of plates from bits of silk used for sutures, after treating them as follows: 
Place some of these pieces (about 5 centimetres long) into a sterilized test-tube, and sterilize them by steam for one hour. At the end of the sterilization remove one piece with sterilized forceps and allow it to brush against your clothing, then make a plate from it; draw another piece across the table and then plate it. Suspend three or four pieces upon a sterilized wire look and let them hang for thirty minutes free in the air, being sure that they touch nothing but the hook; then plate them separately.

Note the results.

In what way do these experiments differ and how can the differences be explained?

Expose to the air six Petri dishes into which either sterilized gelatin or agar-agar has been poured and allowed to solidify; allow them to remain exposed for five, ten, fifteen, twenty, twenty-five, and thirty minutes in a room where no one is at work. Treat a second set in the same way in a room where several persons are moving about. Be careful that nothing touches them, and that they are exposed only to the air. Each dish must be carefully labelled with the time of its exposure.

Do they present different results? What is the reason for this difference?

Which predominate, colonies resulting from the growth of bacteria, or those from common moulds?

How do you account for this condition? 


\section{CHA P T R X V.}

Various experiments in sterilization by steam and by hot air.

PLACE in one of the openings in the cover of the steam sterilizer an accurate thermometer; when the steam has been streaming for a minute or two the thermometer will register $100^{\circ} \mathrm{C}$.; wrap in a bundle of towels or rags or pack tightly in cotton a maximum thermometer; let this thermometer be in the centre of a bundle large enough to quite fill the chamber of the sterilizer. At the end of a few minutes' exposure to the streaming steam remove it; it will be found to indicate a temperature of $100^{\circ} \mathrm{C}$.

Closer study of the penetration of steam has tanght us, however, that the temperature which is found at the centre of such a mass may sometimes be that of the air in the meshes of the material, and not that of steam, and for this reason the sterilization at that point may not be complete, because hot air at $100^{\circ} \mathrm{C}$. has not the sterilizing properties that steam at the same temperature possesses. It is necessary, therefore, that this air should be expelled from the meshes of the material and its place taken by the steam before sterilization is complete. This is insured by allowing the steam to stream through the substances a few minutes before beginning to calculate the time of exposure. There is as yet no absolutely sure means of saying that the temperature at the centre of the mass is that of hot air or of steam, so that the exact length of time that is required for the expulsion 
of the air from the meshes of the material cannot be given.

Determine if the maximum thermometer indicates a temperature of $100^{\circ} \mathrm{C}$. at the centre of a moist bundle in the same way. as when a dry bundle was employed.

To about 50 c.c. of bouillon add about one gramme of chopped hay, and allow it to stand in a warm place for twenty-four hours. At the end of this time it will be found to contain a great variety of organisms. Continue the observation, and a pellicle will be seen to form on the surface of the fluid. This pellicle will be made up of rods which grow as long threads in parallel strands. In many of these rods glistening spores will be seen. After thoroughly shaking, filter the mass through a fine cloth to remove coarser particles.

Pour into each of several test-tubes about 10 c.c. of the filtrate. Allow one tube to remain unmolested in a warm place. Place another in the steam sterilizer for five minutes; a third for ten minutes; a fourth for onehalf hour; a fifth for one hour.

At the end of each of these exposures inoculate a tube of sterilized bouillon from each tube. Likewise make a set of plates or Esmarch tubes upon both gelatin and agar-agar from each tube, and note the results. At the same time prepare a set of plates or Esmarch tubes on agar-agar and on gelatin from the tube which has not been exposed to the action of the steam.

The plates or tubes from the unmolested tube will present colonies of a variety of organisms; separate and study these.

Those from the tube which has been sterilized for five miuutes will present colonies in moderate numbers, 
but, as a rule, they will represent but a single organism. Study this organism in pure cultures.

The same may be predicted for the tube which has been heated for ten minutes, though the colonies will be fewer in number.

The thirty-minute tube may or may not give one or two colonies of the same organism.

The tube which has been heated for one hour is usually sterile.

The bouillon tubes from the first and second tubes which were heated will usually show the presence of only one organism-the bacillus which gave rise to the pellicle-formation in our original mixture. This organism is the bacillus subtilis, and will serve as an object upon which to study the difference in resistance toward steam between the vegetative and spore stages of the same organism.

Inoculate about 100 c.c. of sterilized bouillon with a very small quantity of a pure culture of this organism, and allow it to stand in a warm place for about six hours. Now subject this culture to the action of steam for five minutes; it will be seen that sterilization, as a rule, is complete.

Treat in the same way a second flask of bouillon, inoculated in the same way with the same organism, but after having stood in a warm place for from fortyeight to seventy-two hours-that is, until the spores have formed, and it will be found that sterilization is not complete-the spores of this organism have resisted the action of steam for five minutes.

To determiue if sterilization is complete always resort to the culture methods, as the macroscopic and microscopic methods are deceptive; cloudiness of the media 
or the presence of bacteria microscopically does not always signify that the organisms possess the property of life.

Inoculate in the same way a third flask of bouillon with a very small drop from one of the old cultures upon which the pellicle has formed; mix it well and subject it to the action of steam for two minutes; then place it to one side for from twenty to twenty-four hours, and again heat for two minutes; allow it to stand for another twenty-four hours, and repeat the process on the third day. No pellicle will be formed, and yet spores were present in the original mixture, and, as we have seen, the spores of this organism are not killed by an exposure of five minutes to the steam. How can this result be accounted for?

Saturate several pieces of cotton thread, each about 2 $\mathrm{cm}$. long, in the original decomposed bouillon, and dry them carefully at the ordinary temperature of the room, then at a little higher temperature-about $40^{\circ} \mathrm{C}$. - to complete the process. Regulate the temperature of the hot-air sterilizer for about $100^{\circ} \mathrm{C}$, and subject several pieces of this infected and dried thread to this temperature for the same lengths of time that we exposed the same organisms in bouillon to the steam, viz., five, ten, thirty, and sixty minutes. At the end of each of these periods remove a bit of thread, and prepare a set of plates or Esmareh tubes from it. Are the results analogous to those obtained when steam was employed?

Inerease the temperature of the dry sterilizer and repeat the process. Determine the temperature and time necessary for the destruction of these organisms by the dry heat. These threads should not be simply 
laid upon the bottom of the sterilizer, but should be suspended from a glass rod, which may be placed inside the oven, extending across its top from one side to the other.

Place several of the infected threads in the centre of a bundle of rags. Subject this to a temperature necessary to sterilize the threads by the dry method. Treat another similar bundle to sterilization by steam. In what way do the results of the two processes differ? 


\section{CHAPTER XVI.}

Suppuration-The staphylococcus pyogenes aureus-Staphylococcus pyogenes albus and citreus-Streptococcus pyogenes-Bacillus pyocyaneus-General remarks.

Prepare from the pus of an acute abscess or boil that has been opened under antiseptic precautions a set of plates of agar-agar. Care must be taken that none of the antiseptic fluid gains access to the culture tubes, otherwise its antiseptic effect may be seen and the development of the organisms interfered with. It is best, therefore, to take up a drop of the pus upon the platinum-wire loop after it has been flowing for a few seconds; even then it must be taken from the mouth of the wound and before it has run over the surface of the skin. At the same time prepare two or three coverslips from the pus.

Microscopic examination of these slips will reveal the presence of a large number of pus-cells, both multinucleated and with horseshoe-shaped nuclei, some threads of disintegrated and necrotic connective tissue, and, lying here and there throughout the preparation, small round bodies which will sometimes appear singly, sometimes in pairs, and frequently will be seen grouped together somewhat like clusters of grapes. (See Fig. 54.) They stain readily and are commonly located in the material between the pus-cells; very rarely they may be seen in the protoplasmic body of the cell. (Compare the preparation with a similar one made from 
the pus of gonorrhœea (see Fig. 56, page 259). In what way do the two preparations differ, the one from the other ?)

FIG. 5 .

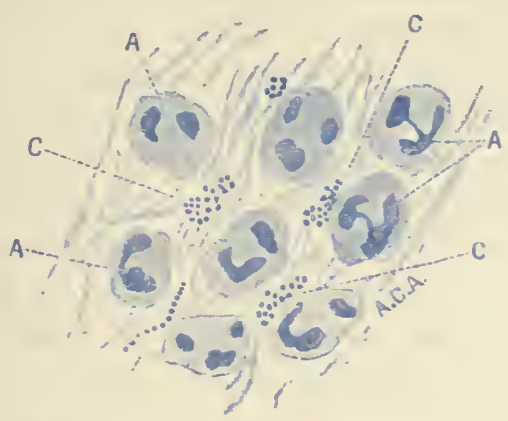

Preparation from pus, showing pus-cells, A, and staphylococci, C.

After twenty-four hours in the incubator the plates will be seen to be studded here and there with yellow or orange-colored colonies, which are usually round, moist, and glistening in the naked-eye appearances. When located in the depths of the medium they are commonly seen to be lozenge or whetstone in shape, while often they appear as irregular stars with blunt points, and again as irregularly lobulated dense masses. In structure they are conspicuous for their density. Under the low objective they appear, when on the surface, as coarsely granular, irregularly round patches, with more or less ragged borders and a dark irregular central mass, which has somewhat the appearance of masses of coarser clumps of the same material as that composing the rest of the colony. Microscopically, these colonies are composed of small round cells, irreg- 
ularly grouped together. They are in every way of the same appearance as those seen upon the original cover-slip preparations.

Prepare from one of these colonies a pure stab-culture in gelatin. After thirty-six to forty-eight hours liquefaction of the gelatin along the track of the needle, most conspicuous at its upper end, will be observed. As growth continues the liquefaction becomes more or less of a stocking-shape, and gradually widens out at its upper end into an irregular funnel. This will continue until the whole of the gelatin in the tube eventually becomes fluid. There can always be noticed at the bottom of the liquefying portion an orange-colored or yellow mass composed of a number of the organisms which have sunk to the bottom of the fluid.

On potato the growth is quite luxuriant, appearing as a brilliant, orange-colored layer, somewhat lobulated and a little less moist than when growing upon agaragar. It does not produce fermentation with gas-production. It belongs to the group of facultative aërobes.

In milk it rapidly brings about coagulation with acid reaction.

It is not motile, and being of the family of micrococci does not form endogenous spores. It possesses, however, a marked resistance toward detrimental agencies.

In bouillon it causes a diffuse clouding, and after a time presents a yellow sedimentation.

This organism is the commonest of the pathogenic bacteria with which we shall meet. It is the staphylococcus pyogenes aureus, and is the organism most frequently concerned in the production of acute, circumscribed, suppurative inflammations. It is almost every- 
where present, and is the organism that causes the surgeon so much annoyance.

In studying its effects upon lower animals a number of points are to be remembered. While it is the etiological factor in the production of most of the suppurative processes in man, still it is with no little difficulty that these conditions can be reproduced in lower animals. Its subcutaneous introduction into their tissues does not always result in abscess-formation, and when it does there seems to have been some coincident interference with the circulation and nutrition of these tissues which renders them less able to resist its inroads. When introduced into the great serous cavities of the lower animals its presence here, too, is not always followed by the production of inflammation. If the abdominal cavity of a dog, for example, be carefully openerl so as to make as slight a wound as possible, and no injury be done to the intestines, large quantities of bouillon cultures or watery suspensions of this organism may be, and repeatedly have been introduced into the peritoneum without the slightest injury to the animal. On the contrary, if some substance which acts as a direct irritant to the intestines-such, for example, as a small bit of potato upon which the organisms are growing - be at the same time introduced, or the intestines be mechanically injured, so that there is a disturbance in their circulation, then the introduction of these organisms is promptly followed by acute and fatal peritonitis. (Halsted. ${ }^{1}$ )

On the other hand, the results which follow their introduction into the circulation are practically constant.

1 Halsted: The Johns Hopkins Hospital Reports. Report in Surgery No. 1, 1991, Vol. II., No. 5, pp. 301-303. 
If one inject into the circulation of the rabbit through one of the veins of the ear, or in any other way, from 0.1 to 0.3 c.c. of a bouillon culture or watery suspension of a virulent variety of this organism, a fatal pyæmia always follows in from two and one-half to three days. A few hours before death the animal is frequently seen to have severe convulsions. Now and then excessive secretion of urine is noticed. The animal may appear in moderately good condition until from eight to ten hours before death. At the autopsy a typical picture presents; the voluntary muscles are seen to be marked here and there by yellow spots, which average the size of a flaxseed, and are of about the same shape. They lie usually with their long axis running longitudinally between the muscle fibres. As the abdominal and thoracic cavities are opened the diaphragm is often seen to be studded by them. Frequently the pericardial sac is distended with a clear gelatinous fluid, and almost constantly the yellow points are to be seen in the myocardium. The kidneys are rarely without them; here they appear on the surface, scattered about as single yellow points, or, again, are seen as conglomerate masses of small yellow points which occupy, as a rule, the area fed by a single vessel. If one make a section into one of these yellow points, it will be seen to extend deep down through the substance of the kidney as a yellow, wedge-shaped mass, the base of the wedge being at the surface of the organ.

It is very rare that these abscesses-for abscesses the yellow points are, as we shall see when we come to study them more closely-are found either in the liver, spleen, or brain; their usual location being, as said, in the kidney, myocardium, and voluntary muscles. 
These minute abscesses contain a dry, cheesy, necrotic centre, in which the staphylococci are present in large numbers, as may be seen upon cover-slips prepared from them. They may also be obtained in pure culture from these suppurating foci.

Preserve in Müller's fluid and in alcohol duplicate bits of all the tissue in which the abscesses are located. When these tissues are hard enough to cut sections should be made through the abscess-points, and the histological changes carefully studied.

Mrcroscopic Study of Cover-slips and Sections. -In cover-slip preparations this organism stains readily with the ordinary dyes.

In tissues, however, it is best to employ some method by means of which contrast-stains may be utilized, and the location and grouping of the organisms in the tissues rendered more conspicuous.

When stained, sections of tissues containing these small abscesses present the following appearances:

To the naked eye will be seen here and there in the section, if the abscesses are very numerous, small, darkly stained areas which range in size from that of a pinpoint up to those having a diameter of from 1 to $2 \mathrm{~mm}$. These points, when in the kidney, may be round or oval in outline, or may appear wedge-shaped, with the base of the wedge toward the surface of the organ. The differences in shape depend frequently upon the direction in which the section has been made through the kidney. In the muscles they are irregularly round or oval.

When quite small they appear in stained sections, to the naked eye, as simple, round or oval, darkly stained points, but when they are more advanced a pale centre can usually be made out. 
When magnified they appear in the earliest stages as minute aggregations of small cells, the nuclei of which stain intensely. Almost always there can be seen about the centre of these cell-accumulations evidences of progressing necrosis. The normal structure of the cells of the tissues will be more or less destroyed; there will be seen a granular condition due to cell-fragmentation; at different points about the centre of this area the tissue will appear cloudy and the tissue-cells will not stain readily. All about and through this spot will be seen the nuclei of pus-cells, many of which are undergoing disintegration. In the smallest of these beginning abscesses the staphylococci are to be seen scattered about the centre of the necrotic tissue, but in a more advanced stage they are commonly seen massed together in very large numbers in the form commonly referred to as emboli of micrococci.

The localized necrosis of the tissues which is seen at the centre of the abscess is the direct result of the action of a poison produced by the bacteria, and represents the starting-point for all abscess-formations.

When the process is farther developed the different parts of the abscess are more easily detected. They then present in sections somewhat the following conditions: at the centre can be seen a dense, granular mass which stains readily with the basic aniline dyes and, when highly magnified, is found to be made up of staphylococei. Sometimes the shape of this mass of staphylococei corresponds to that of the capillary in which the organisms became lodged and developed. Immediately about the embolus of cocci the tissues are seen to be in an advanced stage of necrosis. Their structure is almost completely destroyed, though it is 
seen to be more advanced in some of the elements of the tissues than in others. As we approach the periphery of this faintly stained necrotic area it becomes marked here and there with granular bodies, irregular in size and shape, which stain in the same way as do the nuclei of the pus-cells and represent the result of disintegration going on in these cells.

Beyond this we come upon a dense, deeply stainerl zone, consisting of closely packed pus-cells; of granular detritus resulting from destructive processes acting upon these cells; and of the normal cellular and connectivetissue elements of the part. Here and there through this zone will be seen localized areas of beginning death of the tissues. This zone gradually fades away into the healthy surrounding tissues. It constitutes the socalled "abscess-wall."

Such is the picture presented by the miliary abscess when produced experimentally in the rabbit, and it corresponds throughout with the pathological changes which accompany the formation of larger abscesses in the tissues of human beings.

From these small abscesses in the tissues of the rabbit the staphylococcus pyogenes aureus may again be obtained in pure culture, and will present identically the same characteristics that were possessed by the culture with which the animal was inoculated.

The Less Common Progenic Organisus. - The pus of an acute abscess in the human being may sometimes contain other organisms beside the staphylococcus pyogenes aureus. The staphylococcus pyogenes albus and citreus may be found. The colonies of the former are white, those of the latter are lemon-color. With these exceptions they are in all essential cultural peculi- 
arities similar to the staphylococcus aureus. As a rule, they are not virulent for animals, and when they do possess pathogenic properties it is in a much lower degree than is commonly the case with the golden staphylococcus. The streptococcus pyogenes is also sometimes present. The commonest of the pyogenic organisms, however, is that just described, viz. : the staphylococcus pyogenes aureus. An organism that is almost univer- sally present in the skin, and is often concerned in producing mild forms of inflammation, is the staphylococcus epidernidis albus (Welch), an organism that may readily be confused with the staphylococcus albus. It is distinguished from the latter by the slowness with which it liquefies gelatin and by the comparative absence of pathogenic properties when injected into the circulation of rabbits. Welch regards this organism as a variety of the staphylococcus pyogenes albus. He suggests the above designation for it because of its very limited pyogenic properties.

The Streptococcus Pyogenes.-From a spreading phlegmonous inflammation prepare cover-slips and cultures. What is the predominating organism? Does it appear in the form of regular clusters like those of grapes, or have its individuals a definite regular arrangement? Are its colonies like those of the staphylococcus pyogenes aureus?

Isolate this organism in pure cultures. In these eultures it will be found on microscopic examination to present an arrangement somewhat like a chain of beads. (Fig. 55.)

Determine its peculiarities and describe them accurately. They should be as follows:

Upon microscopic examination a micrococcus should 

be found, but differing in its arrangement from the staphylococei just described. The single cells are not seattered irregularly or arranged in clumps similar to bunches of grapes, but are joined together in chains like strands of beads. These strands are sometimes regular in the arrangement and size of the individual cells composing them, but more commonly certain irregular parts may be seen in them. Here they appear as if two or

Fr6. 55.

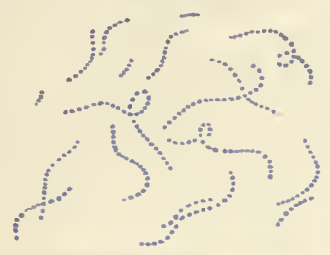

Streptococcus pyogenes.

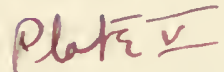

three cells had fused together to form a link, so to speak, in the chain, that is somewhat longer than the remaining links; again, portions of the chain may be thinner than the rest, or may appear broken or ragged. Commonly the individuals comprising this chain of cocei are not round, but appear flattened on the sides adjacent to one another. The chains are sometimes short, consisting of four to six cells, or again they may be much longer, and extend from a half to two-thirds of the way across the field of the microscope.

Under artificial conditions it sometimes grows well, and can be cultivated through many generations, while again it rapidly loses its vitality. When isolated from the diseased area upon artificial media it seems to retain its vitality for a longer period if replanted upon fresh media every day or two for a time; but if the first gen- 
eration is transplanted and is allowed to remain upon the original medium, it is not uncommon to find the organism incapable of further cultivation after a week or ten days.

Under no conditions is the growth of this organism very luxuriant.

On gelatin plates its colonies appear after forty-eight to seventy-two hours as very small, flat, round points, of a bluish-white or opalescent appearance. They do not cause liquefaction of the gelatin, and in size they rarely exceed $0.6-0.8 \mathrm{~mm}$. in diameter. Under low magnifying power they have a brownish or yellowish tinge by transmitted light, and are finely granular. As the colonies become older their regular border may become slightly irregular or notched.

In stab-cultures in gelatin they grow along the entire needle-track as a finely granular line, the granules representing minute colonies of the organism. On the surface the growth does not usually extend beyond the point of puncture.

On agar-agar plates the colonies appear as minute pearly points, which when slightly magnified are seen to be fincly granular, of a light-brownish color, and regular at their margins.

When smeared upon the surface of agar-agar or gelatin slants the growth that results is a thin, pearly, finely granular layer, consisting of minute colonies growing closely side by side. Its growth is most luxuriant on glycerin agar-agar at the temperature of the ineubator $\left(37.5^{\circ} \mathrm{C}\right.$.), and least on gelatin.

On blood-serum its colonies present little that is characteristic; they appear as small, moist, whitish points, from 0.6 to $0.8 \mathrm{~mm}$. in diameter, that are slightly ele- 
vated above the surface of the serum. They do not coalesce to form a layer over the surface, but remain as isolated colonies.

On potato no visible development appears, but after a short time (thirty-six to seventy-two hours) there is a slight increase of moisture about the point inoculated, and microscopic examination shows that a multiplication of the organisms placed at this point has occurred.

In milk its conduct is not always the same, some cultures causing a separation of the milk into a firm clot and colorless whey, while others do not produce this coagulation. The latter, when cultivated in milk of a neutral or slightly alkaline reaction, to which a few drops of litmus tincture have been added, produce a very faint pink color after twenty-four hours at $37.5^{\circ}$ C.; there is no coagulation.

In bouillon it grows as tangled masses or clumps, which upon microscopic examination are seen to consist of long chains of cocci twisted or matted together.

It grows best at the temperature of the body $\left(37.5^{\circ}\right.$ C.), and develops, but less rapidly, at the ordinary room temperature. When virulent, its virulence is said by Petruschky to be preserved by retaining cultures in the ice-chest after they have been growing on gelatin for two days at $22^{\circ} \mathrm{C}$.

It is a facultative anaërobe.

It stains with the ordinary aniline dyes, and is not decolorized when subjected to Gram's method.

It is not motile, and, being a micrococcus, does not form endogenous spores. Under artificial conditions we have no reason to believe that it enters a stage where its resistance to detrimental agencies is increased. In the tissues of the body, howerer, it appears to pos- 
sess a marked tenacity to vitality, for it is not rare to observe recurrences of inflammatory conditions duc to this organism, often at a relatively long time after the primary site of infection is healed.

When introduced into the tissues of lower animals its effects are uncertain. Rosenbach and Passet claimed that protracted, progressive, erysipelatoid inflammations were produced, and Fehleisen, who described a streptococcus in erysipelas that is in all probability identical with the streptococcus pyogenes now under consideration, stated that it produced in the tissues of rabbits (the base of the ear) a sharply defined, migratory reddening without pus-formation. The writer has encountered a culture of this organism that possessed the property of inducing erysipelas when introduced into the skin of the ear, and disseminated absecss-formation when injected into the circulation of rabbits. This observation has an important bearing upon the question concerning the identity of streptococci found in inflammatory conditions. As a rule, it is difficult to obtain any definite pathological alterations in the tissues of animals through the introduction into them of cultures of this organism by any of the methods of inoculation ordinarily praetised. Occasionally, however, cultures are encountered that are conspicuous for their pathogenic powers.

This is the streptococcus pyogenes, and is the organism most commonly found in rapidly spreading suppuration in contradistinction to the staphylococcus pyogenes aureus, which is most frequently found in circumscribed abscess-formations; they nay be found togetler.

If the opportunity presents, obtain cultures from a case of erysipelas. Compare the organism thus obtained 
with the streptococcus just mentioned. Inoculate rabbits both subcutaneously and into the circulation with about 0.2 c.c. of pure cultures of these organisms in bouillon. Do the results correspond, and do they in any way suggest the results obtained with the staphylococcus pyogenes aureus when introduced into animals in the same way? Do these streptococci flourish readily on ordinary media?

The organisms that have just been described are commonly known as the "pyogenic cocci" of Ogston, Rosenbach, and Passet, and up to as late as 1885 were believed to be the specific factors concerned in the production of suppurative inflammations. Since that time, however, considerable modification of this view has taken place, and while they are still known to be the most common causes of suppuration, they are also known not to be the only causes of this process.

With the more general application of bacteriological methods to the study of the manifold conditions coming under the eye of the physician, the surgeon, and the pathologist, observations are constantly being made that do not accord with the view formerly held with regard to the specific relation of the pyogenic cocci to all forms of suppuration. There is an abundance of evidence now at command to justify the opinion that there are a number of organisms not commonly classed as pyogenic which may, under peculiar circumstances, assume this property. For example:

The bacillus of typhoid fever has been found in pure culture in osteomyelitis of the ribs; in acute purulent otitis media; in abscess of the soft parts; in the pus of empyema, and in localized fibro-peritonitis, either during its course or as a sequela of typhoid fever. 
The bacterium coli commune has been found to be present in pure culture in acute peritonitis; in liver abscess; in purulent inflammation of the gall-bladder and ducts; in appendicitis; and Welch has found it in pure culture in fifteen different inflammatory conditions.

The micrococcus lanceolatus (pneumococcus) has been found to be the only organism present in abscess of the soft parts; in purulent infiltration of the tissues about a fracture; in purulent cerebro-spinal meningitis; in suppurative synovitis; in acute pericarditis, and in acute inflammation of the middle ear.

Moreover, many of the less common organisms have been detected in pure cultures in inflammatory conditions with which they were not previously thought to be concerned, and to which they are not usually related etiologically.

In consideration of such evidence as this it is plain that we can no longer adhere rigidly to the opinions formerly held upon the etiology of suppuration, but must subject them to modifications in conformity with this newer evidence. We now know that there exist bacteria other than the "pyogenic cocci," which, though not normally pyogenic, may give rise to tissue-changes indistinguishable from those produced by the ordinary pus organisms. ${ }^{2}$

\section{GONOCOCCUS. MICROCOCCUS GONORRIA:A.}

One observes upon microscopic examination of coverslips prepared from the pus of acute gonorrhœa that

1Welch: "Conditions underlying the Infection of Wounds," Americau Journal of the Medical Selences, November, 1891.

2 lior a more detailed discussion of the subject see "The Faetors Concerued in the Production of Suppuration," International Medieal Magazine, Philadelphía, May, 1892. 
many of the pus-cells contain within their protoplasm numerous small, stained bodies that are usually arranged in pairs. Occasionally a cell is seen that contains only one or two pairs of such bodies; again, a cell will be encountered that is packed with them. Occasionally masses of these small bodies will be seen lying free in the pus. (See Fig. 56.) The majority of the pus-cells do not contain them.

Fig. 56.

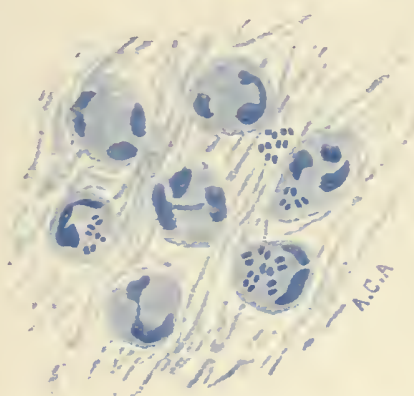

Pus of gonorrhoea, showing diplococei in the bodies of the pus-cells.

$$
\text { aso peote I }
$$

These small, round, or oval bodies are the so-called "gonococci" discovered by Neisser, and more fully studied subsequently by Bumm, to whom we are indebted for much of our knowledge concerning them.

As the name implies, this organism is a micrococcus, and as it is commonly arranged in pairs (flattened at the surface in juxtaposition) it is often designated as diplococcus of gonorrhœa. It is always to be found in gonorrhœal pus, and often persists in the urethral discharges and secretions far into the stage of convalescence. It is not present in inflammatory conditions other than those of gonorrhoeal origin. 
It is easily deteeted mieroscopically in the secretions of acute gonorrhœa. In secondary lesions and in very old, ehronic cases it is difficult of detection and frequently eludes all efforts to find it. It is stained by the ordinary methods, but perhaps most satisfactorily with the alkaline solution of methylene-blue. Most important as a differential test is its failure to stain by the method of Gram. (How does this compare with the behavior of the other pyogenic cocei when treated in the same way?)

It does not grow upon the ordinary nutrient media, and has only been isolated in culture through the employment of special methods. Its growth under artificial circumstances seems to depend upon some partieular nutrient substance that is supplied by blood or blood-serum, and in all the media that have been successfully used for its eultivation this substance is apparently an essential constituent. By the majority of investigators it is thought that only human blood possesses this important ingredient.

It was first isolated in eulture by Bumm, who used for this purpose coagulated human blood-serum obtained from the placenta.

Wertheim improved the method of Bumm by using a mixture of equal parts of sterile human blood-serum and ordinary sterilized nutrient agar-agar, the latter having been liquefied and kept at $50^{\circ} \mathrm{C}$. until after the mixture was made, when it was allowed to cool and solidify.

Other investigators have substituted for human bloodserum certain pathologieal fluids from the human body, such as ascites fluid, fluid from ovarian cysts, and serous effusions from the pleura and from the joint-cavities. 
The method used by Pfeiffer for the cultivation of the bacillus of influenza is also said to have been successfully employed. Abel recommends a needle-prick in the finger as a most convenient source from which to obtain the necessary amount of human blood that is to be smeared over the surface of the slanting agar-agar when Pfeiffer's method is employed.

Wright's modification of Steinschneider's method has given such satisfactory results in his hands that it will be given here with more or less detail. The medium consists of a mixture of urine, blood-serum (human or bovine, either serving the purpose), and nutrient agaragar. The urine and blood-serum are collected without special aseptic precautions, and subsequently freed from bacteria by filtration through unglazed porcelain. Frequently this is the tedious part of the process, as the serum and urine pass very slowly through the porcelain filters that are generally employed in laboratories. Wright recommends a filtering cylinder manufactured by the Boston Filter Company as an apparatus that not only strains out all bacteria, but also permits of a very rapid passage of the fluid.

The details of the method as given by Wright are as follows: "A litre of nutrient agar is prepared in the usual manner, and after filtration it is evaporated to about 600 c.c. This concentration is desirable, so that, after the dilution with the urine and serum, the medium may be sufficiently firm. This concentrated agar is then run into test-tubes and the whole sterilized by steam on three successive days. The quantity of agar placed in each tube is smaller than is usual; this is in order to allow for the subsequent addition of the urine and serum. 
"The blood-serum, which need not be free from corpuscles, is first passed through white sand, which is supported in a funnel by filter-paper, in order to remove as much as possible any partieles in suspension, and is then mixed with half its volume of fresh urine. The mixture of urine and blood-serum is next filtered by suction through an unglazed poreelain cylinder into a receiving flask, such as chemists use for similar purposes by means of a water vacuum pump. This frees the mixture from bacteria.

"The usual precautions are, of course, taken to prevent the contamination of the filtrate, such as the previous sterilization by steam of the cylinder and receiving flask, besides others which will oceur to any bacteriologist.

"To the agar in each test-tube, which is fluid and of a temperature of about $40^{\circ} \mathrm{C}$., there is added about one-third to one-half its volume of the filtered mixture of urine and blood-serum. This is conveniently accomplished by pouring the mixture from the receiving flask through the lateral tube, inserted near its neek directly into the tubes. The preliminary melting of the agar is best effeeted in the steam sterilizer in order that any organisms which have found lodgement in the cotton plugs of the tubes may be destroyed. When the agar is melted it is cooled and kept fluid by placing the tubes in a water-bath at $40^{\circ} \mathrm{C}$. Each tube, after the addition of the urine and serum to the fluid agar, is quickly shaken to insure a good mixture, and is then placed in an inclined position to allow the agar to solidify with a slanting surface. When the medium in the tubes has soliclified the tubes are placed in the incubator for about twenty-four hours to test for eontaminations, after which they are ready for use." 
The successive dilutions are now to be made upon the slanting surface of this mixture, as the mass in the tubes cannot be redissolved without exposure to a degree of heat that apparently interferes with the nutritive value of the serum contained in the medium.

When inoculated with gonorrhœal pus, by smearing a loopful over the surface, the tubes are to be kept at from $37^{\circ}$ to $38^{\circ} \mathrm{C}$. The organism does not develop properly at a temperature below this point.

After twenty-four hours the colonies of the gonococcus appear on the surface of the medium, according to Wright, as very tiny, grayish, semi-translucent points. After forty-eight hours they may be about 1 millimetre or so in diameter, slightly elevated, with a rounded outline, grayish in color, and by transmitted light semi-translucent. By reflected light their surface has the appearance of frosted glass. Later, if few in number, so that their growth is unimpeded, the colonies may attain a diameter of 2 millimetres or more, become thicker and denser, with a faintly brownish tinge about their centres, and a slightly irregular outline.

Under a low power of the microscope a fully developed eolony is seen to consist of a general circular expansion, with thin, translucent, smooth, sharply defined margin, but becoming brownish, granular, and thicker toward the central portion, which is made up of coarse, granular, brown-colored clumps closely packed together.

The appearances coincide with the figure of such a colony given by Wertheim.

If transplanted from the original culture to either glycerin agar-agar or to Lœffler's serum mixture, a 
growth is sometimes observed, more often in the latter than in the former, but of so feeble a nature that these substances cannot be regarded as suitable for its cultivation. As a rule, development does not occur on glycerin agar.

Microscopic examination of colonies of this orgauism reveals the presence of a diplococcus somewhat larger than the ordinary pyogenic cocci. The opposed surfaces of the individual cells that comprise the couplets are flattened and separated by a narrow slit. At times the cocci are arranged as tetrads.

This organism cannot be grown at a temperature lower than that of the human body, and cultures that have been obtained by either of the favorable methods are said to lose their vitality when kept at ordinary room temperature for about two days.

It is killed in a few hours by drying.

Cultures retain their vitality under favorable conditions of nutrition, temperature, and moisture for from three to four weeks.

It is withont pathogenic properties for monkeys, dogs, and horses, as well as for the ordinary smaller animals used for this purpose in the laboratory.

In man typical gonorrhøa has been produced on several occasions by the introduction into the urethra of pure cultures of this organism.

In addition to its causal relation to specific urethritis, it is the cause of gonorrheal prostatitis in man, of gonorrhœal proctitis in both sexes, and of gonorrhoal inflammation of the urethra, of Bartholin's glands, of the cervix uteri, and of the vagina in women and young girls. It is etiologically related to the specific conjunetivitis (ophthalnia neonatorum) of 
young infants, and also occasionally of ophthalmia in adults.

Secondarily, it is concerned in specific inflammations of the tubes and ovaries, of the lymphatics communicating with the genitalia, of the serons surfaces of joints, and of those of the heart, lungs, and abdominal carity.

Positive and Negative Distinguishing PeculiARITIES of the GoNococcus. - Since gonorrhoeal discharges may be contaminated with pyogenic cocci other than those causing the specific inflammation, it is important in efforts to isolate this organism that the differential tests be borne in mind and put into practice. The gonococcus is differentiated from the commoner pyogenic organisms by the following peculiarities:

First, it is practically always seen in the form of diplococci, the pair of individual cells having the appearance of two hemispheres, with the diameters opposed and separated from one another by a narrow, colorless slit. (Is this the case with the staphylococcus or streptococcus pyogenes?)

Second, in the pus it is practically always within the protoplasmic bodies of pus cells. (How does this compare with the conditions found in ordinary pus?)

Third, it stains readily with the ordinary stainingreagents, but loses its color when treated by the method of Gram. (Treat a cover-slip from ordinary pus by this method and note the result.)

Fourth, it does not develop upon any of the ordinary media used in the laboratory; while the common pusorganisms, with perhaps the exception of the streptocoeci, are vigorous growers and are not markedly fastidious as to their nutritive medium.

Fifth, when obtained in pure culture by either of the 
special procedures noted above, its cultivation may be continued upon the same medium; but growth will usually not be observed if it is transplanted to ordinary nutrient gelatin, agar-agar, bouillon, or potato; should it grow under these circumstances its development will be very feeble. (Is this the case with common pus-producers?)

Sixth, it has no pathogenic properties for animals, while several of the pyogenic cocci, notably staphylococcus aureus and streptococcus pyogenes, are usually capable of exciting pathological conditions. (This is less commonly true of streptococcus pyogenes than of staphylococcus aureus.)

\section{BACILLUS PYOCYANEUS (BACILlUS OF GREEN PUS).}

Another common organism that may properly be mentioned at this place, though perhaps not strictly pyogenic, is a bacillus frequently found. in discharges from wounds, viz., the bacillus pyocyaneus, or bacillus of green pus, or of blue pus, or of blue-green pus, as it is commonly called. The bacillus pyocyancus is a delicate rod with rounded or pointed ends. It is actively motile; does not form spores. As seen in preparations made from cultures it is commonly clustered together in irregular masses. It does not form long filaments, there being rarely more than four joined together end to end, and most frequently not even two.

It grows readily on all artificial media, and gives to some of them a bright-green color that is most conspicuous where it is in contact with the air. This green color is not seen in the growth itself to any extent, but is diffused through the medium on which the organism 
is developing. With time this color becomes much darker, and in very old agar-agar cultures may become almost black (sometimes very dark-blue green, at others brownish-black).

FIG. 57.

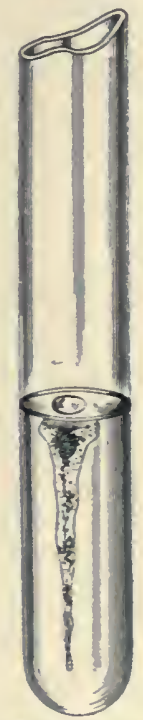

Stab-culture of $b$. pyocyaneus in gelatiu after twentyeight hours at $22^{\circ} \mathrm{C}$.
FIG. 59,

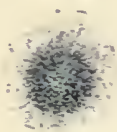

Colony of b. pyocyaneus anter tweuty-fonr hours on gelatin at $20^{\circ}-22^{\circ} \mathrm{C}$.

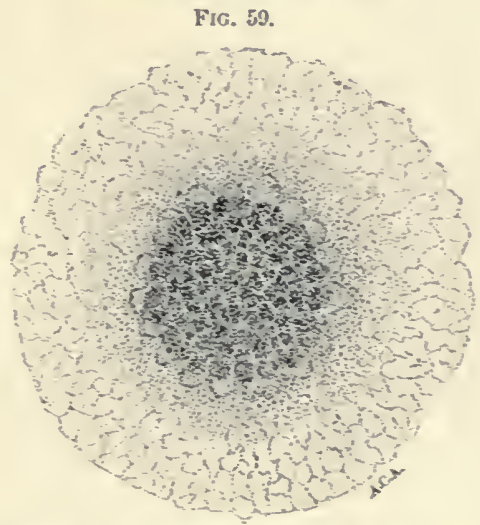

Colony of b. pyocyaneus after forly-two hours on gelatin at $20^{\circ}-220 \mathrm{C}$.

Its growth on gelatin in stab-cultures is accompanied by liquefaction and the diffusion of a bright-green color throughout the unliquefied medium. As liquefaction continues, and the entire gelatin ultimately becomes fluid, the green color is confined to the superficial layers that are in contact with the air. The form taken by the liquefying portion of the gelatin in the earliest stages of 
development is somewhat that of an irregular, slender funnel. (See Fig. 57.)

On gelatin plates the colonies develop rapidly; they are not sharply circumseribed, but- usually present at first a fringe of delicate filaments about their periphery (see Fig. 58). As growth progresses and liquefaction becomes more advanced, the central mass of the colony sinks into the liquefied depression, while at the same time there is an extension of the colony laterally. At this stage the colony, when slightly magnified, may present various appearances, the most common being that shown in Fig. 59.

The gelatin between the growing colonies takes on a bright yellowish-green color; but as growth is comparatively rapid, it is quickly entirely liquefied, and one often sees the colonies floating about in the pale-green fluid.

On agar-agar the growth is dry, sometimes with a slight metallic lustre, and is of a whitish or greenishwhite color, while the surrounding agar-agar is bright green. With time this bright green becomes darker, passing to blue-green, and finally turns almost black.

On potato the growth is brownish, dry, and slightly elevated above the surface. With some cultures the potato about the growth beeomes green; with others this change is not so noticeable. With many eultures a peculiar phenomenon may be produced by lightly touching the growth with a sterilized platinum needle. This phenomenon consists in a change of color from brown to green at the point touched. It is best seen in cultures that have been kept in the ineubator for from seventy-two to ninety-six hours. It occurs in from one to three minutes after touching with the needle, and 
may last from ten minutes to half an hour. This is the "chameleou phenomenon" of Paul Ernst.

In bouillon the green color appears, and the growth is seen in the form of delicate flocculi. A very delicate mycoderma is also produced.

In milk it causes an acid reaction, with coincident coagulation of the casein.

On blood-serum and egg-albumin its growth is accompanied by liquefaction. The growth on coagulated egg-albumin is seen as a dirty-gray deposit surrounded by a narrow brownish zone; the remaining portion of the medium is bright green in color. As the culture becomes older the green may give way to a brown discoloration.

In peptone solution (double strength) it causes a bluish-green color. In one of four cultures from different sources there was a distinct blue color produced.

It produces indol.

It stains with the ordinary dyes, and its flagella may be readily demonstrated by Lœeffler's method of staining.

Inoculation into animals. As a rule, cultures of this organism obtained directly from the discharges of a wound are capable, when introduced into animals, of lighting up diseased conditions; but cultures that are kept on artificial media for a long time may in part, or completely, lose this power.

When guinea-pigs or rabbits are inoculated subcutaneously with 1 c.c. of virulent fluid cultures of this organism, death usually results in from eighteen to thirty-six hours. At the seat of inoculation there is found an extensive purulent infiltration of the tissues and a marked zone of inflammatory œdena.

When introduced directly into the peritoneal cavity 
the results are also fatal, and at autopsy a genuine fibrinous peritonitis is found. There is usually an accumulation of serum in both the peritoneal and pleural cavities. At autopsies after both methods of inoculation the organisms will be found in the blood and internal viscera in pure cultures.

When animals are inoculated with small doses (less than 1 c.c. of a bouillon culture) of this organism death may not ensue, and only a local inflammatory reaction (abscess-formation) may be set up. In these cases the animals are usually protected against subsequent inoculation with doses that would otherwise prove fatal.

Most interesting in connection with bacillus pyocyaneus is the fact, as brought out in the experiments of Bouchard, and of Charrin and others, that its produets possess the power of counteracting the pathogenic activities of bacillus anthracis. That is to say, if an animal be inoculated with a virulent anthrax culture, and soon after be inoculated with a culture of bacillus pyocyaneus, the fatal effeets of the former inoculation may be prevented.

In the literature upon the green-producing organisms that have been found in inflammatory conditions several varieties-believed to be distinet species-have been described, but when cultivated side by side their biologieal differenees are seen to be so slight as to render it probable that they are but modifications of one and the same species.

THE BACILLUS OF BUBONIC PLAGUE.

Before passing from the subject of suppuration it may not be inappropriate to call attention to the light 
that modern methods of investigation have shed upon the etiology of bubonic plague, an epidemic disease characterized by suppuration of the lymphatic glands, and accompanied by a very high rate of mortality.

This pestilence, probably endemic in certain sections of the Orient, is one of the most conspicuous epidemic diseases of history. Since early in the Christian era epidemics and pandemics of plague have made their appearance in Europe at different times. During and after the Middle Ages it was more or less frequent in India, China, Arabia, Northern Africa, Italy, France, Germany, and Great Britain. In history it is variously known as the "Justinian Plague" of the sixth century, the "Black Death" of the fourteenth century, and the "Great Plague of London" of the seventeenth century, though it is difficult to say to what extent these pestilences were uncomplicated manifestations of genuine bubonic plague. During the existence of the Justinian Plague 10,000 people are said to have died in Constantinople in a single day, and Hecker estimates that during the pandenic of the Black Death $25,000,000$ people (a quarter of the entire population of Europe) succumbed to the disease. During the Great Plague of London (1664-'65) the total mortality for one year was 68,596 , out of an estimated population of 460,000 souls.

It is not surprising to learn that it was to guard against the plague that quarantine regulations were first established.

For the most recent, and probably the most exact information concerning the cause and pathology of the plague we are indebted to the investigations of Yersin, 
of Kitasato, and of Aoyama, conducted during the epidemic of 1894 in Hong-Kong, China. 'The results of these studies indicate that bubonic plague is an infectious, not markedly contagious, disease that depends for its existence upon the presence in the tissues of a specific micro-organism - the so-called plague or pest bacillus.

\section{FIg. 60 .}

$A$

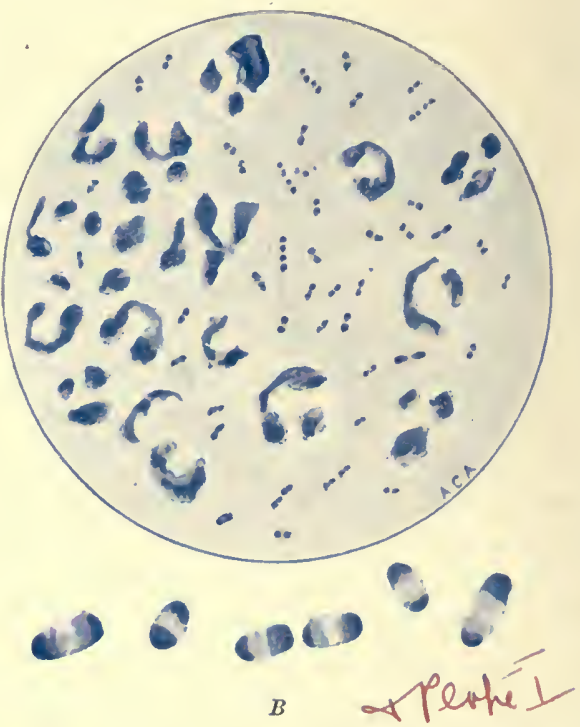

Bacilius of bubonic plague: $A$, in pus from suppurating bubo; $B$, the bacilli very much enlarged to show peculiar polar staining.

This organism is described as a short, oval bacillus, usually secn single, sometimes joined end to end in pairs or threes, less commonly as longer threads. It stains more readily at its ends than at its centre. It is sometimes capsulated; is non-spore-forming; is aërobic, and 
is non-motile. It is found in large numbers in the suppurating glands, and in much smaller numbers in the circulating blood. (Fig. 60.)

It is demonstrable in cover-slip preparations made from the pus and in sections of the glands by the ordinary staining-methods. Yersin states that it retains its color when treated by the method of Gram, while Kitasato says that it at one time stains by this method and at another it becomes decolorized. Aoyama observed that those bacilli within the suppurating glands were decolorized, while those in the blood retained the stain when treated by Gram's method.

Since there is often a mixed infection in these cases it appears likely that the above discrepancy may be attributed to individual peculiarities of different species of bacteria that were under examination.

It may be cultivated upon ordinary nutrient media.

The most favorable temperature for its growth is between $36^{\circ}$ and $39^{\circ} \mathrm{C}$. Its colonies on glycerin agaragar and on coagulated blood-serum are described as iridescent, transparent, and whitish. On gelatin at $18^{\circ}-20^{\circ} \mathrm{C}$. it develops as small, sharply defined, white colonies. In stab-cultures it develops both on the surface and along the track of the needle. Its growth is slow. It does not cause a diffuse clouding of bouillon, but grows rather as irregular, flocculent clumps that adhere to the sides or sink to the bottom of the vessel, leaving the fluid clear.

It is pathogenic for mice, rats, guinea-pigs, rabbits, and sheep. Pigeons are immune. The animals succumb to subcutaneous inoculation in from two to three days. According to Yersin, the site of subcutaneous inoculation becomes odematous and the neighboring 
lymphatics enlarged in a few hours. After twenty-four hours the animal is quiet, the hair is rumpled, tears stream from the eyes, and later convulsions set in which last till death. The results found at autopsy are: bloodstained odema at the site of inoculation, reddening and swelling of the lymphatic glands, bloody extravasation into the abdominal walls, serous effusion into the pleural and peritoneal cavities; the intestine is occasionally hyperæmic, the adrenal bodies congested, and the spleen is enlarged, often showing the presence of grayish points suggestive of miliary tubercles. The plagne, or pest, bacillus is to be detected in large numbers in the local œdema, the lymph glands, the blood, and the internal organs.

As is the case with the group of hemorrhagic septicæmia bacteria, when death does not result promptly after infection there is usually only local evidence of the inoculation, the distribution of the micro-organisms throughout the body being considerably diminished.

It is said that when virulent cultures are employed animals may sometimes be infected by way of the alimentary tract.

This organism is killed by drying at ordinary room temperature in four days. It is killed in three to four hours by direct sunlight. It is destroyed in a half hour by $80^{\circ} \mathrm{C}$., and in a few minutes by $100^{\circ} \mathrm{C}$. (steam). It is killed in one hour by 1 per cent. carbolic acid and in two hours by 1 per cent. milk of lime.

The bacilli apparently lose their virulence after longcontinued cultivation under artificial circumstances, and it is said that from slowly developing, chronic buboes non-virulent or feebly virulent cultures are often obtained. Variations in the degree of virulence 
have been observed in different colonies from the same source.

In man the bacilli are most numerous in the enlarged, suppurating lymphaties. They are present, but in smaller numbers, in the blood and the internal organs.

It has been observed that in the suppurating lymphatic glands of man a variety of organisms may be present, conspicuous among them being the so-called plague bacillus. Occasionally, micrococci predominate.

In these cases of mixed infection the pest bacilli are said to stain less intensely with alkaline methylene-blue than do the streptococei, and more intensely than do the staphylococci that are present. Also, in this event, the streptococei retain the Gram stain, while the pest bacilli and the staphylococci do not. It has been suggested that possibly the organisms found by Kitasato in the blood, and which he describes as pest bacilli, that retained the color when treated by the method of Gram, were pairs of micrococci and not bacilli at all.

It is the opinion of Aoyama that the suppuration of the glands is not caused by the plague bacillus, but is rather the result of the action of the pyogenic cocei with which it is so often associated.

Again, according to Aoyama, the most important and frequent mode of infection in man is through wounds of the skin. He does not regard either the air-passages or the alimentary tract as frequent portals of infection.

The order in which the lymphatics manifest disease appears to depend upon the location of the primary infection. That is to say, if it is upon the feet, as of persons who go barefooted, the superficial and deep inguinal glands are the first to show signs of the dis- 
ease; while if infection occurs through wounds of the hand, the buboes appear first in the axillary region. As a rule, the wound through which infection is received shows little or no inflammatory reaction. ${ }^{1}$

1 The works of Yersin. of Kitasato, and of Aoyama have been exhaustively reviewed by Flexner in the Bulletin of the Johns Hopkins Hospital, vol. v., 1894 , p. 96 , and vol. vii., 1896, p. 180. I am indebted to these reviews for much that is here presented on this subject. 


\section{CHAPTER XVII.}

Sputum septicæmia-Septicæmia resulting from the presence of micrococcus tetragenus in the tissues-Tuberculosis.

OBTAIN from a tuberculous patient a sample of fresh sputum-that of the morning is preferable. Spread it out in a thin layer upon a black glass plate and select one of the small, white, cheesy masses or dense mucous clumps that will be seen scattered through it. With a pointed forceps smear it carefully upon two or three thin cover-slips, dry and fix them in the way given for ordinary cover-slip preparations. Stain one in the ordinary way with Lœftler's alkaline methylene-blue solution, the other by the Gram method, the third after the method given for tubercle bacilli in fluids or sputum.

In that stained by Lœffler's method-slip No. 1will be seen a great variety of organisms-round cells, ovals, short and long rods, perhaps spiral forms. But not infrequently will be seen diplococci, having more or less of a lancet shape; they will be joined together by their broad ends, the points of the lancet being away from the point of juncture of the two cells. There may also be seen masses of cocci which are conspicuous for their arrangement into groups of fours, the adjacent surfaces being somewhat flattened. They are not sarcina, as one can see by the absence of the division in the third direction of space-they divide in only two directions. 
In the slip stained by the Gram method the same groups of the cocci which grow as threes and fours will be seen, but our lancet-shaped diplococci will now present an altered appearance-there can now be detected a capsule surrounding them. This capsule is very delicate in structure, and, though a frequent accompaniment, is not constant. It can sometimes be demonstrated by the ordinary methods of staining, though the method of Gram is most satisfaetory. (Fig. 62.)

In the third slip which has been stained by the method given for tubercle bacilli in sputum, if decolorization has been properly conducted and no contraststain has been employed, the field will be colorless or of only a very pale rose color. None of the numerous organisms seen in the first slip can now be detected, but instead there will be seen scattered through the field very delicate stained rods, which present, in most instances, a conspicuous beaded arrangement of their protoplasm-that is, the staining is not homogeneous, but at tolerably regular intervals along cach rod there are seen alternating intervals of light and color. These rods may be found singly, in groups of twos and threes, or sometimes in clumps consisting of large numbers. When in twos or threes it is not uncommon to find them describing an $\mathrm{X}$ or a $\mathrm{V}$ in their mode of arrangement, or again they will be seen lying parallel the one to the other.

If contrast-stains are used, these rods will be detected and recognized by their retaining the original color with which they have been stained, whereas all other bacteria in the preparation, as well as the tissue-cells which are in the sputum, will take up the contrust-color. (Fig. 61.)

These delicate beaded rods are the bacillus tubercu- 


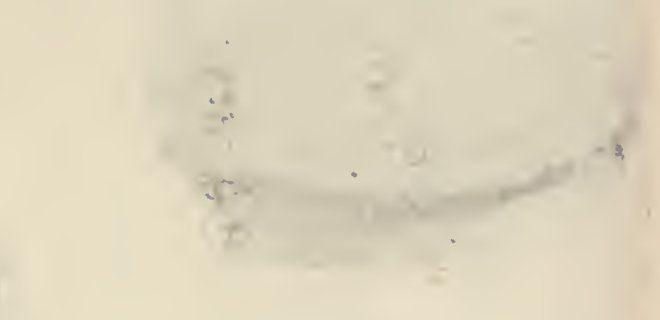

- 


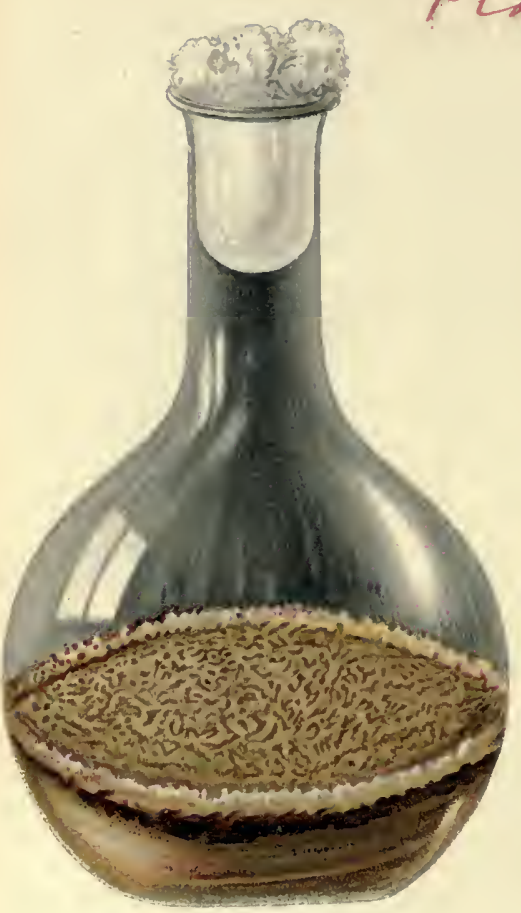

1 BACILLUS TUBERCULOSIS.

Culture in Glycerine Bovillon.

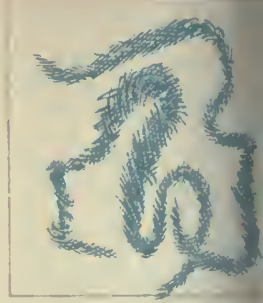

3. BACILLUS TUBERCUL Blood Serum Culture

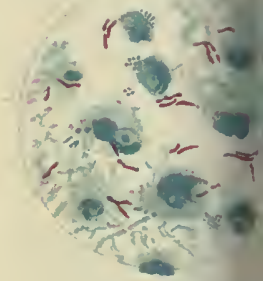

4. BACILLUS TUBERC In Sputum

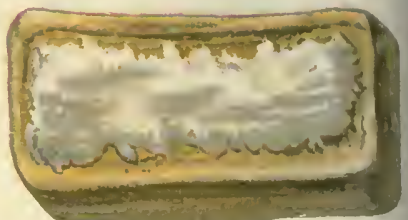

2. BACILLUS ANTHRACIS. Potato Cuiture.

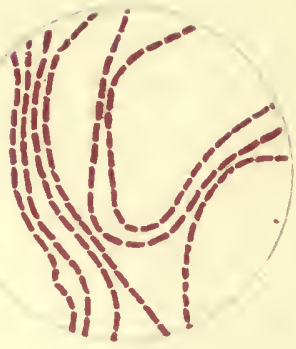

5. BACILLUS ANTHRACIS

From Agar Plate Culture.

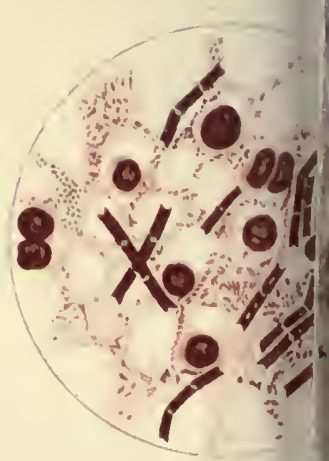


losis. The lancet-shaped diplococei with the capsule are the micrococcus lanceolatus.

FIG. 61 .

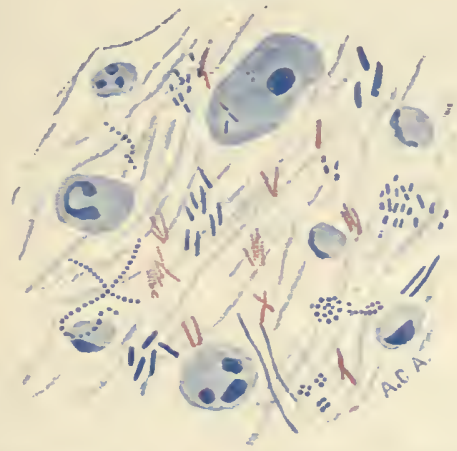

Tuberculous sputum stained by Gabbett's method. Tubercle bacilli seeu as red rods; all else is stained blue.

The cocci grouped in fours are the micrococcus tetragenus.

Ixoculation Experiment. - Inoculate into the subcutaneous tissues of a guinea-pig one of the small white caseous masses similar to that which has beeu examined microscopically. If death ensues, it will be the result of one of the three following forms of infection:

a. Septicæmia ${ }^{1}$ resulting from the introduction into the tissues of an organism frequently present in the sputum. It exists under the various names: micrococcus of sputum septicremia; diplococeus pneumoniæ; pneumococcus of Fränkel; meningococcus; streptococens lanceolatus Pasteuri; micrococeus lanceolatus; micrococcus Pasteuri; coccus lanceolatus; bacillus sali-

1 Septicæmia is that form of infection in which the blood is the chief field of actirity of the organisms. 
varius septicus; bacillus septicus sputigenus; diplococcus lanceolatus capsulatus; micrococcus pneumoniæ crouposæ.

b. A form of septicæmia resulting from the invasion of the tissues by an organism frequently seen in the sputum of tuberculous subjects. It is characterized by its tendency to divide into fours. It is the micrococcus tetragenus.

c. Local or general tuberculosis.

\section{a. SPUTUM SEPTICAMIA.}

If at the end of twenty-four to thirty-six hours the animal be found dead, we may safely suspect that the result was produced by the introduction into the tissues of the organism of sputum septicæmia above mentioned, viz., the micrococeus lanceolatus, which is not uncommonly found in the mouths of healthy individuals as well as in other conditions.

Inspection of the seat of inoculation usually reveals a local reaction. "This may be of a scrous, fibrinous, hemorrhagic, necrotic, or purulent character. Frequently we may find eombinations of these conditions, such as fibro-purulent, fibrino-serous, or sero-hemorrhagic." The most conspicuous naked-eye ehange undergone by the internal organs will be enlargement of the spleen. It is usually swollen, but may at times be normal in appearance. It is sometimes hard, dark red, and dry, or it may be soft and rich in blood. Frequently there is a limited fibrinous exudation over portions of the peritoneum.

1 Welch: Johns Hopkins Hospital Bulletin, December, 1892, vol. ill. No. 27. 
Except in the exudations, the organisms are found only in the lumen of the bloodvessels, where they are usually present in enormous numbers.

In the blood they are practically always free and are but rarely found within the bodies of leucocytes.

In stained preparations from the blood and exudates a capsule is not infrequently seen surrounding the organisms. (Fig. 62.) This, however, is not constant.

FTG. 62.

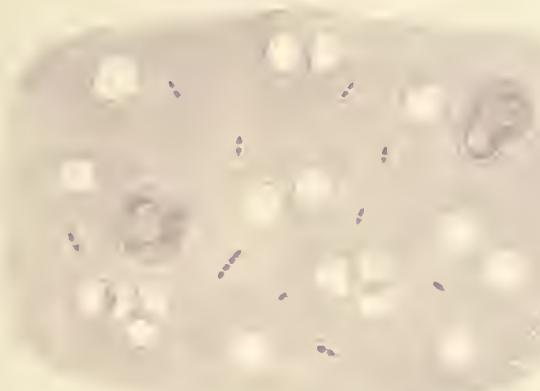

Micrococcus lanceolatus in blood of rabbit. Stained b5 method of Gram. Decolorization not complete.

If a drop of blood from this animal be introduced into the tissues of a second animal (mouse or rabbit), identically the same couditions will be reproduced.

If the organism be isolated from the blood of the animal in pure culture, and a portion of this culture be introduced into the tissues of a susceptible animal, again we shall see the same pathological picture.

It must be remembered, however, that this organism when cultivated for a time on artificial media rapidly loses its pathogenic properties. If, therefore, failure to reproduce the disease after inoculation 
from old cultures should oecur, it is in all probability due to a disappearance of virulence from the organism.

This organism was discovered by Sternberg in 1880 . It was subsequently described by $A$. Frinkel as the etiological factor in the production of acute fibrinous pneumonia.

It is not uncommonly present in the saliva of healthy individuals, having been found by Sternberg in the oral cavities of about 20 per cent. of healthy persons examined by him. It is constantly to be detected in the rusty sputum of patients suffering from acute fibrinous pnenmonia. Its presence has been detected in the middle ear, in the pericardial sac, in the pleura, in the serous cavities of the brain, and indeed it may penetrate from its primary seat in the mouth to almost any of the more distant organs.

The organism is commonly found as a diplococcus, though here and there short chains of four to six individuals joined together may be detected. (Fig. 62, page 281.) The individual cells are more or less oval, or, more strictly speaking, lancet-shaped, for at one end they are commonly pointed. When joined in pairs the junction is always between the broad ends of the ovals, never between the pointed extremities.

As already stated, in preparations directly from the sputum or from the blood of animals, a delicate eapsule may frequently be seen surrounding then. Though fairly constant in preparations directly from the blood of animals and from the sputum or lungs of pneumonic patients, the capsule is but rarely observed in artificial cultures. Occasionally in cultures on blood-serum, in milk, and on agar-agar they can, according to some 
authors, be detected; but this is by no means constant, or even frequent.

This organism grows under artificial conditions very slowly, and frequently not at all.

When successfully grown upon the different media it presents somewhat the following appearance :

On gelatin it grows very slowly, if at all, probably owing in part to the low temperature at which gelatin eultures must be kept. If development occurs, it appears as minute whitish or blue-white points on the plates. These very small colonies are round, finely granular, sharply circumscribed, and slightly elevated above the surface of the gelatin. The growth is very slow, and no liquefaction of the gelatin accompanies it.

If grown in slant- or stab-cultures, the surface-development is very limited; along the needle-track tiny whitish or bluish-white granules appear.

On nutrient agar-agar the colonies are almost transparent; they are more or less glistening and very delicate in structure. On blood-serum development is more marked, though still extremely feeble. Here it also appears as a cluster of isolated fine points growing closely side by side.

A growth on potato is not usually observed. When grown in milk it commonly causes an acid reaction with coincident coagulation of the casein. Some varieties, especially non-virulent ones, do not coagulate milk. ${ }^{1}$

It is not motile.

It grows best at a temperature of from $35^{\circ}$ to $38^{\circ} \mathrm{C}$. Under $24^{\circ} \mathrm{C}$. there is usually no development, but in a few cases it has been seen to grow at as low a tempera- 
ture as $18^{\circ} \mathrm{C}$. From $42^{\circ} \mathrm{C}$. on the development is checked.

Under most favorable conditions the growth is very slow. It grows as well withont as with oxygen. It is, therefore, one of the facultative anaerrobic forms.

The most suceessful efforts at the cultivation of this organism are those seen when the agar-agar-gelatin mixture of Guarniari is employed. (See this medium.)

It may be stained with the ordinary aniline stainingreagents. For demonstrating the eapsule the method of Gram and the acetic acid method give the best results. (See Stainings.)

This organism is eonspicuous for the irregularity of its behavior when grown under artificial conditions; usually it loses its pathogenic properties after a few generations; but again this peculiarity may be retained for a much longer time. Not rarely it fails to grow after three or four transplantations on artificial media, though at times it may be carried through many generations.

Inoculation into animals. The results of inoculations with pure eultures of this organism are also conspicuons for their irregularity. When the organism is of full virulence the form of septicæmia just deseribed is usually produced, but at times it is found to be totally devoid of pathogenic powers; between these extremes eultures may be obtained possessing all variations in the intensity of their disease-producing properties. The principal pathological conditions that may be produced by this organism by inoculations into animals, according to the degree of its virulenee, are aente septicemia, spreading inflammatory exudations, and circumseribed abseesses. All three of these conditions 

may sometimes be produced by inoculating the same cultures into rabbits in varying amounts.

Rabbits, mice, guinea-pigs, dogs, rats, cats, and sheep are susceptible to infection by this organism. Chickens and pigeons are insusceptible. Young animals, as a rule, are more easily infected than old ones. Rabbits and mice are the most susceptible of the animals used for experimental purposes, and in testing the virulence of a culture it is well to inoculate one of each, for with the same cultures it sometimes occurs that it may be virulent for mice and not for rabbits, and vice versa.

If the culture is virulent, intravascular or intraperitoneal injections into rabbits may produce rapid and fatal septicemia, while subcutaneous inoculation of the same material may result in only a localized inflammatory process. On the other hand, subcutaneous inoculation of less virulent cultures may produce a local process, while intravenous inoculation may be without result. This organism is the cause of a number of pathological conditions in human beings that have not hitherto been considered as related to one another etiologically. It is always present in the inflamed area of the lung in acute fibrinous or lobar pneumonia; it is known to cause acute cerebro-spinal meningitis, endo- and peri-carditis, certain forms of pleuritis, arthritis and peri-arthritis, and otitis media.

\section{b. SEPTICAMIA CAUSED BY THE Micrococcus TETRAGENUS.}

Should the death of the animal not occur within the first twenty-eight to thirty hours after inoculation, but be postponed until between the fourth and eighth day, 
it may occur as a result of invasion of the tissnes by the organism now to be described, viz., the micrococcus tetragenus.

This organism was discovered by Gaffky, and was subsequently described by Koch in the account of his experiments upon tuberculosis. It is often present in the saliva of healthy individuals and is commonly present in the sputum of tuberculous patients. Koch found it very frequently in the pulmonary cavities of phthisical patients.

It, however, plays no part in the etiology of tuberculosis.

It is a small round coccus of about $1 \mu$ transverse diameter. It is seen as single cells, joined in pairs and in threes; but its most conspicuous grouping is in fours, from which arrangement it takes its name. In preparations made from cultures of this organism it is not rare to find, here and there, single bodies which are much larger than the other individuals in the field. Close inspection reveals them to be cells in the initial stage of division into twos and fours. A peculiarity of this organism is that the cells are seen to be bound together by a transparent gelatinous substance.

When cultivated artificially it grows very slowly.

Upon gelatin plates the colonies appear as round, sharply circumscribed, punctiform masses which are slightly elevated above the surface of the surrounding medium. Under a low magnifying power they are seen to be slightly grainular and to present a more or less glassy lustre.

The colonies increase but little in size after the third or fourth day. If cultivated as stab-cultures in gelatin, there appears upon the surface at the point of inocula- 
tion a circumscribed white point, slightly elevated above the surface and limited to the immediate neighborhood of the point of inoculation. Down the needle-track the growth is not contiunous, but appears in isolated, round, dense white clumps or beads, which do not develop beyond the size of very small points.

It does not liquefy gelatin.

Upon plates of nutrient agar-agar the colonies appear as small, almost transparent, round points, which have about the same color and appearance as a drop of eggalbumin; they are very slightly opaque. They are moist and glistening. They rarely develop to an extent exceeding 1 to $2 \mathrm{~mm}$. in diameter.

Upon agar-agar as stab- or slant-cultures the surfacegrowth has more or less of a mucoid appearance. It is moist, glistening, and irregularly outlined. The outline of the growth depends upon the moisture of the agar-agar. It is slightly elevated above the surface of the medium.

In contradistinction to the gelatin stab-cultures, the growth in agar-agar is continuous along the track of the needle.

The growth on potato is a thick, irregular, slimylooking patch.

The presence of the transparent gelatinous substance which is seen to surround these organisms renders them coherent, so that efforts to take up a portion of a colony from the agar-agar or potato cultures result usually in drawing out fine, silky threads consisting of organisms imbedded in this gelatinous material.

The organism grows best at from $35^{\circ} \mathrm{C}$. to $38^{\circ} \mathrm{C}$, but can be cultivated at the ordinary room temperature -about $20^{\circ} \mathrm{C}$. 
The growth under all conditions is slow.

It grows both in the presence of and without oxygen.

It is not motile.

It stains readily with all the ordinary aniline dyes. In tissues its presence is readily demonstrated by the staining-method of Gram.

The grouping into fours is partieularly well seen in sections from the organs of animals dead of this form of septicæmia.

In such sections the organisms will always be found within the capillaries.

Inoculation into animals. To the naked eye no alteration can be seen in the organs of animals that have died as a result of inoculation with the micrococcus tetragenus; but microscopic examination of cover-slip preparations from the blood and viscera reveals the presence of the organisms throughout the body-especially is this true of preparations from the spleen. White mice and guinea-pigs are susceptible to the disease. Gray mice, dogs, and rabbits are not susceptible to this form of septicæmia. Subsequent inoculation of healthy animals with a drop of blood, a bit of tissue, or a portion of a pure culture of this organism from the body of an animal dead of the disease, results in a reproduction of the conditions found in the dead animal from which the tissues or eultures were obtained.

It sometimes occurs that in guinea-pigs which have been inoculated with this organism there result local pus-formations, instead of a general septicemia. 'The organisms will then be found in the pus-cavity. 


\section{CHAPTER XVIII.}

Tuberculosis-Microscopic appearance of miliary tubercles-Encapsulation of tnberculons foci-Diffuse caseation-Cavity-formation-Primary infection -Modes of infection-Location of the bacilli in the tissues-Staining-pecnliarities-Organisms with which bacillus tuberculosis may be confoundedPoints of differentiation.

SHovld the animal succumb to neither of the septic processes just described, then its death from tuberculosis may be reasonably expected.

When this disease is in progress alterations in the lymphatic glands nearest the seat of inoculation may be detected by the touch in from two to four weeks. They will then be found to be enlarged. Thongh not constant, tumefaction and subsequent ulceration at the point of inoculation may sometimes be observed. Progressive emaciation, loss of appetite, and difficulty in respiration point to the existence of the general tubercular process. Death ensues in from four to eight weeks after inoculation. At autopsy either general or local tuberculosis may be found. The expressions of the tubercular process are so manifold and in different animals vary so widely the one from the other, that no rigid law as to what will appear at autopsy can d priori be laid down.

The guinea-pig, which is best suited for this experiment because of the greater regularity of its susceptibility to the disease over that of other animals usually found in the laboratory, presents, in the main, changes that are characterized by a condition of coagulation- 
necrosis and caseation. This is particularly the case when the infection is general-i.e., when the process is of the acute miliary type. This pathological-anatomical alteration is best seen in the tissues of the liver and spleen of these animals, where the condition is most pronounced.

In general, the tubereular lesions can be divided into those of strictly focal character - i.e., the miliary and the conglomerate tubercles, and those which are more diffuse in their nature. The latter lesions, although of the same fundamental nature as the miliary tubercles, are much greater in extent and not so sharply circumscribed.

These latter lesions play a greater rôle in the pathology of the disease than do the miliary nodules, although it is to the presence of the miliary nodules that the disease owes its name.

At autopsy the pathological manifestations of the disease are not infrequently seen to be confined to the seat of inoculation and to the neighboring lymphatic glands. These tissues will then present all the characteristies of the tuberculous process in the stage of cheesy degeneration. When the disease is general the degree of its extension varies. Sometimes the small gray nodules-the miliary tubercles-are only to be seen with the naked eye in the tissues of the liver and spleen. Again, they may invade the lungs, and commonly they are distributed over the serous membranes of the intestines, the lungs, the heart, and the brain. These simple gray nodules, as seen by the naked eye, vary in size from that of a pin-point to that of a hempseed, and, as a rule, are, in this stage, the result of the fusion of two or more smaller miliary foci. Though the two terms "miliary" and "conglomerate" . 
exist for the description of the macroscopic appearance of these nodules, yet it is very rarely that any condition other than that due to the fusion together of several of these minute foci can be detected by the naked eye.

The miliary tubercles are of a pale gray color, with a white centre, are slightly elevated above the surface of the tissue in which they exist, and, as stated, vary considerably in dimensions, usually appearing as points which range in size from that of a pin-point to that of a pin-head. They are not only located upon the surface of the organs, but are distributed through the depths of the tissues. To the touch they sometimes present nothing characteristic, but may frequently, when closely packed together in large numbers, give a mealy or sandy sensation to the fingers. Stained sections of these miliary tubercles present a distinctly characteristic appearance, and the disease may be diagnosticated by these histological changes alone, though the crucial test in the diagnosis is the finding of tubercle bacilli in these nodules.

Microscopic Appearaxce of Miliary TuberCLEs. - The simple miliary tubercles under a low magnifying power of the microscope present somewhat the following appearance: there is a central pale area, evidently composed of necrotic tissue because of its incapacity for taking up the nuclear stains commonly employed. Scattered here and there through this necrotic area may be seen granular masses irregular in size and shape; they take up the stains employed, and are evidently the fragments of cell-nuclei in the course of destruction. Through the necrotic area may here and there be seen irregular lines, bands, or ridges, the remains of tissues not yet completely destroyed by the 
necrotic process. Around the periphery of this area may sometimes be noticed large multinucleated cells, the nuclei of which are arranged about the periphery of the cell or grouped irregularly at its poles. The arrangement of these nuclei as observed in sections is usually oval, or somewhat crescentic. In the tubercles from the human subject these large " giant-cells," as they are called, are quite common. They are much less frequent in tubercular tissues from lower animals.

Round about the central focus of necrosis is seen a more or less broad zone of elosely packed small round and oval bodies which stain readily but not homogeneously. They vary in size and shape, and are seen to be imbedded in a delicate network of fibrinous-looking tissue.

This fibrin-like network in which these bodies lie, and which is a common accompaniment of giant-cell formation, is in part composed of fibrin, but is in the main, most probably, the remains of the interstitial fibrous tissue of the part. This zone of which we are speaking is the zone of so-called "granulation tissue," and consists of leucocytes, granulation cells, fibrin, and the fibrous remains of the organ; the irregularly oval, granular bodies which take up the stain are the nuclei of these cells. The zone of granulation tissue surrounds the whole of the tubercular process, and at its periphery fades gradually into the healthy surrounding tissues or fuses with a similar zone surrounding another tubercular focus. This may be taken as a deseription of the typical miliary tuberele.

Diffuse Caseation. - The diffuse caseation, as said, plays a more important rôle in the tuberculous lesion, 
both in the human and experimental forms, than does the formation of miliary tubercles. In this a large area of tissue undergoes the same process of necrosis and caseation as the centre of the miliary tubercle. In some tissues it is more marked than in others. These tissues are the lungs and lymph-glands. In rabbits, particularly, all the changes in the lung frequently come under this head. When this is the case solid masses are found, sometimes as large as a pea, or involving even an entire lobe or the whole lung in some cases. They are of a whitish-yellow, opaque color, and on section are peculiarly dry and hard. Entire lymphatic glands may be changed in this way. The conditions for this caseation of the tissues are probably given when a large number of tubercle bacilli enter the tissue simultaneously and a wide area is involved, instead of the small centre of the miliary tubercle. Necrosis is so rapid that time is not given for those reactive changes to take place in the tissues which result in the formation of the outer zone of the miliary tubercle. In other instances the entire caseous area is surrounded by a granulation zone similar to that around the caseous centre of the miliary tubercles. It is of special importance to recognize the connection between this diffuse caseation and the tubercle bacillus, because until its nature was accurately determined the caseous pneumonia- of the lungs formed the chief obstacle which many encountered in recoguizing the specific infectiousness of tuberculosis.

Cavity-Fonmation. - The production of cavities, which form such a prominent feature in human tuberculosis, particularly in the lungs, is due to softening of the necrotic, caseous masses or of aggregations of 
miliary tubercles. The material softens and is expelled, and a cavity remains. In the wall of this cavity the tuberculous changes still proceed, both as diffuse caseation and formation of miliary tubercles. The whole cavity with the reactive changes in the tissues of its walls may be considered as representing a single tubercle, its wall forming a tissue very analogous to the outer zone of the single tubercle, the cavity itself corresponding to the caseous centre.

In animals used for experiment eavity-formation of this sort is very rare, owing to the greater resistance of the caseous tissue. That it is, however, possible to produce in rabbits pulmonary cavities in all physical respects similar to those seen in the human being has been most beautifully demonstrated by Prudden. He showed that when he had injected into the trachea of rabbits, already affected with tubercular consolidation of the lungs, fluid cultures of streptococcus pyogenes, the result of the mixed infection thus brought about was cavity-formation in eight out of nine lungs subjected to the conditions of the experiment; while in only one out of eleven did cavities form under the influence of the tuberele bacillus alone. ${ }^{1}$

In the contents and in the walls of tubercular eavities in man bacteria other than the tuberele baeillus are found. It is to the influence of some of these, as we have seen, that diseases other than tuberculosis may sometimes be produced by the inoculation. of animals with the sputum from such cases.

1 Prudden : Experimentai Phthisis in Rabbits, with the Formation of Cavities, etc. Transactions of the Association of American Physlcians, 1894, vol. ix. p. 166. 
Excapsulation of Tubercular Foci.-It not uncommonly occurs that round about a necrotic tubercular focus there is formed a fibrous capsule which may completely cut off the diseased from the healthy tissue surrounding it. Or a tubercular focus may, through the resistance of the tissue in which it is located, be more or less completely isolated. In this condition the diseased foci may lie dormant for a long time and give no evidence of their existence, until by some intercurrent interference they are caused to break through their envelopes. With the passage of the bacilli or their spores from the central foci into the vascular or lymphatic circulation the disease may then become general.

It is to some such accident as this that the sudden appearance of general tubercular infeetion in subjects supposed to have recovered from the primary local manifestations may often be attributed. The breakingdown of old caseous lymphatic glands is a common example of this condition.

PriMARY INFECTION.-The primary infection occurs through either the vascular or lymphatic circulation. Through these channels the bacilli gain access to the tissues and become lodged in the finer capillary ramifications or in the more minute lympl-spaces. Here they find conditions favorable to their development, and in the course of their life-processes produce substances of a chemical nature which act directly in bringing about the death of the tissues in their immediate neighborhood. This tissue-death is probably the very first effect of the bacilli in the body, and represents the necrotic centre which can always be seen in even the most minute tubercles. With the production of this progressive necrosis-for progressive it is, as it 
proceeds as long as the bacilli live and continue to produce their poisonous products-there is in addition a reactive change in the surrounding tissues, which consists in the formation of the granulation zone at the outer margins of the dying and dead tissue. This zone consists of small, round granulation cells and of leucocytes, all of which are seen in the meshes of the finer fibrous tissues of the part. At the same time alterations are produced in the walls of the vessels of the locality; these tend to occlude them, and thus the process of tissue-death is favored by a diminution of the amount of nutrition brought to them. These changes may continue until eventually conglomerate tubercles, widespread caseation, or cavity-formation results; or from one cause or another the life-processes of the bacilli may be checked and recovery occur.

Modes of Infection. - Experimentally, tuberculosis may be produced in susceptible animals by subcutaneous inoculation; by direct injection into the circulation; by injection into the peritoneal cavity; by feeding of tuberculous material; by the introduction of the bacilli into the air-passages, and by inoculation into the anterior chamber of the eye.

In the human subject the most common portals of infection are, doubtless, the air-passages, the alimentary tract, and cutaneous wounds. When introduced subentaneously the resulting process finds its most pronounced expression in the lymphatic system. The growing baeilli make their way into the lymphatic spaces of the loose cellular tissue, are taken up in the lymph stream and deposited in the neighboring lymphatic glands. Here they may remain and give rise to no alteration further than that seen in the glands them- 
selves, or they may pass on to neighboring glands, and eventually be disseminated throughout the whole lymphatic system, ultimately reaching the vascular system.

After having gained access to the bloodvessels, the results are the same as those following upon intravascular injection of the bacilli, namely, general tuberculosis quickly follows, with the most conspicuous production of miliary tubercles in the lungs and kidneys, less numerous in the spleen, liver, and bone marrow.

When inhaled into the lungs, if conditions are favorable, multiplication of the bacilli quickly follows. With their growth they are mechanically pressed into the tissues of the lungs. As multiplication continues some are transported from the primary seat of infection to healthy portions of the lung tissue, there to give rise to a further production of the tubercular process.

In the same way infection through the alimentary tract is in the main due to mechanical pressure of the bacilli upon the walls of the intestines. Investigation has shown that lesions of the intestinal coats are not necessary for the entrance of tubercle bacilli from the intestines into the body. They may be transported from the intestinal tract into the lymphatics in the same way that the fat-droplets of the chyle find entrance into the lymphatic circulation.

The evidence produced by Cornet, ${ }^{1}$ together with general statistical evidence, points to the lungs as the most common portal of natural infection for the human being. Unlike most pathogenic organisms, the tubercle bacillus is believed to have the property of forming spores within the tissues. These spores, which are pre-

1 Cornet: Zeit. fur Hygiene, 18s9, Bd. v. S. 191. 
sumably highly resistant and not destroyed by drying, are thrown off from the lungs in the sputum of tuberculous patients in large numbers, and unless special precautions be taken to prevent it the sputum becomes dried, is ground into dust, and sets free in the atmosphere the spores of tuberele bacilli which came with it from the lungs. The frequeney of pulmonary tuberculosis points to this as one of the commonest sourees and modes of infection.

Location of the Bacilli in the Tissufs. - The bacilli will be found to be most numerous in those tissues which are in the active stage of the process.

In the very initial stage of the disease the bacilli will be fewer in number than later. At this time only here and there single rods may be found; later they will be more numerous, and, finally, when the process has advanced to a stage easily recognizable by the naked eye, they will be found in the granulation zones in clumps and seattered about in large numbers.

In the central neerotic masses, which consist of eell detritus, it is rare that the organisms can be demonstrated microseopically. It is at the periphery of these areas and in the progressing granular zone that they are most frequently to be seen.

This apparent absenee of the baeilli from the central necrotic area must not be taken, however, as evidence that this tissue does not contain them. As bacilli, they are difficult to demoustrate here beeause the probabilities are that in this locality, owing to conditions unfavorable to their further growth, they are in the spore-stage, a stage in whieh it is as yet impossible, with our present methods of staining, to render them visible. The fact that this tissue is infective, 
and with it the disease can be reproduced in susceptible animals, speaks for the accuracy of this assumption. A conspicuous example of this condition is seen in old scrofulous glands. These glands usually present a slow process, are commonly caseous, and always possess the property of producing the disease when introduced into the tissues of susceptible animals, and yet they are the most difficult of all tissues in which to demonstrate microscopically the presence of tubercle bacilli.

In tubercles containing giant-cells the bacilli can usually be demonstrated in the granular contents of these cells. Frequently they will be found accumulated at the pole of the cell opposite to that occupied by the nuclei, as if there existed an antagonism between the nuclei and the bacilli. In some of these cells, however, the distribution of the bacilli is seen to be irregular, and they will be found scattered among the nuclei as well as in the necrotic centre of the cell. As the number of bacilli in the giant-cell increases the cell itself is ultimately destroyed.

Tubercular tissues always contain the bacilli or their spores, and are always capable of reproducing the disease when introduced into the body of a susceptible animal. From the tissues of this animal the bacilli may again be obtained and cultivated artificially, and these cultures are capable of again producing the disease when further inoculated. Thus the postulates formulated by Koch, which are necessary to prove the etiological rôle of an organism in the production of a malady, are all fulfilled.

The Tubercle Bacillus.-Of the three pathogenic organisms liable to occur in the sputum of a tu- 
berculous subject, the tuberele bacillus will give us most difficulty in our efforts at cultivation.

It is, in the strict sense of the word, a parasite, and finds conditions entirely favorable to its development only in the animal body. On ordinary artificial media the bacilli taken directly from the animal body grow only very imperfectly, or, in many cases, not at all. From this it seems probable that there is a difference in the nature of individual tubercle bacilli-some appearing to be capable only of growth in the animal tissues, while others are apparently possessed of the power to lead a limited saprophytic existence. It may be, therefore, that those bacilli which we obtain as artificial cultures from the animal body are offsprings from the more saprophytic varieties. At best, one never sees with the tuberele bacillus a saprophytic condition in any way comparable to that possessed by many of the other organisms with which we have to deal.

In efforts to cultivate this organism directly from the tissues of the animal, the method by which one obtains the best results is that recommended by Koch, viz., cultivation upon blood-serum. So strictly is this organism a parasite that very limited alterations in the conditions under which it is growing may result in failure to study it successfully. It is, therefore, necessary that the injunctions for obtaining it in pure culture should be earefully observed.

Preparation of Culdures from Tissues.-Under strictest antiseptic precautions remove from the animal the tubercular organ-the liver, spleen, or a lymplatic gland being preferable. Place the tissue in a sterilized Petri dish and dissect out with sterilized seissors and forceps the small tubercular nodules. Place each nodule 
upon the surface of the blood-serum, one nodule in each tube, and with a heavy, sterilized, looped platinum needle or spatula, rub it carefully over the surface. It is best to dissect away twenty to thirty such tubercles and treat each in the same way. Some of the tubes will remain sterile, others may be contaminated by outside organisms during the manipulation, while a few may give the result desired, viz., a growth of the tubercle bacilli themselves.

The blood-serum upon which the organism is to be cultivated should be comparatively freshly preparedthat is, should not be dry.

After inoculating the tubes they should be carefully sealed up to prevent evaporation and consequent drying. This is done by burning off the superfluous overhanging cotton plug in the gas-flame, and then impregnating the upper layers of the cotton with either sealing-wax or paraffin of a high melting-point; or by inserting over the burned end of the cotton plug a soft, closely fitting cork that has been sterilized in the steam sterilizer just before using (Ghriskey). This precaution is necessary because of the slow growth of the organism. Under the most favorable conditions tubercle bacilli directly from the animal body show no evidence of growth for about twelve days after inoculation upon blood-serum, and, as they must be retained during this time at the body temperature- $37.5^{\circ} \mathrm{C}$. evaporation would take place very rapidly and the medium would become too dry for their development.

If these primary efforts result in the appearance of a culture of the bacilli, further cultivations may be made by taking up a bit of the colony, preferably a moderately large quantity, and transferring it to fresh serum, 
and this in turn is sealed up and retained at the same temperature. Once having obtained the organism in pure culture, its subsequent cultivation may be conducted upon the glycerin-agar-agar mixture-ordinary neutral nutrient agar-agar to which 6 or 7 per cent. of glycerin has been added. This is a very favorable medium for the growth of this organism after it has accommodated itself to its saprophytic mode of existence, though blood-serum is perhaps the best medium to be employed in obtaining the first generation of the organism from the tubercular tissues.

The organism may be cultivated also on neutral milk to which 1 per cent. of agar-agar has been added, also upon the surface of potato, and likewise in meat-infusion bouillon containing 6 or 7 per cent. of glycerin.

Cultures of the tubercle bacillus are characteristic in appearance-after once having seen them there is but little probability of subsequent mistake.

Thcy appear as dry masses, which may develop upon the surface of the medium either as flat scales or as lumps of mealy looking granules. They are never moist, and frequently have the appearance of coarse meal which has been spread upon the surface of the medium. In the lower part of the tube in which they are growing-i.e., that part occupied by a few drops of fluid which has in part been squeezed from the medium during the process of solidification, and is in part water of condensation-the colonies may be seen to float as a thin pellicle upon the surface of the fluid.

The individuals making up the growth adhere so tenacionsly together that it is with the greatest difficulty that they can be completely separated. In even the oldest and dryest cultures pulverization is impos- 
sible. The masses can only be separated and broken up by grinding in a mortar with the addition of some foreign substance, such as very fine, sterilized sand, dust, ete.

The cultures are of a dirty-drab or brownish-gray color when seen on serum or on glycerin-agar-agar.

On potato they grow in practically the same way, though the development is much more limited. They are here of nearly the same color as the potato on which they are growing. When cultivated for a time on potato they are said to lose their pathogenic properties.

On milk-agar-agar they are of so nearly the same color as the medium that, unless they are growing as the mealy looking masses, considerably elevated above the surface, their presence is less conspicuous than when on the other media.

In bouillon they grow as a thin pellicle on the surface. This may fall to the bottom of the fluid and continue to develop, its place on the surface being taken by a second pellicle.

Under all conditions of artificial development the cultures of this organism are always very dry and brittle in appearance, though in truth the individuals adhere tenaciously together by a very glutinous substance.

The tubercle bacillus does not develop on gelatin, because of the low temperature at which this medium must be used.

Microscopic Appearance of the Tubercle BAcullus.-Microscopically the organism itself is a delicate rod, usually somewhat beaded in its structure, though rarely it is seen to be homogeneous. It is either quite straight or somewhat curved or bent on its long 
axis. In some preparations involution-forms, consisting of rods a little clubbed at one extremity or slightly bulging at different points, may be deteeted. Branching forms of this organism have been deseribed. It varies in length-sometimes being seen in very short segments, again much longer, though never as long as threads. On an average, its length is seen to vary from 2 to $5 \mu$. It is commonly deseribed as being in length about one-fourth to one-half the diameter of a red blood-corpuscle. It is very slender. (Fig. 61, page 279.)

These rods usually present, as has been said, an appearance of alternate stained and eolorless portious. It is the latter portions which are believed to be the spores of the organism, thongh as yet no absolute proof of this opinion has been established.

At times these colorless portions are seen to bulge slightly beyond the contour of the rod, and in this way give to the rods the beaded appearance so commonly ascribed to them.

Staining-Peculianities. - A peculiarity of this organism is its behavior toward staining-reagents, and by this means alone it may be easily recognized. The tubercle bacillus does not stain by the ordinary methods. It possesses some peculiarity in its composition that renders it more or less proof against the simpler dyes. It is therefore necessary that more energetic and penetrating reagents than the ordinary watery solutions should be employed. Experience has taught us that certain substances not only increase the solubility of the aniline coloring substances, but by their presence the penetration of the coloring agents is very much increased. Two of these substanees are 
aniline oil and carbolic acid. They are employed in the solutions to about the point of saturation. (For the exact proportions see chapter on Staining-reagents.)

Under the influence of heat these solutions are seen to stain all bacteria very intensely-the tubercle bacilli as weH as the ordinary forms. If we subject our preparation, which may contain a mixture of tubercle bacilli and other forms, to the action of decolorizing-agents, another peculiarity of the tuhercle bacillus will be observed. While all other organisms in the preparation will give up their color and become invisible, the tubercle bacillus retains it with marked tenacity. It stains with great difficulty, but once stained it retains the color even under the influence of strong decolorizing-agents.

ORGANISMS WITH WHICH THE BACILLUS TUBERCULOSIS MAY BE CONFUSED.

Differentral Dragrosis. - While its peculiar micro-chemical reaction is usually considered to be diagnostic of the bacillus tuberculosis, it is well to remember that there are at least three other species of bacilli which, when similarly treated, react in the same way. It is of importance to bear this point in mind, particularly in the microscopic examination of urine and pathological secretions from the genitourinary tract and from the rectum, for of the three species two are frequently found in these localities, viz., the so-called smegma bacillus, located in the smegma and often seen beneath the prepuce and upon the vulva, both normally and in disease, and the socalled bacillus of syphilis, described by Lustgarten as. 
contained in syphilitic manifestations, particularly in primary sores. The third organism of this group-the bacillus of leprosy-because of its rarity is not so likely to cause error in the diagnosis of pathological conditions occurring in these localities.

According to Hueppe, the differential diagnosis between the four organisms depends upon the following reactions: when stained by the carbol-fuchsin method commonly employed in staining the tuberele bacillus the syphilis bacillus becomes almost instantly decolorized by treatment with mineral acids, particularly sulphuric acid, whereas the smegma bacillus resists such treatment for a much longer time, and the lepra and tubercle bacillus for a still longer time. On the other hand, if decolorization is practised with alcohol, instead of acids, the smegma bacillus is the first to lose its color. The bacillus tubereulosis and the bacillus of leprosy are conspicuously retentive of their color even after treatment with both acids and alcohol.

To differentiate, then, between the four organisms he recommends the following order of procedure, based on the above reactions:

1. Treat the preparation, stained with earbol-fuchsin, with sulphuric acid; the syphilis bacillus becomes decolorized, the reaction being almost instantaneous.

2. If it is not at once decolorized, treat with alcohol; if it is the smegma bacillus, this will rob it of its color.

3. If it is still not decolorized, it is either the lepra or tubercle bacillus.

Grethe (Fortschritte der Med., 1896, No. 9) recommends the following as a trustworthy means of distinguishing between the tuberele bacillus and the smegma bacillus : stain in hot carbol-fuchsin solution, wash off
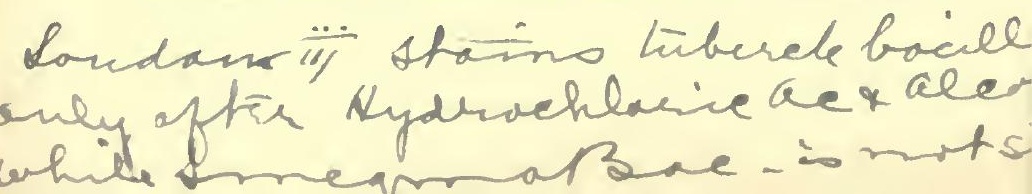
in water, and treat the preparation with a saturated solution of methylene-blue in alcohol. If the questionable organism is the tuberele bacillus, it retains its red color; if the smegma bacillus, the red color is dissolved out by the alcohol and the organism becomes stained blue.

The differential diagnosis between the tubercle bacillus and the lepra bacillus is less satisfactory; they both take on the same stains and both retain them or give them up under treatment with the same decolorizers. The results of investigations, however, indicate differences in the rate of staining and decolorization, and it is accepted by many of those who have compared the two organisms that the lepra bacillus takes up stain very much more readily than does the tubercle bacillus, often staining perfectly by an exposure of only a few minutes to cold watery solutions of the dyes; but when once stained it retains its color much more tenaciously when acted upon by decolorizing-agents than does the latter organism.

According to Baumgarten, the lepra bacillus is stained by an exposure of six to seven minutes to a cold, saturated watery solution of fuchsin, and retains the stain when subsequently treated with acid alcohol (nitric acid, 1 part; alcohol, 10 parts). By similar treatment for the same length of time the bacillus tuberculosis does not ordinarily become stained.

These points, particularly what has been said with reference to the smegma bacillus and the bacillus of syphilis, are of much practical importance, and should always be borne in mind in connection with microscopic examination of materials to which these organisms are liable to gain access. It is hardly necessary to say that 
in the examination of sputum and pathological fluids from other parts of the body the tubercle bacillus is, of the four organisms, always the one most commonly encountered, while the organism described by Lustgarten as the bacillus of syphilis is seen so rarely that many trustworthy investigators question its existence as a species distinct from the ordinary smegma bacillus.

Tuberculin. - The filtered products of growth from old fluid cultures of the tubercle bacillus represent what is known as tuberculin-a group of proteid substances possessing most interesting properties. When injected subcutaneously into healthy subjects tuberculin has no effect; but when introduced into the body of the tuberculous person or animal a pronounced systemic reaction results, consisting of sudden but temporary elevation of temperature, with, at the same time, the occurrence of marked hyperæmia round about the tuberculous focus, a change histologically analogous to that seen in the primary stages of acute inflammation. This zone of hyperæmia, with the coincident exudation and infiltration of cellular elements, probably aids in the isolation or casting off of the tuberculous nodule, the inflammatory zone forming, so to speak, a line of demarcation between the diseased and healthy tissue.

As a curative agent for the treatment of tuberculosis, tuberculin has not merited the confidence that was at first accorded to it. Its greatest field of uscfulness is now admitted to be as an aid to the diagnosis of obseure cases, and more particularly those occurring in cattle, where it has proved itself to be of inestimable value in this particular application.

Susceptrbility of Animals to Tuberculosis. The animals which are known to be susceptible to the 
tubercular processes are man, apes, cattle, horses, sheep, guinea-pigs, pigeons, rabbits, cats, and field mice.

White mice, dogs, and rats possess immunity agaiust the disease.

We have reviewed the three common pathogenic organisms with which we may come in contact in the sputum of tuberculous individuals. Occasionally other forms may be present. The pyogenic forms are not rarely found, and for some time after diphtheria the bacillus of Loeffler is demonstrable in the pharynx, so that it, too, may be present under exceptional circumstances. These latter organisms will be described under their proper heads.

From time to time fowls are known to suffer from a form of tubereulosis that is in many respects similar to human tuberculosis both as regards pathological lesions and etiology. The bacillus causing the disease, while very much like the genuine bacillus tuberculosis morphologically, differs from it in cultural peculiarities, notably in its inability to produce general tuberculosis in rabbits and guinea-pigs; in its growth into long branched forms at $45^{\circ}$ to $50^{\circ} \mathrm{C}$.; and in its never having been detected in human or mammalian tuberculosis.

Anatomical lesions very suggestive of those produced by bacillus tubereulosis have also from time to time been observed in certain rodents. They do not appear to be of speeific nature as regards etiology, for the reason that different authors have described different species of bacilli as the causative agents. The disease suggests tuberculosis only by the more superficial character of its lesions, for in no instance have the organisms detected been in any way similar to the gennine bacillus 
tuberculosis. These affections usually pass under the name pseudo-tnberculosis.

\section{THE BACILLUS OF INFLUENZA.}

An important historic epidemic disease, on the nature of which much light has been shed through modern methods of investigation, is influenza. Quoting Hirsch : the first trustworthy literary records that we have of this disease date from the early part of the twelfth century.

Between 1173 and 1874 it made its epidemic or pandemic appearance on eighty-six different occasions. Its first appearance in this country was in Massachusetts in 1627 ; since that time there have been twenty-two visitations of influenza to the United States. The recent epidemic, namely, that of 1889-'90,.appears to have originated in Central Asia and to have spread pretty much over the entire civilized world. The occurrence of influenza is always remarkable for the rapidity with which it spreads.

During the recent pandemic investigations, having for their object the discovery of its cause, were instituted, with the result of demonstrating in the catarrhal secretions from the air-passages a micro-organism that is claimed to stand in causal relation to inflnenza.

This organism, a bacillus, bacillus infuenzo, as it is called, was discovered, isolated, cultivated, and described by R. Pfeiffer.

It is a very small, slender, non-spore-forming, nonmotile, aërobic bacillus, occurring singly and in pairs, joined end to end. It stains with watery solutions of the ordinary basic aniline dyes; somewhat better with 
alkaline-methylene-blue, but best when treated for five minutes with a dilution of Ziehl's carbol-fuchsin in water (the color of the solution should be pale red). (Fig. 63.) It is decolorized by the method of Gram.

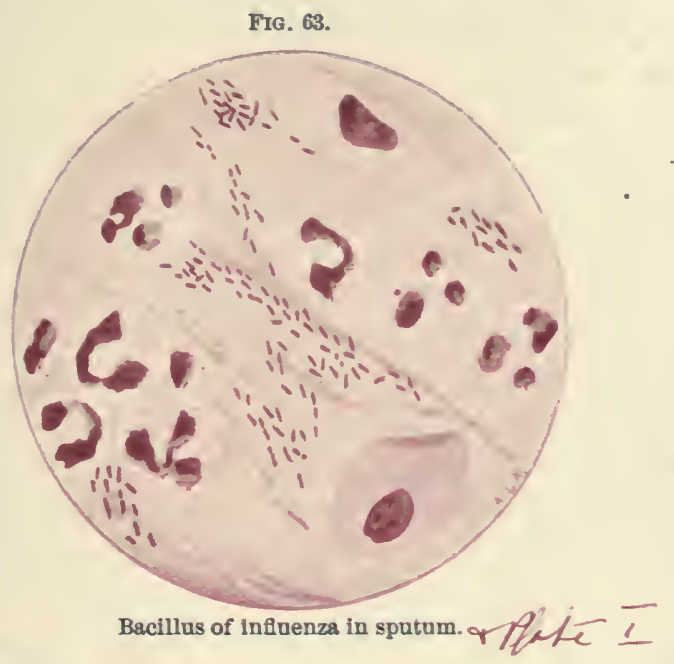

It develops only at temperatures ranging from $26^{\circ}$ to $43^{\circ} \mathrm{C}$. Its optimum temperature for growth is $37^{\circ} \mathrm{C}$. It possesses the peculiarity of developing upon only those artificial culture-media to which blood or blood-coloring-matter has been added. Its cultivation is best conducted and its development most satisfactorily observed by the following procedure: over the surface of a slanted agar tube or over agar-agar solidified in a Petri dish smear a small quantity of sterile blood (not blood-serum). A bit of the mucus from the sputum of the influenza patient is then taken up with sterilized forceps or on a sterilized wire loop, rinsed off in sterile bouillon or water and rubbed over the 
surface of the prepared agar-agar. The plate or tube is then placed in the incubator at $37^{\circ}$ to $38^{\circ} \mathrm{C}$. If influcnza bacilli be present, they will develop as minute, transparent, watery colonies that are without structure and which resemble somewhat minute drops of dew. They are discrete and show little or no tendency to coalesce.

If a small bit of mueus be rubbed over the surface of ordinary nutrient agar-agar, no such colonies develop. In making the diagnosis by this method cultures on both agar-agar containing blood (not bloodserum) and agar-agar coutaining no blood should always be made, for the reason that growth of these peculiar colonies in the former and no such growth in the latter are evidence that one is dealing with materials from a case of influenza.

It may also be cultivated in bonillon to which blood has been added, if kept at body temperature. The growth appears as whitish flakes. Since this organism is a strict aërobe, its cultivation can only be conducted on the surface of the medium used-i.e., where it has frcest access to oxygen. It is therefore inadvisable to prepare plates in the usual way. When its cultivation is attempted in bouillon it is recommended, in order to favor the free diffusion of oxygen, that the depth of fluid be very shallow.

Contrary to what might be supposed, the bacillus of influenza has very little tenacity to life outside of the diseased body. It is destroyed by rapid drying in from two to three hours, and when dried more slowly in from eight to twenty-four hours. Cultures retain their vitality for from two to three weeks. The organism dies in water in a little over a day. As a 
result of these observations, Pfeiffer does not believe the disease to be disseminated by either the air or the water, but rather by direct infection from the catarrhal secretions of the patients.

This organism has not been found outside of the human body. In the influenza patient it is present in the catarrhal secretions, bronchial mucous membrane, and the diseased lung tissues. It may be demonstrated microscopically in the mucus by cover-slip preparations made in the usual way and stained with diluted carbol-fuchsin, referred to above. In the tissues it may be demonstrated in sections stained in the same solution. In the sputum the bacilli are found as masses and as scattered cells. (See Fig. 63.) They are also found within the bodies of leucocytes, especially in the later stages of the disease when convalescence has set in ; at this time they appear as very small, irregular, evidently degenerated bacilli within white bloodcorpuscles. They are also present in the nasal secretions.

At autopsies it is advisable to cut out small pieces of the diseased tissue of about the size of a pea or a bean, rub them well in a small quantity of sterile water or bouillon, and make the cultures from this infusion. By this procedure two advantages are gained: first, a dilution of the number of bacteria present; and, secondly, the tissue furnishes the amount of hæmoglobin that is necessary for the growth of the organism. Under these circumstances it is, of course, not necessary to make a further addition of blood to the culture medium.

The only animal that has been found to be susceptible to inoculation with this organism is the monkey. 
By intratracheal injection Pfeiffer succeded in causing a toxic condition that proved fatal. He does not regard the death of the animals as due to infection, but rather to intoxication. The disease, as seen in man, has not been reproduced in animals. 


\section{CHA P T R XIX.}

Glanders-Characteristics of the disease-Histological structure of the glanders nodule-Susceptibility of different animals to glanders-The bacillus of glanders; its morphological and cultural peculiarities-Diagnosls of glanders.

\section{Syconyus : Rotz (Ger.), Morve (Fr.).}

The disease is generally known as glanders when the mucous membrane of the nostrils is affected, and as farcy when the skiu is the principal site of involvement.

Though most commonly seen in the horse and ass, glanders is not rarely met with in other animals, and is occasionally encountered in man. When occurring spontaneously in the horse its primary seat is usually upon the mucous membrane of the nostrils. It appears in the form of small gray nodules, about which the membrane is congested and swollen. These nodules ultimately coalesce to form ulcers. There is a profuse slimy discharge from the nostrils during the course of the disease. It may extend from its primary seat in the nose to the mouth, larynx, trachea, and ultimately to the lungs. Its secondary manifestations are observed along the lymphatics that communicate with the primary focus; in the lymphatic glands, and as metastatic foci in the internal organs. Less frequently the disease is seen to begin in the skin, particularly in the region of the neck and breast. When in this locality the subcutaneous lymphatics become involved, and are con- 
verted into indurated, knotty cords_"farcy buds"easily discernible from without.

When occurring in man it is usually in individuals who have been in attendance upon animals affected with the disease. It may occur upon the mucous membrane of the nares, but its most conspicuous expressions are in the skin and muscles, where appear abscesses, phlegmons, erysipelas-like inflammations, and local necrosis closely resembling carbuncles. Metastases to the lungs, kidneys, and testicles, as in the horse, may also be seen:

When oceurring upon the mucous membrane glanders is characterized by the presence of small gray nodules, about as large as a pin-head, that closely resemble miliary tubereles in their naked-cye appearance. These consist histologically of granulation tissuei.e., of small round cells, very similar to proliferating leucocytes, of some lymph-cells, and, in the earliest stages, of a small portion of necrotic tissue. As they grow older, and the process advances, there is a tendency toward central necrosis, with the ultimate formation of a soft, yellow, creany, pus-like material. Though strikingly like miliary tubercles in eertain respects in the early stages, they present, nevertheless, decided points of difference when examined more minutely.

The round-cell infiltration of the glanders nodules consists essentially of polynuclear leucocytes, while that of the miliary tubercle partakes more of the nature of a lymphocytic infiltration; in the later stages of the process the glanders nodule breaks down into a soft creamy matter, very analogous to ordinary pus, while in the later stages of the miliary tubercle 
the tendency is toward an amalgamation of its histological constituents, and ultimately to necrosis with caseation. The giant-cell formation common to tuberculosis is never seen in the glanders nodule. As Baumgarten aptly puts it: "The pathological manifestations of glanders, from the histological aspect, stand midway between the acute purulent and the chronic inflammatory processes." $i$ Evidently these differences are only to be explained by differences in the nature of the causes that underlie the several affections. We have studied the characteristics of bacillus tuberculosis; we shall now take up the bacillus of glanders and note the striking differences between them.

The Bacillus of Glanders (bacillus mallei). - In 1882 Lœffler and Schütz discovered in the diseased tissues of animals suffering from glanders a bacillus that,

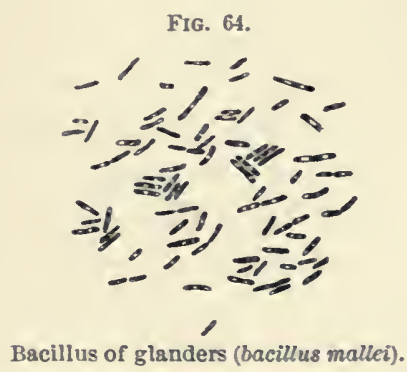

when isolated in pure culture and inoculated into susceptible animals, possesses the property of reproducing the disease with all its clinical and pathological mani-festations. It is therefore the cause of the disease.

1 For a further discussion of the pathology and pathogenesis of this disease see Lehrbuch der pathologischen Mykologie, by Baumgarten, 1590. See, also, Wright: The Histological Lesions of Acute Glanders in Man. Journal of Experimental Sledicine, vol. 1. p. 577. 
It is a short rod, with rounded or slightly pointed ends, that usually takes up the stain somewhat irregularly. (See Fig. 64.) When examined in stained preparations its continuity is marked by alternating darkly and lightly stained areas. It is usually seen as a single rod, bnt may oceur in pairs, and less frequently in longer filaments.

The question as to its spore-forming property is still an open one, though the weight of evidence is in opposition to the opinion that it possesses this peculiarity. Certain observers claim to have demonstrated spores in the bacilli by particular methods of staining, but this statement can have but little weight when compared with the behavior of the organism when subjected to more conclusive tests. For example, it does not, at any stage of development, resist exposure to 3 per cent. carbolic acid solution for longer than five minutes, nor to 1 : 5000 sublimate solution for more than two minutes. It is destroyed in ten minutes in some experiments, and in five in others, by a temperature of $55^{\circ} \mathrm{C}$., and when dried it loses its vitality, according to different observers, in from thirty to forty days; all of which speak directly against this being a spore-bearing bacillus.

It is not motile, and does not, therefore, possess flagella.

It grows readily on the ordinary nutrient media at from $25^{\circ}$ to $38^{\circ} \mathrm{C}$.

Upon nutrient agar-agar, both with and without glycerin, it appears as a moist, opaque, glazed layer, with nothing characteristic about it. This is true both for smear-cultures and for single colonies.

Its growth on gelatin is much less voluminous than 
on media that ean be kept at higher temperature, though it does grow on this media at room temperature without causing liquefaction.

Its growth on blood-serum is seen in the form of a moist, opaque, slimy layer, inclining to a yellowish or dirty, brownish-yellow tinge. It does not liquefy the serum.

- On potato its growth is moderately rapid, appearing at the end of from twenty-four to thirty-six hours at $37^{\circ}$ C. as a moist, amber-yellow, transparent deposit which becomes deeper in color and denser in consistence as growth progresses. It finally takes on a reddishbrown color, and the potato about it becomes darkened.

In bouillon it causes diffuse clouding, with ultimately the formation of a more or less tenacious or ropy sediment.

In milk to which a little litmus has been added it causes the blue color to become red or reddish in from four to five days, and quite red after two weeks at $37^{\circ} \mathrm{C}$. At the same time the milk is separated into a firm clot of casein and clear whey.

Its reactions to heat are very interesting-at $42^{\circ} \mathrm{C}$. it will often grow for twenty days or more. It will not grow at $43^{\circ} \mathrm{C}$., and is killed by exposure to this temperature for forty-eight hours. It is killed in five hours when exposed to $50^{\circ} \mathrm{C}$., and in five minutes by $55^{\circ} \mathrm{C}$.

It grows both with and without oxygen; it is therefore facultative as regards its relation to this gas.

On cover-slips it stains readily with all the basic aniline dyes, and, as a rule, as stated, presents conspicuous irregularities in the way that it takes up the dyes, being usually marked by deeply stained areas that alter- 
nate with points at which it either does not stain at all or only slightly.

The animals that are susceptible to infection by this organism are horses, asses, field mice, guinea-pigs, and cats. Baumgarten records cases of infection in lions and tigers that have been fed, in menageries, with flesh from horses affected with the disease. Rabbits are but slightly susceptible; dogs and sheep still less so. Man is susceptible, and infection not rarely terminates fatally. White mice, common gray house-mice, rats, cattle, and hogs are insusceptible.

Inoculation Experiments. - The most favorable animal upon which to study the pathogenic properties of this organism in the laboratory is the common field mouse. When inoculated subcutaneously with a small portion of a pure culture of the glanders bacillus death ensues in about seventy-two hours. The most conspicuous tissue-changes will be enlargement of the spleen, which is at the same time almost constantly studded with minute gray nodules, the typical glander's nodule. They are rarely present in the lungs, but may frequently be scen in the liver. From these nodules the glanders bacillus may be obtained in pnre culture. With the exception of the characteristic nodule, the disease as seen in this animal presents none of the characteristics that it displays in the horse and ass. The clinical and pathological manifestations resulting from inoculation of guinea-pigs are much more characteristic. The animal lives usually from six to eight weeks after inoculation, and in this time becomes affected with a group of most interesting and peculiar pathological processes. The specific inflammatory condition of the mucous membrane of the nostrils is almost always present. 'The 
joints become swollen and infiltrated to such an extent as often to interfere with the use of the legs. In male animals the testicles become enormously distended witl pus, and on closer examination a true orchitis and epididymitis are seen to be present. The internal organs, particularly the lungs, kidneys, spleen, and liver, are usually the seat of the nodular formations characteristic of the disease. From all of these disease-foci the bacillus causing them can be isolated in pure culture.

Stainisg is Tissues. - Though always present in the diseased tissues, considerable trouble is usually experienced in demonstrating the bacteria by stainingmethods. The difficulty lies in the fact that the bacilli are very easily decolorized, and in tissues stained by the ordinary processes are robbed of their color even by the alcohol with which the tissue is rinsed out and dehydrated. If we will remember not to employ concentrated stains, and not to expose the sections to the stains for too long a time, but little treatment with decolorizing-agents is necessary, and very satisfactory preparations will be obtained. A number of good methods have been suggested for staining the glanders bacilli in tissues, and if what has been said will be borne in mind, no difficulty should be experienced.

Two satisfactory methorls that we have used for this purpose, though perhaps no better than some of the others, are as follows:

(a) Transfer the sections from alcohol to distilled water. This lessens the violence with which the stain subsequently takes hold of the tissues, by diminishing the activity of the diffusion that would occur if they were placed from alcohol into watery solutions of the dyes. Transfer from distilled water to the slide, 
absorb all water with blotting-paper, and stain with two or three drops of

Carbol-suchsin .

Distilled water

for thirty minutes; absorb all superfluous stain with blotting-paper, and wash the section three times with 0.3 per cent. acetic acid, not allowing the acid to act for more than ten seconds each time. Remove all acid from the section by carefully washing in distilled water; absorb all water by gentle pressure with blotting-paper, and finally, at very moderate heat, or with a small bellows (Kühne), dry the section completely on the slide.

When dried clear up in xylol, and mount in xylol balsam.

(b) Transfer sections from alcohol to distilled water; from water to the dilute fuchsin solution, and gently warm (about $50^{\circ} \mathrm{C}$.) for fifteen to twenty minutes. Transfer sections from the staining-solution to the slide, absorb all superfluous stain with blotting-paper, and then treat them with 1 per cent. acetic acid from onehalf to three-quarters of a minute. Remove all trace of acid with distilled water, absorb all water by gentle pressure with blotting-paper, and then treat the seetions with absolute alcohol by allowing it to flow over them drop by drop. For small sections three or four drops are sufficient. Under no eireumstances should the alcohol be allowed to act for more than one-quarter of a minute. Clear up in xylol and mount in xylol-balsam.

In method $b$ the tissues are better preserved than in $a$, where they are dried.

Very good preparations are also obtained by the use of Loffler's alkaline methylene-blue, if eare be taken 
not to stain for too long a time or to decolorize with alcohol too energetically.

No method of contrast-stain for this organism in tissue has been devised.

In properly stained tissues the bacilli will be found most numerous in the centre of the nodules, becoming fewer as we approach the periphery. They usually lie between the cells, but at times may be seen almost filling some of the epithelial cells, of which the nodule contains more or less. They are always present in these nodules in the tissues; they are rarely present in the blood, and, if so, in only small numbers.

Dragrosis of the Disease by the Method of Strauss. - From what has been said the diagnosis of glauders by routine bacteriological methods is certain and relatively easy, but requires time. In clinical work it is of great importance for the diagnosis to be established as quickly as possible. With this in view Strauss devised a method that has given entirely satisfactory results. It consists in introducing iuto the peritoneal cavity of a male guinea-pig a bit of the suspected tissue or culture. If it be from a genuine case of glanders, the testicles begin to swell in about thirty hours, and as this proceeds the skin over them becomes red and shining, desquamation occurs, evidences of pus-formation are seen, and, indeed, the abscess (purulent orchitis) often breaks through the skin. The diagnostic sign is the tumefaction of the testicles.

Malleis.-The filtered products of growth of the glanders bacillus in fluid media represent what is known as mallein-a group of compounds that bear to glanders pretty much the same relation that tuberculin bears to tuberculosis. It is used with considerable success as a 
diagnostic aid in detecting the existence or absence of deep-seated manifestations of the disease, the glanderous animal reacting in from four to ten hours to subcutaneous injections of mallein, while an animal not so affected gives no such reactions.

-It is prepared from old glycerin-bonillon cultures of the glanders bacillus by steaming them for several hours in the sterilizer, after which they are filtered through unglazed porcelain. 


\section{CHA P'TER IX.}

Bacillus dipherix-Its isolation and cultivation-Morphological and cultural peculiarities-Pathogenic properties-Variations in virulence.

From the gray-white deposit on the fauces of a diphtheritic patient prepare a series of cultures in the following way:

Have at hand five or six tubes of Loffler's bloodserum mixture. (See chapter on Media.)

Pass a stout platinum needle, which has been sterilized, into the membrane and twist it around once or twice or brush it gently over the surface of the membrane. Without touching it against anything else rub it carefully over the surface of one of the serum tubes; without sterilizing it pass it over the surface of the second, then the third, fourth, and fifth tube. Place these tubes in the incubator. Then prepare cover-slips from scrapings from the membrane on the fauces. If the case is true diphtheria, the tubes will be ready for examination on the following day.

The reason that plates are not made in the regular way in this examination is that the bacillus of diphtheria develops much more luxuriantly on the serum mixture, from which plates cannot be made, than it does on the media from which they can be made. The method employed, however, insures a dilution in the number of organisms present, and this, in addition to the fact that bacillus diphtherice grows much more quickly on the 
serum mixture than do other organisms, makes its isolation by this method a matter of but little difficulty.

After twenty-four hours in the incubator the tubes will present a characteristic appearance. Their surfaces will be marked at different points by more or less irregular patches of a white or eream-colored growth which is usually more dense at the centre than at its irregular periphery.

Except now and then, when a few orange-colored colonies may be seen, these large irregular patches are the most conspicuous objects on the surface of the serum. Occasionally, almost nothing else appears.

The cover-slips made from the membrane at the time the cultures were prepared will be found on microscopic examination to present, in many cases, a great variety of organisms, but conspicuous among them will be noticed slightly curved bacilli of irregular size and outline. In some cases they will be more or less clubbed at one or both ends; sometimes they appear spindle in shape, again as eurved wedges; now and then they will be seen irregularly segmented. They are rarely or never regular in outline. If the preparation has been stained with Lœffler's alkaline methylene-blue solution, many of these irregular rods are seen to be marked by eircumscribed points in their protoplasm which stain very intensely; they appear almost black. 'This irregularity in outline is the morphologieal characteristic of the bacillus diphtherice of Iswffler.

It must be remembered, however, that the diagnosis of diphtheria should not under all circumstances be made from the examination of cover-slip preparations alone, for there are other organisms present in the mouth cavity, particularly in the moutlis of persons having 
decayed teeth, the morphology of which is so like that of the bacillus of diphtheria that they might easily be mistaken for that organism if subjected to microscopic examination only; and again, the genuine diphtheria bacillus is sometimes found in the mouth cavities of healthy persons in attendauce upon diphtheria cases, who were at the time insusceptible to the pathogenic activities of the organism. In the vast majority of instances, however, where the clinical condition of the patient justifies a suspicion of diphtheria, a microscopic examination alone of the deposit in the throat will serve to confirm or contradict this opinion.

Bacillus diphtheriae, discovered microscopically by Klebs, and isolated in pure culture and proverl to stand in causal relation to diphtheria by Loffler, can readily be identified by its cultural peculiarities and by its pathogenic activity when introduced into tissues of susceptible animals. In guinea-pigs and kittens the results of its growth are histologically identical with those found in the bodies of human beings who have died of diphtheria.

When studied in pure culture its morphological and cultural peculiarities are as follows:

Morphology.-As obtained directly from the diphtheritic deposit in the throat of an individual sick of the disease, it is sometimes comparatively regular in shape, appearing as straight or slightly curved rods with more or less pointed ends. More frequently, however, spindle and club shapes occur, and not rarely many of these rods take up the stain irregularly; in some of them very deeply stained, round or oval points can be detected.

When cultures are examined microscopically it is 
especially characteristic to find irregular, bizarre forms, such as rods with one or both ends swollen, and very frequently rods broken at irregular intervals into short, sharply marked segments, either round, oval, or with straight sides. Some forms stain uniformly, others in various irregular ways, the most common being the appearance of deeply stained granules in a lightly stained bacillus.

By a series of studies upon this organism when eultivated under artificial conditions we have found that its form depends very largely upon the nature of its environment. That is to say, its morphology is always more regular, and it is smaller on glycerin-agar-agar than on other media used for its cultivation; while upon Lœffler's blood-serum the other extremes of development appear: here one sees, instead of the very short, spindle, lancet, club-shaped, always segmented and regularly staining forms as seen upon glyeerin-agaragar, long, irregularly staining threads that are sometimes clubbed and sometimes pointed at their extremities. They are usually marked by areas that stain more intensely than does the rest of the rod, and at times they may be a little swollen at the centre. These differences are so eonspicuous that microseopic preparations from cultures from the same souree, but cultivated in the one ease on glyeerin-agar-agar and in the other upon bloodserum, when placed side by side would hardly be reeognized as of the same organism, unless its peeuliar behavior under these cireumstances was already known. During the past year or so various authors have called attention to branching forms of this organism that are occusionally encountered, especially when cultivated upon albumin. We have never seen the branching 
diphtheria bacilli; and in approximately 6000 bloodserum cultures from cases of diphtheria that have been examined during the past two years by three competent bacteriologists at the laboratory of the Board of Health of Philadelphia, the branching forms of this organism were not observed in a single instance. It is fair to assume, therefore, that this peculiar morploological variation of bacillus diphtheria is comparatively rare.

Fro. 65 .
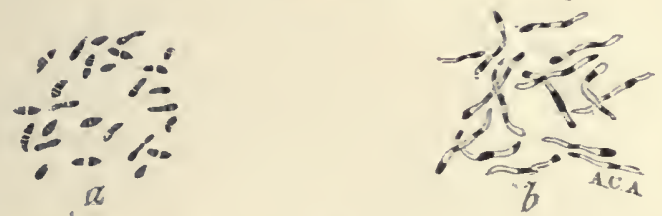

Bacillus diphtherix. a. Its morphology when cultivated on glycerin-agaragar. b. Its morphology as seen In cultures on Loeffler's blood-serum.

On plain nutrient agar-agar (that is, nutrient agaragar without glycerin); on solidified egg-albumin; on a medium consisting of dried albumin, as found in commerce, dissolved in bouillon (about 10 grammes albumin to 100 c.c. of bouillon containing 1 per cent. of grapesugar); in bouillon without glycerin, and in bouillon to which a bit of hard-boiled egg has been added, the morphology of the organism is about intermediate, in both size and outline, between the forms seen upon glycerinagar-agar and upon Lœfller's blood-serum. There will appear about an equal number of short segmented and longer irregularly staining forms, but in general the longest are rarely as long as the long forms seen on blood-sernm, and throughout they are not so conspicuous for the irregularity of their staining. 
In cultures made upon two sets of nutrient agar-agar tubes, differing only in the fact that one set contains glycerin to the extent of 6 per cent., while the others contain none, a noticeable difference in morphology can usually be made out; while the forms on the glycerinagar-agar cultures are throughout small, and pretty regular in size, shape, and staining, those on the plain agar-agar are larger, stain more irregularly, vary more in shape, and when stained by Loeffler's blue are not so uniformly marked by pale transverse lines that give to them the appearance of being made up of numerous short segments.

Though the outline of this organism is more regular under some circumstances than others, it is neverthcless always conspicuons for its manifold variations in shape.

Growth on Serum Mrxture. - The medium upon which it grows most rapidly and luxuriantly, and which is best adapted for determining its presence in diphtheritic exudation, is, as has been stated, the blood-serum mixture of Lœffler. (See chapter on Media.) On the blood-serum mixture the colonies of the bacillus diphtheriæ grow so much more rapidly than the other organisms usually present in secretions and exudations in the throat that at the end of twenty-four hours they are often the only colonies that attract attention; and if others of similar size are present, they are generally of quite a different aspect. Its colonies are large, round, elevated, grayish-white or yellowish, with a centre more opaque than the slightly irregular periphery. The surface of the colony is at first moist, but after a day or two becomes rather dry in appearance.

A blood-serum tube studded over with coalescent or scattered colonics of this organism is so characteristic 
that one familiar with the appearance can anticipate with tolerable certainty the results of microscopic examination.

Glicerix Agar-Agar.-Upon nutrient glycerin agar-agar the colonies likewise present an appearance that may readily be recognized. They are in every way more delicate in their structure than when on the serum mixture. They appear at first, when on the surface, as very flat, almost transparent, dry, non-glistening, round points which are not elevated above the surface upon which they are growing. When slightly magnified they are seen to be granular, and to present an irregular central marking which is denser and darker

FIG. 66.
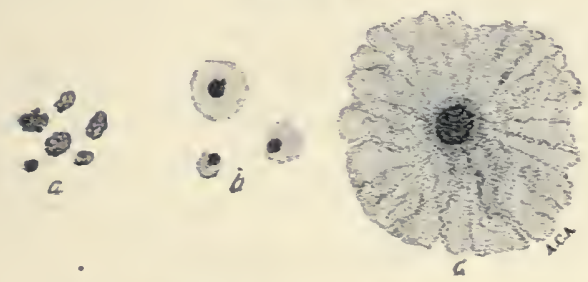

Colonies of bacillus diphtherix on glycerin-agar-agar. a. Colonies located in the depths of the medinm. b. Colonies just breaking out apon the surface of the medium. c. Fally dereloped surface-colony.

by transmitted light than the thin, delieate zone which surrounds it. As the colouy increases in size the thin granular peripheral zone becomes broader, is usually marked by ridges or cracks, and its periphery is notched or scalloped. (Fig. 66, c.) These colonies are always quite dry in appearance. When deep down in the agaragar they are coarsely granular. (Fig. 66, a.) They rarely exceed $3 \mathrm{~mm}$. in diameter. 
Geratin.-On gelatin the colonies develop much more slowly than on the other media that can be retained at a higher temperature. They rarely present their characteristic appearances on gelatin in less than seventy-two hours.

They then appear as flat, dry, translucent points, usually round in outline.

When magnified slightly the centre is seen to be more dense than the surrounding zone or zones, for they are sometimes marked by a concentric arrangement of zones. The periphery is irregularly notched. Like the colonies seen on agar-agar, they are granular, but are much more granular when seen in the depths of the gelatin than when on its surface. On gelatin the colonies rarely become very large; usually they do not reach a diameter of over $1.5 \mathrm{~mm}$.

BouILLoN.-In bouillon it usually grows in fine clumps, which fall to the bottom of the tube, or become deposited on its sides without causing a diffuse clouding of the bouillon. There are sometimes exceptions to this naked-eye appearance: the bouillon may be diffusely clouded; but if one inspect it very closely, particularly if one examine it microscopically as a hanging drop, the arrangement in clumps will always be detected, but they are so small as not to be discernible by the unaided eye.

In bouillon which is kept at a temperature of $35^{\circ}-$ $37^{\circ} \mathrm{C}$. for a long time a soft, whitish pellicle often forms over a part of the surface.

Changes in reactions of the bouillon. The reaction of the bouilion frequently becomes at first acid, and, subsequently, again alkaline, changes which can be observed in cultivations in bouillon to which a little rosolic acid 
has been added. This play of reactions has been attributed to the primary fermentation of muscle sugar that is often present in the bouillon.

Pотато.-On potato at a temperature of $35^{\circ}-37^{\circ} \mathrm{C}$. its growth after several days is entirely invisible, there being only a thin, dry glaze appearing at the point at which the potato was inoculated. Microscopic examination of serapings from the potato, after twenty-four hours at $35^{\circ}-37^{\circ} \mathrm{C}$., reveals a decided increase in the number of individual organisms planted.

Stab- Axd Slant-cultures. - In stab-and slantcultures on both gelatin and glycerin-agar-agar the surface-growth is seen to predominate over that along the track of the needle in the depths of the media.

Isolated colonies on the surface of either of the media in this method of cultivation present the same characteristics that have been given for the colonies on plates.

The growth in simple stab-cultures does not extend laterally very far beyond the point at which the needle entered the medium.

It is a non-motile organism.

It does not form spores.

It is killed in ten minutes by a temperature of $58^{\circ} \mathrm{C}$.

It grows at temperatures ranging from $22^{\circ} \mathrm{C}$. to $37^{\circ}$ C., but most luxuriantly at the latter temperature.

Its growth in the presence of oxygen is more active than when this gas is excluded.

Starvivg. - In cover-slip preparations made either from the fauces of a diphtheritic patient or from a pure culture of the organism it is seen to stain readily with the ordinary aniline dyes. It stains also by the method of Gram, but the best results are those obtained by the use of Lnetler's alkaline methylene-blue solution; this 
brings out the dark points in the protoplasmie body of the bacilli and thus aids in their identification.

For the purpose of demonstrating the Lœeffler bacillus in sections of diphtheritie membrane, both the Gram method and the fibrin method of Weigert give excellent results.

Pathogenic Properties. - When inocnlated subcutaneously into the bodies of suseeptible animals the result is not the produetion of septicæmia, as is seen to follow the introduction into animals of certain other organisms with which we shall have to deal, but the bacillus of diphtheria remains localized at the point of inoculation, rarely disseminating further than the nearest lymphatic glands. It develops at the point in the tissues at which it is deposited, and during its development gives rise to changes in the tissues which result entirely from the absorption of poisonous albumins produced by the bacilli in the course of their development.

In a certain number of cases $^{1}$ diphtheria bacilli have been found in the blood and internal organs of individuals dead of the discase; but all that has been learned from careful study of the secondary manifestations of diphtheria tends to the opinion that they are in no way dependent upon the immediate presence of bacteria, and that the oecasional appearance of diphtheria bacilli in the internal organs is in all probability accidental, and usually unimportant.

By special methods of inoculation ${ }^{2}$ (the injeetion of

1 Frosch : Die Verbreitung des Diphtherie-bacillns im Körper des Menschen Zelt. fur Hygiene und Infectionskrankheiten, 1893, Bd. xlli. pp. 49-52. Booker: Archives of Pediatrics, Aug. 1893. Wright and Stokes : Boston Med. and Surg. Journ., March and April, 1895.

2 Abbott and Ghriskey: A Contribution to the Patbology of Experimental Diphthetria. The Johns Hopkins Hospital Bulletin, No. 30, April, 1893. 



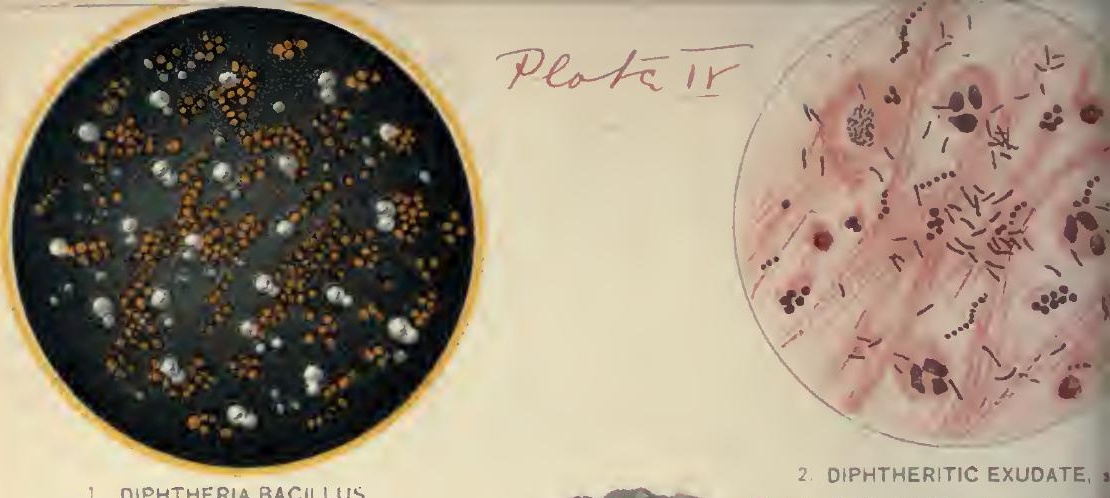

with Staphylococcus Pyogenes Aureus.

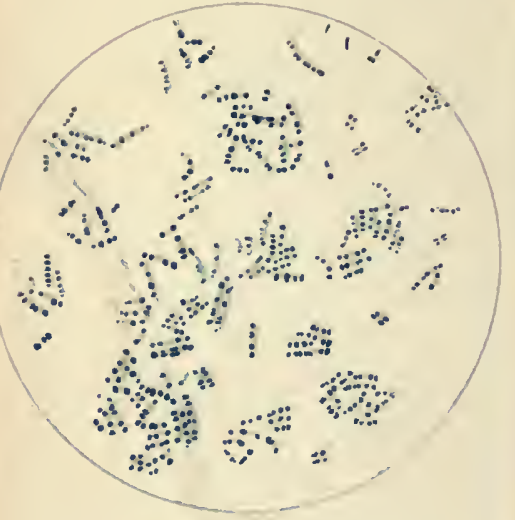

3 DIPHTHERIA BACILLUS. Typical Form.

5. STAPHYLOCOCCUS PYOGENES AUREUS Agar Culture.
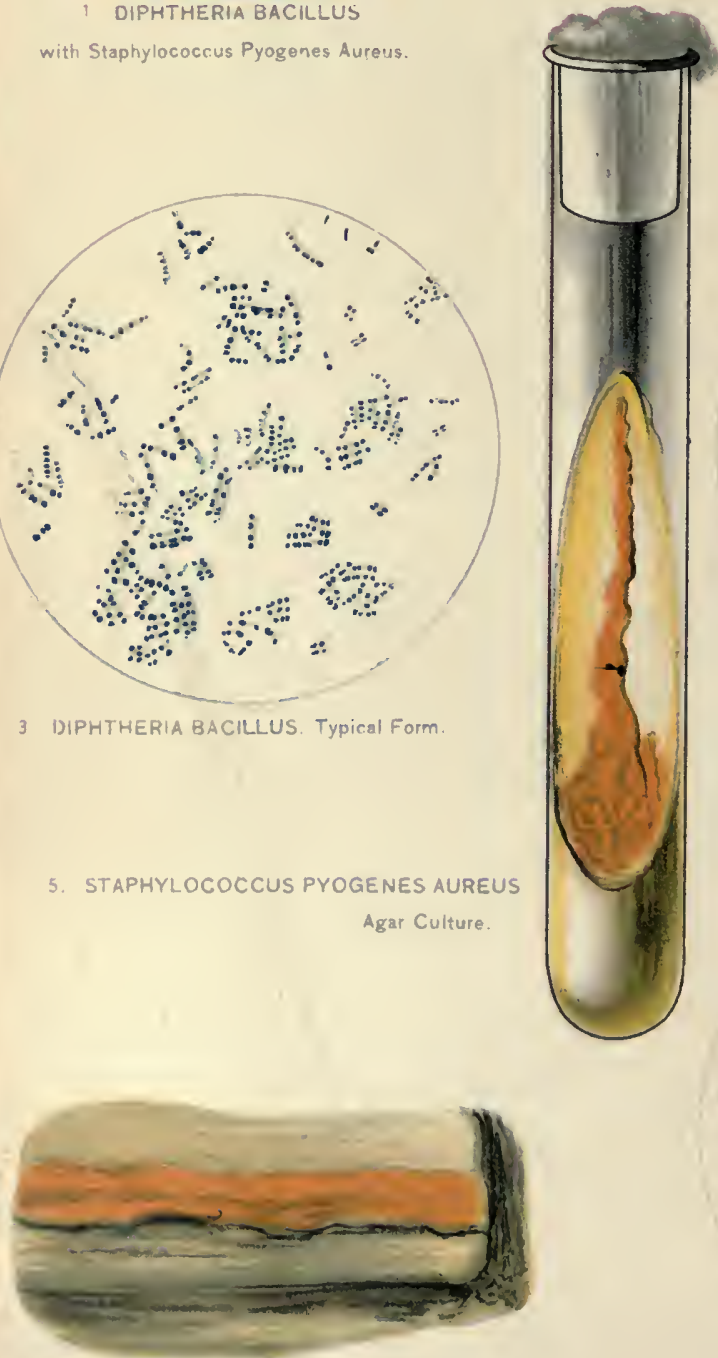

Diphtherie Bacilli, Streptococci and S.

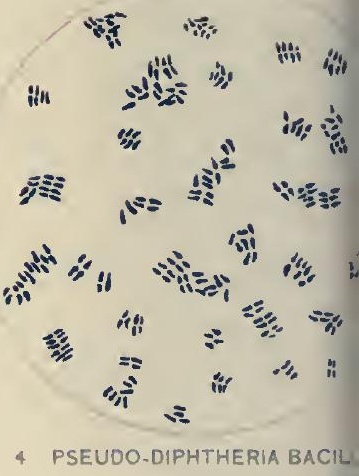


Leeds bruble then

siluen hich

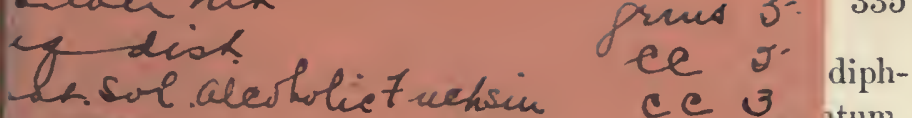

iysagallic ac

की itum, e dis-

\% Sho. Kydrahe (aquend

Yac dest in the 'esults

ablese fachin sre

qreles

- foe $x$ ath of five

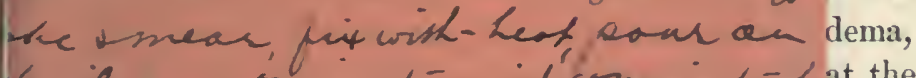
bovil ane minute wish a moniche, tot the oh. Then panr en $\beta$ \& do he sacue phatic - waskg parr an $C$ tor a minute $r$ leura, woth ele drenal wosh thy veyackine. Braillifink somein thadeo, totane or bottecuds myoI in the mididle, brilliauty shining often Kpaint ypear scceding lyshaptcer They nay be above f inocibrinoistance demand not ocytes, e lost well as irregu- 
DIPHTHERIA BAO with Staphylococcus Pyoge

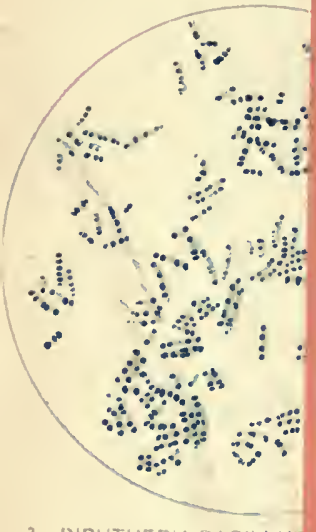

3 DIPHTHERIA BACILLU 
fluid cultures into the testicles of guinea-pigs) diphtheria bacilli can be caused to appear in the omentum, but this is purely an artificial manifestation of the disease and one that is probably never encountered in the natural course of events. More rarely similar results follow upon subcutaneous iuoculation.

If a very minute portion of either a solid or fluid pure culture of this organism be introduced into the sulsentaneous tissues of a guinea-pig or kitten, death of the animal ensues in from twenty-four hours to five days. The usual changes are an extensive local cedema, with more or less hyperemia and ecchymosis at the site of inoculation; swollen and reddened lymphatic glands; increased serous fluid in the peritoneum, pleura, and pericardium; enlarged and hemorrhagic adrenal bodies; occasionally slightly swollen spleen; and sometimes fatty degeneration in the liver, kidney, and myocardium. In guinea-pigs, especially, the liver often shows numerous macroscopic dots and lines on the surface and penetrating the substance of the organ. They vary in size from a pin-point to a pin-head, and may be even larger. They are white and do not project above the surface of the capsule.

The bacilli are always to be found at the seat of inoculation, most abundant in the grayish-white, fibrinopurulent exudate. They become fewer at a distance from this, so that the more remote parts of the cedematous tissues do not contain them. They are found not only free, but contained in large number in leucocytes, some of which have fragmented nuclei, or have lost their nuclei. The bacilli within lencoeytes, as well as some outside, frequently stain very faintly and irregularly, and may appear disintegrated and dead. 
Culture-tubes inoeulated from the blood, spleen, liver, kidneys, adrenal bodies, distant lymphatic glands, and serous transudates, generally yield negative results; and negative results are also obtained when these organs are examined microscopically for the bacilli.

Microscopic examination of the tissues at the seat of inoculation, as well as of the liver, spleen, kidneys, lymphatic glands and elsewhere, reveals the presence of localized foci of cell-death, characterized by a peculiar fragmentation of the nuclei of the cells of these parts.

This destruction of nuclei results in the occurrence of groups of irregularly shaped, deeply staining bodies, having at times the appearance of particles of dust, while again they may be much larger. Some of them are tolerably regular in outline, while others are irregularly crescentic, dumb-bell, flask-shape, whetstone shape, or bladder-like in form. Occasionally nuclei having the appearance of being pinched or drawn out can be seen. At some points the fragments are grouped into isolated masses, indicating the location of the nucleus from the destruction of which they originated. These particles always stain much more intensely than do the normal nuclei of the part. ${ }^{1}$

These peeuliar alterations, as Oertel has shown, in their distribution are eharaeteristic of human diphtheria, and the demonstration of similar changes in animals inoculated with this organism is important additional proof that diphtheria is caused by it.

1 See "The Histological Changes in Experimental Diphtheria," also "The Histological Lesions produced by the Toxalbumin of Diphtheria," by Welch and Flexner. The Johns Hopkins Hospital Bulletin, August, 1891, and March, 1892. 
Au affection may be produced by the inoculation of certain animals that is in all respects identical with the disease diphtheria as it exists in man. If one open the trachea of a kitten and rub upon the mucous membrane a small portion of a pure culture of this organism, the death of the animal usually ensues in from two to four days. At autopsy the wound will be found covered with a grayish, adherent, necrotic, distinetly diphtheritic layer. Around the wound the subcutaneous tissues will be odematous. The lymphatic glands at the angle of the jaws will be swollen and reddened. The mucous membrane of the trachea at the point upon which the bacilli were deposited will be covered with a tolerably firm, grayish-white, loosely attached pseudomembrane in all respects identical with the croupous membrane observed in the same situation in cases of human diphtheria. In the pseudo-membrane and in the oedematous fluid about the skin-wound bacillus diphtherice may be found both in cover-slips and in cultures.

From what we have seen-the localization of the bacilli at the point of inoculation, their absence from the internal organs, and the changes brought about in the cellular elements of the internal organs-there is but one interpretation for this process, viz., that it is due to the production of a soluble poison by the bacteria growing at the seat of inoculation, which, gaining access to the circulation, produces the changes that we observe in the tissues of the internal viscera.

This poison has been isolated from cultures of the bacillus diphtherice, and is found to belong, not to the crystallizable ptomaines, but to the toxic albuminsborlies which, in their chemical composition, are analo- 
gous to the poison of certain venomous serpents. By the introduction of this toxalbumin, as it is called, into the tissues of guinea-pigs and rabbits the same pathological alterations may be produced that we have seen to follow the result of inoculation with the bacilli themselves, except, perhaps, the production of false membranes.

Under the influence of certain circumstances with which we are not acquainted the bacillus diphtherice becomes diminished in virulence or may lose it entirely, so that it is no longer capable of producing death of susceptible animals, but may cause only a transient local reaction from which the animal entirely recovers. Sometimes this reaction is so slight as to be overlooked, and again careful search may fail to reveal evidence of any reaction at all. This exhibition of the extremes of its pathogenic properties, viz., death of the animal, on the one hand, and only very slight local effects on the other, was at one time thought to indicate the existence of two separate and distinct organisms that were alike in cultural and morphological peculiarities, but which differed in their disease-producing power. Further studies on this point have, however, shown that the genuine bacillus diphtherice may possess almost all grades in the degree of its virulence, and that absence of or diminution in virulence can hardly serve to distinguish as separate species these varieties that are otherwise alike; moreover, the histological conditions found at the site of inoculation in animals that have not succumbed, but in which only the local reaction has appeared, are in most cases characterized by the same changes that are seen at autopsy in animals in which the inoculation has proverl fatal. 
In the course of their observations upon a large number of cases Roux and Yersin found that it was not difficult to detect, in the diphtheritic deposits of one and the same individual, bacilli of identical cultural and morphological peculiarities, but of very different degrees of virulence, and that with the progress of the disease toward recovery the less virulent varieties often became quite frequent. ${ }^{1}$

There is, moreover, a mild form of diphtheria affecting only the mucous membrane of the nares, known as membranous rhinitis, from which it is very common to obtain cultures in all respects identical with those from typical diphtheria, save for their inability to kill susceptible animals. On inoculation these cultures produce only local reactions, but they are characterized histologically by the same tissue-changes that follow inoculation with the fully virulent organism.

Clinically, membranous rhinitis is never such an alarming disease as is laryngeal or pharyngeal diphtheria, and, as stated, the organisms causing it are often of a low degree of virulence, though they are, nevertheless, genuine diphtheria bacilli.

For those organisms that are in all respects identical with the virulent bacillus diphtherice, save for their inability to kill guinea-pigs, the designation "pseudo-diphtheritic bacillus" is usually employed; but from such observations as those just cited we are inclined to the opinion that pseudo-diphtheritic, as applied to an organism in all respects identical with the genuine bacillus, except that it is not fatal to susceptible animals, is a

\footnotetext{
1 It must not be assumed from this that the bacilli lose their virulence entirely, or that they all become attenuated with the establishment of convalescence.
} 
misnomer, and that it would be more nearly correct to designate this organism as the attenuated or non-virulent diphtheritic bacillus, reserving the term "pseudodiphtheritic" for that organism or group of organisms (for there are probably several) that is enough like the diphtheria bacillus to attract attention, but is distinguishable from it by certain morphological and cultural peculiarities aside from the question of virulence.

It is a well-known fact that many pathogenic organisms-conspicuous among these being the micrococcus lanceolatus, the staphylococcus pyogenes aureus, and the group of so-called "hemorrhagic septicæmia" organisms-undergo marked variations in the degree of their pathogenic properties, and yet these organisms, when found either devoid of this peculiarity, or possessing it to a diminished degree, are not designated as "pseudo" forms, but simply as the organisms themselves, the virulence of which, from various causes, has been modified.

Note.-Prcpare cover-slip preparations from the mouth-cavities of healthy individuals and from those having decayed teeth. Do they correspoud in any way with those made from diphtheria? Do the same with different forms of sore-throat. Do the peculiarities of any of the organisms suggest those of the bacillus of diphtheria? Wherein is the difference?

In cultures and cover-slips made from both diphtheria and from innocent sore-throats are there any organisms which are almost coustantly present? Which are they, and what are their characteristics?

Which are the predominating organisms in the anginas of scarlet fever?

Do these organisms simulate, in their cultural and 
morphological peculiarities, any of the different species with which you have been working?

Do the diphtheria bacilli disappear from the throat with the disappearance of the membrane? How long do they persist? When obtained from the throat of convalescents are they still pathogenic for guinea-pigs? 


\section{CHAPTER XXI.}

Typhoid fever-Study of the organism concerned in its production. Bacterium coli commune-Its resemblance to the bacillus of typhoid feverIts morphological, cultural, and pathogenic properties-Its differentiation from bacillus typhi abdominalis.

THE organism, discovered by Eberth and by Gaffky, generally recognized as the etiological factor in the production of typhoid fever, may be described as follows:

It is a bacillus about three times as long as it is broad, with rounded ends. It may appear at one time as very short ovals, at another time as long threads, and both forms may occur together. Its breadth remains

Fig. 67.

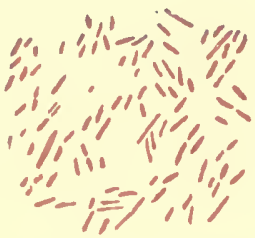

Bacillns lyphi abdominalis from culture twenty-four hours old, on agar-agar.
Fig. 68.

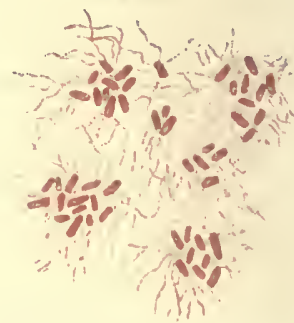

Bacillus typli abdominalis showing flagella stained by Loffler's method.

tolerably constant. Its morphology presents little that will aid in its identification (see lig. 67). It stains a trifle less readily with the aniline dyes than do most of the other organisms. It is very actively motile, and when stained by the special method of Iweffler (see 
this method in chapter on Staining) is seen to possess very delicate locomotive organs in the form of fine, hair-like flagella, which are given off in large numbers from all parts of its surface (see Fig. 68). These flagella are not seen in unstained preparations, nor are they rendered visible by the ordinary methods of staining.

In patients suffering from this disease it has been found during life in the blood, urine, and feces, and at autopsies in the tissues of the spleen, liver, kidneys, intestinal lymphatic glands, and intestines.

Gelatin Plates. - Its growth, when seen in the depths of the medium, presents nothing characteristic, appearing simply as round or oval, finely granular points. "On the surface it develops as very superficial, blue-white colonies, with irregular borders. They are a little denser at the centre than at the periphery.

FIG. 69.

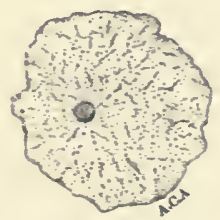

Colony of bacillus typhi abdominalis on gelatin.

When magnified, the colonies present wrinkles or folds, which give to them, in miniature, the appearance seen in the relief maps made to represent mountainous districts (Fig. 69). These colonies have sometimes the appearance of flattened pellicles of glass-wool, and usually present more or less of a pearl-like lustre.

On AgAr-AGAR the colonies present nothing typical. Stab-cultures. - In stab-cultures the growth is 
mostly on the surface, there being only a very limited development down the track made by the needle. The surface-growth has the same appearance in general as that given for the colonies.

PoтAтo. - The growth on potato is usually deseribed as luxuriant but invisible, making its presence evident only by the production of a slight increase of moisture at the inoculated point, and by a limited resistance offered to a needle when it is scraped across the track of growth. While this is true in most cases, yet it cannot be considered as constant, for at times this organism is seen to develop more or less visibly on potato.

Potato Gelatin. - The growth is similar to that upon ordinary nutrient gelatin.

MrLk.-It does not cause coagulation when grown in sterilized milk.

It does not liquefy gelatin.

It grows both with and withont oxygen.

In bouillon it causes a uniform clouding of the medium and brings about a slightly acid reaction.

It does not grow rapidly.

Indor Formation.-It is customary to regard this organism as devoid of the power of forming indol; in fact, this has hitherto been considered as one of its important differential peculiarities. By the usual methods of cultivation and testing the indol reaction is not observed in eultures of the typhoid bacillus. It lias recently been shown, lowever, by Dr. Peckham, that by repeated transplantation, at short intervals, into either Dunham's peptone solution, or, preferably, a freshly prepared alkali-tryptone solution, made from tryptonized beef nuscle, that the indol-producing func- 


tion may be induced in the genuine typhoid bacillus obtained directly from the spleens of typhoid cadavers. ${ }^{1}$

It does not produce gaseous fermentation. On lactoselitmus-agar-agar it grows as pale-blue colonies, causing no reddening of the surrounding medium; though if glucose be substituted for lactose, both the colonies and the surrounding medium become red. In the fermentation-tube, in glucose or lactose bouillon, no evolution of gas as a result of fermentation occurs.

It does not form spores. The irregularities of staining so commonly seen in this organism have in some instances led to the belief that the pale, unstained portions of the bacilli indicate the presence of spores. More reliable tests, however, have demonstrated the error of this opinion. (What is the most trustwortly test of spore-formation?)

It grows at any temperature between $20^{\circ}$ and $38^{\circ} \mathrm{C}$, though more favorably at the latter point.

It is very sensitive to high temperatures, being killed. by an exposure of ten minutes to $60^{\circ} \mathrm{C}$., and in a much shorter time to slightly higher temperatures.

FIG. 70.

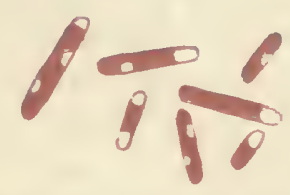

Diagrammatic representation of retraction of protoplasm, with produclion of pale points, in bacillus typhi abdominalis.

Owing to a tendency to retraction of its protoplasm from the cell envelope and the consequent produc-

1 See A. W. Peckbam: The Influence of Environment upon the Biological Functions of the Colon Group of Bacilli. Journal of Experimental Medicine, vol. ii. 1897. 
tion of vacuoles in the bacilli, the staining of this organism is usually more or less irregular. At some points in a single cell marked differences in the intensity of the staining will be seen, and here and there areas quite free from color can commonly be detected. These colorless portions are often so cleanly cut that they look as if they had been punched out with a sharp instrument. (Diagrammatically represented in Fig. 70.)

Presence in Tissufs. - It is not easy to demonstrate this organism in tissues unless it is present in large numbers. The manipulations to which the sections are subjected in being mounted often rob the bacilli of their staining, and render them invisible, or nearly so. If, however, scctions be stained in the carbol-fuchsin solution, either at the ordinary temperature of the room or at a ligher temperature $\left(40^{\circ}\right.$ to $45^{\circ} \mathrm{C}$.), then washed out in absolute alcohol, and cleared up in xylol and mounted in balsam, the bacilli (particularly if the tissue be the liver or spleen) can readily be detected, massed together in their characteristic elumps. If used in the same way, the alkaline methylene-blue solution gives also very satisfactory results.

In searching for the typhoid bacilli in tissues their mode of growth under these circumstances must always be borne in mind, otherwise much labor will be expended in vain. In tissues the typhoid bacilli do not lie scattered about in the same way as do the organisms in tissues from eases of septicemia; they are not regularly distributed along the course of the eapillaries, but are localized in small clumps through the tissues, and it is for these elumps, which are easily detected under a low-power objective, that one should scarch. When the section is prepared for examination, if it be gone 
over with a low-power objective, one will notice at irregular intervals little masses that look in every respect like particles of staining-matter which have been precipitated upon the section at that point. When these little masses are examined with a higher power objective they will be found to consist of small ovals or short rods so closely packed together that the individuals composing the clump can often be seen only at the very periphery of the mass. This is the characteristic appearance of the typhoid organism in tissues. The little masses are usually in the neighborhood of a capillary.

Result of InoculaAtion into Lower Avimals.A great many experiments have been made with the view of reproducing the pathological conditions of this disease, as seen in man, in the tissues of lower animals, but with limited success. Fatal results without the appearance of the typical pathological changes have frequently followed these attempts, but in most cases they could easily be traced to the toxic, ${ }^{1}$ rather than to the truly infective ${ }^{2}$ action of the materials introduced into the inimals.

The most successful efforts for the production of the typical typhoid lesions in lower animals are those reported by Cygnæus. By the introduction of the typhoid bacilli into the tissues of dogs, rabbits, and mice he was able to produce in the small intestine conditions that were histologically and to the naked eye analogous to those found in the human subject.

Of a large number of experiments made by the writer with the same object in view, only one positive result

1 Toxic-Poisonous results not necessarily accompanied by the growth of organisms throughout the tissues.

I Infective or septic-Poisoning of the tissues as a result of the growth of bacteria within them. 
followed the introduction of typhoid bacilli into the circulation of rabbits. In this case the ulcer in the ileum was macroscopically and microscopically identical with those found at autopsy in the small intestine of the human subject dead of this disease. The typhoid bacilli were not only obtained from the spleen of the animal by culture method, but were also demonstrated microscopically in their characteristic clumps in section of the organ.

In connection with the inoculation of animals with bacillus typhi abdominalis observations of a most important nature have been made by Sauarelli ${ }^{1}$ upon the artiticial induction of susceptibility to its pathogenic action. He found that rabbits, guinea-pigs, and mice could be rendered susceptible to infection by this organism by preliminary injections into them of the products of growth of certain saprophytes-proteus vulgaris, bacillus prodigiosus, and bacterium coli commune-and that by whatever means the animal was subsequently inoculated with fresh cultures of the typhoid bacillus, either into the circulation or into the peritoneal cavity, death resulted in from twelve to forty-eight hours, with the most conspicuous pathological alterations in the digestive tract, and particularly in the small intestine. In these cases the infection is general, and the organisms may be recovered from the blood and internal organs. $\mathrm{It}$ is the opinion of Sanarelli that the toxic conditions produced by the preliminary injections of the products of growth of the saprophytic organisms may be considered as analogous to a similar condition that may occur in man from the absorption of abnormal products of fermentation from the intestinal canal-an auto-intoxi- 
cation that so reduces the resistance of the individual as to render him susceptible to infection by the bacillus of typhoid fever, should it gain access to his alimentary tract.

More recently it was reported by Alessi ${ }^{1}$ that rats, guinea-pigs, and rabbits, when compelled to breathe the gaseous products of decomposition from the contents of a cesspool, or from other decomposing matters, gradually became susceptible to infection by the typhoid bacillus; but unfortunately for the integrity of this observation the description given by Alessi of the two cultures of so-called typhoid bacilli used by him for inoculation, was in one case certainly not that of the typhoid organism, and in the other the culture used had been kept under artificial conditions so long as hardly to be reliable for tests of this character.

The importance of these observations in their bearing upon the etiology of typhoid fever, if they are demonstrated by subsequent experiment to be trustworthy, is too obvious to necessitate emphasis, and it is greatly to be desired that they may not be permitted to pass unnoticed, but that others interested may find occasion to institute experiments in the same direction with the hope that some light may be shed upon the mooted question concerning the influence of gaseous products of decomposition upon the health of individuals, and particularly upon the part played by them in diminishing natural resistance to infection. ${ }^{2}$

1 Alessi : Centralblatt für Bakteriologie n. Parasitenkunde, 1894, Bd. xv., กo. 7, p. 228.

2 See paper by the author: "The Effects of the Gaseous Products of Decom. position upon the Health, and Resistance to Infection, of Certain Animals that are forced to Respire Them." Transactions of the Association of American Physicians, 1895, vol. x. pp. 16-44. 
Because of the variations in the morphology and eultural peculiarities of this organism, and because of the difficulty experienced in efforts to reproduce in lower animals the conditions found in the human subject, typhoid fever is bacteriologically one of the most unsatisfactory of the infectious diseases.

There are a number of other organisms which botanically appear to be nearly related to the typhoid bacillus, and which, with our present methods for studying them, so closely simulate it, that the difficulty of identifying this organism is sometimes very great. In addition to this, the variability constantly seen in pure cultures of the typhoid bacillus itself in no way reuders the task more simple.

For example, the morphology of the typhoid bacillus is conspicuously inconstant; its growth on potato, which was formerly described as characteristic, may, with the same organism, at one time appear as the typical invisible development, at another time it may grow in a way easily to be seen with the naked eye; and the change of reaction which it is said to produce in bouillon is sometimes much more intense than at others. The indolproducing function, hitherto regarded as absent from this organism, is now known to be occasionally demonstrable by ordinary methods, and frequently demonstrable by special methods of cultivation. (Peckham, l.c.)

The only properties possessed by it that may be said to be constant are its motility, its inability to eause gascous fermentation of glucose, lactose, or saccharose, its incapacity for coagulating milk, and its growth on gelatin plates; but there are other organisms which approach these same characteristics to a degree that renders their differentiation from the typhoid organisms 
often a matter that requires the careful application of all the different tests.

Probably the most trustwortly, certainly the most recently described, reaction of the typhoid bacillus is that seen when it is brouglit in contact with the bloodserum from liuman beings sick of typhoid fever, or from animals that have survived inoculation with cultures of this organism. This reaction consists of a peculiar alteration in the relation of the organisms to one another in the fluid. As ordinarily seen in a hanging drop of bouillon the typhoid bacillus appears as single, actively motile cells; when to such a drop a drop of dilute serum from a case of typhoid fever is added the motility of the organism gradually becomes lessened, and finally ceases, and the bacteria congregate together in larger and smaller clumps. The reaction may also be made in another way, viz., by adding to about 4 or 5 c.c. of a twenty-four-hour-old bouillon culture of typhoid bacilli in a narrow test-tube about eight drops of serum from a case of typhoid fever, after which the tube is placed in the incubator. After a few hours the normally clouded culture is seen to have undergone a change; instead of the diffuse cloud caused by the growth, the fluid is found to be clear and to contain within it floceulent masses of the bacteria that have agglutinated together as a result of the specific action of the serum used. When employed conversely-i.e., for deciding if the serum used is from a case of typhoid fever or not-the reaction constitutes what is known as "Widal's serum diagnosis of typhoid fever." For this latter purpose it is often necessary to test several cultures of genuine typhoid bacilli, from different sources and of varying degrees of vitality, before a culture is 
finally encountered that gives the reaction most conspicuously and quickly with genuine typhoid serum. This culture is then to be set aside to be used for this test with serums from doubtful cases of the disease. In the hands of all those who have employed this method carefully for the diagnosis of typhoid fever the results are reported to have been almost uniformly satisfactory. The reaction is, so far as experience indicates, speeifici.e., a typical reaction does not oceur between typhoid serum and organisms other than the typhoid bacillus, nor between the typhoid bacillus and serums other than those of typhoid fever. There are, however, confusing reactions-so-called pseudo-reactions-in which more or less elumping of the bacilli and a diminution of motion, without complete cessation, are observed. These have been seen to oceur with normal blood and with blood from other febrile conditions. It is said by Johnston and MeTaggart that they can be prevented if eultures of just the proper degree of vitality are employed. The method is yet in the experimental stage, and there are still numerous features that are not entirely clear. It is, however, in the light of present experienee, fair presumptive evidence that the serum is from a ease of typhoid fever when unmistakable agglutination and cessation of motion are seen in typhoid bacilli that are mixed with the serum of a suspicious febrile condition. For the hanging-drop test sufficient serum may be obtained from a needle-priek in the finger, while for the test-tube reaction a larger amount is needed; this may be obtained from blood drawn from a superficial vein by means of a hypodermic syringe, or from the cleansed skin by a wet-cup, or, better still, from a small cantharides blister.

J Johnston and McTaggart: Montreal Medical Journal, March, 1897. 
All the preceding points should be borne in mind in the examination of drinking-water supposed to be contaminated by typhoid dejections, for the organisms which most nearly approach the typhoid bacillus in growth and morphology are just those organisms which would appear in water contaminated from cesspoolsi.e., the organisms constantly found in the normal intestinal tract. Even in the stools of typhoid-fever patients the presence of these normal inhabitants of the intestinal tract renders the isolation of the typhoid organisms somewhat troublesome.

\section{EISNER'S METHOD OF ISOLATING THE TYPHOID BACILLUS.}

A number of special methorls for the isolation of the typhoid bacillus from mixtures, such as water, feces, etc., that contain it have been recommended, but none of them lias given general satisfaction, and many have proved to be entirely untrustworthy. That which has perhaps attracted the most attention is the recently devised medium of Elsner. It is an acid mixture of gelatin, potato juice, and iodide of potash. It contains no peptone, and no sodium chloride is added. On this medium it is claimed that the ordinary, rapidly growing, liquefying saprophytes do not develop, and that the colon bacillus and typhoid bacillus find it favorable for growth. These are differentiated from one another by the macroscopic and microseopic character of their colonies-i. $e_{\text {. }}$, the growth of the colon colonies differs little or not at all from that seen on ordinary nutrient gelatin, while that of the typhoid colonies is so slow that they are hardly visible at the end of twenty-four hours. After forty-eight hours they appear under the 
low power of the mieroscope as small, pale, finely granular, almost transparent bodies that are easily distinguished from the coarser, brownish colonies of the colon baeilhus.

While the method is useful, it has its limitations, and is not always reliable. At times eolon eolonies will develop in a way that would readily cause one to mistake them for typhoid colonies, while again typhoid eolonies will take on the characteristies of those due to the growth of the eolon bacillus. This is especially the case in plates over forty-eight hours old that have been kept at ordinary room temperature.

In our experience the most serviceable feature of this method is the elimination of many of the common saprophytes usually present in mixtures containing the typhoid and colon baeilli. The majority of them do not grow upon gelatin made by this method, which will now be described.

The description given by Elsner ${ }^{1}$ for the mode of preparation of the medium is so ineomplete and unsatisfactory in most of the important details that praetically all those who have used the method have been obliged to develop their own technique from the general suggestions made in his original communication. The "Elsner medium" that has given satisfaetion in our hands is prepared as follows: grate 1 kilogram of peeled potatoes and allow to stand in the refrigerator over night. Then press out all the juice, using an ordinary meat-press for the purpose. Filter this fresh juice cold, to remove as mueh of the stareh grannles as possible; if this is not done the starch when

1 Elsner: Zelt. fur Hygiene und Infectionskrankheiten, 1896, Bd. 21, p. 25. 
heated swells to such an extent as to render filtration almost impracticable. Boil the filtrate and filter again. Test the filtrate for acidity by titrating 10 c.c. with a decinormal solution of sodium hydroxide, the indicator used being 6 drops of the ordinary $\frac{1}{2}$ per cent. solution of phenolphtalein in 50 per cent. alcohol. The acidity of the juice should be such as to require 3 c.c. of a decinormal sodium hỵdroxide solution to neutralize 10 c.c. of the juice (Potter). If the acidity is found to be greater than this, which is usually the case, dilute with water until the proper degree is reached. If less than this, the juice may be concentrated by evaporation. It is desirable that this acidity should arise from the acids normally present in the potato, and should not be artificially obtained by the addition of other acids. 'Now add 10 per cent. of gelatin (no peptone and no sodium chloride), dissolve by boiling and again test the acidity, using 10 c.c. of the mixture and phenolphtalein as before. Deduct 3 c.c. (the acidity of the potato juice, that is to be maintained) from the number of c.c. of the decinormal sodium hydroxide solntion required to neutralize the $10 \mathrm{c} . \mathrm{c}$. of the gelatin mixture, and from the resulting figure calculate the amount of normal solution of sodium hydroxide needed for the entire volume, and add it. Boil, clarify with an egg, and filter through paper in the usual manner. To the filtrate add potassium iodide in the proportion of 1 per cent. Decant into tubes and sterilize.

The spleen of a patient dead of typhoid fever is the safest source from which to obtain cultures of the typhoid bacillus for study. But it must always be remembered that the same channels through which the 
typhoid bacillus gains access to this viseus are likewise open to other organisms present in the intestines, and for this reason the bacterium coli commune, a normal inhabitant of the colon, may also be found in this locality.

Note.-Obtain a pure culture of typhoid bacilli, and from this make inoculations upon a series of potatoes of different ages and from different sources. Do they all grow alike?

Before sterilizing render another lot of potatoes slightly acid with a few drops of very dilute acetic aeid; render others very slightly alkaline with dilute caustic soda. Do any differences in the growth result?

Make a series of twelve tubes of peptone solution to which rosolic acid has been added. Inoculate them all with as near the same amount of material as possible (one loopful from a bouillon culture into each tube); place them all in the incubator. Is the color-change, as compared with that of the control tube, the same in all cases?

Compare the morphology of cultures of the same age on gelatin, agar-agar, and potato.

Select a culture in which the vacuolations are quite marked. Examine this eulture unstained. Do the organisms look as if they contained spores? How would you demonstrate that the vacuolations are not spores?

Obtain from the normal feces a pure culture of the commonest organism present. Write a full description of it. Now make parallel eultures of this organism and of the typhoid organism on all the different media. How do they differ? In what respeets are they similar? 
Bacterium Coli Commoxe (colon bacillus; bacillus Neapolitanus of Emmerich). -This organism was discovered by Escherich, in 1885, in the intestinal discharges of milk-fed infants. It has since been demonstrated to be a normal inhabitant of the intestines of man and of certain domestic animals (eattle, hogs, dogs).

For a time after its discovery it was considered of but little importance and attracted attention only because of its resemblance, in certain respects, to the bacillus of typhoid fever, with which it was occasionally confounded. In this particular it still serves as a subject for study. Some have even gone so far as to regard them but as varieties of one and the same species, though in the present state of our knowledge this is certainly an assumption for which, as yet, there are not sufficient grounds. That they possess in common certain general points of resemblance and often approach one another in some of their biological peculiarities is true; but, as we shall learn, they each possess peculiari- . ties which, when taken together, render their differentiation from one another a matter of but little difficulty.

With the wider application of bacteriological methods to the study of pathological processes it was occasionally observed that, under favorable circumstances, this organism was disseminated from its normal habitat and appeared in remote organs, often associated with diseased conditions. This was also, at first, considered as of but trifling moment, and its presence in these localities was usually explained as accidental. Its repeated appearance, however, in different parts of the body outside of the intestines, and the frequeney of its association with pathological conditions, ultimately attracted attention to it, and in consequence during the past few 
years a great deal has been written eoncerning the possible pathogenic nature of this organism.

The fact that it is always with us in most intimate association with certain of our life-processes, together with the fact that it is known to appear in organs other than that in which it is normally located, and that its occurrence in diseased conditions is not rare, justifies the opinion that it is one of the most important of the micro-organisms with which we have to deal.

While not generally considered to be a pathogenic organism, there is, nevertheless, sufficient evidence to warrant the statement that, under favorable conditions, with which we are not entirely familiar, this organism may assume pathogenic properties and that its presence in diseased conditions is not always to be considered as accidental, though this is frequently the case.

The morphological and cultural peculiarities of the bacterium coli commune are as follows:

Morphology. In shape it is a rod with rounded ends, sometimes so short as to appear almost spherical, while again it is seen as very much longer threads. Often both forms will be associated in the same culture. It may oceur as single cells or as pairs, joined end-to-end.

There is nothing to be said of its morphology that can aid in its identification, for in this respect it simulates many other organisms. It is usually said to be motile, and undoubtedly is motile in the majority of cases, but its movements are so sluggish that a positive opinion is often difficult.

By Lofller's method of staining, flagella ean be demonstrated, though not in such numbers as are scen to occur on the typhoid fever baeillus.

It does not form spores. 
It grows both with and without oxygen.

On gelatin. When on the surface its colonies appear small, dry, irregular, flat, blue-white points that are commonly somewhat denuded at the margin. They are a trifle denser at the centre than at the periphery, and are often marked at or near the middle by an oval or round nucleus-like mass - the original colony from which the layer on the surface developed. When located in the depths of the gelatin, and examined with a low-power lens, they are at first seen to be finely granular and of a very pale greenish-yellow color; later they become denser, darker, and much more markedly granular. In shape they are round, oval, and lozengelike. When the surface colonies are viewer under a low power of the microscope they present essentially the same appearance as that given for the bacillus of typhoid fever, viz., they resemble flattened pellicles of glass-wall, or patches of finely ground colorless glass. Colonies of this organism on gelatin are frequently enconntered that cannot be distinguished from those resulting from the growth of the bacillus of typhoid fever, though, as a rule, their growth is a little more luxuriant.

In stab- and smear-cultures on gelatin the surfacegrowth is flat, dry, and blue-white or pearl color. Limited growth occurs along the track of the needle in the depths of the gelatin. As the culture becomes older, the gelatin round about the surface-growth may gradually lose its transparency and become cloudy, often quite opaque. In still older cultures small root- or branch-like projections from the surface-growth into the gelatin are sometimes seen to occur.

It does not cause liquefaction of gelatin. 
Its growth on nutrient agar-agar and on blood-serum is luxuriant but not characteristic.

In bouillon it causes diffuse clouding with sedimentation. In some bouillon eultures an attempt at pellicle formation on the surface may be seen, but this is not always the case. In old bouillon cultures the reaction is seen to have become alkaline, and a decided fecal odor may be detected.

It produces indol in bouillon and in peptone solution.

Its growth on potato is rapid and voluminous, appearing after twenty-four to thirty-six hours in the incubator as a more or less lobulated layer of a drab, dark-cream, or brownish-yellow color.

In neutral milk containing a little litmus tincture the blue color is changed to red after from eighteen to twenty-four hours in the incubator, and, in addition, the majority of cultures cause a firm coagulation of the casein in about thirty-six hours, though frequently this takes longer. Very rarely the litmus may indicate the production of acid and no coagulation occur.

In media containing glucose it grows rapidly and causes active fermentation with liberation of earbonic acid and hydrogen. If cultivated in solid media to which glucose ( 2 per cent.) has been added, the gasformation is recognized by the appearance of numerous bubbles along and about the points of growth. If cultivated in fluid media, also eontaining glucose, in the fermentation-tube, evidence of fermentation is given by the colleetion of gas in the closed arm of the tube.

On lactose-litmus-agar-agar its colonies are pink and the color of the surrounding medium is changed from blue to red.

In Dunham's peptone solntion it produces indol in 
from forty-eight to seventy-two hours. It stains with the ordinary aniline dyes. It is decolorized when treated by the method of Gram.

$\mathrm{By}$ comparing what has been said of the bacillus typhi abdominalis and of the bacterium coli commune it will be seen that while they simulate each other in certain respects they still possess individual characteristics by which they may readily be differentiated. The most important of the differential points are:

1. Motility of the bacillus typhi abdominalis is much more conspicuous, as a rule, than is that of the bacterium coli commune.

2. On gelatin the colonies of the typhoid bacillus develop more slowly than do those of the colon bacillus.

3. On potato the growth of the typhoid bacilius is usually invisible (though not always), while that of the colon bacillus is rapid, luxuriant, and always visible.

4. The typhoid bacillus does not cause coagulation of milk with acid reaction. The colon bacillus does this in from thirty-six to forty-eight hours in the incubator.

5. The typhoid bacillus never causes fermentation, with liberation of gas, in media containing glucose, lactose, or saccharose. The colon bacillus is conspicuous for its power of causing fermentation in such solutions.

6. In nutrieut agar-agar or gelatin containing lactose aud litmus tincture, and of a slightly alkaline reaction, the color of the colonies of typhoid bacillus is pale blue, and there is no reddening of the surrounding medium, while the colonies of the colon bacillus are pink and the medium round about them becomes red.

7. The typhoid bacillus does not, as a rule, possess the property of producing indol in solutions of peptone; the growth of the colon bacillus in these solutions is accom- 
panied by the production of indol in from forty-eight to seventy-two hours at $37^{\circ}$ to $38^{\circ} \mathrm{C}$.

8. When twenty-four hours old bouillon cultures of both organisms are brought in contact with the bloodserum from a case of gennine typhoid fever (after the fifth day of the disease), the characteristic agglutination (clumping) of the bacilli occurs in the typhoid culture and not in that of the colon bacillus (Widal's reaction).

Animal inoculations. As with the bacillus of typhoid fever, the results of inoculation of animals with cultures of this organism cannot be safely predicted. According to the observations of Escherich, Emmerich, Weisser, and others, the results that do appear are in most instances to be attributed to the toxic rather than to the infective properties of the culture used.

When introduced into the subcutaneous tissues of mice it has no effect, while similar inoculations of guineapigs are sometimes (not always) followed by abscessformation at the point of injury, or by alterations very similar to those produced by intravasenlar inoculation, viz., death in less than twenty-four hours, accompanied by redness of the peritoneum and marked hyperæmia and ecchymoses of the small intestine; together with swelling of Peyer's patehes. The creum and colon may remain unchanged or present enlarged follicles. There may or may not be an accumulation of fluid in the abdominal eavity, but peritonitis is rarely present. The small intestine may contain bloody muens.

Intravenous inoculation of rabbits may be followed by similar ehanges, with often the occurrence of diarrhoea before death, which may, in the acute cases, result in from three to forty hours. In another group of cases acute fatal intoxication does not result, and the 
animal lives for weeks or months, dying ultimately of what appears to be the effects of a slow or chronic form of infection For a few hours after inoculation these animals present no marked symptoms; exceptionally somnolence and diarrhoa have been observed at this period, indicating acute intoxication from which the animal has recovered. The affection is unattended by fever. The most marked symptom is loss of weight. This is usually progressive from the first or second day after inoculation, with slight fluctuations until death.

At autopsy the animal is found to be emaciated. The subcutaneous tissues and the muscles appear pale and dry. The serous cavities, particularly the pericardial, may contain some excess of serum. The viscera are anæmic. The spleen is small, thin, and pale. Exceptionally ulcers and ecchymoses are observed in the cæecum, but generally there are no lesions of the intestinal tract.

The most striking and constant lesions, those most characteristic of the affection, are in the bile and in the liver; the quantity of bile may not exceed the normal, but in other cases the gall-bladder may be abnormally distended with bile. The bile is nearly colorless or has a pale yellowish or brownish tint, with little or none of a greenish color. Its consistence is much less viscid than normal, being often thin and watery. It usually contains small, opaque, yellowish particles or clumps which can be seen floating in it, even through the walls of the gall-bladder. These clumps consist microscopically of bile-stained, apparently necrotic, epithelial cells; leucocytes in small numbers; amorphous masses of bile pigment, and bacteria often in zoögloa-like clumps. Similar material is found in the larger bile-ducts. 
The liver frequently contains opaque, whitish or yellowish-white spots and streaks of irregular size and shape, which give a peculiar mottling to the organ when present in large number. These areas may be numerous, or only one or two may be found. In size they range from minute points to areas of from 2 to $3 \mathrm{~cm}$. in extent.

By microscopic examination they are found to represent places where the liver cells have undergone necrosis accompanied by emigration of leucocytes, and the cells about them are in a condition of fatty degeneration.

In sections of the liver masses of the bacilli may be discovered in and about the necrotic foci just described.

At these autopsies the colon bacillus is not found generally distributed through the body, but is only to be detected in the bile, liver, and occasionally in the spleen. ${ }^{1}$

1 Consult paper by Blachstein on thls subject. Johns Hopkins Hospital Bulletin, July, 1891. 


\section{CHAPTER XXII.}

The spirillum (comma bacillus) of Asiatic cholera-Its morphological and cultural peculiarities-Pathogenic properties-The bacteriological diagnosis of Asiatic cholera.

At the conference held in Berlin in 1884 for the purpose of discussing the cholera question, it was announced by Koch ${ }^{1}$ that he had discovered in the intestinal evacuations of individuals suffering from Asiatic cholera a micro-organism that he believed to be the cause of the malady. The importance of this statement necessarily attracted widespread attention to the subject, and as one of the results there existed, for a short time following, some skepticism as to the accuracy of Koch's claim. These doubts arose as a result of a series of contributions from other observers who endeavored to prove that the organism found by Koch in cholera evacuations was one that is common to other localities, and not a specific accompaniment of this disease. It was not very long, however, before it was evident that the objections raised by the opponents of Koch were based upon untrustworthy observations, and that by reliable methods of investigation the organism to which he had called attention could be easily differentiated from either and all of those with which it was claimed to be identical.

This organism, known as the spirillum of Asiatic

1 Verhandlungen der Conferenz zur Erürterung der Cholerafrage, 1SS, Berlin. 
cholera, and as Koch's " comma bacillus," beeause of its morphology, is identified by the following peculiarities:

THE MORPHOLOGICAI, AND BIOLOGICAL PECULIARITIES OF THE SPIRILIUM OF ASIATIC CHOI,ERA.

Morphology. It is a slightly curved rod varying from about 0.8 to $2.0 \mu$ in length and from 0.3 to $0.4 \mu$ in thickness-that is to say, it is usually from about onehalf to two-thirds the length of the tubercle bacillus, but is thicker and plumper. Its curve is frequently not more marked than that of a comma, and, indeed, it is often almost straight; at times, though, the curve is much more pronounced, and may even describe a semicircle. Occasionally the curve may be double, one comma joining another, with their convexities pointing in opposite directions, so that a figure similar to the letter $\mathrm{S}$ is produced. In eultures long spiral or undulating threads may often be seen. From these appearances this organism cannot be considered as a bacillus, but rather as an intermediate type between the bacilli and the spirilla. Koch thinks it not improbable that the short eomma forms represent segments of a true spirillum, the normal form of the organism. (Fig. 71.)

It does not form spores, and we have no reliable evidence that it possesses the property of entering, at any time, a stage when its powers of resistance to detrimental agencies are increased.

It is a flagellated organism, but has only a single flagellum attached to one of its ends.

It is actively motile, especially in the comma stage, though the long spiral forms also possess this property. 
Grouping. As found in the slimy flakes in the intestinal discharges from cholera patients, Koch likens its mode of grouping to that seen in a school of small fish when swimming up strean-i.e., they all point in nearly

Fig. 71.

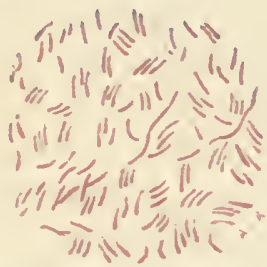

Spirillum of Asiatic cholera. Impression cover-slip from a colony thirty-four hours old.

the same direction and lie in irregularly parallel, linear groups that are formed by one comma being located behind the other without being attached to it.

FIG. 72.
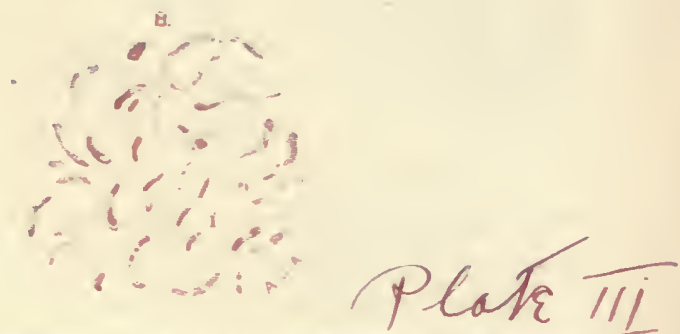

Involution-forms of the spirillnm of Aslatic cholers, as seen in old cultures.

On cover-slip preparations made from cultures in the ordinary way there is nothing characteristic about the grouping, but in impression cover-slips made from young cultures the short commas will nearly always be 
seen in small groups of three or four, lying together in such a way as to have their long axes nearly parallel to one another. (See Fig. 71.)

In old cultures in which development has ceased it undergoes degenerative changes, and the characteristic comma and spiral shapes may entirely disappear, their place being taken by irregular involution-forms that present every variety of outline. (See Fig. 72.) In this stage they take on the stain very feebly, and often not at all.

Cultural peculiarities. On plates of nutrient gelatin that have been prepared from a pure culture of this organism and kept at a temperature of from $20^{\circ}$ to $22^{\circ}$ C., development can often be observed after as short a period as twelve hours, but frequently not beforc sixteen to eighteen hours. This is especially true of the first or "original" plate, containing the largest number of colonies. At this time the plate will present to the naked cye an appearance that has been likened to a ground-glass surface, or to a surface that has been stippled with a very finely pointed ncedle, or one upon which very fine dust has been sprinkled. This appearance is due to the presence of minute eolonies closely packed together upon the surface of the gelatin. In the depth of the gelatin ean also be seen, closely packed, small points, likewise representing growing colonies. As growth progresses liquefaction occurs around the superficial colonies, and in consequence this plate is usually entirely liquid after from twenty-four to thirty hours; the developmental phases through which the colonies páss eannot, therefore, be studied upon it.

On plates 2 and 3 , where the colonies are more widely separated, they can be seen after twenty-four to thirty 
hours as small, round, or oval, white or cream-white points, and when located superficially there can be detected around them a narrow transparent zone of liquefaction. As growth continues this liquefaction extends downward rather than laterally, and the colony ultimately assumes the appearance of a dense, white mass lying at the bottom of a sharply cut pit or funuel containing transparent fluid. This liquefaction is never very widespread nor rapid, and rarely extends for more than one millimetre beyond the colony proper. On plates containing few colonies there is but little or no tendency for them to become confluent, and, as a rule, they do not exceed 2 to $3 \mathrm{~mm}$. as an average diameter.

FIG. 73.
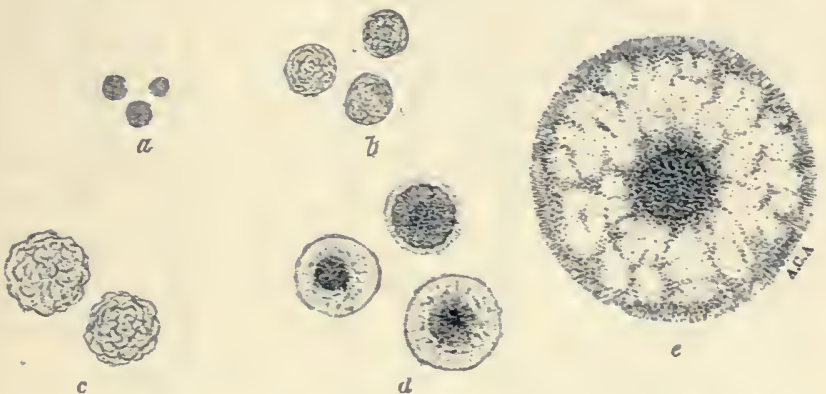

Developmental stages of colonies of the spirillum of Asiatic cholera at $20^{\circ}$ to $22^{\circ} \mathrm{C}$. on gelatin. $X$ about 75 dirmeters.

a. After sixteen to eighteen hours. b. After twenty-fonr to twenty-six hours. c. After thirty-eight to forty hours. d. After forty-eight to finy hours. $e$. After sixty-four to serenty hours.

When examined under a low magnifying lens the very young colonies (sixteen to eighteen hours) appear as pale, translucent, granular globules of a very delicate greenish or yellowish-green color, sharply outlined and not perfectly round. (See $a$, Fig. 73.) As growth pro- 
gresses this homogeneous granular appearance is replaced by an irregular lobulation, and ultimately the sharply cut margin of the eolony becomes dentated or scalloped. (See $b$ and $c$, Fig. 73.) After forty-eight hours (and frequently sooner) liquefaction of the gelatin has taken place to such an extent that the appearance of the colony is entirely altered. Under the magnifying glass the colony proper is now seen to be torn and ragged about its edges, while here and there shreds of the colony can be detected scattered through the liquid into which it is sinking. These shreds evidently represent portions of the colony that became detaehed from its margin as it gradually sank into the liquefied area.

At $d$, in Fig. 73, will be seen a representation of the several appearances afforded by the colonies at this stage. At the end of the second, or during the early part of the third day, the sinking of the colonies into the liquefied pits resulting from their growth is about complete, and under a low lens they now appear as dense, granular masses, surrounded by an area of liquefaction through which can be seen granular prolongations of the colony, usually extending irregularly between the periphery and the central mass. (See $e$, Fig. 73.) If the periphery be examined, it will be seen to be fringed with delicate, cilia-like lines. that radiate from it in much the same way that cilia radiate from the ends of certain columnar epithelial cells.

These are the more marked phases through which the colonies of this organism pass in their development on gelatin plates. With some cultures the various appearances here given appear more quickly, while in eultures from other sources they may be somewhat retarded. 
On plates of nutrient agar-agar the appearance of the colonies is not characteristic. They appear as round or oval patches of growth that are moist and tolerably transparent. The colonies on this medium at $37^{\circ} \mathrm{C}$. naturally grow to a larger size than do those upon gelatin at $22^{\circ} \mathrm{C}$.

FIG. 74.

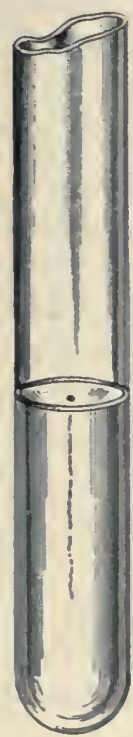

a

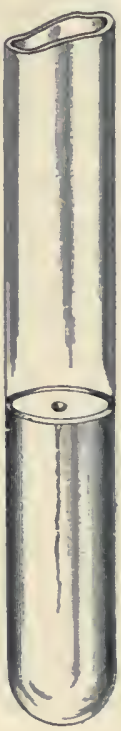

b

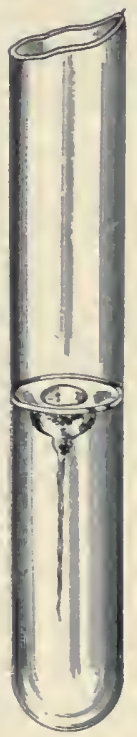

$c$

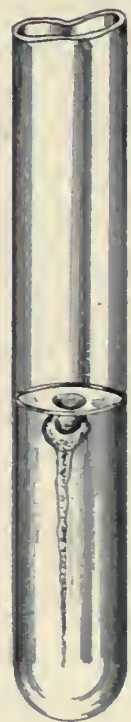

d

Stab-cultures of the spirillum of Aslatic cholera in gelatin, at $18^{\circ}$ to $20^{\circ} \mathrm{C}$.

a. After twenty-four bours. b. After forty-eight hours. c. After serentytwo bours. $d$. After ninety-six hours.

In stab-cultures in gelatin there appears at the top of the needle track after thirty-six to forty-eight hours at $22^{\circ} \mathrm{C}$. a small, funnel-shaped depression. As the growth progresses liquefaction will be seen to occur 
about this point. In the centre of the depression can be distinguished a small, dense, whitish clump, the colony itself. As growth continues the depression increases in extent and ultimately assumes an appearance that consists in the apparent sinking of the liquefied portion in such a way as to leave a perceptible air-space between the top of the liquid and the surface of the solid gelatin. The growth now appears to be capped by a small airbubble. The impression given by it at this stage is not only that there has been a liquefaction, but also a coincident evaporation of the fluid from the liquefied area and a constriction of the superficial opening of the funnel. (See $a, b, c$, and $d$, Fig. 74.) Liquefaction is not especially active along the deeper portions of the track made by the needle, though in stab-cultures in gelatin the liquefaction is much more extensive than that usually seen around colonies on plates. It spreads laterally at the upper portion, and after about a week a large part of the gelatin in the tube may have become fluid, and the growth will have lost its characteristic appearance.

Stab- and smear-eultures on agar-agar present nothing characteristic. They are usually only an exaggeration of the appearance afforded by the single colonies on this medium.

Its growth in bouillon is luxuriant, causing a diffuse clouding and the ultimate produetion of a delicate film upon the surface.

In sterilized milk of a neutral or amphoteric reaction at a temperature of $36^{\circ}-38^{\circ} \mathrm{C}$. it develops actively, and gradually produces an acid reaction with coagulation of the casein. It retains its vitality under these conditions for about three weeks or more. The blue 
color of milk to which neutral litmus tincture has been added is changed to pink after thirty-six or forty-eight hours at body temperature.

Its growth in peptone solution, either that of Dunham (see Special Media) or the one preferred by Koch, viz., 2 parts Witte's peptone, 1 part sodium chloride, and 100 parts distilled water, is accompanied by the production of both indol and nitrites, so that after eight to twelve hours in the incubator at $37^{\circ} \mathrm{C}$. the rose color characteristic of indol appears upon the addition of sulphuric acid alone. (See Indol Reaction.)

(What does the presence of nitrites in these cultures signify ?)

In peptone solution to which rosolic acid has been added the red color is very much intensified after four or five days at $37^{\circ} \mathrm{C}$.

Its growth on potato of a slightly acid reaction is seen after three or four days at $37^{\circ} \mathrm{C}$. as a dull, whitish, non-glistening patch at and about the site of inoculation. It is not elevated above the surface of the potato, and can only be distinctly seen when held to the light in a particular position. Growth on acid potato oceurs, however, only at or near the body temperature, owing probably to the acid reaction, which is sufficient to prevent development at a lower temperature, but does not have this effect when the temperature is more favorable. On solidified blood-serum the growth is usually said to be accompanied by slow liquefaction. I have not succeeded in obtaining this result on Loffler's serum, nor have I detected anything characteristic about its gromth on this medium.

The temperature most favorable for its growth is 
between $35^{\circ}$ and $38^{\circ} \mathrm{C}$. It grows, but more slowly, at $17^{\circ} \mathrm{C}$. Under $16^{\circ} \mathrm{C}$. no growth is visible.

It is not destroyed by freezing. When exposed to $65^{\circ} \mathrm{C}$. its vitality is destroyed in five minutes.

It is strictly aërobic, its development ceasing if the supply of oxygen be cut off.

It does not grow in an atmosphere of carbonic acid, but is not killed by a temporary exposure to this gas. It does not grow in acid media, but flourishes best in media of nentral or slightly alkaline reaction. It is so sensitive to the action of acids that at $22^{\circ} \mathrm{C}$. its development is arrested when an acid reaction equivalent to 0.066 to 0.08 per cent. hydrochloric or nitric acid is present (Kitasato).

In cultures the development of this organism reaches its maximum relatively quickly, then remains stationary for a short period, after which degeneration begins. The dying comma bacilli become altered in appearance and assume the condition known as "involution-forms." (See Fig. 72.) When in this state they take up coloring-reagents very faintly or not at all, and may lose entirely their characteristic shape.

When present with other bacteria, under conditions favorable to growth, the comma bacillus at first grows much more rapidly than do the others; in twenty-four hours it will often so outnumber the other organisms present that microscopic examination would lead one to take the material under consideration to be a pure culture of this organism. This, however, does not last longer than two or three days; they then begin to die, and the other organisms gain the ascendency. This fact has been taken advantage of by Schottelius ${ }^{1}$ in the

1 Deutschę med, Wochensschrift, 1885, No. 14. 
following method devised by him for the bacteriological examination of dejections from cholera patients:

In dejections that are not examined immediately after being passed it is often difficult, because of the large number of other bacteria that may be present, to detect with certainty the cholera organism by microscopic examination. It is advantageous in these cases to mix the dejections with about double their volume of slightly alkaline beef-tea, and allow them to stand for about twelve hours at a temperature of between $30^{\circ}$ and $40^{\circ}$ C. There appears at the end of this time, especially upon the surface of the fluid, a conspicuous increase in the number of comma bacilli, and cover-slip preparations made from the upper layers of the fluid will reveal an almost pure culture of this organism.

It is not improbable that a similar process occurs in the intestines of those suffering from Asiatic cholera, viz., a rapid multiplication of the comma bacilli that have gained access to the intestines takes place, but lasts for only a short time, when the comma bacilli begin to disappear, and after a few days their place is taken by other organisms.

In connection with his experiments upon the poison produced by the cholera organism Pfeiffer $^{1}$ states that in very young cultures, grown under the access of oxygen, there is present a poisonous body that possesses intense toxic properties. This primary cholera-poison stands in very close relation to the material composing the bodies of the bacteria themselves, and is probably an integral constituent of them, for the vitality of the cholera spirilla can be destroyed by means of chloro-

1 Zeitschrift f. Hygiene u. Infectionskrankheiten, Bd. xi. p. 393. 
form and thymol, and by drying, without apparently any alteration of this poisonous body. Absolute alcohol, concentrated solutions of neutral salts, and a temperature of $100^{\circ} \mathrm{C}$., decompose this substance, leaving behind secondary poisons which possess a similar physiological activity, but only when given in from ten to twenty times the dose necessary to produce the same effects with the primary poison.

Other members of the vibrio family also, namely, vibrio Metchnikovi and that of Finkler and Prior (see description of these species), contain, according to Pfeiffer, closely related poisons.

Experiments upon animals. As a result of experiments for the purpose of determining if the disease can be produced in any of the lower animals it is found that white mice, monkeys, cats, dogs, poultry, and many other animals are not susceptible to infection by the methods usually employed in inoculation experiments. When animals are fed on pure cultures of the comma bacillus no effect is produced, and the organisms cannot be obtained from the stomach or intestines; they are destroyed in the stomach and do not reach the intestines; they are not demonstrable in the feces of these animals. Intravascular injections of pure culture into rabbits are followed by a temporary illness, from which the animals usually recover in from two to three days; intraperitoneal injections into white mice are, as a rule, followed by death in from twenty-four to forty-eight hours; the conditions in both instances most probably resulting from the toxic activities of the poisonous products of growth of the organisms that are present in the culture employed. None of the lower animals have ever been known to suffer from $A$ siatic cholera spontaneously. 
The failure to induce cholera in animals by feeding, or by injection of cultures into the stomach, was shown by Nicati and Rietsch ${ }^{1}$ to be due to the destructive action of the acid gastric juice on the bacilli. They showed that if cultures of this organism were introduced into the alimentary tract of certain animals in such a manner that they would not be subjected to the influence of the gastric juice, a condition pathologically closely simulating cholera as it occurs in man could be produced. For this purpose the common bile duct was ligated, after which the cultures were injected directly into the duodenum. Such interference with the flow of bile lessens intestinal peristalsis, and thus pernits the development of the bacilli at the point at which they are deposited - that is, the portion of the intestine having an alkaline reaction and beyond the influence of the acid stomach-juice.

By this method Nicati and Rietsch, Van Ermengem, 2 Koch, ${ }^{3}$ and others were enabled to produce in the animals upon which they operated a condition that was, if not identical, at all events very similar pathologically to that seen in the intestines of subjects dead of the disease.

At a subsequent conference held in Berlin in 1885 Koch ${ }^{4}$ described the following method by means of which he had been able to obtain a relatively high degree of constancy in all his efforts to produce cholera in lower animals: bearing in mind the point made by Nicati and Rietsch as to the effect produced by the acid reaction of the gastric juice, this reaction was first to be

1 Archiv. de Phys. norm. et patb., 1885, xvii., 3e sér., t. vi. Compt-rend., xclx. p. 925. Rev. de Hygiéne, 1835. Rev. de Méd, 1955, v.

2 "Recherches sur le Microbe du Choléra Asiatique," Paris-Bruxelles, 1555. Bull. de l'Acad. roy. de Méd. de Belgique, 3e sér., xviii.

Loc. cit.

4 Loc. cit., 1885. 
neutralized by injecting through a soft catheter passed down the cesophagus into the stomach 5 c.c. of a 5 per cent. solution of sodium carbonate. Ten or fifteen minutes later this was to be followed by the injection into the stomach (also through a soft catheter) of 10 c.c. of a bouillon culture of the cholera spirillum. For the purpose of arresting peristalsis and permitting the bacteria to remain in the stomach and upper part of the duodenum for as long a time as possible, the animal was to receive, immediately following the injection of the culture, an intraperitoneal injection, by means of a hypodermic syringe, of 1 c.c. of tincture of opium for each 200 grammes of its body weight. Shortly after this last injection a deep narcosis sets in and lasts from a half to one hour, after which the animal is again as lively as ever. Of 35 guinea-pigs inoculated in this way by Koch, 30 died of a condition that was, in general, very similar to that seen in Asiatic cholera.

The condition of these animals before death is described as follows: twenty-four hours after the operation the animal appears sick; there is a loss of appetite, and the animal remains quiet in its cage. On the following day a paralytic condition of the hind extremities appears, which, as the day goes on, becomes more pronounced; the animal lies quite flat upon its abdomen or on its side, with its legs extended; respiration is weak and prolonged, and the pulsations of the heart are hardly perceptible; the head and extremities are cold, and the body temperature is frequently subnormal.

The animal usually dies after remaining in this condition for a few hours.

At antopsy the small intestine is found to be deeply injected and filled with a floceulent, colorless fluid. The 
stomach and intestines do not contain solid masses, but fluid; when diarrhoea does not occur, firm scybala may be expected in the rectum. Both by microscopic examination and by cuiture methods comma bacilli are found to be present in the small intestine in practically pure culture.

More recently Pfeiffer $^{1}$ has determined that esseutially similar constitutional effects may be produced in guinea-pigs by the intraperitoneal injection of relatively large numbers of this organism. His plan is to scrape from the surface of a fresh culture on agar-agar as much of the growth as can be held upon a moderatesized wire loop. This is then finely divided in 1 c.c. of bouillon and, by means of a hypodermic syringe, is injected directly into the peritoneal cavity. When virulent cultures have been used this is quickly followed by a fall in the temperature of the animal; this is gradual and continuous until death ensues, which is usually in from eighteen to twenty-four hours after the operation, though exceptionally cases do occur in which the animal recovers, even after having exhibited marked symptoms of most profound toxæmia.

In pursuance of his studies upon this disease Pfeiffer ${ }^{2}$ has demonstrated that it is possible to render an animal tolerant or immune to the poisonous properties of this organism by repeated injections of non-fatal doses of dead cultures (eultures that have been killed by the vapor of chloroform or by heat). He also demonstrated that animals so immnned possess a specific gernicidal action toward the cholera spirillum-i.e., if into the peritoneal cavity of an animal immunized from

1 Zeitschrift fur Hygiene, Bd. xi. and xiv.

2 Zeit. für Hyg. u. Infectionskrankheiten, Bd. ix. Heft. 1. 
Asiatic cholera living cholera spirilla be introduced, they will all be destroyed (disintegrated) within a relatively short time. Furthermore, if the serum of an animal immunized from cholera be injected into the peritoneal cavity of a similar animal not so protected, and immediately afterward living cholera spirilla be introduced, a similar disintegration and destruction of the bacteria will also result. He shows that a more or less definite relation exists between the amount of sernm and the number of organisms introduced. Such a destruction of the comma bacillus by the serum of an immunized animal does not occur outside the animal body - that is, cannot be demonstrated in a test-tube. The specificity of this reaction is suggested by Pfeiffer as a means of differentiating the cholera spirillum from other suspicious species, for no such disintegration of bacterial cells is observed if species other than the cholera spirillum be introduced into the peritoneal cavity of animals immunized from Asiatic cholera.

Pfeiffer has demonstrated that the serum of animals artificially immmized from Asiatic cholera has an agglutinating effect upon fluid cultures of the cholera spirillum similar to that seen when typhoid bacilli are mixed with the serum from typhoid eases, or from animals artificially immunized from typhoid infection or intoxication. (See Agglutinin.)

General considerations. In all cases of Asiatic cholera, and only in this disease, the organism just described can be detected in the intestinal evacuations. The more acute the case and the more promptly the examination is made after the evacnations have been passed from the patient, the less will be the difficulty experienced in detecting the organism. 
In some cases it can be detected in the vomited matters, though by no means so constantly as in the intestinal contents.

As a rule, bacteriological examination fails to reveal the presence of the organisms in the blood and internal organs in this disease, though Nicati and Rietsch claim to have obtained them from the common bile-duct in rapidly fatal cases, and in two out of five cases they were present in the gall-bladder. Doyen and Rasstschewsky ${ }^{1}$ found them in the liver in pure culture, and Tizzoni and Cattani ${ }^{2}$ in both the blood and the gallbladder.

The cholera spirillum is a facultative parasite; that is to say, it apparently finds in certain portions of the world, particularly in those countries in which Asiatic cholera is endemic, conditions that are not entirely unfavorable to its development outside of the body. This has been found to be the fact not only by Koch, who detected the presence of the organism in water-tanks in India, but by many other observers who have succeeded in demonstrating its growth under conditions not embraced in the ordinary methods that are employed for the cultivation of bacteria.

The results of experiments having for their object the determination of the length of time during which this organism may retain its vitality in water are conspicuous for their irregularity. In the transactions of the congress in Berlin, for the discussion of the cholera question, it is stated, in connection with this point, that the experiments made with tank-water in India sometimes resulted in demonstrating the multiplication of

1 Reference to Vratch, 1885, in Allg. Med. Central Zeitung, Berlin.

2 Centralblatt f. die med. Wissenschaften, 1886, No. 43. 
the organisms introdueed into it, while in other cases they died very quiekly.

On February 8, 1884, eomma baeilli were found in the tank at Saheb-Began, in Calcutta, and it was possible to demonstrate them in a living condition up to February 23d.

Koeh states that in ordinary spring-water or wellwater the organisms retained their vitality for thirty days, whereas in the eanal-water (sewage) of Berlin they died after six or seven days; but if this latter were mixed with fecal matters, the organisms retained their vitality for but twenty-seven hours; and in the undiluted contents of cesspools it is impossible to demonstrate them after twelve hours. In the experiments of Nicati and Rietsch they retained their vitality in sterilized distilled water for twenty days; in Marseilles canalwater (sewage), for thirty-eight days; in sea-water, sixty-four days; in harbor-water, eighty-one days; and in bilge-water, thirty-two days.

In the experiments of Hochstetter, on the other hand, they died in distilled water in less than twenty-four hours in five of seven experiments; in one of the two remaining experiments they were alive after a day, and in the other after seven days.

In one experiment with the domestie water-supply of Berlin the organism retained its vitality for 267 days; in another for 382 days, notwithstanding the fact that many other organisms were present at the same time. There is no single ground upon which these variations can be explained, for they depend apparently upon a number of factors which may act singly or together. For example, in general it may be said that the higher the temperature of the water in which these organisms 
are present, up to $20^{\circ} \mathrm{C}$., the longer do they retain their vitality; the purer the water-that is, the poorer in organic matters-the more quickly do the organisms die, whereas the richer it is in organic matter the longer do they retain their vitality.

Still another point that must be considered in this connection is the antagonistic influences under which they find themselves when placed in water containing large numbers of organisms that are, so to speak, at home in water-the so-called normal water-bacteria.

The effect of light upon growing bacteria must not be lost sight of, for it has been shown that a surprisingly large number of these organisms are robbed of their vitality by a relatively short exposure to the rays of the sun, and it is therefore not unlikely that the nonobservance of this fact may be, in part at least, accountable for some of the discrepancies that appear in the results of these experiments.

In his studies upon the behavior of pathogenic and other micro-organisms in the soil Carl Fränkel ${ }^{1}$ found that the cholera spirillum was not markedly susceptible to those deleterious influences that cause the death of a number of other pathogenic organisms. During the months of August, September, and October cultures of the comma bacillus that had been buried in the ground at a depth of three metres retained their vitality; on the other hand, in other months, particularly from April to July, they lost their vitality when buried to the depth of only two metres. At a depth of one and a half metres vitality was not destroyed, and there was a regular development in cultures so placed. 
As a result of experiments performed in the Imperial Health Bureau, at Berlin, it was found that the bodies of guinea-pigs that had died of eholera indueed by Koch's method of inoculation eontained no living eholera spirilla when exhumed after having been buried for nineteen days in wooden boxes, or for twelve days in ziuc boxes. In a few that had been buried in moist earth, without having been encased in boxes, when exhumed after two or three months, the results of examinations for cholera spirilla were likewise negative.

Kitasato, ${ }^{1}$ in his experiments with the eholera organism, found that when mixed with the intestinal evacuations of human beings under ordinary conditions they lost their vitality in from a day and a half to three days. If the evacuations were sterilized before the cultures were mixed with them, the organisms retained their vitality up to from twenty to twenty-five days. $\mathrm{He}$ was unable to eome to any definite conelusion as to the cause of these phenomena.

It was demonstrated by $\mathrm{Hesse}^{2}$ and by Celli $\mathrm{i}^{3}$ that many substances commonly employed as food-stuffs serve as favorable materials for the development of the eholera organism. In his experiments upon its behavior in milk Kitasato ${ }^{4}$ found that at a temperature of $36^{\circ} \mathrm{C}$. the eholera spirillum developed very rapidly during the first three or four hours, and outnumbered the other organisms commonly found in milk. They then diminished in number from hour to lour as the acidity of the milk inereased, until finally their vitality was lost; at the same time the common saprophytic bacteria in-

1 Zeitschrift fur Hygiene, Bd, v. p. 487.

2 Ibid, Bd, v. p. 527.

8 Bolletino della R. Acad. Med. di. Roma, 1888.

4 Zeitschrift fur Hygiene, Bd. v. p. 491. 
creased in number. Relatively the same process occurs at a lower temperature, from $22^{\circ}$ to $25^{\circ} \mathrm{C}$, , but the process is slower, the maximum development of the cholera organisms being reached at about the fifteenth hour, after which time they were overgrown by the ordinary saprophytes present.

From this it would seem that the vitality of the cholera spirillum in milk depends largely upon the reaction: the more quickly the milk becomes sour the more quickly does the organism become inert, while the longer the milk retains its neutral, or only very slightly acid reaction, the longer do the cholera organisms that may be present in it retain their power of multiplication.

According to Laser, ${ }^{1}$ the cholera organism retains its vitality in butter for about seven days; it is therefore possible for the disease to be contracted by the use of butter that has in any way been in contact with cholera material.

In regard to the antagonism between the cholera spirillum and other organisms with which it may come in contact, the experiments of Kitasato ${ }^{2}$ led him to conclude that no organism has been found which, when growing in the same culture medium with it, possessed the power of depriving it of its vitality within a short time. On the other hand, the experiments showed that there were quite a number of other organisms the development of which was checked, and in some cases their vitality was completely destroyed, when growing in the same medium with the cholera spirillum.

From this it would appear that the disappearance of

1 Zeitschrift fur Hygiene, Bd. x. p. 513.

I Ibid., Bd. vi. p. 1. 
the cholera spirillum from mixed cultures and from the evacuations in so short a time as has been mentioned, is due more to unfavorable nutritive conditions than to the direct action of the other organisms present.

When eompletely dried, according to Koch's experiments, the cholera spirillum does not retain its vitality for longer than twenty-four hours, but by others its vitality is said to be destroyed by an absolute drying of three hours. In the moist conditions, as in artificial cultures, vitality may be retained for many months, though repeated observations lead us to believe that, under these circumstances, the virulence is diminished. According to Kitasato, ${ }^{1}$ they retain their vitality when smeared upon thin glass cover-slips and kept in the moist chamber for from 85 to 100 days, and for as long as 200 days when deposited upon bits of silk thread.

In the course of his studies upon the destiny of pathogenic micro-organisms in the dead body von Esmarch ${ }^{2}$ found that, when the cadaver of a guinea-pig dead from the introduction of cholera organisms into the stomach was immersed in water and decomposition allowed to set in, after eleven days, when decomposition was far advanced, it was impossible to find any living cholera spirilla by the ordinary plate methods.

A similar experiment resulted in their disappearance after five days. In another experiment, in which decomposition was allowed to go on without the animal being immersed in water, none could be detected after the fifth day.

Carl Fränkel ${ }^{3}$ has shown that an atmosphere of earbonic acid is directly inhibitory to the development of

1 Zeltschrift für Hyglene, Bd, v. p. 184.

2 Ibid., Bd. vil. p. 1.

8 Ibid., Bd. v. p. 832. 
the cholera spirillum, and Percy Frankland ${ }^{1}$ states that in an atmosphere of this gas it dies in about eight days. In an atmosphere of earbon monoxide its vitality is lost in nine days, and in general the same may be said for it when under the influence of an atmosphere of nitrous oxide gas.

From what has been said we see that the spirillum of Asiatic cholera, while possessing the power of producing in human beings one of the most rapidly fatal forms of the disease with which we are acquainted, is still one of the least resistant of the pathogenic organisms knowu to us. Under conditions most favorable to its growth its development is self-limited; it is conspicuously susceptible to acids, alkalies, other chemical disinfectants, and heat; but when partly dried upon clothing, food, or other objects, it may retain its vitality for a relatively long period of time, and it is more than probable that it is in this way that the disease is often carried from points in which it is epidemic or endemic into localities that are free from the disease.

THE DIAGNOSIS OF ASFATIC CHOLERA BY BACTERIOLOGICAL METHODS.

Because of the manifold channels that are open for the dissemination of this disease it is of the utmost importance that its true nature should be recognized as quickly as possible, for with every moment of delay in its recoguition opportunities for its spread are multiplying. It is essential, therefore, when employing bacteriological means in making the diagnosis, to bear in mind 
those biological and morphological features of the organism that appear most quickly under artificial methods of cultivation, and which, at the same time, may be considered as characteristic of it, viz., its peculiar morphology and grouping; the much greater rapidity of its growth over that of other bacteria with which it may be associated; the characteristic appearance of its colonies on gelatin plates and of its growth in stab-cultures in gelatin; its property of producing indol and coincidently nitrites in from six to eight hours in peptone solution at $37^{\circ}$ to $38^{\circ} \mathrm{C}$; ; and its power of causing the death of guinea-pigs in from sixteen to twenty-four hours when introduced into the peritoneal cavity, death being preceded by symptoms of extreme toxæmia, characterized by prostration and gradual and continuous fall in the temperature of the animal's body.

In a publication made by $\mathrm{Koch}^{1}$ he called attention to a plan of procedure that is employed in this work in the Institute for Infectious Diseases at Berlin. In this scheme the points that have been enumerated are taken into account, and by its employnent the diagnosis can be established in the majority of cases of Asiatic eholera in from eighteen to twenty-two hours. In general, the steps to be taken and points to be borne in mind are as follows: the material should be examined as early as possible after it has been passed.

I. Microscopic examination. From one of the small slimy particles that will be seen in the semi-fluid evacuations prepare a eover-slip preparation in the ordinary way and stain it. If, upon microscopic examination, only curved rods, or curved rods greatly in excess of all

1 Zeitschrift fur Hygiene, 1893, Bd. xiv. 
other forms, are present, the diagnosis of Asiatic cholera is more than likely correct; and particularly is this true if these organisms are arranged in irregular linear groups with the long axes of all the rods pointing in nearly the same direction-that is to say, somewhat as minnows arrange themselves when swimming in schools up stream. (Koch.)

In 1886 Weisser and Frank ${ }^{1}$ expressed their opinion upon the value of microscopic examination in these cases in the following terms:

(a) In the majority of cases microscopic examination is sufficient for the detection of the presence of the comma bacillus in the intestinal evacnations of cholera patients.

(b) Even in the most acute cases, running a very rapid course, the comma bacillus can always be found in the evacuations.

(c) In general, the number of cholera spirilla present is greater the earlier death occurs; when death is postponed, and the disease continues for a longer period, their number is diminished.

(d) Should the patient not die of cholera, but from some other disease, such as typhoid fever, that may be engrafted upon it, the comma bacilli may disappear entirely from the intestines.

II. With another slimy flake prepare a set of gelatin plates. Place them at a temperature of from $20^{\circ}$ to $22^{\circ} \mathrm{C}$, and at sixteen, twenty-two, and thirty-six hours observe the appearance of the colonies. Usually at about twenty-two hours the colonies of this organism can easily be identified by one familiar with them. 
III. With another slimy flake start a culture in a tube of peptone solution-either the solution of Dunham or, as Koch proposes, a solution of double the strength of that of Dunham (Witte's peptone is to be used, as it gives the best and most constant results). Place this at $37^{\circ}$ to $38^{\circ} \mathrm{C}$., and at the end of from six to eight hours prepare cover-slips from the upper layers (without shaking) and examine them microscopically; if comma bacilli were present in the original material, and are capable of multiplication, they will be found in this locality in almost pure culture. After doing this prepare a second peptone culture from the upper layers of the one just examined, also a set of gelatin plates, and with what remains make the test for indol by the addition of ten drops of concentrated sulphuric acid for each ten cubic centimetres of fluid contained in the tube. If comma bacilli are growing in the tube, the rose color characteristic of the presence of indol should appear.

By following this plan "a bacteriologist who is familiar with the morphological and biological peculiarities of this organism should make a more than probable diagnosis at once by microscopic examination alone, and a positive diagnosis in from twenty to, and at the most, twenty-four hours after beginning the examination." (Koch.)

There are certain doubtful cases in which the organisms are present in the intestinal canal in very small numbers, and microscopic examination is not, therefore, of so much assistance. In these eases plates of agaragar, of gelatin, and cultures in the peptone solution should be made.

The plates of agar-agar should not be prepared in 
the usual way, but the agar-agar should be poured into Petri dishes and allowed to solidify, after which one of the slimy particles may be smeared over its surface. The comma bacillus, being markedly aërobic, develops very much more readily when its colonies are located upon the surface than when they are in the depths of the medium. A point to which Koch calls attention, in connection with this step in the manipulation, is the necessity for having the surface of the agar-agar free from the water that is squeezed from it when it solidifies, as the presence of the water interferes with the development of the colonies at isolated points and causes them to become confluent. To obviate this he recommends that the agar-agar be poured into the plates and the water allowed to separate from the surface at the temperature of the incubator before they are used. It is wise, therefore, when one is liable to be called on for such work as this to keep a number of sterilized plates of agar-agar in the incubator ready for use, just as sterilized tubes of media are always ready and at hand. The advantage of using the agar plates is the higher temperature at which they can be kept, and consequently a more farorable condition for the development of the colonies. As soon as isolated colonies appear they should be examined microscopically for the presence of bacteria having the morphology of the one for which we are seeking, and as soon as such is detected gelatin plates and cultures in peptone solution (for the indol reaction) should be made. The peptone cultures started from the original material should be examined microscopically from hour to hour after the sixth hour that they have been in the incubator. The material taken for examination should always come from near the sur- 
face of the fluid, and care should be taken not to shake the tube. As soon as comma bacilli are detected in anything like considerable numbers in the upper layers of the fluid, agar-agar plates and fresh peptone cultures should be made from them. The colonics will develop on the agar-agar plates at $37^{\circ} \mathrm{C}$. in from ten to twelve hours to a size sufficient for recognition by microscopic examination, and from this examination an opinion can usually be given. This opinion should always be controlled by cultures in the peptone solution made from each of several single colonies, and finally the test for the presence or absence of indol in these cultures.

In all doubtful cases in which only a few curved bacilli are present, or in which irregularities in either the rate or mode of their development occur, pure cultures should be obtained by the agar-agar plate method and by the method of cultivation in peptonc solution, as soon as possible, and their virulence tested upon animals. For this purpose eultures upon agar-agar from single colonies must be made. From the surface of one of such cultures a good sized wire-loopful should be scraped and this broken up in about one cubic centimetre of bouillon, and the suspension thus made injected by means of a hypodermic syringe directly into the peritoneal eavity of a guinea-pig of about 350 to 400 grammes weight. For larger animals more material should be used. If the material injected is from a fresh culture of the cholera organism, toxic symptoms at once begin to appear; these have their most pronounced expression in the lowering of temperature, and if one follows this decline in temperature from time to time with the thermometer it will be seen to be gradual and continnous from the time of injection to the death 
THE DIAGNOSIS OF ASIATIC CHOLERA. 393

of the animal (Pfeiffer ${ }^{1}$ ), which occurs in from eighteen to twenty-four hours after the operation.

In general, this is the procedure employed in the Institute for Infectious Diseases, at Berlin, under Koch's direction.

1 Loc. cit. 


\section{CHAPTER XXIII.}

Spirilla of interest, historically and otherwise, that have been confounded with the spirillum of Asiatic cholera-Their peculiaritles and differential features-Vibrio proteus, or bacillus of Finkler and Prior-Spirillum tyrogenum, or cheese spirillum of Deneke-The spirillum of Miller-Vibrio Metchnikovi.

\section{VIBRIO PRO'TEUS (FINKLER-PRIOR BACILLUS).}

Finkler and Prior were the first to contest experimentally the significance of the presence of Koch's comma bacillus in Asiatic cholera, claiming to have found it in the dejections of individuals suffering from other maladies, particularly cholera nostras. The morphological and biological differences between the organism that Finkler and Prior discovered and those of the comma bacillus described by Koch are, however, so pronounced as to warrant the opinion that the confusion arose through imperfect and untrustworthy methods of experimentation. At a somewhat later period Finkler and Prior retracted their claims of identity for the two organisms, and held that the bacterium with which they were dealing was peculiar to cholera nostras-an opinion which, in the light of subsequent work, was also proved to be without foundation in fact.

The characteristies of the spirillum of Finkler and Prior are as follows:

Morphology.-It is thicker and longer than the spirillum of Asiatic cholera; it is often thicker at the middle than at the poles; it forms, like the "comma bacillus," screw-like, twisted threads (Fig. 75). 
It is supplied with a single flagellum at one of its ends, and is, therefore, motile.

FIG. 75.

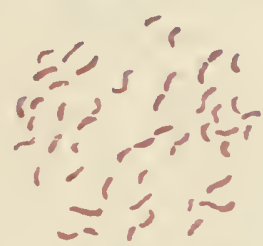

Vibrio proleus, Finkler-Prior bacillus, from culture on agar-agar twentyfour bours old.

It, like the comma bacillus, readily undergoes degenerative changes under conditions unfavorable to growth, and presents the variety of shapes grouped under the head "involution-forms." According to Buchner, this is especially the case when the medium in which they are growing contains glucose ( 5 per cent.) or glycerin ( 2 per cent.).

Cultural Peculiarities.-On gelatin plates the development of its colonies is far more rapid, and liquefaction far more extensive, than in the case of the cholera spirillum. After twenty-two to twenty-four hours in this medium at $20^{\circ}$ to $22^{\circ} \mathrm{C}$. the average size of the colonies is about double that of the comma bacillus. The colonies are darker and denser and do not present under the low lens the same degree of granulation and subsequent lobulation, and they do not become serrated or scalloped around the margin as is the case with Koch's organism. After twenty-two to twentyfour hours they are usually nearly round, regularly granular, and more or less sharply defined. (See Fig. $76, a_{\text {. }}$ At times they may show indefinite markings 
or creases, somewhat suggestive of lobulations. After forty-eight hours on gelatin they usually range from one to three millimetres (some even larger) in diameter, and will appear as sharply cut, saucer-shaped pits of liquefaction, in the most dependent portion of which lies a dense, irregular mass, the colony proper. Under low magnifying power they present at this stage an appearance similar to that shown in Fig. 76, $b$, the central dense mass representing the colony and the irregular

FIG. 76.
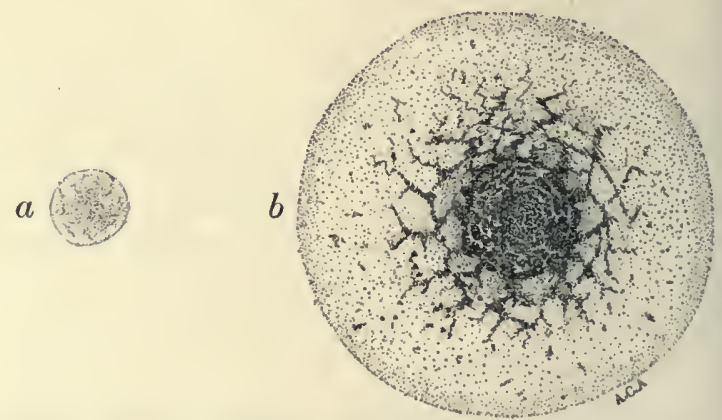

Colonies of the Finkler-Prior bacillus on gelatin. $\times$ about 75 diameters.

a. After twenty-two hours at $20^{\circ}$ to $22^{\circ} \mathrm{C}$. b. After forty-eight bours at $20^{\circ}$ to $22^{\circ} \mathrm{C}$.

ragged lines surrounding it being shreds that have become torn away as it sank into the liquid cansed by its growth. The zone surrounding it, extending to the periphery, is somewhat clondy, and is simply liquefied gelatin. There is a marked tendency for the liquefaction to spread laterally and for the colonies to run together, so that, even on plates containing few colonies, in sixty to seventy-two hours at from $20^{\circ}$ to $22^{\circ} \mathrm{C}$., the entire gelatin is usually couverted into a yellowish- 
white fluid. Under these conditions its growth is accompanied by a marked aromatic odor, impossible to describe; this is especially the case when the liquefaction is far advanced.

FIG. 77.

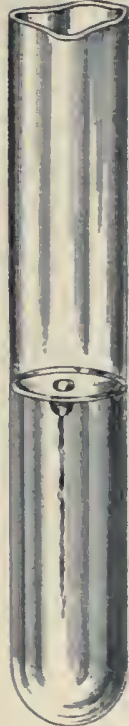

a.

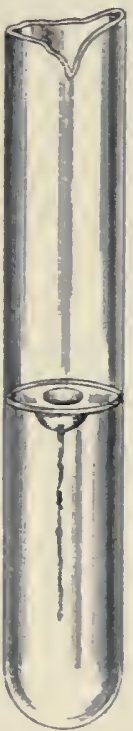

b

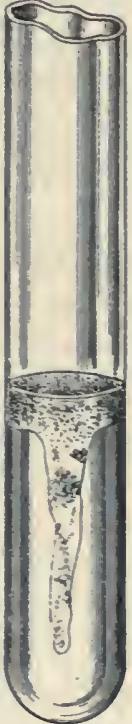

c

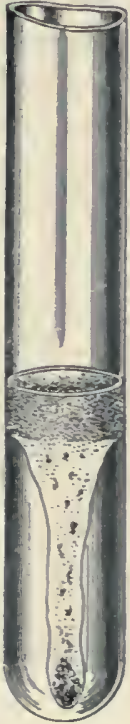

d

Stab-culture of the Finkler-Prior bacillus in gelatin at $15^{\circ}$ to $20^{\circ} \mathrm{C}$. a. After twenty-four hours. b. After forty-eight hours. c. After serenty-two hours. d. After ninety-six hours.

In stab-cultures in gelatin at the room temperature, liquefaction is noticed about the upper part of the needle-track in twenty-four hours. This coudition gradually increases, and at the end of two or three days the entire upper portion of the gelatin has become converted into a cloudy fluid, whereas at the lower part of the canal the liquefaction progresses less rapidly, but is 
still much more marked than that seen as a result of the growth of Koch's spirillum. Indeed, under these circumstances there is no similarity whatever between the growth of the two organisms (see $a, b, c, d$, Fig. 77, and compare these with corresponding cuts in Fig. 74).

It is customary to see scattered through the cloudy liquefied gelatin, ragged, more or less dense masses, fragments of the colony proper.

On nutrient agar-agar there is nothing particularly characteristic about its growth, appearing only as a moist, grayish or yellowish-gray deposit.

On potato, after forty-eight to seventy-two hours, there appears a pale, yellowish-gray deposit; this is moist, glazed, and marked by lobulations, and is surrounded by an irregular, colorless zone of growth that is much less moist than that forming the central area. It grows well on potato at the ordinary temperature of the room.

It causes liquefaction of solidified blood-serum and of coagulated egg-albumin.

In milk to which neutral litmus tincture has been added the blue color takes on a pink tinge in from two to three days at $37^{\circ}$ to $38^{\circ} \mathrm{C}$.

It does not form indol nor does it cause fermentation of glucose.

In peptone solution containing rosolic acid the color is somewhat deepened after four or five days at $37^{\circ} \mathrm{C}$.

Experiments upon Animais. - By ordinary methods of inoculation this organism is without pathogenic properties. Injections, subcutancous and intravascular and directly into the stomach, give negative results. When introduced into the stomach of guinea-pigs by the method employed by Koch in his cholera experi- 
ments, Finkler and Prior had 3 out of 10 animals, and Koch 5 out of 15 animals so treated to die.

The claim of Finkler and Prior that this organism was related etiologically to cholera nostras has been shown by subsequent work to be unjustifiable.

In 1885, 1886, and 1887 Franck $^{1}$ examined seven cases that clinically presented the condition of cholera nostras; in none of these seven cases was the organism of Finkler and Prior, which they claimed to be the cause of the disease, found. In all cases the results of bacteriological examination, in so far as the constant presence of an organism that might stand in causal relation to the disease was concerned, were negative. Only the ordinary intestinal bacteria were found.

\section{SPIRILLUM TYROGEXUM (CHEESE SPIRILLUM OF DENEKE).}

Another spiral form, likewise forming short, commashaped segments in the course of its growth (Fig. 78), is that found by Deneke in old cheese. It is a little smaller than Koch's spirillum. It is motile and has but a single flagellum, attached to one of its ends. It liquefies gelatin more rapidly than does Koch's organism. It possesses no characteristic grouping, as can be seen in impression cover-slips of its colonies. It does not form spores. On gelatin plates its colonies develop very rapidly as saucer-shaped depressions; after twentyfour hours they vary from 1 to $4 \mathrm{~mm}$. in transverse diameter. To the naked eye they are almost transparent, and are usually marked by a denser centre and 
peripheral zone, the space between being quite clear. They are not regularly round in all cases. A peculiar aromatic odor accompanies their growth on gelatin.

FIG. 78.

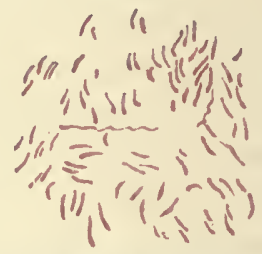

Deneke's cheese spirillum, spirillum tyrogenum. From agar agar culture twenty-four hours old.

Under low magnifying power the smallest colonies are irregularly round in outline, their borders being often rough and broken, and the body of the colony is frequently marked by creases or ridges that give to it a lobulated appearance. The larger colonies under the

FIG. 79.

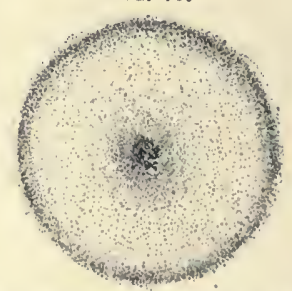

Colony of spirilum tyrogenum on gelatin, twenty-four hours old.

same lens appear as granular patches, a little denser at the periphery and centre than at the intermediate portions. The periphery gradually fades away and no distinct eireumference can be made out. (See Fig. 79.) The colonies of an intermediate size, about which lique- 
faction is just beginning to be apparent, show a dense granular centre, the colony itself, and round about it a delicate, granular developmental zone.

In stab-cultures in gelatin liquefaction is rapid, causing at the end of twenty-four hours a cup-shaped depression at the top of the needle-track, the superficial area of which is about half that of the gelatin in the tube.

FIG. 80.

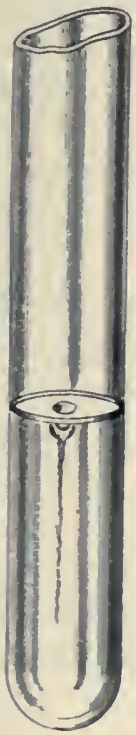

a

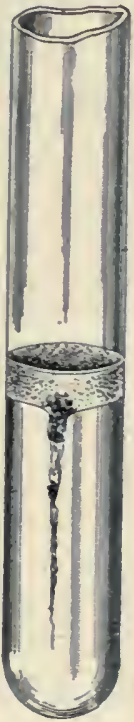

b

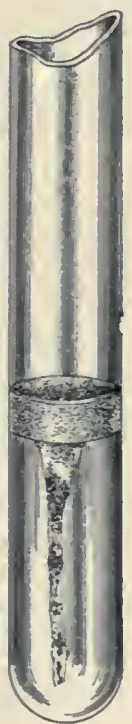

$c$

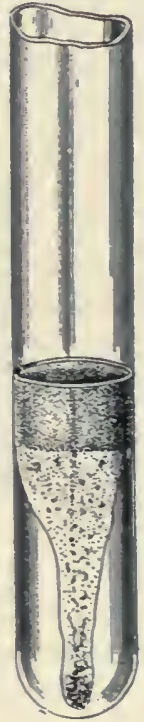

d

Stab-culture of Deneke's cheese spirillum in gelatin, at $18^{\circ}$ to $20^{\circ} \mathrm{C}$.

a. After twenty-four hours. b. After forty-eight hours. c. After seventy-two hours. d. After ninety-six bours.

(Fig. 80,a.) The liquefying process spreads laterally, and at the end of forty-eight hours the whole upper portion of the gelatin may have become liquid. (Fig. $80, b$.) This process continues along the track of the 
needle, and after seventy-two and ninety-six hours the appearances shown in Fig. 80, $c$ and $d$, will be produced.

There is nothing particularly characteristic about its growth upon agar-agar.

On potato there appears a moist, glazed, yellowish, and, at points, brownish-yellow growth that is surrounded by a drier, colorless zone. It is not lobulated.

In milk containing neutral litmus tincture a pink color appears after two to three days at $37^{\circ} \mathrm{C}$; ; after four days the milk is almost decolorized and there is beginning to appear coagulation of the casein with a layer of clear whey above it. During the subsequent twentyfour hours there is complete separation of the contents of the tube into clot and whey.

In Dunham's peptone solution it does not form indol, and the reaction for this body does not appear with either sulphuric acid alone or plus sodium nitrite.

It causes liquefaction of both coagulated blood-serum and egg-albumin.

There is no pellicle formed as a result of its growth in bouillon.

It does not produce fermentation of glucose.

In rosolic-acid-peptone solution its growth causes the red color to become deepened after four or five days at $37^{\circ} \mathrm{C}$.

By Koch's method of introducing enltures into the stomachs of guinea-pigs this organism produced the death of three out of fifteen animals experimented upon-the deaths resulting, most probably, more from the toxic action of the products of growth that were introduced with the organisms than to any pathogenic powers possessed by the organism itself. 
MILLER'S SPIRILLUM.

Another spirillum that has been likened to that of Koch is the one obtained by Miller from a carious tooth. It has so many characteristics in common with the organism of Finkler and Prior that Miller was inclined to consider them identical. In morphology they are indistinguishable. (See Fig. 81.) It grows rapidly, and, like the spirillum of Finkler and Prior, causes rapid liquefaction of gelatin with the coincident production of a peculiar aromatic odor.

FIG. 81 .

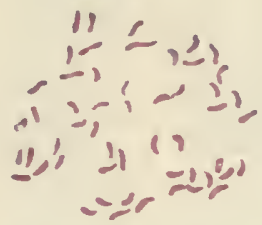

Spirillum of Mfiller. From agar-agar culture twenty-four hours old.

The colonies on gelatin plates appear after twentyfour hours as small, transparent pits of liquefaction, in the centre of which can be seen a minute white point, the colony itself. Under a low lens the largest of these points are uniformly granular and regularly round, and, as a rule, are surrounded by a peripheral zone that is a little darker than the central portion of the colony. The circumference is delicately fringed by short, cilialike prolongations of growth which are not, as a rule, straight, but are twisted in all directions and can only be detected upon very careful examination. (See $a$, Fig. 82.) When located deep in the gelatin the colonies are round, sharply circumscribed, of a pale yel- 
lowish or greenish-yellow eolor, and marked by very delicate irregular lines or ridges. After forty-eight hours the plate eontaining many eolonies is entirely liquefied, while that containing only a few shows the presence of round, sharply cnt, shallow pits of liquefaction that measure from 2 to $10 \mathrm{~mm}$. in diameter. They are a little denser at the eentre than at the periphery, and the dense centre is not sharply circumscribed, but fades off into what has the appearance of a delieate film. (See 6, Fig. 82.) As the colonies become older they are sometimes marked by irregular radii extending from periphery to centre like the spokes of a wheel.

FIG. 82.
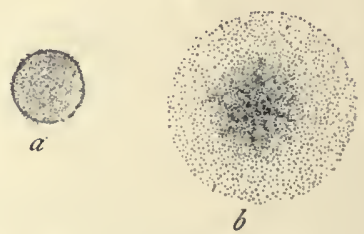

Colonies of Miller's spirillum on gelatin, at $20^{\circ}$ to $22^{\circ} \mathrm{C} . \quad \times$ about 57 diameters.

a. Colony just beneath the surface of the gelatin. $b$. Colony on the surface of the gelatin.

In stab-cultures in gelatin it rapidly produees liquefaction, both at the surface and along the needle-track, and in most respeets gives rise to a eondition very like that resulting from the growth of Finkler and Prior's spirillum, though differing from it in certain details. (See $a, b, c, d$, Fig. 83.)

On agar-agar nothing of special interest appears as a result of its development.

On potato its growth is very like that of the elolera spirillum, vi\%, it appears at $37^{\circ} \mathrm{C}$. as a dry, white pateh 
that lies quite flat upon the surface and can often ouly be seen when the tube is held to the light in a special way.

FIG. 83.

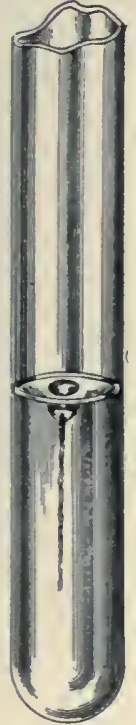

a

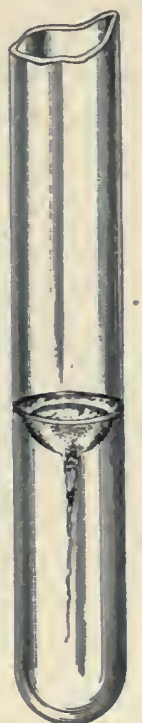

$b$

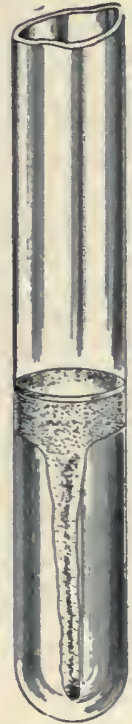

$c$

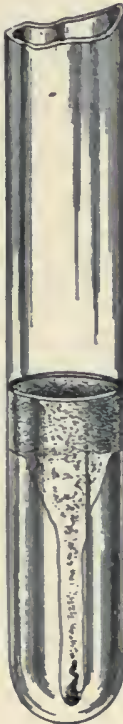

d

Stab-culture of Miller's spirillum in gelatin, at $18^{\circ}$ to $20^{\circ} \mathrm{C}$.

a. After twenty-four honrs. $b$. After forty-eight hours. $c$. After seventy-two hours. d. After ninety-six hours.

Its growth in bouillon is not characteristic. It does not form a pellicle.

It causes liquefaction of both coagulated blood-serum and egg-albumin.

It does not produce indol.

It does not cause fermentation of glucose.

It is non-motile.

In milk containing blue litmus tincture it causes almost complete decolorization in from three to four 
days at $37^{\circ} \mathrm{C}$., with coincident coagulation of the casein and the formation of a layer of clear whey about it.

It causes the red color of rosolic-acid-peptone solution to become somewhat intensified after four or five hours at $37^{\circ} \mathrm{C}$.

Of twenty-one animals treated with this organism by Koch's method of inoculation only four died.

\section{VIBRIO METCHNIKOVI.}

The spirillum that simulates very closely the comma bacillus of cholera in its morphological and cultural peculiarities, but which is still easily distinguished from it, is that described by Gamaleia under the name of

Fra. 84.

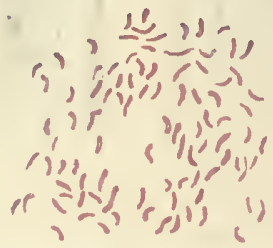

Vibrio Metchnikovi from agar-agar culture, twenty-tour hours old.

vibrio Metchnikovi. It was found post mortem in a number of fowls that had died in the poultry market of Odessa, and the experiments of the discoverer led lim. to believe that it was related etiologically to the gastroenteritis with which the ehickens had been suffering.

In morphology it is seen as short, curved rods and as longer, spiral-like filaments. It is usually thicker than Koch's spirillum and is at times much longer, while 
again it is seen to be shorter. It is usually more distinctly curved than the "comma bacillus." (Fig. 84.)

It is supplied with a single flagellum at one of its extremities, and is, therefore, motile.

It does not form spores.

It is aërobic.

Its growth upon gelatin plates is usually characterized, according to Pfeiffer, by the appearance of two kinds of liquefying colonies, one strikingly like those of the Finkler-Prior organism, the other very similar to those produced by Koch's comma bacillus, though in both cases the liquefaction resulting from the growth of this organism is more energetic than that common to the spirillum of Asiatic cholera. After from twentyfour to thirty hours the medium-sized colonies, when examined under a low power of the microscope, show a yellowish-brown, ragged central mass surrounded by a zone of liquefaction that is marked by a border of delicate radii. (Fig. 85.)

Fio. 85.

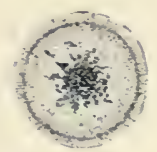

Colony of vibrio Metchnikovi in gelatin, after thirty hours at $20^{\circ}$ to $22^{\circ} \mathrm{C}$. $X$ about 75 diameters.

In gelatin stab-cultures the growth has much the same general appearance as that of the cholera spirillum, but is very much exaggerated in degree. The liquefaction is far more rapid, and the characteristic appearance of the growth is lost in from three to four days. (See $a, b, c, d$, Fig. 86.) Development and liquefaction 
along the deeper parts of the needle-track are much more pronounced than is the case with the "comma bacillus."

Fig. 86 .

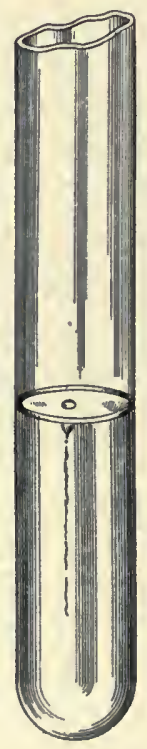

a

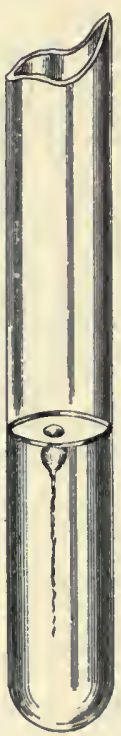

b

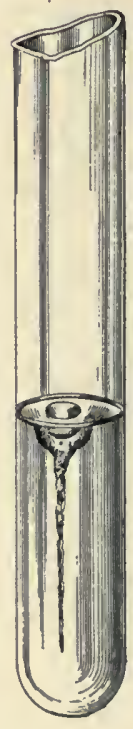

$c$

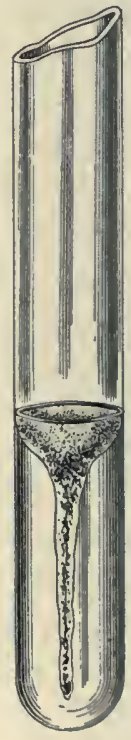

d

Stab-culture of vibrio Metchnikovi In gelatin, at $18^{\circ}$ to $20^{\circ} \mathrm{C}$.

$a$. After twenty-four hours. $b$. After forty-eight hours. c. After seventy-two hours. $d$. After ninety-six hours.

Its growth on agar-agar is rapid, and after twentyfour to forty-eight hours there appears a grayish deposit having a tendency to take on a yellowish tone.

On potato at $37^{\circ} \mathrm{C}$. its growth is seen as a moist, coffee-colored patch surrounded by a much paler zone. The whole growth is so smooth and glistening that it has almost the appearance of being varnished.

In bouillon it quickly causes opacity, with the ulti- 
mate production of a delicate pellicle upon the surface. It causes liquefaction of blood-serum, the liquefied area being covered by a dense, wrinkled pellicle.

When grown in peptone solution it produces indol and coincidently nitrites, so that the rose-colored reaction characteristic of indol is obtained by the addition of sulphuric acid alone. The production of indol by this organism is usually greater than that common to the comma bacillus under the same circumstances.

In milk it causes an acid reaction with coagulation of the casein. The coagulated casein collects at the bottom of the tube in irregular masses, above which is a layer of clear whey. If blue litmus has been added to the milk, the color is changed to pink by the end of twenty-four to thirty hours, and after forty-eight hours decolorization and coagulation occur. The clots of casein are not re-dissolved. After about a week the acidity of the milk is at its maximum, and the organisms quickly die.

It causes the red color of the rosolic-acid-peptone solution to become very much deeper after four or five days at $37^{\circ} \mathrm{C}$.

It does not cause fermentation of glucose with production of gas.

It is killed in five minutes by a temperature of $50^{\circ} \mathrm{C}$. (Sternberg.)

It is pathogenic for chickens, pigeons, and guineapigs. Rabbits and mice are affected only by very large doses.

Chickens affected with the choleraic gastro-enteritis, of which this organism is the cause, are usually seen sitting quietly about with ruffled feathers. They are afflicted with diarrhœa, but do not have any elevation 
of temperature. A hyperæmia of the entire gastrointestinal tract is seen at autopsy. The other internal organs do not, as a rule, present anything abnormal to the naked eye. The intestinal canal contains yellowish fluid with which blood may be mixed. In adult chickens the spirilla are not found in the blood, but in young ones they are usually present in small numbers. By subcutaneous inoculation pigeons suceumb to the pathogenic activities of this organism in from eight to twelve hours. At autopsy pretty much the same condition is seen as was deseribed for chickens, except that large numbers of the spirilla are usually present in the blood. Guinea-pigs usually die in from twenty to twenty-four hours after subcutaneous inoculation. At autopsy an extensive edema of the subcutaneous tissues about the seat of inoculation is seen, and there is usually a necrotic condition of the tissues in the vicinity of the point of puncture. As the blood and internal organs contain the vibrios in large numbers, the infection in these animals takes, therefore, the form of acute, general septicæmia.

Gastro-enteritis may be produced in both chickens and guinea-pigs by feeding them with food in which cultures of this organism have been mixed.

In the antumn of 1896 the anthor isolated from the Schuylkill River at Philadelphia a spirillum that is pathogenic for pigeons and for guinea-pigs, and that possesses so many of the other characteristics common to the gronp of spirilla of which the cholera spirillum forms the most important member, as to justify the opinion that they are members of the same family. That it is not identical with the cholera 
spirillum is evident, for the reason that the latter produces cholera, while the vibrio Schuylkiliensis manifestly does not. ${ }^{1}$

Note. - Since the late epidemic of cholera in Hamburg quite a number of curved or spiral organisms, somewhat like the cholera spirillum, have been discovered. For the descriptions of these the reader is referred to the current bacteriological literature.

1 For the detailed description of this organism see Jonrnal of Experimental Medicine, vol. i. p. 419; also Transactions of the Association of American Physicians, 1S96, vol. xi. p. 394. 


\section{CHAPTER XXIV.}

Study of bacillus anthracis, and the effects produced by its inoculation into animals-Peculiarities of the organism under varying conditions of surroundings.

THE discovery that the blood of animals suffering from splenic fever, or anthrax, always contained minute rod-shaped bodies (Pollender, 1855; Davaine, 1863), led to a closer study of this disease, and has resulted probably in contributing more to our knowledge of bacteriology in general than work upon any of the other infectious maladies.

The outcome of these investigations is that a rodshaped micro-organism, now known as bacillus anthracis, is always present in the blood of animals suffering from this disease; that this organism can be obtained from the tissues of these animals in pure cultures, and that these artificial cultures of bacillus anthracis when introduced into the body of susceptible animals can again produce a condition identical with that found in the animal from which they were obtained.

The disease is a true septicæmia, and after death the capillaries throughout the body will always be found to contain the typical rod-shaped organism in larger or smaller numbers.

This organism, when isolated in pure culture, is seen to be a bacillus which varies considerably in its length, ranging from short rods of 2 to $3 \mu$ in length to longer threads of 20 to $25 \mu$ in length. In breadth it is from 
1 to $1.25 \mu$. Frequently very long threads made up of several rods, joined end to end, are seen.

When obtained directly from the body of an animal it is usually in the form of short rods square at the ends. If highly magnified, the ends are seen to be a trifle thicker than the body of the cell and somewhat indented or concave, peculiarities that help to distinguish it from certain other organisms that are somewhat like it morphologically. (See Fig. 87.)

Fig. S7.
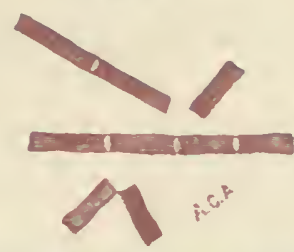

Bacillus anthracis highly magnified to show swellings and concavities at extremities of the single cells.

When cultivated artificially at the temperature of the body the bacillus of anthrax presents a series of very interesting stages.

The short rods develop into long threads, which may be seen twisted or plaited together after the manner of ropes, each thread being marked by the points of juncture of the short rods composing it. (Fig. 88, $a$ and $b$.)

In this condition it remains until alterations in its surroundings, the most conspicuons being diminution in its nutritive supply, favor the production of spores. When this stage begins, changes in the protoplasm of the bacilli may be noticed; they become marked by irregular, granular bodies, which eventually coalesce into glistening, oval spores, one of which lies in nearly every 
segment of the long thread, and gives to the thread the appearance of a string of glistening beads. (Fig. 89.)

F1G. 88.

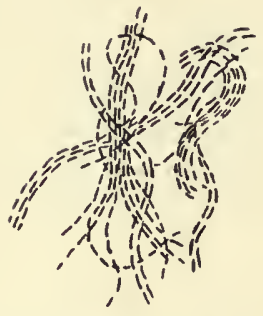

a

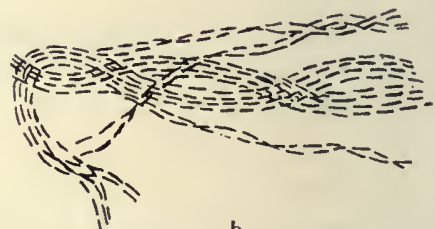

b

Bacillus anthracis. Plaited and twisted threads seen in fresh growing cultures. $\times$ about 400 diameters.

In this stage they remain but a short time. The chains of spores, which are held together by the remains of the cells in which they formed, become broken up, and eventually nothing but free oval spores, and here and there the remains of mature bacilli which have undergone degenerative changes, can be found. In this condition the spores capable of resisting deleterious influ-

FrG. 89.

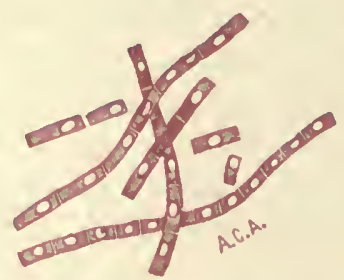

Threads of bacillus anthracis containing spores. $\times$ about 1200 diameters.

ences remain, and, unless their surroundings are altered, have been seen to continue in this living, though inac- 
tive, condition for a very long time. If again placed under favorable conditions, each spore will germinate into a mature cell, and the same series of changes will be repeated until the favorable surroundings become again gradually unfavorable to development, when spore-formation is again seen. Spore-formation takes place ouly at temperatures ranging from $18^{\circ}$ to $43^{\circ} \mathrm{C}$., $37.5^{\circ} \mathrm{C}$. being the most favorable temperature. Under $12^{\circ} \mathrm{C}$. they are not formed. With this organism sporeformation does not occur in the tissues of the living animal, its usual condition at this time being that of short rods. Occasionally, however, somewhat longer forms may be seen.

The bacillus of anthrax is not motile.

Growth ox AGar-AGar. - The colonies of this organism, as seen upon agar-agar, present a very typical appearance, from which they have been likened unto the head of Medusa. From a central point which is

FIG. 90.

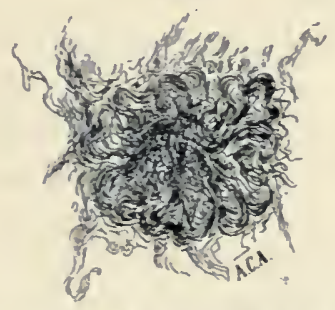

Colony of bacillus anthracis on agar-agar.

more or less dense, consisting of a felt-like mass of long threads matted irregularly together, the growth continues outward upon the surface of the agar-agar. (Fig. 90.) It is made up of wavy bundles in which the threads are seen to lie parallel side by side or are twisted 
in strands like those of a rope-sometimes they have a plaited arrangement. (See Fig. 88.) These bundles twist about and cross in all directions, and eventually disappear at the periphery of the colony. At the extreme periphery of the colonies it is sometimes possible to trace single bundles of these threads for long distances across the surface of the agar-agar. The colony itself is not circumscribed in its appearance, bnt is more or less irregularly fringed or ragged, or scalloped. To the naked eye they look very much like minute pellicles of raw eotton that have been pressed into the surface of the agar-agar.

As the colonies continue to grow they become more and more dense, opaque, and granular and rough on the surface. When touched with a sterilized needle one experiences a sensation that suggests, somewhat, the matted structure of these colonies. The bit that may thus be taken from a colony is always more or less ragged.

Gelatin. - The colonies on gelatin at the earliest stages also present the same wavy appearance; but this characteristic soon becomes in part destroyed by the liquefaction of the gelatin which is produced by the growing organisms. This allows them to sink to the bottom of the fluid, where they lie as an irregular mass.

Through the fluid portion of the gelatin may be seen small clumps of growing bacilli, which look very much like bits of cotton-wool.

BouIlton. - In bouillon the growth is characterized by the formation of flaky masses, which also have very much the appearance of bits of raw cotton. Microscopie examination of one of these flakes reveals the twisted and plaited arrangement of the long threads. 
Porato.-It develops rapidly as a dull, dry, granular, whitish mass, which is more or less limited to the point of inoculation. On potato, at the temperature of the incubator, its spore-formation may easily be observed.

Stab- And Slast-cultures. - Stab- and slant-cultures on agar-agar present in general the appearances given for the colonies, except that the growth is much more extensive. The growth is always more pronounced on the surface than down the track of the needle.

On gelatin it causes liquefaction, which begins on the surface at the point inoculated, and spreads ontward and downward.

It grows best with access to oxygen, and very poorly when the supply of oxygen is interfered with.

Under favorable conditions of aëration, nutrition, and temperature its growth is rapid.

Under $12^{\circ} \mathrm{C}$. and above $45^{\circ} \mathrm{C}$. no growth occurs. The temperature of the body is most favorable to its development.

The spores of the anthrax bacillus are very resistant to heat, though the degree of resistance is seen to vary with spores of different origin. von Esmarch found that anthrax spores from some sources would readily be killed by an exposure of one minute to the temperature of steam, whereas those from other sources resisted this temperature for longer times, reaching in some cases as long as twelve minutes.

Starsing. - The anthrax bacilli stain readily with the ordinary aniline dyes. In tissues their presence may also be demonstrated by the ordinary aniline staining-fluids, or by Gram's method. They may also be 
stained in tissues with a strong watery solution of dahlia, after which the tissue is decolorized in 2 per cent. sodium carbonate solution, washed in water, dehydrated in alcohol, cleared up in xylol, and mounted in balsam. This leaves the bacilli stained, while the tissues are decolorized; or the tissues may be stained a contrastcolor-with eosin, for example-after the dehydration in alcohol, and before the clearing up in xylol. In this case they must be washed out again in alcohol before using the xylol. In the preparation treated in this way the rod-shaped organisms will be of a purple color, and will be seen in the capillaries of the tissues, while the tissues themselves will be of a pale rose color.

Inoculation into Animals.-Introduce into the subcutaneous tissues of the abdominal wall of a guineapig or rabbit a portion of a pure culture of bacillus anthracis. In about forty-eight hours the animal will be found dead. Immediately at the point of inoculation little or no reaction will be noticed, but beyond this, extending for a long distance over the abdomen and thorax, the tissues will be markedly œdematous. Here and there, scattered through this œdematous tissue, small ecchymoses will be seen. The underlying muscles are pale in color. Inspection of the interual viscera reveals no very marked macroscopic changes except in the spleen. This is enlarged, dark in color, and soft. The liver may present the appearance of cloudy swelling; the lungs may be red or pale red in color; the heart is usually filled with blood. There are no changes to be seen by the naked eye.

Prepare cover-slip preparations from the blood and other viscera. They will all be found to contain short 
rods in large numbers. Nowhere can spore-formation be detected. Upon microscopic examination of sections of the organs which have been hardened in alcohol the capillaries are seen to be filled with the bacilli; in some places closely packed together in large numbers, at other points fewer in number. Usually they are present in largest numbers in those tissues having the greatest capillary distribution and at those points at which the circulation is slowest. They are moderately evenly distributed through the spleen. The glomeruli of the kidneys and the capillaries of the

FIG. 91.

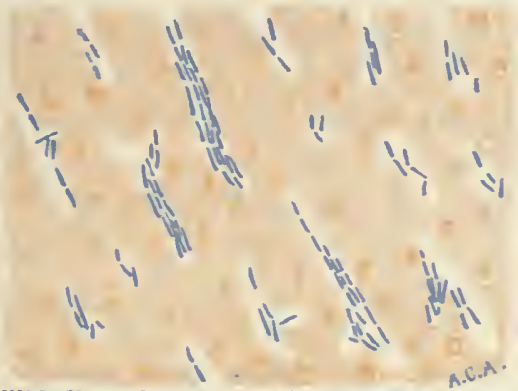

Anthrax bacilli in liver of mouse. $\times$ abont 450 diameters. Bacilli stained

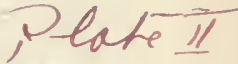
by Gram's method ; tissue stained with Bismarck-brown.

lungs are frequently quite packed with them. The capillaries of the liver contain them in large numbers. (Fig. 91.) Hemorrhages, probably due to rupture of capillaries by the mechanical pressure of the bacilli which are developing within them, not uncommonly occur. When this occurs in the mucous membranes of the alimentary tract the blood may escape through the mouth or anus; when in the kidneys, through the uriniferous tubules. 
Cultures from the different organs or from the oedematous fluid about the point of inoculation result in growth of bacillus anthracis.

The amphibia, dogs, and the majority of birds are not susceptible to this disease. Rats are difficult to infect. Rabbits, guinea-pigs, white mice, gray housemice, sheep, and cattle are susceptible. Infection may occur either through the circulation, through the airpassages, through the alimentary tract, or, as we have just seen, through the subcutaneous tissues.

\section{PROTECTIVE INOCULATION.}

The most noteworthy application of artificially prepared living vaccines to the protection of animals against infection is seen in connection with anthrax in sheep and in bovines.

By a variety of procedures the virulent anthrax bacillus may be in part or totally robbed of its pathogenic properties. It is through the very mild constitutional disturbance, caused in animals vaccinated with such weakened cultures, that protection is often afforded against the severer, frequently fatal, form of the infection.

Without reviewing the various methods that have been employed for attenuating the virulenee of this organism to a degree suitable for protective vaccination, it will suffice to say that the most satisfactory results have been obtained by long-continued cultivation (ten to thirty days) at a temperature of from $42^{\circ}$ to $43^{\circ} \mathrm{C}$. In this procedure the spore-free, virulent bacillus anthracis, obtained direetly from the blood of a recently dead animal, is brought at once into sterile 
nutrient bouillon in about twenty test-tubes, which are immediately placed in an incubator that is carefully regulated to maintain a temperature of $42.5^{\circ} \mathrm{C}$. There should not be a fluctuation of over $0.1^{\circ} \mathrm{C}$.

After about a week a tube is removed from the incubator on each successive day and its virulence tested at once on animals. The degree of attenuation experienced by the cultures grown under these circumstances is determined by tests upon rabbits, guinea-pigs, and mice. The first culture removed may or may not kill rabbits, the most resistant of the three animals used for the test, while it will certainly kill the guinea-pigs and mice; after another two or three days rabbits will no longer suceumb to inoculation with the culture last removed from the incubator, while no diminution will as yet be noticed in its pathogenesis for the other two species. After four to seven days more a culture may be encountered that kills only mice, the guinea-pigs escaping; while ultimately, if the experiment be continued, a degree of attenuation may be reached in which the organism has not even the power of killing a mouse, though it still retains its vitality. Investigation of these attenuations shows them to possess all the characteristics of enfeebled anthrax bacillus; they grow slowly and less vigorously when transplanted; they do not form spores while under a high temperature; and microscopically they present evidences of degeneration. When introduced beneath the skin of animals they disseminate but slightly beyond the site of inoculation, and do not, as a rule, canse the general septicæmia that occurs in susceptible animals after inoculation with normal cultures of this organism. In the practical employment of these attenuated cultures for protective purposes two vaccines 
are employed. These were designated by Pasteur as "first" and "second" vaccines. The "first" is the one that killed only the mice in the preliminary tests, while the "second" is that which killed both mice and guinea-pigs, but failed to kill the rabbit. When larger animals, such as sheep or eattle, are to be protected by vaccination with these vaceines, a subeutaneous inoculation of about 0.3 c.c. of the first vaceine is usually given. This should be practically without noticeable effect, causing neither rise of body-temperature nor other constitutional or local symptoms. After a period of about two weeks the second vaceine is injected in the same way; this may or may not cause disturbance. In the event of its doing so the symptoms are rarely alarming, and, if the vaccines have been properly prepared and tested before use, they disappear within a short time after the injection.

In the large majority of cases sheep, bovines, horses, and mules may be safely protected against anthrax by the careful practice of this method.

\section{EXPERIMENTS.}

Prepare three cultures of anthrax bacilli-one upon gelatin, one upon agar-agar, and one upon potato. Allow the gelatin eulture to remain at the ordinary temperature of the room, place the agar-agar culture in the incubator, and the potato culture at a temperature not above $18^{\circ}$ to $20^{\circ} \mathrm{C}$. Prepare cover-slips from each from day to day. What differences are observed?

Prepare two potato cultures of the anthrax bacillus. Place one in the incubator and maintain the other at a 
temperature of from $18^{\circ}$ to $20^{\circ} \mathrm{C}$. Examine them each day. Do they develop in the same way?

From a fresh culture of anthrax bacilli, in which spore-formation is not yet begun (which is the surest source from which to obtain non-spore-bearing anthrax bacilli), prepare a hanging-drop preparation; also a cover-slip preparation in the usual way and stain it with a strong gentian-violet solution, and another cover-slip preparation which is to be drawn through the flame twelve to fifteen times, stained with aniline gentian-violet, washed off in iodine solution and then in water. Examine these microscopically. Do they all present the same appearance? To what are the differences due?

Do the anthrax threads, as seen in a fresh, growing, hanging drop, present the same morphological appearance as when dried and stained upon a cover-slip? How do they differ?

Liquefy a tube of agar-agar, and when it is at the temperature of $40^{\circ}$ to $43^{\circ} \mathrm{C}$. add a very minute quantity of an anthrax culture which is far advanced in the spore-stage. Mix it thoroughly with the liquid agaragar and from this prepare several hanging drops under strict antiseptic precautions, using the fluid agar-agar for the drops instead of bouillon or salt-solution. Select from among these preparations that one in which the smallest number of spores are present. Under the microscope observe the development of a spore into a mature cell. Describe carefully the developmental - stages. 
Prepare a $1: 1000$ solution of earbolic acid in bouillon. Inoculate this with virulent anthrax spores. If no development occurs after two or three days at the temperature of the thermostat, prepare a solution of $1: 1200$, and continue until the point is reached at which the amount of carbolic acid present just permits of the development of the spores. When the proper dilution is reached prepare a dozen of such tubes and inoculate one of them with virulent anthrax spores. As soon as development is well advanced transfer a loopful from this tube into a second of the carbolic acid tubes; when this has developed, then from this into a third, ete. After five or six generations have been treated in this way study the spore-production of the organisms in that tube. If it is normal, continne to inoculate from one carbolic acid tube to another, and see if it is possible by this means to influence in any way the production of spores by the organism with which you are working. What is the effect, if any?

Prepare two bouillon cultures, each from one drop of blood of an animal dead of anthrax. (Why from the blood of an animal and not from a culture?) Allow one of them to grow for from fourteen to eighteen hours in the incubator; allow the other to grow at the same temperature for three or four days. Remove the first after the time mentioned and subject it to a temperature of $80^{\circ} \mathrm{C}$. for thirty minutes. At the end of this time prepare four plates from it. Make each plate with one drop from the heated bonillon eulture. At the end of three or four days treat the second tube in identically the same way. How do the number of colonies which develop from the two different cultures compare? Was 
there any difference in the time required for their development on the plates?

From a potato culture of anthrax bacilli which has been in the incubator for three or four days scrape away the growth and carefully break it up in 10 c.c. of sterilized physiological salt-solution. The more carefully it is broken up the more accurate will be the experiment. Place this in a bath of boiling water and at the end of one, three, five, seven, and ten minutes make a plate upon agar-agar with one loopful of the contents of this tube. Are the results on the plates alike?

Determine the exact time necessary to sterilize objects, such as silk or cotton threads, on which anthrax spores have been dried, by the steam method and by the hot-air method.

Prepare from the blood of an animal just dead of anthrax a bouillon culture. After this has been in the incubator for from three to four hours subject it to a temperature of $55^{\circ} \mathrm{C}$. for ten minutes. At the end of this time make plates from it and also inoculate a rabbit subcutaneously with it. What are the results? Are the colonies on the plates in every way characteristic?

Inoculate six Erlenmeyer flasks of sterile bonillon, each containing about 35 c.c. of the medium, from either the blood of an animal just dead of anthrax or from a fresh virulent culture in which no spores are formed. 
Place these flasks in the incubator at a temperature of $42.5^{\circ} \mathrm{C}$. At the end of five, ten, fifteen, twenty, twenty-five, etc., days remove a flask. Label each flask as it is taken from the incubator with the exact number of days for which it had been at the temperature of $42.5^{\circ} \mathrm{C}$. Study each flask carefully, both in its culture-peculiarities and in its pathogenie properties when employed on animals.

Are these cultures identical in all respects with those that have been kept at $37^{\circ} \mathrm{C}$.?

If they differ, in what respect is the difference most conspicuous?

Should any of the animals survive the inoculations made from the different cultures in the foregoing experiment, note carefully which one it is, and after ten to twelve days repeat the inoculation, using the same culture; if it again survives, inoculate it with the culture preceding the one just used in the order of removal from the incubator; if it still survives, inoculate it with virulent anthrax. What is the result? How is the result to be explained? Do the cultures which were made from these flasks at the time of their removal from the incubators act in the same way toward animals as the organisms growing in the flasks? Is the action of each of these cultures the same for mice, guinea-pigs, and rabbits?

Prepare a 2 per cent. solution of sulphuric acid in distilled water; suspend in this a number of anthrax spores; at the end of three, six, and nine days at $35^{\circ} \mathrm{C}$. inoculate both a guinca-pig and a rabbit. Prepare cultures from this suspension on the third, sixth, and ninth days; when-the cultures have developed inoculate a 
rabbit and a guinea-pig from the culture made on the ninth day. Should the animals survive, inoculate them again after three or four days with a culture made on the sixth day. Do the results appear in any way peculiar? 


\section{CHAPTER XXV.}

The most important of the organisms found in the soil-The nitrifying bacteria-The bacillus of tetauus-The bacillus of malignant cedema-The bacillus of symptomatic anthrax.

$\mathrm{Br}$ the employment of bacteriological methods in the study of the soil much light has been shed upon the eause and nature of the interesting and momentous biological phenomena that are there constantly in progress. Of these, the one that is of the greatest importance eomprises those changes that accompany the widespread process of disintegration and decomposition, to which reference has already been made (see Chap. I.). This resolution of dead, complex, organie compounds into simpler structures that are assimilable as food for growing vegetation is dependent upon the activities of bacteria located in the superficial layers of the ground. It is not throughout a simple process, brought about by a single, speeifie species of bacteria; but represents a series of metabolic alterations, each definite step of which is most probably the result of the aetivities of different species or groups of species, acting singly or together (symbiotically). Our knowledge upon the subject is not sufficient to permit of our following in detail the manifold alterations undergone by dead organic material in the process of deeomposition that results in its conversion into inorganic eompounds, with the formation of carbonic acid, ammonia, and water as conspicuous end-products. It suffices to 
say that, wherever dead organic matters are exposed to the action of the great group of saprophytic bacteria, in which are found many different species, the alterations through which they pass are ultimately characterized by the appearance of these three bodies. When the process of decomposition occurs in the soil, however, it does not cease at this point, but we find still further alterations-alterations concerning more particularly the ammonia. This change in ammonia is characterized by the products of its oxidation, viz., by the formation of nitrous and nitric acids and their salts; it is not a result of the direct action of atmospheric oxygen upon the ammonia, but occurs through the instrumentality of a special group of saprophytes known as the nitrifying organisms. They are found in the most superficial layers of the ground, and though more common in some places than in others, they are, nevertheless, present over the entire earth's surface. The most conspicuous example of the functional activity of this specific form of soil organism is that seen in the immense saltpetre beds of Chili and Peru, where, through the activities of these microscopic plants, nitrates are produced from the ammonia of the fecal evacuations of sea-fowls in such enormous quantities as to form the source of supply of this article for the commercial world. A more familiar example, though hardly upon such a great scale, is that seen in the decomposition and subsequent nitrification of the organic matters of sewage and other impure waters, in the process of purification by filtration through the soil, a process in which it is possible to follow, by chemical means, the organic matters from their condition as such through their conspicuous modifications to their ultimate conversion into 
ammonia, nitrous and nitric acids. In fact, the same breaking down and building up, resulting ultimately in nitrification, occurs in all nitrogenous matters that are thrown upon the soil and allowed to decay. It is largely through this means that growing vegetation obtains the nitrogen necessary for the nutrition of its tissues, and when viewed from this standpoint we appreciate the importance of this process to all life, animal as well as vegetable, upon the earth.

These very important and interesting nitrifying organisms, of which there appear to be several, have been subjected to considerable study, and are found to possess peculiarities of sufficient interest to justify a more or less detailed description. For a long time all efforts to isolate them from the soils in which they were believed to be present, and to cultivate them by the processes commonly employed in bacteriological work, resulted in failure, and it was not until it was found that the ordinary methods of bacteriological research were in no way applicable to the study of these bacteria that other, and ultimately successful, methods were devised. By these special devices nitrifying bacteria, capable of oxidizing ammonia to nitric acid, have been isolated and cultivated, and the more important of their biological peculiarities recorded by Winogradsky in Switzerland, by G. C. and P. F. Frankland in England, and by Jordan and Richards in this country. From the similarity of the properties, given by these several observers, of the nitrifying organisms isolated by them, it seems likely that they have all been working with either the same organism or very closely allied species.

The organism generally known as the nitro-monas of 
Winogradsky is a short, oval, and frequently almost spherical cell. It divides as usual for bacteria, but there is little tendency for the daughter-cells to adhere together or to form chains. In cultures they are commonly massed together, by a gelatinous material, in the form of zoogloea. They do not form spores, and are probably not motile, though Winogradsky believes he has occasionally detected them in active motion. As has been stated, they do not grow upon the ordinary nutrient media, and cannot, therefore, be isolated by the means commonly employed in separating different species of bacteria. The most astonishing property of this organism is its ability to grow and perform its specific fermentative function in solutions absolutely devoid of organic matter. It is believed to be able to obtain its necessary carbon from carbonic acid. For its isolation and cultivation Winogradsky recommends the following solution:

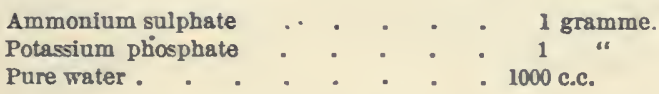

To each flask containing 100 c.c. of this fluid is added from 0.5 to 1.0 gramme of basic magnesium carbonate suspended in a little distilled water and sterilized by boiling. One of the flasks is then to be inoculated with a minute portion of the soil under investigation, and after four to five days a small portion is to be withdrawn, by means of a capillary pipette, from over the surface of the layer of magnesium carbonate and transferred to a second flask, and similarly after four or five days from this to a third flask, and so on. As this medium does not offer conditions favorable to the growth of bacteria requiring organic matter for their 
development, those that were originally introdueed with the soil quickly disappear, and ultimately only the nitrifying organisms remain. These are to be seen as an almost transparent film attached to the clumps and granules of magnesium earbonate on the bottom of the flask.

For their eultivation upon a solid medium be employs a mineral gelatin, the gelatinizing principle of which is silicic acid. A solution of from 3 to 4 per cent. of silicic acid in distilled water, and having a specific gravity of 1.02 , remains fluid and ean be preserved in flasks in this condition (Kühne). By the addition of certain salts to such a solution gelatinization occurs, and will be more or less complete aceording to the proportion of salts added. The salts that have given the best results and the method of mixing them are as follows:

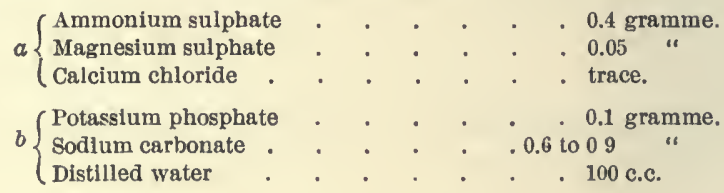

The sulphates and chloride $(a)$ are mixed in 50 c.c. of the distilled water, and the phosphate and earbonate $(b)$ in the remaining 50 e.e., in separate flasks.

Each flask is then sterilized with its contents, which after cooling are mixed together. This represents the solution of mineral salts that is to be added to the silieie acid, little by little, until the proper degree of consistency is obtained (that of ordinary nutrient gelatin). This part of the process is best condueted in the eulture dish. If it is desired to separate the eolonies, as in an ordinary plate, the inoculation and mixing of the material introduced must be done before gelatinization is 
complete; if the material is to be distributed over only the surface of the medium, then the mixture must first be allowed to solidify.

By the use of this silicate-gelatin Winogradsky has isolated from the gelatinous film in the bottom of fluids undergoing nitrification a bacillus which he believes to be associated with the nitro-monas in the nitrifying process.

Our knowlerge of these organisms is as yet too incomplete to permit of a satisfactory description of all their morphological and biological peculiarities. What has been said will serve to indicate the direction in which further studies of the subject should be prosecuted.

For further details the reader is referred to the original contributions. ${ }^{1}$

In addition to the bacteria concerned in decomposition and nitrification there are occasionally present in the soil micro-organisms possessing disease-producing properties. Conspictrous among these may be mentioned the bacillus of malignant oedema (vibrion septique of the French), the bacillus of tetanus, and the bacillus of symptomatic anthrax (Rauschbrand, German; charbon symptomatique, French). It is sometimes due to the presence of one or the other of these organisms that wounds to which soil has had access (crushed wounds from the wheels of cars or wagons, wounds received in agricultural work, etc.) are followed by such grave disturbances of the constitution.

1 Winogradsky: Annales de l'Institut Pasteur, tomes iv., 1890, and v., 1891. Jordan and Richards: Rep. State Board of Health, Mass., "Purification of Sewage and Water," 1590 , vol. ii. p. 864 .

Frankland, G. C. and P. F. : Proc. Royal Soc. London, 1590, xlvii. 
THE BACILLUS OF TETANUS.

In 1884 Nicolaier produced tetanus in mice and rabbits by the subcutaneous inoculation of particles of garden earth, and demonstrated that the pus produced at the point of inoculation was capable of reproducing the disease in other mice and rabbits. He did not succeed in isolating the organism in pure culture. In 1884 Carle and Rattone, and in 1886 Rosenbach, demonstrated the infectious nature of tetanus as it occurs in man by producing the disease in animals through the inoculation of them with the secretions from the wounds of individuals affected with the disease. In $1889 \mathrm{Kita}-$ sato obtained the bacillus of tetanus in pure culture, and described his method of obtaining it and its biological peculiarities as follows:

Method of obtaining it. Inoculate several mice subcutaneously with the secretions from the wound of a case of typical tetanus. This material usually contains not only tetanus bacilli, but other organisms as well, so that at autopsy, if tetanus results, there may be more or less of suppuration at the seat of inoculation in the mice. In order to separate the tetanus bacillus from the others that are present the pus is smeared upon the surface of the several slanted blood-serum or agar-agar tubes and placed at $37^{\circ}$ to $38^{\circ} \mathrm{C}$. After twenty-four hours all the organisms will have developed, and microscopic examination will usually reveal the presence of a few tetanus bacilli, recognizable by their shape, viz., that of a small pin, with a spore representing the head. After forty-eight hours at $38^{\circ} \mathrm{C}$. the culture is subjected to a temperature of $80^{\circ} \mathrm{C}$. in a water-bath for from threc- 
quarters to one hour. At the end of this time series of plates or Esmarch tubes in slightly alkaline gelatin are made with very small amounts of the culture and kept in an atmosphere of hydrogen (see pages 194-199). They are then kept at from $18^{\circ}$ to $20^{\circ} \mathrm{C}$, and at the end of about one week the tetanus bacillus begins to appear in the form of colonies. After about ten days the colonies should not only be examined microscopically, but each colony that has developed in the hydrogen atmosphere should be obtained in pure culture and again grown under the same conditions. The colonies that grow only without oxygen, and which are composed of the pin-shaped organisms, must be tested upon mice. If they represent growths of the tetanus bacillus, the typical clinical manifestations of the disease will be produced in these animals.

In obtaining the organism from the soil much difficulty is experienced. There are a number of sporebearing organisms lhere that are facultative in their relation to oxygen, and are, therefore, very difficult to eliminate; and there is, moreover, one in particular that, like the tetanus bacillus, forms a polar spore. This spore is, however, less round and much more oval than that of the tetanus bacillus, and gives to the organism containing it more the shape of a javelin (or clostridium, properly speaking) than that of a pin, the characteristic shape of the spore-bearing tetanus organism. It is non-pathogenic, and grows both with and without oxygen, and should, consequently, not be mistaken for the latter bacillus. It must also be borne in mind that there are occasionally present in the soil still other bacilli which form polar spores, and which, when in this stage, are almost identical in appearance with 
the tetanus bacillus; but they will usually be found to differ from it in their relation to oxygen, and they are also without disease-producing properties.

Morphology. It is a slender rod with rounded ends. It may appear as single rods, or, in cultures, as long threads. It is motile, though not actively so. The motility is rendered somewhat more conspicuous by examining the organism upon a warm stage.

FIG. 92.

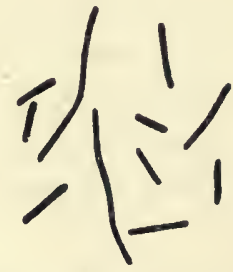

A

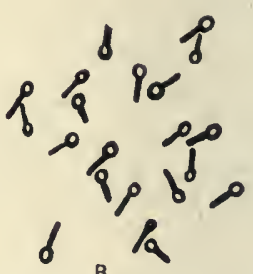

B

Tetanus bacillus. A. Vegetative stage, from gelatin culture. B. Sporestage, showing pin-shapes.

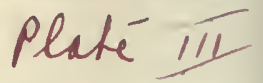

At the temperature of the body it rapidly forms spores. These are round, thicker than the cell, and usually oceupy one of its poles, giving to the rod the appearance of a small pin. (Fig. 92.) When in the spore-stage it is not motile.

It is stained by theordinary aniline staining-reagents. It remains colored under the employment of Gram's method.

Cultural peculiarities. It is an exquisite anaërobe, and cannot be brought to development under the access of oxygen. It grows well in an atmosphere of pure hydrogen, but does not grow under the influence of carbonic acid. 
It grows in ordinary nutrient gelatin and agar-agar of a slightly alkaline reaction. Gelatin is slowly liquefied, with the coincident production of a small amount of gas. Neither agar-agar nor blood-serum is liquefied by its growth.

The addition to the media of from 1.5 to 2 per cent. of glucose, 0.1 per cent. of indigo-sodium sulphate, or 5 per cent. by volume of blue litmus tincture favors its growth.

It grows well in alkaline bouillon under an atmosphere of hydrogen.

It may be cultivated through numerous generations under artificial conditions without loss of virulence.

Appearance of the colonies. The colonies on gelatin under an atmosphere of hydrogen have, in their early stages, somewhat the appearance of the colonies of the common bacillus subtilis, viz., they have a dense, felt-like centre surrounded by a fringe of delicate radii. The liquefaction is so slow that the appearance is retained for a relatively long time, but eventually becomes altered. In very old colonies the entire mass is made up of a number of distinct threads

Fig. 93.

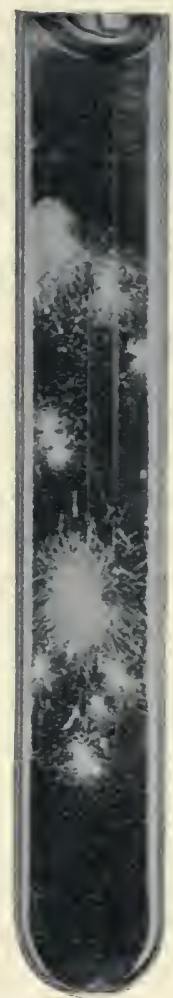

Colonies of the tetanus bacillns four days old,made by distributing the organisms through a tnbe nearly filled with glucose-gelatin. Cultivation under an atmosphere of bydrogen. (From FräneI, and PFEIFFer.) 
that give to it the appearance of a common mould. (See Fig. 93.)

In stab-cultures. In stab-cultures made in tubes about three-quarters filled with gelatin growth begins at about 1.5 to $3 \mathrm{~cm}$. below the surface, and gradually assumes the appearance of a cloudy, linear mass, with prolongations radiating into the gelatin from all sides. Liquefaction with coincident gas production results, and may reach almost to the surface of the gelatiu.

Relation to temperature and to chemical agents. It grows best under a temperature of from $36^{\circ}$ to $38^{\circ} \mathrm{C}$.; gelatin cultures kept at from $20^{\circ}$ to $25^{\circ} \mathrm{C}$. begin to grow after three or four days. In an atmosphere of hydrogen at from $18^{\circ}$ to $20^{\circ} \mathrm{C}$. growth does not usually occur before one week. No growth occurs under $14^{\circ} \mathrm{C}$. At the temperature of the body spores are formed in cultures in about thirty hours, whereas in gelatin cultures at from $20^{\circ}$ to $25^{\circ} \mathrm{C}$. they do not usually appear before a week, when the lower part of the gelatin is quite fluid.

Spores of the tetanus bacillus when dried upon bits of thread over sulphuric acid in the desiccator and subsequently kept exposed to the air, retain their vitality and virulence for a number of months. Their vitality is not destroyed by an exposure of one hour to $80^{\circ} \mathrm{C}$.; on the other hand, an exposure of five minutes to $100^{\circ} \mathrm{C}$. in the steam sterilizer kills them. They resist the action of 5 per cent. carbolic acid for ten hours, but suceumb when exposed to it for fifteen hours. In the same solution, plus 0.5 per cent. hydrochloric acid, they are no longer active after two hours. They are killed when acted upon for three hours by corrosive 
sublimate, $1: 1000$, and in thirty minutes by the same solution plus 0.5 per cent. hydrochloric acid.

Action upon animals. After subcutaneous inoculation of mice with minute portions of a pure culture of this organism tetanus develops in twenty-four hours and ends fatally in from two to three days. Rats, guinea-pigs, and rabbits are similarly affected, but only by larger doses than are required for mice: the fatal dose for a rabbit being from 0.3 to 0.5 c.c. of a welldeveloped bouillon culture. The period of inoculation for rats and guinea-pigs is twenty-four to thirty hours, and for rabbits from two to three days. Pigeons are but slightly, if at all, susceptible.

The tetanic convulsions always appear first in the parts nearest the seat of inoculation, and subsequently become general.

At autopsies upon animals that have succumbed to inoculations with pure cultures ${ }^{1}$ of the tetanus bacillus there is little to be seen by either macroscopic or microscopic examination, and cultures from the seat of inocnlation are usually negative in so far as finding the tetanus bacillus is concerned. At the seat of inoculation there is usually only a hyperæmic condition. In uncomplicated cases there is no suppuration. The internal organs do not present any change, and culture-methods of examination show them to be free from bacteria. The death of the animal results from the absorption of a soluble poison, either produced by the bacteria at the seat of inoculation or, which seems more probable, pro-

1 Animals and human beings that hare become infected with this organism in the natural way commonly present a condition of suppuration at the site of infection; this is probably not due, however, to the tetanus brcillus, but to other bacteria that bave also gained access to the wound at the time of infection. 
dnced by the bacteria in the culture from which they are obtained and introduced with them into the tissues of the animal at the time of the inoculation. In support of the latter hypothesis : mice have been inoculated with pure cultures of this organism; after one hour the point at which the inoculation was made was excised and the tissues cauterized with the hot iron; notwithstanding the short time during which the organisms were in contact with the tissues and the subsequent radical treatment, the animals died after the usual interval and with the regular symptoms of tetanus.

The poison produced by the tetanus bacillus, and to which the symptoms of the disease are due, has been isolated and subjected to detailed study; some of its peculiarities, as given by Kitasato, are as follows : ${ }^{1}$

"When cultures of this organism are robbed of their bacteria by filtration through porcelain the filtrate contains the solnble poison, and is capable, when injected into animals, of causing tetanus.

"Inoculations of other animals with bits of the organs of the animal dead from the action of the tetanus poison produce no result; but similar inoculations with the blood or with the serons exudate from the pleural cavity always result in the appearanee of tetanus. The poison is, therefore, largely present in the circulating fluids.

"The greatest amount of poison is produced by cultivation in fresh neutral bouillon of a very slightly alkaline reaction.

"The activity of the poison is destroyed by an ex- 
posure of one and one-half hours to $55^{\circ} \mathrm{C}$. ; of twenty minutes to $60^{\circ} \mathrm{C}$.; and of five minutes to $65^{\circ} \mathrm{C}$.

"By drying at the temperature of the body under access of air the poison is destroyed; but by drying at the ordinary temperature of the room, or at this temperature in the desiccator over sulphuric acid, it is not destroyed.

"Diffuse daylight diminishes the intensity of the poison. Its intensity is preserved for a much longer time when kept in the dark.

"Direct sunlight robs it of its poisouous properties in from fifteen to eighteen hours.

"Its activity is not diminished by dilutiug a fixed amount with water or nutrient bouillon.

"Mineral acids and strong alkalies lesseu its intensity."

The chemical nature of this poison is not positively known, but according to the recent observations of Brieger and Cohn it is not to be classed with the albumins in the sense in which the word is commonly used. When obtained in a pure, concentrated form its toxic properties are seen to be altered by acids, by alkalies, by sulphuretted hydrogen, and by temperatures above $70^{\circ} \mathrm{C}$. Even when carefully protected from light, moisture, and air it gradually becomes diminished in strength. When feshly prepared by the methods of the authors just cited its potency is almost incredible, $0.000,05$ milligramme being sufficient to cause fatal tetanus in a mouse weighing fifteen grammes.

THE BACILIUS OF MALIGNANT G.DEMA.

The bacillus of malignant œdema, also known as vibrion septique, is another pathogenic form almost 
everywhere present in the soil. In certain respects it is a little like the bacillus of anthrax, and was at one time confounded with it; but it differs in the marked peculiarity of being a strict anaërobe. It was first observed by Pasteur, but it was not until later that Koch, Liborius, Kitt, and others described its peculiarities in detail. It can usually be observed by inserting under the skin of rabbits or guinea-pigs small portions of garden earth, street dust, or decomposing organic substances. There results a widespread œedema, with more or less of gas production in the tissues. In the odematous fluid about the seat of inoculation the organism under consideration may be detected. (Fig. 94, A.)

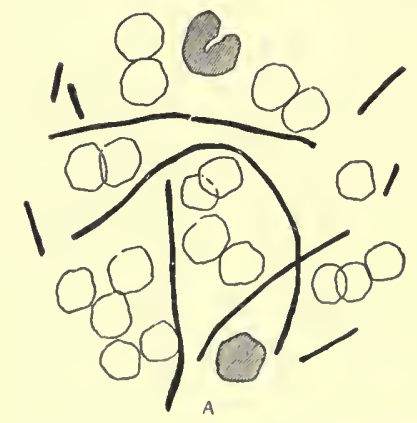

FIG. 94.

Bacilius of malignant odema.

A. Bacilli in short and long threads in oedematous fluid from site of inoculatlon of guinea-pig. (After KocH.)

B. Spore-stage of the organlsm; from culture.

It is a rod of about 3 to $3.5 \mu$ long and from 1 to $1.1 \mu$ thick-i.e., it is about as long as the bacillus anthracis, but is a trifle more slender. It is usually found in pairs, joined end to end, but may occur as longer threads; particularly is this the case in cultures. 
When in pairs the ends that approximate are squarely cut, while the distal extremities are rounded. When occurring singly both ends are rounded. (How does it differ in this respect from bacillus anthracis?) It is slowly motile, and its flagella are located both at the ends and along the sides of the rod. It forms spores that are usually located in or near the middle of the body of the cell. These may cause a swelling of the cell at the point at which they are located and give to it a more or less oval, spindle, or lozenge shape. (Fig. 94, в.)

It is a strict anaërobe, growing on all the ordinary media, but not under the access of oxygen. It grows well in a hydrogen atmosphere. It causes liquefaction of gelatin.

In tubes containing about 20 to 30 c.c. of gelatin that has been liquefied, inoculated with a small amount of the culture, and then rapidly solidified in ice-water, growth appears in the form of isolated colonies at or near the bottom of the tube in from two to three days at $20^{\circ} \mathrm{C}$. These colonies, when of from 0.5 to $1 \mathrm{~mm}$. in diameter, appear as little spheres filled with clear liquid, and are difficult, for this reason,

FIG. 95.

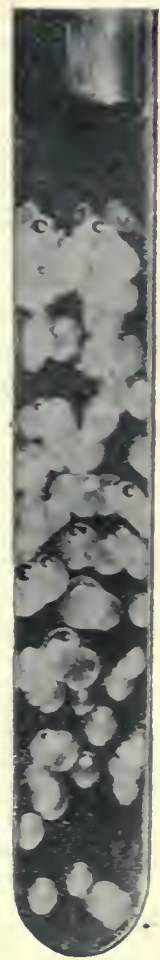

Colonies of the bacillus of malignant oedema in deep gela. tin.. culture. (After FrAskel and PFEIFFER.) to detect. (Fig. 95.)

As they gradually increase in size the contents of the spheres become cloudy and are marked by fine radiating 
stripes, easily to be detected with the aid of a small hand-lens. In deep stab-cultures in agar-agar and gelatin development occurs ouly along the track of puncture at a distance below the surface. Growth is frequently accompanied by the production of gas-bubbles.

It causes rapid liquefaction of blood-serum with production of gas-bubbles, and in two or three days the entire medium may have become converted into at yellowish, semi-fluid mass.

The most satisfactory results in the study of the colonies are obtained by the use of plates of nutrient agaragar kept in a chamber in which all oxygen has been replaced by hydrogen. The colonies appear as dull whitish points, irregular in outline, and when viewed with a low-power lens are seen to be marked by a network of branching and interlacing lines that radiate in an irregular way from the centre toward the periphery.

It grows well at the ordinary temperature of the room, but reaches its highest development at the temperature of the body.

It stains readily with the ordinary aniline dyes. It is decolorized when treated by Gram's method.

Pathogenesis. The animals that are known to be susceptible to inoculation with this organism are man, horses, calves, dogs, goats, sheep, pigs, chickens, pigeons, rabbits, guinea-pigs, and mice. Cases are recorded in which men and horses have developed the disease after injuries, doubtless due to the introduction into the wound, at the time, of soil or dust containing the organism.

If one introduce into a poeket beneath the skin of a susceptible animal about as much garden earth as can be held upon the point of a penknife, the animal fre- 
quently dies in from twenty-four to forty-eight hours. The most conspicuous result found at autopsy is a widespread cedema at and about the seat of inoculation. The œdenatous fluid is at places clear, while again it may be marked with blood; it is usually rich in bacilli (Fig. 94, A) and contains gas-bubbles. Of the internal organs only the spleen shows much change. It is large, dark in color, and contains numerous bacilli. If the autopsy be made immediately after death, bacilli are not commonly found in the blood of the heart; but if deferred for several hours, the organisms will be found in this locality also, a fact that speaks for their multiplication in the body after death. At the moment of death they are present in all the internal viscera and on the serous surfaces of the organs.

Of all animals mice are probably the most susceptible to the action of this organism, and it is not rare to find the organisms in the heart's blood, even immediately after death. They die, as a result of these inoculations, in from sixteen to twenty hours.

Where pure cultures are used for inoculation a relatively large amount must be employed, and it should be introduced into a deep pocket in the subcutaneous tissues some distance from the surface. In continuing the inoculations from animal to animal small portions of organs or a few drops of the edema-fluid should be used. The inoculation may also be successfully made by introducing into a pocket in the skin bits of sterilized thread or paper upon which cultures have been dried.

The methods for obtaining the organism in pure culture, from the cadaver of an animal dead from inoculation, are in all essential respects the same as those given 
for obtaining cultures from tissues in general, but it must be remembered that the organism is a strict anaërobe, and will not grow under the influence of oxygen (see methods of cultivating anaërobic species).

In certain superficial respects this bacillus suggests bacillus anthracis, but differs from it in so many important details that there is no excuse for confounding the two.

Note.-From what has been said of this organism, what are the most important differential points between it and bacillus anthracis? Inoculate several mice with small portions of garden earth and street dust. Isolate the organism that agrees most nearly with the description here given for the bacillus of malignant œelema. Compare its morphological, biological, and pathogenic peculiarities with those of bacillus anthracis under similar circumstances.

Still another pathogenic organism that may be present in the soil is

THE BACILLUS OF SYMPTOMATIC ANTHRAX;

bactérie du charbon symptomatique (French); Bacillus des Rauschbrand (German). It is the organism concerned in the production of the disease of young cattle and sheep commonly known as "black leg," "quarter evil," and "quarter ill," a disease that prevails in certain localities during the warm months, and which is characterized by a peculiar enphysematous swelling of the museular and subcutaneous cellular tissues over the quarters. The muscles and cellular tissues at the points 
affected are seen on section to be saturated with bloody serum, and the muscles, particularly, are of a dark, almost black color. In these areas, in the bloody transudates of the serous cavities, in the bile, and, after death, in the internal organs, the organism to be described can always be detected. It is manifest from this that the soil of localities over which infected herds are grazing may readily become contaminated through a variety of channels, and thus serve as a source of further dissemination of the disease.

The organism was first observed by Feser, and subsequently by Bollinger and others. The most complete description of its morphological and biological peculiarities is that of Kitasato (Zeitschr. für Hygiene, Bd. vi. p. 105 ; Bd. viii. p. 55). The following is from Kitasato's contributions: it is an actively motile rod of

FIG. 96.

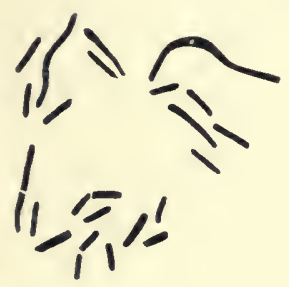

A

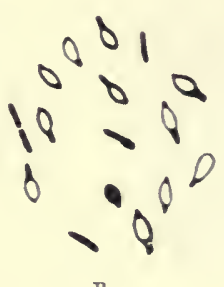

$\mathbf{B}$

Bacillus of symptomatic antbrax. (After Kitasato.)

A. Vegetating forms from a gelatin culture. B. Spore-forms from an agaragar culture.

about 3 to $5 \mu$ long by 0.5 to $0.6 \mu$ thick. It is rounded at its ends, and, as a rule, is seen singly, though now and then pairs joined end to end may occur. It has no tendency to form very long threads. (Fig. 96, A.) 
It forms spores, and when in this stage is seen to be slightly swollen at or near one of its poles, the location in which the spore usually appears. (Fig.

Fio. 97. 96, B.) It is conspienously prone to un-

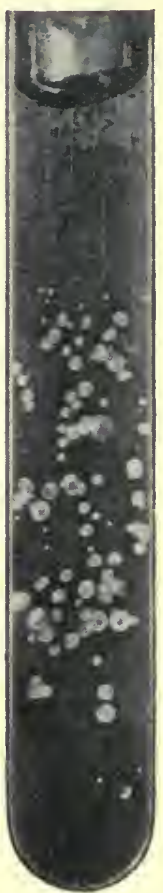

Colonies of the bacillus of symptomatic anthrax, in deep gelatin culture. (After FR $\ddot{A} K E L$ and PFeiffer.) dergo degenerative changes, and involıtion-forms are commonly seen, not only in fresh cultures, but in the tissues of affected animals as well.

Though actively motile when in the vegetative stage, it loses this property and becomes motionless when spores are forming.

It is strictly anaërobic and eannot be cultivated in an atmosphere in which oxygen is present. It grows best under hydrogen, and does not grow under carbonic acid.

The media most favorable to its growth are those containing glucose (1.5 to 2 per cent.), glycerin (4 to 5 per cent.), or some other reducing body, such as indigo-sodium sulphate, sodium formate, ete.

When eultivated upon gelatin plates in an atmosphere of hydrogen the colonies appear as irregular, slightly lobulated masses. After a short time liquefaction of the gelatin occurs and the colony presents a dark, dense, lobulated and broken centre, surrounded by a much more delieate, fringe-like zone.

When distributed through a deep layer of liquefied gelatin that is subsequently caused to solidify colonies develop at only the lower portions of the tube. The 
single colonies appear as discrete globules that cause rapid liquefaction of the gelatin, and ultimately coalesce into irregular, lobulated, liquid areas. In some of the larger colonies an ill-defined, concentric arrangement of alternate clear and cloudy zones can be made out. (Fig. 97.)

In deep stab-cultures in gelatin growth begins after about two or three days at $20^{\circ}$ to $25^{\circ} \mathrm{C}$. It begins usually at about one or two centimetres below the surface, and causes slow liquefaction at and around the track of its development. During the course of its growth gas-bubbles are produced.

In deep stab-cultures in agar-agar at $37^{\circ}$ to $38^{\circ} \mathrm{C}$. growth begins in from twenty-four to forty-eight hours, also at about one or two centimetres below the surface, and is accompanied by the production of gas-bubbles. There is produced at the same time a peculiar, penetrating odor somewhat suggestive of that of rancid butter. Under these conditions spores are formed after about thirty hours.

It grows well in bouillon of very slightly acid reaction under hydrogen, but does not retain its virulence for so long a time as when cultivated upon solid media. In this medium it develops in the form of white floceuli that sink ultimately to the bottom of the glass and leave the supernatant fluid quite clear. If the vessel be now gently shaken, these delicate flakes are distributed homogeneously through it. In bouillon cultures there is often seen a delicate ring of gas-bubbles around the point of contact of the tube and the surface of the bouillon. There is produced also a peculiar, penetrating, sour or rancid odor.

It grows best at the body temperature-i.e., from $37^{\circ}$ 
to $38^{\circ} \mathrm{C}$, but ean also be brought to development at from $16^{\circ}$ to $18^{\circ} \mathrm{C}$. Under $14^{\circ} \mathrm{C}$. no growth is seen. Spore-formation appears much sooner at the higher than at the lower temperatures. When its spores are dried upon bits of thread in the desiccator over sulphuric acid, and then kept under ordinary eonditions, they retain their vitality and virulenee for many months. Similarly, bits of flesh from the affected areas of animals dead of this disease, when eompletely dried, are seen to retain the power of reproducing the disease for a long time. The spores are tolerably resistant to the influence of heat: when subjected to a temperature of $80^{\circ} \mathrm{C}$. for one hour their virulence is not affected, but an exposure to $100^{\circ} \mathrm{C}$. for five minutes completely destroys them. They are also seen to be somewhat resistant to the action of chemicals: when exposed to 5 per cent. carbolic acid they retain their disease-producing properties for about ten hours, whereas the vegetative forms are destroyed in from three to five minutes; in corrosive sublimate solution of the strength of $1: 1000$ the spores are killed in two hours.

When gelatin cultures are examined microseopically the organisms are usually seen as single rods with rounded ends. When cultivated in agar-agar at a higher temperature spores are formed after a short time; the spores are oval, slightly flattened on their sides, thicker than the bacilli, and, as stated, frequently occupy a position inclining to one of the poles of the bacillus, though they are as often seen in the middle. The bacillus containing a spore has usually a elubbed or spindle shape.

It stains readily with the ordinary aniline dyes. It is decolorized by Gram's method. Its spores may be 
stained by the methods usually employed in sporestaining.

Pathogenesis. When susceptible animals, especially guinea-pigs, are inoculated in the deeper subcutaneous cellular tissues with pure cultures of this organism, or with bits of tissue from the affected area of another animal dead of the discase, death ensues in from one to two days. It is preceded by rise of temperature, loss of appetite, and general indisposition. The seat of inoculation is swollen and painful, and drops of bloody serum may sometimes be seen exuding from it. At autopsy the subcutaneous cellular tissues and underlying muscles present a condition of emphysema and extreme odema. The odematous fluid is often bloodstained and the muscles are of a blackish or blackishbrown color. The lymphatic glands are markedly hyperæmic. The internal viscera present but little alteration visible to the naked eye. In the bloodstained serous fluid about the point of inoculation short bacilli are present in large numbers. These often present slight swellings at the middle or near the end. They are not seen as threads, but lie singly in the tissues. Occasionally two will be seen joined end to end. If the autopsy be made immediately after death, these organisms may not be detected in the internal organs; but if not made until after a few hours, they will be found there also. In fresh autopsies only vegetative forms of the organism may be found, but later (in from twenty to twenty-four hours) spore-bearing rods may be detected. (How does this compare with bacillus anthracis?) $\mathrm{By}$ successive inoculations of susceptible animals with the serous fluid from the seat of inoculation of the dead animal the disease may be reproduced. 
Cattle, slicep, goats, guinea-pigs, and mice are susceptible to infection with this organism, and present the conditions above described; whereas horses, asses, and white rats present only local swelling at the site of inoculation. Swine, dogs, cats, rabbits, ducks, chickens, and pigeons are, as a rule, naturally immune from the disease.

Though elosely simulating the bacillus of malignant œedema in many of its peculiarities, this organism can, nevertheless, be readily distinguished from it. It is smaller; it does not develop into long threads in the tissues; it is more actively motile, and forms spores more readily in the tissues of the animal than does the bacillus of malignant œedema. In their relation to animals they also differ, viz., eattle, while conspicuously susceptible to symptomatic anthrax, are practically immune from malignant odema; and while swine, dogs, rabbits, chickens, and pigeons are readily infected with malignant œdema, they are not, as a rule, susceptible to symptomatic anthrax. Horses are affected only locally, and not seriously, by the bacillus of symptomatic anthrax; but they are conspicnously susceptible to both artificial inoculation and natural infection by the bacillus of malignant œdema.

'The distribution of the two organisms over the earth's surface is also quite different. The redema bacillus is present in almost all soils, while the bacillus of symptomatic anthrax appears to be confined to certain localities, especially places over which infeeted herds have been pastured.

A single attack of symptomatic anthrax, if not fatal, affords subsequent proteetion, while infection with the malignant œdema bacillus appears to predispose to recurrence of the disease.

(Baumgarten.) 


\section{CHAPTER XXVI.}

Infection and immunity-The types of infeclion; intimate nature of infection-Septicæmia, toxæmia, va riations in infectious processes-Immunity, natural and acquired-The hypotheses that have been adranced in explanation of immunity-Conclusions.

Ax organism capable of producing disease we call pathogenic or infective, and the process by which it produces disease we know as infection. Diseases, therefore, that depend for their existence upon the presence of bacteria in the tissues are infectious diseases.

What is the intimate nature of this process we call infection? Is it due to the mechanical presence of living bacteria in the body, or does it result from the deposition in the tissues of substances produced by these bacteria that are either locally or generally incompatible with life? Or, is the group of pathological alterations and constitutional symptoms seen in these diseases the result of abstraction from the tissues, by the bacteria growing in them, of substances essential to their vitality? These are some of the more important of the questions that present themselves in the course of analysis of this interesting phenomenon.

Let us look into several typical infectious diseases, note what we find, and see how far the observations thus made will aid us in formulating an opinion. We begin with a study of those diseases in which there is a general infection-i.e., in which there is a general distribution of the infective agents throughout the body. This group comprises the "septicæmias," and of them 
the disease of animals known as anthrax represents a type of the condition. If the cadaver of an animal dead of anthrax be examined by bacteriologieal methods, it will be discovered that there is present in all the organs and tissues an organism, a bacillus, of definite form and biological characteristics; and if the organs, and tissues generally, be subjected to microscopic examination this same organism will be found always present and always located within the capillaries. At many points it will be seen crowded in the capillaries in such numbers as almost, if not quite, to burst them, and very commonly their lumen for a considerable extent is entirely occluded by the growing bacilli. In such a case as this we might be tempted to conclude that death had resulted from mechanical interference with the capillary circulation. Suppose, however, we subject the cultures obtained from this animal to conditions, either chemical or thermal, that are not particularly favorable to their normal development, and from time to time inoculate susceptible animals with the cultures so treated. The result will be that, as we continue to expose our cultures to unfavorable surroundings, the period of time that is required for them to cause the death of animals will, in some cases, gradually become extended, until finally death will not ensue at all after inoculation. If, as these animals die, a careful record of the conditions found at autopsy be kept and compared, it will ultimately be noticed that the animals that die a longer time after inoculation present conditions more or less at variance with those seen in the original animal that died more quickly after having been inoculated. These differences usually consist in a diminution of the number of bacilli that appear upon culture plates from the 
blood and internal organs, and in a lessening in the amount of mechanical obstruction offered to the circulation through plugging of the capillaries by masses of bacilli, as detected by microscopic examination of sections of the organs; indeed, this latter condition may often have almost, if not quite, disappeared. We see here an animal dead from the invasion of the same organism that produced death in the first animal, but with little or none of the appearances to which we were inclined to attribute the death of that animal. It is apparent, then, that this organism with which we have been working can destroy the vitality of an animal in a way other than by mechanically obstructing its bloodressels; it possesses some other means of destroying life. Possibly its growth in the tissues is accompanied by the production of soluble poisons, which when present in the blood are not compatible with life.

Let us see if the study of another group of infections will furnish any evidence in support of such an hypothesis. Introduce into the subcutaneous tissues of a guinea-pig a small amount of pure culture of the bacillus of diphtheria. In three or four days the animal dies. We proceed with our autopsy in exactly the same way that we did with the animals dead of anthrax, and will be astonished to find that the organs, blood, and tissues generally are sterile, ${ }^{1}$ in so far as the presence of the organism with which the animal was inoculated is concerned, and by both culture and microscopic methods it is possible to detect them only at the site of inoculation, where they were deposited. It is very evident that we have here a condition with which mechanical

1 In by far the greater number of cases this is true, but under particnlar circumstances there are exceptions. 
plugging of the capillaries could have had nothing to do, for there are no organisms in the blood to interfere with its circulation. Our hypothesis then with regard to the condition found in our first case of anthrax is again not tenable. Similarly, if an animal that has died of tetanus be examined, we do not find the bacilli in the tissues and circulating fluids generally, and, indeed, often fail to find them at the point of injury. Plainly, these fatal results follo ring upon inoculations with the diphtheria and the tetanus bacillus, with their accompanying tissue-changes, occur from the prescnce of a something that cannot be detected by either cultural or microscopic methods, and this something can be only a soluble substance that is produced by the growing bacteria at the site of inoculation, gains access to the cireulation, and through this channel causes death, for it is hardly to be imagined that the insignificant wound made in the course of inoculation could per se have had this effect. In other words, these latter animals have died from what is called toxcemia (poison in the blood), a condition conspicuously different from septicemia, as seen in our first animal dead of anthrax.

There are, again, other infectious diseases, many of which are known to present variations from what might be considered a typical course, that may still further serve to support the view that infection is a process in which the mechanical effect of organisms in the circulating fluids is of little consequence. Conspicuous among these are the infections that follow upon the introduction into the tissues of susceptible animals of cultures of micrococcus lanceolatus (pneumococens), of the bacillus of chicken cholera, and of the organisms concerned in the production of the so-called "hemor- 
rhagic septicæmias." When running their normal course these organisms cause typical septicæmias in susceptible animals; but often, from causes not entirely clear, the animals die with only local lesions, or with but very few organisms in the internal viscera. We see here conditions analogous to those observed in the two experiments with anthrax, viz., we find a group of diseases that are properly classed as septicaemias, because of the usual general invasion of the body by the organisms concerned in their production, but which frequently assume a purely local character - in both instances proving fatal to the animal infected. From what we have seen it is manifestly probable that, whether these diseases be designated as septicæmias or toxæmias, death is produced in all instances by the poisonous products resulting from the growth of the infecting bacteria. In the case of typical anthrax, and other varieties of septicemia, the production of this poison is associated with the general dissemination of the organisms throughout the body, while in those infections often referred to as toxæmias, of which diphtheria may be taken as a type, the poison is produced by the organisms that remain localized at the site of invasion, and is thence disseminated throughont the body by the circulating fluids.

Infection thus far, then, appears to be a chemical process.

Through special investigations that have been made upon the products of growth of certain pathogenic bacteria this opinion has received further confirmation; it has been found possible by the use of appropriate methods to isolate, from among the mass of material in which certain of these organisms have been artificially 
cultivated, substances which, when separated from the bacteria by which they were produced, possess the power of causing in animals all the constitutional symptoms and pathological tissue-changes that are seen to occur in the course of infection by the organisms themselves. In some instances these poisons-toxins, ${ }^{1}$ as they are collectively called-appear to be the direct result of metabolic changes brought about by bacteria in the medium or tissues in which they may be developing i.e., they are products of nutrition that pass readily into solution, as is conspicuously seen in the case of the bacillus of diphtheria and of tetanus when under both artificial cultivation and in the animal body. Many bacteria which do not possess the power of generating or secreting such poisons may, nevertheless, have intimately associated with their protoplasmic bodies poisonous substances that can only be isolated by particular methods; thus the toxins of bacillus tuberculosis and of spirillum cholerce Asiatica are much more conspicuously present in the protoplasm of these bacteria than in the fluids in which they have grown, and Buchner has isolated from several species of bacteria " bacterioproteins" having the common properties of solubility in alkalies, resistance to the boiling temperature, attraction of leucocytes (positive chemotaxis), and pyogenic powers.

There is as yet little agreement of opinion as to the chemical nature of toxins, but it is probable that the group comprises different bodies of the nature of globulins, nucleo-albumins, peptones, albumoses, and enzymes or ferments.

1 "Toxins" is the term commonly used to designate amorphous poisons of a proteid nature; whlle "ptomaines" is the term used to signify nitrogenous poisons that are crystallizable. 
Toxic ptomaïnes are probably not conspicuously concerned in producing the characteristic symptoms of infection, as they are absent from cultures of certain highly pathogenic bacteria.

In some instances the production of the poisonous principles, even under artificial conditions of cultivation, is of a most astonishing nature, and poisons result that, in the degree of their toxicity, exceed anything hitherto known to us. For instance, the potencies of the poisons that have been isolated from cultures of bacillus diphtherice and of the bacillus of tetanus have been carefully determined by experiments upon animals, and it has been found that 0.4 milligramme of the former is capable of killing eight guinea-pigs, each weighing 400 grammes, or two rabbits, each weighing 3 kilogrammes (Roux and Yersin 1 ); and that 0.0001 milligramme of the latter will produce tetanus in a mouse, with all the characteristic manifestations of the disease (Brieger and $\left.\mathrm{Cohn}^{2}\right)^{3}$

In short, infection may be best conceived as a contest between the invading organisms on the one side and the resisting tissues of the animal body on the other, the weapons of offence of the former being the poisonous products of their growth, the toxins, and the means of defence possessed by the latter being substances which are, so to speak, antidotal to these poisons. To these substances possessed by the animal body for resisting infection the name "alexines" has been given by Buchner, while the name "defensive proteids" is sug-

1 Annales de l'Institut Pastenr, tome iil., 1859, p. 287.

2 Zeitschr. für Hygiene u. Infektionskrankheîten, 1893, Bd. xv. Heft i.

3 Through the nse of more recently devised methods we are enabled to increase still further the toxicity of these poisons; especially is this the case with regard to the diphtheris toxin. 
gested by Hankin. If the tissue-elements are not of sufficient vigor to neutralize the bacterial poisons, the bacteria are victorious and infection results; while, if there be failure to establish a condition of disease, the tissues are victorious, and are said to be resistant or to possess immunity from this particular form of infection.

It is a common observation that certain human beings and animals are more susceptible to the different forms of infection than are others, and that some species of animals are apparently not at all liable to particular diseases; in other words, they are naturally immune from the maladies. The term "natural immunity," as now employed, implies a congenital condition of the individual or species, a condition peculiar to his idioplasm, which has been transmitted to him as a tissue-characteristic through generations of progenitors.

Again, it is often observed that an individual or animal after having recovered from certain forms of infection has thereby acquired protection from subsequent attacks of like character; in other words, they are said to have acquired immunity from this trouble. "Acquired immunity" implies, therefore, a condition of the tissues of an individual, not of necessity peculiar to other members of the race or species, that has resulted during his life from the stimulation of his integral cells by one or another of the specific, infective irritants that may have been purposely introduced, or aecidentally gained aecess to his body.

The problem involving the explanation of these interesting observations has afforded material for reflection and hypothesis for a long time. It is only through investigations that have been condueted during the past few years that it has met with anything approaching 
reasonable solution, and even now there remain a number of important points that are more or less veiled in obscurity.

Conspicuous among the observers who have endeavored to explain the modus operandi of immunity may be mentioned Chauveau, Pasteur, Metchnikoff, Buchner, Flügge and his pupils (Smirnow, Sirotinin, Bitter, Nuttall), Fodor, Hankin, and Pfeiffer. In the following pages we will present briefly the result of investigations by these various authors.

In 1880 Chauveau' suggested an explanation for the phenomenon of immunity that has since been known as the "retention hypothesis." It is, in short, as follows: that the immunity commonly seen to exist in animals that have passed through an attack of infection from a subsequent outbreak of the same malady, and likewise the immunity that has been produced artificially by vaccination, exist by virtue of some bacterial product that has been retained or deposited in the tissues of those auimals, and that this product by its presence prevents the development of the same organisms if they should subsequently gain access to the body.

Bearing upon this view the experiments of Sirotinin, ${ }^{2}$ made with cultures of various pathogenic bacteria, demonstrated that, in so far as culture-experiments were concerned, the only substance produced by growing bacteria that could be in any way inimical to their further development were substances that gave rise to alterations in the reaction of the medium in which they were developing-i.e., acids or alkalies prodnced by the bacteria themselves. So long as the organisms were not 
actually dead from exposure to these substances correction of the abnormal reaction was followed by further development of the organisms. Sirotinin also states that materials eontaining the products of growths of bacteria, so long as they are maintained at a nentral or only slightly alkaline reaction, serve very well as media upon which to cultivate again the same organism that prodnced them, providing the nutritive elements have not been entirely exhausted. He remarks that, if in such a concentrated form as we find the life-products of bacteria in the medium in which they are growing, no inhibitory compounds beyond acids and alkalies are to be detected, it is hardly probable that they are produced in the tissues of the living animal, and retained there, to a degree sufficient to prevent the growth of bacteria that may subsequently gain entrance to these tissues, after the disappearance of the organisms concerned in the primary invasion. On the other hand, Salmon and Smith, ${ }^{1}$ Roux and Chamberland, ${ }^{2}$ and others had demonstrated that a sort of immunity against certain forms of infection may be afforded to susceptible animals by the injection into their tissues of the products of growth of particular organisms which, if themselves introduced into the animal body, would produce fatal results. In the light of subsequent experiments, lowever, the interpretation of this phenomenon is probably not that claimed by the supporters of this hypothesis.

As opposed to the view of Chauveau, Pasteur ${ }^{3}$ and certain of his pupils believed that the immunity frequently afforded to the tissues by an attack of infection,

1 Proc. of the Biol, Soc., Washington, D. C., 1886, vol. 1 il.

2 Annales de l'Institut Pasteur, 1888-'89, tomes 1., 11.

3 Bull, de l'Acad. de Méd., 1881). 
or following upon vaccination against infection, was due rather to an abstraction from the tissues, by the organisms that were concerned in the primary attack, of a something that is necessary to the growth of the infecting organism should it gain entrance to the body at any subsequent time. This view is known as the "exhaustion hypothesis."

As to the exhaustion hypothesis of Pastenr, there is, as yet, no evidence whatever for its support. The work of Bitter, ${ }^{1}$ which was undertaken with the view of determining if, in the process of acquiring immunity, there occurred this exhaustion from the tissues of material necessary to the growth of bacteria that might gain entrance to them at some later date, gave only negative results. The flesh of animals in which immunity had been produced contained all the elements necessary for the growth and nutrition of the bacteria against which the animals had been protected, just as did the flesh of nou-vaccinated animals.

In $188 \pm$ Metchnikoff ${ }^{2}$ published the first of a series of observations upon the relation that is seen to exist between certain of the mesodermal cells of lower animals and insoluble particles that may be present in the tissues of these animals. The outcome of these investigations was the establishment of his well-known doctrine of phagocylosis, the principle of which is that the wandering cells of the animal organism, the leucocytes, possess the property of taking up, rendering inert, and digesting micro-organisms with which they may come in eontact in the tissues. Metchnikoff believed that

Zeitschr. fur Hygiene, 18ss, Bd. iv.

: Arbeiten aus dem Zoülogischeu Institut der Unirersitut Wien., 1S54, Bd. v. Fortschritte der Med., 18s4, Bd. ii. 
in this way immunity from infection may in many, if not all, cases be explained. He believed that susceptibility to or immunity from infection was essentially a matter between the invading bacteria on the one hand, and the leucocytes of the tissues on the other. The success or failure of the lencocytes in protecting the animal against infection depends, according to this doctrine, entirely upon the efficiency of the means possessed by them for destroying bacteria. When these means are of sufficient vigor to bring about the death of the bacteria the tissues are victorious, but when the poisons generated by the bacteria are potent to arrest the phagocytic action of the leucocytes then the tissues succumb and infection results.

Has this doctrine of phagocytosis, as advanced by Metchnikoff, stood the test of experimental criticism ? Evidence that has acerued since the time of its suggestion has rendered questionable the advisability of its unconditional adoption in the strict sense that Metchnikoff propounded it. The later studies of a number of investigators indicate that while the lencocytes play a most important part in the phenomenon of inmunity, it is hardly likely that this always oceurs through their taking up within themselves and digesting invading bacteria, as Metchnikoff believes, but rather that their part in the process is to secrete protective chemical substances that are thrown into the cireulating blood, and which, in part at least, comprise the defensive bodies to which Buchner has given the name "alexines."

The first severe blow that Metehnikoff's theory of phagocytosis received was given by Nuttall, ${ }^{2}$ in his

I See ILahn. Arch. fur Hygiene, 1895, Bd. xxv. p. 105.

2 Zeitschrift fur Hyglenc, 1858, vol. iv. 
work upon the bactericidal action of the animal economy. In these experiments Nuttall showed positively that the destruction of virulent bacteria in the blood of animals was not necessarily dependent upon the immediate presence of living leucocytes, but that the serum of the blood, when quite free from cellular elements, possessed this power to a degree equal to that of the blood when all the constituent parts were present. In the blood, as such, phagocytosis could be seen, but, as a rule, the bacteria presented evidence of having undergone degenerative changes before they had been taken up by the wandering cells.

Contrary to the notions in existence at the time, Traube and Gscheidlen, ${ }^{1}$ as far back as 1874 , demonstrated that considerable quantities of septic material could be injected into the circulating blood without apparently any effect upon the animal. As a result of these experiments, the question that naturally presented itself was: Does the animal organism possess the power of rendering septic organisms inert, and if so, to what extent? Their further work showed that appreciable numbers of living bacteria could be injected into the circulation of warm-blooded animals without producing any noticeable effect. - Particularly was this the case with dogs. If they injected into the circulation of a $\operatorname{dog}$ as much as 1.5 c.e. of decomposing fluid, the blood drawn from the animal after from twenty-four to forty-eight hours showed no especial tendency to decompose, though it was kept under observation for a long time. They believed this power, of rendering living organisms inert, to be possessed by the

1 Jahresbericht đer Schlesischen Ges. für Caltur., 1574; Jahr. iii. p. 179. 
circulating blood to only a limited degree, for, after the injection of much larger amounts of the putrid fluid into the blood of the animal, death usually ensued in from twenty-four to forty-eight hours. The blood drawn from the animal just before death contained the living bacteria of putrefaction, and underwent decomposition. They attributed the germicidal phenomenon to the action of the "ozonized oxygen of the corpuscles of the blood."

In 1882 Rauschenbach ${ }^{1}$ demonstrated that, in the process of coagulation, fibrin was formed not as a specific product of the action of the colorless elements of the blood alone, but also as a result of the combined action between all animal protoplasms and healthy blood plasma, and that in the process there was always a disintegration of the leucocytes that were present. In 1884 Groth $^{2}$ demonstrated further that such a disintegration of leucocytes occurred in normal circulating blood, though here it was not accompanied by coagulation. The results of these observations suggested the question: Does such a disintegration occur when vegetable protoplasm is introduced into the blood? For the purpose of answering this question, Grohmann, ${ }^{3}$ a pupil of Alexander Schmidt, undertook to study the action of the circulating blood upon the vegetable protoplasm of bacteria.

He noticed that elotting of the blood of the horse was very much accelerated by the addition to it of cer-

1 Ueber die Wechselwirkung zwischen Protoplasma und Blutplasma. Dissertation, Dorpat, 1882.

2 Ueber die Schicksale der farblosen Elemente in kreisendem Blut. Dissertation, Dorpat, 1884.

${ }^{3}$ Ueber die Elnwirkung des zellenfreien Blutplasma auf einige planzliche Mikro-organismen. Dissertation, Dorpat, 1884. 
tain bacteria; that at the same time the development of the bacteria was checked, and in the case of the pathogenic varieties their virulence was diminished. This was particularly the case when the anthrax bacillus was employed.

Grohmann seems to have appreciated the significance of this observation, though he took no steps to study the subject more closely. He remarks that the system probably possesses, in the plasma of the blood, a body having disinfectant properties (loc. cit., pp. 6 and 33). This work, however, was not conducted according to the more exact methods of modern bacteriological research, so that the complete demonstration of this phenomenon must be accredited to Nuttall.

Since the publication of Nuttall's work his results have received confirmation from all sides. Fodor, ${ }^{1}$ Buchner, ${ }^{2}$ Lubarsch, ${ }^{3}$ Nissen, ${ }^{4}$ Stern, ${ }^{5}$ Prudden,${ }^{6}$ Charrin and Roger, ${ }^{7}$ and many others have continued in the same line, and have all made practically the same observation.

After the demonstration by Nuttall that the serum of the blood was directly detrimental to the vitality of certain pathogenic bacteria, it became the work of a number of investigators to determine to which element of the serum this property is due, or if it is a function of the serum only as a whole.

In the course of Buchner's experiments it was demonstrated that the serum was robbed of this property by

1 Centr. f. Bakteriologie u. Parasitenkunde, 1890, vol. vii. No. 24.

- Archiv für Hygiene, 1890, rol. x. parts 1 and 2.

3 Centr f. Bakt. n. Parasitenkunde, 1889, vol. vi. No. 18.

+ Zeitschr. für Hygiene, 1859, vol. vi. pert 3.

5 Zeitschr. für klin. Med., 1890, vol. viii. parts 1 and 2.

- N. Y. Med. Record, 1890, rol. xxxvii. pp. 85,86 .

i Soc. de Biol. de Paris. 
an exposure to a temperature of $55^{\circ} \mathrm{C}$. for half an hour; that its efficacy as a germieide was not diminished by alternate freezing and thawing; that by dialysis or extreme dilution with distilled water its germicidal activity was diminished, or completely checked; but that an equal dilution could be made, if sodium chloride solution (0.6-0.7 per cent.) was substituted for the distilled water, without the bactericidal action of the serum losing any of its power. From this lie concluded that the active element in this phenomenon is a living albumin, an essential constituent of which is sodium chloride, and which, when robbed of this salt, either by dialysis or dilution, becomes inert in its behavior toward bacteria. For this or these germicidal constituents of the blood he suggested the name "alexines.",

He found, moreover, that the activity of the serum alone against bacteria was greater than when the cellular elements of the blood were present. This he explains by the assumption that in the serum alone the germicidal element predominates, whereas in the blood, as such, outside of the body, it is still present, but its influence is counteracted by the nutrition offered to the bacteria by the disintegrated cellular elements; so that here the nutritive feature is most conspicuous, and the destructive activity toward bacteria is less effectual.

A closer study of the nature of this germicidal element in the body of animals was made by Hankin and Martin. ' The former isolated from the spleen and lymphatic glands a body-a globulin-which in solution possesses gernicidal properties. 
Similar germicidal, ferment-like globulins have been isolated from the blood by Ogata, ${ }^{1}$ aud in their studies upon tetanus Tizzoni and Cattani $^{2}$ found a body that was antagonistic to the poison produced by the organism of this disease.

According to the observations of Vaughan, ${ }^{3}$ the most important germicidal or protective agents possessed by the body are the nucleins; and Kossel has shown that the cholera vibrio, streptococeus, staphylococcus, and typhoid bacillus are destroyed by 0.5 per cent. solution of nucleinic acid.

Hankin believes the globulins or "defensive proteids" that he has discovered and the albuminoid bodies studied by Buchner to be identical. The most interesting and, in the light of work that has appeared since, the most important, of Hankin's observations were not those upon the power of these globulins to destroy the vitality of living organisms, but rather those upon the relation between them and the poisonous metabolic products of growth of the organisms. For example, if the poisonous products of virulent anthrax bacilli be isolated and mixed with the globulin extracted from normal tissues, the experiments of Hankin showed a directly destructive action on the part of the bacterial products. He found that the amount of poisonous albumose produced by the attenuated anthrax bacilli, that are employed as vaccines, was much less than that produced by the organisms possessing full virulence, and he suggests that perhaps the protective influence of vaccinations that are practised by introducing into the animal the organisms that have been attenuated in vir-

1 Centr. f. Rakt. u. Parasitenkende, 1891, vol. ix. p. 599.

2 Ibid , p. 695 .

3 Vaughan: Medical News, May 20, 159s. 
ulence is dne to a gradual tolerance acquired by the cells of the tissues to the action of the poison when produced in these small quantities; in the same way that a tolerance was acquired by the tissues for the venom of the rattlesnake in the experiments of Sewall ${ }^{1}$ (and more recently in the work of Fraser, Calmette, Weir Mitehell, and others), and similar to that following the injection into the tissues of small quantities of hemialbumose, which in large amounts rapidly proves fatal.

Of utmost importance to these studies of the blood and fluids of the body are the experiments of Belıring and Kitasato ${ }^{2}$ upon the production of immunity to tetanus. In their investigations upon the blood of animals subjected to these experiments it was found that it was not only possible to render animals immune from this disease, but that the serum of the blood of these immunized auimals afforded immunity when injected into the peritoneal cavity of other animals that had not been so protected; and moreover, that this serum possesses curntive powers over the disease after it has, in some cases, been in progress for a time. They found, further, that the serum of animals that had been rendered immune from tetanus, when brought in contact with the poison of tetanns, completely destroyed its poisonous properties, and that the serum from animals or from human beings that do not possess immunity from this disease has no such power.

The demonstration by Behring and Kitasato of the faet that the serum of an immunized animal can not ouly confer immunity to another susceptible animal,

1 Journal of Physiology, 1887, vol. vil. p. 203.

2 Behring and Kitasato: Deutsche med. Woch., 1890. Bd, xvi. p. 1113. 
but in the case of tetanus (and diphtheria, as subsequently demonstrated by Behring and his associates), cure the disease after it is already in progress, is one of the most important steps that has been made in this entire field of study. The subsequent application of the principle involved in that observation by Behring and his colleagues, in their successful efforts to devise a cure for diphtheria in man, has resulted in a triumph which marks an epoch in modern scientific medicine. The same principle has been employed for obtaining curaative agents against other forms of infection and intoxication, notably, of Asiatic cholera, typhoid fever, lobar pueumonia, streptococeus and staphylococcus infection, rabies, tuberculosis, bubonic plagne, syphilis, vaccinia, and serpent venom; but unfortunately, as yet, with but indifferent success; certainly in no case to the same favorable degree as has been seen in the treatment of diphtheria with antitoxic serum.

Another hypothesis in explanation of the immunity acquired by the tissues of the animal organism is that advanced by Buchner, ${ }^{1}$ who suggests that in the primary infection, from which the animal has recovered, there has been produced a reactive change in the integral cells of the body that enables them to protect themselves agaiust subsequent inroads of the same organism. Thongh somewhat more vague at first glance than the other theories in regard to this phenomenon, it is, nevertheless, in the light of subsequent research, most probably the correct explanation of the establishment of immunity in many, if not all, cases. Experiments that bear directly upon this idea have demonstrated that, if

${ }^{1}$ Buchner : Eine nene Theorie über Erzielung von Immunität gegen Infektionskrankheiten. München, 1853. 
animals be subjected to injections of the poisonous products of growth of certain virulent bacteria, they respond to this treatment by more or less pronounced constitutional reactions, and that during this period, and for a short time following, they are protected from the invasion of the virulent bacteria themselves. This observation has, moreover, not been confined to those cases in which injections of the products of growth have been followed by inoculations with the bacteria by which they were produced, but, what is still more interesting and confirmatory of Buclnner's view, it is claimed that a sort of protection from certain specific infections can also be afforded to animals by the injection into them of cultures of entirely different species of bacteria, or their products, and that in some cases these are not of necessity of the disease-producing variety. For instance, Emmerich and Mattei ${ }^{l}$ claim to have rendered rabbits insusceptible to anthrax through injections into them of cultures of the streptococcus of erysipelas.

This, they claim, is not due to any antagonism between the organisms themselves, for in eulture experiments the two organisms grew well together, without any alteration in their pathogenic properties, but rather to the induction of a tissue-activity by which resistance to the inroads of the virulent bacilli was established. Emmerich and Mattei interpret this " reactive tissueeliange" as a power acquired by the integral cells of the body, through the influence of a stimulus, of generating a product that is detrimental to the pathogenic activity of the anthrax bacilli.

1 Emmerich und Matti : Fortschritte der Medizin, 1887, p. 653. 
Pawlowsky, ${ }^{1}$ who obtained similar results from the introduction into the animal of cultures of bacillus prodigiosus, of staphylococcus pyogenes aureus, and of nicrococcus lanceolatus, believes them to be due to the induction of increased energy on the part of the wandering cells, preparing them thus for the difficult task of destroying the more virulent organisms with which the animal is subsequently to be inoculated.

Protection that is afforded in this way apparently contraindicates a specific relation between the morbific elements of particular infections and the protective substances that are present in the body of the animal that has been rendered insusceptible to them. It is probable, however, that this is only apparent, and that the observations of Emmerich and Mattei and of Pawlowsky can be explained in another way : in the blood of animals there is present what may be termed a normal protective (Buchner's alexines) having no specific relations to any particular variety of infection, but serving to protect the animal more or less completely against all bacterial invasion. By the methods employed in the preceding experiments it seems likely, in the light of more recent work, that this normal antidote was simply temporarily accentuated through the tissue-stimulation resultant upon the treatment that the animals had received, for it has never been shown to be possible to bring about as high or as permanent a degree of immunity in an animal from a particular disease as that which can be obtained by the use of the specific microorganism causing the disease, or the products of its growth, especially the latter.

1 Pawlowsky : Virchow's Arch., vol. criii. p. 49. 
A striking illustration of this protective reaction on the part of the animal tissues is brought out in the course of R. Pfeiffer's ${ }^{1}$ experiments on Asiatic cholera. He found that it was possible to confer immunity to animals from this disease; that the blood-serum of such animals protected susceptible animals into which it was injected against what would otherwise be a fatal dose of the cholera spirillum; that the peritoneal fluids of the artificially immunized animal had an almost instantaneous disintegrating, bactericidal action upon living cholera spirilla that were injected into the peritoneal cavity; that the serum from the immune animal had no such effect upon cholera spirilla when tried in the test-tube; but if virulent cholera spirilla be injected into the peritoneum of an animal that was not immune, and this be at once followed by an intraperitoneal injection of serum from an immune animal, almost instantly the peculiar disintegration of the bacteria, as observed in the peritoneum of the immune animal, could be detected. This latter observation is of the utmost importance in its bearing on Buchner's hypothesis, for we see here a serum from an immune animal that is capable of conferring immunity; capable, on injection into a susceptible animal, of endowing its fluids with the peculiar disintegrating, germicidal function noted in the peritoneum of the immune animal from which the serum may have originated; incapable of bactericidal activity outside the body, but the influence of which in the peritoneum of a susceptible animal is to eall forth at once this interesting phenomenon. Manifestly the germicidal substance in this case is neither contained

1 Pfeiffer: Zeit. f. IIyg. u. Infektionskrankheiten, Bd. xviil. p. 1; Ebenda, Bd. xx. p. 198. 
in the protective serum nor in the peritoneum of the susceptible animal before receiving the serum, but is generated by the tissues as a result of the specific irritation of a something contained in this serum; in other words, in consequence of a reaction on the part of the peritoneal tissues, or possibly those of the entire aninal.

The experiments of G. and F. Klemperer' upon acute fibrinous pneumonia, though too limited in extent to be accepted as conclusive, have, nevertheless, offered a number of most significant suggestions, not only in connection with several obsenre features of this discase, but also in relation to the establishment of tissue-resistance.

They found but little diffienlty in affording immunity to animals that are otherwise susceptible to the pathogenic action of the organisms concerned in the production of this disease, ${ }^{2}$ by the introduction into their tissues of the products of growth of the organisms from which the latter had been separated. The immunity thus produced is seen in some cases to last as long as six months; again it is seen to disappear suddenly in a way not to be explained. It was seen in one case to be hereditary, probably having been conferred upon the young, during the nursing-period, through the milk of the mother, as Ehrlich ${ }^{3}$ has shown to oceur in animals artificially immunized from abrin, ricin, and robin.

The energy of the substance that has the power of affording immunity was seen to be very much increased

1 G. and F. Klemperer: Berliner klin. Wochenschr., 1891, Nos. 34 and 35.

- Animals do not, as a rule, present the pnenmonic changes seen in human beings. The introdnction of micrococcus lanceolatus into their tissues resnlts, in the case of susceptible animals, in the prodnction of septicrmia.

Ehrlich : Zeit. für Hygiene n. Infektionskrankheiten, 1992, Bd. xii. p. 183. 
by subjecting it to temperatures somewhat higher than that at which it was produced by the bacteria. The Klemperers found. that if this substance was heated to a temperature of from $41^{\circ}$ to $42^{\circ} \mathrm{C}$. for three or four days, or to $60^{\circ} \mathrm{C}$. for from one to two hours, its intravenous injection was followed by complete immunity in from three to four days; whereas, if the unwarmed material was used, immunity did not appear before fourteen days, and then only after the employment of relatively large amounts. Moreover, when the previously heated products are introduced into the circulation of the animal the systemic reaction is of but short duration; but if the unwarmed substance is employed, immunity is manifest only after the appearance of considerable elevation of temperature, which lasts for a long time.

In explanation of these differences they suggest that, in the latter case, the high fever that is seen to occur in the animal may serve to replace the warming to which the bacterial products had not previously been subjected, and which is necessary before they are in a position to bring about the condition of immunity. They claim that the bacterial products employed to produce immunity in this case are not, in reality, the immunityaffording substance, but that they are only the agents that bring about in the tissues of the animal alterations that result in the production of another body that protects the animal. In support of this, their argument is that several days are necessary for the production of immunity by the introduction into the animal of the bacterial products; whereas, if the blood-serum of this animal, which is now protected, be introduced into the circulation of another animal, no such delay is seen, but instead, the animal is forthwith protected. In the 
former case the actual protecting body had first to be manufactured by the tissues; whereas, in the second it is already prepared, and is introduced as such into the second animal.

They found the serum of artificially immune animals to be not only capable of rendering other animals immune, but that it possessed curative powers when the disease is already in progress. The serum of immunized animals when injected into the circulation of animals in which there was a body-temperature of from $40.4^{\circ}$ to $41^{\circ} \mathrm{C}$. reduced this temperature to normal $\left(37.5^{\circ} \mathrm{C}.\right)$ in twelve consecutive experiments during the first twenty-four hours following its employment.

In their opinion, the crisis seen in pneumonia in human beings indicates the moment at which the poisouous products, manufactured by the bacteria located in the lungs, are present in the circulation in amounts sufficient to stimulate the tissues to the reaction that results in the production of the antidotal substance that has the power of rendering the poisons inert.

At the time of the crisis in pueumonia the bacteria themselves are in no way affected. They remain in the lungs, and can be detected, in full vigor and virulence, in the sputum of patients a long time after the disease is cured. They have lost none of their power of producing poisonous products, and still possess their original pathogenic relations toward susceptible animals. It is only after the crisis that their poisons are neutralized by this antidotal proteid that has been produced by the cells of the tissues, and as this occurs the systemic manifestations gradually disappear. The Klemperers claim to have isolated from cultures of micrococcus lanceolatus a proteid body that is the agent concerned in 
produeing the tissue-reaction which results in the formation of the protecting substance. They likewise isolated from the serum of immunized animals a proteid that possesses the same powers as the serum itself, viz., of affording immunity and curing the disease.

Here, again, it appears that the proeesses of infection and immunity are chemical in their nature, the active poisons of the invading organisms-" the pneumotoxins" - being instrumental in producing the diseased condition, while the antidotal or resisting body of the tissues-" the anti-pneumotoxin" - is the agent by which the poison is neutralized.

Results in general analogous to those of G. and F. Klemperer have also been obtained by Emmerich and Fowitzky. ${ }^{1}$

In the light of these experiments the hypothesis advanced by Buchner, that the establishment of immunity is to be explained by reactive changes in the integral cells of the body, receives additional support, and when we consider the observations of Bitter, ${ }^{2}$ who found that in protective vaccinations against anthrax the vaccines do not disseminate themselves through the body, as is the case when the virulent organisms are introduced, but remain at the point of inoculation, and from this point produce, by the absorption of their chemieal products, the systemic ehanges through which the animal is protected against subsequent infection by the virulent organisms, we feel justified in concluding that the weight of evidence is strongly in favor of this view.

The experiments of the past two or three years indieate the probability of there being present in the blood

1 Emmerich and Fowitzky: Munchener med. Wochenschr., 1891, No. 32.

2 Bitter: Zeltschrift fur Hyglene, 1888, Bd. 1v. 
of animals several different bodies having totally different relations to bacteria and their products, according to the conditions under which they exist. First, there is present in the blood-serum of practically all animals the normal defensive "alexines" already mentioned; second, the antitoxins that are found in the blood of animals artificially immunized from special sorts of infection and intoxication, the functions of which are susceptible of demonstration outside the body as well as within the tissues of the living animal; third, a body possessed of disintegrating bacteriolytic powers-i.e., having the property of actually breaking bacteria to pieces, so that the phenomenon may be observed under the microscope. This phenomenon is especially to be seen within the peritoneum of guinea-pigs that have been rendered immune from Asiatic cholera and from the typhoid and colon infections or intoxications. It is rarely or never seen outside the body, and is not to be confounded with the ordinary bactericidal function of the alexines that is demonstrable in most normal serums; and fourth, a body, the so-called "agglutinin" (Gruber), that is regarded by Widal to represent a "reaction of infection," and not of immunity. The presence of this body in a serum is announced by its peculiar influence on the activity and arrangement of bacteria with which it is brought in contact. In the case of typhoid fever in man, for instance, the serum obtained during the early and middle stages of the disease, when mixed with fluid cultures or suspensions of the typhoid bacillus, causes the bacilli to lose their motility and to congregate (agglutinate) together in masses and clumps, a condition never seen in normal cultures of this organism, and practically never observed when normal serum is 
employed. There are evidences of the presence of "agglutinin" in certain of the antitoxie serums from immune animals, viz., that of animals immune from cholera, pyocyaneus, typhoid and colon infections. So far as experience has gone, this agglutinating influenee is manifested in the great majority of cases only upon the organisms from which the animal is protected. In view of the fact that its presence is regarded as indicative of a reaction of infection, its detection in the blood of immune animals may at first sight appear paradoxical. We should not lose sight of the fact, however, that it is assumed to be totally distinct from the other substances that are concerned in immunity, and its presence in immune animals may, therefore, be reasonably explained as a result of the "reactions of infection" that were coineident with the primary injections into the animal of infective or intoxicating matters necessary to the establishment of the condition of immunity.

The experiments that have been cited afford but an imperfect idea of the enormons amount of work that has been done upon the manifold phases of these important subjects; they may, however, serve to indicate the direction in which the lines of research have been laid. As a result of such investigations, our knowledge upon infeetion and immunity may at present be summarized about as follows :

1. That infection may be considered as a contest between bacteria and living tissues, condueted on the part of the former by means of the poisonous products of their growth, and resisted by the latter through the agency of proteid bodies normally present in and generated by their integral cells.

2. That when infection occurs it may be explained 
either by the excess of vigor of the bacterial products over the antidotal or protective proteids produced by the tissues, or to some cause that has interfered with the normal activity and production of these bodies.

3. That in the serum of the normal circulating blood of many animals there exists a substance that is capable, outside of the body, of rendering inert bacteria that, if introduced into the body of the animal, would prove infective.

4. That immunity is most frequently seen to follow the introduction into the body of the products of growth of bacteria that in some way or other have been modified. This modification may be artificially produced in the products themselves of virulent organisms, and then introduced into the tissues of the animal; or the virulent bacteria may be so treated that they are no longer of full virulence, and when introduced into the body of the animal will produce poisons of a much less vigorous nature than would otherwise be the case.

5. That immunity following the introduction of bacterial products into the tissues is not in all cases the result of the permanent presence of these substances, per se, in the tissues, or of a tolerance acquired by the tissnes to them; but is probably, in certain instances, due to the formation in the tissnes of auother body that acts as a protecting antidote to the poisonous products of invading organisms.

6. That this protecting proteid which is generated by the cells of the tissues need not of necessity be antagonistic to the life of the invading organisms themselves, but in some cases must be looked upon more as an antidote to their poisonons products. 
7. That immunity, as conceived by Ehrlich, may be either "active" or "passive." According to this interpretation, it is "active" when resulting from an ordinary non-fatal attack of infectious disease; or from a mild attack of infection purposely induced through the use of living vaccines; or from the gradual introduction of toxins into the tissues until a conspicuous stage of tolerance is reached. It is "passive" when occurring as a result of the direct transference of the perfected immunizing substance from an immune to a susceptible animal, as by the injection of blood-serum from the former into the latter. "Passive immunity" is, in most cases, conferred at once, without the delay incidental to the usual modes of establishing "active immunity." As a rule, "active" is more lasting than "passive" immunity.

8. That phagocytosis, though frequently observed, is not essential to the establishment of immunity, but is more probably a secondary process, the bacteria being taken up by the leucocytes only after having been modified in virulenee through the normal germicidal activity of the serum of the blood and of other fluids in the body. It is, however, probable that important factors in the establishment of immunity are substances secreted and thrown into the circulating fluids by the living leucocytes.

9. That, of the hypotheses advanced in explanation of aequired immunity, the one worthy of greatest confidence is that which assumes immunity to be due to reactive changes ou the part of the tissues that result in the formation in these tissues of antitoxic substances capable of neutralizing the poisons produced by the bacteria against which the animal has been immunized; 
though in certain cases the establishment of immunity is accompanied by the assumption of germicidal as well as antitoxic properties by the fluids and tissues, and in a few instances the germicidal is more pronounced than the antitoxic function. 


\section{CHAPTER XXVII.}

Bacteriological study of water-Methods employed - Precautions to be observed-Apparatus used, and methods of using them-Methods of investigating air and soil.

THe conditions that favor the epidemic outbreak of typhoid fever, Asiatic cholera, and other maladies of which these may be taken as types, have served as a subject for discussion by sanitarians for a long time.

Of the hypotheses that have been advanced in cxplanation of the existence and dissemination of these diseases, two stand pre-eminent and are worthy of consideration. They are the "ground-water" theory of von Pettenkofer and his pupils, and the "drinkingwater" theory of the school of bacteriologists of which Koch stands at the head.

The adherents to the "ground-water" view explain the presence of these diseases in epidenic form throngh alterations in the soil resulting from fluctuations in the level of the soil-water, and assign to the drinking-water either a very insignificant rôle, or, as is most frequently the ease, ignore it entirely. On the other hand, those who have been instrumental in developing the drinkingwater hypothesis elaim that alterations in the soil play little or no part in favoring the appearance of these diseases in a neighborhood; but that, as a rule, they appear as a result of direet infection, through the nse of waters that are contaminated with materials containing the specific organisms that are known to cause such diseases. 
As a result of many observations on both sides of the question, the evidence is greatly in favor of the opinion that polluted water is primarily the underlying cause of these epidemics, and this too, very often, when the state of the soil-water, in the light of the "groundwater" hypothesis, is just the reverse of what it should be in order to render it answerable for them. It is manifest, therefore, that the careful bacteriological study of water intended for domestic use is of the greatest importance, and should be a routine procedure in all communities receiving their water-supply from sources that are liable to pollution.

The object aimed at in such investigations should be to determine if the water approaches constancy in the number and kind of bacteria contained in it-for all waters, except deep ground-water, contain bacteria; if sudden fluctuations in the number and kind of bacteria occur in these waters, and if so, to what are they due; and finally, and most important, does the water contain constantly, or at irregular periods, bacteria that can be traced to human excrement, not of necessity pathogenic varieties, but bacteria that are known to be present normally in the intestinal canal? For, if conditions are favorable to the presence of these varieties, the same conditions would favor the admission to the water of other forms of bacteria that are concerned in the production of diseases of the intestines.

In considering water from a bacteriological standpoint it must always be borne in mind that comparisons with any general fixed standard are not of much value, for just as normal waters from different sources are seen to present variations in their chemical composition, without being unfit for use, so may the relative 
number of bacteria in water from one source be always greater or smaller than in that from another, and yet no difference may be seen to result from their employment. For this reason the proper study of any water, from this point of view, should begin with the establishment of what may be called its normal mean number of bacteria, as well as the character of the prevailing species; and in order to do this the investigations must cover a long period of time through all the seasonal variations of weather. From data obtained in this way it may be possible to predict approximately the normal baeteriological condition of water at any season. Marked deviations from these "means," either in the quantity or quality of the organisms present, can then be considered as indicative of the existence of some unusual, disturbing element, the nature of which should be investigated. Similarly, it is impossible to formulate an opinion of much value from a single chemical analysis of a water, for the results thus obtained indicate only the state of the water at the time the sample was procured, and give no indication as to whether it differed at that time from its usual condition, or from the normal condition of the water throughont the immediate neighborhood.

The interpretation of the results of both chemical and bacteriological analysis of a sample of water acquires its full value only through comparison, either with "means" that have been determined for this water, or with the results of simultaneous analyses of a number of samples from the other sources of supply of the locality.

The aid of the baeteriologist is frequently sought in connection with investigations upon waters that are sup- 
posed to be concerned in the production of disease, particularly typhoid fever, either in isolated cases or in widespread epidemic outbreaks, and almost as often do reliable bacteriologists fail to detect the bacillus that is the cause of typhoid fever in these waters.

The failure to find the organisms of typhoid fever in water by the usual methods of analysis does not by any meaus prove that they are not present or have not been present. The means that are ordinarily employed in the work admit of such very small volume of water being used in the test that we can readily understand how these organisms might be present in moderate numbers and yet none of them be included in the drop or two of the water that are taken for study. The conditions are not those of a solution, each drop of which contains exactly as much of the dissolved material as do all other drops of equal volume, but are rather those of a suspension in each drop or volume of which the number of suspended particles is liable to the greatest degree of variation. Furthermore, there are other reasons that would, a priori, preclude our expecting to find the typhoid bacilli in water in which we may have reason to believe they had been deposited, viz., attention is not usually directed to the water until the presence of the disease has become conspicuous, usually in from three to four weeks after the time when the pollution probably occurred. Three or four weeks are ordinarily sufficient time for the delicate, non-resistant bacillus of typhoid fever to succumb to the unfarorable conditions under which it finds itself in water. By unfavorable conditions are meant the absence of suitable nutrition; unfavorable temperature; probably the antagonistic influence of more hardy saprophytic bacteria, particu- 
larly the so-called "water-bacteria," and of more highly organized water-plants; the effect of mechanical precipitation; and, of great importance, the disinfecting action of direct sunlight.

Though it is so rare as to be almost never, that typhoid bacilli are found in drinking-water, it must, nevertheless, not be supposed that bacteriological analyses of suspicious waters shed no light upon the existence of pollution and the suitability or non-suitability of the water for drinking-purposes.

In the normal intestinal tract of all human beings, and of many other mammals, as well as associated with the specific disease-producing bacterium in the intestines of typhoid-fever patients, is an organism that is frequently found in polluted drinking-waters, and whose presence is proof positive of pollution by either normal or diseased intestinal contents; and though efforts may result in failure to detect the specific bacillus of typhoid fever, the finding of the other organism, the bacterium coli commune, justifies one in expressing the opinion that the water under consideration has been polluted by intestinal evacuations from either human beings or animals. Waters so located as to be liable to such pollution can never be considered as other than a continuous source of danger to those using them.

Another point to be remembered is in conneetion with the value of chlorine as indicative of contamination by human excrement. It is commonly taught that an excessive amount of chlorine in water points to contamination by human exereta. This may or may not be true according to circumstances. A high proportion of this substance in a sample of water fron a locality, the neighboring waters of which are poor in chlorine, is 
unquestionably a suspicious indication, but in a district close to the sea or near salt deposits, for instance, where the water generally is high in chlorine, the value of the indications thus afforded is very much diminished unless the amount found in the sample under examination greatly exceeds the normal "mean," previously determined, for the amount of chlorine in the waters of the neighborhood.

A striking example of such a condition as the latter recently occurred in the experience of the writer while inspecting a group of water-supplics on the east coast of Florida. In each instance the water was obtained from properly protected artesian wells, ranging from 200 to 400 feet deep, and located within a few hundred yards of the sea. The first sample that was subjected to chemical analysis revealed such an unusually high proportion of chlorine that, had this sample alone been considered, the opinion that it was polluted by human excreta might have been advanced. To prevent such an error samples of water from a number of wells in the neighborhood were examined, and they were all found to contain from ten to twelve times the amount of chlorine that ordinarily appears in inland waters, the excess being eridently due to leakage through the soil into the wells of water from the sea. In short, the presence of an excess of chlorine in water, while often indicating pollution from human evacuations, may, nevertheless, sometimes arise from other sources; but the presence in water of bacteria normally found in the intestinal canal can manifestly admit of but one interpretation, viz., that fecal matters have at some time and place been deposited in this water, and that while no specific disease-producing organisms may have been 
detected, still, waters in which such pollutions are possible are a constant source of menace to the health of those who use them for domestic purposes.

A sudden variation from the normal, mean number of bacteria, or from the normal chemical composition of a water, calls at once for a thorongh inspection of the supply, while at the same time the characters of the organisms present are to be subjected to the most careful study.

The Qualitative Bacteriological Analysis OF WATER. - The qualitative bacteriological analysis of water entails much labor, as it requires not only that all the different species of organism found in the water should be isolated, but that each representative should be subjected to systematic study, and its pathogenic or non-pathogenic properties determined.

For this purpose a knowledge of the methods for the isolation of individual species, which have already been deseribed, and of the means of studying these species when isolated, is indispensable.

For this analysis certain precautions essential to accuracy are always to be observed.

The sample is to be collected under the most rigid precautions that will exclude organisms from sources other than that under consideration. If drawn from a spigot, it should never be collected until the water has been flowing for fifteen to twenty minutes in a full stream. If obtained from a stream or a spring, it should be collected, not from the surface, but rather from about one foot bencath the surface.

It should always be collected in vessels which have previously been thoroughly freed from all dirt and organie particles, and then sterilized; and the plates 
should be made as quickly as possible after collecting the sample.

When circumstances permit, all water analyses should be made on the spot at which the sample is taken, as it is known that during transportation, unless the samples are kept packed in ice, a multiplication of the organisms contained in it always occurs.

For the purpose of qualitative analysis it is necessary that a small portion of the water-one, two, three, five drops-should first be employed as the amounts from which plates are to be made. In this way one forms some idea as to the approximate number of organisms in the water, and can, in consequence, determine the amount of water necessary to use for each set of plates. Duplicate plates are always to be made-one set upon agar-agar, which are to be kept in the incubator at body temperature, and one set upon gelatin, to be kept at from $18^{\circ}$ to $20^{\circ} \mathrm{C}$.

As soon as the colonies have developed the plates are to be carefully compared and studied. It is to be noted if any difference in the appearance of the organisms on corresponding plates exists, and if so, to what is it due? It is to be particularly noted which plates contain the greater number of colonies, those kept at the higher or those at the lower temperature. In this way the temperature best suited for the growth of the majority of these organisms may be determined.

As a rule, the greater number of colonies appear upon the gelatin plates that are kept at $18^{\circ}$ to $20^{\circ} \mathrm{C}$., and from this it would seem that many of the normal waterbacteria do not find the higher temperature so favorable to their development as do the organisms not naturally present in water, particularly the pathogenic varieties. 
NoтE. - In determining if the organisms found are possessed of pathogenic properties, in what way will your tests be influenced by this observation?

From recent investigations upon this subject it appears that the difference in behavior toward heat of bacteria present in water may have a very important application. Dr. Theobald Smith has recently suggested a method by which it is easily possible to isolate, from waters in which they are present, certain organisms that are of the utmost importance in influencing our judgment upon the fitness of the water for domestic use. By the addition of small quantities, one, two, or three drops of the suspicious water to fermentation tubes (see article on Fermentation Tube) containing bouillon to which 2 per cent. of glucose has been added, and keeping them at the temperature of the body, $37^{\circ}$ to $38^{\circ} \mathrm{C}$., the growth of the intestinal bacteria that may be present in the water is favored, while that of the water-organisms is not; in consequence, after from thirty-six to forty-eight hours the fermentation characteristic of most of these organisms is evidenced by the accumulation of gas in the closed end of the tube. From these tubes the growing bacteria can then be easily isolated by the plate method, and it will not be infrequent to find intestinal bacteria present in pure culture.

Another method for the same object is to collect a sample of about 100 c.c. of the water to be tested in a sterilized flask, and add to this about 25 c.c. of sterilized bouillon of four times the usual strength. This is then placed in the incubator at $37^{\circ}$ to $38^{\circ} \mathrm{C}$., for thirty-six to forty-eight hours, after which plates are to 
be made from it in the usual way; the results will often be a pure culture of some single organism, either one of the intestinal variety or a closely allied species. By a method analogous to the latter the spirillum of Asiatic cholera has been isolated from water; and by taking advantage of the effect of elevated temperature upon the bacteria of water Dr. Vaughan, of Michigan, has succeeded in isolating from suspicious waters a group of organisms very closely allied to the bacillus of typhoid fever.

The Quantitative Estimatiox of Bacteria in WATER.—Quantitative analysis requires more care in the measurement of the exact volume of water employed, for the results are to be expressed in terms of the number of individual organisms to a definite volume. The necessity for making the plates at the place at which the sample is collected is to be particularly accentuated in this analysis, for the multiplication of the organisms during transit is so great that the results of analyses made after the water has been in a vessel for a day or two are often very different from those that would have been obtained on the spot.

Note. - Inoculate a tube containing about ten cubic centimetres of sterilized distilled or tap water with a very small quantity of a solid culture of some one of the organisms with which you have been working, taking care that none of the culture medium is introduced into the water-tube and that the bacteria are evenly distributed through it. Make plates at once, and on each succeeding day, from this tube, and determine by counts whether there is an increase or diminution in the number of organisms-i.e., if they are 
growing or dying. Represent the results graphically, and it will be noticed that in many eases there is at first, during the first three or four days, a multiplication, after which there is a rapid diminution; and, if the organism does not form spores, usually complete death in from ten to twelve days. This is not true for all organisms, but does hold for many.

Where it is not convenient, however, to make the analysis on the spot, the sample of water should be collected and packed in iee and kept on ice until ready for use, which should in all cases be as soon after its collection as possible.

For the collection of water for this purpose, a convenient vessel to be employed is a glass bulb (Fig. 98) or balloon, which one soon learns to make for one's self from glass tubing.

FIG. 98.

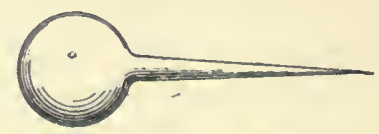

Glass bulb for collecting samples of water.

It consists simply of a round glass sphere blown on the end of a glass tube, which latter is subsequently drawn ont into a fine capillary stem and sealed while hot. As it cools, the contraction of the air within the bulb results in the production of a negative pressure.

If the point of the stem be broken off under water, the water is pressed up into the bulb, bccause of the existence of the negative pressure within. The negative pressure obtained in this way is frequently insufficient to permit of the bulb being completely filled, 
and often only a few drops of fluid can be obtained. To obviate this bulbs may be blown and allowed to cool, but not sealed. After a sufficient number of them are prepared they are taken, one at a time, and gently warmed over the flame; while still warm the extremity of the stem is dipped into distilled water and held there until a few drops have passed up into the bulb; this is then carefully boiled, or, rather, completely vaporised, over the flame, and while the steam is still escaping the point is sealed in the gas-flame. All air will thus have been replaced by water vapor, and if the point of the stem be now broken off under the water the bulb will fill quickly and completely. It is not desirable to fill them completely, but rather to only about three-fourths of their capacity, as when full it is difficult to empty them without contaminating the contents. They are emptied by genlly warming over a gas or alcohol flame.

A number of them may be made, sealed, and kept on band. They are sterile so long as they are sealed, because of the heat that is employed in their manufacture.

When a sample of water is to be taken, the point of a bulb is simply broken off with sterilized forceps under water at the place from which the sample is to be procured and held there until the necessary amount has been obtained. This may serve as a sample from which to prepare plates or Esmarch tubes on the spot, or the tip of the stem may be resealed in the flame of an alcohol lamp, the bulb packed in ice, and transported in this condition to the laboratory.

Another very simple and useful device for collecting water samples is that recommended by Kirschner. It consists of a piece of glass tubing of about 5 or $6 \mathrm{~mm}$. 
inside diameter, and $36 \mathrm{~cm}$. long, bent in the form of a $\mathrm{U}$, with either extremity of the arms bent again at right angles in the same plane and drawn out to a point and sealed. They are sterilized in the flame as they are made. The sample is collected by breaking off both points, immersing one of them in water and sucking on the other until the tube is filled. Then both points are again sealed in the flame and the tube packed in ice. The objection to this tube is the danger of contaminating its contents with saliva during the act of filling by suction, though this danger is not so great as might at first appear, as we shall learn in our efforts to cultivate bacteria from the mouth-cavity.

Nore.-Make cover-slips from your own mouth; make plates on both gelatin and agar-agar, at the same time. Compare the number of bacteria found by mieroscopic examination of the cover-slips with the number of colonies that develop on the plates.

In beginning the quantitative analysis of water with which one is not acquainted there are certain preliminary steps that are essential.

It is necessary to know approximately the number of organisms contained in any fixed volume, so as to determine the quantity of water to be employed for the plates or tubes. This is usually done by making preliminary plates from one drop, two drops, 0.25 e.c., 0.5 c.c., and 1 c.c. of the water. After each plate has been labelled with the amount of water used in making it, it is placed aside for development. When this has oceurred one selects the plate upon which the eolonies are only moderate in number-about 200 to 300 colonies presenting 
- and employs in the subsequent analysis the same amount of water that was used in making this plate.

If the original water contained so many organisms that there developed on a plate or tube made with one drop too many colonies to be easily counted, then the sample must be diluted with one, ten, twenty-five, fifty, or one hundred volumes, as the case may require, of sterilized distilled water. This dilution must be accurate, and its exact extent noted, so that subsequently the number of organisms per volume in the original water may be calculated.

The use of a drop is not sufficiently accurate. The dilution should therefore always be to a degree that will admit of the employment of a volume of water that may be exactly measured, 0.25 and 0.5 c.c. being the amounts most convenient for use.

Duplicate plates should always be made and the mean of the number of colonies that develop upon them taken as the basis from which to calculate the number of organisms per volume in the original water.

For example: from a sample of water 0.25 c.c. is added to a tube of liquefied gelatin, carefully mixed and poured out as a plate. When development occurs the number of colonies are too numerous to be accurately counted. One cubic centimetre of the original water is then to have added to it, under precautions that prevent contamination from without, 99 c.c. of sterilized distilled water-that is, we have now a dilution of $1: 100$. Again, 0.25 c.c. of this dilution is plated, and we find 180 colonies on the plate. Assuming that each colony develops from an individual bacterium, though this is perhaps not strictly true, we had 180 organisms in 0.25 c.c. of our $1: 100$ dilution, therefore 
in 0.25 c.c. of the original water we had $180 \times 100=$ 18,000 bacteria, which will be 72,000 bacteria per cnbic centimetre $(0.25=18,000,1$ c.c. $=18,000 \times 4=72,000)$. The results are always to be expressed in terms of the number of bacteria per cubic centimetre of the original water.

Another point of very great importance (already mentioned) is the effect of temperature upon the number of colonies of bacteria that will develop on plates made from water. It must always be remembered that a larger number of colonies appear on gelatin plates made from water and kept at $18^{\circ}$ to $20^{\circ} \mathrm{C}$. than on agar-agar plates kept in the incubator. The following table, illustrative of this point, gives the results of parallel analyses of the same waters, the one series of counts having been made upon gelatin plates at the ordinary temperature of the room, the other upon plates of agar-agar kept for the same length of time in the incubator at from $37^{\circ}$ to $38^{\circ} \mathrm{C}$. It will be seen from the table that much the larger number of colonies-i.e., much higher results, are always obtained when gelatin is employed. The importance of this point in the quantitative bacteriological analysis of water is too apparent to require further comment. 
TABLE ILLUSTRATING THE PROPORTION BETWEES THE RESULTS OBTAINED BY THE USE OF GELATIN AND AGAR-AGAR IN QUANTITATIVE BACTERIOLOGICAL ANALYSIS OF WATER. RESULTS RECORDED ARE THE NCMBER OF COLONIES THAT DEVELOPED FROM THE SAME AMOUNT OF WATER IN EACH SERIES.I

NUMBer of COLONIES FroY WATER THAT DEVELOPED tTON-

Gelatin plates at $15^{\circ}$ to $20^{\circ} \mathrm{C}$.

\begin{tabular}{|c|c|c|c|c|c|}
\hline 310 & & •• & & & \\
\hline 250 & & . & . & . & \\
\hline 310$\}$ & & . & - & 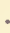 & - \\
\hline 340$\}$ & . & • & • & 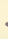 & . \\
\hline 650$\}$ & . & • & • & . & . \\
\hline 630$\}$ & . & $\cdot$ & • & & - \\
\hline 350$\}$ & . & • & • & & . \\
\hline 400$\}$ & • & $\bullet$ & • & & . \\
\hline 1000$\}$ & . & . & • & & . \\
\hline 890$\}$ & . & $\theta$ & & & . \\
\hline 310 & . & - & • & & . \\
\hline 70$\}$ & • & • & & & • \\
\hline 90 & . & . & & & \\
\hline 0 & & & & & \\
\hline
\end{tabular}

Another point of equal importance in its influence upon the number of colonies that develop is the reaction of the gelatin. A marked excess of either alkalinity or acidity always has a retarding effect upon many species found in water. Experience at Lawrence has shown that gelatin of such a degree of acidity as to require the further addition of from 15 to 20 c.c. per litre of a normal canstic alkali solution to bring it to the phenolphtalein neutral point gives, on the whole, the best results. Thus, by way of illustration, Fuller found that a sample of Merrimac River water gave 5800 colonies per c.c. on phenolphtalein neutral gelatin, 15,000 colonies on gelatin that would need 20 c.c. of normal alkali solution to bring it up to the phenol-

1 I am indebted to Dr. James Homer Wright, Thomas Scott Fellow in Hygiene (1892-'93), University of Pennsylvanis, for the results preseuted in this table. 
phtalein neutral point-i.e., a feebly acid nutrient gelatin, and 500 colonies on a gelatin so alkaline as to require 20 c.c. of a normal acid solution to bring it back to the phenolphtalein neutral point.

Throughout this part of the work it is to be borne in mind that when one refers to plates it is not to a set, as in the isolation experiments, bnt to a single plate.

Method of Counting the Colonies on Plates. -For convenience in counting colonies on plates or in tubes it is customary to divide the whole area of the gelatin occupied by colonies into smaller areas, and either count all the colonies in each of these areas and add the several sums together for the total, or to count the number of colonies in each of several areas, ten or twelve, take the mean of the results and multiply this by the number of areas containing colonies. This latter procedure obtains, of course, only when all the areas are of the same size.

By this latter method, however, the results vary so much in different counts of the same plate that they cannot be considered as more than rough approximations.

Note.-Prepare a plate; calculate the number of colonies upon it by this latter method. Now repeat the ealculation, making the average from another set of squares. Now actually count the entire number of colonies on the plate. Compare the results.

For facilitating the counting of colonies several very convenient devices exist. 
WOLFFH ÜGEL'S COUNTING-APPARATUS.-This apparatus (Fig. 99) consists of a flat wooden stand, the centre of which is cut out in such a way that either a black or white glass plate may be placed in it. These form a background upon which the colonies may more easily be seen when the plate to be counted is placed upon it.

Fio. 99.

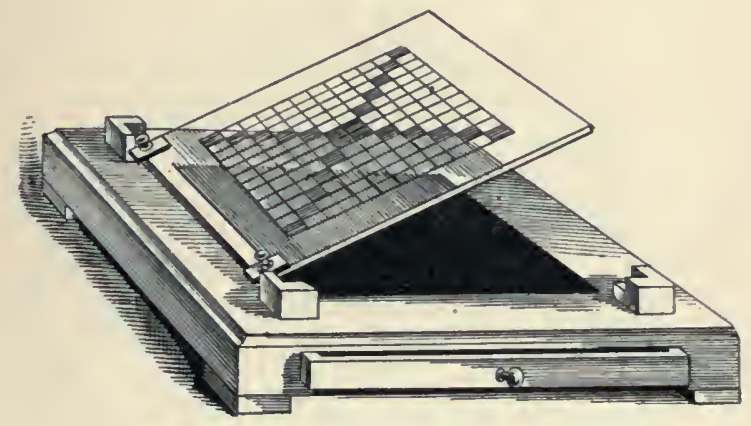

Wolff hügel's apparatus for counting colonies.

When the gelatin plate containing the colonies has been placed upon this background of glass, it is then covered by a transparent glass plate which swings on a hinge. This plate, which is ruled in square centimetres and subdivisions, when in position is just above the colonies, without touching them.

The gelatin plate is moved about until it rests under the centre of the area occupied by the ruled lines.

The number of colonies in each square centimetre is then counted, and the sum-total of the colonies in all these areas gives the number of colonies on the plate; or, as has already been indicated, if the number of colonies be very great a mean may be taken of the number 
in several (6. or 8) squares; this is to be multiplied by the total number of squares occmpied by the gelatin. The result is an approximation of the total number of colonies.

When the colonies are quite small, as is frequently the ease, the counting may be rendered easier by the use of a small hand-lens.

FIG. 100.

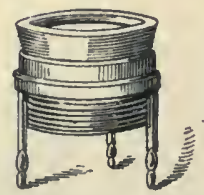

Lens for counting colonies.

In Fig. 100 is seen the form of hand-lens commonly employed with this apparatus. Several useful modifications of this apparatus have been introduced. The most important is that of Lafar (Centralblatl für Bakteriologie und Parasilenkunde, 1891, Band xv. p. 331). I Lafar's counter consists of a glass disk of the dimmeter of ordinary size Petri dishes. It is supplied with a collar or flange that fits around the bottom of the Petri dish, and thus holds the counter in position. The disk is ruled with concentric circles and its area divided into seetors of such size that the spaces between the concentric circles and the radii forming the sectors are of equal size. Three of the sectors are subdivided into smaller areas of equal size for convenience in counting when the colonies are very numerous. The prineiples involved are similar to those of the preceding apparatus, but the circular form of the apparatus admits of 
more exactness wheu counting colonies on a circular plate. ${ }^{1}$

Parks (Journ. Bact. and Path., 1896, vol. iv. No. 1) has introduced a cheap and convenient modification of Lafar's apparatus. ${ }^{2}$ It consists of a sheet of white paper on which is printed a black disk that is ruled

Fig. 101.

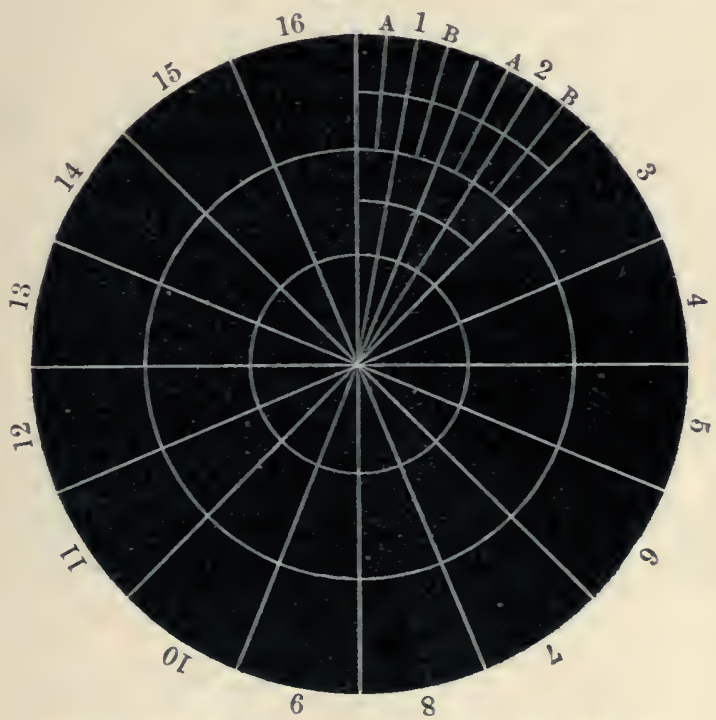

Park's apparatus for counting colonies (reduced one-third).

with white lines, in somewhat the same fashion as is Lafar's counter, though the areas of the smallest subdivisions are not of one size and do not bear a constant

1 Lafar's apparatus is to be obtained from F. Mollentopf, 10 Thorstrasse, Stuttgart, who holds the patent for it. Its price is about 8 or 9 marks.

2 Copies of this apparatus are to be had of Ash \& Co.. 42 Southwark Street, London, or of Lentz \& Sons, North Elerenth Street, Philadelphia, Pa. (The cost is but a few ceuts per copy.) 
relation to each other. To use this apparatus (Fig. 101) the Petri dish is placed centrally upon it, the cover of the dish is removed, and the colonies are counted as they lie over the spaces bounded by the white lines on the black disk beneath. When the plate is centred over the black disk the portion lying over one sector is exactly one-sixteenth of the whole plate.

Esmarch's Counter. - Esmarch has devised a counter (Fig. 102) for estimating the number of colonies present when they are upon a cylindrical surface, as when in rolled tubes. The principles and methods of estimation are practically the same as those given for Wolff hügel's apparatus.

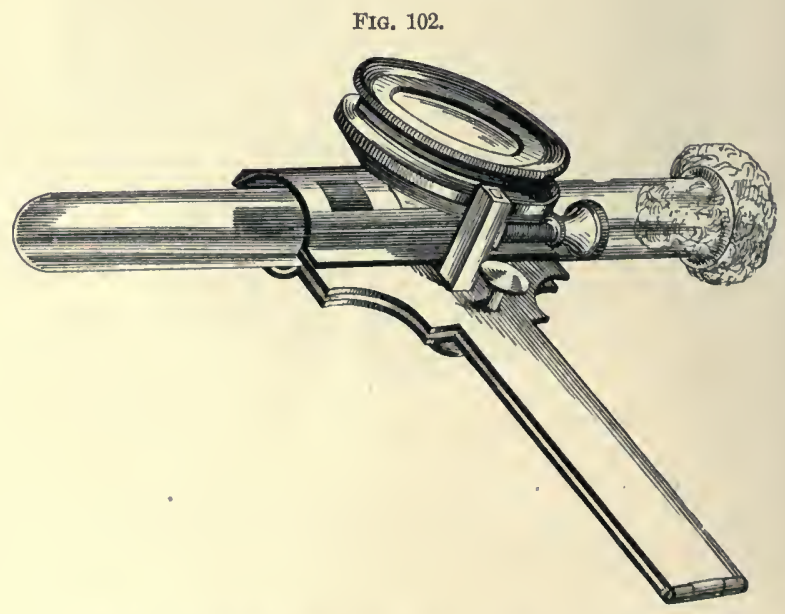

Esmareh's apparatus for counting colonies in rolled tubes.

A simpler method than by the use of Esmarch's apparatus may be employed for counting the colonies in 
rolled tubes. It consists in dividing the tube by lines into four or six longitudinal areas, which are subdivided by transverse lines drawn about 1 or $2 \mathrm{~cm}$. apart. The lines may be drawn with pen and ink. They need not be exactly the same distance apart nor exactly straight. Beginning with one of these squares at one end of the tube, which may be marked with a cross, the tube is twisted with the fingers, always in one direction, and the exact number of colonies in each square as it appears in rotation is counted, care being taken not to count a square more than once; the sums are then added together, and the result gives the number of colonies in the tube. This method may be facilitated by the use of a hand-lens.

In all these methods there is one error that is difficult to eliminate: it is assumed that each colony represents the outgrowth from a single organism. This is probably not always the case, as there may exist clumps of bacteria which represent hundreds or even thousands of individuals, but which still give rise to but a single colony-this is usually estimated as a single organism in the water under analysis.

Where grounds exist for suspecting the presence of these clumps they may in part be broken up by shaking the original water with sterilized sand.

What has been said for the bacteriological examination of water holds good for all fluids which are to be subjected to this form of analysis.

BaCteriologica i، Air Axa lysis. - Quite a number of methods for the bacteriological study of the air exist.

In the main they consist either of allowing air to pass over solid nutrient media (Koch, Hesse) and observing the colonies which develop upon the media, 
or of filtering the bacteria from the air by means of porous and liquid substances, and studying the organisms thus obtained. (Miguel, Petri, Stranss, Würz, Sedgwick-Tucker.)

The former methods have given place almost entirely to the latter for reasons of greater exactness possessed by the latter.

In some of the methods which provide for the filtration of bacteria from the air by means of liquid substances a measured volume of air is aspirated through liquefied gelatin; this is then rolled into an Esmarch tube and the number of colonies counted, just as was done in the water analysis. This is the simplest procedure. An objection raised against it is that organisms

FIG. 103.

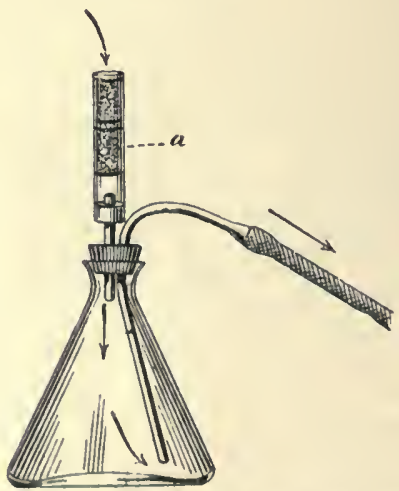

Petri's apparatus for bacteriological analysis of air. The tube packed with sand is seen at the point $a$.

may be lost, and not eome into the calculation, by passing throngh the medium in the centre of an air-bubble without being arrested by the fluid-an objection that appears to have more of speculative than of real value. 
The methods of filtration through porons substances appear, on the whole, to give the best results. Petri recommends the aspiration of a measured volume of air through glass tubes into which sterilized sand is packed. (Fig. 103.) When the aspiration is finished the sand is mixed with liquefied gelatin, plates are made, and the number of developing colonies counted, the results giving the number of organisms contained in the volume of air aspirated throngh the sand.

The main objection to this method is the possibility of mistaking a sand granule for a colony. This objection has been overcome by Sedgwick and Tucker, who employ granulated sugar instead of the sand; this, when brought into the liquefied gelatin, dissolves, and no such error as that possible in the Petri method can be made.

Sedgwick-Tucker Method.-Ou the whole, the method proposed by Sedgwick and Tucker gives such uniform results that it is to be recommended above the others. It is as follows :

The apparatus employed by them consists essentially of three parts :

1. A glass tube of a special form, to which the name cierobioscope has been given.

2. A stout copper eylinder of about sixteen litres capacity, provided with a vacuum-gange.

3. An air-pump.

The aërobioscope (Fig. 104) is about $35 \mathrm{~cm}$. in its entire length; it is $15 \mathrm{~cm}$. long and $4.5 \mathrm{~cm}$. in diameter at its expanded part; one end of the expanded part is narrowed down to a neck $2.5 \mathrm{~cm}$. in diameter and $2.5 \mathrm{~cm}$. long. To the other end is fused a glass tube $15 \mathrm{~cm}$. long and $0.5 \mathrm{~cm}$. inside diameter, in which is to be placed the filtering material. 
Upon this narrow tube, $5 \mathrm{~cm}$. from the lower end, a mark is made with a file, and up to this mark a small roll of brass-wire gauze $(a)$ is inserted; this serves as a stop for the filtering material which is to be placed over it. Beneatl the gauze (at b), and also at the large end $(c)$, the apparatus is plugged with cotton.

FIG. 104.

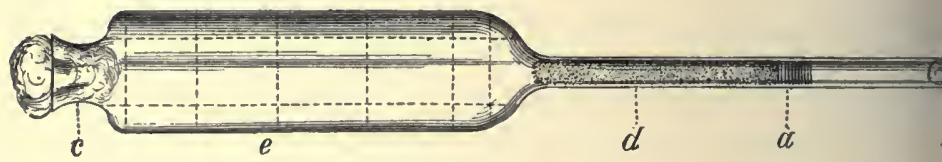

The Sedgwick-Tucker aërobioscope.

When thoroughly cleaned, dried, and plugged, the apparatus is to be sterilized in the hot-air sterilizer. When cool, the cotton plug is removed from the large end (c), and thoroughly dried and sterilized No. 50 granulated sugar is poured in until it just fills the 10 $\mathrm{cm}$. (d) of the narrow tube above the wire-gauze. This columu of sugar is the filtering material employed to engage and retain the bacteria. After pouring in the sugar the cotton-wool plug is replaced, and the tube is again sterilized at $120^{\circ} \mathrm{C}$. for several hours.

Taking the air sample. In order to measure the amount of air used the value of each degree on the vacuum-gauge is determined in terms of air by means of an air-meter, or by calculation from the known capacity of the eylinder. This fact ascertained, the negative pressure indicated by the needle on exhausting the cylinder slows the volume of air which must pass into it in order to fill the vacuum. By means of the airpump one exhausts the cylinder until the needle reaches 
the mark corresponding to the amount of air required. ${ }^{1}$

A sterilized aërobioscope is now to be fixed in the upright position and its small end connected by a rubber tube with a stopcock on the cylinder, or to a glass tube tightly fixed in the neck of an aspirating bottle by means of a perforated rubber stopper. The cottou plug is then moved from the upper end of the aerrobioscope, and the desired amount of air is aspirated through the sugar. Dust particles and bacteria will be held back by the sugar. During manipulation the cotton plug is to be protected from contamination.

When the required amount of air has been aspirated through the sugar the cotton plug is replaced, and by gently tapping the aerrobioscope while held in an almost horizontal position, the sugar, and with it the bacteria, are brought into the large part (e) of the apparatus. When all the sugar is thus shaken down into this part of the apparatus about 20 c.c. of liquefied, sterilized gelatiu is poured in through the opening at the end $c$, the sugar dissolves, and the whole is then rolled on ice, just as is done in the preparation of an ordinary Esmarch tube.

The gelatin is most easily poured into the aerobioscope by the use of a small, sterilized, cylindrical funnel (Fig. 105), the stem of which is bent to an angle of about $110^{\circ}$ with the long axis of the body.

The larger part of the aerobioscope is divided into squares, to facilitate the counting of the colonies.

1 Such a cylinder and air-pump are not necessary. A pair of ordinary aspirating bottles of known capacity graduated into litres and fractions thereof answer perfectly well. Or one can determine by the weight of water that has flowed from the aspirator the rolume of air that has passed in to take Its place-i. e., the volume of air that has passed through the aërobioscope. 
By the employment of this apparatus one can make these analyses at any place, and can, without fear of contamination, carry the tubes to the laboratory, where the eultivation part of the work may be done.

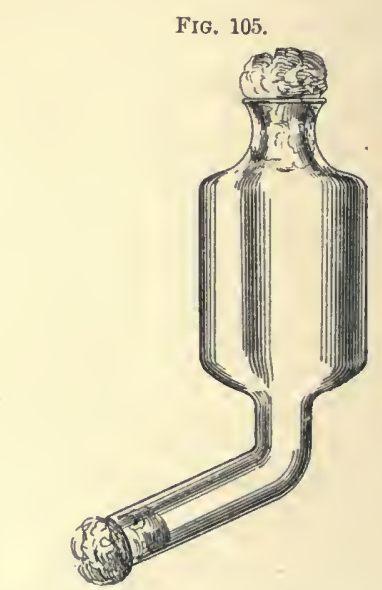

Bent funnel for use with aërobioscope.

Aside from this advantage, the filter being soluble only the insoluble bacteria are left imbedded in the gelatin.

For general use this method is to be preferred to the others that have been mentioned.

Bacteriological Study of the Soll.-Bacteriological study of the soil may be made by either breaking up small particles of earth in liquefied media and making plates directly from this, or by what is perhaps a better method, as it gets rid of insoluble particles which may give rise to errors: breaking up the soil in sterili\%ed water and then making plates immediately from the water. 
It must be borne in mind that many of the ground organisms belong to the anaërobic group, so that in these studies this point should be remembered and the methods for the cultivation of such organisms practised in connection with the ordinary methods. It must also be remembered that the nitrifying organisms, everywhere present in the ground, cannot be isolated by the ordinary methods, and will not appear in plates made after either of the above plans. The special devices that have been arranged for their cultivation will be found in the chapter on soil organisms. 


\section{CHAPTER XXVIII.}

Methods of testing disinfectants and antiseptics-Experiments illustrating the precautions to be taken-Experiments in skin disinfection.

There are several ways of determining the germicidal value of chemical substances, the most common being to expose organisms dried upon bits of silk thread to the disinfectant for different lengths of time, and then, after removing, and carefully washing the threads in water, to place them in nutrient media at a favorable temperature, and notice if any growth appears. If no growth results, the disinfection is presumably successful. Another method is to mix fluid cultures of bacteria with the disinfectant in varying proportions, and, after different intervals of time, to determine if disinfection is in progress by transferring a portion of the mixture to nutrient media, just as in the other methor of work.

By the former process the bits of thread, usually about 1 to $2 \mathrm{~cm}$. long, are placed in a dry test-tube provided with a cotton plug and carefully sterilized, either by the dry method or in the steam sterilizer, before using. They are then immersed in a pure bouillon eulture or in a salt solution suspension of the organism upon which the disinfectant is to be tested. I say "pure culture" because it is always desirable in testing a new germicide to determine its value as such on several different resistant species of bacteria, both in the vegetating and in the spore stage. After the threads have remained in the eulture or suspension for 
from five to ten minutes they are removed under antiseptic precautions and carefully separated and spread out upon the bottom of a sterilized Petri dish. This is then placed either in the incubator at a temperature not exceeding $38^{\circ} \mathrm{C}$. until the excess of fluid has evaporated, or in a desiccator over sulphuric acid, calcium chloride, or any other drying agent, but they are not left there until absolutely dry, only until the excess of moisture has disappeared. When sufficiently dry they ean then be employed in the test. This is done by immersing them in solutions of the disinfectant of different but known strengths for a fixed interval of time, say one or two hours, after which they are removed, rinsed off in sterilized distilled water to remove the excess of disinfectant adhering to them, and placed in fresh sterilized culture media, which is then placed in the incubator at from $37^{\circ}$ to $38^{\circ} \mathrm{C}$. If after twentyfour, forty-eight, or seventy-two hours a growth occurs at or about the bit of thread, and this growth consists of the organism upon which the test was made, manifestly there has been no disinfection; if no growth occurs after, at most, ninety-six honr's, it is safe to presume that the bacteria have been killed, unless our efforts at rinsing off the excess of disinfectant from the thread have not been successful, and a small amount of disinfectant is now active in preventing development-i.e., is acting as an antiseptic.

By the latter process, in which cultures or suspensions of the organisms are mixed with different but known strengths of the disinfectant, a small portion of the mixture, usually a loopful or a drop, is transferred at the end of a definite time to the fresh medium which is to determine whether the organisms have 
been killed or not. This is commonly a tube of fluid agar-agar, which is poured out into a Petri dish, allowed to solidify, and placed in the incubator, as in the other experiment.

After the minimum strength of disinfectant necessary to destroy the vitality of the organisms with which we are working has been determined, for any fixed time, it then remains for us to decide what is the shortest time in which this strength will have the same effect. We then work with a constant dilution of the disinfectant, but with different intervals of exposure-one, five, ten minutes, etc. - until we have decided not only the minimum amount of disinfectant required for the destruction of the bacteria, but the shortest time necessary for this under known conditions.

A factor not to be lost sight of is the temperature under which these experiments are conducted, for it must always be borne in mind that the action of a disinfectant is usually more energetic at a higher than at a lower temperature.

Now in both of these methods it is easy to see that unless special precautions are taken a minute portion of the disinfectant may be carried along with the thread, or drop, into the medium which is to determine whether the organisins do or do not possess the power of growth, and here have a restraining or antiseptic action. For organisms in their normal condition-that is, those which have never been exposed to the action of a disinfectant, the amount of certain disinfectants that is necessary to restrain growth is very small indeed, and for organisms that have already been exposed for a time to such agents this amount is even much less. It is plain, then, that if the test is to be an accurate 
one, precautions must be taken against admitting this minute trace of disinfectant to the medium with which we are to determine if the bacteria that have been exposed to the disinfectant have been killed or not.

The precautions that have hitherto been taken to prevent this accident are, where the threads are employed, washing them in sterilized distilled water and then in alcohol; or, where fluid cultures were mixed with the disinfectant in solution, an effort was usually made to dilute the amount of disinfectant earried over to a point at which it loses its inhibiting power.

While such precautions are sufficient in many cases, they do not answer for all. Certain chemicals have the property of combining so firmly with the threads upon which the bacteria are located as to require other special means of ridding the threads of them; and in solutions in which proteid substances are present along with the bacteria a similar union between them and the disinfectant may likewise take place. In both instances this amount of disinfectant adhering to the silk threads or in combination with the proteids must be gotten rid of, otherwise the results of the test may be fallacious. A partial solution of the problem comes from studies that have been made upon corrosive sublimate in its various applications for disinfecting purposes, and in this connection it has been shown by Shaefer ${ }^{1}$ that it is impossible to rid silk threads of the corrosive sublimate adhering to them by simple washing, as the sublimate acts as a mordant and forms a firm union with the tissues of the threads. Braatz ${ }^{2}$ found the same to hold good for catgut. For example, he found that catgut

1 Shaefer: Berliner klin. Woch., 1590, No. 3, p. 50.

- Brastz: Centr. f. Bakt. und Parasitenkunde, Bd. viii, No. 1, p. 8. 
which had been immersed in solutions of sublimate gave the characteristic reactions of the salt after having been immersed in distilled water, which had been repeatedly renewed, for five weeks.

He remarks that a similar firm combination between sublimate and cotton will take place after a longer time, but it occurs so slowly that it cannot interfere with disinfection experiments in the same way as he believes the employment of silk to act.

The most successful attempt at removing all traces of sublimate from the threads or from the proteid substances in which are located the bacteria whose vitality is to be tested is that made by Geppert, who subjected them to the action of ammonium sulphide in solntion. By this procedure the mercury is converted into insoluble sulphide, and does not now have an inhibiting effect upon the growth of those bacteria that may not have succumbed to its action when in the form of the bichloride.

In the second method of testing disinfectants, mentioned above-that is, when cultures of bacteria and solutions of the disinfectant are mixed, and after a time a drop of the mixture is removed and added to sterile nutrient media, the inhibiting amount of disinfectunt ean readily be gotten rid of by dilution-that is to say, instead of trunsporting the drop directly to the fresh merlium, add it to 10 or 12 c.c. of sterilized salt-solution (0.6-0.7 per cent. of $\mathrm{NaCl}$ in distilled water), or distilled water, and after thoroughly shaking add a drop of this to the medium in which the power of development of the bacteria is to be determined.

Another important point to be borne in mind in testing disinfectants is the necessity of so arranging the conditions that each individual organisms will be ex- 
posed to the action of the agent used. When clumps of bacteria exist we are not always assured of this, for only those on the surface of the clump may be affected, while those in the centre of the mass may entirely escape. being protected by those surrounding them. These clumps and minute masses are especially liable to be present in fluid cultures and in suspensions of the bacteria, and must be eliminated before the test is begun, if this is to be made by mixing them with solutions of the agent to be tested. This is best accomplished in the following way: the organisms should be cultivated in bouillon containing sand or finely divided particles of glass; after growing for a sufficient length of time they are then to be shaken thoroughly, in order that all clumps may be mechanically broken up by the sand. The culture is then filtered through a tube containing closely packed glass wool.

The filtration may be accomplished without fear of contamination of the culture by the employment of an Allihin tube, which is practically nothing more than a thick-walled test-tube drawn out to a finer tube at its blunt end so as to convert it into a sort of cylindrical funnel. The tube when finished and ready for use has the appearance given in Fig. 106.

Cylindrical funnel used for filtering cultures on which disinfectants are to be tested.

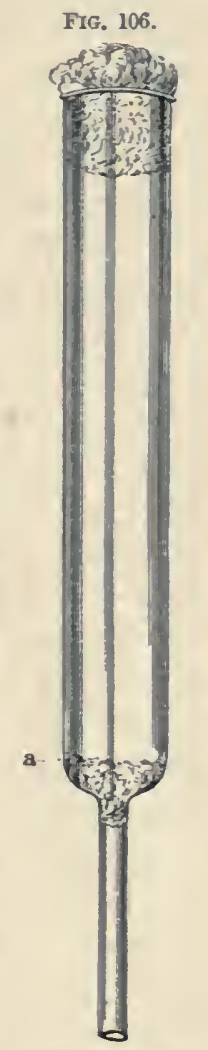


The whole tube, after being plugged at the bottom with glass wool and at its wide open extremity with cotton wool (a, Fig. 106), is placed vertically, small end down, into an Erlenmeyer flask of about 100 c.c. capacity and sterilized in a steam sterilizer for the proper time. It is kept in the covered sterilizer until it is to be used, which should be as soon as possible after sterilization.

The watery suspension or bouillon culture of the organisms is now to be filtered repeatedly through the glass wool into sterilized flasks until a degree of transparency is reached which will permit the reading of moderately fine print through a layer of the fluid about $2 \mathrm{~cm}$. thick-i.e., through an ordinary test-tube full of it. It can then be subjected to the action of the disinfectant, and, as a rule, the results are far more uniform than when no attention is paid to the existence of clumps. It is hardly necessary to say that in the practical employment of disinfectants outside the laboratory no such precautions are taken, but in laboratory work, where it is desired to determine exactly the value of different substances as germicides, all the precautions that have been mentioned will be found essential to precision.

The disinfectant value of gases and vapors is determined by their influence upon test-objects in elosed chambers. The object is to determine the proportion of the gas, when mixed with air, that is required to destroy the bacteria exposed to its action in a given time. For this purpose the test is commonly made as follows: under a sterilized bell glass of known capacity the test-objects are placed. Into the chamber is then admitted sufficient of a known mixture of air and the 
gas under consideration to eliminate completely all the air; or, the pure gas itself may be introduced in the amount necessary to give the desired dilution when mixed with the air in the chamber. After the time decided upon for the test the infected articles are removed and the vitality of the bacteria upon them is determined.

In the case of the vapors of volatile fluids, such, for instance, as formaline, the fluid is placed under the bell glass in all open dish; in another open dish the testobjects are placed. The bell glass is then sealed to an underlying ground-glass plate by vaseline or paraffin, and the fluid is allowed to vaporize at ordinary room temperature. The point here to be decided is the rolume or weight of such a fluid that it is necessary to expose in an air chamber of known cubic capacity in order that bacteria may be destroyed by its vapor in a given time.

In determining the germicidal value of different chemical agents upon certain pathogenic bacteria, susceptible animals are sometimes inoculated with the organisms after they have been exposed to the disinfectant. If no pathological condition results, disinfection is presumed to have been successful; while if the condition characteristic of the activities of the given organism in the tissues of this animal appears, the reverse is the case. The objections to this method that have been raised are: "First. The test-organisms may be modified as regards reproductive activity without being killed; and in this case a modified form of the disease may result from the inoculation, of so mild a character as to escape observation. Second. An animal that has suffered this modified form of the disease enjoys protection, more or less perfect, from future at- 
tacks, and if used for a subsequent experiment may, by its immunity from the effects of the pathogenic testorganism, give rise to the mistaken assumption that this had been destroyed by the action of the germicidal agent to which it had been subjected." (Sternberg.)

\section{DETERMINATION OF ANTISEPTIC PROPERTIES.}

In this test sterile media are employed and are usually arranged in two groups : the one to remain normal in composition and to serve as controls, while to the other is to be added the substance to be tested in different but known strengths. It is customary to employ test-tubes each containing an exact amount of bouillon, gelatin, or agar-agar, as the case may be. To each tube a definite amount of the antiseptic is added, and if it is not of a volatile nature or not injured by heat, they may then be sterilized. After this they are to be inoculated with the organism upon which the test is to be made, and at the same time one of the "control" tubes (one of those to which no antiseptic has been added) is inoculated. They are all then to be placed in the incubator and kept under observation. If at the end of twenty-four, forty-eight, or seventy-two hours no growth appears in any but the "control" tubes, it is evident that the antiseptic must be added in smaller amounts, for we are to determine the point at which it is not as well as that at which it is capable of preventing development. The experiment is then repeated, using smaller amounts of the antiseptic until we reach a point at which growth just oceurs notwithstanding the presence of the antiseptic; its antiscptic strength then lies a trifle above the amount present in this tube. 
If, for example, there was no development in the tubes in which the antiseptic was present in the proportion of $1: 1000$ and growth in the one in which it was present in $1: 1400$, the experiment would be repeated with strength of the antiseptic corresponding to $1: 1000$, $1: 1100,1: 1200,1: 1300,1: 1400$, and in this way one gradually strikes the point at which growth is just prevented. This point represents the antiseptic value of the substance used for the organism upon which it has been tested.

\section{EXPERIMENTS.}

Into each of three tubes containing 10 c.c.-one of normal salt-solution, another of bouillon, a third of fluid blood-serum-add as much of a culture of the staphylococcus pyogenes aureus as can be held upon the looped platinum needle. Mix this thoroughly, so that no clumps exist, and then add exactly 10 c.c. of 1 : 500 solution of corrosive sublimate. Mix it thoroughly; and at the end of three minutes transfer a drop from each tube into a tube of liquefied agar-agar, and pour this into a Petri dish. Label each dish carefully and place them in the incubator. Are the results the same in all the plates? How are the differences to be explained? To what strength of the disinfectant were the organisms exposed in the experiment?

Into each of two tubes containing 10 c.c.-the one of normal salt-solution, the other of bouillon-add as much of a spore-containing culture of anthrax bacilli as can be held upon the loop of the platinum wire. Mix this thoroughly so that no clumps exist, and then add exactly 10 c.c. of a $1: 500$ solution of corrosive sublimate. Mix thoroughly, and at the end of five 
minutes transfer a drop from each tube into a tube of liquefied agar-agar. Pour this immediately into a Petri dish. Label each dish carefully and place them in the incubator. Note the results at the end of twentyfour, forty-eight, and seventy-two hours. How do you explain them?

Make identically the same experiment with the same spore-containing culture of anthrax bacilli, except that the drop from the mixture is to be transferred to 10 c.c. of a mixture of equal parts of ammonium sulphide and sterilized distilled water. After remaining in this for about half a minute, a drop is to be transferred to a tube of liquefied agar-agar, poured into Petri dishes, labelled, and placed in the incubator. Note the results. Do they correspond with those obtained in the preceding experiment? How are the differences explained?

Prepare a $1: 1000$ solution of corrosive sublimate. To each of twelve tubes containing exactly 10 c.c. of bouillon add one drop to the first, two drops to the second, and so on until the last tube has had twelve drops added to it. Mix thoroughly and then inoculate each with one wire-loopful of a bonillon culture of staphylococcus pyogenes aureus. Place them all in the incubator after carefully labelling them. Note the order in which growth appears.

Do the same with anthrax spores, with spores of bacillus subtilis and with the typhoid bacillus, and see how the results compare. From these experiments what will be the strength of corrosive sublimate neces- 
sary to act as an antiseptic under these conditions for the organisms employed?

Make a similar series of experiments, using a 5 per cent. solution of carbolic acid.

Determine the antiseptic point of the common disinfectants for the organisms with which you are working.

Determine the time necessary for the destruction of the organisms with which you are working, by corrosive sublimate in $1: 1000$ solution, under different conditions-with and without the presence of albuminous bodies other than the bacteria, and under varying conditions of temperature.

In making these experiments be careful to guard against the introduction of enough sublimate into the agar-agar from which the Petri plate is to be made to inhibit the growth of the organisms which may not have been destroyed by the sublimate. This may be done by transferring two drops from the mixture of sublimate and organism into not less than 10 c.c. of sterilized physiological salt-solution, in which they may be thoroughly shaken for from one to two ninutes, or into the solution of ammonium sulphide of the strength given.

To 10 c.c. of a bouillon culture of staphylococcus pyogenes aureus, or anthrax spores, add 10 c.c. of corrosive sublimate in 1:500 solution, and allow it to remain in contact with the organisms for only one-half the time necessary to destroy them (use an organism for which this has been determined). Then transfer a drop of the mixture to each of three liquefied agar-agar tubes and pour them into Petri dishes. Place them in the incubator and observe them for twenty-four, forty- 
eight, and seventy-two hours. No growth occurs. How is this to be accounted for?

At the end of seventy-two hours inoculate all of these plates with a culture of the same organism which has not been exposed to sublimate, by taking up bits of culture on the needle and drawing it across the plates. A growth now results. We have here an experiment in which organisms which have been exposed to sublimate for a much shorter time than necessary to destroy them, when transferred directly to a favorable culture medium do not grow, and yet, when the same organism which has not been exposed to sublimate at all is planted upon the same medium it does grow. How is this to be accounted for?

Slin-disinfection. With a sterilized knife serape from the skin of the hands, at the root of the nails, and under the nails, small particles of epidermis. Prepare plates from them. Note the results.

Wash the hands carefully for ten minutes in hot water and scrub them during this time with soap and a sterilized brush. Rinse them in hot water. Again prepare plates from scrapings of the skin on the fingers, at the root of the nails, and under the nails. Note the results.

$\Lambda$ gain, wash as before in hot water with soap and brush, rinse in lot water, then soak the hands for five minutes in 1:1000 corrosive sublimate solution, and, as before, prepare plates from scrapings from the same localities. Note the results.

Repeat this latter procedure in exactly the same way, but before taking the scrapings let some one pour anmonimm sulphide over the points from which the scrap- 
ings are to be made. After it has been on the hands about three minutes again scrape, and note the result upon plates made from the scrapings.

Wash as before in hot water and soap, rinse in clean hot water, immerse for a minute or two in alcohol, after this in 1:1000 sublimate solution, and finally in ammonium sulphide, and then prepare plates from scrapings from the points mentioned.

In what way do the results of these experiments differ one from another?

To what are these differences due?

What have these experiments taught?

In making the above experiments it must be remembered that the strictest care is necessary in order to prevent the access of germs from without into our media. The haud upon which the experiment is being performed must be held away from the body and must not touch any object not concerned in the experiment. The scraping should be done with the point of a knife that has been sterilized in the flame and allowed to cool. The scrapings may be transferred directly from the knife-point to the gelatin by means of a sterilized platinum wire loop.

The brush used should be thoroughly cleansed and always kept in 1: 1000 solution of corrosive sublimate. It should be washed in hot water before using. 



\section{APPENDIX.}

LisT of apparatus and materials required in a beginner's bacteriological laboratory:

\section{MICROSCOPE AND ACCESSORIES.}

Microscope with coarse and fine adjustment and heavy, firm base; Abbe sub-stage condensing system, arranged either as the "simple" or as the regular Abbe condenser, in either case to be provided with iris diaphragm; objectives equivalent, in the English nomenclature, to about one-fourth inch and one-sixth inch dry, and one-twelfth inch oil-immersion system; a triple revolving nose-piece; three oculars, varying in magnifying porver; and a bottle of immersion oil.

Glass slides, English shape and size and of colorless glass.

Six slides with depressions in centre of about 6 to 8 $\mathrm{mm}$. in diameter.

Cover-slips, 15 by $15 \mathrm{~mm}$. square and from 0.15 to $0.18 \mathrm{~mm}$. thick.

Forceps. One pair of fine-pointed forceps and one pair of the Cornet or Stewart pattern, for holding cover-slips.

Platinum needles in glass handles. One straight, of about $4 \mathrm{~cm}$. long; one looped at the end of about 4 
$\mathrm{cm}$. long; and one straight of about $8 \mathrm{~cm}$. long. Glass handles to be of about $3 \mathrm{~mm}$. thickness and from 15 to $17 \mathrm{~cm}$. long.

\section{STAINING- AND MOUNTING-REAGENTS.}

200 c.c. of saturated alcoholic solution of fuchsin. 200 c.c. of saturated alcoholic solution of gentian violet.

200 c.c. of saturated alcoholic solution of methyleneblue.

200 grammes of pure aniline.

200 grammes of C. P. carbolic acid.

500 grammes of C. P. nitric acid.

500 grammes of C. P. sulphuric acid.

200 grammes of C. P. glacial acetic acid.

1 litre of ordinary 93-95 per cent. alcohol.

1 litre of absolute alcohol.

500 grammes of ether.

500 grammes of pure xylol.

50 grammes of Canada balsam dissolved in xylol.

100 grammes of Schering's celloidin.

10 grammes of iodine and 30 grammes of iodide of potassium in substance.

100 grammes of tannic acid.

100 grammes of ferrous sulphate.

Distilled water.

FOR NUTRIENT MEDIA.

14 pound Liebig's or $\Lambda$ rmour's beef extract.

250 grammes Witte's peptone.

2 kilogrammes of gold label gelatin (Hesteberg's). 
100 grammes of agar-agar in substance.

200 grammes of sodium chloride (ordiuary table salt).

500 grammes of pure glycerin.

50 grammes of pure glucose.

20 grammes of pure lactose.

100 grammes of caustic potash.

200 c.c. of litmus tincture.

10 grammes of rosolic acid (corallin).

Blue and red litmus paper; curcuma paper.

5 grammes of phenolphtalein in substance.

Filter paper, the quality ordinarily used by druggists. 100 grammes of pyrogallic acid.

1 kilogramme C. P. granulated zinc.

\section{GLASSWARE.}

200 best quality test-tubes, slightly heavier than those sold for chemical work, about 12 to $13 \mathrm{~cm}$. long and 12 to $14 \mathrm{~mm}$. inside diameter.

15 Petri double dishes about 8 or $9 \mathrm{~cm}$. in diameter and from 1 to $1.5 \mathrm{~cm}$. deep.

6 Florence flasks, Bohemian glass, 1000 c.c. capacity.

6 Florence flasks, Bohemian glass, 500 c.c. capacity.

12 Erlenmeyer flasks, Bohemian glass, 100 c.c. capacity.

1 graduated measuring cylinder, 1000 c.c. capacity.

1 graduated measuring cylinder, 100 c.c. capacity.

25 bottles, 125 c.c. capacity, narrow necks with ground glass stoppers.

25 bottles, 125 c.c. capacity, wide mouths, with ground glass stoppers.

1 anatomical or preserving jar, with tightly fitting 
cover, of about 4 litres capacity, for collecting bloodserum.

2 battery jars of about 2 litres capacity, provided with loosely fitting, weighted, wire-net covers, for mice.

10 feet of soft glass tubing, 2 or $3 \mathrm{~mm}$. inside diameter.

20 feet of soft glass tubing, $4 \mathrm{~mm}$. inside diameter.

6 glass rods, 18 to $20 \mathrm{~cm}$. long and 3 or $4 \mathrm{~mm}$. in diameter.

6 pipettes of 1 c.c. each, divided into tenths.

2 pipettes of 10 c.c. each, divided into cubic centimetres and fractions.

1 burette of 50 c.c. capacity, divided into cubic centimetres and fractions.

1 separating funnel of 750 c.c. capacity, for filling tubes.

2 glass funnels, best quality, about $15 \mathrm{~cm}$. in diameter.

2 glass funnels, best quality, about $8 \mathrm{~cm}$. in diameter.

2 glass funnels, best quality, about 4 or $5 \mathrm{~cm}$. in diameter.

2 porcelain dishes, 200 c.c. capacity.

6 ordinary water tumblers for holding test-tubes.

1 ruled plate for counting colonies.

1 gas generator, 600 c.c. capacity, pattern of Kipl or v. Wartha.

BURNERS, TUBING, FTC.

2 Bunsen burners, single flame.

1 Rose burner.

1 Koch safety burner, single flame.

6 feet of white rubber gas-tubing. 
12 feet of pure red rubber tubing of 5 to $6 \mathrm{~mm}$. inside . diameter.

1 thermo-regulator, pattern of L. Meyer or Reichert.

2 thermometers, graduated in degrees Centigrade, registering from $0^{\circ}$ to $100^{\circ}$, graduated on the stem.

1 thermometer graduated in tenths and registering from $0^{\circ}$ to $50^{\circ} \mathrm{C}$.

1 thermometer registering to $200^{\circ} \mathrm{C}$.

\section{INSTRUMENTS, ETC.}

1 microtome, pattern of Schanze, with knife.

1 razor strop.

6 cheap quality scalpels, assorted sizes. 2 pairs heavy dissecting-forceps.

1 pair medium-size straight scissors.

1 pair small-size straight scissors.

1 hypodermic syringe that will stand steam sterilization.

2 teasing-needles.

1 pair long-handled crucible tongs for holding mice.

1 wire mouse-holder.

2 small pine boards on which to tack animals for autopsy.

2 covered stone jars for disinfectants and for receiving infected materials.

\section{IYCUBATORS AND STERILIZERS.}

1 incubator, simple square form, either entirely of copper, or of galvanized iron with copper bottom.

1 medium-size hot-air sterilizer with double walls, asbestos jacket, and movable false bottom of copper plates. 
1 medium-size steam sterilizer; either the pattern of Koch, or that known as the Arnold steam sterilizer, preferably the latter.

\section{MISCELLANEOUS.}

1 pair of balances, capacity 1 kilogramme; aceurate to 0.2 gramme.

1 set of cork borers.

1 hand-lens.

1 wooden filter-stand.

2 iron stands with rings and clamps.

3 round, galvanized iron wire baskets to fit loosely into steam sterilizer.

3 square, galvanized iron wire baskets to fit loosely into hot-air sterilizer.

1 sheet-iron box for sterilizing pipettes, etc.

1 covered, agatc-ware saucepan, 1200 e.c. capacity.

2 iron tripods.

1 yard of moderately heavy wire gauze.

2 test-tube racks, each holding 24 tubes, 12 in a row.

1 constant-level, east-irou water-batll.

2 potato-knives.

2 test-tube brushes with reed handles.

Cotton batting.

Copper wire, wire nippers.

Round and triangular files.

Labels.

Towels and spouges. 


\section{IN DE X.}

BBE, substage condensing sysA tem of, 26

Abscess, histological study of, 249 production of, 247, 248, 249

Aērobic bacteria, 33

Aērobioscope, 508

Agar-agar, preparation of (sce Media). properties of, 76,77

Agglutinin, 497

Air, bacteriological analysis of, 505-510

Petri's method for, 507

Sedgwick-Tucker method, $507-510$

Alexines, 459, 464

Anaërobic bacteria, 33

methods of cultivating, 194-200

Buchner's, 196

Esmarch's 199

Frānkel's, 196

Hesse's, 194

Kitasato andWeil's, 199

Koch's, 194

Liborius's, 194

Aniline dyes for differentiating bacteria, 190

Animals, fluctuations in weight and temperature of, 221227

inoculation of, 206-221

apparatus used in, 208, $209,210,217$

intralymphatic, 218

intraocular, 220

intraperitoneal and plenral, 218

intravascular, 212
Animals, inoculation of, subcutaneous, 206

obserrations of, after inoculation, 221-227

post-mortem examination of, 228-233

cultures from tissues at, 230

disinfection of implements after, 232

disposal of remains from, 232

external inspection, 228

incision through skin, 228

Nuttall's spear for use at, 230

opening the body cavities, 229

position of animal, 228 precautions during, 228 preservation of tissues from, 231

Anthrax, 412-427

animals that are susceptible to, 420

bacillus of, $412-427$

biology of, 415-4is

discovery of, 17,412

experiments with, $422-$ 427

morphology of, 412415

pathogenesis of, 418$420^{\circ}$

protective inoculation against, $420-422$

spore - formation, 413415

staining of, 417 
Anthrax, symptomatic, bacillus Bacteria, constancy in morpholof, $446-452$

Antiseptic, definition of, 71

Antiseptics, tests of, 520

Apparatus necessary to bacteriological work, $526-532$

preparation of, 109

Appendix, list of apparatus, 526

BACILLI, 36-38

B differentiation from spores, 40

flagella upon, 45

involution-forms of, 39

life-cycle of, 38

mode of multiplication, 4144

motility of, 45

spore-formation in, 38,39

Bacillus anthracis, 412-427

coli communis, 357-364

"comma," 365-393

diphtheriæ, 325-341

Finkler-Prior, 394-399

influenzæ, 310-314

lepræ, 306, 307

mallei (of glanders),315-324

Neapolitanus, 357-364

nitrifying, 428-433

cedematis maligni, 441-446

of bubonic plague, 269-275

pyocyaneus, $265-269$

pseudo-diphtheria, 339

smegma, 305-307

subtilis, 241

symptomatic anthrax, 446

syphilis, 305-307

tetani, 434-441

tuberculosis, 299-308

typhi abdominalis, $342-356$

Bacteria, aërobic, 33

anaërobic, 33

methods of cultivating, 194-200

behavior toward staining-reagents, 190

capsule surrounding, 152

chromogenic, 29

classification of, 36

conditions necessary to growth of, 35

ogy of, 39

definition of, 27

denitrifying, 30

discovery of, $13-15$

facultative, 34

fermentation by, 191

apparatus for testing, 192

gases resulting from, 193

flagellated forms of, 45

identification of, 177

involution-forms of, 39

isolation of, in pure culture, 72-76

principles of, 72-74

on slanted media, 123 , 124

microscopic examination of, 178-185

modes of multiplication of, 41-43

morphology of, 36-46

motility of, $45-46$

nitrifying, $30,428-433$

nutrition of, 31-33

photogenic, 30

points to be observed in describing, 203

reaction produced by, 189

relation to man, 28,29

relation to temperature, 34 , 35

results of growth, 29, 30

rôle in nature, 28

saprogenic, 30

spore-formation of, 38-41, 43,44

study of, 185

staining-reactions of, 190

systematic study of, 177

thermophilic, 34,35

thiogenic, 30

zymogenic, 30

Bacteriology, application of metl1ods of, 235

Bacterium coli commune, 357364

characteristics of cultural, 359-361 
Bacterium coli commune, char- Cholera Asiatica, beharior of acteristics of, morphological, 358 pathogenic, 362 differentiation of, from bac. trph. abdom., 361 where found, 357

Behring and Kitasato, 470

Billroth, 23 and Tiegel, 24

Birch-Hirschfeld, 22

Black leg (see Symptomatic Anthrax).

Blood, relations to bacteria and to toxins, 467

Blood-serum as culture medium (see Media).

germicidal element of, 467469 action of, 464

Bolton's potato method, 93

Bonnet, 20

Booker's modification of Esmarch's method, 121

Bouillon (see Media).

Brieger and Cohn, 441-459

Brooding-oven, 125-127

Brownian motion, 184

Buchner, 467-471

Bulb for water samples, 494

Burdon-Sanderson, 25

Burner, Koch's safety, for use with incubator, 127

CARBOLIC acid as disinfectCholera Asiatica, beharior of
spirillum of, in
water, $381-383$ characteristics of, cultural, 368 $-376$

morphological, 366-368

destiny of, in dead body, 384-386

effects of drying, 386

existence outside the body, 381

experiments upon animals with, 376 $-380$

general considerations upon, 380

location in the body, 380,381

poisons produced by, 375

relation to gases, $374,386-387$ to other bacteria, 374,385 to pntrefaction, 384 to sunlight, 383 specific reaction of immuned animals to, 379 toxin of, 458

Chromogenic bacteria, 30

Classen, 23

Cohn, 21

Colon bacillus (see Bacterium Colli Commune).

Colonies, counting of, 500 study of, 133-135

Chauveau, 461

Cherreul and Pasteur, 19

Chlorophyll, 27, 28

Cholera Asiatica, diagnosis of, 387-393 method of Schottelius, 374,375 spirillum of, 365 behavior of, in butter, 385 in milk, 384 in soil, 383

Comma bacillus (see Cholera Asiatica).

Cornet, 297

Corrosive sublimate as disinfectant, 66-68

Cooling-stage, 115,120

Cover-slips, cleaning of, 140 impression, 144

microscopic examination of, 181 
Cover-slips, preparation of, 141 steps in making, 141

Cultures, gelatin, 187 hanging-drop, 183 potato, 188 pure, 135 reactions of, 189 stab- and smear-, 135

Cygnæus, 347

\section{DECOLORIZING solutions,}

Decomposition, 27

Defensive proteids, 469

Deneke's cheese spirillum (see Spirillum tyrogenum).

Denitrifying bacteria, 30

Diphtheria, bacillus of, 325-341 cultural peculiarities of, 330-334

experiments upon, 340

location in tissues, 335 337

method of obtaining, 325

modification in virulence, 338

morphology of, 327

pathogenesis of, 334340

poison produced by, 337 potency of, 459

principles of immunizing against, 430, 431

pseudo-diphtheria bac, 339

histological clianges accomnanying, 336

Diplococci, 38

Disinfectants and antiseptics, experiments with, 521

general considerations, 64-71 methods of testing, 512

precautions to be observed, 514

use of animals as test-objects for, 519

use in the laboratory, 70-71

Disinfection, general considerations, $64-71$
Disinfection, influence of temperature on, 67

inorganic salts in, 65-67

in the laboratory, 70,71

modus operandi, 66

reliable agents for purposes of, 69,70

selection of agents to be used in, 65,66

Dunham's solution, 104

\section{TBERTH, 23}

14 Ehrlich, 23

Emmerich and Fowitzky, 478 and Mattei, 472

Erysipelas, 256

Escherich, 357

Esmarch tubes, 120, 123

Booker's method of rolling, 121

made of agar-agar, 122

Exposure and contact-experiments upon, 236

FACULTATIVE bacteria, 34

fi use of the term, 34

Fehleisen, 23

Fermentation, 27, 191 gases resulting from, 193 particular forms of, 30 -tube, 192 method of using, 192194

Filter, method of folding, 85-87

Finkler-Prior bacillus, 394-399

Flagella, 45, 46

methods of staining, 155159

Bunge's, 157

Lœfler's 155

van Ermengem's, 158

Flagellated organisms, 45

Frankland, G. and P. F., 430

Funnel for filling aêrobioscope, 510

for filling test tubes, 110 , 111

for filtering cultures, 517 lot water, 87 
GAS-PRESSURE regulator, G 131

Gelatin, cultures in, 187

their characteristics, 187,188

preparation of (see Media). properties, $76-78$

Geppert, 66, 67, 516

Glanders, 315-324

bacillus of, 317

cultivation of, 315-320

inoculation with, 320

morphology, 317, 318

staining of, in tissues, 321

diagnosis of, by use of mallein, 323

by Strauss's method, 323

manifestations of, 315-317

histology of, 316,317

susceptibility of animals to, 320

synonyms, 315

Gonococcus, 258-266

appearance in pus, 258

cultivation of, 260

Bumm's method for the, 260

Wertheim's method for the, 260

Wright's method for the, 261

distinguishing features of, 265

morphology of, 259

pathogenesis, 264-265

vitality of, 264

Gonorrhœa, pus of, 259

Green pus bacillus (see Bacillus pyocyaneus).

Guarniari's agar-gelatin, 107

HALSTED, 219, 246

HI Hanging-drop, 183

Hankin, 468, 469 and Martin, 468

Henle, 18

Hoffmann, 19

Hot-water funnel, 87

Hydrogen, test for purity of, 198
Hypodermic syringes and needles, 213,217

[MBEDDING of tissues, 164, 165

Immunity, 460 acquired, 460 blood in, 467

conclusions concerning, 480, 483

earlier studies on blood relative to, 465

"exhaustion" hypothesis, 463 experiments of the Klemperers on, 475

humoral theory of, 464

hypothesis of Buchner, 471 evidence in favor of, 472

natural, 460

nature of protective bodies, 467,469

observations of Behring and Kitasato, 470

"retention" hypothesis, 461 theory of Metchnikoff, 463

Incubator, 125, 127

burner for heating, 127

Indol, production of, by bacteria, 200

method of detecting, 201

Infection, 453, 460

chemical nature of, 457

conclusions concerning, 459 modus operandi, 457

poisons present in, 456,459

study of types of, 453,458

Influenza, bacillus of, 310,314 cultivation of, 311,312 dissemination of, 313 isolation of, from tissues, 313

morphology of, 310

occurrence in tissues, 313

staining of, 310,311

susceptibility of animals to, 313

vitality of, 312

Inoculation of animals, 206-221 intraocular, 220 
Inoculation, intraperitoneal and Malignant œedema, bacillus of, pleural, 218

intravascular, 212

subcutaneous, 206

intralymphatic, 218

apparatus used in, 208, 209, 210,217

Introduction, 13-26

Involution-forms of bacteria, 39

Isolation of colonies on slanted media in tubes, 123, 124

JORDAN and Richards, 430

KLEBS, 23-25, 327

I Klemperer, F. and G., work on pneumonia, 475

Koch, fundamental researches of, 25,26

postulates of, 298

safety burner of, 127

ACTOSE-LITMUS agar-agar

1 or gelatin (see Media).

Leeuwenhoek, 13-16

Lens for counting colonies, 502

Lepra bacillus, 306, 307

staining-peculiarities of, 306,307

Letzerich, 23

$$
23
$$

Levelling-tripod, 115

Lime, chloride of, 71

milk of, 69,71

Litmus milk, 103

Loefller's alkaline methyleneblue, 147

blood-serum mixture, 107

isolation of the bacillus of diphtheria, 327

stain for flagella, 46,155

Loefler and Schütz, discovery of the bacillus of glanders, 317

Lukomsky, 23

MALIGNANT odema, bacillus of, 441,446

cultural peculiarities of, 443,444

Mallein, 323

morphology of, 442

pathogenesis of, 444

susceptibility of ani-

mals to, 444

Meat-extracts in culture media, 84

-infusion, 107

Media, culture, 79

agar-agar, 89

clarification of, 90

filtration of, 90

glycerin, 91

neutralization of, $79-84$

solution of, 89,91

blood-serum, 95

Councilman - Mallory method, 99

mixture of Lœfller, 107

Nuttall's method, 100

original method of Koch, 95-99

preservation of, 99 , 102

by chloroform, 102

sterilization and so-

lidification of, 97 99

bouillon, 79

neutralization of, $79-84$

gelatin, 84

clarification of, 87

filtration of, $85-$ 87

solution of, 85

sterilization of, 84 , 85

Guarniari's agar-agar gelatin, 107

lactose-litmus agar-agar or gelatin, $106^{\circ}$

litmus milk, 103

meat-infusion, 107

milk, 103

-agar-agar, 89 
Media-, culture, peptone solution, Dunham's, 104 rosolic-acid- reptone solution, 105

potatoes, 92

Bolton's method, 93

Esmarch's method, 94

mashed, 94

original method, 92

Metchnikoff, 463

Milk (see Media).

Micrococci, 36, 37

mode of multiplication, 41,42

Micrococcus lanceolatus, 280 285

irregularities in development, 282, 284

morphological peculiarities, 281

results of inoculation with, 284,285

staining of, 284

susceptibility of animals to, 285

variations in virulence, 285

where found, 282

Micrococcus tetragenus, 279, 285$28 \mathrm{~s}$

cultural peculiarities of, 286-288

morphology of, 286 susceptibility of animals to, 288

where found, 286

Microscope, parts of, 178-181

Microtome, 163

N̈̈GELI, 31

1 Nassiloff, 23

Needham, 18

Nitrification, 428

Nitrifying bacteria, 428-433

Nitrites, test for, 202

Nitro-monas of Winogradsky, $430-433$

cultural peculiarities of, 431-433

morphology of, 431
Normal solution, 196

Nuttall, 230, 464-467

OL immersion system, use of, 180

Oertel, 23, 386

Ozanam, 17

PARASITE, 27

1 Pasteur, 17, 19, 25, 442, 462

Peptone, test of purity of, 104, 105

with rosolic acid, 105

Peritenitis, production of, 247

Petri's dishes, 119

Pfeiffer, $375,379,350$

Phagocy tosis, 463

Photogenic bacteria, 30

Plague, bubonic, bacillus of, 270276

cultivation of, 273

mode of infection with, 275

morphology of, 272

occurrence in tissues, 273,275

pathogenesis, 273,274

vitality of, 274

Plates, apparatus employed in making, 113-120

Esmarch's modification, 120

Booker's modification of, 121

Koch's fundamental observations, $72-73$

materials used in making, 113

Petri's modification, 119

principles involved, $72-76$

technique of making, 113116

Platinum needles and loops, 114 Plenciz, 16

Post-mortem examination of animals, 228-233

cultures from tissues at, 230

disinfection of implements after, 232 
Post-mortem examination, disposal of remains from, 232

external inspection at, 228

incision through the skin at, 228

Nuttall's spear for use at, 230

opening of the body cavities, 229

position of animal during, 228

precautions during, 228

preparation of coverslips at, 231

preservation of materials, 231

Postulates of Koch, 298

Potato, characteristics of cultures on, 188

preparation for culture purposes (see Media).

Prudden, 293, 467

Pseudo-diphtheria bacillus, 339 -tuberculosis, 309

Pure culture, 135

Pus, microscopic appearance of, 243

Putrefaction, 27

Pyæmia, production of, 248

Pyocyaneus, bacillus, 266-270

chameleon phenomena of, 269

pathogenic properties of, 269,270

protective properties of, 270

QUARTER evil or quarter ill (see Symptomatic Anthrax).

RECKLINGHAUSEN, 22, 23

R Regulator, gas-pressure, 131

Rindfleisch, 22 thermo-, 128-131

Rosolic - acid - peptone solution (see Media).

Roux and Yersin, 459
SAPROGENIC bacteria, 30

S Saprophyte, 27 rôle in nature, 28

Sarcinæ, 38

mode of multiplication, 42

Schottelius's method of examining cholera evacuations, 374, 375

Schröder and Dusch, 19!

Schulze, 19

Schwann, 19

Section-cutting, 162

Septicæmia, 279, 280, 285

from micrococcus tetragenus, 285

from sputum, 280

Skin-disinfection, experiments in, 524

Smear-cultures, 135

Smegma bacillus, staining-peculiarities of, 306-308

Soil, bacteriological analysis of, 510

nitrifying bacteria in, 428 organisms present in, 433

phenomena in operation in, 428-430

Spallanzani, 18, 19

Spirilla, 36-41

Spirillum of Asiatic cholera 'see Cholera :

of Deneke, 394-403

biology of, 399-402

morphology of, 399 pathogenesis of, 402

of Finkler-Prior (see Vibrio Proteus).

of Metchnikoff (see Vibrio

Metchnikovi).

of Miller, 403-406 biology of, 403-406 morphology of, 403 pathogenesis of, 406

tyrogenum (see Spirillum of Deneke).

undula, 45

Spores, formation of, 43-45 method of studying, 38$41,43,44,185$

mode of development, 43,44 recognition of, 40,44 
Spores, staining of, 152

Sputum, inoculations with, 279

microscopic examination of, 277,278

pathogenic properties of, 279 , 280

septicremias, 280, 285

tuberculosis, 289

tubercular, 277

Stab-cultures, 135

Staining, methods and solutions used in, 139-162

acetic acid, 152

Bunge's, $15 \tilde{\imath}$

Gabbett's, 151

general remarks on, 159

Gram's, 151

Gray's, 173

Koch-Ehrlich's, 14i, 14S, 172

Kuehne's, 170

Løeftler's blue, 147

Loffler's flagellar, 155

Mceller's, 154

ordinary solutions used, 145 bottles for holding, 146

ran Ermengem's, $15 \mathrm{~s}$

Weigert's, 17 I

Ziehl-Neelsen, 147, 172

Staphylococcus pyogenes albus, 251

aureus, $244-251$ cultural peculiarities of, $245-24 i$ pathogenesis, 247 where to be excitreus, 25$]$ pected, 246

Sterilization, chemical, $64-\bar{\imath} 1$ direct, $56-5 \mathrm{~S}$

experiments upon, 239-243

by heat, $49-64$ principles involved, 52 by hot air, 63,64 apparatus used, 63

by steam, 51-61 apparatns used, $58-62$ under pressure, 56, 61, 62 intermittent, $52-55$ at low temperature, 55 principles involved, $47-70$
Sterilization, use of the term, $47-$ 49

Sternberg, 282

Strauss's method for diagnosis of glanders, 323

Streptococci, 38

mode of multiplication, 42

Streptococcus pyogenes, $252-25 \pi$

biology of, $252-256$

effects of inoculation with, 256

morphology of, 253

where to be expected, $25 \%, 256$

Subtilis bacillus, 241

Suppuration, 244

bacteria common to, 246

general remarks upon, 257

less common causes of, 251, $25 \%, 258$

microscopic appearance of pns, 244

Symptomatic anthrax, bacillus of, 446-452

biology of, 448-451

differentiation from bacillus of malignant redema, 452

morphology of, 447

pathogenesis, 451

susceptibility of animals to, 452

Syphilis bacillus, staining of, $304-30 i$

TEST-TUBES, cleaner for, 109

1 cleaning of, 109

filling with media, 110 apparatus for, 111 plugging with cotton, 110 position after flling, 112 sterilization of, 110

Tetanus, bacillus of, 434-441 biology of, $436-439$ effects on animals, 439 method of obtaining, 434 morphology of, 436 poison produced by, 440, 441

toxin, potency of, 441 
Tetrads, 38

Thermophilic bacteria, $30,54,55$

Thermo-regulator, 128

Thermostat (see Incubator).

Thiogenic bacteria, 30

Tissues, cultures from, at autop. sies, 230

Nuttall's spear for making, 230

cutting sections of, 162

hardening of, 162

imbedding of, 164, 165

in celloidin, 164

in paraffin, 165

preservation of, 162

staining of bacteria in, $165-$ 176

special methods, 168176

dahlia, 170

dry, 173

Ehrlich's, 172

Gram's, 168

Gray's, 173

Kuehne's, 170

Weigert's, 171

'Zieh I-Neelsen's, 172

Toxæmia, 457

steps in the process, 168

Toxins, 456-459

Traube and Gscheidlen, 465

Treviranus, 19

Tripod for levelling plates, 115

Tube, Esmarch, 120, 123

Tuberculin, 308

Tuberculosis, 289-310

cavity-formation in, 293, 294 conditions simulating, 309 diffuse caseation of, 292 encapsulation of tubercular foci, 295 giant cells in, 292

location of bacilli in, 298

manifestations in experimental, 290

miliary tubercles, structure of, 291

modes of infection, 296

primary infection, 295

pseudo, 309, 310

sputum in, 277
Tuberculosis sputum, inoculation of animals with, 279

microscopic appearance of, 278,279

staining of, 148

susceptibility of animals to, 308

Tuberculosis, bacillus of, 299$30 \mathrm{~s}$

appearance of cultures, 302

cultivation from tissues, 300

methods of staining, 148 dry method, 173

Gabbett's, 151

Gray's, 173

Koch-Ehrlich's, 148, 172

Nuttall's modification, 150

Ziehl-Neelsen's, 172

microscopic appearance of, 303

organisms that simulate it, 305

differential diagnosis of, 305

staining of, in tissues, 172-176

staining-peculiarities of, 304

toxin of, 458

Tyndall, 20

Typhoid fever, bacillus of, $342-$ 356

constant properties of, 350

cultivation of, 343 346

difficulty in identifying, 350

differentiation from bacillus coli comInunis, 361

Elsner's medium for isolating, 353

experiments with, 356

inoculations with, 347 
Typhoid ferer, bacillus of, location of, in tissues, 346

morphology, 342

reaction of, with typhoid sernm,35̃ 1

source from which to obtain, 343,3 j̄j

water as a carrier of, 484,488

Widal's reaction with, 351

VAUGHAN, 469

Vibrio Metchnikovi, 406410

characteristics of, cultural, 407-409

morphological, 406 pathogenesis of, 409, 410

Vibrio proteus of Finkler and Prior, 394-399

cultivation of, 395

morphology of, 394

pathogenesis of, 398

relation to cholera nostras, 394,399

Vibrion septique, $\mathbf{4 4 1 - 4 4 6}$

WALDEYER, 22

Water, general observations upon bacteriological study of, 484 qualitative bacteriological analysis of, 490
Water, qualitative bacteriological analysis of, precautions in obtaining sample, 490

prelimnary steps in, 491

quantitative bacteriological analysis of, 493

counting of colonies in, 500 apparatus for, 501-505

dilution of sample in, 497

obtaining sample for, 494

selection of proper medium for, 498500

source of error, 505

relation to epidemics, 484,485 typhoid bacilli in, 486-488

value of bacteriological examination of, $487-490$

value of chemical examination of, 486,489

Weigert, 26

Welch, 25S, 280, 283

Widal's reaction, 351

Wilde, 23

Winogradsks, nitro-monas of, 430-433

Wound infection, 22-26

Wurtz's agar-agar and gelatin, 106

7OOGLEEA of bacteria, 40

L Zymogenic bacteria, 30 



\section{LEA BROTHERS \& COMPANY,}

706, 708 $\& 10$ gansom St., Philadelphia.

111 Fifth Ave. (Cor. 18th St.), New York.

The books in the snnered list will be sent by mail, post-paid, to any Post-Office in the United States, on receipt of the printed prices.

\section{IN $\mathbf{D} \mathbf{E} \mathbf{X}$.}

ANATOMY. Gray, p. 11 ; Allen, 3 ; Treves, 30 ; Gerrish, 11; Ellis, 9. DICTIONARIES. Dunglison, p. 8; Duane, 8 ; National, 4.

PHYSICS. Draper, p. 8; Robertson, 24.
[Schofield, 25. PHYSIOLOGY. Foster, p. 10 ; Dalton, 7 ; Chapman, 5 ; Powers, 23 ; CHEMISTRY. Simon, p. 26 ; Attfield, 3 ; Fornes, 10; Chalres, 5 ; PHARMACY. Caspari, p. 5. [Luff, 19 ; Remsen, 24.

MATERIA MEDICA. Culbretb, p. 6 ; Maisch, 19; Farquharson, 9 ; DISPENSATORY. National, p. 21.

[Bruce, 4.

THERAPEUTICS. Hare, p. 13 ; Fothergill, 10 ; Whitla, 31 ; YearBook. 31 ; Hayem \& Hare, 14 ; Bruce, 4.

PRACTICE. Flint, p. 9 ; Loomis \& Thompson, 19; Lyman, 19.

DIAGNOSIS. IIusser, p. 21; Hare, 12; Simon, 25; Herrick, 15; Hutchison \& Rainey, 16.

CLIMATOLOGY. Solly, p. 26 ; Hayem \& Hare, 14. [Hamilton, 12. NERVOUS DISEASES. Dercum, p. 7; Gray, 11; Mitchell, 20 ; MENTAL DISEASES. Clouston, p. 6 ; Sarage, 24 ; Folsom, 10.

BACTERIOLOGY. Abbott, p. 2 ; Vanghan \& Nory. 30 ; Senn's (Surgical), 25.

[Dunham, 8 .

HISTOLOGY. Klein, p. 18; Schafer's Essen., 25 ; Schafer's Pract., 25;

PATHOLOGY. Green, p. 12; Gibbes, 10; Coats, 6; Pepper (Surgical), 23

SURGERY. Park, p. 22; Dennis, 7; Roberts, 24; Ashhurst, 3; Treves, 29; Bryant, 5 ; Druitt, 8.

SURGERY-OPERATIVE. Stimson, p. 27 ; Smith, 26 ; Treves, 29.

SURGERY-ORTHOPEDIC. Young, p. 31 ; Gibney, 10.

SURGERY - MINOR. Wharton, p. 30.

FRACTURES and DISLOCATIONS. Hamilton, p. 12; Stimason, 27. OPHTH ALMOLOGY. Norris \& Oliver, p. 21; Nettleship, 21; Juler, 17; OTOLOGY. Politzer, p. 23 ; Burnett, 5 ; Field, 9; Bacon, 4. [Berry, 4. LARYNGOLOGY and RHINOLOGY. Browne, p. 4, Coakley, 6. DENTISTRY. Essig (Prosthetic), p. 9 ; Kirk (Operative), 17 ; American System, 2; Coleman, 6; Burchard 5.
[Morris, 20. URINARY DISEASES. Roberts, p. 24; Black, 4; Purdy, 23; VENEREAL DISEASES. Taylor, p. 28; Hayden, 14 ; Culver \& Hayden, 6 ; Cornil, 6.

SEXUAL DISORDERS. Fuller, p. 10 ; Taylor, 29.

DERMATOLOGY. Hyde, p. 16 ; Jackson, 16 ; Pye-Smith, 24 ; Morris, 20 ; Jamieson, 17 ; Hardaway, 12.

GYNECOLOGY. American System, p. 3; Thomas \& Mandé, 29 Emmet, 9 ; Tait, 28 ; Davenport, 7 ; May, 20 ; Dudley, 8.

OBSTETRICS. American System, p. 3; Daris, 7; Parvin, 22 ; Playfair, 23 ; King, 17 ; Jerrett, 17 ; Herman, 15 ; Barnes, 3.

PEDIATRICS. J. Lewis Smith, p 26; Owen, 22; Thomson, 29.

HYGIENE. Egbert, p. 9; Richardson, 24.

MEDICAI JURISPRUDENCE. Taylor, p. 28.

QUIZ SERIES and MANUALS. Pp. 25 and 27.

5111899 
ABBOTT (A. O.). PRINCIPLES OF BACTERIOLOGY: a Practical Manual for Students and Physicians. New (4th) edition thoroughly revised and greatly enlarged. In one handsome $12 \mathrm{mo}$, vol. of 543 pages, with 106 engravings, of which 19 are colored. Cloth, $\$ 2.75$.

One of its most attractive charac- $\mid$ cessfully. To those who require a teristics is that the directions are so condensed yet nevertheless complete clearly given that anyone with a work upon Bacteriology we most moderate amount of laboratory train- cordially recommend it.-The Theraing can, with a little care as to peutie Gazette. detail, make his experiments suc-

AMERICAN SYSTEM OF PRACTICAL MEDICINE. A SYSTEM OF PRACTICAL MEDICINE. In contributions by Various American Authors. Edited by ALFRED L. LOOMIS, M.D., LL.D., and W. GilmaN Thompson, M. D. In four very handsome oetavo volumes of about 900 pages each, fully illustrated. Completc work now ready. Per volume, eloth, $\$ 5$; leather, $\$ 6$; half Morocco, $\$ 7$. For sale by subscription only.

Prospectus free on application to the Publishers.

Every chapter is a masterpiece of cine" is a work of which every completeness, and is particularly ex- American pliysician may reasonably cellent in regard to treatment, many feel proud, and in which every praccriginal prescriptions, formulæ, titioner will find a safe and trustcharts and tables being given for the worthy counsellor in the daily reguidance of the practitioner.

"The American System of Medi- Mredical Journal.

AMOERICAN SYSTEM OF DENTISTRY. In treatises by various authors. Edited by WILBOR F. LITCH, M.D., D.D.S. In four very handsome super-royal octavo volumes, containing about 4000 pages, with about 2200 illustrations and many full-page plates. Volume IV., preparing. Per vol., cloth, \$6; leather, $\$ 7$; half Morocco, $\$ 8$. For sale by subscription only. Prospectus free on application to the Publishers.

AMERICAN TEXT-BOOKS OF DENTISTRY. In Contributions by Eminent American Authorities. In two very handsome octavo volumes, richly illustrated :

PROSTHETIC DENTISTRY. Edited by CharLes J. Essig, M.D., D.D.S., Professor of Mechanical Dentistry and Metallurgy, Department of Dentistry, University of Pennsylvania, Philadelphia. 760 pages, 983 engravings. Cloth, $\$ 6$; leather, $\$ 7$. Net.

No more thorough production will It is up to date in every particular. be found either in this country or in It is a practieal course on prosthetics any country where dentistry is un. which any student ean take up durderstood as a part of civilization.- ing or after college.-Dominion DenThe International Dental Journal. tal Journal.

OPERATIVE DENTISTRY. Edited by FowarD C. KIRK, D.D.S., Professor of Clinical Dentistry, Department of Dentistry, University of Pennsylvania. 699 pages, 751 engravings. Cloth, $\$ 5.50$; leather, $\$ 6.50$. Net. Just ready. See also page 17 .

Written by a number of practi- . It is repletc in every particular tioners as well known at the chair and treats the subject in a progressive as in journalistic literature, many of manner. It is a book that every them teachers of eminence in our progressive dentist should possess, colleges. It should be included in and we can heartily recommend it the list of text-books set down as to the profession.-The Ohio Dentrl most usefil to the college student.- Journal.

The Dental News. 
AMERICAN SYSTEMS OF GYNECOLOGY AND OBSTETRICS. In treatises by the most eminent American specialists. Gynecology edited by MatTHEW D. MANx, A. M., M. D., and Obstetrics edited by BARTox C. HIRST, M.D. In four large octavo volumes comprising 3612 pages, with 1092 engravings, and 8 colored plates. Per volume, cloth, $\$ 5$; lesther, $\$ 6$; half Russia, $\$ 7$. For sale by subscription only. Prospectus free on application to the Publishers.

AMERICAN TEXT-BOOK OF ANATOMY. See Gerrish, page 11.

ALLEN (HARRISON). A SYSTEM OF HUMAN ANATOMY; WITH AN INTRODUCTORY SECTION ON HISTOLOGY, by E. O. Shax EsPEARE, M.D. Comprising $\$ 13$ double-columned quarto pages, with 350 engravings on stone, 109 plates, and 241 wood cuts in the text. In six sections, each in a portfolio. Price per section, $\$ 3.50$. Also, bound in one volume, cloth, $\$ 23$. Sold by subscription only.

A TEXT-BOOK OF OBSTETRICS BY AMERICAN ACTHORS. See Jexcett, page 17.

A TREATISE ON SURGERY BY AMERICAN AUTHORS. FOR STUDENTS AND PRACTITIONERS OF SURGERY AND MEDICINE. Edited by Roswell Park, M.D. See page 22.

ASHRURST (JOHN, JR.). THE PRINCIPLES AND PRACTICE OF SURGERY. For the use of Students and Practitioners. Sixth and revised edition. In one large and handsome octavo volume of 1161 pages, with 656 engravings. Cloth, $\$ 6$; leather, $\$ 7$.

As a masterly epitome of what has | text-book, we do not know its equal. been said and done in surgery, as $\mathbf{z}$. It is the best single text-book of succinct and logical statement of the surgery that we have yet seen in this principles of the subject, as a model country.-New York Post-Graduate.

A SYSTEM OF PRACTICAL MEDICINE BY AMERICAN AUTHORS. Edited by WilliaM PePper, M. D., LL. D. In five large octavo volumes, containing 5573 pages and $19 \mathrm{~S}$ illustrations. Price per volume, cloth, $\$ 5$; leather $\$ 6$; half Russia, $\$ 7$. Sold by subscription only. Prospectus free on application to the Publishers.

ATPFIELD (JOHN). CHEMISTRY; GENERAL, MEDICAL AND PHARMACEUTICAL. Fourteenth edition, specially revised by the Anthor for America. In one handsome 12mo. volume of 794 pages, with 83 illustrations. Cloth, $\$ 2.75$; leather, $\$ 3.25$.

It is replete with the latest inform- | been adopted, bringing the work into ation, and considers the chemistry of close touch with the latest United every substance recognized officially States Pharmacopaia, of which it is or in general practice. The modern a worthy companion.-The Pittsburg scientific chemical nomenclature has iredieal Revievo.

BALL (CHARIAS B.). THE RECTUM AND ANUS, THEIR DISEASES AND TREATMENT. Second edition. In one $12 \mathrm{mo}$. volume of 453 pages, with $60^{\circ}$ engravings and 4 colored plates. Cloth, $\$ 2.25$. See Series of Clinical Manuals, page 25.

BARNES (ROBERT AND FANCOURT). A SYSTEM OF OBSTETRIC MEDICINE AND SURGERY, THEORETICAL AND CLINICAL. The Section on Embryology by PrOF. Minves MARsHALL. Octavo, $\$ 72$ pages, with 231 illus. Cloth, $\$ 5$; leather, $\$ 6$. 
BACON (GORHAM) AND BLAKE (CIAARENCE J.). ON THE EAR. One $12 \mathrm{mo}$. volume, 350 pages. Fully illustrated. In press.

BARTHOLOW (ROBERTS). CHOLERA; ITS CAUSATION, PREVENTION AND TREATMENT. In one $12 \mathrm{mo}$. volume of 127 pages, with 9 illustrations. Clotl, $\$ 1.25$.

BARTHOLOW (ROBERTS). MEDICAL ELECTRICITY. A PRACTICAL TREATISE ON THE APPLICATIONS OF ELECTRICITY TO MEDICINE AND SURGERY. Third edition. In one octavo volume of 308 pages, with 110 illustrations.

BELL (F. JEFFREY). COMPARATIVE ANATOMY AND PHYSIOLOGY. In one $12 \mathrm{mo}$. volume of 561 pages, with 229 engravings. Cloth, \$2. See Students' Series of Manuals, page 27.

BERRY (GEORGE A.). DISEASES OF THE EYE ; A PRACTICAL TREATISE FOR STUDENTS OF OPHTHALMOLOGY. Second edition. Very handsome octavo vol. of 745 pages, with 197 original illus. in the text, of which 87 are exquisitely colored. Cloth, $\$ 8$.

BILLINGS (JOHN-S.). THE NATIONAL MEDICAL DICTIONARY. Including in one alphabet English, French, German, Italian and Latin Technical Terms used in Medicine and the Collateral Sciences. In two very handsome imperial octavo volumes containing 1574 pages and two colored plates. Per volume, cloth, $\$ 6$; leather, $\$ 7$; half Morocco, $\$ 8.50$. For sale by subscription only. Specimen pages on application to the publishers.

BLACK (D. CAMPBELL). THE URINE IN HEALTH AND DISEASE, AND URINARY ANALYSIS, PHYSIOLOGICALLY AND PATHOLOGICALLY CONSIDERED. In one 12mo. volume of 256 pages, with 73 engravings. Cloth, $\$ 2.75$.

A concise, yet complete manual, An excellent presentation of uroltreating of the subject from a prac- ogy in its latest phase, concise, practical and clinical standpoint. Its tical, clinical, well illustrated and usefulness should insure it a wel- well printed.-Maryland Medical come.-The Ohio Medical Journal. Journal.

BIOXAM (C. I.). CHEMISTRY, INORGANIC AND ORGANIC. With Experiments. New Amcrican from the fifth London edition. In one liandsome octavo volume of 727 pages, with 292 illustrations. Cloth, $\$ 2$; leather, $\$ 3$.

BRICINEIR (SAMUEI, M.). THE SURGICAL PATIENT. Treatment Before and After Operation. One handsome volume of about 400 pages, fully illustrated. Preparing.

BROA DBENT (W. H.). THE PULSE. In one 12mo. vol. of 317 pages, with 59 engravings. Cloth, $\$ 1.75$. Sce Series of Clinical Manuals, p. 25 .

BIROWNE (LENNOX). THE TIIROAT AND NOSE AND THEIR DISFASES. New (4th) and enlarged edition. In one imperial octavo volume of 751 pages, with 235 engravings and 120 illustrations in color. Cloth, $\$ 6.50$.

—_ KOCI'S REMEDY IN RELATION ESPECIALLY TO TIROAT CONSUMPTION. In one octavo volume of 121 pages, with 45 illustrations, 4 of which are colored, and 17 charts. Cloth, \$1.50.

BRUCE (y. MITCHELI). MATERIA MEDICA AND THERAPEUTICS. Fifth edition. In one 12mo. volume of 591 pages. Cloth, \$1.50. See Student's Series of Manuals, page. 27.

- PRINCIPLES OF TREATMENT. In one octavo volume. Preparing. 
BRUNTON (T. LAUDER). A MANUAL OF PHARMACOLOGY, THERAPEUTICS AND MATEPIA MEDICA; including the Pharmacy, the Phrsiological Action and the Therapeutical Uses of Drugs. In one octaro volnme.

BRYANT (THOMAS). THE PRACTICE OF SURGERY. Fourth American from the fourth English edition. In one imperial octaro vol. of 1040 pages, with 727 illustrations. Cloth, 6.50 ; leather, $\$ 7.50$.

BUYSTEAD (F. J.) AND TAYTOR (R. W.). THE PATHOLOGY AND TREATMENT OF VENEREAL DISEASES. See Taylor on Venereal Diseases, page $2 S$.

BURCHARD (HENRY H.). DENTAL PATHOLOGY AND THEPAPEUTICS. Handsome octaro, 575 pages, with 400 illustrations. Just ready. Cloth, net, $\$ 5.00$; leather, net, $\$ 6.00$.

BURNETT (CHARLES H.)。 THE EAR: ITS ANATOMY, PHYSIOLOGY AND DISEASES. A Practical Treatise for the Use of Students and Practitioners. Second edition. In one 8vo. volume of 5\$0 pages, with 107 illustrations. Cloth, $\$ 4$; leather, $\$ 5$.

BUTLIN (HENRY T.). DISEASES OF THE TONGUE. In one pocket-size $12 \mathrm{mo}$. rol. of 456 pages, with 8 col'd plates and 3 engrs. Limp cloth, \$3.50. See Series of Clinical Manuals, page 25.

CARTER (R. BRUDENELU) AND FROST (W. ADAJS). OPHTHALMIC SURGERY. In one pocket-size $12 \mathrm{mo}$. volume of 559 pages, with 91 engravings and one plate. Cloth, \$2.25. See Series of Clinical IIanuals, page 25.

CASPARI (CHARLES JR.). A TREATISE ON PHAPMACY.

For Students and Pharmacists. In one handsome octavo volume of 680 pages, with $2 S S$ illustrations. Cloth, $\$ 4.50$.

A work of more than ordinary / macy, and his contact with students merit, which impresses one with its made him aware of their exact orderly arrangement of subjects, wants in the matter of a manual. eminent practicality, but over and His rork is admirable, and the above all with the author's intimate student who cannot understand must knowledge of details. - The Amer. be dull indeed. The book is full of Druggist and Pharm. Record.

The author's duties as Professor of Theory and Practice of Pharmacr in the Maryland College of Pharnew, clean, sharp illustrations, which tell the story frequently at a glance. The index is full and accurate.National Druggist.

CHAPMAN (HENRY C.). A TREATISE ON HUMAN PHYSIOLOGY. In one octaro rolume of 925 pages, with 605 illustrations. Cloth, \$5.50; leather, \$6.50.

In every respect the work fulfils |mirable work of reference for the its promise, whether as a complete physician.- Worth Carolina Medical treatise for the student or as an ad- Journal.

CHARLES (T. CRANSTOUN). THE ELEMENTS OF PHYSIO. LOGICAL AND PATHOLOGICAL CHEMISTRY. In one hand. some octaro rolume of 451 pages, with 39 engravings and 1 colored plate. Cloth, $\$ 3.50$.

CHEYNE (W. WATSON). THE TREATMENT OF WOUNDS, ULCERS AND ABSCESSES. In one $12 \mathrm{mo}$. volume of 207 pages. Cloth, \$1.25.

One will be surprised at the need at any moment. The sections amount of practical and useful in- devoted to ulcers and abscesses are formation it contains; information indispensable to any physician.that the practitioner is likely to The Charlotte Medical Journal. 
Lea Brothers \& Co., Phrta Delphia and New York.

CHURCHUL (FLEETWOOD). ESSAYS ON THE PUERPERAL FEVER. In one octavo volume of 464 pages. Cloth, $\$ 2.50$.

CHARKE (W. B.) AND LOCKWOOD (C. B.). THE DISSECTOI'S MANUAL. In one $12 \mathrm{mo}$. volume of 396 pages, with 49 engravings. Cloth, $\$ 1.50$. See Students' Series of Mranuals, page 27.

CLFLAND (JOHN). A DIRECTORY FOR THE DISSECTION OF THE HUMAN BODY. In one $12 \mathrm{mo}$. vol. of 178 pages. Cloth, $\$ 1.25$.

CLINICAL MANUALS. See Series of Clinical Manuals, page 25.

CLOUSTON (THOMAS S.). CLINICAL LECTURES ON MENTAL DISEASES. New (4th) edition. In one octavo volume of 736 pages, with 15 colored plates. Cloth, $\$ 4.75$.

ToLsou's Abstract of Laws of U.S. on Custody of Insane, octavo, $\$ 1.50$, is sold in conjunction with Clouston on Mental Diseases for $\$ 5.50$ for the two works.

CLOWES (FRANK). AN ELEMENTARY TREATISE ON PRACTICAL CHEMISTRY AND QUALITATIVE INORGANIC ANALYSIS. From the fourth English edition. In one handsome $12 \mathrm{mo}$. volume of 387 pages, with 55 engravings. Cloth, $\$ 2.50$.

COAKLEY (CORNELIUS G.). THE DIAGNOSIS AND TREATMENT OF DISEASES OF THE NOSE, TIIROAT, NASOPHARYNX AND TRACHEA. In one $12 \mathrm{mo}$. volume of about 400 pages, fully illustrated. Preparing.

COATS (JOSEPH). A TREATISE ON PATHOLOGY. In one vol. of 829 pages, with 339 engravings. Cloth, $\$ 5.50$; leather, $\$ 6.50$.

COIEMAN (ALFRED). A MANUAL OF DENTAL SURGERY AND PATHOLOGY. With Notes and Additions to adapt it to American Practice. By Thos. C. Stellw wagen, M.A., M.D., D.D.S. In one handsome octavo vol. of 412 pages, with 331 engravings. Cloth, $\$ 3.25$.

CONDUE (D. FRANCIS). A PRACTICAL TREATISE ON THE DISEASES OF CHILDREN. Sixth edition, revised and enlarged. In one large 8vo. volume of 719 pages. Cloth, $\$ 5.25$; leather, $\$ 6.25$.

CORNL (V.). SYPIILIS: ITS MORBID ANATOMY, DIAGNOSIS AND TREATMENT. Translated, with Notes and Additions, by J. IIENRY C. Simes, M.D. and J. WilliaM WIIITE, M.D. In one 8vo. volume of 461 pages, with 84 illustrations. Cloth, $\$ 3.75$.

CULBRETH (DAVU M. R.). MATERIA MEDICA AND PHARMACOLOGY. In one handsome octavo volume of 812 pages, with 445 illustrations, Cloth, $\$ 4.75$.

A thorough, authoritative and adopted as the text-book in all colsystematic exposition of its most leges of pharmacy and medicine. important domain. - The Canada It is one of the most valuable works Lancet.

This work ought to be at once Medical Journal.

CULVER (E. M.) AND HAXUEN (J. 12.). MANUAL OF VENE. REAL DISEASES. In one $12 \mathrm{mo}$. volume of 289 pages, with 33 engravings. Cloth, $\$ 1.75$. 
DAITYON (JOHN C.). A TREATISE OY HUMAN PHYSIOLOGY. Seventh edition, thoroughly revised and greatly improved. In one very handsome octavo volume of 722 pages, with 252 engravings. Cloth, $\$ 5$; leather, $\$ 6$.

DOCTRINES OF THE CIRCULATION OF THE BLOOD. In one handsome $12 \mathrm{mo}$. volnme of 293 pages. Cloth, $\$ 2$.

DAVENPORT (F. H.). DISEASES OF WOMEN. A Manual of Gynecology. For the use of Students and Practitioners. New (3d) edition. In one handsome $12 \mathrm{mo}$. volume with many engravings. shortly.

DAIIS (EDWARD P.). A TREATISE OY OBSTETRICS. FOR STUDENTS AND PRACTITIONEPS. In one very handsome octaro volume of 546 pages, with 217 engravings and 30 full-page plates in colors and monochrome. Cloth, $\$ 5$; leather, $\$ 6$.

This work must become the prac- books on the subject. It is exceptiontitioner's text-book as well as the ally useful from every standpoint.student's. It is up to date in every Nashville Jour.of Med.and Surgery. respect.-Va. Med. Semi-Monthly. From a practical standpoint the

A work nuequalled in excellence. work is all that could be desired. A - The Chicago Clinical Reviero.

Decidedly one of the best text- treatise on obstetrics.-Med. News.

DAVIS (F. H.). LECTURES ON CLINICAL MEDICINE. Second edition. In one $12 \mathrm{mo}$. volume of 287 pages. Cloth, $\$ 1.75$.

DE LA BECHE'S GEOLOGICAL OBSERVER. In one large octavo volume of 700 pages, with 300 engravings. Cloth, $\$ 4$.

DENNTS (FREDERIC S.) AND BULINGS (JOHN S.). A SYSTEM OF SURGERY. In contributions by American Authors. Complete work in four very handsome octavo volumes, containing 3652 pages, with 1585 engravings and 45 full-page plates in colors and monochrome. Just ready. Per volume, cloth, $\$ 6$; leather, $\$ 7$; half Morocco, gilt back and top, $\$ 8.50$. For sale by subscription only. Full prospectus free on application to the publishers.

It is worthy of the position which American surgery and is thoronghly surgery has attained in the great practical.-Annals of Surgery.

Republic whence it comes. - The No work in English can be conLondon Lancet.

sidered as the rival of this.-The the most advanced condition of Sciences.

DERCUM (FRANCIS $\boldsymbol{x}$., EDITOR). A TEXT-BOOK ON NERVOUS DISEASES. By American Authors. In one handsome octavo volume of 1054 pages, with 341 engravings and 7 colored plates. Cloth, $\$ 6.00$; leather, $\$ 7.00$. Net.

Representing the actual status of The work is representative of the our knowledge of its subjects, and best methods of teaching, as develthe latest and most fully up-to-date oped in the leading medical colleges of any of its class.-Jour. of Amer- of this country.-Alienist and Neu-ican Med. Association.

The most -thoroughly up-to-date treatise that we have on this subject. - American Journal of Insanity. rologist.

The best text-book in any language.-The Medical Fortnightly.

DE SCHWEINTY (GEORGE E.). THE TOXIC AMBLYOPIAS. Their Classification, History, Symptoms, Pathology and Treatment. Very handsome octavo, 240 pages, 46 engravings, and 9 full-page plates in colors. Limited edition, de luxe binding, \$4. Net. 
DRAPER (JOHN C.). MEDICAL PHYSICS. A Text-book for Stu. dents and Practitioners of Medicine. In one handsome octavo volume of 734 pages, with 376 engravings. Cloth, $\$ 4$.

DRUITY (ROBERT). THE PRINCIPLES AND PRACTICE OF MODERN SURGERY. A new' American, from the twelfth London edition, edited by STANLEX BOYD, F.R.C.S. In one large octavo volume of 965 pages, with 373 engravings. Cloth, $\$ 4$; leather, $\$ 5$.

DUANE (ALEXANDER). THE STUDENT'S DICTIONARY OF MEDICINE AND THE ALLIED SCIENCES. New edition. Com. prising the Pronunciation, Derivation and Full Explanation of Medical Terms, with much Collateral Descriptive Matter. Numerous Tables, etc. Square octavo of 658 pages. Cloth, $\$ 3.00$; half leather, $\$ 3.25$; full sheep, $\$ 3.75$. Thumb-letter Index, 50 cents extra.

Medical students are here provided The book is brought accurately to with full information concerning date. It is a model of conciseness, every word they will meet in acquir- convenience and thoroughness, ing their professional education.-. Medical Record.

Nat. Med. Review.

Far superior to any dictionary for the medical student that we know of. -Western Med. and Surg. Reporter.

The standard dictionary for medi: cal students. - Charlotte IIed. Jour.

The best student's dietionary.Canada Lancet.

DUDIEY (E. C.). THE PRINCIPLES AND PRACTICE OF GYNECOLOGY. Handsome octavo of 725 pages, with +25 illustrations in black and colors. In press.

DUNCAN (J. MATTHEUVS). CLINICAL LECTURES ON THE DISEASES OF WOMEN. Delivered in St. Bartholomew's Hospital. In one octavo volume of 175 pages. Cloth, $\$ 1.50$.

DUNGLISON (ROBLEY). A DICTIONARY OF MEDICAL SCIENCE. Containing a full explanation of the various subjects and terms of Anatomy, Physiology, Medical Chemistry, Pharmacy, Pharmacology, Therapentics, Medicine, Hygiene, Dietetics, Pathology, Surgery, Ophthalmology, Otology, Laryngology, Dermatology, Gynecology, Obstetries, Pediatrics, Medical Jurispridence, Dentistry, ctc., etc. By Roblex Duxglison, M.D., LI. D., late Professor of Institutes of Medicine in the Jefferson Medical College of Philadelphia. Edited by RICHARD J. DUnGlison, A. M., M. D. Twenty-first edition, thoroughly revised and greatly enlarged and improved, with the Pronunciation, Accentuation and Derivation of the Terms. With Appendix. In one magnificent imperial octavo volume of 1225 pages. Cloth, $\$ 7$; leather, \$8. Thumb-letter Index for quick use, 75 cents extra.

The most satisfactory and authori- Pronunciation is indicated by the tative guide to the derivation, defini- phonetic system. The definitions are tion and pronunciation of medical unusually clear and concise. The terms. - The Charlotte Med Journal. book is wholly satisfactory.-UniCovering the entire field of medi- versity Medical Magazine.

cine, surgery and the collateral The volume is entitled to be called sciences, its range of usefuluess can an cncyclopredia rather than a dicscarcely be mensured.-Med. Record. tionary.-Brooklyn Med. Journal.

DUNHAM (EDWARI) K.). MORIBID AND NORMAL, IISTOI,()(iY. Octavo, 400 pages, riclily illustrated. Preparing.

FDES (ROIBERT T.). TEXT-BOOK OF THERAPEUTICS AND MATERIA MEDICA. In one 8 vo, volume of 544 pages. Cloth, $\$ 3.50$; leather, $\$ 4.50$.

HDLS (ARTHUR W.). DISEASES OF WOMEN. A Manual for Students and Practitioners. In one handsome 8 vo. volume of 576 pages, with 148 engravings. Cloth, $\$ 3$; lesther, \$4. 
FGBERT (SFNECA). A MANUAL OF HYGIENE AND SANITATION. In one $12 \mathrm{mo}$. volume of 359 pages, with 63 illustrations. Just ready. Cluth, I't, \$2.25.

EWIS (GEORGE VINER). DEMONSTRATIONS IN ANATOMY. Being a Guide to the Knowledge of the Human Body by Dissection. From the eighth and revised English edition. In one octavo volume of 716 pages, with 249 engravings. Cloth, $\$ 4.25$; leather, $\$ 5.25$.

EMINET (THOMAS ADDIS). THE PRINCIPLES AND PRACTICE OF GYN ECOLOGY, for the use of Students and Practitioners. Third edition, enlarged and revised. In one large 8 vo. volume of 880 pages, with 150 original engravings. Cloth, $\$ 5$; leather, $\$ 6$.

ERICHSEN (JOHN E.). THE SCIENCE AND ART OF SURGERY. A new American from the eighth enlarged and revised London edition. In two large octaro volumes containing 2316 pages, with 984 engravings. Cloth, $\$ 9$; leather, $\$ 11$.

ESSIG (CHARLES J.). PROSTHETIC DENTISTRY. See American Text-Books of Dentistry, page 2.

FARQUHARSON (ROBERT). A GUIDE TO THERAPEUTICS. Fourth American from fourth English edition, revised by Frask WoodBdry, M.D. In one $12 \mathrm{mo}$. volume of 581 pages. Cloth, $\$ 2.50$.

FIELD (GEORGE P.). A MANUAL OF DISEASES OF THE EAR. Fourth edition. In one octavo volnme of 391 pages, with 73 engravings and 21 colored plates. Cloth, $\$ 3.75$.

To those who desire a concise It is just such a work as is needed work on diseases of the ear, clear by every general practitioner. and practical, this manual com- Americun Practitioner and Ners. mends itself in the highest degree.

FLLNT (AUSTIN). A TREATISE ON THE PRINCIPLES AND PRACTICE OF MEDICINE. Seventh edition, thoroughly revised by FrEderick P. HENRY, M. D. In one large Svo, volume of 1143 pages, with engravings. Cloth. $\$ 5.00$; leather, $\$ 6.00$.

The work has well earned its lead- $\quad$ The leading text-book on general ing place in medical literature. The medicine in the medical sehools of element of treatment is by no means the United States. - Northwestern neglected; in fact, by the editor a Lancet.

fresh stimulus is given to this peces- The best of American text-books sary department by a comprehensive on Practice.-Amer. Jedico-Surgical study of all the new and leading the- Bulletin.

rapeutic agents.-Mredical Record.

A MANUAL OF AUSCULTATION AND PERCUSSION; of the Physical Diagnosis of Diseases of the Lungs and Heart, and of Thoracic Aneurism. Fifth edition, revised by JAMES C. WILSON, M. D. In one handsome $12 \mathrm{mo}$. volnme of 274 pages, with 12 engravings. - A PRACTICAL TREATISE ON THE DIAGNOSIS AND TREATMENT OF DISEASES OF THE HEART. Second edition enlarged. In one octavo volume of 550 pages. Cloth, \$4.

A PRACTICAL TREATISE ON THE PHYSICAL EXPLORATION OF THE CHEST, AND THE DIAGNOSIS OF DISEASES AFFECTING THE RESPIRATORY ORGATS. Second and revised edition. In one octavo volume of 591 pages. Cloth, $\$ 4.50$. MEDICAL ESSAYS. In one $12 \mathrm{mo}$. vol. of 210 pages. Cloth, $\$ 1.38$. ON PHTHISIS: ITS MORBID ANATOMY, ETIOLOGY, ETC. A Series of Clinical Lectures. In one 8 ro. volume of 442 pages. Cloth, $\$ 3.50$. 
FOLSOM (C. F.). AN ABSTRACT OF STATUTES OF U. S. ON CUSTODY OF THE INSANE. In one 8vo. vol, of 108 pages. Cloth, \$1.50. With Clouston on Mental Diseases (new edition, see page 6) $\$ 5.50$ for the two works.

FOSTER (MICHAEL). A TEXT-BOOK OF PHYSIOLOGY. New (6th) and revised American from the sixth English edition. In one large octavo volume of 923 pages, with 257 illustrations. Cloth, $\$ 4.50$; leather, $\$ 5.50$.

Unquestionably the best book that course, and all that the physician can be placed in the student's hands, will need as well.-Dominion Med. and as a work of reference for the Monthly.

busy physician it can scarcely be For physician, student, or teacher exeelled.-The Phila. Polyclinic. this is and long will remain the

This single volume contains all standard, up-to-date work on physithat will be necessary in a college ology.-Virginia Medical Monthly.

FOTHERGILL (J. MUNER). THE PRACTITIONER'S HANDBOOK OF TREATMENT. Third edition. In one handsome octavo volume of 664 pages. Cloth, $\$ 3.75$; leather, $\$ 4.75$.

To have a deseription of the clearly stated, cannot fail to prove normal pliysiological processes of an a great convenience to many thoughtorgan and of the methods of treat- ful but busy pliysicians. The pracment of its morbid conditions tieal value of the volume is greatly brought together in a single chapter, increased by the introduction of many and the relations between the two preseriptions-New York Med. Jour.

FOWNES (GEORGE). A MANUAL OF ELEMENTARY CHEMISTRY (INORGANIC AND ORGANIC). Twelfth edition. Embodying WATTS' Physical and Inorganic Chemistry. In one royal 12mo. volume of 1061 pages, with 168 engravings, and 1 colored plate. Cloth, $\$ 2.75$; leather, $\$ 3.25$.

FRANKI,AND (E.) AND JAPP (F.R.). INORGANIC CHEMISTRY. In one handsome octavo volume of 677 pages, with 51 engravings and 2 plates. Cloth, $\$ 3.75$; leather, $\$ 4.75$.

FULLER (EUGENE). DISORDERS OF THE SEXUAL ORGANS IN THE MALE. In one very handsome octavo volume of 238 pages, with 25 engravings and 8 full-page plates. Cloth, $\$ 2$.

It is an interesting work, and one tive and brings views of sound which, in view of the large and pathology and rational treatment to profitable amount of work done in many cases of sexual disturbance this field of late years, is timely and whose treatment has been too of en well needed.-Dedical Fortnightly. fruitless for good. - Annals of

The book is valnable and instruc- Surgery.

FULLER (HENRY). ON DISLASES OF THE LUNGS AND AIR PASSAGES. Their Pathology, Physieal Diagnosis, Symptoms and Treatment. From second English edition. In one 8 vo. volume of 475 pages. Cloth, $\$ 3.50$.

GANT (FRFDERICK JAMES). THE STUDENT'S SURGERY. A Multum in Parvo. In one square octavo volume of 845 pages, with 159 engravings. Cloth, $\$ 3.75$.

GIBBES (HENEAGE). PRACTICAL PATHOLOGY AND MOR. BID UISTOLOGY. In one very handsome octavo volume of 314 pages, with 60 illustrations, mostly photographic. Cloth, $\$ 2.75$.

GIBNEY (V. P.). ORTHOPEDIC SURGERY. For the use of Praettioners and Students. In one 8vo, vol, profusely illus. Preparing. 
GERTISH (FREDERIC H.). A TEXT-BOOK OF ANATOMY. By American Authors. Edited by Frederic H. Gerrish, M. D. In one imp. octaro volume, richly illustrated. Preparing.

GOUUD (A. PEARCE). SURGICAL DIAGNOSIS. In one $12 \mathrm{mo}$. vol. of 589 pages. Cloth, $\$ 2$. See Student's Series of Manuals, p. 27.

GRAY (HENRY). ANATOMY, DESCRIPTIVE AND SURGICAL. New and thoroughly revised American edition, much enlarged in text, and in engravings in black and colors. In one imperial octavo volume of 1239 pages, with 772 large and elaborate engravings on wood. Price of edition with illnstrations in colors: cloth, \$i; leather. $\$ S$. Price of edition with illustrations in black : cloth, $\$ 6$; leather, $\$ 7$.

This is the best single volume upon Anatomy in the Euglish language. A thorough knowledge of the subject is acquired without consulting other books. As a work of reference for the surgeon or practitioner it has no superior.-University Medical Magazine.

This edition has been revised to adapt it thoroughly to the requirements of teachers and students of the present day. The illustrations in Gray's Anatomy have always been one of its especial features; each bone, ligament, muscle, nerve, artery and tissue has been appropriately labelled, and in late editions have appeared in colors where essential. Gray's Anatomy affords the student more satisfaction than any other treatise with which we are familiar.-Buffalo Mred. Journal.

The most largely used anatomical text-book published in the English language.-Annals of Surgery.
Gray's Anatomy, in spite of the efforts which have been made from time to time to displaee it, still holds first place in the esteem of both teachers and students. - The Brooklyn Medical Journal.

Particular stress is laid upon the practical side of anatomical teach. ing, and especially the Surgical Anatomy.-Chicago Med. Recorder. The foremost of all medical textbooks.-Medical Fortuightly.

Gray's inatomy should be the first work whieh a medical student should purchase, nor should he be without a cops throughout his professional career.-Pitisburg Medical Reviero.

This new edition of Gray is a matchless treatise upon Human Anatomy. Medical students and practitioners desiring a complete library on anatomy will make no mistake in purchasing Gray.-Tri-Shte Med. Journal.

GRAY (HANDON CARTER). A TREATISE ON NERVOUS AND MENTAL DISEASES. For Students qnd Practitioners of Medicine. New (2d) edition. In one handsome octavo volume of 728 pages, with 172 engravings and 3 colored plates. Cloth, $\$ 4.75$; leather, $\$ 5.75$.

We have here what has so often but also those hygienic and dietetic been desired-an up-to-date text- measures which are often the physibook upon nerrous and mental diseases combined. A well-written, terse, explicit, and authoritative volume treating of both subjects is a step in the direetion of popular demand.-The Chicago Clinical Reviers.

"The word treatment," says the author, "has been construed in the broadest sense to include not only medicinal and non-medicinal agents, cian's best reliance."-The Journal of the American Medical Association.

The descriptions of the various diseases are aecurate and the symptoms and differential diagnosis are set before the student in such a way as to be readily comprehended. The author's long experience renders his views on therapeutics of great value. - The Journal of Nervous and Mental Disease. 
GREEN (T. HENRY). AN INTRODUCTION TO PATHOLOGY AND MORBID ANATOMY. Seventh American from the eighth London edition. In one handsome oetavo volume of 595 pages, with 224 engravings and a colored plate. Cloth, $\$ 2.75$.

A work that is the text-book of of the day-as mueh so almost as probably four-fifths of all the stu- Gray's Anatomy. It is fully up-todents of pathology in the United date in the reeord of fact, and so proStates and Great Britain stands in fusely illustrated as to give to each no need of commendation. The work detail of text suffieient explanation. preeisely meets the needs and wishes The work is an essential to the pracof the general practitioner.-The titioner-whether as surgeon or physAmerican Practitioner and News. ician. It is the best of up-to-date Green's Pathology is the text-book text-books.-VirginiaMed.Monthly.

GREENE (WILLIAM H.). A MANUAL OF MEDICAL CHEMISTRY. For the Use of Students. Based upon BowMaN's Medical Chemisiry. In one $12 \mathrm{mo}$. vol. of 310 pages, with 74 illus. Cloth, $\$ 1.75$.

GROSS (SAMUEL D.). A PRACTICAL TREATISE ON THE DISEASES, INJURIES AND MALFORMATIONS OF THE URINARY BLADDER, THE PROSTATE GLAND AND THE URETHRA. Third edition, thoroughly revised and edited by SAMUEL W. GROSS, M. D. In one oetavo vol. of 574 pages, with 170 illus. Cloth, $\$ 4.50$.

HABERSHON (S. O.). ON THE DISEASES OF THE ABDOMEN, comprising those of the Stomach, Esophagus, Caecum, Intestiues and Peritoneum. Second American from the third English edition. In one octavo volume of 554 pages, with 11 engravings. Cloth, $\$ 3.50$.

HAMUTON (ALLAN MCLANE). NERVOUS DISEASES, THEIR DESCRIPTION AND TREATMENT. Seeond and revised edition. In one octavo volume of 598 pages, with 72 engravings. Cloth, $\$ 4$.

HAMULTON (FRANIK H.). A PRACTICAL TREATISE ON FRAC. TURES AND DISLOCATIONS. Eighth edition, revised and edited by STEPHEN SMITI, A. M., M. D. In one handsome octavo volume of 832 pages, with 507 engravings. Cloth, $\$ 5.50$; leather, $\$ 6.50$.

Its numerous editions are convin- cent revision make it a work thorcing proof of its value and popular- oughly in accordance with moderu ity. It is preëminently the authority practice theoretically, meehanically, on fractures and dislocations. 'The aseptically. - Boston Medical and additions it has received by its re- Surgical Journal.

HARDAWAY (W.A.). MANUAL OF SKIN DISEASES. New (2d) edition. In one $12 \mathrm{mo}$, volume. Shortly.

HARE (HOBART AMORY). PRACTICAL DIAGNOSIS. TIE USE OF SYMPTOMS IN TIIE DIAGNOSIS OF DISEASE. New (2d) edition. In one octavo volume of 598 pages, with 201 engravings and 13 full-page colored plates. Cloth, $\$ 4.75$.

It is unique in miny respects, and he will beeome a hetter diagnostithe author has introduced rudical cian. This is $n$ companion to Prac. changes which will be weleomed by ticul Therapcutics, by the same all. Anyone who reads this book author, and it is diffienlt to conceive will become a more acute observer, of any two works of greater practical will pay more attention to the simple utility.-Medical Review. yet indicative signs of disease, and 


\section{HARE (HOBART AMORY). A TEXT-BOOK OF PRACTICAL} THERAPEUTICS, with Special Reference to the Application of Remedial Measures to Disease and their Employment upon a Rational Basis. With, articles on various subjects by well-known specialists. Sixth and revised edition. In one cetaro volume of 758 pages. Cloth, $\$ 3.75$; leather, $\$ 4.75$.

Its classifications are inimitable, it can be readily used in connection and the readiness with which any- with Hare's Practical Diagnosis. thing can be found is the most won. For the needs of the stndent and derful achievement of the art of in- general practitioner it has no equal. dexing. This edition takes in all -Medical Sentinel.

the latest discorered remedies.- The best planued therapeutic work The St. Louis Clinique.

The great value of the work lies in the fact that precise indications for administration are given. A complete index of diseases and renedies makes it an easy refereuce work. It has been arranged so that

of the century.-American Practitioner and lexes.

It is a book precisely adapted to the needs of the busy practitioner, who can rely upon finding exactly what he needs. - The National Medical Revievo.

\section{HARE (HOBART AMORY, EDITOR). A SYSTEM OF PRAC-} TICAL THERAPEUTICS. In a series of contributions by eminent practitioners. In four large octavo volumes comprising about 4500 pages, with about 550 engravings. Vol. IV., just ready. For sale by subscription only. Full prospectus free on application to the Publishers. Regular price, Vol. IV., cloth, $\$ 6$; leather, $\$ 7$; half Russia, $\$$. Price Vol. IV. to former or new subscribers to complete work, cloth, $\$ 5$; leather, $\$ 6$; half Russia, $\$ 7$. Complete work, eloth, $\$ 20$; leather, $\$ 24$; half Russia, $\$ 28$.

The great value of Hare's System of Practical Therapeutics has led to a widespread demand for a new volume to represent advances in treatment made since the publication of the first three. More than fulfilling this request the Editor has secured contributions from practically a new corps of equally eminent authors, so that entirely fresh and original matter is. ensured. The plan of the work, which prored so successful, has been followed in this new volume, which will be found to present the latest devel. opments and applications of this most practical branch of the medical art. Prescriptions indicative of the manner in which particular drugs are administered are frequently inserted, with the aim of making the work as helpful as possible; and especial care is devoted to such precision of detail as will render it a safe guide in the use of the newer and less familiar agents. This volume will therefore be indispensable to the many thousands of subscribers to the original work, and it will be of no less value by itself, since it reflects the whole position of each subject. The entire System is an unrivalled encyclopæedia on the practical parts of medicine, and merits the great success it has won for that reason. 
HARTSHORNE (HENRY). ESSENTIALS OF THE PRINCIPLES AND PRACTICE OF MEDICINE. Fifth edition. In one $12 \mathrm{mo.}$ volume, 669 pages, with 144 engravings. Cloth, $\$ 2.75$; half bound, $\$ 3$.

A HANDBOOK OF ANATOMY AND PHYSIOLOGY. In one $12 \mathrm{mo}$. volume of 310 pages, with 220 engravings. Cloth, $\$ 1.75$.

A CONSPECTUS OF THE MEDICAL SCIENCES. Comprising Manuals of Anatomy, Physiology, Chemistry, Materia Medica, Practice of Medicine, Surgery and Obstetrics. Second edition. In one royal $12 \mathrm{mo}$. vol. of 1028 pages, with 477 illus. Cloth, $\$ 4.25$; leather, $\$ 5$.

\section{HA YDEN (JAMES R.). A MANUAL OF VENEREAL DISEASES.}

In one $12 \mathrm{mo}$. volume of 263 pages, with 47 engravings. Cloth, $\$ 1.50$.

It is practical, concise, definite ticularly thorough, and may be and of sufficient fulness to be satis- relied upon as a guide in the manfactory.-Chicago Clinical Review. agement of this class of diseases.-

This work gives all of the prac- Northwestern Lancet.

tically essential information about It is well written, up to date, and the three venereal diseases, gon- will be found very useful.-Interorrhœa, the chancroid and syphilis. national Medical Magazine.

In diagnosis and treatment it is par-

HAYEM (GEORGES) AND HARE (H. A.). PHYSICAL AND NA'TURAL THERAPEUTICS. The Remedial Use of Heat, Electricity, Modifications of Atmospheric Pressure, Climates and Mineral Waters. Edited by Prof. H. A. HARE, M. D. In one octavo volume of 414 pages, with 113 engravings. Cloth, $\$ 3$.

This well-timed up-to-date volume recognition. Within this large is particularly adapted to the re- range of applicability, physical quirements of the general practi- agencies when compared with drugs tioner. The section on mineral are more dircet and simple in their waters is most scientific and prac- results. Medical literature has long tical. Some 200 yages are given up been rich in treatises upon medical to electricity and evidently embody agents, but an authoritative work the latest scientific information on upon the other great branch of the subject. Altogether this work therapentics has until now been a is the clearest and most practical aid desideratum. The section on climate, to the study of nature's therapeutics rewritten by Prof. Iare, will, for that has yet eome under our obser- the first tiue, place the alundant vation.-The Medical Fortnightly. resources of our country at the in-

For many diseases the most potent telligent command of American remedies lie outside of the materin practitioners. - The Kansas City medica, a fact yearly receiving wider

Medical Index.

HEIRMAN (G. ERNEST). FIRST LINES IN MIDWIFERY. In one $12 \mathrm{mo}$. vol. of 198 pages, with 80 engravings. Cloth, $\$ 1.25$. See Student's Series of Manuals, page 27.

HERMANN (L.). EXPERTMENTAL PIIARMACOLOGY. A Handbook of the Methods for Determining the Pliysiological Actions of Drugs. Translated by Robert Meade Smitu, M.D. In one $12 \mathrm{mo.}$ volume of 199 pages, with 32 engravings. Cloth, $\$ 1.50$. 
HERRICK (JAMES B.). A HANDBOOK OF DIAGNOSIS. In one handsome $12 \mathrm{mo}$. volume of 429 pages, with 80 engravings and 2 colored plates. Cloth, $\$ 2.50$.

Excellently arranged, practical, microscopical examination to be emconcise, up-to-date, and eminently plosed in each class. The technique well fitted for the use of the prac- of blood examination, including color titioner as.well as of the student.- analysis, is very clearly stated. Chicago Med. Recorder.

This volume accomplishes its objects more thoronghly and completely than any similar work yet published. Each section devoted to diseases of special systems is preceded with an exposition of the methods of physical, chemical and Uranalysis receives adequate space and care.-New York Med. Journal.

We commend the book not only to the undergraduate, but also to the physician who desires a ready means of refreshing his knowledge of diagnosis in the exigencies of professional life.-Memphis Medical Monthly.

HUWL (BERKELEY). SYPHILIS AND LOCAL CONTAGIOUS DISORDERS. In one 8 ro, volume of 479 pages. Cloth, $\$ 3.25$.

HILLIER (THOMAS). A HANDBOOK OF SKIN DISEASES. Second edition. In one royal $12 \mathrm{mo}$. rolume of 353 pages, with two plates. Cloth, $\$ 2.25$.

HIRST (BARTON C.) AND PIERSOL (GEORGE A.). HUMAN MONSTROSITIES. Magnificent folio, containing $2: 0$ pages of text and illustrated with 123 engravings and 39 large photographic plates from nature. In four parts, price each, $\$ 5$. Limited edition. For sale by subscription only.

HOBLYN (RICHARD D.). A DICTIONARY OF THE TERMS USED IN MEDICINE AND THE COLLATERAL SCIENCES. In one $12 \mathrm{mo}$. volume of 520 double-columned pages. Cloth, $\$ 1.50$; leather, $\$ 2$.

HODGE (HUGH L.). ON DISEASES PECULIAR TO WOMEN, INCLUDING DISPLACEMENTS OF THE UTERUS. Second and revised edition. In one Svo. vol. of $519 \mathrm{pp}$, with illus. Cloth, \$4.50.

HOFFMANN (FREDERICK) AND POWVER (FREDERICK B.). A MANUAL OF CHEMICAL ANALYSIS, as Applied to the Examination of Medicinal Chemicals and their Preparations. Third edition, entirely rew ritten and much enlarged. In one handsome octaro volume of 621 pages, with 179 engrarings. Cloth, $\$ 4.25$.

HOLDEN (LUTHER). LANDMARKS, IEDICAL AND SURGI. CAL. From the third English edition. With additions by W. W. KEEN, M. D. In one royal $12 \mathrm{mo}$. volume of 148 pages. Cloth, $\$ 1$.

HOLMES (TLMOTHY). A TREATISE ON SURGERY. Its Principles and Practice. A new American from the fifth English edition. Edited by T. PICK EriYg PICK, F.R.C.S. In one handsome octavo rolume of 1008 pages, with 428 engravings. Cloth, $\$ 6$; leather, $\$ 7$.

A SYSTEM OF SURGERY. With notes and additions by various American authors. Edited by JoHN H. PACKARD, M. D. In three very handsome 8 vo. volumes containing 3137 double-columned pages, with 979 engravings and 13 lithographic plates. Per volume; cloth, $\$ 6$; leather, $\$ 7$; half Russis, $\$ 7.50$. For sale by nutscription onily. 
HORNER (WULLAM E.). SPECIAL ANATOMY AND HISTOLOGY. Eighth edition, revised and modified. In two large 8vo. volumes of 1007 pages, containing 320 engravings. Cloth, $\$ 6$.

HUDSON (A.). LECTURES ON THE STUDY OF FEVER. In one octavo volume of 308 pages. Cloth, $\$ 2.50$.

HUTCHISON (ROBERT) AND RAINY (HARRY). CLINICAL METHODS. A GUIDE TO THE PRACTICAL STUDY OF MEDICINE. In one $12 \mathrm{mo}$. volume of 562 pages, with 137 engravings and 8 colored plates. Cloth, $\$ 3.00$. Just ready.

HUTCHINSON (JONATHAN). SYPHILIS. In one pocket-size $12 \mathrm{mo}$. volume of 542 pages, with 8 chromo-lithographic plates. Cloth, $\$ 2.25$. See Series of Clinical Manuals, p. 25.

HYDE (JAMIES NEVINS). A PRACTICAL TREATISE ON DIS. EASES OF THE SKIN. New (4th) edition, thoroughly revised. In one octavo volume of 815 pages, with 110 engravings and 12 fullpage plates, 4 of which are colored. Cloth, $\$ 5.25$; leather, $\$ 6.25$.

This edition has been carefully re- culcated throughout is sound as well vised, and every real advance has as practical.-The American Jourbeen recognized. The work answers the needs of the general practitioner, the specialist, and the student, and th is a happy example of the fact that such a wide range of adaptation can be given within the compass of a volume of convenient size and price. -The Ohio Med. Jour.

A treatise of exceptional merit characterized by conscipntious care and scientific accuracy. - Buffalo Med. Journal.

Those who wish the latest views may confidently consult its pages.University Med. Magazine.

A complete exposition of our ar knowledge of cutaneous medicine as it exists to-day. The teaching in-1

JACISON (GEORGE THOMAS), TIE READY-IREERENCF HANDBOOK OF DISEASES OF TIIE SKIN. New (2d) edition. In one $12 \mathrm{mo}$. volume of 589 pages, with 69 illustrations and a colored plate. Cloth, $\$ 2.75$.

A prompt and ready source of knowledge on all points of terminology, symptoms, varieties, etiology, pathology, diagnosis, treatment and prognosis of dermal affections. Tables of differential diagnosis and standard prescriptions will be found scattered through the text, and the work ends with an appendix of well. tried formula. The serics of illustrations is rich and instructive.Memphis Med. Monthly.

The text is clear and sufficiently full. The subject of treatment inual of the Medical Sciences.

It is the best one-volume work that we know. The student who gets this book will find it a useful investment, as it will well serve him when he goes into practice.-Virginia Medical Semi-Monthly.

A full and thoroughly moidern text-book on dermatology. - The Pittsburg Medical Review.

All new facts have been considered in detail, and in every way this book represents the Dermatolcgy of today. It is the most practical handbook on dermatology with which we are acquainted.-The Chicago Med-

ical Recorder.

cludes all the newer methods and remedies of proved value. It is a thoroughly satisfactory and clcar expression of cutaneous diseases.American Journal of the Medical Sciences.

The work is fair and accurate, full and completc, and it cmbodies the recent additions to our information. Above all, it is eminently practical. The reviewer has found it a good book for students, and believes it is equally good for the practitioner.Chicago Clinical Review. 
JAMTESON (W. AUTAN). DISEASES OF THE SKIN, Third edition. In one octaro volume of 656 pages, with 1 engraving and 9 double-page chromo-lithographic plates. Cloth, 8 .

JEWETT (CHARYES). ESSENTIALS OF OBSTETRICS. In one $12 \mathrm{mo}$. rolume of 356 pages, with 80 engravings and 3 colored plates. Cloth, $\$ 2.25$. Just ready.

An exceedingly useful manual for $/$ ing it in attractive and easily tangistudent and practitioner. The au- ble form. The book is well illusthor has succeeded unusually well trated throughout.-Nashville Jour. in condensing the text and in arrang- of Medicine and Surgery.

A TEXT-BOOK OF OBSTETRICS. By American Anthors. One large octaro volume, profusely illustrated. In press.

JONES (C. HANDFIELD). CLINICAL OBSERVATIONS ON FUNCTIONAL NERVOUS DISORDERS. Second American edition. In one octaro volume of 340 pages. Cloth, $\$ 3.25$.

\section{JULER (HENRY). A HANDBOOK OF OPHTHALMIC SCIENCE} AND PRACTICE. Second edition. In one octaro volume of 549 pages, with 201 engravings, 17 chromo-lithographic plates, test-types of Jaeger and Snellen, and Holmgren's Color-Blindness Test. "Cloth, $\$ 5.50$; lesther, $\$ 6.50$.

The continuous approval mani- at the same time concise, and couchfested toward this work testifies to the success with which the author has produced concise descriptions and typical illustrations of all the important affections of the ere. The volume is particularly rich in matter of practical ralue, such as directions for diagnosing, use of instruments, testing for glasses, for color blindness, etc. The sections deroted to treatment are singularly full, and in language that eannot fail to be understood. This edition likewise embodies such revisions and changes as were necessary to render it thoroughly representative, and moreorer it bas been enriched by the addition of 100 pages and 75 engravings. All told, there are 201 engravings, exclusive of 17 handsomely colored.The Medical Age.

KING (A. F.A.). A MANUAL OF ORSTETRICS. Seventh edition. In one $12 \mathrm{mo}$. rolume of 573 pages, with 223 illustrations. Cloth, $\$ 2.50$.

The student world, particularly, seems thoroughly conversant with the merits of this manual, for there is certainly no work upon obstetrics more in demand by them. This edition has been thoroughly revised in an endeavor to represent recent adrances in its subject, special attention being devoted to aseptic midwifery. The book is increased in size, and a number of illustrations has been added to its already rich store.-Memphis Med. Monthly.

From first to finish it is thoroughly practical, concise in expression, well illustrated, and iucludes a statement of nearly every fact of importance discussed in obstetric treatises or cyclopedias. Its arrangement is progressire, which makes it most suitable for the class-room. The well arranged index renders the hook useful to the practitioner who is in haste to refresh his memory.Yirginia Medical Semi-Monthly.

KIRK (FDWARD C.). OPERATIVE DENTISTRY. Handsome octaro of 700 pages, with 751 illustrations. Just ready. See American Tert-Books of Dentistry, page 2. 
KLEIN (E.). ELEMENTS OF HISTOLOGY. Fourth edition. In one pocket-size 12mo. volume of 376 pages, with 194 engravings. Cloth, \$1.75. See Student's Series of Manuals, page 27.

It is the most complete and con- / which it is written, the absence or cise work of the kind that has yet debatable matter, and of conflicting emanated from the press, and is views, the convenient size of the invaluable to the active as well as book and its moderate price, will to the embryo practitioner. The account for its undoubted success,illustrations are vastly superior to those in most works of its class.The Medical Age.

The cloar and concise manner in tology.-Canadian Practitioner.

LANDIS (HENRY G.). THE MANAGEMENT OF LABOR. In one handsome $12 \mathrm{mo}$. volume of 329 pages, with 28 illus. Cloth, \$1.75.

LA ROCHE (R.). YELLOW FEVER. In two 8vo. volumes of 1468 pages. Cloth, $\$ 7$.

PNEUMONIA. In one 8vo. volume of 490 pages. Cloth, $\$ 3$.

LAURENCE (J. Z.) AND MOON (ROBERT C.). A IIANDYBOOK OF OPHTHALMIC SURGERY. Secoud edition. In one octavo volume of 227 pages, with 66 engravings. Cloth, $\$ 2.75$.

LEA (HENRY C.). A HISTORY OF AURICULAR CONFESSION AND INDULGENCES IN THE LATIN CHURCH. In three octavo volumes of about 500 pages each. Per volume, cloth, $\$ 3.00$. Complete work just ready.

CHAPTERS FROM THE RELIGIOUS HISTORY OF SPAIN ; CENSORSHIP OF THE PRESS; MYSTICS AND ILLUMINATITHE ENDEMONIADAS; EL SÁNTO NIÑO DE LA GUARDIA; BRIANDA DE BARDAXI. In one $12 \mathrm{mo}$. volume of 522 pages. Cloth, $\$ 2.50$.

FORMULARY OF TIIE PAPAL PENITENTIARY. In one octavo volume of 221 pages, with frontispiece. Cloth, $\$ 2.50$.

- SUPERSTITION AND FORCE; ESSAYS ON THE WAGER OF LAW, THE WAGER OF BATTLE, THE ORDEAL AND TORTURE. Fourth edition, thoroughly revised. In one handsome royal $12 \mathrm{mo}$. volume of 629 pages. Cloth, $\$ 2.75$.

STUDIES IN CHURCH HISTORY. The Rise of the Temporal Power-Benefit of Clergy-Excomnunication. New edition. In one handsome $12 \mathrm{mo}$. volume of 605 pares. Cloth, $\$ 2.50$.

AN HISTORICAL SKETCH OF SACERDO'TAL CELIBACY IN TIEE CIIRISTIAN CHURCH. Second edition. In one handsome octavo volume of 685 pages. Clotli, $\$ 4.50$.

LEE (HENRY) ON SYPIILIS. In one 8vo. volume of 246 pages. Cloth, $\$ 2.25$.

LEHMANN (C. G.). A MANUAL OF CHEMICAL PHYSIOLOQY. In one 8 ro. volume of 327 pages, witl 41 engravings. Cloth, $\$ 2.25$. 
LOOMTS (ALFRED L.) AND THOMPSON (W. GHLMAN, EDITORS). A SYSTEM OF PRACTICAL MEDICINE. In Contributions by Varions American Authors. In four very bandsome octaro rolumes of abont 900 pages each, fully illustrated in in black and colors. Complete work now ready. Per volume, cloth, $\$ 5$; lesther, \$6; half Morocco, \$7. For sale by subscription only. Full prospectus free on applicstion to the Publishers. See American System of Practical Medicine, page 2.

LUDLOW (J. L.). A MANUAL OF EXAMINATIONS UPON ANATOMY, PHYSIOLOGY, SURGERY, PRACTICE OF MEDICINE, OBSTETRICS, MATERIA MEDICA, CHEMISTRY, PHAR. MACY AND THERAPEUTICS. To which is added a Medical Formulary. Third edition. In one roral-12mo. rolume of 816 pages, with 370 engravings. Cloth, $\$ 3.25$; leather, $\$ 3.75$.

LUFF (ARTHUR P.). MANUAL OF CHEMISTRY, for the use of Students of Medicine. In one $12 \mathrm{mo}$, volnme of 522 pages, with 36 engravings. Cloth, \$2. See Student's Series of Manuals, page 27.

LYMAN (HENRY M.). THE PRACTICE OF MFDICINE. In one very handsome octaro volume of 925 pages, with 170 engravings. Cloth, $\$ 4.75$; leather, $\$ 5.75$.

An excellent treatise on the practice of medicine, Fritten br one who is not only familiar with his subject, but who has also learned through practical experience in teaching what are the needs of the student and how to present the facts to his mind in the most resdily assimilable form. The practical and busy physician, who wants to ascertain in a short time all the necessary facts concerning the pathology or

treatment of any disease will find here a safe and convenient guide.The Charlotte Medical Journal.

Complete, concise, fully abreast ot the times and needed by all students and practitioners.-Univ. Jed. Mag. An exceedingly raluable text-book. Practical, systematic, complete and well balanced.-Chicago Med. Recorder.

Represents fully the most recent knowledge.-Monitreal Med. Jour.

LYONS (ROBERT D.). A TREATISE ON FEVER. In one octaro volume of 362 pages. Cloth, $\$ 2.25$.

MACKENZIE (JOHN NOLAND). ON THE NOSE AND THROAT. Handsome nctavo, abont 600 pages, richly illustrated. Preparing.

MAISCH (JOHN M.). A MANUAL OF ORGANIC MATERIA MEDICA. New (6th) edition, thoroughly revised by H. C. C. MAISCH, $\mathrm{Ph}$. G., $\mathrm{Ph}$. D. In one very handsome $12 \mathrm{mo}$. volume of 509 pages, with 285 engravings. Cloth, $\$ 3$.

The best handbook upon pharmacognosy of any published in this country.-Boston Med. \& Sur. Jour.

Noted on both sides of the Atlantic and esteemed as much in Germany as in America. The work has no equal. -Dominion Med. Monthly.

Used as text-book in every college of pharmacy in the United States and recommended in medical colleges.-American Therapist.

New matter has been added, and the whole work has receired careful revision, so as to conform to the new United States Pharmacopcia.-Virginia Medical Monthly.

This standard text-book is a work of such well-tried merit that it stands in no danger of being superseded.-Amer. Druggist and Pharm. Record. 
MANUALS. See Student's Quiz Series, page 27, Student's Series of Manuals, page 27, and Series of Clinical Manuals, page 25.

MARSH (HOWARD). DISEASES OF THE JOINTS. In one $12 \mathrm{mo.}$ volume of 468 pages, with 64 engravings and a colored plate. Cloth, $\$ 2$. See Series of Clinical Manuals, page 25.

MARTIN (EDWARD). A MANUAL OF SURGICAT, DIAGNOSIS. In one $12 \mathrm{mo}$. volume of about $400 \mathrm{pp}$., fully illustrated. Preparing.

MAY (C. H.). MANUAL OF THE DISEASES OF WOMEN. For the use of Students and Practitioners. Second edition, revised by $\mathrm{L}$. $\mathrm{S}$. RAD, M. D. In one $12 \mathrm{mo}$. volume of 360 pages, with 31 engravings. Cloth, $\$ 1.75$.

MTTCHELL (S. WEIR). CLINICAL LESSONS ON NERVOUS DISEASES. In one $12 \mathrm{mo}$. volume of 299 pages, with 19 engravings and 2 colored plates. Cloth, $\$ 2.50$. Of the hundred numbered copies with the Anthor's signed title page a few remain; these are offered in green cloth, gilt top, at $\$ 3.50$, net.

There is no question as to the interest of the clinical pictures presented in this volume. Many rare examples of spurious troubles (hysteria) are given and irregular types of other "nervous" affections. The study of these types, from the author's clear notes and deductions, will be of value to the student of neurology.-The Chicago Clinical Review.

This is a book by a master and if we mistake not it will prove a very

popular one. The book treats of bysteria, recurrent melancholia, disorders of sleep, choreic movements, false sensations of cold, ataxia, hemiplegic pain, treatment of sciatica, erythromelalgia, reflex ocular neurosis, hysteric contractions, rotary movements in the feeble minded, etc. Few can speak with more authority than the author.The Tournal of the American Medical Association.

MTTCHELL (JOHN K.). REMOTE CONSEQUENCES OF INJURIES OF NERVES AND THEIR TREATMENT. In one handsome $12 \mathrm{mo}$. volume of 239 pages, with 12 illustrations. Cloth, $\$ 1.75$.

Injuries of the nerves are of fre- government documents, and has quent occurrence in private practice, skilfully utilized his opportunities. and often the cause of intractable This work will doubtless take a and painful conditions, conse- prominent place in medical literaquently this volume is of especial ture among the special monograplis interest. Doctor Mitchell has had which throw light into obseure access to hospital records for the last places and contribute to the advance thirty years, as well as to the of medical science.-The Med. Age.

MORRIS (HENTY). SURGICAL DIREASES OF THE KIDNEY. In one $12 \mathrm{mo}$. volume of 554 pages, with 40 engravings and 6 colored plates. Cloth, \$2.25. See Series of Clinical Mamuals, page 25.

MORRIS (MATCOLM). DISEASES OF THE SKIN. In one square 8vo. volume of 572 pages, with 19 chromo-lithographic figures and 17 engravings. Cloth, $\$ 3.50$.

MÜLUER (J.). PRINCIPLES OF PHYSICS AND METEOROLOGY. In one large 8 vo. rol. of 623 pages, with 538 cuts. Cloth, $\$ 4.50$ 
MUSSER (JOEN H.). A PRACTICAL TREATISE ON MEDICAL DIAGNOSIS, for Students and Physicians. New (2d) edition, thoroughly revised. In one octavo volume of 931 pages, with 177 engravings and 11 full-page colored plates. Cloth, $\$ 5$; leather, $\$ 6$.

We have no work of equal value in English. - University Medical Magazine.

Every real advance that has been made in this rapidly progressing department of medicine is here recorded. There is no half knowledge. His descriptions of the diagnostic manifestations of diseases are accurate. This work will meet all the requirements of student and physician.-The Medical Neros.

From its pages may be made the diagnosis of every malady that aftlicts the human body, including those which in general are dealt with only by the specialist. The early demand for the new edition speaks volumes for the book's popularity.-Northoestern Lancet.

It so thoroughly meets the precise demands incident to modern research that it has been already adopted as a leading text-book by the medical colleges of this country. - North American Practitioner.

Occupies the foremost place \&s a thorough, systematic treatise.-Ohio Medical Journal.

The best of its kind, invaluable to the student, general practitioner and teacher.-IIontreal MedicalJournal.

NATIONAL DISPENSATORY. See Stillé, Maisch \& Caspari, p. 27.

NATIONAT, FORMULARY. See Stillê, Maisch \& Carpari's National Dispensatory, page 27 .

NATIONAI MEDIOAL DIÖIONARY. See Billings, page 4.

NETTLESHIP (E.). DISEASES OF THE EYE. Ner (5th) American from sixth English edition, thoroughly revised. In one $12 \mathrm{mo}$. volume of 521 pages, with 161 engravings, and 2 colored plates, test-types, formuliz and color-blindness test. Cloth, $\$ 2.25$. Just ready.

By far the best student's text-book English language. - Journal of on the subject of ophthalmology and Medicine and Science.

is conreniently and concisely ar- The present edition is the result ranged.-The Clinical Revieve.

of revision both in England and

It has been conceded by ophthal- America, and therefore contains the mologists generally that this work latest and best ophthalmological for compactness, practicality and ideas of both continents. - The Phyclearness has no superior in the sician and Surgeon.

NORRIS (WAT. F.) AND OLIVER (CHAS. A.). TEXT-BOOK OF OPHTHALMOLOGY. In one octavo volume of 641 pages, with 357 engravings and 5 colored plates. Cloth, $\$ 5$; leather, $\$ 0$.

We take pleasure in commending, to the every-day student and the the "Text-book" to students and scientific observer is offered to the practitioners as a safe and admir- reader. Rules and procedures are able guide, well qualified to furnish made so plain and so evident, that them, as the authors intended it any student-can easily understand should, with "a working knowl- and employ them. It is practical in edge of ophthalmology." - John its teachings. We unreservedly enHopkins Hospital Bulletin.

The furst text-book of diseases of the eye written by American authors for American colleges and students. Every method of ocnlar precision that can be of any clinical advantage dorse it as the best, the safest and the most comprehensive volume upon the subject that has ever been offered to the American medical public.Annals of Ophthalmology and Otology. 
OWEN (EDMUND). SURGICAL DISEASES OF CHILDREN. In one $12 \mathrm{mo}$. volume of 525 pages, with 85 engravings and 4 colored plates. Cloth, $\$ 2$. See Series of Clinical Manuals, page 25 .

PARK (ROSWELL). A TREATISE ON SURGERY BY AMERICAN AUTHORS. In two handsome octavo volumes. Volume I., General Surgery, 799 pages, with 356 engravings and 21 full-page plates, in colors and monochrome. Volume II., Special Surgery, 800 pages, with 430 engravings and 17 full-page plates, in colors and monochrome. Per volume, cloth, $\$ 4.50$; leather, $\$ 5.50$. Net. Complete work now ready.

The work is fresh, clear and practical, covering the ground thoroughly yet briefly, and well arranged for rapid reference, so that it will be of special value to the student and busy practitioner. The pathology is broad, clear and scientific, while the suggestions upon treatment are clear-cut, thoroughly modern and admirably resourceful.-Johns Hopkins Hospital Bulletin.

The latest and best work written upon the science and art of surgery. Columbus Medical Journal.

Its special field of application is in practical, every-day use. It well deserves a place in every medical man's library.-The Pittsburg Medical Review. The illustrations are almost en- leans Med. and Surgical Journal. tirely new and executed in such a way that they add great force to the text. It gives us unusual pleasure to recommend this work to students and practitioners alike.-The Chi. cago Medical Recorder.

The various writers have embodicd the teachings accepted at the present hour and the methods now in vogue, both as regards causes and treatment.-The North American Practitioner.

Both for the student and practitioner it is most valuable. It is thoroughly practical and yet thoroughly scientific.-Medical News.

A truly modern surgery, not only in pathology, but also in sound surgical therapeutics. - New Or-

PARRY (JOHN S.). EXTRA-UTERINE PREGNANCY, ITS CLINICAL HISTORY, DIAGNOSIS, PROGNOSIS AND TREATMENT. In one octavo volume of 272 pages. Cloth, $\$ 2.50$.

PARVIN (THEOPHILUS). THE SCIENCE AND ART OF OBSTETRICS. Third edition. In one handsome octavo volume of 677 pages, with 267 engravings and 2 colored plates. Cloth, $\$ 4.25$; leatlier, $\$ 5.25$.

In the foremost rank anong the most practical and scientific medical
works of the day.-Medical News.

It ranks second to none in the English language.-Annals of Gynecology and P'ediatry.

The book is complete in every department, and contains all the necessary detail required by the modern practising obstetrician. - International Melical Mragazine.

In breadth and scope the work is adapted to the needs of the advanced scholar and specialist. The con- sideration of every subject is in reality brought up to the hour when the copy went to print.-Medicine. Parvin's work is practical, concise and comprehensive. Wc com. mend it as first of its class in the English language.-Medical Fortnightly.

Parvin's classical work now occunies the front rank of modern text-books. It is an admirable textbook in every sense of the word.Nashville Journal of Bledicine and Surgery. 
Lea Brothers \& Co., Philadelphia aNd New York.

PAYNE (JOSEPH FRANK). A MANUAL OF GENERAL PATHOLOGY. Designed as an Introduction to the Practice of Medicine. In one octaro volume of 524 pages, with 153 engravings and 1 colored plate.

PEPPER'S SYSTEM OF MEDICINE. See page 3.

PEPPER (A.J.). FORENSIC MEDICINE. In press. See Student's Series of Manuals, page 27.

SURGICAL PATHOLOGY. In one $12 \mathrm{mo}$. volume of 511 pages, with 81 engravings. Cloth, $\$ 2$. See S'udent's Series of Manuals, p. 27.

PICK (T. PICKERING). FRACTURES AND DISLOCATIONS. In one $12 \mathrm{mo}$. volume of 530 pages, with 93 engravings. Cloth, $\$ 2$. See Series of Clinical Manuals, page 25.

PLAYFAIR (W. S.). A TREATISE ON THE SCIENCE AND PRACTICE OF MIDWIFERY. Sixth American from the eighth English edition. Edited, with additions, by R. P. HARris, M. D. In one octavo volume of 697 pages, with 217 engravings and 5 plates. Cloth, \$4; leather, \$5.

In the numerous editions which / obstetrician. It holds a place among have appeared it has been kept constantly in the foremost rank. It is a work which can be conscientiously recommended to the profession.The Albany Medical Annals.

This work must occupy a foremost place in obstetric medicine as a safe guide to both student and the ablest English-speaking authorities on the obstotric art.-Buffalo Medical and Surgical Journal.

An epitome of the science and practice of midwifery, which embodies all recent advances. - The Medical Fortnightly.

THE SYSTEMATIC TREATMENT OF NERVE PROSTRATION AND HYSTERIA. In one 12mo. volume of 97 pages. Cloth, $\$ 1$.

POLITZER (ADAM). A TEXT-BOOK OF THE DISEASES OF THE EAP AND ADJACENT ORGANS. Second American from the third German edition. Translated by Oscar DoDD, M. D., and edited by SiR WiLLIAN DALBY, F.R.C.S. In one octavo volume of 748 pages, with 330 original engravings. Cloth, $\$ 5.50$.

The anatomy and physiology of ment are clear and reliable. We each part of the organ of hearing can confidently recommend it, for it are carefully considered, and then contains all that is known upon the follows an enumeration of the dis- subject.-London Lancet.

eases to which that special part of A safe and elaborate guide into the anditory apparatus is especially every part of otology.-American liable. The indications for treat- Journal of the Medical Sciences.

POWER (HENRY). HUMAN PHYSIOLOGY. Second edition. In one 12mo. volume of 396 pages, with 47 engravings. Cloth, $\$ 1.50$. See Student's Series of Nanials, page 27.

PURDY (CHARLES W.). BRIGHT'S DISEASE AND ALLIED AFFECTIONS OF THE KIDNEY. In one octavo volume of 288 pages, with 18 engravings. Cloth, $\$ 2$. 
PYE-SMITH (PHILP H.). DISEASES OF THE SKIN. In one $12 \mathrm{mo}$. vol. of $407 \mathrm{pp}$., with 28 illus., 18 of which are colored. Cloth, $\$ 2$.

QUIZ SERIES. See Student's Quiz Series, page 27.

RALFE (CHARLES H.). CLINICAL CHEMISTRY. In one $12 \mathrm{mo}$. volume of 314 pages, with 16 engravings. Cloth, $\$ 1.50$. See Student's Series of DIanuals, page 27.

RAMSBOTHAM (FRANCIS H.). THE PRINCIPLES AND PRACTICE OF OBSTETRIC MEDICINE AND SURGERY. In one imperial octavo volume of 640 pages, with 64 plates and numerous engravings in the text. Strongly bound in leather, $\$ 7$.

REICHERT (EDWARD T.). A TEXT-BOOK ON PHYSIOLOGY. In one handsome octavo volume of about 800 pages, richly illustrated. Preparing.

REMSEN (IRA). THE PRINCIPLES OF THEORETICAL CHEMISTRY. New (5th) edition, thoroughly revised. In one $12 \mathrm{mo}$. volume of 326 pages. Cloth, $\$ 2$.

A clear and concise explanation that the work has met with general of a difficult subject. We cordially favor. This is further established recommend it.-The London Lancet. by the fact that it has been trans-

The book is equally adapted to the lated into German and Italian. The student of chemistry or the practi- treatise is especially adapted to the tioner who desires to broaden his laboratory student. It ranks unusutheoretical knowledge of chemistry. ally high among the works of this - New Orleans Med, and Surg. Jour. class. This edition has been brought The appearance of a fifth edition fully up to the times.-American of this treatise is in itself a guarantee Medico-Surgical Bulletin.

RICHARDSON (BENJAMIN WARD). PREVENTIVE MEDICINE. In one octavo volume of 729 pages. Cloth, $\$ 4$; leather, $\$ 5$.

ROBERTS (JOHN B.). THE PRINCIPLES AND PRACTICE OF MODERN SURGERY. In one octavo volume of 780 pages, with 501 engravings. Cloth, $\$ 4.50$; leather, $\$ 5.50$.

THE COMPEND OF ANATOMY. For use in the Dissecting Room and in preparing for Examinations. In one $16 \mathrm{mo}$. volume of 196 pages. Limp cloth, 75 cents.

ROBERTS (SIR WILLLAM). A PRACTICAL TREATISE ON URINARY AND RENAL DISEASES, INCLUDING URINARY DEPOSITS. Fourth American from the fourth London cdition. In one very handsome 8 vo. vol. of 609 pp., with 81 illus. Cloth, $\$ 3.50$.

ROIBERTSON (J. MCGREGOR). PHYSIOLOGICAL PHYSICS. In one $12 \mathrm{mo}$. volume of 537 pages, with 219 engravings. Cloth, $\$ 2$. See Student's Series of Manuals, page 27.

ROSS (JAMES). A HANDBOOK OF THE DISEASES OF THE NERVOUS SYSTEM. Iu one handsome octavo volume of 726 pages, with 184 engravings. Cloth, $\$ 4.50$; leather, $\$ 5.50$.

SAVAGE (GEORGE H.). INSANITY AND ALLIED NEUROSES, PRACTICAL AND CLINICAL. In one $12 \mathrm{mo}$. volume of 551 pages, with 18 typical engravings. Cloth, $\$ 2$. See Series of Clinical Manuals, page 25. 
SCHAFER (EDWARD A.). THE ESSENTIALS OF HISTOLOGY. DESCRIPTIVE AND PRACTICAL. For the use of Students. New (4th) edition. In one handsome octavo volume of 311 pages, with 325 illustrations. Cloth, $\$ 3$.

Nowhere else will the same very | The most satisfactory elementary moderate outlay secure as thoroughly tert-book of histology in the Enguseful and interesting an atlas of lish language.-The Boston Med. and structural anatomy.-The American Sur. Jour.

Journal of the Medical Sciences.

A COURSE OF PRACTICAL HISTOLOGY. New (2d) edition.

In one $12 \mathrm{mo}$. volume of 307 pages, with 59 engravings. Cloth, $\$ 2.25$.

The book very nearly approaches overpraised. It bears eloquent tesperfection. Methods are given with timony to the wide knowledge and an accuracy of detail and prevision untiring industry of its author.of difticnlties which can hardly be The Scottish Med. and Surg. Jour.

SOHMTTZ AND ZUNPT'S CLASSICAT SERIES. Advanced Latin Exercises. Cloth, 60 cts. Schmidt's Elementary Latin Exercises. Cloth, 50 cents. Sillust. Cloth, 60 cents. Nepos. Cloth, 60 cents. Virgil. Cloth, 85 cents. Curtius. Cloth, 80 cents.

SCHOFIELD (ALFRED T.). ELEMENTARY PHYSIOLOGY FOR STUDENTS. In one $12 \mathrm{mo}$. volume of 380 pages, with 227 engravings and 2 colored plates. Cloth, $\$ 2$.

SCHREIBER (JOSEPH). A MANUAL OF TREATMENT BY MASSAGE AND METHODICAL MUSCLE EXERCISE. Translated by WALTER Mendersos, M. D., of New York. In one handsome octavo volume of 274 pages, with 117 fine engravings.

SENN (NICHOLAS). SURGICAL BACTERIOLOGY. Second edition. In one octavo rolume of 268 pages, with 13 plates, 10 of which are colored, and 9 engravings. Cloth, $\$ 2$.

SERIES OF CLINTCAY MLNUATS. A Series of Authoritative Monographs on Important Clinical Subjects, in $12 \mathrm{mo}$. volumes of about 550 pages, well illustrated. The following volumes are now ready: BroAdBENT on the Pulse, $\$ 1.75$; YeO on Food in Health and Disease, new (2d) edition, $\$ 2.50$; CARTER and Frost's Ophthalmic Surgery, $\$ 2.25$; Hutchinson on Syphilis, \$2.25; MARsh on Diserses of the Joints, \$2; MORRIS on Surgical Diseases of the Kidney, \$2.25; OWEN on Surgical Diseases of Children, $\$ 2$; PICK on Fractures and Dislocations, $\$ 2$; BUTLIN on the Tongue, $\$ 3.50$; SAYAGE on Insanity and Allied Neuroses, $\$ 2$; and Treves on Intestinal Obstruction, $\$ 2$. The following is in press: LUCAS on Diseases of the Urethra.

For separate notices, see under various authors' names.

SERIES OF STUDENT'S MANUALS. See page 27.

SLMON (CHARLES E.). CLINICAL DIAGNOSIS, BY MICROSCOPICAL AND CHEMICAL METHODS. New (2d) edition. In one very handsome octaro volume of 530 pages, with 135 engravings and 14 full-page colored plates. Cloth, \$3.50. Just ready.

This book thoroughly deserves its In all respects entirely up to date. success. It is a very complete, authentic and nseful manual of the microscopical and chemical methods which are employed in diagnosis. Very excellent colored plates illustrate this work. - Nee York Medical -Wedical Fiecord.

The chapter on examination of the urine is the most complete and advanced that we know of in the English language.-Canadian PracJourial. 
SIMON (W.). MANUAL OF CHEMISTRY. A Guide to Lectures and Laboratory Work for Beginners in Chemistry. A Text-book specially adapted for Students of Pharmacy and Medicine. Fifth edition. In one 8 vo. volume of 501 pages, with 44 engravings and 8 plates showing eolors of 64 tests. Cloth, $\$ 3.25$.

It is diffieult to see how a better the covers of this book.-The Northbook could be constructed. No man western Lancet.

who devotes himself to the practice Its statements are all elear and its of medicine need know more about teachings are practical.-Virginia chemistry than is eontained between Med. Monthly.

SLADE (D. D.). DIPHTHERIA; ITS NATURE AND TREATMENT. Seeond edition. In one royal $12 \mathrm{mo}$. vol., $158 \mathrm{pp}$. Cloth, $\$ 1.25$.

SMITH (EDWARD). CONSUMPTION ; ITS EARLY AND REMEDIABLE STAGES. In one 8vo. volume of $253 \mathrm{pp}$. Cloth, $\$ 2.25$.

SMITH (J. LEWIS). A TREATISE ON THE DISEASES OF INFANCY AND CHILDHOOD. Eighth edition, thoroughly revised and rewritten and mueh enlarged. In one large 8vo. volume of 983 pages, with 273 engravings and 4 full-page plates. Cloth, $\$ 4.50$; leather, $\$ 5.50$.

The most eomplete and satisfactory text-book with which we are acquainted.-American Gynecological and Obstetrical Journal.

It truly is the most evenly balaneed, elear in deseription and thorough in detail of any of the books published in this country on this subjeet.-Medical Fortnightly.

A treatise which in every respeet

ean more than hold its own against any other work treating of the same subjeet.-American Medico-Surgical Bulletin.

A safe guide for students and physicians.-The Am.Jour. of Obstetrics. For years the leading text-book on ehildren's diseases in America.Chicago IIedical Recorder.

SMITH (STEPHEN). OPERATIVE SURGERY. Second and thoroughly revised edition. In one oetavo volume of 892 pages, with 1005 engravings. Cloth, $\$ 4$; leatber, $\$ 5$.

One of the most satisfactory works $\mid$ dium for the modern surgeon.-Boson modern operative surgery yet ton Medical and Surgical Joumal. published. The book is a compen-

SOILY (S. HDWIN). A HANDBOOK OF MEDICAL CLIMATOJOGY. In one handsome oetavo volume of 462 pages, with engravings and 11 full-page plates, 5 of whieh are in colors. Clotl, $\$ 4.00$. Just ready.

A clear and lucid summary of an accurate observer and practical what is known of climate in relation to its influence upon liuman beings. - The Therapeutic Gazette.

The book is admirably planned, elearly written, and the anthor speaks from an experience of thirty years as therapeutist.-Maryland Med.Jour. Every prietitioner of medieine shonld possess limaself of a copy and study it, and we are sure lie will never regret it.-St. Louis Medical and Surgical Journal.

STILLÉ (ALFREI). CHOLERA; ITS ORIGIN, IIISTORY, CAUSATION, SYMPTOMS, LESIONS, PREVEN'ION AND TREATMENT. In one $12 \mathrm{mo}$. volume of 163 pages, with a chart showing routes of previous epidenies. Cloth, $\$ 1.25$.

-THERAPEUTICS AND MATERIA MEDICA. Fourth and revised edition. In two octuvo volumes, eontaining 1936 pages. Cloth, \$10; leather, \$12. 
STULE (ALFRED), MAISCH (JOHN M.) AND CASPARI (CHAS. JR.). THE NATIONAL DISPENSATORY: Containing the Natural History, Chemistry, Pharmacy, Actions and Uses of Medicines, including those recognized in the latest Pharmacopoeias of the United States, Great Britain and Germany, with numerous references to the French Codex. Fifth edition, revised and enlarged, including the new U.S. Pharmacopoia, Seventh Decennial Revision. With Supplement containing the new edition of the National Formulary. In one magnificent imperial octaro volume of about 2025 pages. with 320 engravings. Cloth, \$7.25; leather, $\$ 5$. With ready reference Thumb-letter Index. Cloth, $\$ 7.75$; leather, $\$ 5.50$.

Recommended most highly for the amount of information contained in physician, and invaluable to the this work is made arailable is indidruggist.-Therapeutic Gazetle.

It is the official guide for the Medical and Pharmaceatical professions. -Buffalo Med. and Sur.Jour.

cated by the twenty-five thousand references in the two indexes.-Boston Medical and Surgical Journal. Should be recognized as a national

The readiness with which the vast I standard.-North Am. Practitioner.

STLMSON (LEWTS A.). A MANUAL OF OPERATIVE SURGERY. New (3d) edition. In one royal $12 \mathrm{mo}$. volume of 614 pages, with 306 engravings. Cloth, $\$ 3.75$.

A useful and practical guide for The book is worth the price for the all students and practitioners.-Am. illustrations alone.-Ohio Medical Joumal of the Medical Sciences.

STIMSON (LEWTS A.). A TREATISE ON FRACTURES AND DISLOCATIONS. In two handsome octavo volumes. Vol. I., FracTURES, 582 pages, 360 engrarings. Fol. II., Dislocatioss, 540 pages, 163 engravings. Complete work, cloth, $\$ 5.50$; leather, $\$ 7.50$. Either volume separately, Cloth, $\$ 3$; leather, $\$ 4$.

STUDENT'S QUIZ SERLES. A New Series of Mannals in question and answer for Students and Practitioners, covering the essentials of medical science. Thirteen volumes, pocket size, convenient, authoritative, well illnstrated, handsomely bound in limp cloth, and issued at a low price. 1. Anatomy (double number); 2. Physiologr; 3. Chemistry and Physics; 4. Histology, Pathology and Bacteriology; 5 . Materia Medica and Therapeutics; 6. Practice of Medicine; 7. Surgery (donble number); 8. Genito-Urinary and Venereal Diseases; 9. Diseases of the Skin; 10. Diseases of the Eye, Ear, Throat and Nose; 11. Obstetries; 12. Gynecology; 13. Diseases of Children. Price, \$1 each, except Nos. 1 and 7, Anatomy and Surgery, which being double numbers are priced at $\$ 1.75$ each. Full specimen circular on application to publishers.

STUDENT'S SERIES OF MANUALS. A Series of Fifteen Manuals by Eminent Teachers or Examiners. The volumes are pocket-size 12 mos. of from $300-540$ pages, profusely illustrated, and bound in red limp cloth. The following volnmes may now be annonnced: HERMAX's First Lines in Midwifery, \$1.25; LuFF's Manual of Chemistry, $\$ 2$; BruCE's Materia Medica and Therapeuties (fifth edition), $\$ 1.50$; BELL's Comparative Anatomy and Physiology, \$2; ROBERTsox's Phrsiological Phrsics, 2 ; Gould's Surgical Diagnosis, 2 ; KLEIX's Elements of Histology (4th edition), 1.75 ; PEPPER's Surgical Pathology, \$2; Treves' Surgical Applied Anatomy, $\$ 2$; Power's Human Physiology (2d editiou), \$1.50; RAlfE's Clinical, Chemistry, $\$ 1.50$; and CLARKE and Lock wOOD's Dissector's Manual \$1.50. The following is in press: PEPPER'S Forensic Medicine.

For separate notices, see under various author's names. 
STURGES (OCTAVIUS). AN INTRODUCTION TO THE STUDY OF CLINICAL MEDICINE. In one 12mo. volume. Cloth, $\$ 1.25$.

SUTTON (JOHN BLAND). " SURGICAL DISEASES OF THE OVARIES AND FALLOPIAN TUBES. Including Abdominal Pregnancy. In one $12 \mathrm{mo}$. volume of 513 pages, with 119 engravings and 5 colored plates. Cloth, $\$ 3$.

TUMORS, INNOCENT AND MAIIGNANT. Their Clinical Features and Appropriate Trement. In one 8vo. vol. of $526 \mathrm{pp}$., with 250 engravings and 9 full-page plates. Cloth, $\$ 4.50$. Just reudy.

TAIT (LAWSON). DISEASES OF WOMEN AND ABDOMINAL SURGERY. In two handsome octavo volumes. Vol. I. contain: 546 pages and 3 plates. Cloth, $\$ 3$. Vol. II., preparing.

TANNER (THOMAS HAWKES) ON THE SIGNS AND DISEASES OF PREGNANCY. From the second English edition. In one octavo volume of 490 pages, with 4 colored plates and 16 engravings. Cloth, $\$ 4.25$.

TAYLOR (ALFRED S.). MEDICAL JURISPRUDENCE. NeW American from the twelfth English edition, specially revised by CLARK BELL, Esq., of the N. Y. Bar. In one 8vo. vol. of 831 pages, with 54 engrs. and 8 full-page plates. Cloth, $\$ 4.50$; leather, $\$ 5.50$ Jusi ready.

To the student, as to the physician, nesses, it strongly behooves them to we would say, get Taylor first, and be prepared according to the princithen add as means and inclination ples and practice everywhere acenable you.-American Practitioner cepted. The work will be found to and Neus.

It is the authority sccepted as final by the courts of all Englishspeaking countries. This is the important consideration for medical men, sinee in the event of their being summoned as experts or wit- Pacific Medical Journal.

be thorough, authoritative and modern.-Albany Law Journal.

Probably the best work on the subject written in the English language. The work has been thoroughly revised and is up to date.ON POISONS IN RELATION TO MEDICINE AND MEDICAL JURISPRUDENCE. Third American from the third London edition. In one octavo volume of 788 pages, with 104 illustrations. Cloth, $\$ 5.50$; leather, $\$ 6.50$.

TAYLOR (ROBERT W.). THE PATHOLOGY AND TREATMENT OF VENEREAL DISEASES. In one very liandsome ootavo volume of 1002 pages, with 230 engravings and 7 colored plates. Cloth, $\$ 5.00$; leather, $\$ 6.00$. ( $\mathrm{Net}$.)

By loug odds the best work on venereul diseases. - Louisville Medical Monthly.

In the observation and treatment of venereal diseases his experience has been greater probably thau that of any other practitioner of this contiuent.-Nero York Medical Joumul.

The clearest, most unbiased and ably presented treatise as yet published on this vast subject.-The Medical News.

Decidedly the most important and authoritative treatise on venereal

diseases that has in reeent years appeared in English.-American Journal of the Medical Sciences.

It is a veritable storchouse of our knowledge of the venereal diseases. It is commended as a conservative, practical, full exposition of the greatest value.-Chicago Clinical Review.

The best work on venereal diseases in the English language. It is certainly above everything of the kind.-The St. Lowis Medical and Surgical Journal. 
TAYLOR (ROBERT W.). A PRACTICAL TREATISE ON SEXUAL DISORDERS IN THE MALE AND FEMALE. In one Svo. vol. of 448 pp., with 73 engravings and 8 colored plates. Cloth, \$3. Net. Just ready.

It is a timely boon to the medical the female is presented in an exhausprofession that an observer of Dr. tive manner, all of the causes proTaylor's skill and experience has ducing it being described. The written a work on this hitherto author bas presented to the profesneglected and little understood class sion the ablest and most scientific of diseases which places them on a work as ret published on sexual scientific basis and renders them so disorders, and one which, if carefully clear that the physician who reads followed, will be of unlimited ralue its pages can treat this class of to both physician and patient.patients intelligently. Sterility in Medical Neics.

A CLINICAL ATLAS OF VENEREAL AND SKIN DISEASES. Including Diagnosis, Prognosis and Treatment. In eight large folio parts, measuring $14 \times 18$ inches, and comprising 213 besutiful figures on 58 full-page chromo-lithographic plates, 85 fine engravings and 425 pages of text. Complete work now ready. Price per part, sewed in heary embossed paper, $\$ 2.50$. Bound in one rolume, half Russia, $\$ 27$; half Turkey Morocco, $\$ 28$. For sale by subscription only. Address the publishers. Specimen plates by mail on receipt of ten cents.

TAYLOR (SEYMOUR). INDEX OF MEDICINE. A Manual for the use of Senior Students and others. In one large $12 \mathrm{mo}$. volume of 802 pages. Cloth, $\$ 3.75$.

THOMAS (T. GAILLARD) AND MUNDE (PAUL F.). A PRACTICAL TREATISE ON THE DISEASES OF WOIEN. Sixth edition, thoroughly revised by PAUL F. MUNDE, M. D. In one large and handsome octavo rolume of 824 pages, with 347 engravings. Cloth, $\$ 5$; leather, $\$ 6$.

The best practical treatise on the This work, which has already gone subject in the English language. through five large editions, and has It will be of especial value to the been translated into French, Gergeneral practitioner as well as to the man, Spanish and Italian, is the specialist. The illustrations are very most practical and at the same time satisfactory. Many of them are new the most complete treatise upon the and are particularly clear and attrac-. subject.-The Archives of Gynecoltive.-Boston Ifed. and Sur. Jour.

ogy, Obstetrics and Pediatrics.

THOMPSON (SIR HENRY). CLINICAL LECTURES ON DIS. EASES OF THE URINARY ORGANS. Second and revised edition. In one octavo vol. of 203 pp., with 25 engravings. Cloth, $\$ 2.25$. THE PATHOLOGY AND TREATMENT OF STRICTURE OF THE URETHRA AND URINARY FISTULE. From the third English edition. In one octaro rolume of 359 pages, with 47 engravings and 3 lithographic plates. Cloth, $\$ 3.50$.

THOMSON (JOHN). DISEASES OF CHILDREN. In one crown octavo volume of about 350 pages, with 52 illustrations. In press.

TODD (ROBERT BENTIEY). CLINICAL LECTURES ON CER. TAIN ACUTE DISEASES. In one 8vo. vol. of $320 \mathrm{pp}$., cloth, $\$ 2.50$.

TREVES (FREDERICK). OPERATIVE SURGERY. In two 8vo. vols. containing 1550 pp., with 422 illus. Cloth, $\$ 9$; leath., $\$ 11$.

$\triangle$ SYSTEM OF SURGERY. In Contributions by Twenty-five English Surgeons. In two large octavo volumes. Yol. I., 1178 pages, with 463 engravings and 2 colored plates. Vol. II., 1120 pages, with 487 engravings and 2 colored plates. Price per volume, cloth, $\$ 8$. Complete Work now ready. 
TREVES (FREDERICK). THE STUDENTS' HANDBOOK OF SURGICAL OPERATIONS. In one $12 \mathrm{mo}$. volume of $508 \mathrm{pp}$., with 94 illustrations. Cloth, $\$ 2.50$.

SURGICAL APPLIED ANATOMY. In one 12mo. vol. of $540 \mathrm{pp}$., with 61 engravings. Cloth, $\$ 2$. See Student's Series of Ifanuals, p. 27 .'

INTESTINAL OBSTRUCTION. In one 12mo. volume of 522 pages, with 60 illus. Cloth, $\$ 2$. See Series of Clinical Manuals, p. 25.

TUKE (DANIEL HACK). THE INFLUENCE OF THE MIND UPON THE BODY IN HEALTH AND DISEASE. Second edition. In one 8 vo. volume of 467 pages, with 2 colored plates. Cloth, $\$ 3$.

VAUGHAN (VICTOR C.) AND NOVY (FREDERICK G.). PTOMAINS, LEUCOMAINS, TOXINS AND ANTITOXINS, or the Chemical Factors in the Causation of Disease. New (3d) edition. In one $12 \mathrm{mo}$. volume of 603 pages. Cloth, $\$ 3$.

The work has been brought down | The present edition has been not to date, and will be found entirely only thoroughly revised throughout satisfactory.-Journal of the Ameri- but also greatly enlarged, ample can Medical Association.

The most exhaustive and most recent presentation of the subject.American Jour. of the Med. Sciences. consideration being given to the new subjects of toxins and antitoxins.Tri-Strute Medical Journal.

VISITING LIST. THE MEDICAL NEWS VISITING LIST for 1898 . Four styles: Weekly (dated for 30 patients); Monthly (undated for 120 patients per month); Perpetual (undated for 30 patients each week); and Perpetual (undated for 60 patients each week). The 60patient book consists of 256 pages of assorted blanks. The first three styles contain 32 pages of important data, thoroughly revised, and 160 pages of assorted blanks. Each in one volume, price, \$1.25. With thumb-letter index for quick use, 25 cents extra. Special rates to advance-paying subscribers to THE MEDICAL NEws or THE american Journal of the Medical Sciences, or both. See p. 32.

WATSON (THOMAS). LECTURES ON THE PRINCIPLES AND PRACTICE OF PHYSIC. A new American from the fifth and enlarged English edition, with additions by H. HARTSHORNE, M. D. In two large 8vo. vols. of 1840 pp., with 190 cuts. Cloth, $\$ 9$; leather, $\$ 11$.

WEST (CHARLES). LECTURES ON THE DISEASES PECULIAR TO WOMEN. Third American from the third English edition. In one octavo volume of 543 pages. Cloth, $\$ 3.75$; leather, $\$ 4.75$.

- ON SOME DISORDERS OF TIIE NERVOUS SYSTEM IN CHILDHOOD. In one small $12 \mathrm{mo}$. volume of 127 pages. Cloth, $\$ 1$.

WHARTON (HENRY R.). MINOR SURGERY AND BANDAGING. New (3d) edition. In one $12 \mathrm{mo}$. vol. of 594 pages, with 475 engravings, many of which are photographic. Cloth, \$3.

We know of no book which more/work of ready reference for surthorouglily, or more satisfactorily geons.-North Amer. Practitioner. covers the ground of Minor Surgery The part devoted to bandaging is and Bandaging.-Brooklyn Medical perhaps the best exposition of the Journal.

Well written, conveniently arranged and amply illustrated. It covers the field so fully as to render 1t a valuable text-book, as well as a subject in the English language. It can be lighly commended to the student, the practitioner and the specialist.-The Chicago Medical Recorder. 
WHTTLA (WTLLAM). DICTIONARY OF TREATMENT, OR THERAPEUTIC INDEX. Including Medical and Surgical Therapeutics. In one square nctaro volume of 917 pages. Cloth, $\$ 4$.

WHSON (ERASMUS). A SYSTEM OF HUMAY AYATOMY. A new and revised American from the last English edition. Illustrated with 397 engravings. In one octaro rolume of 616 pages. Cloth, \$4; leather, $\$ 5$.

THE STUDENT'S BOOK OF CUTANEOUS MEDICINE. In one $12 \mathrm{mo}$. volume. Cloth, $\$ 3.50$.

WINCKEL ON PATHOLOGY AND TREATMENT OF CHILDBED. Translated by JAMES R. CHADWICK, A. M., M. D. With additions by the Anthor. In one octavo volume of $4 \$ 1$ pages. Cloth, $\$ 4$.

WÖHLER'S OUTLINES OF ORGANIC CHEMISTRY. Translated from the eighth German edition, by IRA REsisex, M.D. In one 12mo. rolume of 550 pages. Cloth, \$3.

YEAR-BOOK OF TPEATMENT FOR 1895. A Critical Review for Practitioners of Medicine and Surgery. In contributions by 2.5 wellknown medical writers. 12 mo., $455^{\circ}$ pages. Cloth, $\$ 1.50$. In combination with THE MEDICAL NEWs and THE AMERICAN Jodrxal OF THE MEDICAL SCIRYCES, 75 cents. See page 32.

To repeat the praises bestowed on the hands of a practical and recogprevious issues is not to do full jus- nized authority. The whole field of tice, as every rear shows improve- medicine is in this way trarersed, ment and adrances which make the and a critical estimate formed of all work one of increasing ntility to the that is substantial and meritorious physician. The work this year con- in recent progress,-The Physician sists of twenty-five chapters, each in and Surgeon.

YEAR-BOOKS OF TREATMENT FOR 1892, 1893, 1896 and 1897, similar to above. Each, cloth, \$1.50.

YEO (I. BURNEY). FOOD IN HEALTH AND DISEASE. New (2d) edition. In one 12 mo. volume of 592 pages, with 4 engravings. Cloth, \$2.50. See Series of Clinical Manuals, page 26.

We doubt whether any book on / work of Dr. Yeo's. The value of dietetics has been of greater or more the work is not to be orerestimated. widespread usefuluess than has this -New York Medicul Journal. much-quoted and much-consulted

A MAYUAL OF MEDICAL TREATMENT, OR CLINICAL. THERAPEUTICS. Two rolumes containing 1275 pages. Cloth, $\$ 5.50$.

YOUNG (JAMES K.). ORTHOPEDIC SUPGERY. In one Sro. volume of 475 pages, with 286 illustrations. Cloth, $\$$; leather, $\$ 5$.

In studying the different chapters, surgical specialty and every page one is impressed with the thorough- abounds with evidences of pracness of the work. The illustrations ticality. It is the clearest and most are numerons - the book thoroughly moderin work upon this growing depractical-Medical Neacs.

It is a thorongh, a very comprehensive work upon this legitimate partment of surgery, - The Chicago Clinical Revievo. 


\section{Mpdical Reriodicals and Combinations.}

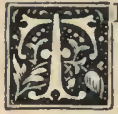

HE student cannot begin too early in his conrse a habit of rearling current medical literature. In this way he will best acquire ar intelligent interest in the vital questions of his profession, secure a vast fund of information which will constantly supplement the knowledge gained from text-books, and become finiliar with the approved methods of calling public attention to such additions as he may make to medleal science during lis professional life. For these purposes the following periodicals are most admirably adapted:

\section{THE MEDICAL NEWS (Weekly, $\$ 4.00$ per Annum).}

THE NEw's contains each weck thirty-two quarto pages, comprising original articles, clivical lectures and notes on practicil aulvances, latest hospital methods, summaries of progress condensed from the best medical journals of the world, full abstracts of important articles, able enlitorials on eurrent topics, book reviews, medical correspondence from important cen. tres, and news items of interest. Published for fifty years, Trie NEws is familiar with the necds of medical men and the best methods of meeting them.

\section{THE AMERICAN JOURNAL OF THE MEDICAL SCIENCES}

\section{(Monthly, $\$ 4.00$ per Annum).}

'THE AMERICAN Journal is a medical magazine affording, in the $12 \&$ pages of each issue, ample space for elaborate original articles on important medical discoveries, diseriminating reviews on valuable medical literature, and classified summaries of progress. According to the highest literary authority of the profession, "from this file alone, were all otlier" publica tions of the press for the last fifty years destroyed, it would be possible to reproduce the great majority of the real contributions of the worli to medical science during that period."

\section{COMMUTATION RATE.}

Taken together, TIE JourxaI, and NeW' form a peculiarly usefin combination, and afford their readers the assurance that nothing of value in the progress of medical matters shall escape attention. To lead every reader to prove this personally the commutation rate has been placed at the exceedingly low figure of $\$ 7.50$.

\section{SPECIAL COMBINATION OFFERS.}

The Menicat, News Visitixg List (regular price, \$1.25), or The Year-Book of Treatment (regnlar price, $\$ 1.50$, will be furnished to advance-paying subseribers to either or both of these periodicals for 75 cents apiece; or.Journal, News, Visiting List and Year-Book, \$8.50. Circulars free on application.

IEA BROTHERS \& CO $\overline{\text { Publishers }}$ 706, 708 \& 710 Sansom St., Phila.

LEA BROTHERS \& CO,, Publishers, ill Fifth Ave.(Cor.18th S1.), New York. H 


$$
\text { - }
$$


973 





\section{DEC 1219831}

\section{PLEASE DO NOT REMOVE CARDS OR SLIPS FROM THIS POCKET}

\section{UNIVERSITY OF TORONTO LIBRARY}

Biological

\& Medical 
